\title{
I(III)-Catalyzed Oxidative Cyclization-Migration Tandem Reactions of Unactivated Anilines
}

\author{
Tianning Deng, Emily Shi, Elana Thomas and Tom G. Driver* \\ Department of Chemistry, University of Illinois at Chicago 845 West Taylor Street, Chicago, Illinois, 60607-7061
}

\section{Supporting Information}

\section{Contents:}

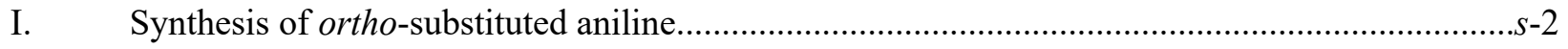

II. Formation of $3 H$-indoles from ortho-substituted anilines.......................................................29

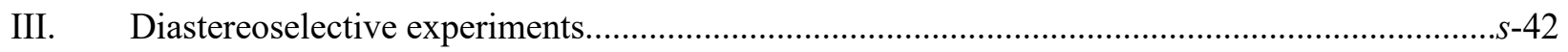

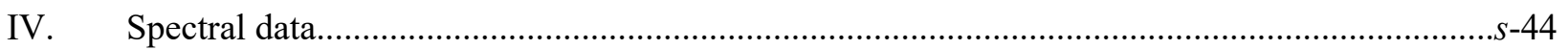

General. ${ }^{1} \mathrm{H}$ NMR and ${ }^{13} \mathrm{C}$ NMR spectra were recorded at ambient temperature using $500 \mathrm{MHz}$ or $300 \mathrm{MHz}$ spectrometers. The data are reported as follows: chemical shift in ppm from internal tetramethylsilane on the $\delta$ scale, multiplicity (br $=$ broad, $\mathrm{s}=$ singlet, $\mathrm{d}=$ doublet, $\mathrm{t}=$ triplet, $\mathrm{q}=$ quartet, $\mathrm{m}=$ multiplet $)$, coupling constants $(\mathrm{Hz})$ and integration. Highresolution mass spectra were obtained by peak matching. Melting points are reported uncorrected. Infrared spectroscopy was obtained using a diamond attenuated total reflectance (ATR) accessory. Analytical thin layer chromatography was performed on $0.25 \mathrm{~mm}$ extra hard silica gel plates with UV254 fluorescent indicator. Liquid chromatography was performed using forced flow (flash chromatography) of the indicated solvent system on $60 \AA(40-60 \mu \mathrm{m})$ mesh silica gel $\left(\mathrm{SiO}_{2}\right)$. Medium pressure liquid chromatography (MPLC) was performed to force flow the indicated solvent system down columns that had been packed with $60 \AA(40-60 \mu \mathrm{m})$ mesh silica gel $\left(\mathrm{SiO}_{2}\right)$. All reactions were carried out under an atmosphere of nitrogen in glassware, which had been ovendried. Unless otherwise noted, all reagents were commercially obtained and, where appropriate, purified prior to use. Acetonitrile, Methanol, Toluene, THF, $\mathrm{Et}_{2} \mathrm{O}$, and $\mathrm{CH}_{2} \mathrm{Cl}_{2} \mathrm{were}$ dried by filtration through alumina according to the procedure of Grubbs. ${ }^{1}$ Metal salts were stored in a nitrogen atmosphere dry box.

1. Pangborn, A. B.; Giardello, M. A.; Grubbs, R. H.; Rosen, R. K.; Timmers, F. J. Organometallics 1996, 15, 1518. 


\section{Synthesis of ortho-substituted aniline.}

\section{A. Substrate synthesis overview.}

The 2-substituted aniline substrates were constructed from ortho-substituted anilines following the process outlined in Scheme s1 and s2.

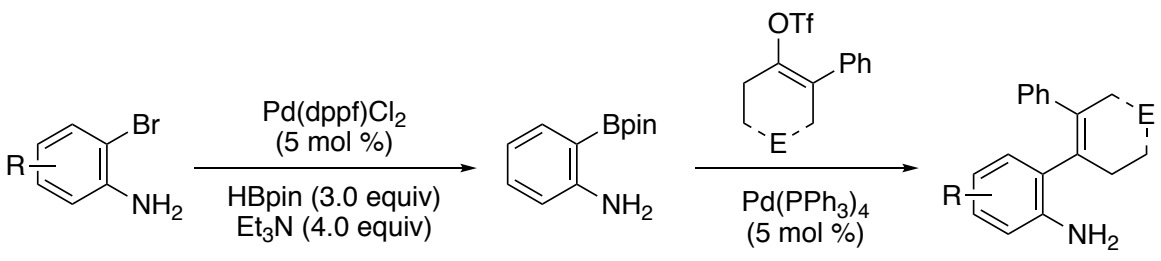

Scheme s1. Synthesis of 2-Substituted aniline substrates.

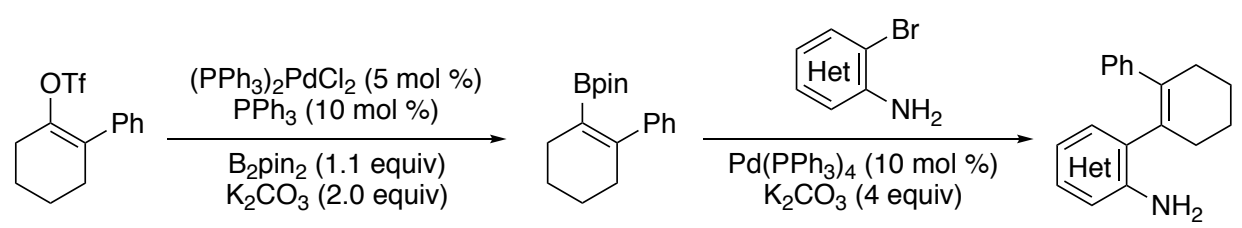

Scheme s2. Synthesis of 2-substituted heterocyclic aniline substrates from boronic ester and aryl halide partners

\section{B. Synthesis of 2-aminoarylboronic acid pinacol esters.}

1. General Procedure.

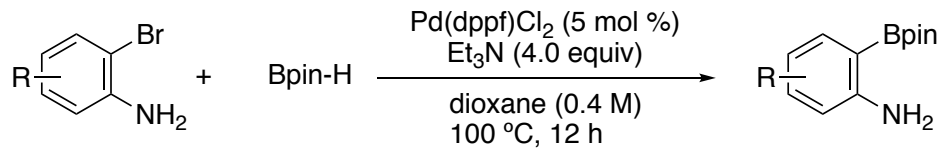

To a mixture of 2-bromoaniline (4.0 mmol, 1.0 equiv), $\mathrm{Et}_{3} \mathrm{~N}$ (16.0 mmol, 4.0 equiv), (dppf) $\mathrm{PdCl}_{2}(0.20 \mathrm{mmol}, 5 \mathrm{~mol} \%)$ in 1,4-dioxane $(0.4 \mathrm{M})$, was added 4,4,5,5-tetramethyl-1,3,2-dioxaborolane (12.0 mmol, 30 equiv) dropwise. The resultant mixture was heated to $100{ }^{\circ} \mathrm{C}$ using an oil bath. After $12 \mathrm{~h}$, the mixture was cooled to room temperature and diluted with $20 \mathrm{~mL}$ of a saturated aq soln of $\mathrm{NH}_{4} \mathrm{Cl}$. The resulting mixture was extracted with $3 \times 10 \mathrm{~mL}$ of $\mathrm{CH}_{2} \mathrm{Cl}_{2}$. The combined organic phases were washed with brine. The resulting organic phase was dried over $\mathrm{Na}_{2} \mathrm{SO}_{4}$, filtered, and was concentrated in vacuo. Purification using MPLC afforded the product.

2. Characterization data for 2-aminoarylboronic acid pinacol esters.<smiles>Nc1ccccc1Br</smiles>

2-(3,3,4,4-Tetramethylborolan-1-yl)aniline s1a. ${ }^{2}$ The general procedure was followed using $1.38 \mathrm{~g}$ of 2-bromoaniline (8.0 $\mathrm{mmol}), 3.50 \mathrm{~mL}$ of HBpin (24.0 mmol), $4.48 \mathrm{~mL}$ of Et $3 \mathrm{~N}(32.0 \mathrm{mmol}), 0.320 \mathrm{~g}$ of (dppf)PdCl $2(0.40 \mathrm{mmol})$ in $20 \mathrm{~mL}$ of 1,4-dioxane (0.4 M). Purification by MPLC (2:98 to 15:85 EtOAc:hexanes) afforded the product as a yellow solid (1.23 $\mathrm{g}$, $70 \%)$. The spectral data matched that reported by Driver and co-workers: ${ }^{2}{ }^{1} \mathrm{H} \mathrm{NMR}\left(500 \mathrm{MHz}, \mathrm{CDCl}_{3}\right) \delta 7.63(\mathrm{dd}, J=7.4$ $\mathrm{Hz}, 1.3 \mathrm{~Hz}, 1 \mathrm{H}), 7.23(\mathrm{td}, J=7.7 \mathrm{~Hz}, 1.5 \mathrm{~Hz}, 1 \mathrm{H}), 6.69(\mathrm{t}, J=7.3 \mathrm{~Hz}, 1 \mathrm{H}), 6.61(\mathrm{~d}, J=8.1 \mathrm{~Hz}, 1 \mathrm{H}), 4.75(\mathrm{br} \mathrm{s}, 2 \mathrm{H}), 1.36$ $(\mathrm{s}, 12 \mathrm{H}) ;{ }^{13} \mathrm{C}$ NMR $\left(125 \mathrm{MHz}, \mathrm{CDCl}_{3}\right) \delta 163.9(\mathrm{C}), 153.7(\mathrm{C}), 136.8(\mathrm{CH}), 132.8(\mathrm{CH}), 116.9(\mathrm{CH}), 114.8(\mathrm{CH}), 83.5(\mathrm{C})$, $25.0\left(\mathrm{CH}_{3}\right)$ only visible peaks.

2. Sun, K.; Liu, S.; Bec, P. M.; Driver, T. G. Angew. Chem. Int. Ed. 2011, 50, 1702. 


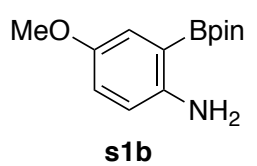

4-Methoxy-2-(3,3,4,4-tetramethylborolan-1-yl)aniline $\mathbf{s} 1 \mathbf{b} .^{2}$ The general procedure was followed using $0.812 \mathrm{~g}$ of 2 bromo-4-methoxyaniline (4.0 mmol), $1.75 \mathrm{~mL}$ of HBpin (12.0 mmol), $2.24 \mathrm{~mL}$ of Et ${ }_{3} \mathrm{~N}(16.0 \mathrm{mmol}), 0.140 \mathrm{~g}(\mathrm{dppf}) \mathrm{PdCl} \mathrm{d}_{2}$ $(0.20 \mathrm{mmol})$, in $10 \mathrm{~mL}$ of 1,4-dioxane $(0.4 \mathrm{M})$. Purification by MPLC (2:98 to 20:80 EtOAc:hexanes) afforded product as a dark red solid $(0.837 \mathrm{~g}, 84 \%)$. The spectral data matched that reported by Driver and co-workers: ${ }^{2}{ }^{1} \mathrm{H} \mathrm{NMR}(500 \mathrm{MHz}$, $\left.\mathrm{CDCl}_{3}\right) \delta 7.15(\mathrm{~d}, J=3.1 \mathrm{~Hz}, 1 \mathrm{H}), 6.85(\mathrm{dd}, J=8.7,3.1 \mathrm{~Hz}, 1 \mathrm{H}), 6.58(\mathrm{~d}, J=8.8 \mathrm{~Hz}, 1 \mathrm{H}), 3.90(\mathrm{br} \mathrm{s}, 2 \mathrm{H}), 3.76(\mathrm{~s}, 3 \mathrm{H})$, $1.34(\mathrm{~s}, 12 \mathrm{H}) ;{ }^{13} \mathrm{C}$ NMR $\left(125 \mathrm{MHz}, \mathrm{CDCl}_{3}\right) \delta 151.4(\mathrm{C}), 147.9(\mathrm{C}), 120.6(\mathrm{CH}), 119.6(\mathrm{CH}), 116.5(\mathrm{CH}), 83.6(\mathrm{C}), 56.0$ $\left(\mathrm{CH}_{3}\right), 24.9\left(\mathrm{CH}_{3}\right)$ only visible peaks.

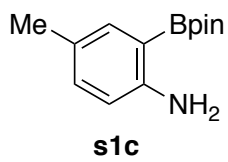

4-Methyl-2-(3,3,4,4-tetramethylborolan-1-yl)aniline s1c. ${ }^{3}$ The general procedure was followed using $0.372 \mathrm{~g}$ of 2 bromo-4-methylaniline (2.0 mmol), $0.88 \mathrm{~mL}$ of HBpin $(6.0 \mathrm{mmol}), 1.14 \mathrm{~mL}$ of Et $3 \mathrm{~N}(8.0 \mathrm{mmol}), 0.082 \mathrm{~g}$ of $(\mathrm{dppf}) \mathrm{PdCl}_{2}$ $(0.10 \mathrm{mmol})$, in $5 \mathrm{~mL}$ of 1,4-dioxane $(0.4 \mathrm{M})$. Purification by MPLC (2:98 to 15:85 EtOAc:hexanes) afforded the product as a yellow solid $(0.324 \mathrm{~g}, 71 \%)$. The spectral data matched that reported by Driver and co-workers: ${ }^{3}{ }^{1} \mathrm{H} \mathrm{NMR}(500 \mathrm{MHz}$, $\left.\mathrm{CDCl}_{3}\right) \delta 7.51(\mathrm{~s}, 1 \mathrm{H}), 7.09(\mathrm{~d}, J=7.9 \mathrm{~Hz}, 1 \mathrm{H}), 6.58(\mathrm{~d}, J=8.0 \mathrm{~Hz}, 1 \mathrm{H}), 4.68(\mathrm{br} \mathrm{s}, 2 \mathrm{H}), 2.28(\mathrm{~s}, 3 \mathrm{H}), 1.40(\mathrm{~s}, 12 \mathrm{H}) ;{ }^{13} \mathrm{C}$ NMR (125 MHz, $\left.\mathrm{CDCl}_{3}\right) \delta 151.6(\mathrm{C}), 136.8(\mathrm{CH}), 133.7(\mathrm{CH}), 125.7(\mathrm{C}), 115.1(\mathrm{CH}), 83.5(\mathrm{C}), 25.0\left(\mathrm{CH}_{3}\right), 20.3\left(\mathrm{CH}_{3}\right)$ only visible peaks.

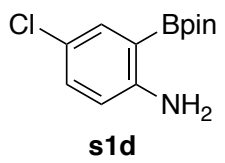

4-Chloro-2-(3,3,4,4-tetramethylborolan-1-yl)aniline s1 d. ${ }^{4}$ The general procedure was followed using $1.04 \mathrm{~g}$ of 2-bromo4-chloroaniline (5.0 mmol), $2.20 \mathrm{~mL}$ of HBpin (15.0 mmol), $2.80 \mathrm{~mL}$ of Et $3 \mathrm{~N}(20.0 \mathrm{mmol}), 0.204 \mathrm{~g}$ of (dppf)PdCl $2(0.25$ $\mathrm{mmol})$, in $12 \mathrm{~mL}$ of 1,4-dioxane $(0.4 \mathrm{M})$. Purification by MPLC (2:98 to 15:85 EtOAc:hexanes) afforded the product as a yellow solid $(0.837 \mathrm{~g}, 66 \%)$. The spectral data matched that reported by Maleczka, Smith and co-workers: ${ }^{4} \mathrm{H}$ NMR (500 $\left.\mathrm{MHz}, \mathrm{CDCl}_{3}\right) \delta 7.55(\mathrm{~d}, J=2.5 \mathrm{~Hz}, 1 \mathrm{H}), 7.13(\mathrm{dd}, J=8.6,2.6 \mathrm{~Hz}, 1 \mathrm{H}), 6.52(\mathrm{~d}, J=8.6 \mathrm{~Hz}, 1 \mathrm{H}), 4.68(\mathrm{br}$ s, $2 \mathrm{H}), 1.34(\mathrm{~s}$, $12 \mathrm{H}) ;{ }^{13} \mathrm{C}$ NMR $\left(125 \mathrm{MHz}, \mathrm{CDCl}_{3}\right) \delta 152.1(\mathrm{C}), 135.8(\mathrm{CH}), 132.5(\mathrm{CH}), 121.6(\mathrm{C}), 116.2(\mathrm{CH}), 83.9(\mathrm{C}), 24.9\left(\mathrm{CH}_{3}\right)$ only visible peaks.

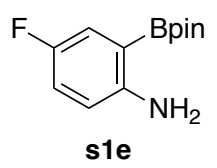

4-Fluoro-2-(3,3,4,4-tetramethylborolan-1-yl)aniline s1e. ${ }^{5}$ The general procedure was followed using $1.14 \mathrm{~g}$ of 2-bromo4-fluoroaniline (6.0 mmol), $2.60 \mathrm{~mL}$ of HBpin (18.0 mmol), $3.40 \mathrm{~mL}$ of Et $3 \mathrm{~N}(24.0 \mathrm{mmol}), 0.245 \mathrm{~g}$ of $\left(\mathrm{dppf}^{\mathrm{P}} \mathrm{PdCl}_{2}(0.30\right.$ $\mathrm{mmol})$, in $15 \mathrm{~mL}$ of 1,4-dioxane $(0.4 \mathrm{M})$. Purification by MPLC (2:98 to 15:85 EtOAc:hexanes) afforded the product as a brown solid (1.02 g, 71\%). The spectral data matched that reported by Driver and co-workers: ${ }^{1} \mathrm{H} \mathrm{NMR}\left(500 \mathrm{MHz}, \mathrm{CDCl}_{3}\right)$ $\delta 7.30(\mathrm{dd}, J=9.1,3.2 \mathrm{~Hz}, 1 \mathrm{H}), 6.92(\mathrm{td}, J=8.5,3.1 \mathrm{~Hz}, 1 \mathrm{H}), 6.52(\mathrm{dd}, J=8.8,4.2 \mathrm{~Hz}, 1 \mathrm{H}), 4.62(\mathrm{br} \mathrm{s}, 2 \mathrm{H}), 1.34(\mathrm{~s}, 12 \mathrm{H})$;

3. Nguyen, Q.; Sun, K.; Driver, T. G. J. Am. Chem. Soc. 2012, 134, 7262.

4. Preshlock, S. M.; Plattner, D. L.; Maligres, P. E.; Krska, S. W.; Maleczka, R. E.; Smith, M. R. Angew. Chem., Int. Ed. 2013, 52, 12915.

5. Kong, C.; Jana, N.; Driver, T. G. Org. Lett. 2013, 15, 824. 
${ }^{13} \mathrm{C} \mathrm{NMR}\left(125 \mathrm{MHz}, \mathrm{CDCl}_{3}\right) \delta 155.2\left(\mathrm{~d}, J_{C F}=234.6 \mathrm{~Hz}\right), 150.0(\mathrm{C}), 121.5\left(\mathrm{~d}, J_{C F}=19.9 \mathrm{~Hz}, \mathrm{CH}\right), 119.7\left(\mathrm{~d}, J_{C F}=22.8 \mathrm{~Hz}\right.$, $\mathrm{CH}), 116.0\left(\mathrm{~d}, J_{C F}=6.6 \mathrm{~Hz}, \mathrm{CH}\right), 83.9(\mathrm{C}), 24.9\left(\mathrm{CH}_{3}\right)$ only visible peaks; ${ }^{19} \mathrm{~F}$ NMR $\left(282 \mathrm{MHz}, \mathrm{CDCl}_{3}\right) \delta-129.6$.

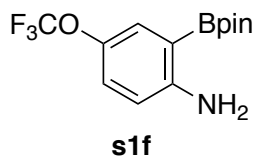

2-(3,3,4,4-Tetramethylborolan-1-yl)-4-(trifluoromethoxy)aniline s1. ${ }^{6}$ The general procedure was followed using $1.28 \mathrm{~g}$ of 2-bromo-4-(trifluoromethoxy)aniline (5.0 mmol), $2.30 \mathrm{~mL}$ of HBpin $(15.0 \mathrm{mmol}), 2.80 \mathrm{~mL}$ of Et $3 \mathrm{~N}(20.0 \mathrm{mmol}), 0.204$ $\mathrm{g}$ of (dppf)PdCl $\mathrm{Pd}_{2}(0.25 \mathrm{mmol})$, in $12 \mathrm{~mL}$ of 1,4-dioxane $(0.4 \mathrm{M})$. Purification by MPLC (2:98 to 15:85 EtOAc:hexanes) afforded the product as a yellow solid $(1.05 \mathrm{~g}, 70 \%)$. The spectral data matched that reported by Driver and co-workers: ${ }^{6}$ ${ }^{1} \mathrm{H}$ NMR $\left(500 \mathrm{MHz}, \mathrm{CDCl}_{3}\right) \delta 7.44(\mathrm{~d}, J=3.3 \mathrm{~Hz}, 1 \mathrm{H}), 7.05(\mathrm{ddd}, J=8.9,3.0,1.1 \mathrm{~Hz}, 1 \mathrm{H}), 6.55(\mathrm{~d}, J=8.8 \mathrm{~Hz}, 1 \mathrm{H}), 4.78$ (br s, 2H), $1.34(\mathrm{~s}, 12 \mathrm{H}) ;{ }^{13} \mathrm{C}$ NMR $\left(125 \mathrm{MHz}, \mathrm{CDCl}_{3}\right) \delta 152.4(\mathrm{C}), 140.0(\mathrm{C}), 129.0(\mathrm{CH}), 126.0(\mathrm{CH}), 120.7\left(\mathrm{q}, J_{C F}=\right.$ $254.8 \mathrm{~Hz}, \mathrm{C}), 115.5(\mathrm{CH}), 84.0(\mathrm{C}), 24.9\left(\mathrm{CH}_{3}\right)$ only visible peaks; ${ }^{19} \mathrm{~F}$ NMR $\left(282 \mathrm{MHz}, \mathrm{CDCl}_{3}\right) \delta-58.8$.

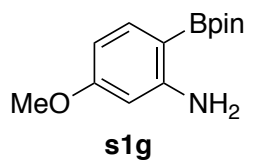

5-Methyoxy-2-(3,3,4,4-tetramethylborolan-1-yl)aniline s1g. ${ }^{6}$ The general procedure was followed using $2.02 \mathrm{~g}$ of 2 bromo-5-methoxyaniline $(10.0 \mathrm{mmol}), 4.40 \mathrm{~mL}$ of HBpin $(30.0 \mathrm{mmol}), 5.80 \mathrm{~mL}$ of $\mathrm{Et}_{3} \mathrm{~N}(40.0 \mathrm{mmol}), 0.351 \mathrm{~g} \mathrm{of}$ $\left(\mathrm{PPh}_{3}\right)_{2} \mathrm{PdCl}_{2}(0.50 \mathrm{mmol})$, in $25 \mathrm{~mL}$ of 1,4-dioxane $(0.4 \mathrm{M})$. Purification by MPLC (2:98 to 20:80 EtOAc:hexanes) afforded the product as a black oil $(1.57 \mathrm{~g}, 63 \%)$. The spectral data matched that reported by Driver and co-workers: ${ }^{6} \mathrm{H}$ NMR (500 $\left.\mathrm{MHz}, \mathrm{CDCl}_{3}\right) \delta 7.56(\mathrm{~d}, J=8.3 \mathrm{~Hz}, 1 \mathrm{H}), 6.28(\mathrm{dd}, J=8.4,2.3 \mathrm{~Hz}, 1 \mathrm{H}), 6.12(\mathrm{~d}, J=2.3 \mathrm{~Hz}, 1 \mathrm{H}), 4.74(\mathrm{br} \mathrm{s}, 2 \mathrm{H}), 3.76(\mathrm{~s}$, $3 \mathrm{H}), 1.34(\mathrm{~s}, 12 \mathrm{H}) ;{ }^{13} \mathrm{C}$ NMR $\left(125 \mathrm{MHz}, \mathrm{CDCl}_{3}\right) \delta 163.7(\mathrm{C}), 155.6(\mathrm{C}), 138.4(\mathrm{CH}), 103.8(\mathrm{CH}), 99.4(\mathrm{CH}), 83.3(\mathrm{C}), 54.9$ $\left(\mathrm{CH}_{3}\right), 24.9\left(\mathrm{CH}_{3}\right)$ only visible peaks.

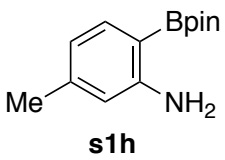

5-Methyl-2-(3,3,4,4-tetramethylborolan-1-yl)aniline $\mathbf{s} 1 \mathbf{h}^{6}{ }^{6}$ The general procedure was followed using $1.86 \mathrm{~g}$ of 2-bromo5 -methylaniline (10.0 mmol), $4.40 \mathrm{~mL}$ of HBpin $(30.0 \mathrm{mmol}), 5.80 \mathrm{~mL}$ of Et $3 \mathrm{~N}$ (40.0 mmol), $0.408 \mathrm{~g}$ of (dppf)PdCl $2(0.50$ $\mathrm{mmol})$, in $25 \mathrm{~mL}$ of 1,4-dioxane $(0.4 \mathrm{M})$. Purification by MPLC (2:98 to 15:85 EtOAc:hexanes) afforded the product as a orange solid $(1.53 \mathrm{~g}, 66 \%)$. The spectral data matched that reported by Driver and co-workers: ${ }^{6}{ }^{1} \mathrm{H} \mathrm{NMR}\left(500 \mathrm{MHz}, \mathrm{CDCl}_{3}\right)$ $\delta 7.75(\mathrm{~d}, J=7.5 \mathrm{~Hz}, 1 \mathrm{H}), 6.66(\mathrm{~d}, J=7.6 \mathrm{~Hz}, 1 \mathrm{H}), 6.47(\mathrm{~s}, 1 \mathrm{H}), 4.92(\mathrm{br} \mathrm{s}, 2 \mathrm{H}), 2.40(\mathrm{~s}, 3 \mathrm{H}), 1.46(\mathrm{~s}, 12 \mathrm{H}) ;{ }^{13} \mathrm{C} \mathrm{NMR}(125$ $\left.\mathrm{MHz}, \mathrm{CDCl}_{3}\right) \delta 154.3(\mathrm{C}), 143.0(\mathrm{C}), 137.1(\mathrm{CH}), 118.1(\mathrm{CH}), 115.6(\mathrm{CH}), 83.4(\mathrm{C}), 25.0\left(\mathrm{CH}_{3}\right), 21.8\left(\mathrm{CH}_{3}\right)$ only visible peaks.

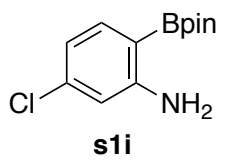

5-Chloro-2-(3,3,4,4-tetramethylborolan-1-yl)aniline s1i. ${ }^{4}$ The general procedure was followed using $1.04 \mathrm{~g}$ of 2-bromo5-chloroaniline (5.0 mmol), $2.20 \mathrm{~mL}$ of HBpin $(15.0 \mathrm{mmol}), 2.80 \mathrm{~mL}$ of Et $3 \mathrm{~N}(20.0 \mathrm{mmol}), 0.204 \mathrm{~g}$ of $(\mathrm{dppf}) \mathrm{PdCl}_{2}(0.25$ $\mathrm{mmol})$, in $12 \mathrm{~mL}$ of 1,4-dioxane $(0.4 \mathrm{M})$. Purification by MPLC (2:98 to 15:85 EtOAc:hexanes) afforded the product as a yellow oil $(0.919 \mathrm{~g}, 73 \%)$. The spectral data matched that reported by Maleckza, Smith and co-workers: ${ }^{4} \mathrm{H}$ NMR (500 $\left.\mathrm{MHz}, \mathrm{CDCl}_{3}\right) \delta 7.53(\mathrm{~d}, J=8.0 \mathrm{~Hz}, 1 \mathrm{H}), 6.64(\mathrm{dd}, J=8.0,1.9 \mathrm{~Hz}, 1 \mathrm{H}), 6.57$ (d, $\left.J=1.9 \mathrm{~Hz}, 1 \mathrm{H}\right), 4.84($ br s, $2 \mathrm{H}), 1.34$ (s,

6. Jana, N.; Nguyen, Q.; Driver, T. G. J. Org. Chem. 2014, 79, 2781. 
12H); ${ }^{13} \mathrm{C}$ NMR $\left(125 \mathrm{MHz}, \mathrm{CDCl}_{3}\right) \delta 154.8(\mathrm{C}), 138.6(\mathrm{C}), 138.1(\mathrm{CH}), 117.0(\mathrm{CH}), 114.3(\mathrm{CH}), 83.7(\mathrm{C}), 24.9\left(\mathrm{CH}_{3}\right)$ only visible peaks.

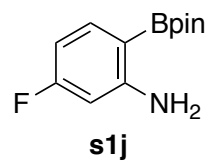

5-Fluoro-2-(3,3,4,4-tetramethylborolan-1-yl)aniline $\mathbf{s 1 j .}{ }^{7}$ The general procedure was followed using $1.90 \mathrm{~g}$ of 2-bromo5-fluoroaniline (10.0 mmol), $4.40 \mathrm{~mL}$ of HBpin $(30.0 \mathrm{mmol}), 5.80 \mathrm{~mL}$ of $\mathrm{Et}_{3} \mathrm{~N}(40.0 \mathrm{mmol}), 0.408 \mathrm{~g}$ of $\left(\mathrm{dppf}^{\mathrm{N}} \mathrm{PdCl}_{2}(0.50\right.$ $\mathrm{mmol})$, in $25 \mathrm{~mL}$ of 1,4-dioxane $(0.4 \mathrm{M})$. Purification by MPLC (2:98 to 15:85 EtOAc:hexanes) afforded the product as a brown oil $(1.81 \mathrm{~g}, 76 \%)$. The spectral data matched that reported by Zhou and Driver: ${ }^{1}{ }^{1} \mathrm{H}$ NMR $\left(500 \mathrm{MHz}, \mathrm{CDCl}_{3}\right) \delta 7.67$ (t, $J=7.8 \mathrm{~Hz}, 1 \mathrm{H}), 6.41(\mathrm{dt}, J=8.4,5.5 \mathrm{~Hz}, 1 \mathrm{H}), 6.27(\mathrm{dd}, J=11.7,2.4 \mathrm{~Hz}, 1 \mathrm{H}), 5.00(\mathrm{br} \mathrm{s}, 2 \mathrm{H}), 1.35(\mathrm{~s}, 12 \mathrm{H}) ;{ }^{13} \mathrm{C} \mathrm{NMR}$ $\left(125 \mathrm{MHz}, \mathrm{CDCl}_{3}\right) \delta 166.5\left(\mathrm{~d}, J_{C F}=247.5 \mathrm{~Hz}\right), 156.2\left(\mathrm{~d}, J_{C F}=11.3 \mathrm{~Hz}, \mathrm{C}\right), 139.1\left(\mathrm{~d}, J_{C F}=10.5 \mathrm{~Hz}, \mathrm{CH}\right), 104.0\left(\mathrm{~d}, J_{C F}=\right.$ $20.9 \mathrm{~Hz}, \mathrm{CH}), 100.9\left(\mathrm{~d}, J_{C F}=23.4 \mathrm{~Hz}, \mathrm{CH}\right), 83.6(\mathrm{C}), 24.8\left(\mathrm{CH}_{3}\right)$ only visible peaks; ${ }^{19} \mathrm{~F}$ NMR $\left(282 \mathrm{MHz}, \mathrm{CDCl}_{3}\right) \delta$ 108.6 .

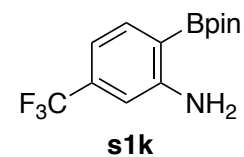

2-(3,3,4,4-Tetramethylborolan-1-yl)-5-(trifluoromethyl)aniline s1k. ${ }^{3}$ The general procedure was followed using $2.40 \mathrm{~g}$ of 2-bromo-5-(trifluoromethyl)aniline $(10.0 \mathrm{mmol}), 4.40 \mathrm{~mL}$ of HBpin $(30.0 \mathrm{mmol}), 5.80 \mathrm{~mL}$ of Et $3 \mathrm{~N}(40.0 \mathrm{mmol}), 0.408$ $\mathrm{g}$ of (dppf) $\mathrm{PdCl}_{2}(0.50 \mathrm{mmol})$, in $25 \mathrm{~mL}$ of 1,4-dioxane $(0.4 \mathrm{M})$. Purification by MPLC (2:98 to 15:85 EtOAc:hexanes) afforded the product as a yellow solid $(1.87 \mathrm{~g}, 64 \%)$. The spectral data matched that reported by Driver and co-workers: ${ }^{3}$ ${ }^{1} \mathrm{H}$ NMR $\left(500 \mathrm{MHz}, \mathrm{CDCl}_{3}\right) \delta 7.80(\mathrm{~d}, J=7.8 \mathrm{~Hz}, 1 \mathrm{H}), 6.94(\mathrm{~d}, J=7.7 \mathrm{~Hz}, 1 \mathrm{H}), 6.83(\mathrm{~s}, 1 \mathrm{H}), 5.05($ br s, $2 \mathrm{H}), 1.38(\mathrm{~s}, 12 \mathrm{H})$; ${ }^{13} \mathrm{C} \mathrm{NMR}\left(125 \mathrm{MHz}, \mathrm{CDCl}_{3}\right) \delta 153.9(\mathrm{C}), 137.6(\mathrm{CH}), 134.3\left(\mathrm{q}, J_{C F}=31.7 \mathrm{~Hz}, \mathrm{C}\right), 124.3\left(\mathrm{q}, J_{C F}=272.4 \mathrm{~Hz}, \mathrm{C}\right), 112.6(\mathrm{q}$, $\left.J_{C F}=3.8 \mathrm{~Hz}\right), 110.9\left(\mathrm{q}, J_{C F}=4.1 \mathrm{~Hz}\right), 84.0(\mathrm{C}), 24.7\left(\mathrm{CH}_{3}\right)$ only visible peaks.

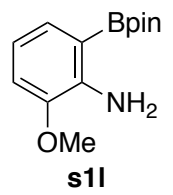

2-Methoxy-6-(3,3,4,4-tetramethylborolan-1-yl)aniline $\mathbf{s 1 1 .}{ }^{8}$ The general procedure was followed using $2.03 \mathrm{~g}$ of 2 bromo-6-methoxyaniline $(10.0 \mathrm{mmol}), 4.40 \mathrm{~mL}$ of HBpin $(30.0 \mathrm{mmol}), 5.80 \mathrm{~mL}$ of $\mathrm{Et}_{3} \mathrm{~N}(40.0 \mathrm{mmol}), 0.408 \mathrm{~g}$ of (dppf) $\mathrm{PdCl}_{2}(0.50 \mathrm{mmol})$, in $25 \mathrm{~mL}$ of 1,4-dioxane $(0.4 \mathrm{M})$. Purification by MPLC (2:98 to 15:85 EtOAc:hexanes) afforded the product as a white solid $(0.998 \mathrm{~g}, 40 \%)$. The spectral data matched that reported by Smith and co-workers: ${ }^{8}{ }^{1} \mathrm{H}$ NMR $\left(500 \mathrm{MHz}, \mathrm{CDCl}_{3}\right) \delta 7.33(\mathrm{~d}, J=7.3 \mathrm{~Hz}, 1 \mathrm{H}), 6.88(\mathrm{~d}, J=7.7 \mathrm{~Hz}, 1 \mathrm{H}), 6.70(\mathrm{t}, J=7.6 \mathrm{~Hz}, 1 \mathrm{H}), 5.07($ br s, $2 \mathrm{H}), 3.87(\mathrm{~s}$, $3 \mathrm{H}), 1.40(\mathrm{~s}, 12 \mathrm{H}) .{ }^{13} \mathrm{C}$ NMR $\left(125 \mathrm{MHz}, \mathrm{CDCl}_{3}\right) \delta 146.5(\mathrm{C}), 144.1(\mathrm{C}), 128.2(\mathrm{CH}), 116.2(\mathrm{CH}), 113.0(\mathrm{CH}), 83.5(\mathrm{C})$, $55.5\left(\mathrm{CH}_{3}\right), 25.0\left(\mathrm{CH}_{3}\right)$ only visible peaks.

7. Zhou, F.; Driver, T. G. Org. Lett. 2014, 16, 2916.

8. Smith, M. R., III.; Bisht, R.; Haldar, C.; Pandey, G.; Dannatt, J. E.; Ghaffari, B.; Maleczka, R. E., Jr.; Chattopadhyay, B. ACS Catal. 2018, 8,6216 . 


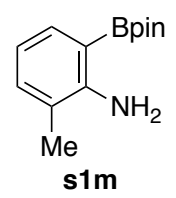

2-Methyl-6-(3,3,4,4-tetramethylborolan-1-yl)aniline $\mathbf{s} 1 \mathrm{~m}^{8}{ }^{8}$ The general procedure was followed using $1.86 \mathrm{~g}$ of 2-bromo6-methylaniline (10.0 mmol), $4.40 \mathrm{~mL}$ of HBpin $(30.0 \mathrm{mmol}), 5.80 \mathrm{~mL}$ of $\mathrm{Et}_{3} \mathrm{~N}(40.0 \mathrm{mmol}), 0.408 \mathrm{~g}$ of $\left(\mathrm{dppf}^{\mathrm{PdCl}} \mathrm{Pd}_{2}(0.50\right.$ $\mathrm{mmol})$, in $25 \mathrm{~mL}$ of 1,4-dioxane $(0.4 \mathrm{M})$. Purification by MPLC (2:98 to 15:85 EtOAc:hexanes) afforded the product as an orange solid $(1.10 \mathrm{~g}, 47 \%)$. The spectral data matched that reported by Smith and co-workers: ${ }^{1} \mathrm{H} \mathrm{NMR}\left(500 \mathrm{MHz}, \mathrm{CDCl}_{3}\right)$ $\delta 7.67(\mathrm{~d}, J=7.4 \mathrm{~Hz}, 1 \mathrm{H}), 7.23(\mathrm{~d}, J=7.2 \mathrm{~Hz}, 1 \mathrm{H}), 6.75(\mathrm{t}, J=7.4 \mathrm{~Hz}, 1 \mathrm{H}), 4.88(\mathrm{br} \mathrm{s}, 2 \mathrm{H}), 2.22(\mathrm{~s}, 3 \mathrm{H}), 1.44(\mathrm{~s}, 12 \mathrm{H}) .{ }^{13} \mathrm{C}$ NMR $\left(125 \mathrm{MHz}, \mathrm{CDCl}_{3}\right) \delta 152.0(\mathrm{C}), 134.9(\mathrm{CH}), 133.9(\mathrm{CH}), 121.4(\mathrm{C}), 116.9(\mathrm{CH}), 83.6(\mathrm{C}), 25.0\left(\mathrm{CH}_{3}\right), 17.7\left(\mathrm{CH}_{3}\right)$ only visible peaks.

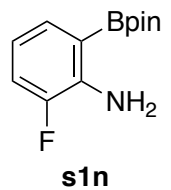

2-Fluoro-6-(3,3,4,4-tetramethylborolan-1-yl)aniline s1n. ${ }^{8}$ The general procedure was followed using $1.90 \mathrm{~g}$ of 2-bromo6-fluoroaniline (10.0 mmol), $4.40 \mathrm{~mL}$ of HBpin (30.0 mmol), $5.80 \mathrm{~mL}^{\circ} \mathrm{Et}_{3} \mathrm{~N}(40.0 \mathrm{mmol}), 0.408 \mathrm{~g}$ of $(\mathrm{dppf}) \mathrm{PdCl}_{2}(0.50$ $\mathrm{mmol})$, in $25 \mathrm{~mL}$ of 1,4-dioxane $(0.4 \mathrm{M})$. Purification by MPLC (2:98 to 15:85 EtOAc:hexanes) afforded the product as a pale yellow solid $(1.39 \mathrm{~g}, 58 \%)$. The spectral data matched that reported by Smith and co-workers: ${ }^{8}{ }^{1} \mathrm{H} \mathrm{NMR}(500 \mathrm{MHz}$, $\left.\mathrm{CDCl}_{3}\right) \delta 7.45-7.40(\mathrm{~m}, 1 \mathrm{H}), 7.10-7.02(\mathrm{~m}, 1 \mathrm{H}), 6.62(\mathrm{td}, J=7.7,4.5 \mathrm{~Hz}, 1 \mathrm{H}), 4.87(\mathrm{br} \mathrm{s}, 2 \mathrm{H}), 1.37(\mathrm{~s}, 12 \mathrm{H}) ;{ }^{13} \mathrm{C} \mathrm{NMR}$ $\left(125 \mathrm{MHz}, \mathrm{CDCl}_{3}\right) \delta 151.2\left(\mathrm{~d}, J_{C F}=239 \mathrm{~Hz}, \mathrm{C}\right), 141.8\left(\mathrm{~d}, J_{C F}=11.1 \mathrm{~Hz}, \mathrm{C}\right), 131.7\left(\mathrm{~d}, J_{C F}=3.7 \mathrm{~Hz}, \mathrm{CH}\right), 117.7\left(\mathrm{~d}, J_{C F}=\right.$ $18.5 \mathrm{~Hz}, \mathrm{CH}), 116.4\left(\mathrm{~d}, J_{C F}=6.4 \mathrm{~Hz}, \mathrm{CH}\right), 83.8(\mathrm{C}), 24.9\left(\mathrm{CH}_{3}\right)$ only visible peaks.
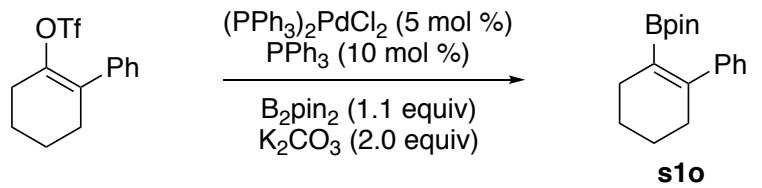

(s4)

4,4,5,5-Tetramethyl-2-(3,4,5,6-tetrahydro-[1,1'-biphenyl]-2-yl)-1,3,2-dioxaborolane s1o. ${ }^{9}$ A mixture of $0.613 \mathrm{~g}$ of vinyl triflate (2.0 mmol, 1.0 equiv), $0.559 \mathrm{~g}$ of $\mathrm{B}_{2} \mathrm{pin}_{2}(2.2 \mathrm{mmol}), 0.553 \mathrm{~g}$ of $\mathrm{K}_{2} \mathrm{CO}_{3}(4.0 \mathrm{mmol}), 0.070 \mathrm{~g}$ of $\left(\mathrm{PPh}_{3}\right)_{2} \mathrm{PdCl}_{2}(0.10$ $\mathrm{mmol})$, in $20 \mathrm{~mL}$ of 1,4-dioxane $(0.1 \mathrm{M})$ was heated to $90{ }^{\circ} \mathrm{C}$ using an oil bath. After $16 \mathrm{~h}$, the mixture was cooled to room temperature and diluted with $20 \mathrm{~mL}$ of a saturated aq soln of $\mathrm{NH}_{4} \mathrm{Cl}$. The resulting mixture was extracted with $3 \times 10 \mathrm{~mL}$ of $\mathrm{CH}_{2} \mathrm{Cl}_{2}$. The combined organic phases were washed with brine. The resulting organic phase was dried over $\mathrm{Na}_{2} \mathrm{SO}_{4}$, filtered, and was concentrated in vacuo. Purification using MPLC afforded the product as a colorless oil $(0.298 \mathrm{~g}, 52 \%)$. The spectral data matched that reported by Reichle and Breit: ${ }^{1} \mathrm{H}$ NMR $\left(500 \mathrm{MHz}, \mathrm{CDCl}_{3}\right) \delta{ }^{1} \mathrm{H} \mathrm{NMR}\left(501 \mathrm{MHz}, \mathrm{CDCl}_{3}\right)$ $\delta 7.28-7.18(\mathrm{~m}, 5 \mathrm{H}), 2.41-2.34(\mathrm{~m}, 2 \mathrm{H}), 2.32-2.25(\mathrm{~m}, 2 \mathrm{H}), 1.81-1.73(\mathrm{~m}, 2 \mathrm{H}), 1.73-1.63(\mathrm{~m}, 2 \mathrm{H}), 1.11(\mathrm{~s}, 12 \mathrm{H})$; ${ }^{13} \mathrm{C} \mathrm{NMR}\left(125 \mathrm{MHz}, \mathrm{CDCl}_{3}\right) \delta 148.8(\mathrm{C}), 145.5(\mathrm{C}), 127.7(\mathrm{CH}), 127.6(\mathrm{CH}), 126.6(\mathrm{CH}), 82.9(\mathrm{C}), 31.7\left(\mathrm{CH}_{2}\right), 28.1\left(\mathrm{CH}_{2}\right)$, $24.6\left(\mathrm{CH}_{3}\right), 23.1\left(\mathrm{CH}_{2}\right), 22.2\left(\mathrm{CH}_{2}\right)$ only visible peaks.
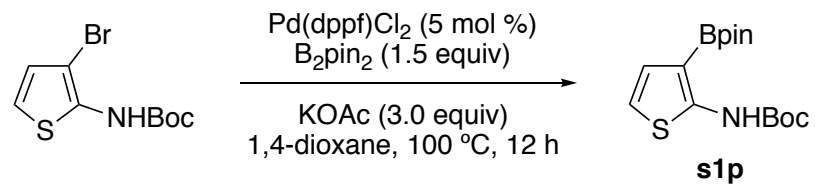

(s5)

tert-Butyl (3-(4,4,5,5-tetramethyl-1,3,2-dioxaborolan-2-yl)thiophen-2-yl)carbamate s1p. A mixture of $1.39 \mathrm{~g}$ of tertbutyl (3-bromothiophen-2-yl)carbamate (5.0 mmol, 1.0 equiv), $1.90 \mathrm{~g}$ of $\mathrm{B}_{2} \mathrm{pin}_{2}(7.5 \mathrm{mmol}), 1.47 \mathrm{~g}$ of $\mathrm{K}_{2} \mathrm{CO}_{3}(15.0 \mathrm{mmol})$, $0.204 \mathrm{~g}$ of $\mathrm{Pd}(\mathrm{dppf}) \mathrm{Cl}_{2}(0.25 \mathrm{mmol})$, in $25 \mathrm{~mL}$ of 1,4-dioxane $(0.2 \mathrm{M})$ was heated to $100{ }^{\circ} \mathrm{C}$ using an oil bath. After $12 \mathrm{~h}$,

9. Reichle, M. A.; Breit, B. Angew. Chem., Int. Ed. 2012, 51, 5730. 
the mixture was cooled to room temperature and diluted with $20 \mathrm{~mL}$ of a saturated aq soln of $\mathrm{NH}_{4} \mathrm{Cl}$. The resulting mixture was extracted with $3 \times 10 \mathrm{~mL}$ of $\mathrm{CH}_{2} \mathrm{Cl}_{2}$. The combined organic phases were washed with brine. The resulting organic phase was dried over $\mathrm{Na}_{2} \mathrm{SO}_{4}$, filtered, and was concentrated in vacuo. Purification using MPLC (0:100 to 2:98 EtOAc:hexanes) afforded the product as a green oil $(0.518 \mathrm{~g}, 32 \%):{ }^{1} \mathrm{H}$ NMR $\left(500 \mathrm{MHz}, \mathrm{CDCl}_{3}\right) \delta 8.78(\mathrm{br}, 1 \mathrm{H}), 7.00(\mathrm{~d}$, $J=5.4 \mathrm{~Hz}, 1 \mathrm{H}), 6.68(\mathrm{~d}, J=5.5 \mathrm{~Hz}, 1 \mathrm{H}), 1.52(\mathrm{~s}, 9 \mathrm{H}), 1.30(\mathrm{~s}, 12 \mathrm{H})$. The crude thiophene boronic acid ester was used in the subsequent cross-coupling reaction without additional purification.
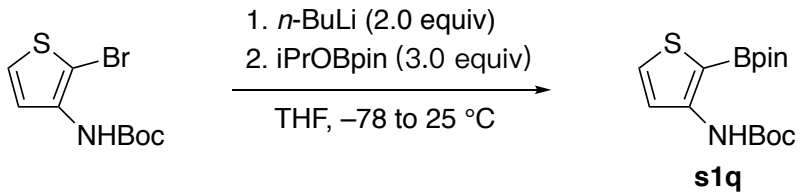

tert-Butyl (2-(4,4,5,5-tetramethyl-1,3,2-dioxaborolan-2-yl)thiophen-3-yl)carbamate s1q. To a solution of $1.67 \mathrm{~g}$ of tertbutyl (2-bromothiophen-3-yl)carbamate $(6.0 \mathrm{mmol})$ in $30 \mathrm{~mL}$ of THF under an argon atmosphere was added dropwise 4.8 $\mathrm{mL}$ of a $2.5 \mathrm{M}$ solution of $n$-BuLi $(12.0 \mathrm{mmol})$ in hexanes at $-78^{\circ} \mathrm{C}$. After stirring for $2 \mathrm{~h}, 3.7 \mathrm{~mL}$ of iPrOBPin $(18.0 \mathrm{mmol})$ was added over $20 \mathrm{~min}$, and the reaction was allowed to warm to room temperature. After $12 \mathrm{~h}$, the reactives in the reaction mixture were quenched with $50 \mathrm{~mL}$ of water. The resulting mixture was extracted with $3 \times 20 \mathrm{~mL}$ of $\mathrm{CH}_{2} \mathrm{Cl}_{2}$. The combined organic phases were washed with brine. The resulting organic phase was dried over $\mathrm{Na}_{2} \mathrm{SO}_{4}$, filtered, and was concentrated in vacuo. The product was afforded as red oil $\left(87 \%\right.$, determined by ${ }^{1} \mathrm{H}$ NMR because the thiophencarbamate s1q readily decomposes in air or upon exposure to silica gel). The crude thiophen boronic acid ester was used in the subsequent crosscoupling reaction without additional purification.

\section{Preparation of $\alpha$-aryl ketones}

1. General procedures

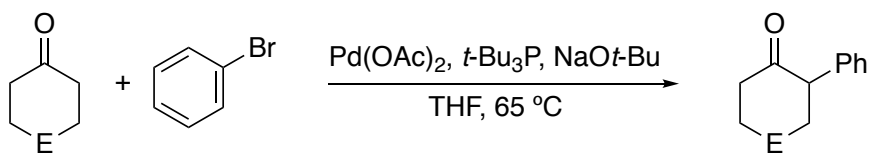

Method A: To a mixture of hetero-cyclohexanone (10.0 mmol, 1.0 equiv), bromobenzene (20.0 mmol, 2.0 equiv), $\mathrm{Pd}(\mathrm{OAc})_{2}$ $(0.50 \mathrm{mmol}, 5 \mathrm{~mol} \%), t$-Bu $\mathrm{P}(0.65 \mathrm{mmol}, 6.5 \mathrm{~mol} \%)$ in $20 \mathrm{~mL}$ of THF $(0.50 \mathrm{M})$, was added $\mathrm{NaO} t$-Bu $(15.0 \mathrm{mmol}, 1.5$ equiv). The resultant mixture was heated to $65^{\circ} \mathrm{C}$ using an oil bath. After $24 \mathrm{~h}$, the mixture was cooled to room temperature and diluted with $20 \mathrm{~mL}$ of a saturated aq soln of $\mathrm{NaHCO}_{3}$. The resulting mixture was extracted with $3 \times 10 \mathrm{~mL}$ of EtOAc. The combined organic phases were washed with brine. The resulting organic phase was dried over $\mathrm{Na}_{2} \mathrm{SO}_{4}$, filtered and was concentrated in vacuo. Purification using MPLC afforded the product. The method was not optimized.

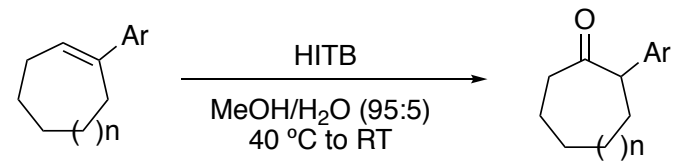

Method B: Following the report by Justik and Koser, ${ }^{10}$ to a mixture of 1 -arylcyclo alkenes (11.0 mmol, 1.1 equiv) in 43 $\mathrm{mL}$ of methanol and $2 \mathrm{~mL}$ of water, was added [hydroxy(tosyloxy)iodo]benzene (HTIB, $10.0 \mathrm{mmol}, 1.0$ equiv). The resultant mixture was heated to $40^{\circ} \mathrm{C}$ using an oil bath, turning to colorless solution rapidly. After $1 \mathrm{~h}$ at room temperature, the mixture was concentrated in vacuo. The resulting mixture was extracted with $3 \times 10 \mathrm{~mL}$ of DCM. The combined organic phases were washed with brine. The resulting organic phase was dried over $\mathrm{Na}_{2} \mathrm{SO}_{4}$, filtered, and was concentrated in vacuo. Purification using MPLC afforded the product. The method is not optimized.

10. Justik, M. W.; Koser, G. F. Tetrahedron Lett. 2004, 45, 6159. 
2. Characterization data for $\alpha$-aryl ketones<smiles>O=C1CCC([18OH])CC1Cc1ccccc1</smiles>

4-(Tert-butyl)-2-phenylcyclohexan-1-one s2a. ${ }^{11}$ Method A was followed using $1.54 \mathrm{~g}$ of 4-(tert-butyl)cyclohexan-1-one $(10.0 \mathrm{mmol}), 2.10 \mathrm{~mL}$ of $\mathrm{PhBr}(20.0 \mathrm{mmol}), 0.112 \mathrm{~g}$ of $\mathrm{Pd}(\mathrm{OAc})_{2}(0.5 \mathrm{mmol}), 0.132 \mathrm{~g}$ of $t-\mathrm{Bu} \mathrm{P}_{3} \mathrm{P}(0.65 \mathrm{mmol})$ and $1.44 \mathrm{~g}$ of $\mathrm{NaO} t$-Bu $(15.0 \mathrm{mmol})$ in $20 \mathrm{~mL}$ of THF $(0.5 \mathrm{M})$. Purification by MPLC (2:98 to 5:95 EtOAc:hexanes) afforded the product as a brown oil $(0.574 \mathrm{~g}, 25 \%)$. The spectral data matched that reported by Aggarwal and Olofsson: ${ }^{11}{ }^{1} \mathrm{H} \mathrm{NMR}(500 \mathrm{MHz}$, $\left.\mathrm{CDCl}_{3}\right) \delta 7.37(\mathrm{t}, J=7.5 \mathrm{~Hz}, 2 \mathrm{H}), 7.29(\mathrm{t}, J=7.3 \mathrm{~Hz}, 1 \mathrm{H}), 7.18(\mathrm{~d}, J=7.5 \mathrm{~Hz}, 2 \mathrm{H}), 3.64(\mathrm{dd}, J=12.3,5.6 \mathrm{~Hz}, 1 \mathrm{H}), 2.66-$ $2.45(\mathrm{~m}, 2 \mathrm{H}), 2.32(\mathrm{dt}, J=9.9,4.3 \mathrm{~Hz}, 1 \mathrm{H}), 2.21(\mathrm{ddt}, J=13.1,6.5,3.3 \mathrm{~Hz}, 1 \mathrm{H}), 1.80(\mathrm{t}, J=11.4 \mathrm{~Hz}, 2 \mathrm{H}), 1.52(\mathrm{~d}, J=14.2$ $\mathrm{Hz}, 1 \mathrm{H}), 0.99(\mathrm{~s}, 9 \mathrm{H}) ;{ }^{13} \mathrm{C} \mathrm{NMR}\left(125 \mathrm{MHz}, \mathrm{CDCl}_{3}\right) \delta 210.3(\mathrm{C}), 139.2(\mathrm{C}), 128.8(\mathrm{CH}), 128.4(\mathrm{CH}), 127.0(\mathrm{CH}), 57.0(\mathrm{CH})$, $47.4(\mathrm{CH}), 41.7\left(\mathrm{CH}_{2}\right), 37.0\left(\mathrm{CH}_{2}\right), 32.6(\mathrm{C}), 28.6\left(\mathrm{CH}_{2}\right), 27.8\left(\mathrm{CH}_{3}\right)$.
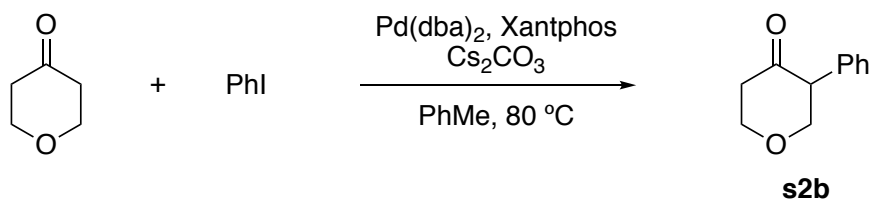

(s9)

3-Phenyltetrahydro-4H-pyran-4-one s2b. ${ }^{12}$ Following the report by Christoffers and co-workers, to a mixture of $0.92 \mathrm{~g}$ of tetrahydro- $4 \mathrm{H}$-pyran-4-one ( $10.0 \mathrm{mmol}, 1.0$ equiv.), $2.25 \mathrm{~mL}$ of iodobenzene (20.0 mmol, 2.0 equiv.), $0.288 \mathrm{~g}$ of $\mathrm{Pd}(\mathrm{dba})_{2}$ (0.50 mmol, $5 \mathrm{~mol} \%), 0.694 \mathrm{~g}$ Xantphos $(1.20 \mathrm{mmol}, 12 \mathrm{~mol} \%)$ in $10 \mathrm{~mL}$ of toluene $(1.0 \mathrm{M})$, was added $4.25 \mathrm{~g}$ of $\mathrm{Cs}_{2} \mathrm{CO}_{3}$ (13.0 mmol, 1.3 equiv.). The resultant mixture was heated to $80^{\circ} \mathrm{C}$ using an oil bath. After $24 \mathrm{~h}$, the mixture was cooled to room temperature and diluted with $20 \mathrm{~mL}$ of a saturated aq soln of $\mathrm{NaHCO}_{3}$. The resulting mixture was extracted with $3 \times$ $10 \mathrm{~mL}$ of EtOAc. The combined organic phases were washed with brine. The resulting organic phase was dried over $\mathrm{Na}_{2} \mathrm{SO}_{4}$, filtered, and was concentrated in vacuo. Purification of the resulting residue by MPLC (2:98 to 5:95 EtOAc:hexanes) afforded the product as a black oil $(0.210 \mathrm{~g}, 12 \%)$. The spectral data matched that reported by Christoffers and co-workers: ${ }^{12}$ ${ }^{1} \mathrm{H}$ NMR $\left(500 \mathrm{MHz}, \mathrm{CDCl}_{3}\right) \delta 7.34(\mathrm{~d}, J=7.6 \mathrm{~Hz}, 2 \mathrm{H}), 7.29(\mathrm{~d}, J=7.3 \mathrm{~Hz}, 1 \mathrm{H}), 7.24(\mathrm{~d}, J=7.7 \mathrm{~Hz}, 2 \mathrm{H}), 4.21$ (hextet, $J=$ $5.5 \mathrm{~Hz}, 2 \mathrm{H}), 3.96(\mathrm{q}, J=10.4 \mathrm{~Hz}, 2 \mathrm{H}), 3.78(\mathrm{t}, J=7.5 \mathrm{~Hz}, 1 \mathrm{H}), 2.63(\mathrm{q}, J=7.7 \mathrm{~Hz}, 1 \mathrm{H}), 2.55(\mathrm{~d}, J=13.9 \mathrm{~Hz}, 1 \mathrm{H}) ;{ }^{13} \mathrm{C}$ NMR $\left(125 \mathrm{MHz}, \mathrm{CDCl}_{3}\right) \delta 205.6(\mathrm{C}), 135.1(\mathrm{C}), 129.0(\mathrm{CH}), 128.7(\mathrm{CH}), 127.6(\mathrm{CH}), 73.1\left(\mathrm{CH}_{2}\right), 68.5\left(\mathrm{CH}_{2}\right), 58.0(\mathrm{CH})$, $41.9\left(\mathrm{CH}_{2}\right)$.

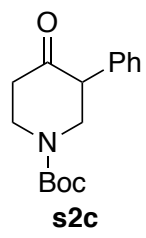

Tert-butyl 4-oxo-3-phenylpiperidine-1-carboxylate s2c. ${ }^{12}$ Method A was followed using $1.99 \mathrm{~g}$ of tert-butyl 4oxopiperidine-1-carboxylate $(10.0 \mathrm{mmol}), 2.10 \mathrm{~mL}$ of $\mathrm{PhBr}(20.0 \mathrm{mmol}), 0.112 \mathrm{~g}$ of $\mathrm{Pd}(\mathrm{OAc})_{2}(0.5 \mathrm{mmol}), 0.132 \mathrm{~g}$ of $t$ $\mathrm{Bu}_{3} \mathrm{P}(0.65 \mathrm{mmol})$ and $1.44 \mathrm{~g}$ of $\mathrm{NaOt}-\mathrm{Bu}(15.0 \mathrm{mmol})$ in $20 \mathrm{~mL}$ of THF $(0.5 \mathrm{M})$. Purification by MPLC (2:98 to 5:95 EtOAc:hexanes) afforded the product as a yellow solid $(1.24 \mathrm{~g}, 45 \%)$. The spectral data matched that reported by Christoffers and co-workers: ${ }^{12}{ }^{1} \mathrm{H}$ NMR $\left(500 \mathrm{MHz}, \mathrm{CDCl}_{3}\right) \delta 7.26(\mathrm{t}, J=7.4 \mathrm{~Hz}, 2 \mathrm{H}), 7.20(\mathrm{t}, J=7.5 \mathrm{~Hz}, 1 \mathrm{H}), 7.11(\mathrm{~d}, J=$ $7.5 \mathrm{~Hz}, 2 \mathrm{H}), 4.33-3.98(\mathrm{~m}, 2 \mathrm{H}), 3.72-3.29(\mathrm{~m}, 3 \mathrm{H}), 2.53-2.38(\mathrm{~m}, 2 \mathrm{H}), 1.44(\mathrm{~s}, 9 \mathrm{H}) ;{ }^{13} \mathrm{C} \mathrm{NMR}(125 \mathrm{MHz}, \mathrm{CDCl} 3) \delta$ $206.6(\mathrm{C}), 154.3(\mathrm{C}), 135.7(\mathrm{C}), 128.6(\mathrm{CH}), 128.5(\mathrm{CH}), 127.4(\mathrm{CH}), 80.4(\mathrm{C}), 56.2(\mathrm{CH}), 49.0\left(\mathrm{CH}_{2}\right), 43.2\left(\mathrm{CH}_{2}\right), 40.5$ $\left(\mathrm{CH}_{2}\right), 28.4\left(\mathrm{CH}_{3}\right)$.

11. Aggarwal, V. K.; Olofsson, B. Angew Chem. Int. Ed. 2005, 44, 5516.

12. Rosiak, A.; Frey, W.; Christoffers, J. Eur. J. Org. Chem. 2006, 4044. 


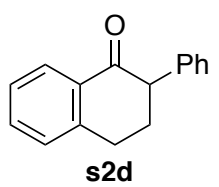

2-Phenyl-3,4-dihydronaphthalen-1(2H)-one s2d. ${ }^{13}$ Method A was followed using $1.35 \mathrm{~mL}$ of $\alpha$-tetralone $(10.0 \mathrm{mmol})$, $2.10 \mathrm{~mL}$ of $\mathrm{PhBr}(20.0 \mathrm{mmol}), 0.112 \mathrm{~g}$ of $\mathrm{Pd}(\mathrm{OAc})_{2}(0.5 \mathrm{mmol}), 0.132 \mathrm{~g}$ of $t-\mathrm{Bu}_{3} \mathrm{P}(0.65 \mathrm{mmol})$ and $1.44 \mathrm{~g}$ of $\mathrm{NaO} t-\mathrm{Bu}$ $(15.0 \mathrm{mmol})$ in $20 \mathrm{~mL}$ of THF $(0.5 \mathrm{M})$. Purification by MPLC (2:98 to 5:95 EtOAc:hexanes) afforded the product as a white solid $(0.782 \mathrm{~g}, 35 \%)$. The spectral data matched that reported by Zhou and co-workers: ${ }^{13}{ }^{1} \mathrm{H} \mathrm{NMR}\left(500 \mathrm{MHz}, \mathrm{CDCl}_{3}\right) \delta$ $8.23(\mathrm{~d}, J=7.8 \mathrm{~Hz}, 1 \mathrm{H}), 7.54(\mathrm{t}, J=7.5 \mathrm{~Hz}, 1 \mathrm{H}), 7.41(\mathrm{q}, J=8.6,8.0 \mathrm{~Hz}, 3 \mathrm{H}), 7.38-7.34(\mathrm{~m}, 1 \mathrm{H}), 7.32(\mathrm{~d}, J=8.0 \mathrm{~Hz}$, $1 \mathrm{H}), 7.28(\mathrm{~d}, J=7.5 \mathrm{~Hz}, 2 \mathrm{H}), 3.83(\mathrm{dd}, J=10.2,5.9 \mathrm{~Hz}, 1 \mathrm{H}), 3.20-3.00(\mathrm{~m}, 1 \mathrm{H}), 2.44$ (pentet, $J=5.3 \mathrm{~Hz}, 2 \mathrm{H}) ;{ }^{13} \mathrm{C} \mathrm{NMR}$ $\left(125 \mathrm{MHz}, \mathrm{CDCl}_{3}\right) \delta 198.1(\mathrm{C}), 144.3(\mathrm{C}), 140.1(\mathrm{C}), 133.6(\mathrm{CH}), 133.0(\mathrm{C}), 129.1(\mathrm{CH}), 128.7(\mathrm{CH}), 128.6(\mathrm{CH}), 127.8$ $(\mathrm{CH}), 127.0(\mathrm{CH}), 126.9(\mathrm{CH}), 54.5\left(\mathrm{CH}_{2}\right), 31.3\left(\mathrm{CH}_{2}\right), 28.9\left(\mathrm{CH}_{2}\right)$.

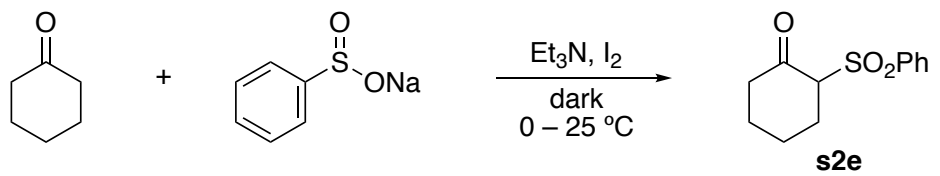

(s10)

2-(Phenylsulfonyl)cyclohexan-1-one s2e. ${ }^{14}$ Following the report of Sreedhar and co-workers, $2.10 \mathrm{~mL}$ of cyclohexanone (20 mmol, 1.0 equiv) in the presence of $3.35 \mathrm{~mL}$ of $\mathrm{Et}_{3} \mathrm{~N}(24 \mathrm{mmol}, 1.2 \mathrm{mmol})$ were stirred in $20 \mathrm{~mL}$ of MeOH. After 30 min, $3.94 \mathrm{~g}$ of benzenesulfinic acid sodium salt ( $24 \mathrm{mmol}, 1.2$ equiv), $5.08 \mathrm{~g}$ of molecular iodine ( $20 \mathrm{mmol}, 1.0$ equiv) was added and the reaction vessel was wrapped with aluminum foil to exclude light. After $16 \mathrm{~h}$, the resulting mixture was diluted with $20 \mathrm{~mL}$ of $\mathrm{H}_{2} \mathrm{O}$, and the resulting mixture was extracted with $3 \times 10 \mathrm{~mL}$ of EtOAc. The combined organic phases were washed with brine. The resulting organic phase was dried over $\mathrm{Na}_{2} \mathrm{SO}_{4}$, filtered, and was concentrated in vacuo. Purification of the resulting residue using MPLC (2:98 EtOAc:hexanes to 20: 80 acetone:hexane) afforded the product as a red oil (0.932 g, 20\%). The spectral data matched that reported by Sreedhar and co-workers: ${ }^{14}{ }^{1} \mathrm{H}$ NMR $\left(500 \mathrm{MHz}, \mathrm{CDCl}_{3}\right) \delta 7.88(\mathrm{~d}, J=$ $7.3 \mathrm{~Hz}, 2 \mathrm{H}), 7.65$ (t, $J=7.5 \mathrm{~Hz}, 1 \mathrm{H}), 7.55$ (t, $J=7.8 \mathrm{~Hz}, 2 \mathrm{H}), 3.90-3.79(\mathrm{~m}, 1 \mathrm{H}), 2.79$ (ddd, $J=14.9,9.7,5.6 \mathrm{~Hz}, 1 \mathrm{H})$, $2.58-2.49(\mathrm{~m}, 1 \mathrm{H}), 2.42(\mathrm{dt}, J=14.5,5.7 \mathrm{~Hz}, 1 \mathrm{H}), 2.28-2.17(\mathrm{~m}, 2 \mathrm{H}), 2.03-1.95(\mathrm{~m}, 1 \mathrm{H}), 1.82(\mathrm{dtd}, J=14.2,9.4,4.9$ $\mathrm{Hz}, 1 \mathrm{H}), 1.74$ (ddt, $J=13.1,6.7,3.4 \mathrm{~Hz}, 1 \mathrm{H}) ;{ }^{13} \mathrm{C} \mathrm{NMR}\left(125 \mathrm{MHz}, \mathrm{CDCl}_{3}\right) \delta 202.3(\mathrm{C}), 138.3(\mathrm{C}), 134.2(\mathrm{CH}), 129.2(\mathrm{CH})$, $129.0(\mathrm{CH}), 72.8(\mathrm{CH}), 41.7\left(\mathrm{CH}_{2}\right), 27.6\left(\mathrm{CH}_{2}\right), 26.6\left(\mathrm{CH}_{2}\right), 22.1\left(\mathrm{CH}_{2}\right)$.

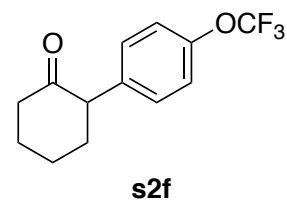

2-(4-(Trifluoromethoxy)phenyl)cyclohexan-1-one s2f. Method A was followed using $1.24 \mathrm{~mL}$ of cyclohexanone (12.0 $\mathrm{mmol}), 2.41 \mathrm{~g}$ of 1-bromo-4-(trifluoromethoxy)benzene (10.0 mmol), $0.112 \mathrm{~g}$ of $\mathrm{Pd}(\mathrm{OAc})_{2}(0.5 \mathrm{mmol}), 0.132 \mathrm{~g}$ of $t$ - $\mathrm{Bu} 33$ $(0.65 \mathrm{mmol})$ and $1.44 \mathrm{~g}$ of $\mathrm{NaO} t-\mathrm{Bu}(15.0 \mathrm{mmol})$ in $20 \mathrm{~mL}$ of THF $(0.5 \mathrm{M})$. Purification by MPLC (2:98 to 5:95 EtOAc:hexanes) afforded the product as a yellow oil $(1.45 \mathrm{~g}, 56 \%)$. The crude $\alpha$-aryl ketone was used in the subsequent vinyl triflate preparation reaction without additional purification.

13. Huang, Z.; Lim, L. H.; Chen, Z.; Li, Y.; Zhou, F.; Su, H.; Zhou, J. Angew. Chem. Int. Ed. 2013, 52, 4906.

14. Rawat, V. S.; Reddy, P. L. M.; Sreedhar, B. RSC Adv., 2014, 4, 5165. 


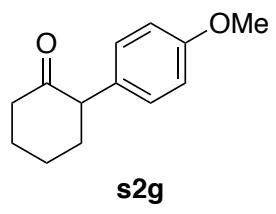

2-(4-Methoxyphenyl)cyclohexan-1-one s2g. ${ }^{13}$ Method A was followed using $1.24 \mathrm{~mL}$ of cyclohexanone (12.0 mmol), 1.25 $\mathrm{mL}$ of 4-bromoanisole $(10.0 \mathrm{mmol}), 0.112 \mathrm{~g}$ of $\mathrm{Pd}(\mathrm{OAc})_{2}(0.5 \mathrm{mmol}), 0.132 \mathrm{~g}$ of $t-\mathrm{Bu}_{3} \mathrm{P}(0.65 \mathrm{mmol})$ and $1.44 \mathrm{~g}$ of $\mathrm{NaO} t-$ $\mathrm{Bu}(15.0 \mathrm{mmol})$ in $20 \mathrm{~mL}$ of THF $(0.5 \mathrm{M})$. Purification by MPLC (2:98 to 5:95 EtOAc:hexanes) afforded the product as a yellow solid $(1.11 \mathrm{~g}, 54 \%)$. The spectral data matched that reported by Zhou and co-workers: ${ }^{13}{ }^{1} \mathrm{H} \mathrm{NMR}\left(500 \mathrm{MHz}, \mathrm{CDCl}_{3}\right)$ $\delta 7.07(\mathrm{~d}, J=6.5 \mathrm{~Hz}, 2 \mathrm{H}), 6.88(\mathrm{~d}, J=6.9 \mathrm{~Hz}, 2 \mathrm{H}), 3.78(\mathrm{~s}, 3 \mathrm{H}), 3.57(\mathrm{dd}, J=12.0,5.5 \mathrm{~Hz}, 1 \mathrm{H}), 2.55-2.40(\mathrm{~m}, 2 \mathrm{H}), 2.28$ $-2.18(\mathrm{~m}, 1 \mathrm{H}), 2.18-2.07(\mathrm{~m}, 1 \mathrm{H}), 2.06-1.90(\mathrm{~m}, 2 \mathrm{H}), 1.90-1.73(\mathrm{~m}, 2 \mathrm{H}) ;{ }^{13} \mathrm{C} \mathrm{NMR}\left(125 \mathrm{MHz}, \mathrm{CDCl}_{3}\right) \delta 210.7(\mathrm{C})$, $158.5(\mathrm{C}), 131.0(\mathrm{C}), 129.5\left(\mathrm{CH}_{2}\right), 113.8\left(\mathrm{CH}_{2}\right), 56.5\left(\mathrm{CH}_{3}\right), 55.2\left(\mathrm{CH}_{3}\right), 42.2\left(\mathrm{CH}_{2}\right), 35.3\left(\mathrm{CH}_{2}\right), 27.9\left(\mathrm{CH}_{2}\right), 25.4\left(\mathrm{CH}_{2}\right)$.

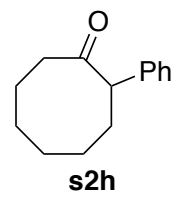

2-Phenylcyclooctan-1-one s2h. ${ }^{13}$ Method B was followed using $2.05 \mathrm{~g}$ of 1-phenylcycloocene (11.0 mmol), $3.93 \mathrm{~g}$ of HTIB $(10.0 \mathrm{mmol})$ in $43 \mathrm{~mL}$ of $\mathrm{MeOH}$ and $2 \mathrm{~mL}$ of water. Purification by MPLC (2:98 to 5:95 EtOAc:hexanes) afforded the product as a pale yellow oil $(0.552 \mathrm{~g}, 27 \%)$. The spectral data matched that reported by Zhou and co-workers: ${ }^{13}{ }^{1} \mathrm{H}$ NMR $\left(500 \mathrm{MHz}, \mathrm{CDCl}_{3}\right) \delta 7.36-7.31(\mathrm{~m}, 2 \mathrm{H}), 7.27(\mathrm{t}, J=7.6 \mathrm{~Hz}, 2 \mathrm{H}), 7.19(\mathrm{t}, J=7.4 \mathrm{~Hz}, 1 \mathrm{H}), 3.77(\mathrm{dd}, J=12.4,3.0 \mathrm{~Hz}, 1 \mathrm{H})$, $2.57(\mathrm{td}, J=12.6,3.5 \mathrm{~Hz}, 1 \mathrm{H}), 2.38-2.26(\mathrm{~m}, 1 \mathrm{H}), 2.25-2.17(\mathrm{~m}, 1 \mathrm{H}), 1.99-1.90(\mathrm{~m}, 2 \mathrm{H}), 1.88-1.80(\mathrm{~m}, 1 \mathrm{H}), 1.77-$ $1.67(\mathrm{~m}, 2 \mathrm{H}), 1.55(\mathrm{t}, J=11.1 \mathrm{~Hz}, 2 \mathrm{H}), 1.49-1.36(\mathrm{~m}, 2 \mathrm{H}) ;{ }^{13} \mathrm{C}$ NMR $\left(125 \mathrm{MHz}, \mathrm{CDCl}_{3}\right) \delta 216.0(\mathrm{C}), 139.5(\mathrm{C}), 128.5$ $(\mathrm{CH}), 127.8(\mathrm{CH}), 127.0(\mathrm{CH}), 57.4(\mathrm{CH}), 40.3\left(\mathrm{CH}_{2}\right), 31.7\left(\mathrm{CH}_{2}\right), 26.85\left(\mathrm{CH}_{2}\right), 26.81\left(\mathrm{CH}_{2}\right), 26.77\left(\mathrm{CH}_{2}\right), 24.7\left(\mathrm{CH}_{2}\right)$.

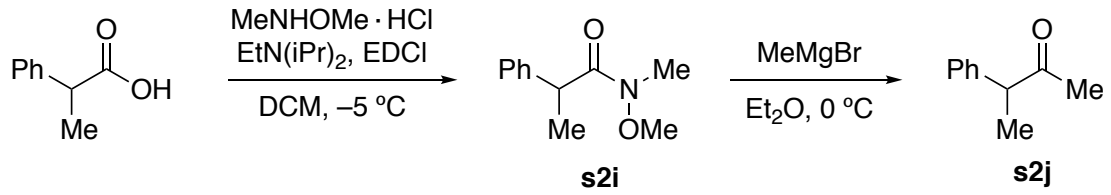

$(s 11)$

3-Phenylbutan-2-one s2j. ${ }^{15}$ Following the report by Valdés and co-workers, To a cooled $-5{ }^{\circ} \mathrm{C}$ solution of $1.36 \mathrm{~mL}$ of 2 phenylpropanoic acid (10.0 mmol, 1.0 equiv) in $70 \mathrm{~mL}$ of $\mathrm{CH}_{2} \mathrm{Cl}_{2}, 0.975 \mathrm{~g}$ of $\mathrm{N}, \mathrm{O}$-dimethylhydroxylamine hydrochloride (10.0 mmol, 1.0 equiv.), $1.74 \mathrm{~mL}$ of $\operatorname{EtN}(i \operatorname{Pr})_{2}(10.0 \mathrm{mmol}, 1.0$ equiv) were added. Then, a solution of $\mathrm{EDCl}(1.96 \mathrm{~g}, 10$ $\mathrm{mmol}$ ) in $20 \mathrm{~mL}$ of $\mathrm{CH}_{2} \mathrm{Cl}_{2}$ was added dropwise. After $1 \mathrm{~h}$ at $-5^{\circ} \mathrm{C}$, the mixture is washed with $2 \times 30 \mathrm{~mL}$ of a $1 \mathrm{M}$ aq soln of $\mathrm{HCl}$. The organic layers are collected, dried over $\mathrm{Na}_{2} \mathrm{SO}_{4}$, filtered and concentrated under reduced pressure to afford the Weinreb amide $\mathbf{s} 2 \mathbf{i}$, which was carried on to the next step without any additional purification or characterization.

To a cooled $0{ }^{\circ} \mathrm{C}$ solution of $\mathbf{s} 2 \mathbf{i}$ in $50 \mathrm{~mL}$ of $\mathrm{Et}_{2} \mathrm{O}$, was added dropwise $10 \mathrm{~mL}$ of methylmagnesium bromide (3 $\mathrm{M}$ solution in $\mathrm{Et}_{2} \mathrm{O}, 30.0 \mathrm{mmol}, 3$ equiv). A white precipitate quickly appeared. After $1 \mathrm{~h}$, the reactives were quenched with by the addition of a saturated aq soln of $\mathrm{NH}_{4} \mathrm{Cl}$ aqueous $(2 \times 100 \mathrm{~mL})$, extracted by ethyl acetate $(3 \times 20 \mathrm{~mL})$. The combined organic phases dried over $\left.\mathrm{Na}_{2} \mathrm{SO}_{4}\right)$, filtered and concentrated under reduced pressure. Purification of the resulting residue using MPLC (2:98 to 5:95 EtOAc:hexanes) to afford the product as a colorless oil (1.03 g, 70\% over 2 steps). The spectral data matched that reported by Valdés and co-workers: ${ }^{15}{ }^{1} \mathrm{H}$ NMR $\left(500 \mathrm{MHz}, \mathrm{CDCl}_{3}\right) \delta 7.25(\mathrm{t}, J=7.4 \mathrm{~Hz}, 2 \mathrm{H}), 7.19-7.13$ $(\mathrm{m}, 3 \mathrm{H}), 3.68(\mathrm{q}, J=7.0 \mathrm{~Hz}, 1 \mathrm{H}), 1.95(\mathrm{~s}, 3 \mathrm{H}), 1.32(\mathrm{~d}, J=7.1 \mathrm{~Hz}, 3 \mathrm{H}) ;{ }^{13} \mathrm{C}$ NMR $\left(125 \mathrm{MHz}, \mathrm{CDCl}_{3}\right) \delta 208.3(\mathrm{C}), 140.7$ $(\mathrm{C}), 128.9(\mathrm{CH}), 127.8(\mathrm{CH}), 127.1(\mathrm{CH}), 53.6(\mathrm{CH}), 28.2\left(\mathrm{CH}_{3}\right), 17.2\left(\mathrm{CH}_{3}\right)$.

15. Pérez-Aguilar, M. C.; Valdés, C. Angew. Chem. Int. Ed. 2015, 54, 13729. 


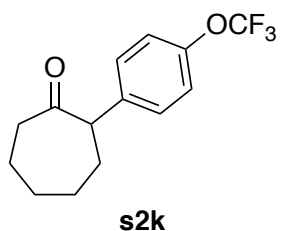

2-(4-(Trifluoromethoxy)phenyl)cycloheptan-1-one $\mathbf{s 2 k}$. Method B was followed using 2.82 g of 1-(4(trifluoromethoxy)phenyl)cyclohept-1-ene (11.0 mmol), $3.93 \mathrm{~g}$ of HTIB $(10.0 \mathrm{mmol})$ in $43 \mathrm{~mL}$ of MeOH and $2 \mathrm{~mL}$ of water. Purification by MPLC (2:98 to 5:95 EtOAc:hexanes) afforded the product as a colorless solid $(0.960 \mathrm{~g}, 35 \%): \mathrm{mp}=44{ }^{\circ} \mathrm{C}$; ${ }^{1} \mathrm{H}$ NMR $\left(500 \mathrm{MHz}, \mathrm{CDCl}_{3}\right) \delta 7.24(\mathrm{~d}, J=8.7 \mathrm{~Hz}, 2 \mathrm{H}), 7.16(\mathrm{~d}, J=8.4 \mathrm{~Hz}, 2 \mathrm{H}), 3.76(\mathrm{dd}, J=11.3,3.9 \mathrm{~Hz}, 1 \mathrm{H}), 2.69-$ $2.60(\mathrm{~m}, 1 \mathrm{H}), 2.60-2.52(\mathrm{~m}, 1 \mathrm{H}), 2.15-2.08(\mathrm{~m}, 1 \mathrm{H}), 2.07-1.88(\mathrm{~m}, 4 \mathrm{H}), 1.73-1.60(\mathrm{~m}, 1 \mathrm{H}), 1.54-1.41(\mathrm{~m}, 2 \mathrm{H}) ;{ }^{13} \mathrm{C}$ NMR (125 MHz, $\left.\mathrm{CDCl}_{3}\right) \delta 212.8(\mathrm{C}), 148.1(\mathrm{C}), 139.2(\mathrm{C}), 129.3(\mathrm{CH}), 120.9(\mathrm{CH}), 120.5\left(\mathrm{q}, J_{C F}=256.8 \mathrm{~Hz}, \mathrm{C}\right), 57.8$ $(\mathrm{CH}), 42.9\left(\mathrm{CH}_{2}\right), 32.3\left(\mathrm{CH}_{2}\right), 29.7\left(\mathrm{CH}_{2}\right), 28.8\left(\mathrm{CH}_{2}\right), 24.9\left(\mathrm{CH}_{2}\right) ;{ }^{19} \mathrm{~F}$ NMR $\left(282 \mathrm{MHz}, \mathrm{CDCl}_{3}\right) \delta-58.3$. ATR-FTIR (thin film): 2932, 2859, 1704, 1508, 1254, 1213, 1157, 1020, 937, $848 \mathrm{~cm}^{-1}$. HRMS (ESI) $\mathrm{m} / z$ calculated for $\mathrm{C}_{14} \mathrm{H}_{16} \mathrm{O}_{2} \mathrm{~F}_{3}(\mathrm{M}+\mathrm{H})^{+}$: 273.1102, found: 273.1095 .

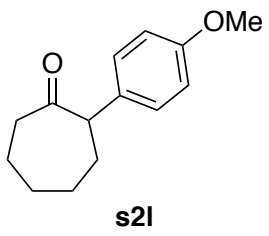

2-(4-Methoxyphenyl)cycloheptan-1-one s2l. ${ }^{16}$ Method B was followed using $2.22 \mathrm{~g}$ of 1-(4-methoxyphenyl)cyclohept-1ene (11.0 mmol), $3.93 \mathrm{~g}$ of HTIB $(10.0 \mathrm{mmol})$ in $43 \mathrm{~mL}$ of MeOH and $2 \mathrm{~mL}$ of water. Purification by MPLC (2:98 to 5:95 EtOAc:hexanes) afforded the product as a yellow oil $(0.902 \mathrm{~g}, 41 \%)$. The spectral data matched that reported by Roche and co-workers: ${ }^{16}{ }^{1} \mathrm{H}$ NMR $\left(500 \mathrm{MHz}, \mathrm{CDCl}_{3}\right) \delta 7.13(\mathrm{~d}, J=8.1 \mathrm{~Hz}, 2 \mathrm{H}), 6.83(\mathrm{~d}, J=8.5 \mathrm{~Hz}, 2 \mathrm{H}), 3.75(\mathrm{~s}, 3 \mathrm{H}), 3.65(\mathrm{dd}, J=$ $11.6,4.1 \mathrm{~Hz}, 1 \mathrm{H}), 2.65(\mathrm{td}, J=12.7,3.0 \mathrm{~Hz}, 1 \mathrm{H}), 2.50-2.43(\mathrm{~m}, 1 \mathrm{H}), 2.13-2.05(\mathrm{~m}, 1 \mathrm{H}), 2.04-1.84(\mathrm{~m}, 4 \mathrm{H}), 1.67-$ $1.55(\mathrm{~m}, 1 \mathrm{H}), 1.48-1.36(\mathrm{~m}, 2 \mathrm{H}) ;{ }^{13} \mathrm{C}$ NMR $\left(125 \mathrm{MHz}, \mathrm{CDCl}_{3}\right) \delta 213.6(\mathrm{C}), 158.5(\mathrm{C}), 132.5(\mathrm{C}), 128.8(\mathrm{CH}), 113.9(\mathrm{CH})$, $57.9(\mathrm{CH}), 55.2\left(\mathrm{CH}_{3}\right), 42.4\left(\mathrm{CH}_{2}\right), 32.0\left(\mathrm{CH}_{2}\right), 30.0\left(\mathrm{CH}_{2}\right), 28.5\left(\mathrm{CH}_{2}\right), 25.4\left(\mathrm{CH}_{2}\right)$.

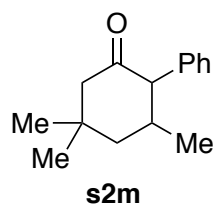

3,5,5-Trimethyl-2-phenylcyclohexan-1-one s2 m. Method A was followed using $1.90 \mathrm{~mL}$ of 3,3,5-trimethylcyclohexanone (12.0 mmol), $1.05 \mathrm{~mL}$ of bromobenzene (10.0 mmol), $0.112 \mathrm{~g}$ of $\mathrm{Pd}(\mathrm{OAc})_{2}(0.5 \mathrm{mmol}), 0.132 \mathrm{~g}$ of $t-\mathrm{Bu}_{3} \mathrm{P}(0.65 \mathrm{mmol})$ and $1.44 \mathrm{~g}$ of $\mathrm{NaOt}$-Bu $(15.0 \mathrm{mmol})$ in $20 \mathrm{~mL}$ of THF $(0.5 \mathrm{M})$. Purification by MPLC (2:98 to 5:95 EtOAc:hexanes) afforded the product as a yellow solid ( $2.02 \mathrm{~g}, 93 \%)$. The crude $\alpha$-aryl ketone was used in the subsequent vinyl triflate preparation reaction without additional purification.

\section{Preparation of vinyl triflates.}

1. General procedure.

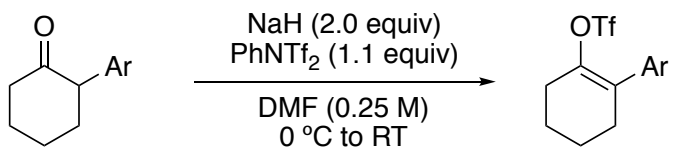

$(\mathrm{s} 12)$

16. Jeedimalla, N.; Jacquet, C.; Bahneva, D.; Youte Tendoung, J.- J.; Roche, S. P. J. Org. Chem. 2018, 83, 12357. 
To a cooled $0{ }^{\circ} \mathrm{C}$ suspension of $\mathrm{NaH}(60 \%$ dispersed in mineral oil, $20 \mathrm{mmol}, 2.0$ equiv) in $40 \mathrm{~mL}$ of DMF $(0.25 \mathrm{M})$ was added 2-arylcyclohexanone (10 mmol, 1.0 equiv). The mixture was warmed to room temperature. After 30 minutes, $N$ phenyl-bis(trifluoromethanesulfonamide) $(11 \mathrm{mmol}, 1.1$ equiv) was added. After an additional 12 hours, the reaction mixture was diluted with water and ethyl acetate. The phases were separated, and the organic phase was washed with brine and water. The resulting organic phase was dried over $\mathrm{Na}_{2} \mathrm{SO}_{4}$, filtered, and concentrated in vacuo. The resulting mixture was purified using MPLC to afford the product.

2. Characterization data for vinyl triflates.

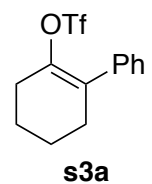

3,4,5,6-Tetrahydro-[1,1'-biphenyl]-2-yl trifluoromethanesulfonate s3a. ${ }^{17}$ The general method was followed using 1.75 $\mathrm{g}$ of 2-phenylcyclohexanone $(10.0 \mathrm{mmol}), 0.800 \mathrm{~g}$ of $\mathrm{NaH}(20.0 \mathrm{mmol}, 60 \% \mathrm{w} / \mathrm{w}), 3.93 \mathrm{~g}$ of $N$-phenylbis(trifluoromethanesulfonamide) $(11.0 \mathrm{mmol})$ in $40 \mathrm{~mL}$ of DMF $(0.25 \mathrm{M})$. Purification by MPLC (2:98 to 5:95 EtOAc:hexanes) afforded the product as colorless oil $(2.06 \mathrm{~g}, 67 \%)$. Triflate s3a was reported by Rigby and Qabar: ${ }^{17} \mathrm{H}$ NMR $\left(500 \mathrm{MHz}, \mathrm{CDCl}_{3}\right) \delta 7.41(\mathrm{t}, J=7.2 \mathrm{~Hz}, 2 \mathrm{H}), 7.35(\mathrm{~d}, J=7.1 \mathrm{~Hz}, 1 \mathrm{H}), 7.31(\mathrm{~d}, J=7.1 \mathrm{~Hz}, 2 \mathrm{H}), 2.54-2.52(\mathrm{~m}, 4 \mathrm{H})$, $1.91-1.89(\mathrm{~m}, 2 \mathrm{H}), 1.83-1.81(\mathrm{~m}, 2 \mathrm{H}) ;{ }^{13} \mathrm{C}$ NMR $\left(125 \mathrm{MHz}, \mathrm{CDCl}_{3}\right) \delta 143.9(\mathrm{C}), 137.0(\mathrm{C}), 131.2(\mathrm{C}), 128.3(\mathrm{CH}), 128.1$ $(\mathrm{CH}), 127.9(\mathrm{CH}), 118.2\left(\mathrm{q}, J_{C F}=317 \mathrm{~Hz}, \mathrm{C}\right), 31.3\left(\mathrm{CH}_{2}\right), 28.2\left(\mathrm{CH}_{2}\right), 23.0\left(\mathrm{CH}_{2}\right), 22.1\left(\mathrm{CH}_{2}\right)$.

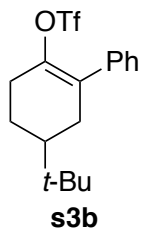

5-(Tert-butyl)-3,4,5,6-tetrahydro-[1,1'-biphenyl]-2-yl trifluoromethanesulfonate $\mathbf{s 3 b}{ }^{18}$ The general method was followed using $0.921 \mathrm{~g}$ of 4-(tert-butyl)-2-phenylcyclohexan-1-one (4.0 mmol), $0.320 \mathrm{~g}$ of NaH $(8.0 \mathrm{mmol}, 60 \% \mathrm{w} / \mathrm{w}), 1.57$ g of $N$-phenyl-bis(trifluoromethanesulfonamide) $(11.0 \mathrm{mmol})$ in $16 \mathrm{~mL}$ of DMF $(0.25 \mathrm{M})$. Purification by MPLC (2:98 to 3:97 EtOAc:hexanes) afforded the product as yellow oil $(0.826 \mathrm{~g}, 57 \%)$. The spectral data matched that reported by Driver and co-workers: ${ }^{18}{ }^{1} \mathrm{H}$ NMR $\left(500 \mathrm{MHz}, \mathrm{CDCl}_{3}\right) \delta 7.38(\mathrm{t}, J=7.3 \mathrm{~Hz}, 2 \mathrm{H}), 7.34-7.30(\mathrm{~m}, 1 \mathrm{H}), 7.27(\mathrm{~d}, J=7.4 \mathrm{~Hz}, 2 \mathrm{H})$, $2.63-2.44(\mathrm{~m}, 3 \mathrm{H}), 2.31-2.21(\mathrm{~m}, 1 \mathrm{H}), 2.09-1.98(\mathrm{~m}, 1 \mathrm{H}), 1.59-1.44(\mathrm{~m}, 2 \mathrm{H}), 0.94(\mathrm{~s}, 9 \mathrm{H}) ;{ }^{13} \mathrm{C} \mathrm{NMR}(125 \mathrm{MHz}$, $\left.\mathrm{CDCl}_{3}\right) \delta 143.7(\mathrm{C}), 137.1(\mathrm{C}), 131.0(\mathrm{C}), 128.4(\mathrm{CH}), 128.1(\mathrm{CH}), 127.9(\mathrm{CH}), 118.1\left(\mathrm{q}, J_{C F}=320 \mathrm{~Hz}, \mathrm{C}\right), 43.7(\mathrm{CH}), 32.9$ $\left(\mathrm{CH}_{2}\right), 32.2\left(\mathrm{CH}_{2}\right), 29.1\left(\mathrm{CH}_{2}\right), 27.2\left(\mathrm{CH}_{3}\right), 24.4\left(\mathrm{CH}_{2}\right)$. The crude vinyl triflate was used in the subsequent cross-coupling reaction without additional purification.

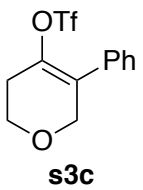

5-Phenyl-3,6-dihydro-2H-pyran-4-yl trifluoromethanesulfonate s3c. ${ }^{19}$ The general method was followed using $0.705 \mathrm{~g}$ of 3-phenyltetrahydro- $4 \mathrm{H}$-pyran-4-one $(4.0 \mathrm{mmol}), 0.320 \mathrm{~g}$ of $\mathrm{NaH}(8.0 \mathrm{mmol}, 60 \% \mathrm{w} / \mathrm{w}), 1.57 \mathrm{~g}$ of $N$-phenylbis(trifluoromethanesulfonamide) $(11.0 \mathrm{mmol})$ in $16 \mathrm{~mL}$ of DMF $(0.25 \mathrm{M})$. Purification by MPLC (2:98 to 5:95 EtOAc:hexanes) afforded the product as colorless oil (0.604 g, 49\%). Triflate s3c was reported by Driver and co-workers: ${ }^{19}$

17. Rigby, J. H.; Qabar, M. J. Am. Chem. Soc. 1991, 113, 8975.

18. Deng, T.; Mazumdar, W.; Ford, R. L.; Jana, N.; Izar, R.; Wink, D. J.; Driver, T. G. J. Am. Chem. Soc. 2020, 142, 4456.

19. Su, N.; Deng, T.; Wink, D. J.; Driver, T. G. Org. Lett. 2017, 19, 3990. 
${ }^{1} \mathrm{H}$ NMR $\left(500 \mathrm{MHz}, \mathrm{CDCl}_{3}\right) \delta 7.39(\mathrm{dt}, J=12.9,7.1 \mathrm{~Hz}, 3 \mathrm{H}), 7.30(\mathrm{~d}, J=7.3 \mathrm{~Hz}, 2 \mathrm{H}), 4.43(\mathrm{~d}, J=3.3 \mathrm{~Hz}, 2 \mathrm{H}), 4.00(\mathrm{t}, J$ $=5.7 \mathrm{~Hz}, 2 \mathrm{H}), 2.72-2.57(\mathrm{~m}, 2 \mathrm{H}) ;{ }^{13} \mathrm{C} \mathrm{NMR}\left(125 \mathrm{MHz}, \mathrm{CDCl}_{3}\right) \delta 140.4(\mathrm{C}), 132.2(\mathrm{C}), 130.2(\mathrm{C}), 128.8(\mathrm{CH}), 128.6(\mathrm{CH})$, $128.3(\mathrm{CH}), 118.1\left(\mathrm{q}, J_{C F}=320 \mathrm{~Hz}, \mathrm{C}\right), 68.4\left(\mathrm{CH}_{2}\right), 64.7\left(\mathrm{CH}_{2}\right), 28.3\left(\mathrm{CH}_{2}\right)$. The crude vinyl triflate was used in the subsequent cross-coupling reaction without additional purification.

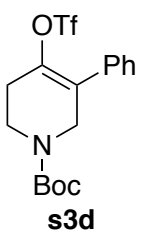

Tert-butyl 5-phenyl-4-(((trifluoromethyl)sulfonyl)oxy)-3,6-dihydropyridine-1(2H)-carboxylate s3d. ${ }^{18}$ The general method was followed using $1.10 \mathrm{~g}$ of tert-butyl 4-oxo-3-phenylpiperidine-1-carboxylate (4.0 mmol), $0.320 \mathrm{~g}$ of $\mathrm{NaH}(8.0$ mmol, $60 \% \mathrm{w} / \mathrm{w}), 1.57 \mathrm{~g}$ of $N$-phenyl-bis(trifluoromethanesulfonamide) $(11.0 \mathrm{mmol})$ in $16 \mathrm{~mL}$ of DMF $(0.25 \mathrm{M})$. Purification by MPLC (2:98 to 5:95 EtOAc:hexanes) afforded the product as yellow oil $(0.978 \mathrm{~g}, 60 \%)$. The spectral data matched that reported by Driver and co-workers: ${ }^{18}{ }^{1} \mathrm{H}$ NMR $\left(500 \mathrm{MHz}, \mathrm{CDCl}_{3}\right) \delta 7.38-7.30(\mathrm{~m}, 3 \mathrm{H}), 7.27(\mathrm{~d}, J=7.5 \mathrm{~Hz}$, $2 \mathrm{H}), 4.21(\mathrm{~s}, 2 \mathrm{H}), 3.74-3.62(\mathrm{~m}, 2 \mathrm{H}), 2.56(\mathrm{td}, J=6.0,3.2 \mathrm{~Hz}, 2 \mathrm{H}), 1.47(\mathrm{~s}, 9 \mathrm{H}) ;{ }^{13} \mathrm{C} \mathrm{NMR}\left(125 \mathrm{MHz}, \mathrm{CDCl}_{3}\right) \delta 154.2$ $(\mathrm{C}), 141.5(\mathrm{C}), 133.3(\mathrm{C}), 129.2(\mathrm{C}), 128.7(\mathrm{CH}), 128.5(\mathrm{CH}), 128.3(\mathrm{CH}), 118.1(\mathrm{q}, J=320.0 \mathrm{~Hz}), 80.5(\mathrm{C}), 60.2\left(\mathrm{CH}_{2}\right)$, $46.8\left(\mathrm{~d}, J=66.8 \mathrm{~Hz}, \mathrm{CH}_{2}\right), 40.6\left(\mathrm{~d}, J=109.8 \mathrm{~Hz}, \mathrm{CH}_{2}\right), 28.2\left(\mathrm{CH}_{3}\right)$. The crude vinyl triflate was used in the subsequent cross-coupling reaction without additional purification.<smiles>c1ccc2c(c1)CCCC2</smiles>

2-Phenyl-3,4-dihydronaphthalen-1-yl trifluoromethanesulfonate s3e. Th general method was followed using $0.889 \mathrm{~g}$ of 2-phenyl-3,4-dihydronaphthalen-1(2H)-one (4.0 mmol), $0.320 \mathrm{~g}$ of NaH $(8.0 \mathrm{mmol}, 60 \% \mathrm{w} / \mathrm{w}), 1.57 \mathrm{~g}$ of $N$-phenylbis(trifluoromethanesulfonamide) $(11.0 \mathrm{mmol})$ in $16 \mathrm{~mL}$ of DMF $(0.25 \mathrm{M})$. Purification by MPLC (2:98 to $3: 97$ EtOAc:hexanes) afforded the product as white solid $(1.01 \mathrm{~g}, 71 \%)$. The crude vinyl triflate was used in the subsequent cross-coupling reaction without additional purification.

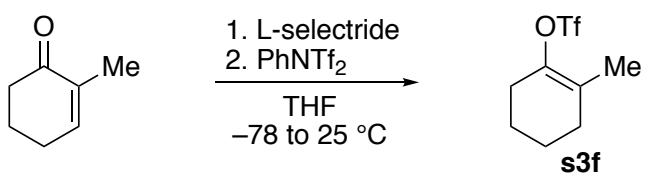

2-Methylcyclohex-1-en-1-yl trifluoromethanesulfonate s3f. ${ }^{20}$ To a cold $-78{ }^{\circ} \mathrm{C}$ solution of $0.55 \mathrm{~g}$ of 2 methylcyclohexenone (5.0 mmol, 1.0 equiv) in $13 \mathrm{~mL}$ of THF was added $5.3 \mathrm{~mL}$ of a $1 \mathrm{M}$ solution of L-Selectride ${ }^{\circledR}$ in THF (5.25 mmol, 1.05 equiv). After $1 \mathrm{~h}, 1.79 \mathrm{~g}$ of $N$-phenyl-bis(trifluoromethanesulfonamide) (10.1 mmol, 1.01 equiv) in $13 \mathrm{~mL}$ of THF was added. The resulting solution was then allowed to warm to room temperature. After stirring for overnight, the solution was diluted with $50 \mathrm{~mL}$ of hexanes and washed with $3 \times 50 \mathrm{~mL}$ of water. The combined aqueous phases were extracted with $2 \times 25 \mathrm{~mL}$ of hexanes. The combined organic phases were washed with $3 \times 50 \mathrm{~mL}$ of a $10 \%$ aq soln of $\mathrm{NaOH}$, $2 \times 50 \mathrm{~mL}$ of brine. The resulting organic phase was dried over $\mathrm{Na}_{2} \mathrm{SO}_{4}$, filtered and the filtrate was concentrated in vacuo. Purification by MPLC (2:98 EtOAc:hexanes) afforded the product as colorless oil $(0.733 \mathrm{~g}, 60 \%)$. The spectral data matched that reported by Crisp and Scott: ${ }^{20}{ }^{1} \mathrm{H}$ NMR $\left(500 \mathrm{MHz}, \mathrm{CDCl}_{3}\right) \delta 2.30(\mathrm{ddd}, J=6.6,4.4,2.2 \mathrm{~Hz}, 2 \mathrm{H}), 2.15-2.07(\mathrm{~m}, 2 \mathrm{H})$, $1.77-1.69(\mathrm{~m}, 5 \mathrm{H}), 1.64-1.57(\mathrm{~m}, 2 \mathrm{H}) ;{ }^{13} \mathrm{C} \mathrm{NMR}\left(125 \mathrm{MHz}, \mathrm{CDCl}_{3}\right) \delta 143.3(\mathrm{C}), 126.4(\mathrm{C}), 118.4\left(\mathrm{q}, J_{C F}=319 \mathrm{~Hz}, \mathrm{C}\right)$, $30.6\left(\mathrm{CH}_{2}\right), 27.5\left(\mathrm{CH}_{2}\right), 23.2\left(\mathrm{CH}_{2}\right), 21.7\left(\mathrm{CH}_{2}\right), 16.6\left(\mathrm{CH}_{3}\right)$. The crude vinyl triflate was used in the subsequent crosscoupling reaction without additional purification.

20. Crisp, G. T.; Scott, William J.; Synthesis. 1985, 3, 335. 


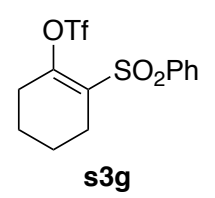

2-(Phenylsulfonyl)cyclohex-1-en-1-yl trifluoromethanesulfonate s3g. The general method was followed using $0.953 \mathrm{~g}$ of 2-(phenylsulfonyl)cyclohexan-1-one $(4.0 \mathrm{mmol}), 0.320 \mathrm{~g}$ of $\mathrm{NaH}(8.0 \mathrm{mmol}, 60 \% \mathrm{w} / \mathrm{w}), 1.57 \mathrm{~g}$ of $\mathrm{N}$-phenylbis(trifluoromethanesulfonamide) $(11.0 \mathrm{mmol})$ in $16 \mathrm{~mL}$ of DMF $(0.25 \mathrm{M})$. Purification by MPLC (2:98 to 3:97 EtOAc:hexanes) afforded the product as yellow oil $(1.01 \mathrm{~g}, 71 \%):{ }^{1} \mathrm{H}$ NMR $\left(500 \mathrm{MHz}, \mathrm{CDCl}_{3}\right) \delta 7.95(\mathrm{~d}, J=7.7 \mathrm{~Hz}, 2 \mathrm{H})$, $7.66(\mathrm{t}, J=7.5 \mathrm{~Hz}, 1 \mathrm{H}), 7.56(\mathrm{t}, J=7.7 \mathrm{~Hz}, 2 \mathrm{H}), 2.55(\mathrm{tt}, J=5.7,2.7 \mathrm{~Hz}, 2 \mathrm{H}), 2.45(\mathrm{td}, J=5.9,2.9 \mathrm{~Hz}, 2 \mathrm{H}), 1.73(\mathrm{t}, J=5.9$ $\mathrm{Hz}, 2 \mathrm{H}), 1.68$ (q, $J=6.9,4.7 \mathrm{~Hz}, 2 \mathrm{H}) ;{ }^{13} \mathrm{C} \mathrm{NMR}\left(125 \mathrm{MHz}, \mathrm{CDCl}_{3}\right) \delta 152.3(\mathrm{C}), 140.0(\mathrm{C}), 134.1(\mathrm{CH}), 129.6(\mathrm{C}), 129.3$ $(\mathrm{CH}), 128.0(\mathrm{CH}), 118.23(\mathrm{q}, J=320.6 \mathrm{~Hz}), 28.8\left(\mathrm{CH}_{2}\right), 26.1\left(\mathrm{CH}_{2}\right), 21.9\left(\mathrm{CH}_{2}\right), 21.3\left(\mathrm{CH}_{2}\right)$. The crude vinyl triflate was used in the subsequent cross-coupling reaction without additional purification.
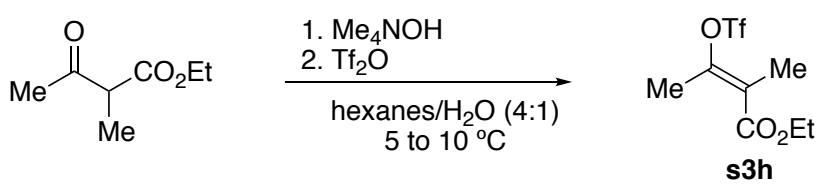

Ethyl (E)-2-methyl-3-(((trifluoromethyl)sulfonyl)oxy)but-2-enoate $\mathbf{s 3 h} .{ }^{21}$ Following the report by Frantz and coworkers, to a cooled $0{ }^{\circ} \mathrm{C}$ solution of $0.63 \mathrm{~mL}$ of ethyl 2-methyl-3-oxobutanoate (4.0 mmol, 1.0 equiv) in $20 \mathrm{~mL}$ of hexanes $(0.2 \mathrm{M})$ and $5 \mathrm{~mL}$ of water was added $7.18 \mathrm{~mL}$ of a $25 \mathrm{wt} \%$ aq soln of tetramethylammonium hydroxide $(20 \mathrm{mmol}, 5.0$ equiv) in one portion. After 5 minutes, $1.68 \mathrm{~mL}$ of triflic anhydride (10 mmol 2.5 equiv) was added dropwise. After $15 \mathrm{~min}$, the biphasic solution was diluted with $5 \mathrm{~mL}$ of $\mathrm{H}_{2} \mathrm{O}$ and the layers were separated. The aqueous layer was extracted with 2 $\times 10 \mathrm{~mL}$ of EtOAc. The combined organic layers were washed with $5 \mathrm{~mL}$ of $\mathrm{H}_{2} \mathrm{O}, 5 \mathrm{~mL}$ of brine, and dried over $\mathrm{Na}_{2} \mathrm{SO}_{4}$. The organic layer was filtered, and the filtrate was concentrated in vacuo to yield the vinyl triflate as a colorless oil (1.04 g, 47\%). Triflate s3i was previously reported by Frantz and co-workers: ${ }^{21}{ }^{1} \mathrm{H}$ NMR $\left(500 \mathrm{MHz}, \mathrm{CDCl}_{3}\right) \delta 4.23(\mathrm{q}, J=7.1 \mathrm{~Hz}$, 2H), $2.45-2.33(\mathrm{~m}, 3 \mathrm{H}), 1.97(\mathrm{q}, J=4.4,2.3 \mathrm{~Hz}, 3 \mathrm{H}), 1.30(\mathrm{t}, J=7.1 \mathrm{~Hz}, 3 \mathrm{H}) ;{ }^{13} \mathrm{C} \mathrm{NMR}\left(125 \mathrm{MHz}, \mathrm{CDCl}_{3}\right) \delta 166.3(\mathrm{C})$, $154.5(\mathrm{C}), 122.8(\mathrm{C}), 118.2\left(\mathrm{q}, J_{C F}=319 \mathrm{~Hz}, \mathrm{C}\right), 61.4\left(\mathrm{CH}_{2}\right), 18.9\left(\mathrm{CH}_{3}\right), 13.9\left(\mathrm{CH}_{3}\right), 13.7\left(\mathrm{CH}_{3}\right)$. The crude vinyl triflate was used in the subsequent cross-coupling reaction without additional purification.

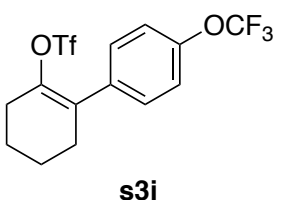

4'-(Trifluoromethoxy)-3,4,5,6-tetrahydro-[1,1'-biphenyl]-2-yl trifluoromethanesulfonate s3i. The general method was followed using $1.03 \mathrm{~g}$ of 2-(4-trifuoromethoxyphenyl)cyclohexan-1-one $(4.0 \mathrm{mmol}), 0.320 \mathrm{~g}$ of NaH $(8.0 \mathrm{mmol}, 60 \% \mathrm{w} / \mathrm{w})$, $1.57 \mathrm{~g}$ of $\mathrm{N}$-phenyl-bis(trifluoromethanesulfonamide) $(11.0 \mathrm{mmol})$ in $16 \mathrm{~mL}$ of DMF $(0.25 \mathrm{M})$. The crude vinyl triflate was used in the subsequent cross-coupling reaction without additional purification.

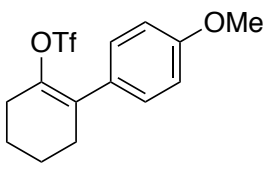

s3j

4'-Methoxy-3,4,5,6-tetrahydro-[1,1'-biphenyl]-2-yl trifluoromethanesulfonate s3j. The general method was followed using $0.817 \mathrm{~g}$ of 2-(4-methoxyphenyl)cyclohexan-1-one (4.0 mmol), $0.320 \mathrm{~g}$ of NaH $(8.0 \mathrm{mmol}, 60 \% \mathrm{w} / \mathrm{w}), 1.57 \mathrm{~g}$ of $\mathrm{N}$ -

21. Babinski, D.; Soltani, O.; Frantz, D. E. Org. Lett. 2008, 10, 2901. 
phenyl-bis(trifluoromethanesulfonamide) $(11.0 \mathrm{mmol})$ in $16 \mathrm{~mL}$ of DMF $(0.25 \mathrm{M})$. The crude vinyl triflate was used in the subsequent cross-coupling reaction without additional purification.

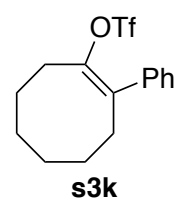

(Z)-2-Phenylcyclooct-1-en-1-yl trifluoromethanesulfonate s3k. ${ }^{18}$ The general method was followed using $0.809 \mathrm{~g}$ of 2 phenylcyclooctanone $(4.0 \mathrm{mmol}), 0.320 \mathrm{~g}$ of $\mathrm{NaH}(8.0 \mathrm{mmol}, 60 \% \mathrm{w} / \mathrm{w}), 1.57 \mathrm{~g}$ of $\mathrm{N}$-phenyl-bis(trifluoromethanesulfonamide) $(11.0 \mathrm{mmol})$ in $16 \mathrm{~mL}$ of DMF $(0.25 \mathrm{M})$. Purification by MPLC (2:98 to 3:97 EtOAc:hexanes) afforded the product as a yellow oil $(0.628 \mathrm{~g}, 47 \%)$. The spectral data matched that reported by Driver and co-workers: $:{ }^{18} \mathrm{H} \mathrm{NMR}(500$ $\left.\mathrm{MHz}, \mathrm{CDCl}_{3}\right) \delta 7.36(\mathrm{t}, J=7.5 \mathrm{~Hz}, 2 \mathrm{H}), 7.33-7.28(\mathrm{~m}, 1 \mathrm{H}), 7.24(\mathrm{~d}, J=7.7 \mathrm{~Hz}, 2 \mathrm{H}), 2.66(\mathrm{t}, J=6.2 \mathrm{~Hz}, 2 \mathrm{H}), 2.54(\mathrm{t}, J=$ $5.7 \mathrm{~Hz}, 2 \mathrm{H}), 1.84$ (pentet, $J=5.6 \mathrm{~Hz}, 2 \mathrm{H}), 1.66(\mathrm{dq}, J=13.9,6.7,6.0 \mathrm{~Hz}, 6 \mathrm{H}) ;{ }^{13} \mathrm{C} \mathrm{NMR}\left(125 \mathrm{MHz}, \mathrm{CDCl}_{3}\right) \delta 145.0(\mathrm{C})$, $137.4(\mathrm{C}), 133.3(\mathrm{C}), 128.4(\mathrm{CH}), 128.3(\mathrm{CH}), 127.8(\mathrm{CH}), 118.1\left(1, J_{C F}=319.5 \mathrm{~Hz}, \mathrm{C}\right), 33.1\left(\mathrm{CH}_{2}\right), 30.7\left(\mathrm{CH}_{2}\right), 28.5\left(\mathrm{CH}_{2}\right)$, $28.5\left(\mathrm{CH}_{2}\right), 26.4\left(\mathrm{CH}_{2}\right), 26.0\left(\mathrm{CH}_{2}\right)$. The crude vinyl triflate was used in the subsequent cross-coupling reaction without additional purification.

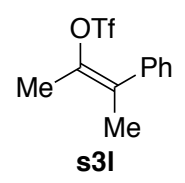

(Z)-3-Phenylbut-2-en-2-yl trifluoromethanesulfonate $\mathbf{s 3 1 .}{ }^{22}$ The general method was followed using $0.953 \mathrm{~g}$ of 3 phenylbutan-2-one (4.0 mmol), $0.320 \mathrm{~g}$ of NaH $(8.0 \mathrm{mmol}, 60 \% \mathrm{w} / \mathrm{w}), 1.57 \mathrm{~g}$ of $N$-phenyl-bis(trifluoromethanesulfonamide) $(11.0 \mathrm{mmol})$ in $16 \mathrm{~mL}$ of DMF $(0.25 \mathrm{M})$. Purification by MPLC (2:98 to 3:97 EtOAc:hexanes) afforded the product as yellow oil $(1.01 \mathrm{~g}, 71 \%)$. Triflate $\mathbf{s 3 1}$ was previously reported by Driver and co-workers: ${ }^{22}{ }^{1} \mathrm{H} \mathrm{NMR}\left(500 \mathrm{MHz} \mathrm{CDCl}_{3}\right) \delta$ $7.40(\mathrm{t}, J=7.5 \mathrm{~Hz}, 2 \mathrm{H}), 7.33(\mathrm{t}, J=7.3 \mathrm{~Hz}, 1 \mathrm{H}), 7.28(\mathrm{~d}, J=7.6 \mathrm{~Hz}, 2 \mathrm{H}), 2.25(\mathrm{~s}, 3 \mathrm{H}), 2.10(\mathrm{~s}, 3 \mathrm{H}) ;{ }^{13} \mathrm{C} \mathrm{NMR}(125 \mathrm{MHz}$, $\left.\mathrm{CDCl}_{3}\right) \delta 164.0(\mathrm{C}), 140.7(\mathrm{C}), 138.1(\mathrm{C}), 128.3(\mathrm{CH}), 128.1(\mathrm{CH}), 127.8(\mathrm{CH}), 118.2\left(\mathrm{q}, J_{C F}=320 \mathrm{~Hz}, \mathrm{C}\right), 20.0\left(\mathrm{CH}_{2}\right)$, $17.2\left(\mathrm{CH}_{2}\right)$. The crude vinyl triflate was used in the subsequent cross-coupling reaction without additional purification.

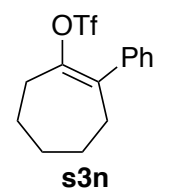

2-Phenylcyclohept-1-en-1-yl trifluoromethanesulfonate $\mathbf{s 3 m .} .^{22}$ The general method was followed using $0.753 \mathrm{~g}$ of $2-$ phenylcycloheptan-1-one $\quad(4.0 \mathrm{mmol}), \quad 0.320 \mathrm{~g}$ of $\mathrm{NaH} \quad(8.0 \mathrm{mmol}, \quad 60 \% \mathrm{w} / \mathrm{w}), \quad 1.57 \mathrm{~g}$ of $\mathrm{N}$-phenylbis(trifluoromethanesulfonamide) $(11.0 \mathrm{mmol})$ in $16 \mathrm{~mL}$ of DMF $(0.25 \mathrm{M})$. Purification by MPLC (2:98 to 3:97 EtOAc:hexanes) afforded the product as an orange oil $(0.679 \mathrm{~g}, 53 \%)$. Triflate $\mathbf{s 3 m}$ was previously reported by Driver and co-workers: ${ }^{22}{ }^{1} \mathrm{H}$ NMR $\left(500 \mathrm{MHz}, \mathrm{CDCl}_{3}\right) \delta 7.36(\mathrm{t}, J=7.5 \mathrm{~Hz}, 2 \mathrm{H}), 7.30(\mathrm{~m}, 1 \mathrm{H}), 7.25(\mathrm{~m}, 2 \mathrm{H}), 2.70(\mathrm{~m}, 2 \mathrm{H}), 2.56(\mathrm{~m}$, 2H), $1.84-1.77(\mathrm{~m}, 6 \mathrm{H}) ;{ }^{13} \mathrm{C}$ NMR $\left(125 \mathrm{MHz}, \mathrm{CDCl}_{3}\right) \delta 147.5(\mathrm{C}), 139.1(\mathrm{C}), 131.1(\mathrm{C}), 128.2(\mathrm{CH}), 127.9(\mathrm{CH}), 127.6$ $(\mathrm{CH}), 118.1\left(\mathrm{q}, J_{C F}=317.5 \mathrm{~Hz}, \mathrm{CF}_{3}\right), 34.0\left(\mathrm{CH}_{2}\right), 35.4\left(\mathrm{CH}_{2}\right), 30.9\left(\mathrm{CH}_{2}\right), 25.9\left(\mathrm{CH}_{2}\right), 24.5\left(\mathrm{CH}_{2}\right)$. The crude vinyl triflate was used in the subsequent cross-coupling reaction without additional purification.

22. Jana, N.; Zhou, F.; Driver, T. G. J. Am. Chem. Soc. 2015, 137, 6738. 


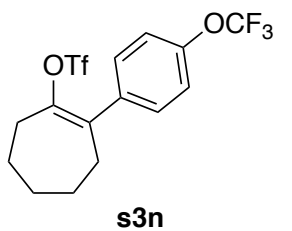

2-(4-(Trifluoromethoxy)phenyl)cyclohept-1-en-1-yl trifluoromethanesulfonate $\mathbf{s 3 n}{ }^{18}$ The general method was followed using $1.09 \mathrm{~g}$ of 2-(4-(trifluoromethoxy)phenyl)cyclohept-1-ome $(4.0 \mathrm{mmol}), 0.320 \mathrm{~g}$ of NaH $(8.0 \mathrm{mmol}, 60 \%$ w/w), $1.57 \mathrm{~g}$ of $N$-phenyl-bis(trifluoromethanesulfonamide) $(11.0 \mathrm{mmol})$ in $16 \mathrm{~mL}$ of DMF $(0.25 \mathrm{M})$. Purification by MPLC (2:98 to 3:97 EtOAc:hexanes) afforded the product as a yellow oil $(0.469 \mathrm{~g}, 30 \%)$. The spectral data matched that reported by Driver and co-workers: ${ }^{18}{ }^{1} \mathrm{H}$ NMR $\left(500 \mathrm{MHz}, \mathrm{CDCl}_{3}\right) \delta 7.27(\mathrm{~d}, J=8.3 \mathrm{~Hz}, 2 \mathrm{H}), 7.20(\mathrm{~d}, J=8.3 \mathrm{~Hz}, 2 \mathrm{H}), 2.84-2.62$ $(\mathrm{m}, 2 \mathrm{H}), 2.59-2.44(\mathrm{~m}, 2 \mathrm{H}), 2.01-1.69(\mathrm{~m}, 6 \mathrm{H}) ;{ }^{13} \mathrm{C}$ NMR $\left(125 \mathrm{MHz}, \mathrm{CDCl}_{3}\right) \delta 148.6(\mathrm{C}), 148.1(\mathrm{C}), 137.7$ (C), 134.9 $(\mathrm{C}), 129.5(\mathrm{CH}), 120.7(\mathrm{CH}), 120.5\left(\mathrm{q}, J_{C F}=257 \mathrm{~Hz}, \mathrm{C}\right), 118.1$ (q, $\left.J_{C F}=320 \mathrm{~Hz}, \mathrm{C}\right), 33.8\left(\mathrm{CH}_{2}\right), 33.4\left(\mathrm{CH}_{2}\right), 30.7\left(\mathrm{CH}_{2}\right)$, $25.8\left(\mathrm{CH}_{2}\right), 24.3\left(\mathrm{CH}_{2}\right)$. The crude vinyl triflate was used in the subsequent cross-coupling reaction without additional purification.

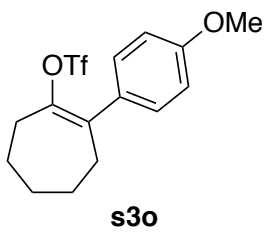

2-(4-Methoxyphenyl)cycloheptan-1-en-1-yl trifluoromethanesulfonate s3o. ${ }^{18}$ The general method was followed using $0.873 \mathrm{~g}$ of 2-(4-methoxyphenyl)cycloheptan-1-one $(4.0 \mathrm{mmol}), 0.320 \mathrm{~g}$ of NaH $(8.0 \mathrm{mmol}, 60 \% \mathrm{w} / \mathrm{w}), 1.57 \mathrm{~g}$ of $\mathrm{N}$-phenylbis(trifluoromethanesulfonamide) $(11.0 \mathrm{mmol})$ in $16 \mathrm{~mL}$ of DMF $(0.25 \mathrm{M})$. Purification by MPLC (2:98 to 3:97 EtOAc:hexanes) afforded the product as a yellow oil $(0.448 \mathrm{~g}, 32 \%)$. The spectral data matched that reported by Driver and co-workers: ${ }^{18}{ }^{1} \mathrm{H}$ NMR $\left(500 \mathrm{MHz}, \mathrm{CDCl}_{3}\right) \delta 7.20(\mathrm{~d}, J=8.8 \mathrm{~Hz}, 2 \mathrm{H}), 6.90(\mathrm{~d}, J=8.7 \mathrm{~Hz}, 2 \mathrm{H}), 3.81(\mathrm{~s}, 3 \mathrm{H}), 2.69(\mathrm{dd}, J=$ 7.0, $3.6 \mathrm{~Hz}, 2 \mathrm{H}), 2.59-2.48(\mathrm{~m}, 2 \mathrm{H}), 1.87-1.71(\mathrm{~m}, 6 \mathrm{H}) ;{ }^{13} \mathrm{C}$ NMR (125 MHz, $\left.\mathrm{CDCl}_{3}\right) \delta 159.1(\mathrm{C}), 147.2(\mathrm{C}), 131.0(\mathrm{C})$, $130.0(\mathrm{C}), 129.3(\mathrm{CH}), 118.2\left(\mathrm{q}, J_{C F}=320 \mathrm{~Hz}, \mathrm{C}\right), 113.6(\mathrm{CH}), 55.1\left(\mathrm{CH}_{3}\right), 34.1\left(\mathrm{CH}_{2}\right), 33.4\left(\mathrm{CH}_{2}\right), 30.9\left(\mathrm{CH}_{2}\right), 25.9\left(\mathrm{CH}_{2}\right)$, $24.5\left(\mathrm{CH}_{2}\right)$. The crude vinyl triflate was used in the subsequent cross-coupling reaction without additional purification.

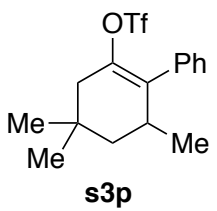

4,4,6-Trimethyl-3,4,5,6-tetrahydro-[1,1'-biphenyl]-2-yl trifluoromethanesulfonate s3p. The general method was followed using $0.865 \mathrm{~g}$ of 3,5,5-trimethyl-2-phenylcyclohexan-1-one $(4.0 \mathrm{mmol}), 0.320 \mathrm{~g}$ of NaH $(8.0 \mathrm{mmol}, 60 \% \mathrm{w} / \mathrm{w})$, $1.57 \mathrm{~g}$ of $\mathrm{N}$-phenyl-bis(trifluoromethanesulfonamide) $(11.0 \mathrm{mmol})$ in $16 \mathrm{~mL}$ of DMF $(0.25 \mathrm{M})$. The crude vinyl triflate was used in the subsequent cross-coupling reaction without additional purification.

\section{Preparation of ortho-substituted aniline.}

1. General procedure.

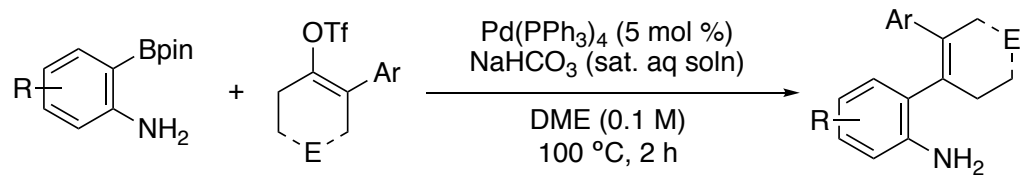

Method A: To a mixture of vinyl triflate ( $2.0 \mathrm{mmol}, 1.0$ equiv), 2-aminophenylboronic acid pinacol ester (2.4 mmol, 1.2 equiv), and $\mathrm{Pd}\left(\mathrm{PPh}_{3}\right)_{4}(0.1 \mathrm{mmol}, 5 \mathrm{~mol} \%)$ in dimethoxyethane $(0.1 \mathrm{M})$ was added a saturated aq soln of $\mathrm{NaHCO}_{3}(2$ 
$\mathrm{mL} / \mathrm{mmol}$ of boronic ester). The resulting mixture was heated to $100^{\circ} \mathrm{C}$ using an oil bath. After $2 \mathrm{~h}$, the mixture was cooled to room temperature and diluted with a saturated aq soln of $\mathrm{NH}_{4} \mathrm{Cl}$. The resulting mixture was extracted with $3 \times 10 \mathrm{~mL}$ of ethyl acetate, and the combined organic phases were washed with $30 \mathrm{~mL}$ of brine. The resulting organic phase was dried over $\mathrm{Na}_{2} \mathrm{SO}_{4}$, filtered and the filtrate was concentrated in vacuo. Purification using MPLC afforded the product.

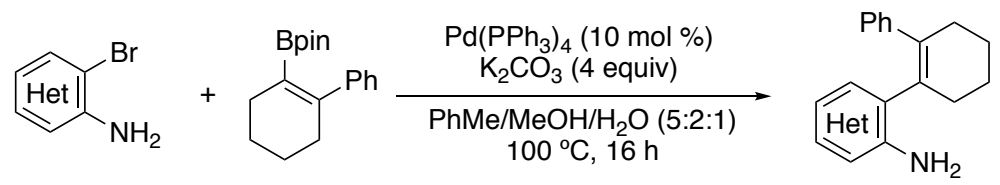

Method B: Following the report by Ortgies and Breder, ${ }^{23}$ to a mixture of heterocyclic bromide (2.0 mmol, 1.0 equiv), vinyl boronic acid ester (2.2 mmol, 1.1 equiv), and $\mathrm{Pd}\left(\mathrm{PPh}_{3}\right)_{4}(0.2 \mathrm{mmol}, 10 \mathrm{~mol} \%)$ in $25 \mathrm{~mL}$ of toluene, $10 \mathrm{~mL}$ of methanol and $5 \mathrm{~mL}$ of water was added $\mathrm{K}_{2} \mathrm{CO}_{3}\left(8.0 \mathrm{mmol}, 4\right.$ equiv). The resulting mixture was heated to $100{ }^{\circ} \mathrm{C}$ using an oil bath. After $16 \mathrm{~h}$, the mixture was cooled to room temperature and diluted with a saturated aq soln of $\mathrm{NH}_{4} \mathrm{Cl}$. The resulting mixture was extracted with $3 \times 10 \mathrm{~mL}$ of ethyl acetate, and the combined organic phases were washed with $30 \mathrm{~mL}$ of brine. The resulting organic phase was dried over $\mathrm{Na}_{2} \mathrm{SO}_{4}$, filtered and the filtrate was concentrated in vacuo. Purification using MPLC afforded the product.

2. Characterization data for ortho-substituted anilines.

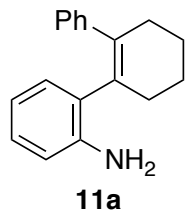

$3^{\prime}, 4^{\prime}, 5^{\prime}, 6^{\prime}$-Tetrahydro-[1,1':2',1''-terphenyl]-2-amine 11a. ${ }^{18}$ Method A was followed using $0.612 \mathrm{~g}$ of vinyl triflate (2.00 $\mathrm{mmol}), 0.526 \mathrm{~g}$ of 2-aminophenylboronic acid pinacol ester $(2.40 \mathrm{mmol}), 0.116 \mathrm{~g}$ of $\mathrm{Pd}\left(\mathrm{PPh}_{3}\right)_{4}(0.10 \mathrm{mmol}) \mathrm{in} 20 \mathrm{~mL}$ of dimethoxyethane $(0.1 \mathrm{M})$. Purification by MPLC (2:98 to 15:85 EtOAc:hexanes) afforded the product as a yellow oil ( 0.497 g, 99\%). The spectral data matched that reported by Driver and co-workers: ${ }^{18}{ }^{1} \mathrm{H}$ NMR $\left(500 \mathrm{MHz}, \mathrm{CDCl}_{3}\right) \delta 7.21-7.14(\mathrm{~m}$, $5 \mathrm{H}), 7.04-7.01(\mathrm{~m}, 2 \mathrm{H}), 6.74(\mathrm{t}, J=7.3 \mathrm{~Hz}, 1 \mathrm{H}), 6.56(\mathrm{~d}, J=7.8 \mathrm{~Hz}, 1 \mathrm{H}), 3.57$ (br s, 2H), $2.70-2.66(\mathrm{~m}, 1 \mathrm{H}), 2.54-2.46$ $(\mathrm{m}, 3 \mathrm{H}), 1.96(\mathrm{~m}, 4 \mathrm{H}) ;{ }^{13} \mathrm{C}$ NMR $\left(125 \mathrm{MHz}, \mathrm{CDCl}_{3}\right) \delta 143.1(\mathrm{C}), 142.8(\mathrm{C}), 136.2(\mathrm{C}), 132.8(\mathrm{C}), 130.0(\mathrm{CH}), 129.7(\mathrm{C})$, $128.0(\mathrm{CH}), 127.7(\mathrm{CH}), 127.4(\mathrm{CH}), 126.3(\mathrm{C}), 118.4(\mathrm{CH}), 115.4(\mathrm{CH}), 31.7\left(\mathrm{CH}_{2}\right), 31.4\left(\mathrm{CH}_{2}\right), 23.6\left(\mathrm{CH}_{2}\right), 23.4\left(\mathrm{CH}_{2}\right)$. ATR-FTIR (thin film): 3375, 3054, 2926, 2855, 1611, 1492, 1447, 1295, 747, $698 \mathrm{~cm}^{-1}$. HRMS (ESI) $\mathrm{m} / z$ calculated for $\mathrm{C}_{18} \mathrm{H}_{20} \mathrm{~N}(\mathrm{M}+\mathrm{H})^{+}: 250.1596$, found: 250.1596 .

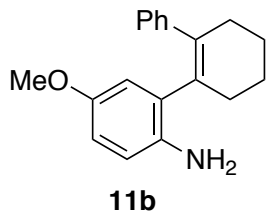

5-Methoxy-3',4',5',6'-tetrahydro-[1,1':2',1''-terphenyl]-2-amine 11b. ${ }^{18}$ Method A was followed using $0.612 \mathrm{~g}$ of vinyl triflate $(2.00 \mathrm{mmol}), 0.600 \mathrm{~g}$ of 4-methoxy-2-(3,3,4,4-tetramethylborolan-1-yl)aniline $(2.40 \mathrm{mmol}), 0.116 \mathrm{~g}$ of $\mathrm{Pd}\left(\mathrm{PPh}_{3}\right)_{4}$ $(0.10 \mathrm{mmol})$ in $20 \mathrm{~mL}$ of dimethoxyethane $(0.1 \mathrm{M})$. Purification by MPLC (2:98 to 20:80 EtOAc:hexanes) afforded the product as a pink solid $(0.447 \mathrm{~g}, 80 \%)$. The spectral data matched that reported by Driver and co-workers: ${ }^{18} \mathrm{mp}=120{ }^{\circ} \mathrm{C}$; ${ }^{1} \mathrm{H}$ NMR $\left(500 \mathrm{MHz}, \mathrm{CDCl}_{3}\right) \delta 7.19-7.13(\mathrm{~m}, 4 \mathrm{H}), 7.13-7.06(\mathrm{~m}, 1 \mathrm{H}), 6.62-6.55(\mathrm{~m}, 2 \mathrm{H}), 6.47(\mathrm{~d}, J=8.4 \mathrm{~Hz}, 1 \mathrm{H}), 3.70$ (s, 3H), 3.30 (br s, 2H), $2.70-2.57(\mathrm{~m}, 1 \mathrm{H}), 2.52-2.34(\mathrm{~m}, 3 \mathrm{H}), 1.97-1.82(\mathrm{~m}, 4 \mathrm{H}) ;{ }^{13} \mathrm{C}$ NMR $\left(125 \mathrm{MHz}, \mathrm{CDCl}_{3}\right) \delta 152.4$ $(\mathrm{C}), 143.0(\mathrm{C}), 136.5(\mathrm{C}), 136.1(\mathrm{C}), 132.8(\mathrm{C}), 131.0(\mathrm{C}), 127.9(\mathrm{CH}), 127.7(\mathrm{CH}), 126.3(\mathrm{CH}), 116.6(\mathrm{CH}), 115.4(\mathrm{CH})$, $113.1(\mathrm{CH}), 55.7\left(\mathrm{CH}_{3}\right), 31.6\left(\mathrm{CH}_{2}\right), 31.3\left(\mathrm{CH}_{2}\right), 23.5\left(\mathrm{CH}_{2}\right), 23.2\left(\mathrm{CH}_{2}\right)$. ATR-FTIR (thin film): $2928,2855,1599,1499$,

23. Ortgies, S.; Breder, A. Org. Lett. 2015, 17, 2748. 
$1465,1278,1169,1278,1169,1041,810,759,699 \mathrm{~cm}^{-1}$. HRMS (ESI) $m / z$ calculated for $\mathrm{C}_{19} \mathrm{H}_{22} \mathrm{NO}(\mathrm{M}+\mathrm{H})^{+}: 280.1701$, found: 280.1698 .

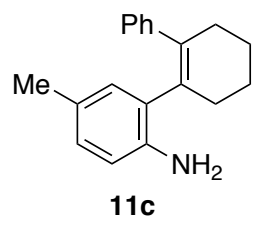

5-Methyl-3',4',5',6'-tetrahydro-[1,1':2',1'-terphenyl]-2-amine 11c. ${ }^{18}$ Method A was followed using 0.306 g of vinyl triflate $(1.00 \mathrm{mmol}), 0.280 \mathrm{~g}$ of 4-methyl-2-(3,3,4,4-tetramethylborolan-1-yl)aniline $(1.20 \mathrm{mmol}), 0.058 \mathrm{~g}$ of $\left.\mathrm{Pd}_{(\mathrm{PPh}}\right)_{4}$ $(0.05 \mathrm{mmol})$ in $10 \mathrm{~mL}$ of dimethoxyethane $(0.1 \mathrm{M})$. Purification by MPLC (2:98 to 15:85 EtOAc:hexanes) afforded the product as a brown solid $(0.172 \mathrm{~g}, 65 \%)$. The spectral data matched that reported by Driver and co-workers: $:^{18} \mathrm{mp}=90{ }^{\circ} \mathrm{C}$; ${ }^{1} \mathrm{H}$ NMR $\left(500 \mathrm{MHz}, \mathrm{CDCl}_{3}\right) \delta 7.19-7.04(\mathrm{~m}, 5 \mathrm{H}), 6.80(\mathrm{~s}, 1 \mathrm{H}), 6.77(\mathrm{~d}, J=8.3 \mathrm{~Hz}, 1 \mathrm{H}), 6.43(\mathrm{~d}, J=8.0 \mathrm{~Hz}, 1 \mathrm{H}), 3.38(\mathrm{br}$ $\mathrm{s}, 2 \mathrm{H}), 2.67-2.55(\mathrm{~m}, 1 \mathrm{H}), 2.46-2.32(\mathrm{~m}, 3 \mathrm{H}), 2.21(\mathrm{~s}, 3 \mathrm{H}), 1.94-1.81(\mathrm{~m}, 4 \mathrm{H}),{ }^{13} \mathrm{C} \mathrm{NMR}\left(125 \mathrm{MHz}, \mathrm{CDCl}_{3}\right) \delta 143.1$ (C), $140.0(\mathrm{C}), 135.7(\mathrm{C}), 132.8(\mathrm{C}), 130.3(\mathrm{CH}), 129.8(\mathrm{C}), 127.9(\mathrm{CH}), 127.6(\mathrm{CH}), 127.4(\mathrm{C}), 126.2(\mathrm{CH}), 115.5(\mathrm{CH})$, $31.8\left(\mathrm{CH}_{2}\right), 31.3\left(\mathrm{CH}_{2}\right), 23.5\left(\mathrm{CH}_{2}\right), 23.3\left(\mathrm{CH}_{2}\right), 20.6\left(\mathrm{CH}_{3}\right)$ only visible peaks. ATR-FTIR (thin film): 3017, 2924,2855 , $2832,1616,1501,1442,1289,1151,811,758,698 \mathrm{~cm}^{-1}$. HRMS (ESI) $m / z$ calculated for $\mathrm{C}_{19} \mathrm{H}_{22} \mathrm{~N}(\mathrm{M}+\mathrm{H})^{+}: 264.1752$, found: 264.1748 .

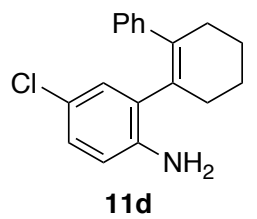

5-Chloro-3',4',5',6'-tetrahydro-[1,1':2',1'-terphenyl]-2-amine 11d. ${ }^{18}$ Method A was followed using $0.612 \mathrm{~g}$ of vinyl triflate (2.00 mmol), $0.686 \mathrm{~g}$ of 4-chloro-2-(3,3,4,4-tetramethylborolan-1-yl)aniline (2.40 mmol), $0.116 \mathrm{~g}$ of $\mathrm{Pd}\left(\mathrm{PPh}_{3}\right)_{4}(0.10$ $\mathrm{mmol})$ in $20 \mathrm{~mL}$ of dimethoxyethane $(0.1 \mathrm{M})$. Purification by MPLC (2:98 to 15:85 EtOAc:hexanes) afforded the product as a yellow solid $(0.554 \mathrm{~g}, 98 \%)$. The spectral data matched that reported by Driver and co-workers: $:{ }^{18} \mathrm{mp}=67{ }^{\circ} \mathrm{C} ;{ }^{1} \mathrm{H} \mathrm{NMR}$ $\left(500 \mathrm{MHz}, \mathrm{CDCl}_{3}\right) \delta 7.24-7.14(\mathrm{~m}, 5 \mathrm{H}), 7.07(\mathrm{~d}, J=2.6 \mathrm{~Hz}, 1 \mathrm{H}), 6.96(\mathrm{dd}, J=8.6,2.6 \mathrm{~Hz}, 1 \mathrm{H}), 6.41(\mathrm{~d}, J=8.5 \mathrm{~Hz}, 1 \mathrm{H})$, $3.58(\mathrm{br} \mathrm{s}, 2 \mathrm{H}), 2.72-2.63(\mathrm{~m}, 1 \mathrm{H}), 2.53-2.46(\mathrm{~m}, 1 \mathrm{H}), 2.46-2.38(\mathrm{~m}, 2 \mathrm{H}), 1.93(\mathrm{dq}, J=13.2,7.1,6.6 \mathrm{~Hz}, 4 \mathrm{H}) ;{ }^{13} \mathrm{C}$ NMR (125 MHz, $\left.\mathrm{CDCl}_{3}\right) \delta 142.8(\mathrm{C}), 141.5(\mathrm{C}), 137.2(\mathrm{C}), 131.6(\mathrm{C}), 131.2(\mathrm{C}), 129.5(\mathrm{CH}), 127.9(\mathrm{CH}), 127.9(\mathrm{CH})$, $127.2(\mathrm{CH}), 126.6(\mathrm{CH}), 122.6(\mathrm{C}), 116.5(\mathrm{CH}), 31.5\left(\mathrm{CH}_{2}\right), 31.4\left(\mathrm{CH}_{2}\right), 23.4\left(\mathrm{CH}_{2}\right), 23.2\left(\mathrm{CH}_{2}\right)$. ATR-FTIR (thin film): $2928,2856,1611,1487,1403,1288,1147,811,759,699,607 \mathrm{~cm}^{-1}$. HRMS (ESI) $\mathrm{m} / z$ calculated for $\mathrm{C}_{18} \mathrm{H}_{19} \mathrm{NCl}(\mathrm{M}+\mathrm{H})^{+}$: 284.1206, found: 284.1202 .

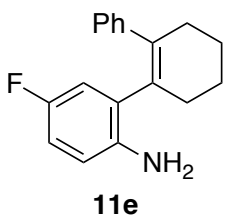

5-Fluoro-3',4',5',6'-tetrahydro-[1,1':2',1'-terphenyl]-2-amine 11e. ${ }^{18}$ Method A was followed using $0.612 \mathrm{~g}$ of vinyl triflate (2.00 mmol), $0.569 \mathrm{~g}$ of 4-fluoro-2-(3,3,4,4-tetramethylborolan-1-yl)aniline (2.40 mmol), $0.116 \mathrm{~g}$ of $\mathrm{Pd}\left(\mathrm{PPh}_{3}\right)_{4}(0.10$ $\mathrm{mmol})$ in $20 \mathrm{~mL}$ of dimethoxyethane $(0.1 \mathrm{M})$. Purification by MPLC (2:98 to 15:85 EtOAc:hexanes) afforded the product as a brown oil $(0.492 \mathrm{~g}, 92 \%)$. The spectral data matched that reported by Driver and co-workers: ${ }^{18}{ }^{1} \mathrm{H} \mathrm{NMR}(500 \mathrm{MHz}$, $\left.\mathrm{CDCl}_{3}\right) \delta 7.20-7.10(\mathrm{~m}, 5 \mathrm{H}), 6.74(\mathrm{dd}, J=9.5,3.0 \mathrm{~Hz}, 1 \mathrm{H}), 6.68(\mathrm{td}, J=8.5,3.0 \mathrm{~Hz}, 1 \mathrm{H}), 6.43(\mathrm{dd}, J=8.8,4.9 \mathrm{~Hz}, 1 \mathrm{H})$, 3.42 (br s, 2H), $2.69-2.57(\mathrm{~m}, 1 \mathrm{H}), 2.52-2.31(\mathrm{~m}, 3 \mathrm{H}), 1.97-1.84(\mathrm{~m}, 4 \mathrm{H}) ;{ }^{13} \mathrm{C} \mathrm{NMR}\left(125 \mathrm{MHz}, \mathrm{CDCl}_{3}\right) \delta 156.12(\mathrm{~d}$, $\left.J_{C F}=235.9 \mathrm{~Hz}, \mathrm{C}\right), 142.8(\mathrm{C}), 138.9(\mathrm{C}), 136.9(\mathrm{C}), 131.9(\mathrm{C}), 130.98\left(\mathrm{~d}, J_{C F}=7.0 \mathrm{~Hz}, \mathrm{C}\right), 127.8(\mathrm{CH}), 127.8(\mathrm{CH}), 126.5$ $(\mathrm{CH}), 116.2(\mathrm{CH}), 116.1\left(\mathrm{~d}, J_{C F}=16.4 \mathrm{~Hz}, \mathrm{CH}\right), 113.8\left(\mathrm{~d}, J_{C F}=22.2 \mathrm{~Hz}, \mathrm{CH}\right), 31.33\left(\mathrm{CH}_{2}\right), 23.36\left(\mathrm{CH}_{2}\right), 23.15\left(\mathrm{CH}_{2}\right)$ only peaks visible; ${ }^{19} \mathrm{~F}$ NMR $\left(282 \mathrm{MHz}, \mathrm{CDCl}_{3}\right) \delta$-127.4. ATR-FTIR (thin film): 2928, 2857, 2832, 1600, 1494, 1423, 1259, $1163,1013,892,810,757,699 \mathrm{~cm}^{-1}$. HRMS (ESI) $\mathrm{m} / z$ calculated for $\mathrm{C}_{18} \mathrm{H}_{19} \mathrm{NF}(\mathrm{M}+\mathrm{H})^{+}: 268.1502$, found: 268.1498 . 


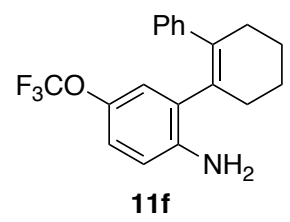

5-(Trifluoromethoxy)-3',4',5',6'-tetrahydro-[1,1':2',1''-terphenyl]-2-amine 11f. ${ }^{18}$ Method A was followed using 0.612 $\mathrm{g}$ of vinyl triflate $(2.00 \mathrm{mmol}), 0.725 \mathrm{~g}$ of 2-(3,3,4,4-tetramethylborolan-1-yl)-4-(trifluoromethoxy)aniline (2.40 mmol), $0.116 \mathrm{~g}$ of $\mathrm{Pd}\left(\mathrm{PPh}_{3}\right)_{4}(0.10 \mathrm{mmol})$ in $20 \mathrm{~mL}$ of dimethoxyethane $(0.1 \mathrm{M})$. Purification by MPLC (2:98 to 15:85 EtOAc:hexanes) afforded the product as a red oil $(0.605 \mathrm{~g}, 91 \%)$. The spectral data matched that reported by Driver and coworkers: ${ }^{18}{ }^{1} \mathrm{H}$ NMR $\left(500 \mathrm{MHz}, \mathrm{CDCl}_{3}\right) \delta 7.26-7.13(\mathrm{~m}, 5 \mathrm{H}), 6.97(\mathrm{~d}, J=2.8 \mathrm{~Hz}, 1 \mathrm{H}), 6.88(\mathrm{dd}, J=8.7,2.8 \mathrm{~Hz}, 1 \mathrm{H}), 6.45$ $(\mathrm{d}, J=8.7 \mathrm{~Hz}, 1 \mathrm{H}), 3.62(\mathrm{br} \mathrm{s}, 2 \mathrm{H}), 2.73-2.64(\mathrm{~m}, 1 \mathrm{H}), 2.57-2.38(\mathrm{~m}, 3 \mathrm{H}), 1.96(\mathrm{dh}, J=13.4,6.0 \mathrm{~Hz}, 4 \mathrm{H}) ;{ }^{13} \mathrm{C} \mathrm{NMR}$ $\left(125 \mathrm{MHz}, \mathrm{CDCl}_{3}\right) \delta 142.7(\mathrm{C}), 141.8(\mathrm{C}), 141.0(\mathrm{C}), 137.6(\mathrm{C}), 131.7(\mathrm{C}), 130.4(\mathrm{C}), 127.9(\mathrm{CH}), 127.8(\mathrm{CH}), 126.6(\mathrm{CH})$, $123.1(\mathrm{CH}), 120.9\left(\mathrm{q}, J_{C F}=255 \mathrm{~Hz}, \mathrm{C}\right), 120.4(\mathrm{CH}), 115.6(\mathrm{CH}), 31.4\left(\mathrm{CH}_{2}\right), 31.1\left(\mathrm{CH}_{2}\right), 23.4\left(\mathrm{CH}_{2}\right), 23.1\left(\mathrm{CH}_{2}\right) ;{ }^{19} \mathrm{~F} \mathrm{NMR}$ $\left(282 \mathrm{MHz}, \mathrm{CDCl}_{3}\right) \delta$-58.9. ATR-FTIR (thin film): 2930, 1617, 1499, 1252, 1211, 1152, 817, 758, 699, $617 \mathrm{~cm}^{-1}$. HRMS (ESI) $\mathrm{m} / z$ calculated for $\mathrm{C}_{19} \mathrm{H}_{19} \mathrm{NOF}_{3}(\mathrm{M}+\mathrm{H})^{+}: 334.1419$, found: 334.1421 .

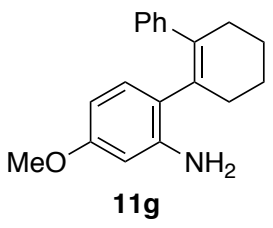

4-Methoxy-3', $4^{\prime}, 5^{\prime}, 6^{\prime}$-tetrahydro-[1,1':2',1'-terphenyl]-2-amine 11g. ${ }^{18}$ Method A was followed using $0.612 \mathrm{~g}$ of vinyl triflate $(2.00 \mathrm{mmol}), 0.598 \mathrm{~g}$ of 5-methoxy-2-(3,3,4,4-tetramethylborolan-1-yl)aniline $(2.40 \mathrm{mmol}), 0.116 \mathrm{~g} \mathrm{of} \mathrm{Pd}\left(\mathrm{PPh}_{3}\right)_{4}$ $(0.10 \mathrm{mmol})$ in $20 \mathrm{~mL}$ of dimethoxyethane $(0.1 \mathrm{M})$. Purification by MPLC (2:98 to 20:80 EtOAc:hexanes) afforded the product as a yellow foam $(0.556 \mathrm{~g}, 99 \%)$. The spectral data matched that reported by Driver and co-workers: ${ }^{18}{ }^{1} \mathrm{H}$ NMR $\left(500 \mathrm{MHz}, \mathrm{CDCl}_{3}\right) \delta 7.14-7.05(\mathrm{~m}, 5 \mathrm{H}), 6.84(\mathrm{~d}, J=8.3 \mathrm{~Hz}, 1 \mathrm{H}), 6.24(\mathrm{dd}, J=8.3,2.5 \mathrm{~Hz}, 1 \mathrm{H}), 6.06(\mathrm{~d}, J=2.5 \mathrm{~Hz}, 1 \mathrm{H})$, 3.69 (s, 3H), 3.41 (br s, 2H), $2.57(\mathrm{~d}, J=17.1 \mathrm{~Hz}, 1 \mathrm{H}), 2.40(\mathrm{~d}, J=17.6 \mathrm{~Hz}, 1 \mathrm{H}), 2.33(\mathrm{dq}, J=6.1,2.8 \mathrm{~Hz}, 2 \mathrm{H}), 1.85$ (dhextet, $J=12.3,6.6,5.7 \mathrm{~Hz}, 4 \mathrm{H}) ;{ }^{13} \mathrm{C}$ NMR $\left(125 \mathrm{MHz}, \mathrm{CDCl}_{3}\right) \delta 159.0(\mathrm{C}), 143.7(\mathrm{C}), 143.2(\mathrm{C}), 136.2(\mathrm{C}), 132.3(\mathrm{C})$, 130.6 (CH), $127.9(\mathrm{CH}), 127.6(\mathrm{CH}), 126.1(\mathrm{CH}), 122.7(\mathrm{C}), 104.0(\mathrm{CH}), 100.8(\mathrm{CH}), 55.0\left(\mathrm{CH}_{3}\right), 31.9\left(\mathrm{CH}_{2}\right), 31.3\left(\mathrm{CH}_{2}\right)$, $23.4\left(\mathrm{CH}_{2}\right), 23.3\left(\mathrm{CH}_{2}\right)$. ATR-FTIR (thin film): 2927, 2831, 1615, 1575, 1506, 1444, 1294, 1205, 1168, 1033, 758, 699,590 $\mathrm{cm}^{-1}$. HRMS (ESI) $\mathrm{m} / z$ calculated for $\mathrm{C}_{19} \mathrm{H}_{22} \mathrm{NO}(\mathrm{M}+\mathrm{H})^{+}: 280.1701$, found: 280.1699 .

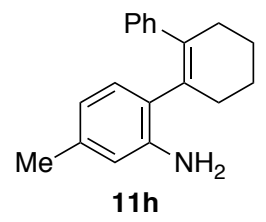

4-Methyl-3',4',5',6'-tetrahydro-[1,1':2',1'-terphenyl]-2-amine 11h. ${ }^{18}$ Method A was followed using $0.612 \mathrm{~g}$ of vinyl triflate $(2.00 \mathrm{mmol}), 0.560 \mathrm{~g}$ of 5-methyl-2-(3,3,4,4-tetramethylborolan-1-yl)aniline $(2.40 \mathrm{mmol}), 0.116 \mathrm{~g}$ of $\mathrm{Pd}\left(\mathrm{PPh}_{3}\right)_{4}$ $(0.10 \mathrm{mmol})$ in $20 \mathrm{~mL}$ of dimethoxyethane $(0.1 \mathrm{M})$. Purification by MPLC (2:98 to 15:85 EtOAc:hexanes) afforded the product as a yellow oil $(0.430 \mathrm{~g}, 82 \%)$. The spectral data matched that reported by Driver and co-workers: ${ }^{18}{ }^{1} \mathrm{H} \mathrm{NMR}(500$ $\left.\mathrm{MHz}, \mathrm{CDCl}_{3}\right) \delta 7.32-7.25(\mathrm{~m}, 4 \mathrm{H}), 7.22(\mathrm{dq}, J=5.8,2.8 \mathrm{~Hz}, 1 \mathrm{H}), 7.02(\mathrm{~d}, J=7.6 \mathrm{~Hz}, 1 \mathrm{H}), 6.65(\mathrm{~d}, J=7.6 \mathrm{~Hz}, 1 \mathrm{H}), 6.44$ $(\mathrm{s}, 1 \mathrm{H}), 3.59(\mathrm{br} \mathrm{s}, 2 \mathrm{H}), 2.82-2.71(\mathrm{~m}, 1 \mathrm{H}), 2.66-2.50(\mathrm{~m}, 3 \mathrm{H}), 2.34(\mathrm{~s}, 3 \mathrm{H}), 2.04(\mathrm{dh}, J=12.6,6.5,5.4 \mathrm{~Hz}, 4 \mathrm{H}) ;{ }^{13} \mathrm{C}$ NMR (125 MHz, $\left.\mathrm{CDCl}_{3}\right) \delta 143.4(\mathrm{C}), 142.7(\mathrm{C}), 137.0(\mathrm{C}), 136.1(\mathrm{C}), 132.9(\mathrm{C}), 129.9(\mathrm{CH}), 128.1(\mathrm{CH}), 127.8(\mathrm{CH})$, $127.0(\mathrm{C}), 126.4(\mathrm{CH}), 119.4(\mathrm{CH}), 116.3(\mathrm{CH}), 32.0\left(\mathrm{CH}_{2}\right), 31.5\left(\mathrm{CH}_{2}\right), 23.7\left(\mathrm{CH}_{2}\right), 23.5\left(\mathrm{CH}_{2}\right), 21.4\left(\mathrm{CH}_{3}\right)$. ATR-FTIR (thin film): 2925, 2856, 2832, 1619, 1575, 1506, 1490, 1441, 1297, 796, 758, $698 \mathrm{~cm}^{-1}$. HRMS (ESI) $\mathrm{m} / z$ calculated for $\mathrm{C}_{19} \mathrm{H}_{22} \mathrm{~N}(\mathrm{M}+\mathrm{H})^{+}: 264.1752$, found: 264.1749 . 


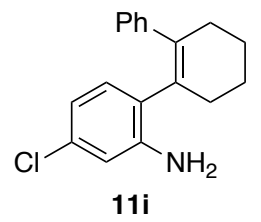

4-Chloro-3',4',5',6'-tetrahydro-[1,1':2',1'-terphenyl]-2-amine 11i. ${ }^{18}$ Method A was followed using $0.612 \mathrm{~g}$ of vinyl triflate $(2.00 \mathrm{mmol}), 0.609 \mathrm{~g}$ of 5-chloro-2-(3,3,4,4-tetramethylborolan-1-yl)aniline $(2.40 \mathrm{mmol}), 0.116 \mathrm{~g}$ of $\mathrm{Pd}\left(\mathrm{PPh}_{3}\right)_{4}(0.10$ $\mathrm{mmol})$ in $20 \mathrm{~mL}$ of dimethoxyethane $(0.1 \mathrm{M})$. Purification by MPLC (2:98 to 15:85 EtOAc:hexanes) afforded the product as a yellow oil $(0.548 \mathrm{~g}, 97 \%)$. The spectral data matched that reported by Driver and co-workers: ${ }^{18}{ }^{1} \mathrm{H} \mathrm{NMR}(500 \mathrm{MHz}$, $\left.\mathrm{CDCl}_{3}\right) \delta 7.27-7.18(\mathrm{~m}, 5 \mathrm{H}), 6.98(\mathrm{~d}, J=8.1 \mathrm{~Hz}, 1 \mathrm{H}), 6.74(\mathrm{~d}, J=8.1 \mathrm{~Hz}, 1 \mathrm{H}), 6.55(\mathrm{~s}, 1 \mathrm{H}), 3.67(\mathrm{br}, 2 \mathrm{H}), 2.73-2.66$ $(\mathrm{m}, 1 \mathrm{H}), 2.58-2.51(\mathrm{~m}, 1 \mathrm{H}), 2.48-2.41(\mathrm{~m}, 2 \mathrm{H}), 1.97(\mathrm{dt}, J=12.5,6.4 \mathrm{~Hz}, 4 \mathrm{H}) ;{ }^{13} \mathrm{C}$ NMR $\left(125 \mathrm{MHz}, \mathrm{CDCl}_{3}\right) \delta 144.1$ $(\mathrm{C}), 143.0(\mathrm{C}), 137.2(\mathrm{C}), 132.6(\mathrm{C}), 131.8(\mathrm{C}), 131.1(\mathrm{CH}), 128.1(\mathrm{C}), 128.0(\mathrm{CH}), 126.6(\mathrm{CH}), 118.2(\mathrm{CH}), 115.0(\mathrm{CH})$, $31.6\left(\mathrm{CH}_{2}\right), 31.5\left(\mathrm{CH}_{2}\right), 23.5\left(\mathrm{CH}_{2}\right), 23.3\left(\mathrm{CH}_{2}\right)$ only visible peaks. ATR-FTIR (thin film): 3384, 2927, 2856, 2832, 1610, $1566,1488,1414,1260,1094,900,794,758,699 \mathrm{~cm}^{-1}$. HRMS (ESI) $\mathrm{m} / z$ calculated for $\mathrm{C}_{18} \mathrm{H}_{19} \mathrm{NCl}(\mathrm{M}+\mathrm{H})^{+}: 284.1206$, found: 284.1204 .

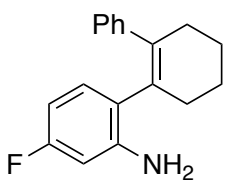

11j

4-Fluoro-3', $\mathbf{4}^{\prime}, \mathbf{5}^{\prime}, \mathbf{6}^{\prime}$-tetrahydro-[1,1':2',1'-terphenyl]-2-amine $11 \mathbf{j} .{ }^{18}$ Method A was followed using $0.612 \mathrm{~g}$ of vinyl triflate (2.00 mmol), $0.569 \mathrm{~g}$ of 5-fluoro-2-(3,3,4,4-tetramethylborolan-1-yl)aniline $(2.40 \mathrm{mmol}), 0.116 \mathrm{~g}$ of $\left.\mathrm{Pd}_{(\mathrm{PPh}}\right)_{4}(0.10$ $\mathrm{mmol})$ in $20 \mathrm{~mL}$ of dimethoxyethane $(0.1 \mathrm{M})$. Purification by MPLC (2:98 to 15:85 EtOAc:hexanes) afforded the product as a red oil $(0.457 \mathrm{~g}, 85 \%)$. The spectral data matched that reported by Driver and co-workers: ${ }^{18}{ }^{1} \mathrm{H} \mathrm{NMR}\left(500 \mathrm{MHz}, \mathrm{CDCl}_{3}\right)$ $\delta 7.23-7.17(\mathrm{~m}, 2 \mathrm{H}), 7.16-7.09(\mathrm{~m}, 3 \mathrm{H}), 6.92(\mathrm{t}, J=7.5 \mathrm{~Hz}, 1 \mathrm{H}), 6.40(\mathrm{td}, J=8.5,2.6 \mathrm{~Hz}, 1 \mathrm{H}), 6.23(\mathrm{dd}, J=10.8,2.6$ $\mathrm{Hz}, 1 \mathrm{H}), 3.62(\mathrm{br} \mathrm{s}, 2 \mathrm{H}), 2.67-2.59(\mathrm{~m}, 1 \mathrm{H}), 2.53-2.44(\mathrm{~m}, 1 \mathrm{H}), 2.42-2.30(\mathrm{~m}, 2 \mathrm{H}), 1.98-1.85(\mathrm{~m}, 4 \mathrm{H}) ;{ }^{13} \mathrm{C} \mathrm{NMR}(125$ $\left.\mathrm{MHz}, \mathrm{CDCl}_{3}\right) \delta 162.38\left(\mathrm{~d}, J_{C F}=242 \mathrm{~Hz}, \mathrm{C}\right), 144.3\left(\mathrm{~d}, J_{C F}=10.8 \mathrm{~Hz}, \mathrm{C}\right), 143.0(\mathrm{C}), 137.0(\mathrm{C}), 131.9(\mathrm{C}), 131.0\left(\mathrm{~d}, J_{C F}=9.7\right.$ $\mathrm{Hz}, \mathrm{CH}), 127.9(\mathrm{CH}), 127.8(\mathrm{CH}), 126.4(\mathrm{CH}), 104.8\left(\mathrm{~d}, J_{C F}=21.3 \mathrm{~Hz}, \mathrm{CH}\right), 101.8\left(\mathrm{~d}, J_{C F}=24.3 \mathrm{~Hz}, \mathrm{CH}\right), 31.7\left(\mathrm{CH}_{2}\right), 31.4$ $\left(\mathrm{CH}_{2}\right), 23.4\left(\mathrm{CH}_{2}\right), 23.3\left(\mathrm{CH}_{2}\right) ;{ }^{19} \mathrm{~F}$ NMR $\left(282 \mathrm{MHz}, \mathrm{CDCl}_{3}\right) \delta$-116.5. ATR-FTIR (thin film): 3477, 3386, 2928, 2857, 1615, $1591,1502,1433,1283,1163,1068,974,838,758,699 \mathrm{~cm}^{-1}$. HRMS (ESI) $m / z$ calculated for $\mathrm{C}_{18} \mathrm{H}_{19} \mathrm{NF}(\mathrm{M}+\mathrm{H})^{+}: 268.1502$, found: 268.1496 .

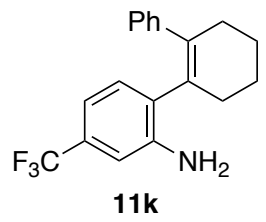

4-(Trifluoromethyl)-3',4',5',6'-tetrahydro-[1,1':2',1'-terphenyl]-2-amine 11k. ${ }^{18}$ Method A was followed using $0.612 \mathrm{~g}$ of vinyl triflate $(2.00 \mathrm{mmol}), 0.689 \mathrm{~g}$ of 2-(3,3,4,4-tetramethylborolan-1-yl)-5-(trifluoromethyl)aniline $(2.40 \mathrm{mmol}), 0.116$ $\mathrm{g}$ of $\mathrm{Pd}\left(\mathrm{PPh}_{3}\right)_{4}(0.10 \mathrm{mmol})$ in $20 \mathrm{~mL}$ of dimethoxyethane $(0.1 \mathrm{M})$. Purification by MPLC (2:98 to 15:85 EtOAc:hexanes) afforded the product as a yellow solid $(0.633 \mathrm{~g}, 100 \%)$. The spectral data matched that reported by Driver and co-workers: ${ }^{18}$ $\mathrm{mp}=63{ }^{\circ} \mathrm{C} ;{ }^{1} \mathrm{H} \mathrm{NMR}\left(500 \mathrm{MHz}, \mathrm{CDCl}_{3}\right) \delta 7.29-7.20(\mathrm{~m}, 5 \mathrm{H}), 7.18(\mathrm{~d}, J=8.0 \mathrm{~Hz}, 1 \mathrm{H}), 7.04(\mathrm{~d}, J=7.9 \mathrm{~Hz}, 1 \mathrm{H}), 6.81(\mathrm{~s}$, $1 \mathrm{H}), 3.79(\mathrm{br} \mathrm{s}, 2 \mathrm{H}), 2.78-2.68(\mathrm{~m}, 1 \mathrm{H}), 2.63-2.55(\mathrm{~m}, 1 \mathrm{H}), 2.51-2.41(\mathrm{~m}, 2 \mathrm{H}), 2.08-1.91(\mathrm{~m}, 4 \mathrm{H}) ;{ }^{3} \mathrm{C}$ NMR $(125$ $\left.\mathrm{MHz}, \mathrm{CDCl}_{3}\right) \delta 143.3(\mathrm{C}), 142.8(\mathrm{C}), 137.5(\mathrm{C}), 133.0(\mathrm{C}), 131.7(\mathrm{C}), 130.5(\mathrm{CH}), 129.5\left(\mathrm{q}, J_{C F}=31.8 \mathrm{~Hz}, \mathrm{C}\right), 128.0(\mathrm{CH})$, $127.9(\mathrm{CH}), 126.7(\mathrm{CH}), 124.6\left(\mathrm{q}, J_{C F}=270 \mathrm{~Hz}, \mathrm{C}\right), 114.7\left(\mathrm{q}, J_{C F}=4.2 \mathrm{~Hz}, \mathrm{CH}\right), 111.6\left(\mathrm{q}, J_{C F}=4.0 \mathrm{~Hz}, \mathrm{CH}\right), 31.5\left(\mathrm{CH}_{2}\right)$, $31.3\left(\mathrm{CH}_{2}\right), 23.4\left(\mathrm{CH}_{2}\right), 23.2\left(\mathrm{CH}_{2}\right) ;{ }^{19} \mathrm{~F}$ NMR $\left(282 \mathrm{MHz}, \mathrm{CDCl}_{3}\right) \delta$-63.0. ATR-FTIR (thin film): 2931, 1618, 1434, 1334, $1257,1163,1118,1087,910,760,670 \mathrm{~cm}^{-1}$. HRMS (ESI) $m / z$ calculated for $\mathrm{C}_{19} \mathrm{H}_{19} \mathrm{NF}_{3}(\mathrm{M}+\mathrm{H})^{+}: 318.1470$, found: 318.1461 . 


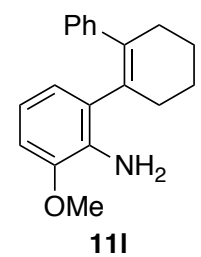

3-Methoxy-3',4',5',6'-tetrahydro-[1, $1^{\prime}: 2^{\prime}, 1^{\prime \prime}$-terphenyl]-2-amine $111 .{ }^{18}$ Method A was followed using $0.612 \mathrm{~g}$ of vinyl triflate $(2.00 \mathrm{mmol}), 0.598 \mathrm{~g}$ of 2-methoxy-6-(3,3,4,4-tetramethylborolan-1-yl)aniline $(2.40 \mathrm{mmol}), 0.116 \mathrm{~g}$ of $\mathrm{Pd}\left(\mathrm{PPh}_{3}\right)_{4}$ $(0.10 \mathrm{mmol})$ in $20 \mathrm{~mL}$ of dimethoxyethane $(0.1 \mathrm{M})$. Purification by MPLC (2:98 to 20:80 EtOAc:hexanes) afforded the product as a yellow oil $(0.400 \mathrm{~g}, 72 \%)$. The spectral data matched that reported by Driver and co-workers: ${ }^{18}{ }^{1} \mathrm{H}$ NMR $(500$ $\mathrm{MHz}^{\mathrm{CDCl}} \mathrm{CD}_{3} \delta 7.14-7.08(\mathrm{~m}, 4 \mathrm{H}), 7.08-7.02(\mathrm{~m}, 1 \mathrm{H}), 6.63-6.54(\mathrm{~m}, 3 \mathrm{H}), 3.76(\mathrm{~s}, 3 \mathrm{H}), 3.68(\mathrm{br} \mathrm{s}, 2 \mathrm{H}), 2.61-2.53(\mathrm{~m}$, $1 \mathrm{H}), 2.49-2.40(\mathrm{~m}, 1 \mathrm{H}), 2.40-2.28(\mathrm{~m}, 2 \mathrm{H}), 1.86(\mathrm{td}, J=11.5,11.0,5.7 \mathrm{~Hz}, 4 \mathrm{H}) ;{ }^{13} \mathrm{C}$ NMR $\left(125 \mathrm{MHz}, \mathrm{CDCl}_{3}\right) \delta 147.2$ $(\mathrm{C}), 143.2(\mathrm{C}), 136.1(\mathrm{C}), 132.7(\mathrm{C}), 129.8(\mathrm{C}), 128.0(\mathrm{C}), 127.9(\mathrm{CH}), 127.7(\mathrm{CH}), 126.3(\mathrm{CH}), 122.2(\mathrm{CH}), 117.4(\mathrm{CH})$, $108.3(\mathrm{CH}), 55.4\left(\mathrm{CH}_{3}\right), 31.4\left(\mathrm{CH}_{2}\right), 31.4\left(\mathrm{CH}_{2}\right), 23.6\left(\mathrm{CH}_{2}\right), 23.4\left(\mathrm{CH}_{2}\right)$. ATR-FTIR (thin film): 2928, 1833, 1568, 1472, $1440,1276,1195,1175,1056,1010,775,755,731,699 \mathrm{~cm}^{-1}$. HRMS (ESI) $m / z$ calculated for $\mathrm{C}_{19} \mathrm{H}_{22} \mathrm{NO}(\mathrm{M}+\mathrm{H})^{+}: 280.1701$, found: 280.1699 .

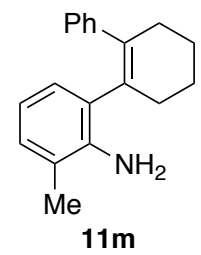

3-Methyl-3',4',5',6'-tetrahydro-[1,1':2',1'-terphenyl]-2-amine 11m. ${ }^{18}$ Method A was followed using $0.612 \mathrm{~g}$ of vinyl triflate $(2.00 \mathrm{mmol}), 0.560 \mathrm{~g}$ of 2-methyl-6-(3,3,4,4-tetramethylborolan-1-yl)aniline $(2.40 \mathrm{mmol}), 0.116 \mathrm{~g} \mathrm{of} \mathrm{Pd}\left(\mathrm{PPh}_{3}\right)_{4}$ $(0.10 \mathrm{mmol})$ in $20 \mathrm{~mL}$ of dimethoxyethane $(0.1 \mathrm{M})$. Purification by MPLC (2:98 to 15:85 EtOAc:hexanes) afforded the product as a yellow oil $(0.440 \mathrm{~g}, 84 \%)$. The spectral data matched that reported by Driver and co-workers: ${ }^{18}{ }^{1} \mathrm{H}$ NMR (500 $\left.\mathrm{MHz}, \mathrm{CDCl}_{3}\right) \delta 7.21(\mathrm{~d}, J=4.4 \mathrm{~Hz}, 4 \mathrm{H}), 7.18-7.12(\mathrm{~m}, 1 \mathrm{H}), 6.94(\mathrm{t}, J=6.8 \mathrm{~Hz}, 2 \mathrm{H}), 6.70(\mathrm{t}, J=7.5 \mathrm{~Hz}, 1 \mathrm{H}), 3.54($ br s, $2 \mathrm{H}), 2.75-2.65(\mathrm{~m}, 1 \mathrm{H}), 2.56(\mathrm{~d}, J=17.5 \mathrm{~Hz}, 1 \mathrm{H}), 2.52-2.41(\mathrm{~m}, 2 \mathrm{H}), 2.13(\mathrm{~s}, 3 \mathrm{H}), 1.97(\mathrm{dt}, J=20.1,6.2 \mathrm{~Hz}, 4 \mathrm{H}) ;{ }^{13} \mathrm{C}$ NMR (125 MHz, $\left.\mathrm{CDCl}_{3}\right) \delta 143.2(\mathrm{C}), 141.0(\mathrm{C}), 136.2(\mathrm{C}), 133.2(\mathrm{C}), 129.4(\mathrm{C}), 128.6(\mathrm{CH}), 127.9(\mathrm{CH}), 127.8(\mathrm{CH})$, $127.7(\mathrm{CH}), 126.3(\mathrm{CH}), 122.2(\mathrm{C}), 117.9(\mathrm{CH}), 31.7\left(\mathrm{CH}_{2}\right), 31.5\left(\mathrm{CH}_{2}\right), 23.6\left(\mathrm{CH}_{2}\right), 23.4\left(\mathrm{CH}_{2}\right), 17.9\left(\mathrm{CH}_{3}\right)$. ATR-FTIR (thin film): 3381, 3018, 2927, 2854, 1609, 1491, 1475, 1461, 1435, 1274, 1174, 1074, 753, 744, $698 \mathrm{~cm}^{-1}$. HRMS (ESI) $m / z$ calculated for $\mathrm{C}_{19} \mathrm{H}_{22} \mathrm{~N}(\mathrm{M}+\mathrm{H})^{+}: 264.1752$, found: 264.1748 .

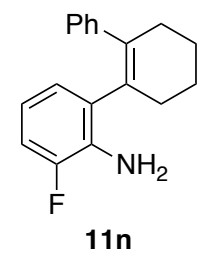

3-Fluoro-3', $4^{\prime}, 5^{\prime}, 6^{\prime}$-tetrahydro-[1,1':2',1'-terphenyl]-2-amine 11n. ${ }^{18}$ Method A was followed using $0.612 \mathrm{~g}$ of vinyl triflate $(2.00 \mathrm{mmol}), 0.569 \mathrm{~g}$ of 2 -fluoro-6-(3,3,4,4-tetramethylborolan-1-yl)aniline $(2.40 \mathrm{mmol}), 0.116 \mathrm{~g} \mathrm{of} \mathrm{Pd}\left(\mathrm{PPh}_{3}\right)_{4}(0.10$ $\mathrm{mmol})$ in $20 \mathrm{~mL}$ of dimethoxyethane $(0.1 \mathrm{M})$. Purification by MPLC (2:98 to 15:85 EtOAc:hexanes) afforded the product as a yellow oil $(0.513 \mathrm{~g}, 96 \%)$. The spectral data matched that reported by Driver and co-workers: ${ }^{18}{ }^{1} \mathrm{H} \mathrm{NMR}(500 \mathrm{MHz}$, $\left.\mathrm{CDCl}_{3}\right) \delta 7.25-7.14(\mathrm{~m}, 5 \mathrm{H}), 6.91-6.83(\mathrm{~m}, 2 \mathrm{H}), 6.66(\mathrm{td}, J=7.9,5.3 \mathrm{~Hz}, 1 \mathrm{H}), 3.68(\mathrm{br} \mathrm{s}, 2 \mathrm{H}), 2.75-2.66(\mathrm{~m}, 1 \mathrm{H}), 2.54$ $(\mathrm{d}, J=18.2 \mathrm{~Hz}, 1 \mathrm{H}), 2.48(\mathrm{qt}, J=5.7,3.7,2.9 \mathrm{~Hz}, 2 \mathrm{H}), 1.98(\mathrm{ddd}, J=16.0,8.0,5.0 \mathrm{~Hz}, 4 \mathrm{H}) ;{ }^{13} \mathrm{C} \mathrm{NMR}\left(125 \mathrm{MHz}, \mathrm{CDCl}_{3}\right)$ $\delta 151.9\left(\mathrm{~d}, J_{C F}=238 \mathrm{~Hz}, \mathrm{C}\right), 142.9(\mathrm{C}), 137.0(\mathrm{C}), 131.9\left(\mathrm{~d}, J_{C F}=3.2 \mathrm{~Hz}, \mathrm{C}\right), 131.7\left(\mathrm{~d}, J_{C F}=2.6 \mathrm{~Hz}, \mathrm{C}\right), 131.4\left(\mathrm{~d}, J_{C F}=\right.$ $12.1 \mathrm{~Hz}, \mathrm{C}), 127.9(\mathrm{CH}), 126.6(\mathrm{CH}), 125.3\left(\mathrm{~d}, J_{C F}=2.9 \mathrm{~Hz}, \mathrm{CH}\right), 117.6\left(\mathrm{~d}, J_{C F}=7.6 \mathrm{~Hz}, \mathrm{CH}\right), 113.0\left(\mathrm{~d}, J_{C F}=18.9 \mathrm{~Hz} \mathrm{CH}\right)$, $31.5\left(\mathrm{CH}_{2}\right), 31.4\left(\mathrm{CH}_{2}\right), 23.5\left(\mathrm{CH}_{2}\right), 23.3\left(\mathrm{CH}_{2}\right)$; only visible peaks; ${ }^{19} \mathrm{~F}$ NMR $\left(282 \mathrm{MHz}, \mathrm{CDCl}_{3}\right) \delta-135.1$. ATR-FTIR (thin 
film): $3477,3387,2928,2857,2833,1622,1597,1470,1272,1172,880,775,755,730,699 \mathrm{~cm}^{-1}$. HRMS (ESI) $\mathrm{m} / z$ calculated for $\mathrm{C}_{18} \mathrm{H}_{19} \mathrm{NF}(\mathrm{M}+\mathrm{H})^{+}: 268.1502$, found: 268.1494 .

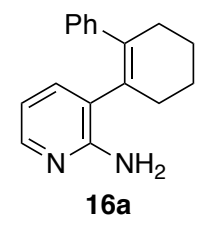

3-(3,4,5,6-Tetrahydro-[1,1'-biphenyl]-2-yl)pyridin-2-amine 16a. ${ }^{18}$ Method B was followed using $0.415 \mathrm{~g}$ of 3bromopyridin-2-amine $(2.00 \mathrm{mmol}), 0.569 \mathrm{~g}$ of vinyl boronic acid ester $(2.20 \mathrm{mmol}), 0.232 \mathrm{~g}$ of $\left.\mathrm{Pd}_{\left(\mathrm{PPh}_{3}\right)}\right)_{4}\left(0.20 \mathrm{mmol}^{2}\right.$, $1.10 \mathrm{~g} \mathrm{~K}_{2} \mathrm{CO}_{3}(8.0 \mathrm{mmol})$ in $25 \mathrm{~mL}$ of toluene, $10 \mathrm{~mL}$ of methanol and $5 \mathrm{~mL}$ of water. Purification by MPLC (2:98 to 40:60 EtOAc:hexanes) afforded the product as an orange solid $(0.215 \mathrm{~g}, 43 \%)$. The spectral data matched that reported by Driver and co-workers: ${ }^{18} \mathrm{mp}=88{ }^{\circ} \mathrm{C} ;{ }^{1} \mathrm{H}$ NMR $\left(500 \mathrm{MHz}, \mathrm{CDCl}_{3}\right) \delta 7.84(\mathrm{dd}, J=5.0,1.9 \mathrm{~Hz}, 1 \mathrm{H}), 7.15-7.09(\mathrm{~m}, 3 \mathrm{H}), 7.09-$ $7.06(\mathrm{~m}, 1 \mathrm{H}), 7.06-7.01(\mathrm{~m}, 2 \mathrm{H}), 6.51(\mathrm{dd}, J=7.3,5.0 \mathrm{~Hz}, 1 \mathrm{H}), 4.35(\mathrm{br} \mathrm{s}, 2 \mathrm{H}), 2.56-2.34(\mathrm{~m}, 3 \mathrm{H}), 2.32-2.16(\mathrm{~m}, 1 \mathrm{H})$, $1.95-1.74(\mathrm{~m}, 4 \mathrm{H}) ;{ }^{13} \mathrm{C}$ NMR $\left(125 \mathrm{MHz}, \mathrm{CDCl}_{3}\right) \delta 155.2(\mathrm{C}), 146.1(\mathrm{CH}), 142.5(\mathrm{C}), 137.7(\mathrm{CH}), 131.3(\mathrm{C}), 127.9(\mathrm{CH})$, $127.7(\mathrm{CH}), 126.6(\mathrm{CH}), 123.8(\mathrm{C}), 114.0(\mathrm{CH}), 31.4\left(\mathrm{CH}_{2}\right), 30.8\left(\mathrm{CH}_{2}\right), 23.2\left(\mathrm{CH}_{2}\right), 23.0\left(\mathrm{CH}_{2}\right)$ only visible peaks. ATRFTIR (thin film): 3296, 3167, 2927, 2856, 1598, 1573, 1440, 1211, 1136, 1069, 996, 757, $699 \mathrm{~cm}^{-1}$. HRMS (ESI) $\mathrm{m} / \mathrm{z}$ calculated for $\mathrm{C}_{17} \mathrm{H}_{19} \mathrm{~N}_{2}(\mathrm{M}+\mathrm{H})^{+}: 251.1548$, found: 251.1543 .

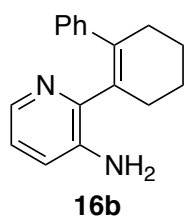

2-(3,4,5,6-Tetrahydro-[1,1'-biphenyl]-2-yl)pyridin-3-amine 16b. ${ }^{18}$ Method B was followed using $0.415 \mathrm{~g}$ of 2bromopyridin-3-amine $(2.00 \mathrm{mmol}), 0.569 \mathrm{~g}$ of 4,4,5,5-tetramethyl-2-(3,4,5,6-tetrahydro-[1,1'-biphenyl]-2-yl)-1,3,2dioxaborolane $(2.20 \mathrm{mmol}), 0.232 \mathrm{~g}$ of $\mathrm{Pd}\left(\mathrm{PPh}_{3}\right)_{4}(0.20 \mathrm{mmol}), 1.10 \mathrm{~g} \mathrm{~K} \mathrm{CO}_{3}(8.0 \mathrm{mmol})$ in $25 \mathrm{~mL}$ of toluene, $10 \mathrm{~mL}$ of methanol and $5 \mathrm{~mL}$ of water. Purification by MPLC (2:98 to 30:70 EtOAc:hexanes) afforded the product as a yellow solid $(0.297 \mathrm{~g}, 59 \%)$. The spectral data matched that reported by Driver and co-workers: ${ }^{18} \mathrm{mp}=101{ }^{\circ} \mathrm{C} ;{ }^{1} \mathrm{H} \mathrm{NMR}(500 \mathrm{MHz}$, $\left.\mathrm{CDCl}_{3}\right) \delta 7.95(\mathrm{~d}, J=4.7 \mathrm{~Hz}, 1 \mathrm{H}), 7.06-7.00(\mathrm{~m}, 5 \mathrm{H}), 6.76(\mathrm{dd}, J=8.0,4.6 \mathrm{~Hz}, 1 \mathrm{H}), 6.58(\mathrm{~d}, J=7.9 \mathrm{~Hz}, 1 \mathrm{H}), 3.43(\mathrm{br} \mathrm{s}$, $2 \mathrm{H}), 2.80-2.52(\mathrm{~m}, 2 \mathrm{H}), 2.38-2.23(\mathrm{~m}, 1 \mathrm{H}), 2.22-2.10(\mathrm{~m}, 1 \mathrm{H}), 1.96-1.69(\mathrm{~m}, 4 \mathrm{H}) ;{ }^{13} \mathrm{C} \mathrm{NMR}\left(125 \mathrm{MHz}, \mathrm{CDCl}_{3}\right) \delta$ $148.2(\mathrm{C}), 142.4(\mathrm{C}), 139.2(\mathrm{CH}), 138.8(\mathrm{C}), 136.7(\mathrm{C}), 132.6(\mathrm{C}), 127.9(\mathrm{CH}), 127.7(\mathrm{CH}), 126.6(\mathrm{CH}), 122.2(\mathrm{CH}), 121.9$ $(\mathrm{CH}), 30.8\left(\mathrm{CH}_{2}\right), 29.9\left(\mathrm{CH}_{2}\right), 23.2\left(\mathrm{CH}_{2}\right), 22.8\left(\mathrm{CH}_{2}\right)$. ATR-FTIR (thin film): 3302, 3170, 2928, 2856, 2832, 1607, 1580, $1449,1309,1284,1140,797,756,700 \mathrm{~cm}^{-1}$. HRMS (ESI) $\mathrm{m} / z$ calculated for $\mathrm{C}_{17} \mathrm{H}_{19} \mathrm{~N}_{2}(\mathrm{M}+\mathrm{H})^{+}: 251.1548$, found: 251.1542.

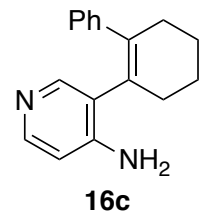

3-(3,4,5,6-Tetrahydro-[1,1'-biphenyl]-2-yl)pyridin-4-amine 16c. ${ }^{18}$ Method B was followed using $0.415 \mathrm{~g}$ of 2bromopyridin-3-amine (2.00 mmol), $0.569 \mathrm{~g}$ of 4,4,5,5-tetramethyl-2-(3,4,5,6-tetrahydro-[1,1'-biphenyl]-2-yl)-1,3,2dioxaborolane $(2.20 \mathrm{mmol}), 0.232 \mathrm{~g}$ of $\mathrm{Pd}\left(\mathrm{PPh}_{3}\right)_{4}(0.20 \mathrm{mmol}), 1.10 \mathrm{~g}$ of $\mathrm{K}_{2} \mathrm{CO}_{3}(8.0 \mathrm{mmol})$ in $25 \mathrm{~mL}$ of toluene, $10 \mathrm{~mL}$ of methanol and $5 \mathrm{~mL}$ of water. Purification by MPLC (2:98 EtOAc:hexanes to 20:80 MeOH:EtOAc) afforded the product as a yellow foam $(0.344 \mathrm{~g}, 69 \%)$. The spectral data matched that reported by Driver and co-workers: ${ }^{18} \mathrm{mp}=55{ }^{\circ} \mathrm{C} ;{ }^{1} \mathrm{H} \mathrm{NMR}$ $\left(500 \mathrm{MHz}, \mathrm{CDCl}_{3}\right) \delta 7.87(\mathrm{~s}, 1 \mathrm{H}), 7.81(\mathrm{~d}, J=5.5 \mathrm{~Hz}, 1 \mathrm{H}), 7.09-6.97(\mathrm{~m}, 5 \mathrm{H}), 6.23(\mathrm{~d}, J=5.7 \mathrm{~Hz}, 1 \mathrm{H}), 4.42(\mathrm{br} \mathrm{s}, 2 \mathrm{H})$, $2.49(\mathrm{~d}, J=18.4 \mathrm{~Hz}, 1 \mathrm{H}), 2.38(\mathrm{~d}, J=18.0 \mathrm{~Hz}, 1 \mathrm{H}), 2.31-2.16(\mathrm{~m}, 2 \mathrm{H}), 1.89-1.69(\mathrm{~m}, 4 \mathrm{H}) ;{ }^{13} \mathrm{C} \mathrm{NMR}\left(125 \mathrm{MHz}, \mathrm{CDCl}_{3}\right)$ $\delta 149.9(\mathrm{C}), 149.5(\mathrm{CH}), 147.4(\mathrm{CH}), 142.6(\mathrm{C}), 138.9(\mathrm{C}), 128.8(\mathrm{C}), 127.8(\mathrm{CH}), 127.7(\mathrm{CH}), 126.5(\mathrm{CH}), 124.4(\mathrm{C}), 109.2$ (C), $31.4\left(\mathrm{CH}_{2}\right), 31.2\left(\mathrm{CH}_{2}\right), 23.2\left(\mathrm{CH}_{2}\right), 23.0\left(\mathrm{CH}_{2}\right)$. ATR-FTIR (thin film): 3054, 2927, 2857, 1691, 1622, 1591, 1492, $1120,1170,1131,827,758,719,700 \mathrm{~cm}^{-1}$. HRMS (ESI) $\mathrm{m} / z$ calculated for $\mathrm{C}_{17} \mathrm{H}_{19} \mathrm{~N}_{2}(\mathrm{M}+\mathrm{H})^{+}: 251.1548$, found: 251.1543 . 


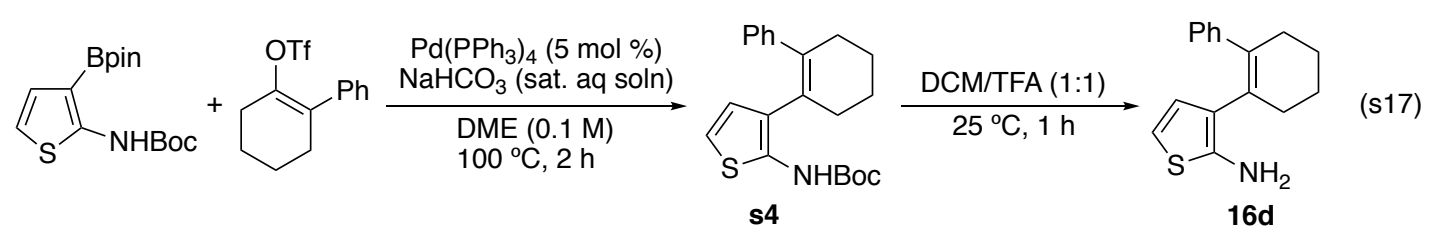

3-(3,4,5,6-Tetrahydro-[1,1'-biphenyl]-2-yl)thiophen-2-amine 16d. Method A was followed using $0.612 \mathrm{~g}$ of vinyl triflate (2.00 mmol), $0.569 \mathrm{~g}$ of tert-butyl (3-(4,4,5,5-tetramethyl-1,3,2-dioxaborolan-2-yl)thiophen-2-yl)carbamate (2.40 mmol), $0.116 \mathrm{~g}$ of $\mathrm{Pd}\left(\mathrm{PPh}_{3}\right)_{4}(0.10 \mathrm{mmol})$ in $20 \mathrm{~mL}$ of dimethoxyethane $(0.1 \mathrm{M})$. After $2 \mathrm{~h}$, the mixture was cooled to room temperature and diluted with a saturated aq soln of $\mathrm{NH}_{4} \mathrm{Cl}$. The resulting mixture was extracted with $3 \times 10 \mathrm{~mL}$ of ethyl acetate, and the combined organic phases were washed with $30 \mathrm{~mL}$ of brine. The resulting organic phase was dried over $\mathrm{Na}_{2} \mathrm{SO}_{4}$, filtered and the filtrate was concentrated in vacuo to afford $\mathbf{s 4}$, which was submitted to the Boc-deprotection without purification or characterization. The concentrate was diluted with $3 \mathrm{~mL}$ of DCM, and $3 \mathrm{~mL}$ of trifluoroacetic acid, and the resulting mixture was stirred at room temperature. After $1 \mathrm{~h}$, the reactives in the reaction mixture were neutralized by the additional of a saturated aq soln of $\mathrm{NaHCO}_{3}$. The resulting mixture was extracted with $3 \times 10 \mathrm{~mL}$ of DCM, and the combined organic phases were washed with $30 \mathrm{~mL}$ of brine. The resulting organic phase was dried over $\mathrm{Na}_{2} \mathrm{SO}_{4}$, filtered and the filtrate was concentrated in vacuo. Purification using MPLC (2:98 to 5:95 EtOAc:hexanes) afforded the product as a dark green oil $\left(0.133 \mathrm{~g}, 26 \%\right.$ over two steps): ${ }^{1} \mathrm{H}$ NMR $\left(500 \mathrm{MHz}, \mathrm{CDCl}_{3}\right) \delta 7.24-7.19(\mathrm{~m}, 2 \mathrm{H}), 7.17-7.12(\mathrm{~m}, 3 \mathrm{H}), 6.71(\mathrm{~d}$, $J=5.6 \mathrm{~Hz}, 1 \mathrm{H}), 6.46(\mathrm{~d}, J=5.6 \mathrm{~Hz}, 1 \mathrm{H}), 3.18(\mathrm{br} \mathrm{s}, 2 \mathrm{H}), 2.56-2.49(\mathrm{~m}, 2 \mathrm{H}), 2.42-2.34(\mathrm{~m}, 2 \mathrm{H}), 1.89-1.81(\mathrm{~m}, 4 \mathrm{H})$; ${ }^{13} \mathrm{C}$ NMR $\left(125 \mathrm{MHz}, \mathrm{CDCl}_{3}\right) \delta 143.6(\mathrm{C}), 135.5(\mathrm{C}), 129.2(\mathrm{C}), 128.0(\mathrm{CH}), 127.93(\mathrm{C}), 127.89(\mathrm{CH}), 127.7(\mathrm{CH}), 126.4$ $(\mathrm{CH}), 124.6(\mathrm{C}), 111.2(\mathrm{CH}), 31.6\left(\mathrm{CH}_{2}\right), 31.2\left(\mathrm{CH}_{2}\right), 23.4\left(\mathrm{CH}_{2}\right), 23.1\left(\mathrm{CH}_{2}\right)$. ATR-FTIR (thin film): 2926, 1732, 1435, $1262,966,760,700 \mathrm{~cm}^{-1}$. HRMS (ESI) $\mathrm{m} / z$ calculated for $\mathrm{C}_{16} \mathrm{H}_{18} \mathrm{NS}(\mathrm{M}+\mathrm{H})^{+}: 256.1160$, found: 256.1156 .

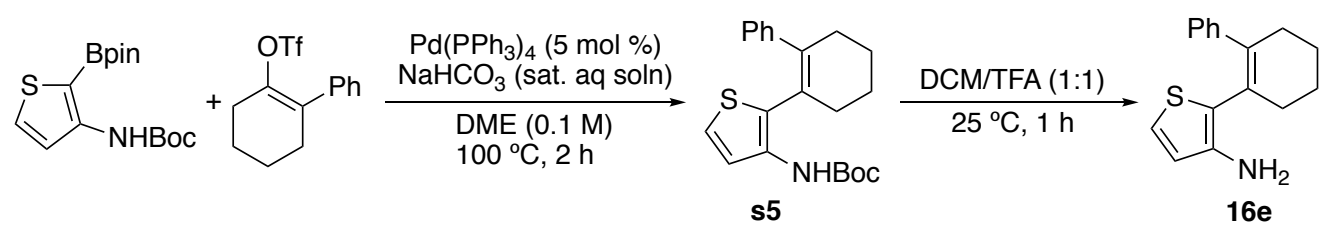

(s18)

2-(3,4,5,6-Tetrahydro-[1,1'-biphenyl]-2-yl)thiophen-3-amine 16e. Method A was followed using $0.612 \mathrm{~g}$ of vinyl triflate (2.00 mmol), $0.569 \mathrm{~g}$ of tert-butyl (2-(4,4,5,5-tetramethyl-1,3,2-dioxaborolan-2-yl)thiophen-3-yl)carbamate $(2.40 \mathrm{mmol})$, $0.116 \mathrm{~g}$ of $\mathrm{Pd}\left(\mathrm{PPh}_{3}\right)_{4}(0.10 \mathrm{mmol})$ in $20 \mathrm{~mL}$ of dimethoxyethane $(0.1 \mathrm{M})$. After $2 \mathrm{~h}$, the mixture was cooled to room temperature and diluted with a saturated aq soln of $\mathrm{NH}_{4} \mathrm{Cl}$. The resulting mixture was extracted with $3 \times 10 \mathrm{~mL}$ of ethyl acetate, and the combined organic phases were washed with $30 \mathrm{~mL}$ of brine. The resulting organic phase was dried over $\mathrm{Na}_{2} \mathrm{SO}_{4}$, filtered and the filtrate was concentrated in vacuo to afford $\mathbf{5 5}$, which was submitted to the Boc-deprotection without purification or characterization. The concentrate was diluted with $3 \mathrm{~mL}$ of DCM and $3 \mathrm{~mL}$ of trifluoroacetic acid was added. After $1 \mathrm{~h}$, the reactives in the reaction mixture were neutralized by the addition of a saturated aq soln of $\mathrm{NaHCO}_{3}$. The resulting mixture was extracted with $3 \times 10 \mathrm{~mL}$ of DCM, and the combined organic phases were washed with $30 \mathrm{~mL}$ of brine. The resulting organic phase was dried over $\mathrm{Na}_{2} \mathrm{SO}_{4}$, filtered and the filtrate was concentrated in vacuo. Purification using MPLC (2:98 to 5:95 EtOAc:hexanes) afforded the product as a yellow oil $\left(0.281 \mathrm{~g}, 55 \%\right.$ over two steps): ${ }^{1} \mathrm{H}$ NMR $\left(500 \mathrm{MHz}, \mathrm{CDCl}_{3}\right) \delta 7.28-7.21(\mathrm{~m}, 4 \mathrm{H}), 7.18(\mathrm{q}, J=4.4 \mathrm{~Hz}, 1 \mathrm{H}), 7.01(\mathrm{~d}, J=5.2 \mathrm{~Hz}, 1 \mathrm{H}), 6.31(\mathrm{~d}, J=5.2 \mathrm{~Hz}, 1 \mathrm{H}), 3.01$ (br s, 2H), $2.55(\mathrm{t}, J=4.9 \mathrm{~Hz}, 2 \mathrm{H}), 2.49(\mathrm{t}, J=5.4 \mathrm{~Hz}, 2 \mathrm{H}), 1.87$ (pentet, $J=4.4 \mathrm{~Hz}, 4 \mathrm{H}) ;{ }^{13} \mathrm{C} \mathrm{NMR}\left(125 \mathrm{MHz}, \mathrm{CDCl}_{3}\right) \delta$ $143.5(\mathrm{C}), 139.1(\mathrm{C}), 137.3(\mathrm{C}), 128.1(\mathrm{CH}), 128.0(\mathrm{CH}), 127.2(\mathrm{C}), 126.6(\mathrm{CH}), 122.9(\mathrm{CH}), 121.5(\mathrm{CH}), 120.5(\mathrm{C}), 33.6$ $\left(\mathrm{CH}_{2}\right), 31.6\left(\mathrm{CH}_{2}\right), 23.31\left(\mathrm{CH}_{2}\right), 23.28\left(\mathrm{CH}_{2}\right)$. ATR-FTIR (thin film): 3434, 3350, 2927, 2856, 1606, 1557, 1402, 758, 721 $\mathrm{cm}^{-1}$. HRMS (ESI) $\mathrm{m} / z$ calculated for $\mathrm{C}_{16} \mathrm{H}_{18} \mathrm{NS}(\mathrm{M}+\mathrm{H})^{+}: 256.1160$, found: 256.1158 . 


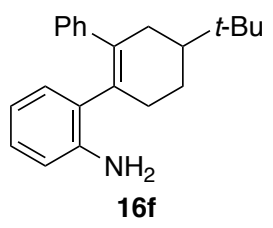

4'-(tert-Butyl)-3',4',5',6'-tetrahydro-[1,1':2',1''-terphenyl]-2-amine 16f. ${ }^{18}$ Method A was followed using $0.725 \mathrm{~g}$ of vinyl triflate $(2.00 \mathrm{mmol}), 0.526 \mathrm{~g}$ of 2-aminophenylboronic acid pinacol ester $(2.40 \mathrm{mmol}), 0.116 \mathrm{~g}$ of $\mathrm{Pd}\left(\mathrm{PPh}_{3}\right)_{4}(0.10 \mathrm{mmol})$ in $20 \mathrm{~mL}$ of dimethoxyethane $(0.1 \mathrm{M})$. Purification by MPLC (2:98 to 20:80 EtOAc:hexanes) afforded the product, a yellow foam, as a $1: 1$ ratio of diastereomers $(0.346 \mathrm{~g}, 57 \%)$. The spectral data matched that reported by Driver and co-workers: ${ }^{18}$ Diagnostic peaks for the one isomer: ${ }^{1} \mathrm{H}$ NMR $\left(500 \mathrm{MHz}, \mathrm{CDCl}_{3}\right) \delta 7.26-7.20(\mathrm{~m}, 4 \mathrm{H}), 7.17(\mathrm{q}, J=4.0 \mathrm{~Hz}, 1 \mathrm{H}), 7.09(\mathrm{~d}$, $J=7.6 \mathrm{~Hz}, 0.5 \mathrm{H}), 6.78(\mathrm{t}, J=7.4 \mathrm{~Hz}, 0.5 \mathrm{H}), 6.60(\mathrm{~d}, J=7.9 \mathrm{~Hz}, 0.5 \mathrm{H}), 3.69(\mathrm{br} \mathrm{s}, 2 \mathrm{H}), 2.86-2.65(\mathrm{~m}, 0.5 \mathrm{H}), 2.60-2.46$ $(\mathrm{m}, 3 \mathrm{H}), 2.21-2.06(\mathrm{~m}, 1 \mathrm{H}), 1.83-1.65(\mathrm{~m}, 1 \mathrm{H}), 1.10(\mathrm{~s}, 9 \mathrm{H}),{ }^{13} \mathrm{C} \mathrm{NMR}\left(125 \mathrm{MHz}, \mathrm{CDCl}_{3}\right) \delta 143.4(\mathrm{C}), 142.8(\mathrm{C}), 136.4$ (C), $132.8(\mathrm{C}), 132.4(\mathrm{C}), 130.4(\mathrm{CH}), 129.7(\mathrm{CH}), 128.3(\mathrm{CH}), 127.8(\mathrm{CH}), 126.4(\mathrm{CH}), 118.5(\mathrm{CH}), 115.4(\mathrm{CH}), 45.2$ $(\mathrm{CH}), 33.4\left(\mathrm{CH}_{2}\right), 33.2\left(\mathrm{CH}_{2}\right), 32.5(\mathrm{C}), 27.5\left(\mathrm{CH}_{3}\right), 24.7\left(\mathrm{CH}_{2}\right)$. Diagnostic peaks for the other isomer: ${ }^{1} \mathrm{H} \mathrm{NMR}(500 \mathrm{MHz}$, $\left.\mathrm{CDCl}_{3}\right) \delta 7.04(\mathrm{q}, J=7.1 \mathrm{~Hz}, 1 \mathrm{H}), 7.00(\mathrm{~d}, J=7.5 \mathrm{~Hz}, 0.5 \mathrm{H}), 6.74(\mathrm{t}, J=7.5 \mathrm{~Hz}, 0.5 \mathrm{H}), 6.55(\mathrm{~d}, J=8.0 \mathrm{~Hz}, 0.5 \mathrm{H}), 2.42-$ $2.27(\mathrm{~m}, 0.5 \mathrm{H}), 1.64-1.48(\mathrm{~m}, 1 \mathrm{H}) ;{ }^{13} \mathrm{C}$ NMR $\left(125 \mathrm{MHz}, \mathrm{CDCl}_{3}\right) \delta 143.2(\mathrm{C}), 142.6(\mathrm{C}), 136.3(\mathrm{C}), 129.5(\mathrm{CH}), 127.8$ $(\mathrm{CH}), 127.4(\mathrm{CH}), 126.4(\mathrm{CH}), 118.4(\mathrm{CH}), 44.9(\mathrm{CH}), 33.2\left(\mathrm{CH}_{2}\right), 32.8\left(\mathrm{CH}_{2}\right), 27.5\left(\mathrm{CH}_{3}\right), 24.6\left(\mathrm{CH}_{2}\right)$. ATR-FTIR (thin film): $2958,1611,1492,1450,1365,1212,1145,746,698 \mathrm{~cm}^{-1}$. HRMS (ESI) $\mathrm{m} / z$ calculated for $\mathrm{C}_{22} \mathrm{H}_{28} \mathrm{~N}(\mathrm{M}+\mathrm{H})^{+}: 306.2222$, found: 306.2216 .

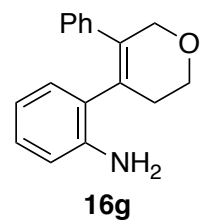

2-(5-Phenyl-3,6-dihydro-2H-pyran-4-yl)aniline 16g. ${ }^{18}$ Method A was followed using $0.308 \mathrm{~g}$ of vinyl triflate (1.00 mmol), $0.263 \mathrm{~g}$ of 2-aminophenylboronic acid pinacol ester $(1.20 \mathrm{mmol}), 0.058 \mathrm{~g}$ of $\mathrm{Pd}\left(\mathrm{PPh}_{3}\right)_{4}(0.05 \mathrm{mmol})$ in $10 \mathrm{~mL} \mathrm{of}$ dimethoxyethane $(0.1 \mathrm{M})$. Purification by MPLC (2:98 to 30:70 EtOAc:hexanes) afforded the product as a red solid (0.224 g, 90\%). The spectral data matched that reported by Driver and co-workers: $:^{18} \mathrm{mp}=72{ }^{\circ} \mathrm{C} ;{ }^{1} \mathrm{H} \mathrm{NMR}\left(500 \mathrm{MHz} \mathrm{CDCl}_{3}\right) \delta$ $7.21-7.14(\mathrm{~m}, 3 \mathrm{H}), 7.14-7.10(\mathrm{~m}, 2 \mathrm{H}), 7.04-6.95(\mathrm{~m}, 2 \mathrm{H}), 6.71(\mathrm{t}, J=7.5 \mathrm{~Hz}, 1 \mathrm{H}), 6.54(\mathrm{~d}, J=7.8 \mathrm{~Hz}, 1 \mathrm{H}), 4.64-4.38$ $(\mathrm{m}, 2 \mathrm{H}), 4.12-3.99$ (m, 2H), 3.61 (br s, 2H), $2.91-2.35$ (m, 2H); ${ }^{13} \mathrm{C}$ NMR $\left(125 \mathrm{MHz}, \mathrm{CDCl}_{3}\right) \delta 142.9(\mathrm{C}), 138.2(\mathrm{C})$, $135.3(\mathrm{C}), 130.3(\mathrm{C}), 129.8(\mathrm{CH}), 128.1(\mathrm{CH}), 127.99(\mathrm{CH}), 127.95(\mathrm{CH}), 127.3(\mathrm{CH}), 127.2(\mathrm{C}), 118.4(\mathrm{CH}), 115.4(\mathrm{CH})$, $68.8\left(\mathrm{CH}_{2}\right), 65.0\left(\mathrm{CH}_{2}\right), 30.6\left(\mathrm{CH}_{2}\right)$. ATR-FTIR (thin film): 3363, 2923, 2851, 1613, 1492, 1448, 1296, 1138, 1098, 987 , $876,750,699 \mathrm{~cm}^{-1}$. HRMS (ESI) $\mathrm{m} / z$ calculated for $\mathrm{C}_{17} \mathrm{H}_{18} \mathrm{NO}(\mathrm{M}+\mathrm{H})^{+}: 252.1388$, found: 252.1384 .

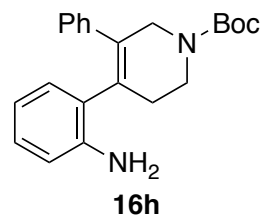

tert-Butyl 4-(2-aminophenyl)-5-phenyl-3,6-dihydropyridine-1(2H)-carboxylate $16 \mathrm{~h} .{ }^{18}$ Method A was followed using $0.815 \mathrm{~g}$ of vinyl triflate $(2.00 \mathrm{mmol}), 0.526 \mathrm{~g}$ of 2 -aminophenylboronic acid pinacol ester $(2.40 \mathrm{mmol}), 0.116 \mathrm{~g} \mathrm{of} \mathrm{Pd}\left(\mathrm{PPh}_{3}\right)_{4}$ $(0.10 \mathrm{mmol})$ in $20 \mathrm{~mL}$ of dimethoxyethane $(0.1 \mathrm{M})$. Purification by MPLC (2:98 to 30:70 EtOAc:hexanes) afforded the product, a yellow foam, as a 1:1 ratio of slow rotamers $(0.470 \mathrm{~g}, 67 \%)$. The spectral data matched that reported by Driver and co-workers: ${ }^{18} \mathrm{mp}=118{ }^{\circ} \mathrm{C}$. Diagnostic peaks for the one rotamer: ${ }^{1} \mathrm{H}$ NMR $\left(500 \mathrm{MHz}, \mathrm{CDCl}_{3}\right) \delta 7.19-7.09(\mathrm{~m}, 5 \mathrm{H})$, $6.96(\mathrm{t}, J=7.7 \mathrm{~Hz}, 1 \mathrm{H}), 6.91(\mathrm{~d}, J=7.5 \mathrm{~Hz}, 1 \mathrm{H}), 4.58-4.10(\mathrm{~m}, 2 \mathrm{H}), 3.72(\mathrm{~s}, 2 \mathrm{H}), 2.52(\mathrm{br} \mathrm{s}, 1 \mathrm{H}), 1.54(\mathrm{~s}, 9 \mathrm{H}) ;{ }^{13} \mathrm{C} \mathrm{NMR}$ $\left(125 \mathrm{MHz}, \mathrm{CDCl}_{3}\right) \delta 155.0(\mathrm{C}), 142.7(\mathrm{C}), 139.2(\mathrm{C}), 133.7(\mathrm{C}), 131.5(\mathrm{C}), 129.8(\mathrm{CH}), 128.1(\mathrm{CH}), 128.0(\mathrm{CH}), 127.9$ $(\mathrm{CH}), 127.6(\mathrm{C}), 127.2(\mathrm{CH}), 118.4(\mathrm{CH}), 115.4(\mathrm{CH}), 80.0(\mathrm{C}), 47.2\left(\mathrm{CH}_{2}\right), 41.4\left(\mathrm{CH}_{2}\right), 31.0\left(\mathrm{CH}_{2}\right), 28.6\left(\mathrm{CH}_{3}\right)$. Diagnostic peaks for the other rotamer: ${ }^{1} \mathrm{H}$ NMR $\left(500 \mathrm{MHz}, \mathrm{CDCl}_{3}\right) \delta 6.65(\mathrm{t}, J=7.5 \mathrm{~Hz}, 1 \mathrm{H}), 6.51(\mathrm{~d}, J=8.0 \mathrm{~Hz}, 1 \mathrm{H}), 3.53(\mathrm{~s}, 2 \mathrm{H})$, 2.47 (br s, 1H); ${ }^{13} \mathrm{C}$ NMR (125 MHz, $\left.\mathrm{CDCl}_{3}\right) \delta 40.2\left(\mathrm{CH}_{2}\right)$. ATR-FTIR (thin film): 3360, 2975, 2928, 1683, 1613, 1417, 
$1365,1335,1241,1164,1130,1098,986,866,746,698 \mathrm{~cm}^{-1}$. HRMS (ESI) $m / z$ calculated for $\mathrm{C}_{22} \mathrm{H}_{27} \mathrm{~N}_{2} \mathrm{O}_{2}(\mathrm{M}+\mathrm{H})^{+}$: 351.2073, found: 351.2063 .

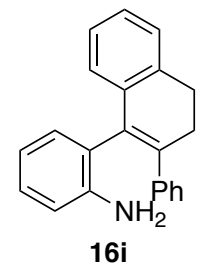

2-(2-Phenyl-3,4-dihydronaphthalen-1-yl)aniline 16i. ${ }^{18}$ Method A was followed using $0.709 \mathrm{~g}$ of vinyl triflate $(2.00 \mathrm{mmol})$, $0.526 \mathrm{~g}$ of 2-aminophenylboronic acid pinacol ester $(2.40 \mathrm{mmol}), 0.116 \mathrm{~g}$ of $\mathrm{Pd}\left(\mathrm{PPh}_{3}\right)_{4}(0.10 \mathrm{mmol})$ in $20 \mathrm{~mL}$ dimethoxyethane $(0.1 \mathrm{M})$. Purification by MPLC (2:98 to 20:80 EtOAc:hexanes) afforded the product as a yellow solid $(0.527 \mathrm{~g}, 89 \%)$. The spectral data matched that reported by Driver and co-workers: ${ }^{18} \mathrm{mp}=144{ }^{\circ} \mathrm{C} ;{ }^{1} \mathrm{H} \mathrm{NMR}(500 \mathrm{MHz}$, $\left.\mathrm{CDCl}_{3}\right) \delta 7.35(\mathrm{~d}, 1 \mathrm{H}), 7.32-7.24(\mathrm{~m}, 5 \mathrm{H}), 7.23-7.15(\mathrm{~m}, 3 \mathrm{H}), 6.97(\mathrm{~d}, J=7.4 \mathrm{~Hz}, 1 \mathrm{H}), 6.93(\mathrm{~d}, J=7.7 \mathrm{~Hz}, 1 \mathrm{H}), 6.76(\mathrm{t}$, $J=8.7 \mathrm{~Hz}, 2 \mathrm{H}), 3.62(\mathrm{~s}, 2 \mathrm{H}), 3.15(\mathrm{q}, J=7.2,6.3 \mathrm{~Hz}, 2 \mathrm{H}), 3.04(\mathrm{td}, J=13.3,10.6,6.6 \mathrm{~Hz}, 1 \mathrm{H}), 2.93(\mathrm{dt}, J=15.2,7.1 \mathrm{~Hz}$, $1 \mathrm{H}) ;{ }^{13} \mathrm{C}$ NMR $\left(125 \mathrm{MHz}, \mathrm{CDCl}_{3}\right) \delta 144.7(\mathrm{C}), 142.6(\mathrm{C}), 138.9(\mathrm{C}), 135.7(\mathrm{C}), 135.5(\mathrm{C}), 132.5(\mathrm{C}), 132.1(\mathrm{CH}), 128.2$ $(\mathrm{CH}), 127.8(\mathrm{CH}), 127.5(\mathrm{CH}), 127.4(\mathrm{CH}), 127.3(\mathrm{CH}), 126.8(\mathrm{CH}), 126.6(\mathrm{CH}), 125.4(\mathrm{CH}), 125.1(\mathrm{C}), 118.6(\mathrm{CH}), 115.3$ $(\mathrm{CH}), 30.5\left(\mathrm{CH}_{2}\right), 28.6\left(\mathrm{CH}_{2}\right)$. ATR-FTIR (thin film): 3463, 3374, 3055, 3019, 2933, 2883, 2830, 1612, 1489, 1451, 1297, $759,727,698 \mathrm{~cm}^{-1}$. HRMS (ESI) $\mathrm{m} / z$ calculated for $\mathrm{C}_{22} \mathrm{H}_{20} \mathrm{~N}(\mathrm{M}+\mathrm{H})^{+}: 298.1596$, found: 298.1594 .

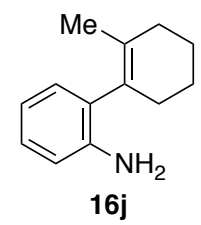

6'-Methyl-2',3',4',5'-tetrahydro-[1,1'-biphenyl]-2-amine 16j. ${ }^{18}$ Method A was followed using $0.488 \mathrm{~g}$ of vinyl triflate $(2.00 \mathrm{mmol}), 0.526 \mathrm{~g}$ of 2-aminophenylboronic acid pinacol ester $(2.40 \mathrm{mmol}), 0.116 \mathrm{~g}$ of $\mathrm{Pd}\left(\mathrm{PPh}_{3}\right)_{4}(0.10 \mathrm{mmol})$ in $20 \mathrm{~mL}$ of dimethoxyethane $(0.1 \mathrm{M})$. Purification by MPLC (2:98 to 20:80 EtOAc:hexanes) afforded the product as a brown oil $(0.258 \mathrm{~g}, 69 \%)$. The spectral data matched that reported by Driver and co-workers: ${ }^{18}{ }^{1} \mathrm{H}$ NMR $\left(500 \mathrm{MHz}, \mathrm{CDCl}_{3}\right) \delta 7.10(\mathrm{t}$, $J=7.6 \mathrm{~Hz}, 1 \mathrm{H}), 6.96(\mathrm{~d}, J=7.4 \mathrm{~Hz}, 1 \mathrm{H}), 6.79(\mathrm{t}, J=7.4 \mathrm{~Hz}, 1 \mathrm{H}), 6.74(\mathrm{~d}, J=7.9 \mathrm{~Hz}, 1 \mathrm{H}), 3.67(\mathrm{br} \mathrm{s}, 2 \mathrm{H}), 2.23-2.18(\mathrm{~m}$, 2H), $2.17-2.11(\mathrm{~m}, 2 \mathrm{H}), 1.85-1.70(\mathrm{~m}, 4 \mathrm{H}), 1.53(\mathrm{~s}, 3 \mathrm{H}) ;{ }^{13} \mathrm{C}$ NMR $\left(125 \mathrm{MHz}, \mathrm{CDCl}_{3}\right) \delta 143.1(\mathrm{C}), 131.3(\mathrm{C}), 129.9(\mathrm{C})$, $129.2(\mathrm{C}), 129.1(\mathrm{CH}), 127.3(\mathrm{CH}), 118.4(\mathrm{CH}), 114.9(\mathrm{CH}), 31.2\left(\mathrm{CH}_{2}\right), 30.9\left(\mathrm{CH}_{2}\right), 23.6\left(\mathrm{CH}_{2}\right), 23.3\left(\mathrm{CH}_{2}\right), 20.3\left(\mathrm{CH}_{3}\right)$. ATR-FTIR (thin film): 2923, 2854, 2830, 1610, 1492, 1447, 1293, 1276, 1143, 744, $668 \mathrm{~cm}^{-1}$. HRMS (ESI) $\mathrm{m} / z$ calculated for $\mathrm{C}_{13} \mathrm{H}_{18} \mathrm{~N}(\mathrm{M}+\mathrm{H})^{+}: 188.1439$, found: 188.1433 .

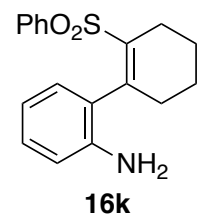

6'-(Phenylsulfonyl)-2',3', $\mathbf{4}^{\prime}, 5^{\prime}$-tetrahydro-[1,1'-biphenyl]-2-amine 16k. ${ }^{18}$ Method A was followed using $0.741 \mathrm{~g}$ of vinyl

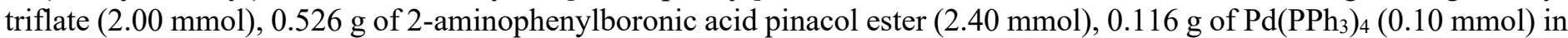
$20 \mathrm{~mL}$ of dimethoxyethane $(0.1 \mathrm{M})$. Purification by MPLC (2:98 to 20:80 EtOAc:hexanes) afforded the product as a yellow foam $(0.525 \mathrm{~g}, 84 \%)$. The spectral data matched that reported by Driver and co-workers: ${ }^{18}{ }^{1} \mathrm{H} \mathrm{NMR}\left(500 \mathrm{MHz}, \mathrm{CDCl}_{3}\right) \delta$ $7.40-7.30(\mathrm{~m}, 3 \mathrm{H}), 7.19(\mathrm{t}, J=7.6 \mathrm{~Hz}, 2 \mathrm{H}), 6.91(\mathrm{t}, J=7.8 \mathrm{~Hz}, 1 \mathrm{H}), 6.49-6.42(\mathrm{~m}, 2 \mathrm{H}), 6.37(\mathrm{~d}, J=7.5 \mathrm{~Hz}, 1 \mathrm{H}), 3.47$ (br s, 2H), $2.66-2.56(\mathrm{~m}, 1 \mathrm{H}), 2.55-2.47(\mathrm{~m}, 1 \mathrm{H}), 2.34(\mathrm{dt}, J=19.3,5.9 \mathrm{~Hz}, 1 \mathrm{H}), 2.07-1.97(\mathrm{~m}, 1 \mathrm{H}), 1.74(\mathrm{dq}, J=11.2$, 4.3, 3.7 Hz, 1H), $1.68-1.57(\mathrm{~m}, 2 \mathrm{H}), 1.57-1.50(\mathrm{~m}, 1 \mathrm{H}) ;{ }^{13} \mathrm{C}$ NMR $\left(125 \mathrm{MHz}, \mathrm{CDCl}_{3}\right) \delta 148.7(\mathrm{C}), 143.1(\mathrm{C}), 141.1(\mathrm{C})$, 139.0 (C), $132.8(\mathrm{CH}), 128.60(\mathrm{CH}), 128.57(\mathrm{CH}), 128.1(\mathrm{CH}), 127.6(\mathrm{CH}), 126.2(\mathrm{C}), 118.2(\mathrm{CH}), 115.9(\mathrm{CH}), 33.7\left(\mathrm{CH}_{2}\right)$, $26.0\left(\mathrm{CH}_{2}\right), 22.5\left(\mathrm{CH}_{2}\right), 22.0\left(\mathrm{CH}_{2}\right)$. ATR-FTIR (thin film): 3365, 2932, 2858, 1622, 1493, 1447, 1289, 1144, 1013, 748, $721,688,609,596 \mathrm{~cm}^{-1}$. HRMS (ESI) $m / z$ calculated for $\mathrm{C}_{18} \mathrm{H}_{20} \mathrm{NO}_{2} \mathrm{~S}(\mathrm{M}+\mathrm{H})^{+}: 314.1215$, found: 314.1209 . 


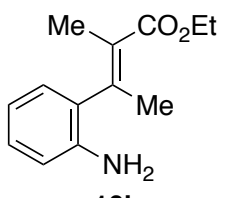

161

Ethyl (E)-3-(2-aminophenyl)-2-methylbut-2-enoate 161. ${ }^{18}$ Method A was followed using $0.552 \mathrm{~g}$ of vinyl triflate $(2.00$ $\mathrm{mmol}), 0.526 \mathrm{~g}$ of 2-aminophenylboronic acid pinacol ester $(2.40 \mathrm{mmol}), 0.116 \mathrm{~g}$ of $\mathrm{Pd}\left(\mathrm{PPh}_{3}\right)_{4}(0.10 \mathrm{mmol})$ in $20 \mathrm{~mL}$ of dimethoxyethane $(0.1 \mathrm{M})$. Purification by MPLC (2:98 to 20:80 EtOAc:hexanes) afforded the product, a yellow oil, as a single isomer $(0.395 \mathrm{~g}, 90 \%)$. The spectral data matched that reported by Driver and co-workers: ${ }^{18}{ }^{1} \mathrm{H} \mathrm{NMR}(500 \mathrm{MHz}$, $\left.\mathrm{CDCl}_{3}\right) \delta 7.07(\mathrm{t}, J=7.7 \mathrm{~Hz}, 1 \mathrm{H}), 6.89(\mathrm{~d}, J=7.5 \mathrm{~Hz}, 1 \mathrm{H}), 6.75(\mathrm{t}, J=7.5 \mathrm{~Hz}, 1 \mathrm{H}), 6.69(\mathrm{~d}, J=8.1 \mathrm{~Hz}, 1 \mathrm{H}), 4.26(\mathrm{q}, J=$ $7.1 \mathrm{~Hz}, 2 \mathrm{H}), 3.66$ (br s, 2H), $2.22(\mathrm{~s}, 3 \mathrm{H}), 1.73(\mathrm{~s}, 3 \mathrm{H}), 1.34(\mathrm{t}, J=7.0 \mathrm{~Hz}, 3 \mathrm{H}) ;{ }^{13} \mathrm{C} \mathrm{NMR}\left(125 \mathrm{MHz}, \mathrm{CDCl}_{3}\right) \delta 169.5(\mathrm{C})$, $143.1(\mathrm{C}), 141.9(\mathrm{C}), 128.6(\mathrm{C}), 128.2(\mathrm{CH}), 127.6(\mathrm{CH}), 127.0(\mathrm{C}), 118.4(\mathrm{CH}), 115.3(\mathrm{CH}), 60.4\left(\mathrm{CH}_{2}\right), 21.9\left(\mathrm{CH}_{3}\right), 16.9$ $\left(\mathrm{CH}_{3}\right), 14.3\left(\mathrm{CH}_{3}\right)$. ATR-FTIR (thin film): 3375, 2981, 1703, 1614, 1492, 1448, 1290, 1237, 1133, 1094, 1055, 772, 746, $668 \mathrm{~cm}^{-1}$. HRMS (ESI) $\mathrm{m} / z$ calculated for $\mathrm{C}_{13} \mathrm{H}_{18} \mathrm{NO}_{2}(\mathrm{M}+\mathrm{H})^{+}: 220.1338$, found: 220.1334 .

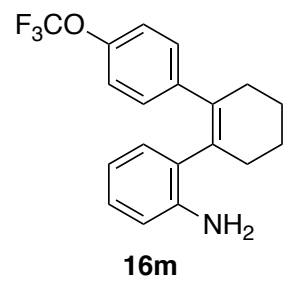

4"-(Trifluoromethoxy)-3',4',5',6'-tetrahydro-[1,1':2',1''-terphenyl]-2-amine $16 \mathbf{m} .{ }^{18}$ Method A was followed using $0.390 \mathrm{~g}$ of vinyl triflate $(1.00 \mathrm{mmol}), 0.263 \mathrm{~g}$ of 2 -aminophenylboronic acid pinacol ester $(1.20 \mathrm{mmol}), 0.058 \mathrm{~g}$ of $\left.\mathrm{Pd}_{(\mathrm{PPh}}\right)_{4}$ $(0.05 \mathrm{mmol})$ in $10 \mathrm{~mL}$ of dimethoxyethane $(0.1 \mathrm{M})$. Purification by MPLC (2:98 to 20:80 EtOAc:hexanes) afforded the product as a yellow oil $(0.299 \mathrm{~g}, 90 \%)$. The spectral data matched that reported by Driver and co-workers: ${ }^{18}{ }^{1} \mathrm{H}$ NMR $(500$ $\left.\mathrm{MHz}, \mathrm{CDCl}_{3}\right) \delta 7.16(\mathrm{~d}, J=8.5 \mathrm{~Hz}, 2 \mathrm{H}), 7.00(\mathrm{~d}, J=7.9 \mathrm{~Hz}, 3 \mathrm{H}), 6.96(\mathrm{~d}, J=7.5 \mathrm{~Hz}, 1 \mathrm{H}), 6.71(\mathrm{t}, J=7.4 \mathrm{~Hz}, 1 \mathrm{H}), 6.56$ $(\mathrm{d}, J=7.9 \mathrm{~Hz}, 1 \mathrm{H}), 3.57($ br s, $2 \mathrm{H}), 2.68-2.54(\mathrm{~m}, 1 \mathrm{H}), 2.52-2.34(\mathrm{~m}, 3 \mathrm{H}), 2.04-1.77(\mathrm{~m}, 4 \mathrm{H}) ;{ }^{13} \mathrm{C} \mathrm{NMR}(125 \mathrm{MHz}$, $\left.\mathrm{CDCl}_{3}\right) \delta 147.5(\mathrm{C}), 142.6(\mathrm{C}), 141.7(\mathrm{C}), 135.0(\mathrm{C}), 133.8(\mathrm{C}), 129.8(\mathrm{CH}), 129.3(\mathrm{CH}), 129.1(\mathrm{C}), 127.6(\mathrm{CH}), 120.55(\mathrm{q}$, $\left.J_{C F}=256.5 \mathrm{~Hz}, \mathrm{C}\right), 120.0(\mathrm{CH}), 118.4(\mathrm{CH}), 115.3(\mathrm{CH}), 31.6\left(\mathrm{CH}_{2}\right), 31.2\left(\mathrm{CH}_{2}\right), 23.4\left(\mathrm{CH}_{2}\right), 23.1\left(\mathrm{CH}_{2}\right) .{ }^{19} \mathrm{~F} \mathrm{NMR}(282$ $\left.\mathrm{MHz}, \mathrm{CDCl}_{3}\right) \delta-58.1$. ATR-FTIR (thin film): 2930, 1611, 1506, 1254, 1155, $746 \mathrm{~cm}^{-1}$. HRMS (ESI) $\mathrm{m} / z$ calculated for $\mathrm{C}_{19} \mathrm{H}_{19} \mathrm{~F}_{3} \mathrm{NO}(\mathrm{M}+\mathrm{H})^{+}: 334.1419$, found: 334.1422 .

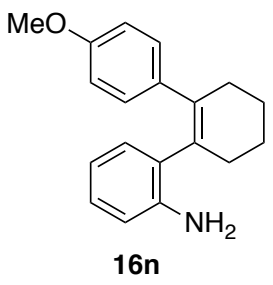

4'-Methoxy-3',4',5',6'-tetrahydro-[1,1':2',1'-terphenyl]-2-amine 16n. ${ }^{18}$ Method A was followed using $0.168 \mathrm{~g}$ of vinyl

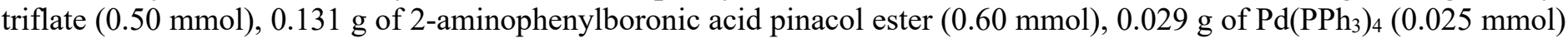
in $5 \mathrm{~mL}$ of dimethoxyethane $(0.1 \mathrm{M})$. Purification by MPLC (2:98 to 20:80 EtOAc:hexanes) afforded the product as a yellow oil $(0.116 \mathrm{~g}, 83 \%)$. The spectral data matched that reported by Driver and co-workers: ${ }^{18}{ }^{1} \mathrm{H}$ NMR $\left(500 \mathrm{MHz}, \mathrm{CDCl}_{3}\right) \delta 7.03$ $(\mathrm{d}, J=8.3 \mathrm{~Hz}, 2 \mathrm{H}), 7.00-6.93(\mathrm{~m}, 2 \mathrm{H}), 6.72-6.63(\mathrm{~m}, 3 \mathrm{H}), 6.52(\mathrm{~d}, J=7.9 \mathrm{~Hz}, 1 \mathrm{H}), 3.71(\mathrm{~s}, 3 \mathrm{H}), 3.52(\mathrm{br} \mathrm{s}, 2 \mathrm{H}), 2.64-$ $2.53(\mathrm{~m}, 1 \mathrm{H}), 2.48-2.26(\mathrm{~m}, 3 \mathrm{H}), 1.94-1.75(\mathrm{~m}, 4 \mathrm{H}) ;{ }^{13} \mathrm{C}$ NMR $\left(125 \mathrm{MHz}, \mathrm{CDCl}_{3}\right) \delta 157.8(\mathrm{C}), 142.6(\mathrm{C}), 135.4(\mathrm{C})$, $135.3(\mathrm{C}), 132.0(\mathrm{C}), 130.0(\mathrm{C}), 129.9(\mathrm{CH}), 128.9(\mathrm{CH}), 127.2(\mathrm{CH}), 118.4(\mathrm{CH}), 115.4(\mathrm{CH}), 113.0(\mathrm{CH}), 55.0\left(\mathrm{CH}_{3}\right)$, $31.8\left(\mathrm{CH}_{2}\right), 31.3\left(\mathrm{CH}_{2}\right), 23.5\left(\mathrm{CH}_{2}\right), 23.3\left(\mathrm{CH}_{2}\right)$. ATR-FTIR (thin film): 2927, 2832, 1608, 1610, 1243, $748 \mathrm{~cm}^{-1}$. HRMS (ESI) $\mathrm{m} / z$ calculated for $\mathrm{C}_{19} \mathrm{H}_{22} \mathrm{NO}(\mathrm{M}+\mathrm{H})^{+}: 280.1701$, found: 280.1701. 


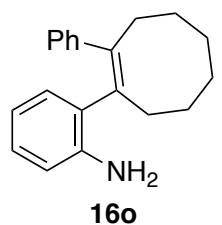

(Z)-2-(2-Phenylcyclooct-1-en-1-yl)aniline 16o. ${ }^{18}$ Method A was followed using $0.669 \mathrm{~g}$ of vinyl triflate $(2.00 \mathrm{mmol}), 0.526$ $\mathrm{g}$ of 2-aminophenylboronic acid pinacol ester $(2.40 \mathrm{mmol}), 0.116 \mathrm{~g}$ of $\mathrm{Pd}\left(\mathrm{PPh}_{3}\right)_{4}(0.10 \mathrm{mmol})$ in $20 \mathrm{~mL}$ of dimethoxyethane (0.1 M). Purification by MPLC (2:98 to 20:80 EtOAc:hexanes) afforded the product as a yellow oil $(0.422 \mathrm{~g}, 76 \%)$. The spectral data matched that reported by Driver and co-workers: ${ }^{18}{ }^{1} \mathrm{H}$ NMR $\left(500 \mathrm{MHz}, \mathrm{CDCl}_{3}\right) \delta 7.20-7.13(\mathrm{~m}, 4 \mathrm{H}), 7.10$ $(\mathrm{dd}, J=7.9,4.4 \mathrm{~Hz}, 1 \mathrm{H}), 6.98(\mathrm{t}, J=8.6 \mathrm{~Hz}, 2 \mathrm{H}), 6.65(\mathrm{t}, J=7.4 \mathrm{~Hz}, 1 \mathrm{H}), 6.56(\mathrm{~d}, J=7.8 \mathrm{~Hz}, 1 \mathrm{H}), 3.57(\mathrm{br} \mathrm{s}, 2 \mathrm{H}), 2.98-$ $2.89(\mathrm{~m}, 1 \mathrm{H}), 2.85(\mathrm{dt}, J=13.8,6.9 \mathrm{~Hz}, 1 \mathrm{H}), 2.60(\mathrm{dt}, J=13.9,4.3 \mathrm{~Hz}, 1 \mathrm{H}), 2.46(\mathrm{dt}, J=13.6,4.4 \mathrm{~Hz}, 1 \mathrm{H}), 1.96-1.84(\mathrm{~m}$, 4H), $1.80-1.71(\mathrm{~m}, 4 \mathrm{H}) ;{ }^{13} \mathrm{C}$ NMR $\left(125 \mathrm{MHz}, \mathrm{CDCl}_{3}\right) \delta 144.0(\mathrm{C}), 142.8(\mathrm{C}), 139.6(\mathrm{C}), 135.5(\mathrm{C}), 130.1(\mathrm{CH}), 130.0(\mathrm{C})$, $128.0(\mathrm{CH}), 127.6(\mathrm{CH}), 127.2(\mathrm{CH}), 126.1(\mathrm{CH}), 117.9(\mathrm{CH}), 115.2(\mathrm{CH}), 33.6\left(\mathrm{CH}_{2}\right), 32.7\left(\mathrm{CH}_{2}\right), 30.4\left(\mathrm{CH}_{2}\right), 28.9\left(\mathrm{CH}_{2}\right)$, $27.2\left(\mathrm{CH}_{2}\right), 26.9\left(\mathrm{CH}_{2}\right)$. ATR-FTIR (thin film): 2919, 2849, 1611, 1490, 1468, 1447, 1293, 1092, 747, $698 \mathrm{~cm}^{-1}$. HRMS (ESI) $m / z$ calculated for $\mathrm{C}_{20} \mathrm{H}_{24} \mathrm{~N}(\mathrm{M}+\mathrm{H})^{+}: 278.1909$, found: 278.1911 .

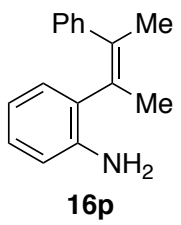

(Z)-2-(3-Phenylbut-2-en-2-yl)aniline 16p. ${ }^{18}$ Method A was followed using $0.560 \mathrm{~g}$ of vinyl triflate $(2.00 \mathrm{mmol}), 0.526 \mathrm{~g}$ of 2-aminophenylboronic acid pinacol ester $(2.40 \mathrm{mmol}), 0.116 \mathrm{~g}$ of $\mathrm{Pd}\left(\mathrm{PPh}_{3}\right)_{4}(0.10 \mathrm{mmol})$ in $20 \mathrm{~mL}$ of dimethoxyethane $(0.1 \mathrm{M})$. Purification by MPLC (2:98 to 20:80 EtOAc:hexanes) afforded the product, an orange oil, as a 94:6 mixture of Zand $E$-isomers $(0.353 \mathrm{~g}, 79 \%)$. The spectral data matched that reported by Driver and co-workers: ${ }^{18}{ }^{1} \mathrm{H} \mathrm{NMR}(500 \mathrm{MHz}$, $\left.\mathrm{CDCl}_{3}\right) \delta 7.15-7.05(\mathrm{~m}, 5 \mathrm{H}), 6.96(\mathrm{t}, J=7.7 \mathrm{~Hz}, 1 \mathrm{H}), 6.91(\mathrm{~d}, J=7.5 \mathrm{~Hz}, 1 \mathrm{H}), 6.65(\mathrm{t}, J=7.5 \mathrm{~Hz}, 1 \mathrm{H}), 6.53(\mathrm{~d}, J=7.9$ $\mathrm{Hz}, 1 \mathrm{H}), 3.66$ (br s, 2H), 2.25 (s, 3H), $2.15(\mathrm{~s}, 3 \mathrm{H}) ;{ }^{13} \mathrm{C}$ NMR (125 MHz, CDCl 3 ) $\delta 143.9$ (C), $142.6(\mathrm{C}), 134.3(\mathrm{C}), 130.6$ $(\mathrm{C}), 130.5(\mathrm{C}), 130.0(\mathrm{CH}), 128.0(\mathrm{CH}), 127.6(\mathrm{CH}), 127.3(\mathrm{CH}), 126.1(\mathrm{CH}), 118.4(\mathrm{CH}), 115.4(\mathrm{CH}), 20.8\left(\mathrm{CH}_{3}\right), 20.7$ $\left(\mathrm{CH}_{3}\right)$. ATR-FTIR (thin film): 3378, 3019, 2919, 1611, 1490, 1449, 1292, 1067, 1027, 763, 747, $699 \mathrm{~cm}^{-1}$. HRMS (ESI) $m / z$ calculated for $\mathrm{C}_{16} \mathrm{H}_{18} \mathrm{~N}(\mathrm{M}+\mathrm{H})^{+}: 224.1439$, found: 224.1435 .

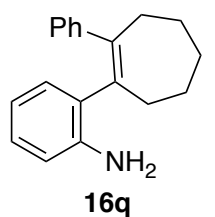

2-(2-Phenylcyclohept-1-en-1-yl)aniline 16q. ${ }^{18}$ Method A was followed using $0.640 \mathrm{~g}$ of vinyl triflate $(2.00 \mathrm{mmol}), 0.526$ $\mathrm{g}$ of 2-aminophenylboronic acid pinacol ester $(2.40 \mathrm{mmol}), 0.116 \mathrm{~g}$ of $\mathrm{Pd}\left(\mathrm{PPh}_{3}\right)_{4}(0.10 \mathrm{mmol})$ in $20 \mathrm{~mL}$ of dimethoxyethane (0.1 M). Purification by MPLC (2:98 to 20:80 EtOAc:hexanes) afforded the product as a yellow solid $(0.525 \mathrm{~g}, 99 \%)$. The spectral data matched that reported by Driver and co-workers: ${ }^{18} \mathrm{mp}=60{ }^{\circ} \mathrm{C} ;{ }^{1} \mathrm{H} \mathrm{NMR}\left(500 \mathrm{MHz}, \mathrm{CDCl}_{3}\right) \delta 7.19-7.13(\mathrm{~m}$, $2 \mathrm{H}), 7.13-7.07(\mathrm{~m}, 3 \mathrm{H}), 6.98(\mathrm{t}, J=7.7 \mathrm{~Hz}, 1 \mathrm{H}), 6.89(\mathrm{~d}, J=7.3 \mathrm{~Hz}, 1 \mathrm{H}), 6.65(\mathrm{t}, J=7.5 \mathrm{~Hz}, 1 \mathrm{H}), 6.57(\mathrm{~d}, J=7.9 \mathrm{~Hz}$, 1H), 3.61 (br s, 2H), $2.86(\mathrm{ddd}, J=14.8,9.2,2.4 \mathrm{~Hz}, 1 \mathrm{H}), 2.80-2.70(\mathrm{~m}, 2 \mathrm{H}), 2.62(\mathrm{ddd}, J=14.3,8.1,2.4 \mathrm{~Hz}, 1 \mathrm{H}), 2.06$ (dtt, $J=14.6,7.1,4.1 \mathrm{~Hz}, 1 \mathrm{H}), 1.96(\mathrm{ddq}, J=12.9,8.5,3.9,3.4 \mathrm{~Hz}, 1 \mathrm{H}), 1.88(\mathrm{qd}, J=10.5,7.0 \mathrm{~Hz}, 2 \mathrm{H}), 1.81-1.70(\mathrm{~m}$, $2 \mathrm{H}) ;{ }^{13} \mathrm{C}$ NMR $\left(125 \mathrm{MHz}, \mathrm{CDCl}_{3}\right) \delta 144.5(\mathrm{C}), 142.7(\mathrm{C}), 142.6(\mathrm{C}), 138.8(\mathrm{C}), 131.6(\mathrm{C}), 129.9(\mathrm{CH}), 127.8(\mathrm{CH}), 127.6$ $(\mathrm{CH}), 127.2(\mathrm{CH}), 126.0(\mathrm{CH}), 118.4(\mathrm{CH}), 115.3(\mathrm{CH}), 36.3\left(\mathrm{CH}_{2}\right), 36.2\left(\mathrm{CH}_{2}\right), 32.9\left(\mathrm{CH}_{2}\right), 27.5\left(\mathrm{CH}_{2}\right), 26.8\left(\mathrm{CH}_{2}\right)$. ATRFTIR (thin film): 2917, 2846, 1610, 1489, 1447, 1292, 1028, 971, 745, 699, $668 \mathrm{~cm}^{-1}$. HRMS (ESI) $\mathrm{m} / z$ calculated for $\mathrm{C}_{19} \mathrm{H}_{22} \mathrm{~N}(\mathrm{M}+\mathrm{H})^{+}: 264.1752$, found: 264.1748 . 


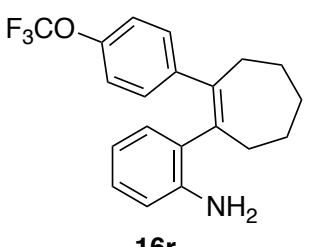

2-(2-(4-(Trifluoromethoxy)phenyl)cyclohept-1-en-1-yl)aniline 16r. ${ }^{18}$ Method A was followed using $0.809 \mathrm{~g}$ of vinyl triflate $(2.00 \mathrm{mmol}), 0.526 \mathrm{~g}$ of 2-aminophenylboronic acid pinacol ester $(2.40 \mathrm{mmol}), 0.116 \mathrm{~g}$ of $\mathrm{Pd}\left(\mathrm{PPh}_{3}\right)_{4}(0.10 \mathrm{mmol})$ in $20 \mathrm{~mL}$ of dimethoxyethane (0.1 M). Purification by MPLC (2:98 to 20:80 EtOAc:hexanes) afforded the product as an orange oil $(0.694 \mathrm{~g}, 99 \%)$. The spectral data matched that reported by Driver and co-workers: ${ }^{18}{ }^{1} \mathrm{H} \mathrm{NMR}\left(500 \mathrm{MHz}, \mathrm{CDCl}_{3}\right) \delta 7.13$ $-7.08(\mathrm{~m}, 2 \mathrm{H}), 7.02-6.95(\mathrm{~m}, 3 \mathrm{H}), 6.82(\mathrm{dd}, J=7.6,1.6 \mathrm{~Hz}, 1 \mathrm{H}), 6.62(\mathrm{td}, J=7.4,1.1 \mathrm{~Hz}, 1 \mathrm{H}), 6.58(\mathrm{~d}, J=7.9 \mathrm{~Hz}, 1 \mathrm{H})$, 3.59 (br s, 2H), 2.83 (ddd, $J=14.8,9.6,2.0 \mathrm{~Hz}, 1 \mathrm{H}), 2.72$ (dddd, $J=14.1,11.0,8.7,2.2 \mathrm{~Hz}, 2 \mathrm{H}), 2.60$ (ddd, $J=14.2,8.3$, $2.2 \mathrm{~Hz}, 1 \mathrm{H}), 2.11-2.01(\mathrm{~m}, 1 \mathrm{H}), 1.99-1.68(\mathrm{~m}, 5 \mathrm{H}) ;{ }^{13} \mathrm{C} \mathrm{NMR}\left(125 \mathrm{MHz}, \mathrm{CDCl}_{3}\right) \delta 147.2(\mathrm{C}), 143.2(\mathrm{C}), 142.7(\mathrm{C})$, $141.4(\mathrm{C}), 139.8(\mathrm{C}), 131.0(\mathrm{C}), 129.8(\mathrm{CH}), 129.1(\mathrm{CH}), 127.4(\mathrm{CH}), 120.5\left(\mathrm{q}, J_{C F}=256.8 \mathrm{~Hz}, \mathrm{C}\right), 119.9(\mathrm{CH}), 118.4(\mathrm{CH})$, 115.2 $(\mathrm{CH}), 36.1\left(\mathrm{CH}_{2}\right), 36.0\left(\mathrm{CH}_{2}\right), 32.7\left(\mathrm{CH}_{2}\right), 27.3\left(\mathrm{CH}_{2}\right), 26.6\left(\mathrm{CH}_{2}\right) ;{ }^{19} \mathrm{~F} \mathrm{NMR}\left(282 \mathrm{MHz}, \mathrm{CDCl}_{3}\right) \delta-58.3$. ATR-FTIR (thin film): 2921, 2850, 1612, 1505, 1491, 1449, 1220, 1205, 1162, 849, 810, $747 \mathrm{~cm}^{-1}$. HRMS (ESI) $\mathrm{m} / z$ calculated for $\mathrm{C}_{20} \mathrm{H}_{21} \mathrm{NOF}_{3}(\mathrm{M}+\mathrm{H})^{+}:$348.1575, found: 348.1567 .

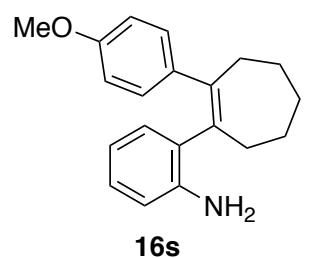

2-(2-(4-Methoxyphenyl)cyclohept-1-en-1-yl)aniline $\mathbf{1 6 s} .{ }^{18}$ Method A was followed using $0.701 \mathrm{~g}$ of vinyl triflate (2.00

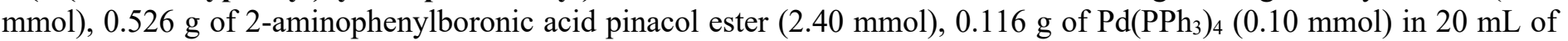
dimethoxyethane $(0.1 \mathrm{M})$. Purification by MPLC (2:98 to 20:80 EtOAc:hexanes) afforded the product as an orange oil $(0.306 \mathrm{~g}, 52 \%)$. The spectral data matched that reported by Driver and co-workers: ${ }^{18} \mathrm{mp}=100{ }^{\circ} \mathrm{C} ;{ }^{1} \mathrm{H} \mathrm{NMR}(500 \mathrm{MHz}$, $\left.\mathrm{CDCl}_{3}\right) \delta 7.04-6.98(\mathrm{~m}, 2 \mathrm{H}), 6.97-6.92(\mathrm{~m}, 1 \mathrm{H}), 6.91-6.85(\mathrm{~m}, 1 \mathrm{H}), 6.69-6.60(\mathrm{~m}, 3 \mathrm{H}), 6.55(\mathrm{~d}, J=7.9 \mathrm{~Hz}, 1 \mathrm{H}), 3.72$ (s, 5H), 2.79 (ddd, $J=14.7,8.7,2.3 \mathrm{~Hz}, 1 \mathrm{H}), 2.74-2.63(\mathrm{~m}, 2 \mathrm{H}), 2.56$ (ddd, $J=14.3,8.0,2.7 \mathrm{~Hz}, 1 \mathrm{H}), 2.00$ (dddd, $J=$ $14.9,10.9,7.0,4.1 \mathrm{~Hz}, 1 \mathrm{H}), 1.95-1.86(\mathrm{~m}, 1 \mathrm{H}), 1.86-1.76(\mathrm{~m}, 2 \mathrm{H}), 1.71(\mathrm{p}, J=3.7 \mathrm{~Hz}, 2 \mathrm{H}) ;{ }^{13} \mathrm{C} \mathrm{NMR}\left(125 \mathrm{MHz}, \mathrm{CDCl}_{3}\right)$ $\delta 157.7(\mathrm{C}), 142.6(\mathrm{C}), 141.9(\mathrm{C}), 137.7(\mathrm{C}), 136.8(\mathrm{C}), 131.8(\mathrm{C}), 129.8(\mathrm{CH}), 128.9(\mathrm{CH}), 127.0(\mathrm{CH}), 118.5(\mathrm{CH}), 115.3$ $(\mathrm{CH}), 113.0(\mathrm{CH}), 55.1\left(\mathrm{CH}_{3}\right), 36.4\left(\mathrm{CH}_{2}\right), 36.2\left(\mathrm{CH}_{2}\right), 32.8\left(\mathrm{CH}_{2}\right), 27.4\left(\mathrm{CH}_{2}\right), 26.8\left(\mathrm{CH}_{2}\right)$. ATR-FTIR (thin film): 2917 , $2846,1608,1508,1490,1449,1290,1244,1177,1032,826,748 \mathrm{~cm}^{-1}$. HRMS (ESI) $\mathrm{m} / z$ calculated for $\mathrm{C}_{20} \mathrm{H}_{24} \mathrm{NO}(\mathrm{M}+\mathrm{H})^{+}$: 294.1858, found: 294.1851 .

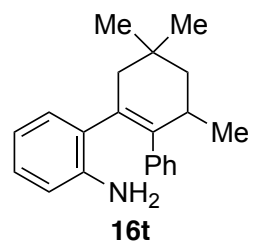

3',5',5'-Trimethyl-3',4',5',6'-tetrahydro-[1,1':2',1'-terphenyl]-2-amine 16t. Method A was followed using $0.348 \mathrm{~g}$ of vinyl triflate $(1.00 \mathrm{mmol}), 0.263 \mathrm{~g}$ of 2-aminophenylboronic acid pinacol ester $(1.20 \mathrm{mmol}), 0.058 \mathrm{~g}$ of $\mathrm{Pd}\left(\mathrm{PPh}_{3}\right)_{4}(0.05$ $\mathrm{mmol})$ in $10 \mathrm{~mL}$ of dimethoxyethane $(0.1 \mathrm{M})$. Purification by MPLC (2:98 to 20:80 EtOAc:hexanes) afforded the product, a yellow oil, as a 2:1 ratio of rotamers $(0.261 \mathrm{~g}, 90 \%)$; Diagnostic peaks for the major rotamer: ${ }^{1} \mathrm{H} \mathrm{NMR}\left(500 \mathrm{MHz}^{\mathrm{CDCl}} 3\right)$ $\delta 7.24-7.09(\mathrm{~m}, 5.45 \mathrm{H}), 7.01(\mathrm{t}, J=7.7 \mathrm{~Hz}, 0.67 \mathrm{H}), 6.80(\mathrm{t}, J=7.5 \mathrm{~Hz}, 0.65 \mathrm{H}), 6.69-6.61(\mathrm{~m}, 0.65 \mathrm{H}), 6.48(\mathrm{~d}, J=7.9$ $\mathrm{Hz}, 0.62 \mathrm{H}), 3.42(\mathrm{~s}, 1.15 \mathrm{H}), 2.96(\mathrm{dt}, J=12.3,6.4 \mathrm{~Hz}, 1 \mathrm{H}), 2.46(\mathrm{dd}, J=17.5,3.8 \mathrm{~Hz}, 0.60 \mathrm{H}), 2.05(\mathrm{~d}, J=18.0 \mathrm{~Hz}, 0.59 \mathrm{H})$, $1.90-1.81(\mathrm{~m}, 0.92 \mathrm{H}), 1.54-1.46(\mathrm{~m}, 1.03 \mathrm{H}), 1.28(\mathrm{~s}, 1.82 \mathrm{H}), 1.19(\mathrm{~s}, 1.99 \mathrm{H}), 0.98(\mathrm{~d}, J=7.0 \mathrm{~Hz}, 2.02 \mathrm{H}) ;{ }^{13} \mathrm{C} \mathrm{NMR}(125$ $\left.\mathrm{MHz}, \mathrm{CDCl}_{3}\right) \delta 142.0(\mathrm{C}), 141.3(\mathrm{C}), 139.6(\mathrm{C}), 131.8(\mathrm{C}), 129.9(\mathrm{CH}), 129.4(\mathrm{C}), 128.6(\mathrm{CH}), 127.5(\mathrm{CH}), 127.4(\mathrm{CH})$, 
$126.1(\mathrm{CH}), 118.0(\mathrm{CH}), 115.6(\mathrm{CH}), 46.2\left(\mathrm{CH}_{2}\right), 45.9\left(\mathrm{CH}_{2}\right), 32.3(\mathrm{CH}), 31.7\left(\mathrm{CH}_{3}\right), 29.7(\mathrm{C}), 25.7\left(\mathrm{CH}_{3}\right), 21.2\left(\mathrm{CH}_{3}\right)$. Diagnostic peaks for minor rotamer: ${ }^{1} \mathrm{H}$ NMR $\left(500 \mathrm{MHz}, \mathrm{CDCl}_{3}\right) \delta 7.24-7.09(\mathrm{~m}, 5.45 \mathrm{H}), 6.97(\mathrm{t}, J=7.7 \mathrm{~Hz}, 0.38 \mathrm{H}), 6.56$ (t, $J=7.4 \mathrm{~Hz}, 0.33 \mathrm{H}), 3.80$ (br s, 0.53H), 2.62 (dd, $J=17.2,3.5 \mathrm{~Hz}, 0.29 \mathrm{H}), 1.99$ (d, $J=17.2 \mathrm{~Hz}, 0.28 \mathrm{H}), 1.27(\mathrm{~s}, 1.05 \mathrm{H})$, $1.20(\mathrm{~s}, 0.94 \mathrm{H}), 1.03(\mathrm{~d}, J=6.9 \mathrm{~Hz}, 1.03 \mathrm{H}) ;{ }^{13} \mathrm{C} \mathrm{NMR}\left(125 \mathrm{MHz}, \mathrm{CDCl}_{3}\right) \delta 143.5(\mathrm{C}), 141.8(\mathrm{C}), 141.2(\mathrm{C}), 132.0(\mathrm{C})$, $130.8(\mathrm{C}), 129.7(\mathrm{CH}), 128.0(\mathrm{CH}), 127.6(\mathrm{CH}), 127.0(\mathrm{CH}), 126.0(\mathrm{CH}), 118.6(\mathrm{CH}), 114.9(\mathrm{CH}), 46.0\left(\mathrm{CH}_{2}\right), 44.9\left(\mathrm{CH}_{2}\right)$, $32.8(\mathrm{CH}), 31.5\left(\mathrm{CH}_{3}\right), 29.9(\mathrm{C}), 25.6\left(\mathrm{CH}_{3}\right), 21.3\left(\mathrm{CH}_{3}\right)$. ATR-FTIR (thin film): 2951, 2899, 2866, 2359, 2339, 1611, 1492, $1453,747,700 \mathrm{~cm}^{-1}$. HRMS (ESI) $\mathrm{m} / z$ calculated for $\mathrm{C}_{21} \mathrm{H}_{26} \mathrm{~N}(\mathrm{M}+\mathrm{H})^{+}:$292.2065, found: 292.2059.

\section{Formation of $\mathbf{3 H}$-indoles from ortho-substituted aniline}

\section{A. Screening of reaction conditions}
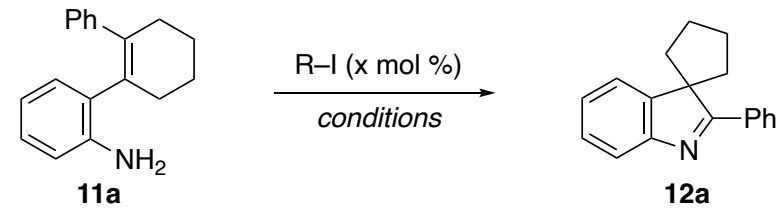

$12 a$ (s19)

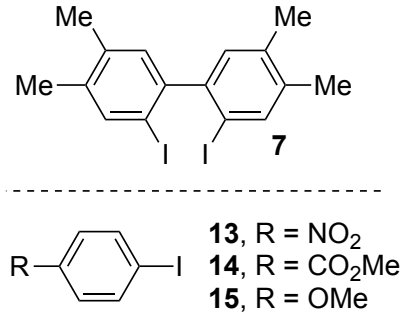

To a solution of anilines 11a (1.0 equiv), iodide ( $20 \mathrm{~mol} \%$ ), and additive in $2 \mathrm{~mL}$ of solvent, was added the oxidant in one portion. The resulting mixture was sealed. After $3 \mathrm{~h}$, the reactives were quenched by the addition of $3 \mathrm{~mL}$ of a saturated aq soln of $\mathrm{Na}_{2} \mathrm{~S}_{2} \mathrm{O}_{3}$ and $3 \mathrm{~mL}$ of a saturated aq soln of $\mathrm{NaHCO}_{3}$. The resulting mixture was extracted with $3 \times 5 \mathrm{~mL}$ of EtOAc. The combined organic phases were washed with $10 \mathrm{~mL}$ of brine. The resulting organic phase was dried over $\mathrm{Na}_{2} \mathrm{SO}_{4}$ and filtered through Celite. The filtrate was concentrated under reduced pressure, and the residue was analyzed using ${ }^{1} \mathrm{H} N M R$ spectroscopy using $\mathrm{CH}_{2} \mathrm{Br}_{2}$ as the internal standard. 
Table s1. Optimization of I(III)-catalyzed oxidative cyclization of 2-aminostyrenes

\begin{tabular}{|c|c|c|c|c|c|c|c|c|c|}
\hline entry & $\mathrm{R}-\mathrm{I}$ & $\mathrm{mol} \%$ & oxidant & equiv & additive & equiv & solvent & $\mathrm{T}\left({ }^{\circ} \mathrm{C}\right)$ & $\begin{array}{c}\text { yield, } \\
\%^{\mathrm{a}}\end{array}$ \\
\hline 1 & $\mathrm{PhI}$ & 20 & $\mathrm{mCPBA}$ & 1.7 & $\ldots$ & $\ldots$ & TFE & 0 to $25^{\circ} \mathrm{C}$ & 44 \\
\hline 2 & $\mathrm{PhI}$ & 10 & mCPBA & 1.7 & $\ldots$ & $\ldots$ & HFIP & 0 to $25^{\circ} \mathrm{C}$ & 60 \\
\hline 3 & $\mathrm{PhI}$ & 20 & Selectfluor & 2.0 & TFA & 2.0 & HFIP & $25^{\circ} \mathrm{C}$ & 42 \\
\hline 4 & $\mathrm{PhI}$ & 20 & Selectfluor & 2.0 & TFA & 2.0 & $\mathrm{HFIP} / \mathrm{H}_{2} \mathrm{O}(100: 1)$ & $25^{\circ} \mathrm{C}$ & 77 \\
\hline 5 & $\mathrm{PhI}$ & 20 & Selectfluor & 2.0 & TFA & 2.0 & $\mathrm{HFIP} / \mathrm{H}_{2} \mathrm{O}(10: 1)$ & $25^{\circ} \mathrm{C}$ & 100 \\
\hline 6 & $\mathrm{PhI}$ & 20 & Selectfluor & 2.0 & TFA & 2.0 & $\mathrm{HFIP} / \mathrm{H}_{2} \mathrm{O}(1: 1)$ & $25^{\circ} \mathrm{C}$ & 100 \\
\hline 7 & $\mathrm{PhI}$ & 20 & Selectfluor & 2.0 & TFA & 2.0 & $\mathrm{HFIP} / \mathrm{H}_{2} \mathrm{O}(1: 10)$ & $25^{\circ} \mathrm{C}$ & 60 \\
\hline 8 & $\mathrm{PhI}$ & 20 & Selectfluor & 1.3 & TFA & 2.6 & $\mathrm{HFIP} / \mathrm{H}_{2} \mathrm{O}(10: 1)$ & $25^{\circ} \mathrm{C}$ & 100 \\
\hline 9 & $\mathrm{PhI}$ & 20 & Selectfluor & 1.3 & $\mathrm{Me}_{3} \mathrm{SiOAc}$ & 2.6 & $\mathrm{HFIP} / \mathrm{H}_{2} \mathrm{O}(10: 1)$ & $25^{\circ} \mathrm{C}$ & 72 \\
\hline 10 & $\mathrm{PhI}$ & 20 & Selectfluor & 1.3 & $\mathrm{Me}_{3} \mathrm{SiO}_{2} \mathrm{CCF}_{3}$ & 2.6 & $\mathrm{HFIP} / \mathrm{H}_{2} \mathrm{O}(10: 1)$ & $25^{\circ} \mathrm{C}$ & 90 \\
\hline 11 & none & $\ldots$ & Selectfluor & 1.3 & TFA & 2.6 & $\mathrm{HFIP} / \mathrm{H}_{2} \mathrm{O}(10: 1)$ & $25^{\circ} \mathrm{C}$ & 22 \\
\hline 12 & $\mathrm{PhI}$ & 20 & Selectfluor & 1.3 & $\ldots$ & $\ldots$ & $\mathrm{HFIP} / \mathrm{H}_{2} \mathrm{O}(10: 1)$ & $25^{\circ} \mathrm{C}$ & 84 \\
\hline 13 & $\mathrm{PhI}$ & 10 & Selectfluor & 1.3 & TFA & 2.6 & $\mathrm{HFIP} / \mathrm{H}_{2} \mathrm{O}(10: 1)$ & $25^{\circ} \mathrm{C}$ & 99 \\
\hline 14 & $\mathrm{PhI}$ & 10 & Selectfluor & 1.3 & $\mathrm{Me}_{3} \mathrm{SiO}_{2} \mathrm{CCF}_{3}$ & 2.6 & $\mathrm{HFIP} / \mathrm{H}_{2} \mathrm{O}(10: 1)$ & $25^{\circ} \mathrm{C}$ & 99 \\
\hline 15 & $\mathrm{PhI}$ & 5 & Selectfluor & 1.3 & TFA & 2.6 & $\mathrm{HFIP} / \mathrm{H}_{2} \mathrm{O}(10: 1)$ & $25^{\circ} \mathrm{C}$ & 99 \\
\hline 16 & $\mathrm{PhI}$ & 1 & Selectfluor & 1.3 & TFA & 2.6 & $\mathrm{HFIP} / \mathrm{H}_{2} \mathrm{O}(10: 1)$ & $25^{\circ} \mathrm{C}$ & 99 \\
\hline 15 & 7 & 20 & Selectfluor & 1.3 & TFA & 2.6 & $\mathrm{HFIP} / \mathrm{H}_{2} \mathrm{O}(10: 1)$ & $25^{\circ} \mathrm{C}$ & 91 \\
\hline 16 & 13 & 20 & Selectfluor & 1.3 & TFA & 2.6 & $\mathrm{HFIP} / \mathrm{H}_{2} \mathrm{O}(10: 1)$ & $25^{\circ} \mathrm{C}$ & 30 \\
\hline 17 & 14 & 20 & Selectfluor & 1.3 & TFA & 2.6 & $\mathrm{HFIP} / \mathrm{H}_{2} \mathrm{O}(10: 1)$ & $25^{\circ} \mathrm{C}$ & 73 \\
\hline 18 & 15 & 20 & Selectfluor & 1.3 & TFA & 2.6 & $\mathrm{HFIP} / \mathrm{H}_{2} \mathrm{O}(10: 1)$ & $25^{\circ} \mathrm{C}$ & 70 \\
\hline 19 & $n$-BuI & 20 & Selectfluor & 1.3 & TFA & 2.6 & $\mathrm{HFIP} / \mathrm{H}_{2} \mathrm{O}(10: 1)$ & $25^{\circ} \mathrm{C}$ & 100 \\
\hline 20 & TBAI & 20 & Selectfluor & 1.3 & TFA & 2.6 & $\mathrm{HFIP} / \mathrm{H}_{2} \mathrm{O}(10: 1)$ & $25^{\circ} \mathrm{C}$ & 32 \\
\hline 21 & $\mathrm{I}_{2}$ & 20 & Selectfluor & 1.5 & None & None & HFIP & $25^{\circ} \mathrm{C}$ & 0 \\
\hline 22 & None & $\ldots$ & NIS & 1.5 & None & None & HFIP & $25^{\circ} \mathrm{C}$ & 18 \\
\hline 23 & None & $\ldots$ & NIS & 1.5 & None & None & $\mathrm{HFIP} / \mathrm{H}_{2} \mathrm{O}(10: 1)$ & $25^{\circ} \mathrm{C}$ & 0 \\
\hline 24 & None & $\ldots$ & OXONE & 1.5 & None & None & $\mathrm{HFIP} / \mathrm{H}_{2} \mathrm{O}(10: 1)$ & $25^{\circ} \mathrm{C}$ & 0 \\
\hline 25 & None & $\ldots$ & CAN & 1.5 & None & None & $\mathrm{HFIP} / \mathrm{H}_{2} \mathrm{O}(10: 1)$ & $25^{\circ} \mathrm{C}$ & 0 \\
\hline 26 & None & $\ldots$ & DDQ & 1.5 & None & None & $\mathrm{HFIP} / \mathrm{H}_{2} \mathrm{O}(10: 1)$ & $25^{\circ} \mathrm{C}$ & 0 \\
\hline 27 & None & $\ldots$ & $\mathrm{SeO}_{2}$ & 1.5 & None & None & $\mathrm{HFIP} / \mathrm{H}_{2} \mathrm{O}(10: 1)$ & $25^{\circ} \mathrm{C}$ & 0 \\
\hline
\end{tabular}

a As determined using ${ }^{1} \mathrm{H}$ NMR spectroscopy using $\mathrm{CH}_{2} \mathrm{Br}_{2}$ as an internal standard. b The product decomposed in the reaction condition on entry 2 . c $93 \%$ starting material remained.

\section{B. Optimized reaction procedure.}
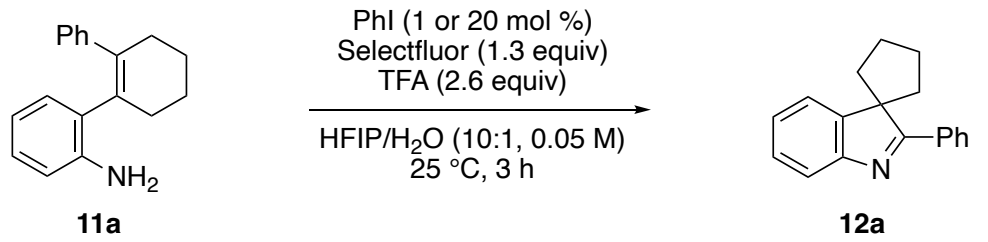

(s20)

To a solution of 2 -substituted aniline ( $0.1 \mathrm{mmol}, 1.0$ equiv), iodobenzene ( 1 or $20 \mathrm{~mol} \%$ ), and trifluoroacetic acid (2.6 equiv) in $2 \mathrm{~mL}$ of HFIP and $0.2 \mathrm{~mL}$ of $\mathrm{H}_{2} \mathrm{O}(0.05 \mathrm{M})$ was added Selectfluor $(0.2 \mathrm{mmol}, 2.0$ equiv) in portion. After $3 \mathrm{~h}$, the reactives were quenched by the addition of $3 \mathrm{~mL}$ of a saturated aq soln of $\mathrm{Na}_{2} \mathrm{~S}_{2} \mathrm{O}_{3}$ and $3 \mathrm{~mL}$ of a saturated aq soln of $\mathrm{NaHCO}_{3}$. The resulting mixture was extracted with $3 \times 5 \mathrm{~mL}$ of EtOAc. The combined organic phases were washed with $10 \mathrm{~mL}$ of brine, dried over $\mathrm{Na}_{2} \mathrm{SO}_{4}$ and filtered through Celite, the filtrate was concentrated in vacuo. Purification using MPLC afforded the product. 


\section{Characterization data for $\mathbf{3 H}$-indoles.}

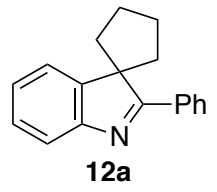

2'-Phenylspiro[cyclopentane-1,3'-indole] 12a. ${ }^{22}$ The optimized procedure was followed using $0.025 \mathrm{~g}$ of aniline $11 \mathrm{a}(0.10$ $\mathrm{mmol}), 0.11 \mu \mathrm{L}$ of iodobenzene $(0.001 \mathrm{mmol}), 20 \mu \mathrm{L}$ of trifluoroacetic acid $(0.26 \mathrm{mmol}), 0.046 \mathrm{~g}$ of Selectfluor $(0.13 \mathrm{mmol})$ in $2 \mathrm{~mL}$ of 1,1,1,3,3,3-hexafluoropropan-2-ol and $0.2 \mathrm{~mL}$ of water $(0.05 \mathrm{M})$. Purification by MPLC (2:98 to 5:95 EtOAc:hexanes) afforded the product as a yellow oil $(0.024 \mathrm{~g}, 98 \%)$. The spectral data matched that reported by Driver and co-workers: ${ }^{22}{ }^{1} \mathrm{H}$ NMR $\left(500 \mathrm{MHz}, \mathrm{CDCl}_{3}\right) \delta 8.15-8.04(\mathrm{~m}, 2 \mathrm{H}), 7.69(\mathrm{~d}, J=7.7 \mathrm{~Hz}, 1 \mathrm{H}), 7.53-7.45(\mathrm{~m}, 3 \mathrm{H}), 7.40(\mathrm{~d}, J$ $=7.4 \mathrm{~Hz}, 1 \mathrm{H}), 7.35(\mathrm{t}, J=7.6 \mathrm{~Hz}, 1 \mathrm{H}), 7.24(\mathrm{t}, J=7.5 \mathrm{~Hz}, 1 \mathrm{H}), 2.52-2.39(\mathrm{~m}, 2 \mathrm{H}), 2.31-2.16(\mathrm{~m}, 4 \mathrm{H}), 2.00-1.88(\mathrm{~m}$, $2 \mathrm{H}) ;{ }^{13} \mathrm{C}$ NMR $\left(125 \mathrm{MHz}, \mathrm{CDCl}_{3}\right) \delta 182.7(\mathrm{C}), 153.0(\mathrm{C}), 150.2(\mathrm{C}), 132.9(\mathrm{C}), 130.4(\mathrm{CH}), 128.6(\mathrm{CH}), 128.3(\mathrm{CH}), 127.4$ $(\mathrm{CH}), 125.9(\mathrm{CH}), 121.0(\mathrm{CH}), 120.7(\mathrm{CH}), 63.3(\mathrm{C}), 36.8\left(\mathrm{CH}_{2}\right), 27.6\left(\mathrm{CH}_{2}\right)$. ATR-FTIR (thin film): 2956, 2872, 1520, $1493,1465,1455,1442,1343,1265,1018,770,750,694 \mathrm{~cm}^{-1}$. HRMS (ESI) $\mathrm{m} / z$ calculated for $\mathrm{C}_{18} \mathrm{H}_{18} \mathrm{~N}(\mathrm{M}+\mathrm{H})^{+}: 248.1439$, found: 248.1435 .

$1 \mathrm{~mol}$ scale reaction to produce 2 '-phenylspiro[cyclopentane-1,3'-indole] 12a. To a solution of $0.250 \mathrm{~g}$ of aniline 11a (1.00 mmol), $23 \mu \mathrm{L}$ of iodobenzene $(0.20 \mathrm{mmol})$, and $200 \mu \mathrm{L}$ of trifluoroacetic acid $(2.6 \mathrm{mmol})$ in $20 \mathrm{~mL}$ of HFIP and 2.0 $\mathrm{mL}$ of $\mathrm{H}_{2} \mathrm{O}(0.05 \mathrm{M}), 0.460 \mathrm{~g}$ of selectfluor was added in portion. After $3 \mathrm{~h}$, the reactives were quenched by the addition of $30 \mathrm{~mL}$ of a saturated aq soln of $\mathrm{Na}_{2} \mathrm{~S}_{2} \mathrm{O}_{3}$ and $30 \mathrm{~mL}$ of a saturated aq soln of $\mathrm{NaHCO}_{3}$. The resulting mixture was extracted with $3 \times 50 \mathrm{~mL}$ of EtOAc. The combined organic phases were washed with $100 \mathrm{~mL}$ of brine, dried over $\mathrm{Na}_{2} \mathrm{SO}_{4}$ and filtered through Celite, the filtrate was concentrated in vacuo. Purification using MPLC (2:98 to 5:95 EtOAc:hexanes) afforded the product as a yellow oil $(0.23 \mathrm{~g}, 94 \%)$.

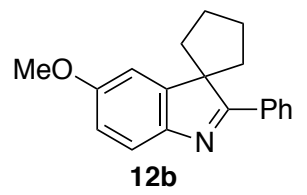

5'-Methoxy-2'-phenylspiro[cyclopentane-1,3'-indole] 12b. ${ }^{22}$ The optimized procedure was followed using $0.028 \mathrm{~g}$ of aniline $11 \mathrm{~b}(0.10 \mathrm{mmol}), 0.11 \mu \mathrm{L}$ of iodobenzene $(0.001 \mathrm{mmol}), 20 \mu \mathrm{L}$ of trifluoroacetic acid $(0.26 \mathrm{mmol}), 0.046 \mathrm{~g}$ of Selectfluor $(0.13 \mathrm{mmol})$ in $2 \mathrm{~mL}$ of 1,1,1,3,3,3-hexafluoropropan-2-ol and $0.2 \mathrm{~mL}$ of water $(0.05 \mathrm{M})$. Purification by MPLC (2:98 to 10:90 EtOAc:hexanes) afforded the product as a yellow oil $(0.028 \mathrm{~g}, 100 \%)$. The spectral data matched that reported by Driver and co-workers: ${ }^{22}{ }^{1} \mathrm{H}$ NMR $\left(500 \mathrm{MHz} \mathrm{CDCl}_{3}\right) \delta 8.11-7.98(\mathrm{~m}, 2 \mathrm{H}), 7.58(\mathrm{~d}, J=8.4 \mathrm{~Hz}, 1 \mathrm{H}), 7.54-7.38(\mathrm{~m}$, $3 \mathrm{H}), 6.95(\mathrm{~d}, J=2.8 \mathrm{~Hz}, 1 \mathrm{H}), 6.87(\mathrm{dd}, J=8.3,2.6 \mathrm{~Hz}, 1 \mathrm{H}), 3.86(\mathrm{~s}, 3 \mathrm{H}), 2.54-2.38(\mathrm{~m}, 2 \mathrm{H}), 2.28-2.13(\mathrm{~m}, 4 \mathrm{H}), 1.98-$ $1.90(\mathrm{~m}, 2 \mathrm{H}) ;{ }^{13} \mathrm{C}$ NMR $\left(125 \mathrm{MHz}, \mathrm{CDCl}_{3}\right) \delta 180.8(\mathrm{C}), 158.5(\mathrm{C}), 151.9(\mathrm{C}), 146.9(\mathrm{C}), 133.0(\mathrm{C}), 130.1(\mathrm{CH}), 128.6(\mathrm{CH})$, $128.0(\mathrm{CH}), 120.9(\mathrm{CH}), 111.6(\mathrm{CH}), 108.2(\mathrm{CH}), 63.4(\mathrm{C}), 55.8\left(\mathrm{CH}_{3}\right), 37.0\left(\mathrm{CH}_{2}\right), 27.5\left(\mathrm{CH}_{2}\right)$. ATR-FTIR $($ thin film): 2954, 2872, 2833, 1591, 1520, 1466, 1440, 1288, 1272, 1211, 1119, 1032, 813, 771, $695 \mathrm{~cm}^{-1}$. HRMS (ESI) $\mathrm{m} / z$ calculated for $\mathrm{C}_{19} \mathrm{H}_{20} \mathrm{NO}(\mathrm{M}+\mathrm{H})^{+}: 278.1545$, found: 278.1537 .

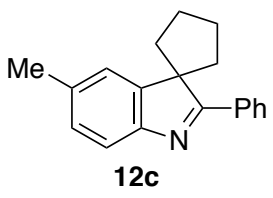

5'-Methyl-2'-phenylspiro[cyclopentane-1,3'-indole] 12c. ${ }^{22}$ The optimized procedure was followed using $0.026 \mathrm{~g}$ of aniline $11 \mathrm{c}(0.10 \mathrm{mmol}), 0.11 \mu \mathrm{L}$ of iodobenzene $(0.001 \mathrm{mmol}), 20 \mu \mathrm{L}$ of trifluoroacetic acid $(0.26 \mathrm{mmol}), 0.046 \mathrm{~g}$ of Selectfluor $(0.13 \mathrm{mmol})$ in $2 \mathrm{~mL}$ of 1,1,1,3,3,3-hexafluoropropan-2-ol and $0.2 \mathrm{~mL}$ of water $(0.05 \mathrm{M})$. Purification by MPLC (2:98 to 5:95 EtOAc:hexanes) afforded the product as a white oil $(0.026 \mathrm{~g}, 100 \%)$. The spectral data matched that reported 
by Driver and co-workers: ${ }^{22}{ }^{1} \mathrm{H}$ NMR $\left(500 \mathrm{MHz}, \mathrm{CDCl}_{3}\right) \delta 8.14-8.02(\mathrm{~m}, 2 \mathrm{H}), 7.57(\mathrm{~d}, J=7.8 \mathrm{~Hz}, 1 \mathrm{H}), 7.53-7.39(\mathrm{~m}$, $3 \mathrm{H}), 7.20(\mathrm{~s}, 1 \mathrm{H}), 7.16(\mathrm{~d}, J=7.8 \mathrm{~Hz}, 1 \mathrm{H}), 2.49-2.36(\mathrm{~m}, 5 \mathrm{H}), 2.28-2.13(\mathrm{~m}, 4 \mathrm{H}), 1.93(\mathrm{dt}, J=12.6,5.6 \mathrm{~Hz}, 2 \mathrm{H}) ;{ }^{33} \mathrm{C}$ NMR (125 MHz, $\left.\mathrm{CDCl}_{3}\right) \delta 181.8(\mathrm{C}), 150.9(\mathrm{C}), 150.4(\mathrm{C}), 135.7(\mathrm{C}), 133.0(\mathrm{C}), 130.2(\mathrm{C}), 128.6(\mathrm{CH}), 128.2(\mathrm{CH}), 128.0$ $(\mathrm{CH}), 121.8(\mathrm{CH}), 120.2(\mathrm{CH}), 63.1(\mathrm{C}), 36.9\left(\mathrm{CH}_{2}\right), 27.6\left(\mathrm{CH}_{2}\right), 21.7\left(\mathrm{CH}_{3}\right)$. ATR-FTIR (thin film): 2955, 2872, 1520, $1490,1463,1443,1340,1220,1019,819,771,694 \mathrm{~cm}^{-1}$. HRMS (ESI) $\mathrm{m} / z$ calculated for $\mathrm{C}_{19} \mathrm{H}_{20} \mathrm{~N}(\mathrm{M}+\mathrm{H})^{+}: 262.1596$, found: 262.1591 .

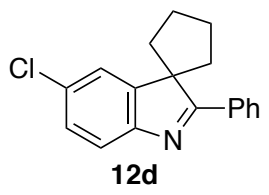

5'-Chloro-2'-phenylspiro[cyclopentane-1,3'-indole] $12 \mathrm{~d}^{22}$ The optimized procedure was followed using $0.028 \mathrm{~g}$ of aniline $11 \mathrm{~d}(0.10 \mathrm{mmol}), 0.11 \mu \mathrm{L}$ of iodobenzene $(0.001 \mathrm{mmol}), 20 \mu \mathrm{L}$ of trifluoroacetic acid $(0.26 \mathrm{mmol}), 0.046 \mathrm{~g}$ of Selectfluor $(0.13 \mathrm{mmol})$ in $2 \mathrm{~mL}$ of 1,1,1,3,3,3-hexafluoropropan-2-ol and $0.2 \mathrm{~mL}$ of water $(0.05 \mathrm{M})$. Purification by MPLC (2:98 to 5:95 EtOAc:hexanes) afforded the product as a white solid $(0.024 \mathrm{~g}, 85 \%)$. The spectral data matched that reported by Driver and co-workers: ${ }^{22}{ }^{1} \mathrm{H}$ NMR $\left(500 \mathrm{MHz}, \mathrm{CDCl}_{3}\right) \delta 8.13-8.01(\mathrm{~m}, 2 \mathrm{H}), 7.58(\mathrm{~d}, J=8.2 \mathrm{~Hz}, 1 \mathrm{H}), 7.52-7.44(\mathrm{~m}$, $3 \mathrm{H}), 7.35(\mathrm{~s}, 1 \mathrm{H}), 7.31(\mathrm{~d}, J=8.1 \mathrm{~Hz}, 1 \mathrm{H}), 2.50-2.38(\mathrm{~m}, 2 \mathrm{H}), 2.26-2.11(\mathrm{~m}, 4 \mathrm{H}), 1.98-1.86(\mathrm{~m}, 2 \mathrm{H}) ;{ }^{13} \mathrm{C}$ NMR $(125$ $\left.\mathrm{MHz}, \mathrm{CDCl}_{3}\right) \delta 183.0(\mathrm{C}), 151.8(\mathrm{C}), 151.6(\mathrm{C}), 132.4(\mathrm{C}), 131.4(\mathrm{C}), 130.7(\mathrm{CH}), 128.3(\mathrm{CH}), 128.3(\mathrm{CH}), 127.5(\mathrm{CH})$, $121.5(\mathrm{CH}), 121.5(\mathrm{CH}), 63.7(\mathrm{CH}), 36.8\left(\mathrm{CH}_{2}\right), 27.5\left(\mathrm{CH}_{2}\right)$. ATR-FTIR (thin film): 2957, 2874, 1520, 1442, 1337, 1251, $1077,823,772,694 \mathrm{~cm}^{-1}$. HRMS (ESI) $\mathrm{m} / z$ calculated for $\mathrm{C}_{18} \mathrm{H}_{17} \mathrm{NCl}(\mathrm{M}+\mathrm{H})^{+}: 282.1050$, found: 282.1046 .

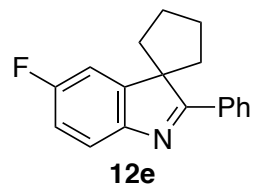

5'-Chloro-2'-phenylspiro[cyclopentane-1,3'-indole] $12 \mathrm{e} .^{22}$ The optimized procedure was followed using $0.027 \mathrm{~g}$ of aniline $11 \mathrm{e}(0.10 \mathrm{mmol}), 0.11 \mu \mathrm{L}$ of iodobenzene $(0.001 \mathrm{mmol}), 20 \mu \mathrm{L}$ of trifluoroacetic acid $(0.26 \mathrm{mmol}), 0.046 \mathrm{~g}$ of Selectfluor ( $0.13 \mathrm{mmol})$ in $2 \mathrm{~mL}$ of 1,1,1,3,3,3-hexafluoropropan-2-ol and $0.2 \mathrm{~mL}$ of water $(0.05 \mathrm{M})$. Purification by MPLC (2:98 to 5:95 EtOAc:hexanes) afforded the product as a yellow oil $(0.026 \mathrm{~g}, 97 \%)$. The spectral data matched that reported by Driver and co-workers: ${ }^{22}{ }^{1} \mathrm{H}$ NMR $\left(500 \mathrm{MHz}, \mathrm{CDCl}_{3}\right) \delta 8.09-8.00(\mathrm{~m}, 2 \mathrm{H}), 7.60(\mathrm{dd}, J=8.4,4.7 \mathrm{~Hz}, 1 \mathrm{H}), 7.53-7.42$ $(\mathrm{m}, 3 \mathrm{H}), 7.09(\mathrm{~d}, J=8.4 \mathrm{~Hz}, 1 \mathrm{H}), 7.03(\mathrm{t}, J=9.1 \mathrm{~Hz}, 1 \mathrm{H}), 2.54-2.36(\mathrm{~m}, 2 \mathrm{H}), 2.30-2.12(\mathrm{~m}, 4 \mathrm{H}), 1.98-1.87(\mathrm{~m}, 2 \mathrm{H})$; ${ }^{13} \mathrm{C}$ NMR $\left(125 \mathrm{MHz}, \mathrm{CDCl}_{3}\right) \delta 182.5(\mathrm{C}), 161.6\left(\mathrm{~d}, J_{C F}=242.0 \mathrm{~Hz}, \mathrm{C}\right), 152.0(\mathrm{C}), 149.1(\mathrm{C}), 132.6(\mathrm{C}), 130.5(\mathrm{CH}), 128.6$ $(\mathrm{CH}), 128.1(\mathrm{CH}), 121.3\left(\mathrm{~d}, J_{C F}=8.8 \mathrm{~Hz}, \mathrm{CH}\right), 114.0\left(\mathrm{~d}, J_{C F}=23.8 \mathrm{~Hz}, \mathrm{CH}\right), 108.7\left(\mathrm{~d}, J_{C F}=23.8 \mathrm{~Hz}, \mathrm{CH}\right), 63.8(\mathrm{CH}), 36.8$ $\left(\mathrm{CH}_{2}\right), 27.4\left(\mathrm{CH}_{2}\right) ;{ }^{19} \mathrm{~F}$ NMR $\left(282 \mathrm{MHz}, \mathrm{CDCl}_{3}\right) \delta-116.7$. ATR-FTIR (thin film): 2957, 2874, 1596, 1523, 1460, 1443, $1345,1263,1190,822,772,694 \mathrm{~cm}^{-1}$. HRMS (ESI) $\mathrm{m} / z$ calculated for $\mathrm{C}_{18} \mathrm{H}_{17} \mathrm{NF}(\mathrm{M}+\mathrm{H})^{+}: 266.1345$, found: 266.1340 .

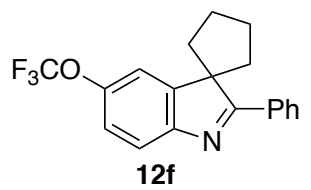

2'-Phenyl-5'-(trifluoromethoxy)spiro[cyclopentane-1,3'-indole] 12f. $^{18}$ The optimized procedure was followed using $0.033 \mathrm{~g}$ of aniline $11 \mathrm{f}(0.10 \mathrm{mmol}), 0.11 \mu \mathrm{L}$ of iodobenzene $(0.001 \mathrm{mmol}), 20 \mu \mathrm{L}$ of trifluoroacetic acid $(0.26 \mathrm{mmol}), 0.046$ $\mathrm{g}$ of Selectfluor $(0.13 \mathrm{mmol})$ in $2 \mathrm{~mL}$ of 1,1,1,3,3,3-hexafluoropropan-2-ol and $0.2 \mathrm{~mL}$ of water $(0.05 \mathrm{M})$. Purification by MPLC (2:98 to 5:95 EtOAc:hexanes) afforded the product as a white solid $(0.030 \mathrm{~g}, 91 \%)$. The spectral data matched that reported by Driver and co-workers: ${ }^{22} \mathrm{mp}=80{ }^{\circ} \mathrm{C} ;{ }^{1} \mathrm{H} \mathrm{NMR}\left(500 \mathrm{MHz}, \mathrm{CDCl}_{3}\right) \delta 8.18-8.02(\mathrm{~m}, 2 \mathrm{H}), 7.65(\mathrm{~d}, J=8.3 \mathrm{~Hz}$, $1 \mathrm{H}), 7.55-7.45(\mathrm{~m}, 3 \mathrm{H}), 7.32-7.15(\mathrm{~m}, 2 \mathrm{H}), 2.55-2.36(\mathrm{~m}, 2 \mathrm{H}), 2.28-2.16(\mathrm{~m}, 4 \mathrm{H}), 1.93(\mathrm{dt}, J=11.7,5.8 \mathrm{~Hz}, 2 \mathrm{H})$; ${ }^{13} \mathrm{C}$ NMR $\left(125 \mathrm{MHz}, \mathrm{CDCl}_{3}\right) \delta 183.8(\mathrm{C}), 151.6(\mathrm{C}), 151.5(\mathrm{C}), 147.3(\mathrm{C}), 132.4(\mathrm{C}), 130.8(\mathrm{CH}), 128.7(\mathrm{CH}), 128.3(\mathrm{CH})$, $121.2(\mathrm{CH}), 120.6\left(\mathrm{q}, J_{C F}=253.8 \mathrm{~Hz}, \mathrm{C}\right), 120.3(\mathrm{CH}), 114.5(\mathrm{CH}), 63.9(\mathrm{CH}), 36.8\left(\mathrm{CH}_{2}\right), 27.5\left(\mathrm{CH}_{2}\right) .{ }^{19} \mathrm{~F} \mathrm{NMR}(282 \mathrm{MHz}$, 
$\left.\mathrm{CDCl}_{3}\right) \delta-58.3$. ATR-FTIR (thin film): 2960, 1522, 1461, 1255, 1222, 1163, 772, $694 \mathrm{~cm}^{-1}$. HRMS (ESI) $\mathrm{m} / z$ calculated for $\mathrm{C}_{19} \mathrm{H}_{17} \mathrm{NOF}_{3}(\mathrm{M}+\mathrm{H})^{+}: 332.1262$, found: 332.1251 .

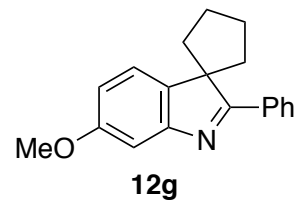

6'-Methoxy-2'-phenylspiro[cyclopentane-1,3'-indole] 12g. ${ }^{22}$ The optimized procedure was followed using $0.028 \mathrm{~g}$ of aniline $11 \mathrm{~g}(0.10 \mathrm{mmol}), 0.55 \mu \mathrm{L}$ of iodobenzene $(0.005 \mathrm{mmol}), 20 \mu \mathrm{L}$ of trifluoroacetic acid $(0.26 \mathrm{mmol}), 0.046 \mathrm{~g}$ of Selectfluor $(0.13 \mathrm{mmol})$ in $2 \mathrm{~mL}$ of 1,1,1,3,3,3-hexafluoropropan-2-ol and $0.2 \mathrm{~mL}$ of water $(0.05 \mathrm{M})$. Purification by MPLC (2:98 to 5:95 EtOAc:hexanes) afforded the product as a brown oil $(0.014 \mathrm{~g}, 50 \%)$. The spectral data matched that reported by Driver and co-workers: ${ }^{22}{ }^{1} \mathrm{H}$ NMR $\left(500 \mathrm{MHz}, \mathrm{CDCl}_{3}\right) \delta 8.07(\mathrm{dd}, J=6.8,2.8 \mathrm{~Hz}, 2 \mathrm{H}), 7.50-7.45(\mathrm{~m}, 3 \mathrm{H}), 7.27(\mathrm{~d}, J=$ $8.5 \mathrm{~Hz}, 2 \mathrm{H}), 6.79(\mathrm{dd}, J=8.2,2.5 \mathrm{~Hz}, 1 \mathrm{H}), 3.86(\mathrm{~s}, 3 \mathrm{H}), 2.46-2.38(\mathrm{~m}, 2 \mathrm{H}), 2.23-2.13(\mathrm{~m}, 4 \mathrm{H}), 1.94-1.87(\mathrm{~m}, 2 \mathrm{H}) ;{ }^{13} \mathrm{C}$ NMR (125 MHz, $\left.\mathrm{CDCl}_{3}\right) \delta 183.9(\mathrm{C}), 159.6(\mathrm{C}), 154.2(\mathrm{C}), 142.5(\mathrm{C}), 132.8(\mathrm{C}), 130.5(\mathrm{CH}), 128.6(\mathrm{CH}), 128.3(\mathrm{CH})$, $121.2(\mathrm{CH}), 112.1(\mathrm{CH}), 106.0(\mathrm{CH}), 62.9(\mathrm{C}), 55.6\left(\mathrm{CH}_{3}\right), 37.0\left(\mathrm{CH}_{2}\right), 27.4\left(\mathrm{CH}_{2}\right)$. ATR-FTIR (thin film): 2954, 2872, $1614,1478,1439,1272,1150,1141,1028,771,695 \mathrm{~cm}^{-1}$. HRMS (ESI) $\mathrm{m} / z$ calculated for $\mathrm{C}_{19} \mathrm{H}_{20} \mathrm{NO}(\mathrm{M}+\mathrm{H})^{+}: 278.1545$, found: 278.1538 .

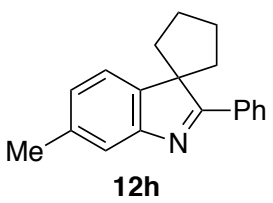

6'-Methyl-2'-phenylspiro[cyclopentane-1,3'-indole] $12 \mathbf{h} .^{22}$ The general procedure was followed using $0.026 \mathrm{~g}$ of aniline $11 \mathrm{~h}(0.10 \mathrm{mmol}), 0.55 \mu \mathrm{L}$ of iodobenzene $(0.005 \mathrm{mmol}), 20 \mu \mathrm{L}$ of trifluoroacetic acid $(0.26 \mathrm{mmol}), 0.046 \mathrm{~g}$ of Selectfluor $(0.13 \mathrm{mmol})$ in $2 \mathrm{~mL}$ of 1,1,1,3,3,3-hexafluoropropan-2-ol and $0.2 \mathrm{~mL}$ of water $(0.05 \mathrm{M})$. Purification by MPLC (2:98 to 5:95 EtOAc:hexanes) afforded the product as a yellow oil $(0.023 \mathrm{~g}, 88 \%)$. The spectral data matched that reported by Driver and co-workers: ${ }^{22}{ }^{1} \mathrm{H}$ NMR $\left(500 \mathrm{MHz}, \mathrm{CDCl}_{3}\right) \delta 8.08(\mathrm{dd}, J=6.7,3.0 \mathrm{~Hz}, 2 \mathrm{H}), 7.51(\mathrm{~s}, 1 \mathrm{H}), 7.48(\mathrm{dd}, J=5.1,2.0 \mathrm{~Hz}, 3 \mathrm{H})$, $7.28(\mathrm{~d}, J=7.5 \mathrm{~Hz}, 1 \mathrm{H}), 7.05(\mathrm{~d}, J=7.5 \mathrm{~Hz}, 1 \mathrm{H}), 2.51-2.34(\mathrm{~m}, 5 \mathrm{H}), 2.27-2.13(\mathrm{~m}, 4 \mathrm{H}), 2.00-1.83(\mathrm{~m}, 2 \mathrm{H}) ;{ }^{13} \mathrm{C} \mathrm{NMR}$ $\left(125 \mathrm{MHz}, \mathrm{CDCl}_{3}\right) \delta 182.9(\mathrm{C}), 153.3(\mathrm{C}), 147.4(\mathrm{C}), 137.2(\mathrm{C}), 133.0(\mathrm{C}), 130.3(\mathrm{CH}), 128.6(\mathrm{CH}), 128.3(\mathrm{CH}), 126.6$ $(\mathrm{CH}), 121.4(\mathrm{CH}), 120.6(\mathrm{CH}), 63.0(\mathrm{C}), 36.9\left(\mathrm{CH}_{2}\right), 27.5\left(\mathrm{CH}_{2}\right), 21.5\left(\mathrm{CH}_{2}\right)$. ATR-FTIR (thin film): 2955, 2872, 1519, $1484,1454,1326,1146,1018,809,771,694 \mathrm{~cm}^{-1}$. HRMS (ESI) $\mathrm{m} / z$ calculated for $\mathrm{C}_{19} \mathrm{H}_{20} \mathrm{~N}(\mathrm{M}+\mathrm{H})^{+}: 262.1596$, found: 262.1590 .

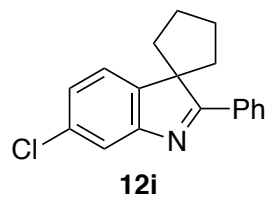

6'-Chloro-2'-phenylspiro[cyclopentane-1,3'-indole] $12 \mathbf{i}^{18}{ }^{18}$ The optimized procedure was followed using $0.028 \mathrm{~g}$ of aniline $11 \mathrm{i}(0.10 \mathrm{mmol}), 0.55 \mu \mathrm{L}$ of iodobenzene $(0.005 \mathrm{mmol}), 20 \mu \mathrm{L}$ of trifluoroacetic acid $(0.26 \mathrm{mmol}), 0.046 \mathrm{~g}$ of Selectfluor $(0.13 \mathrm{mmol})$ in $2 \mathrm{~mL}$ of $1,1,1,3,3,3$-hexafluoropropan-2-ol and $0.2 \mathrm{~mL}$ of water $(0.05 \mathrm{M})$. Purification by MPLC (2:98 to 5:95 EtOAc:hexanes) afforded the product as a pale pink oil $(0.023 \mathrm{~g}, 82 \%)$. The spectral data matched that reported by Driver and co-workers: ${ }^{18}{ }^{1} \mathrm{H}$ NMR $\left(500 \mathrm{MHz}, \mathrm{CDCl}_{3}\right) \delta 8.07(\mathrm{dd}, J=6.8,3.0 \mathrm{~Hz}, 2 \mathrm{H}), 7.65(\mathrm{~d}, J=1.9 \mathrm{~Hz}, 1 \mathrm{H}), 7.49(\mathrm{dd}, J$ $=5.0,2.0 \mathrm{~Hz}, 3 \mathrm{H}), 7.29(\mathrm{~d}, J=7.9 \mathrm{~Hz}, 1 \mathrm{H}), 7.20(\mathrm{dd}, J=8.0,2.0 \mathrm{~Hz}, 1 \mathrm{H}), 2.43(\mathrm{dt}, J=13.2,8.0 \mathrm{~Hz}, 2 \mathrm{H}), 2.26-2.12(\mathrm{~m}$, 4H), $1.98-1.85(\mathrm{~m}, 2 \mathrm{H}) ;{ }^{13} \mathrm{C} \mathrm{NMR}\left(125 \mathrm{MHz}, \mathrm{CDCl}_{3}\right) \delta 184.3(\mathrm{C}), 154.3(\mathrm{C}), 148.5(\mathrm{C}), 132.9(\mathrm{C}), 132.4(\mathrm{C}), 130.8(\mathrm{CH})$, $128.7(\mathrm{CH}), 128.4(\mathrm{CH}), 125.7(\mathrm{CH}), 121.6(\mathrm{CH}), 121.0(\mathrm{CH}), 63.1(\mathrm{C}), 36.9\left(\mathrm{CH}_{2}\right), 27.5\left(\mathrm{CH}_{2}\right)$. ATR-FTIR (thin film): 2956, 2874, 1600, 1519, 1454, 1442, 1325, 1065, 811, 771, $693 \mathrm{~cm}^{-1}$. HRMS (ESI) $\mathrm{m} / z$ calculated for $\mathrm{C}_{18} \mathrm{H}_{17} \mathrm{NCl}(\mathrm{M}+\mathrm{H})^{+}$: 282.1050, found: 282.1044 . 


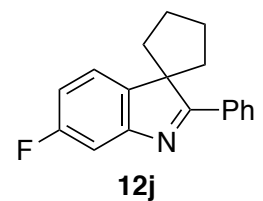

6'-Fluoro-2'-phenylspiro[cyclopentane-1,3'-indole] $\mathbf{1 2 j} .^{22}$ The general procedure was followed using $0.027 \mathrm{~g}$ of aniline $11 \mathrm{j}(0.10 \mathrm{mmol}), 0.55 \mu \mathrm{L}$ of iodobenzene $(0.005 \mathrm{mmol}), 20 \mu \mathrm{L}$ of trifluoroacetic acid $(0.26 \mathrm{mmol}), 0.046 \mathrm{~g}$ of Selectfluor $(0.13 \mathrm{mmol})$ in $2 \mathrm{~mL}$ of $1,1,1,3,3,3$-hexafluoropropan-2-ol and $0.2 \mathrm{~mL}$ of water $(0.05 \mathrm{M})$. Purification by MPLC (2:98 to 5:95 EtOAc:hexanes) afforded the product as a white oil $(0.020 \mathrm{~g}, 76 \%)$. The spectral data matched that reported by Driver and co-workers: ${ }^{22}{ }^{1} \mathrm{H}$ NMR $\left(500 \mathrm{MHz}, \mathrm{CDCl}_{3}\right) \delta 8.08(\mathrm{dd}, J=6.8,3.0 \mathrm{~Hz}, 2 \mathrm{H}), 7.52-7.44(\mathrm{~m}, 3 \mathrm{H}), 7.37(\mathrm{dd}, J=9.1,2.4$ $\mathrm{Hz}, 1 \mathrm{H}), 7.30(\mathrm{dd}, J=8.2,5.2 \mathrm{~Hz}, 1 \mathrm{H}), 6.92(\mathrm{td}, J=8.7,2.4 \mathrm{~Hz}, 1 \mathrm{H}), 2.43(\mathrm{dt}, J=13.3,7.8 \mathrm{~Hz}, 2 \mathrm{H}), 2.23-2.17(\mathrm{~m}, 4 \mathrm{H})$, $1.91(\mathrm{dd}, J=13.4,6.2 \mathrm{~Hz}, 2 \mathrm{H}) ;{ }^{13} \mathrm{C}$ NMR $\left(125 \mathrm{MHz}, \mathrm{CDCl}_{3}\right) \delta 184.7(\mathrm{C}), 162.6\left(\mathrm{~d}, J_{C F}=243.0 \mathrm{~Hz}, \mathrm{C}\right), 154.3(\mathrm{C}), 145.8$ $(\mathrm{C}), 132.5(\mathrm{C}), 130.8(\mathrm{CH}), 128.6(\mathrm{CH}), 128.4(\mathrm{CH}), 121.3\left(\mathrm{~d}, J_{C F}=9.6 \mathrm{~Hz}, \mathrm{CH}\right), 112.3\left(\mathrm{~d}, J_{C F}=23.1 \mathrm{~Hz}, \mathrm{CH}\right), 108.1(\mathrm{~d}$, $\left.J_{C F}=23.6 \mathrm{~Hz}, \mathrm{CH}\right), 63.0(\mathrm{C}), 37.0\left(\mathrm{CH}_{2}\right), 27.5\left(\mathrm{CH}_{2}\right) ;{ }^{19} \mathrm{~F}$ NMR $\left(282 \mathrm{MHz}, \mathrm{CDCl}_{3}\right) \delta-116.1$. ATR-FTIR (thin film): 2958 , $2874,1602,1519,1479,1472,1258,1128,956,771,694 \mathrm{~cm}^{-1}$. HRMS (ESI) $\mathrm{m} / z$ calculated for $\mathrm{C}_{18} \mathrm{H}_{17} \mathrm{NF}(\mathrm{M}+\mathrm{H})^{+}: 266.1345$, found: 266.1340 .

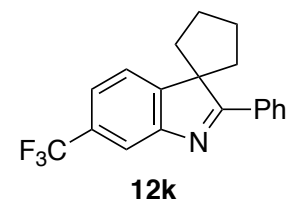

2'-Phenyl-6'-(trifluoromethyl)spiro[cyclopentane-1,3'-indole] $12 \mathbf{k} .{ }^{18}$ The optimized procedure was followed using 0.032 $\mathrm{g}$ of aniline $11 \mathrm{k}(0.10 \mathrm{mmol}), 0.11 \mu \mathrm{L}$ of iodobenzene $(0.001 \mathrm{mmol}), 20 \mu \mathrm{L}$ of trifluoroacetic acid $(0.26 \mathrm{mmol}), 0.046 \mathrm{~g}$ of Selectfluor $(0.13 \mathrm{mmol})$ in $2 \mathrm{~mL}$ of 1,1,1,3,3,3-hexafluoropropan-2-ol and $0.2 \mathrm{~mL}$ of water $(0.05 \mathrm{M})$. Purification by MPLC (2:98 to 5:95 EtOAc:hexanes) afforded the product as a white solid $(0.029 \mathrm{~g}, 91 \%)$. The spectral data matched that reported by Driver and co-workers: $:^{18} \mathrm{mp}=66{ }^{\circ} \mathrm{C} ;{ }^{1} \mathrm{H} \mathrm{NMR}\left(500 \mathrm{MHz}, \mathrm{CDCl}_{3}\right) \delta 8.10(\mathrm{dd}, J=6.5,2.9 \mathrm{~Hz}, 2 \mathrm{H}), 7.93(\mathrm{~s}, 1 \mathrm{H}), 7.54-$ $7.45(\mathrm{~m}, 5 \mathrm{H}), 2.51-2.41(\mathrm{~m}, 2 \mathrm{H}), 2.24(\mathrm{td}, J=8.5,7.1,4.8 \mathrm{~Hz}, 4 \mathrm{H}), 1.94(\mathrm{dtd}, J=9.2,6.9,6.2,3.7 \mathrm{~Hz}, 2 \mathrm{H}) ;{ }^{13} \mathrm{C} \mathrm{NMR}$ $\left(125 \mathrm{MHz}_{\mathrm{CDCl}}\right) \delta 184.3(\mathrm{C}), 153.7(\mathrm{C}), 153.4(\mathrm{C}), 132.3(\mathrm{C}), 131.0(\mathrm{CH}), 130.0\left(\mathrm{q}, J_{C F}=32.0 \mathrm{~Hz}, \mathrm{C}\right), 128.7(\mathrm{CH}), 128.4$ $(\mathrm{CH}), 124.4\left(\mathrm{q}, J_{C F}=272.3 \mathrm{~Hz}, \mathrm{C}\right), 122.8\left(\mathrm{q}, J_{C F}=3.9 \mathrm{~Hz}, \mathrm{CH}\right), 121.1(\mathrm{CH}), 117.6\left(\mathrm{q}, J_{C F}=3.8 \mathrm{~Hz}, \mathrm{CH}\right), 63.5(\mathrm{C}), 36.8$ $\left(\mathrm{CH}_{2}\right), 27.6\left(\mathrm{CH}_{2}\right) ;{ }^{19} \mathrm{~F} \mathrm{NMR}\left(282 \mathrm{MHz}, \mathrm{CDCl}_{3}\right) \delta-62.4$. ATR-FTIR (thin film): 2959, 2876, 1522, 1426, 1349, 1323, 1165 , $1122,1056,891,828,772,694 \mathrm{~cm}^{-1}$. HRMS (ESI) $\mathrm{m} / z$ calculated for $\mathrm{C}_{19} \mathrm{H}_{17} \mathrm{NF}_{3}(\mathrm{M}+\mathrm{H})^{+}: 316.1313$, found: 316.1308 .

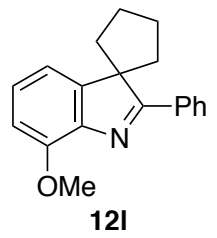

7'-Methoxy-2'-phenylspiro[cyclopentane-1,3'-indole] $121 .^{18}$ The optimized procedure was followed using $0.028 \mathrm{~g}$ of aniline $111(0.10 \mathrm{mmol}), 0.11 \mu \mathrm{L}$ of iodobenzene $(0.001 \mathrm{mmol}), 20 \mu \mathrm{L}$ of trifluoroacetic acid $(0.26 \mathrm{mmol}), 0.046 \mathrm{~g}$ of Selectfluor $(0.13 \mathrm{mmol})$ in $2 \mathrm{~mL}$ of 1,1,1,3,3,3-hexafluoropropan-2-ol and $0.2 \mathrm{~mL}$ of water $(0.05 \mathrm{M})$. Purification by MPLC (2:98 to 5:95 EtOAc:hexanes) afforded the product as a colorless oil $(0.024 \mathrm{~g}, 86 \%)$. The spectral data matched that reported by Driver and co-workers: ${ }^{18}{ }^{1} \mathrm{H}$ NMR $\left(500 \mathrm{MHz}, \mathrm{CDCl}_{3}\right) \delta 8.15-8.10(\mathrm{~m}, 2 \mathrm{H}), 7.47-7.42(\mathrm{~m}, 3 \mathrm{H}), 7.20(\mathrm{t}, J=7.8 \mathrm{~Hz}$, $1 \mathrm{H}), 7.01(\mathrm{~d}, J=7.5 \mathrm{~Hz}, 1 \mathrm{H}), 6.89(\mathrm{~d}, J=8.2 \mathrm{~Hz}, 1 \mathrm{H}), 4.03(\mathrm{~s}, 3 \mathrm{H}), 2.43(\mathrm{dt}, J=13.0,7.5 \mathrm{~Hz}, 2 \mathrm{H}), 2.25-2.16(\mathrm{~m}, 4 \mathrm{H})$, $1.92(\mathrm{dt}, J=12.5,5.7 \mathrm{~Hz}, 2 \mathrm{H}) ;{ }^{13} \mathrm{C}$ NMR $\left(125 \mathrm{MHz}, \mathrm{CDCl}_{3}\right) \delta 181.2(\mathrm{C}), 152.4(\mathrm{C}), 151.6(\mathrm{C}), 141.1(\mathrm{C}), 132.8(\mathrm{C}), 130.2$ $(\mathrm{CH}), 128.42(\mathrm{CH}), 128.37(\mathrm{CH}), 127.2(\mathrm{CH}), 113.4(\mathrm{CH}), 110.1(\mathrm{CH}), 63.6(\mathrm{C}), 56.0\left(\mathrm{CH}_{3}\right), 36.9\left(\mathrm{CH}_{2}\right), 27.5\left(\mathrm{CH}_{2}\right)$. ATRFTIR (thin film): 2955, 2873, 1610, 1589, 1483, 1272, 1256, 1105, 750, $695 \mathrm{~cm}^{-1}$. HRMS (ESI) $\mathrm{m} / z$ calculated for $\mathrm{C}_{19} \mathrm{H}_{20} \mathrm{NO}$ $(\mathrm{M}+\mathrm{H})^{+}: 278.1545$, found: 278.1537 . 


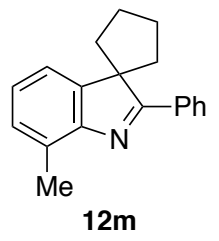

7'-Methyl-2'-phenylspiro[cyclopentane-1,3'-indole] $\mathbf{1 2 m} .^{18}$ The optimized procedure was followed using $0.026 \mathrm{~g}$ of aniline $11 \mathrm{~m}(0.10 \mathrm{mmol}), 0.11 \mu \mathrm{L}$ of iodobenzene $(0.001 \mathrm{mmol}), 20 \mu \mathrm{L}$ of trifluoroacetic acid $(0.26 \mathrm{mmol}), 0.046 \mathrm{~g}$ of Selectfluor ( $0.13 \mathrm{mmol}$ ) in $2 \mathrm{~mL}$ of 1,1,1,3,3,3-hexafluoropropan-2-ol and $0.2 \mathrm{~mL}$ of water (0.05 M). Purification by MPLC (2:98 to 5:95 EtOAc:hexanes) afforded the product as a colorless oil $(0.024 \mathrm{~g}, 92 \%)$. The spectral data matched that reported by Driver and co-workers: ${ }^{18}{ }^{1} \mathrm{H}$ NMR $\left(500 \mathrm{MHz}, \mathrm{CDCl}_{3}\right) \delta 8.13-8.06(\mathrm{~m}, 2 \mathrm{H}), 7.51-7.43(\mathrm{~m}, 3 \mathrm{H}), 7.22(\mathrm{~d}, J=6.9 \mathrm{~Hz}$, $1 \mathrm{H}), 7.19-7.10(\mathrm{~m}, 2 \mathrm{H}), 2.68(\mathrm{~s}, 3 \mathrm{H}), 2.48-2.35(\mathrm{~m}, 2 \mathrm{H}), 2.27-2.12(\mathrm{~m}, 4 \mathrm{H}), 1.92(\mathrm{dt}, J=12.4,5.4 \mathrm{~Hz}, 2 \mathrm{H}) ;{ }^{13} \mathrm{C} \mathrm{NMR}$ $\left(125 \mathrm{MHz}, \mathrm{CDCl}_{3}\right) \delta 181.5(\mathrm{C}), 151.6(\mathrm{C}), 150.1(\mathrm{C}), 133.3(\mathrm{C}), 130.5(\mathrm{C}), 130.1(\mathrm{CH}), 128.6(\mathrm{CH}), 128.5(\mathrm{CH}), 128.3$ $(\mathrm{CH}), 125.8(\mathrm{CH}), 118.3(\mathrm{CH}), 63.5(\mathrm{C}), 36.8\left(\mathrm{CH}_{2}\right), 27.5\left(\mathrm{CH}_{2}\right), 16.9\left(\mathrm{CH}_{3}\right)$. ATR-FTIR (thin film): 3054, 2955, 2873, $1521,1494,1324,1072,762,694 \mathrm{~cm}^{-1}$. HRMS (ESI) $\mathrm{m} / z$ calculated for $\mathrm{C}_{19} \mathrm{H}_{20} \mathrm{~N}(\mathrm{M}+\mathrm{H})^{+}: 262.1596$, found: 262.1586.

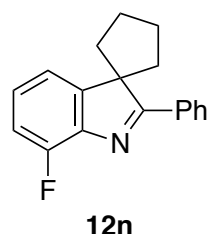

7'-Fluoro-2'-phenylspiro[cyclopentane-1,3'-indole] $12 \mathrm{n} .{ }^{18}$ The optimized procedure was followed using $0.027 \mathrm{~g}$ of aniline $11 \mathrm{n}(0.10 \mathrm{mmol}), 0.11 \mu \mathrm{L}$ of iodobenzene $(0.001 \mathrm{mmol}), 20 \mu \mathrm{L}$ of trifluoroacetic acid $(0.26 \mathrm{mmol}), 0.046 \mathrm{~g}$ of Selectfluor $(0.13 \mathrm{mmol})$ in $2 \mathrm{~mL}$ of 1,1,1,3,3,3-hexafluoropropan-2-ol and $0.2 \mathrm{~mL}$ of water $(0.05 \mathrm{M})$. Purification by MPLC (2:98 to 5:95 EtOAc:hexanes) afforded the product as a yellow oil $(0.027 \mathrm{~g}, 90 \%)$. The spectral data matched that reported by Driver and co-workers: ${ }^{18}{ }^{1} \mathrm{H}$ NMR $\left(500 \mathrm{MHz}, \mathrm{CDCl}_{3}\right) \delta 8.13(\mathrm{dd}, J=6.8,3.0 \mathrm{~Hz}, 2 \mathrm{H}), 7.51-7.44(\mathrm{~m}, 3 \mathrm{H}), 7.21-7.15$ $(\mathrm{m}, 2 \mathrm{H}), 7.06(\mathrm{ddd}, J=9.5,6.8,2.4 \mathrm{~Hz}, 1 \mathrm{H}), 2.45(\mathrm{dt}, J=13.4,8.0 \mathrm{~Hz}, 2 \mathrm{H}), 2.28-2.13(\mathrm{~m}, 4 \mathrm{H}), 2.01-1.87(\mathrm{~m}, 2 \mathrm{H}) ;{ }^{13} \mathrm{C}$ NMR (125 MHz, $\left.\mathrm{CDCl}_{3}\right) \delta 182.9(\mathrm{C}), 155.4(\mathrm{C}), 153.5\left(\mathrm{~d}, J_{C F}=28.8 \mathrm{~Hz}, \mathrm{C}\right), 140.0(\mathrm{C}), 132.4(\mathrm{C}), 130.8(\mathrm{CH}), 128.6(\mathrm{CH})$, $128.5(\mathrm{CH}), 127.2\left(\mathrm{~d}, J_{C F}=7.5 \mathrm{~Hz}, \mathrm{CH}\right), 116.6\left(\mathrm{~d}, J_{C F}=3.8 \mathrm{~Hz}, \mathrm{CH}\right), 114.4\left(\mathrm{~d}, J_{C F}=18.7 \mathrm{~Hz}, \mathrm{CH}\right), 64.0(\mathrm{C}), 37.0\left(\mathrm{CH}_{2}\right)$, $27.5\left(\mathrm{CH}_{2}\right) ;{ }^{19} \mathrm{~F}$ NMR $\left(282 \mathrm{MHz}, \mathrm{CDCl}_{3}\right) \delta-127.5$. ATR-FTIR (thin film): 2957, 2874, 1623, 1478, 1243, 1096, 789, 772, $751,694 \mathrm{~cm}^{-1}$. HRMS (ESI) $\mathrm{m} / z$ calculated for $\mathrm{C}_{18} \mathrm{H}_{17} \mathrm{NF}(\mathrm{M}+\mathrm{H})^{+}: 266.1345$, found: 266.1343 .

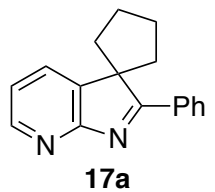

2'-Phenylspiro[cyclopentane-1,3'-pyrrolo[2,3-b]pyridine] $17 \mathbf{a}^{18}{ }^{18}$ The optimized procedure was followed using $0.025 \mathrm{~g}$ of 2-amino-3-vinylpyridine $16 \mathrm{a}(0.10 \mathrm{mmol}), 2.3 \mu \mathrm{L}$ of iodobenzene $(0.020 \mathrm{mmol}), 20 \mu \mathrm{L}$ of trifluoroacetic acid $(0.26$ $\mathrm{mmol}), 0.046 \mathrm{~g}$ of Selectfluor $(0.13 \mathrm{mmol})$ in $2 \mathrm{~mL}$ of 1,1,1,3,3,3-hexafluoropropan-2-ol and $0.2 \mathrm{~mL}$ of water $(0.05 \mathrm{M})$. Purification by MPLC (2:98 to 35:65 EtOAc:hexanes) afforded the product as a yellow oil $(0.024 \mathrm{~g}, 97 \%)$. The spectral data matched that reported by Driver and co-workers: ${ }^{18}{ }^{1} \mathrm{H}$ NMR $\left(500 \mathrm{MHz}, \mathrm{CDCl}_{3}\right) \delta 8.46(\mathrm{~d}, J=5.0 \mathrm{~Hz}, 1 \mathrm{H}), 8.19(\mathrm{dd}, J$ $=7.3,2.4 \mathrm{~Hz}, 2 \mathrm{H}), 7.64(\mathrm{~d}, J=7.4 \mathrm{~Hz}, 1 \mathrm{H}), 7.52-7.41(\mathrm{~m}, 3 \mathrm{H}), 7.08(\mathrm{dd}, J=7.4,5.0 \mathrm{~Hz}, 1 \mathrm{H}), 2.50-2.37(\mathrm{~m}, 2 \mathrm{H}), 2.27$ $-2.10(\mathrm{~m}, 4 \mathrm{H}), 1.88(\mathrm{dt}, J=12.2,5.1 \mathrm{~Hz}, 2 \mathrm{H}) ;{ }^{13} \mathrm{C}$ NMR $\left(125 \mathrm{MHz}, \mathrm{CDCl}_{3}\right) \delta 186.1(\mathrm{C}), 166.6(\mathrm{C}), 148.1(\mathrm{CH}), 142.6(\mathrm{C})$, $131.9(\mathrm{C}), 131.4(\mathrm{CH}), 128.9(\mathrm{CH}), 128.8(\mathrm{CH}), 128.6(\mathrm{CH}), 120.5(\mathrm{CH}), 62.2(\mathrm{C}), 36.7\left(\mathrm{CH}_{2}\right), 27.5\left(\mathrm{CH}_{2}\right)$. ATR-FTIR (thin film): 2954, 2873, 1583, 1520, 1399, 1233, 791, 773, $694 \mathrm{~cm}^{-1}$. HRMS (ESI) $\mathrm{m} / z$ calculated for $\mathrm{C}_{17} \mathrm{H}_{17} \mathrm{~N}_{2}(\mathrm{M}+\mathrm{H})^{+}: 249.1392$, found: 249.1387. 


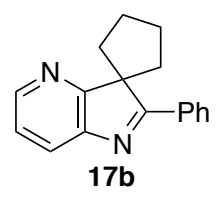

2'-Phenylspiro[cyclopentane-1,3'-pyrrolo[3,2-b]pyridine] $\mathbf{1 7 b}$. $^{18}$ The optimized procedure was followed using $0.025 \mathrm{~g}$ of 3-amino-2-vinylpyridine $\mathbf{1 6 b}(0.10 \mathrm{mmol}), 0.11 \mu \mathrm{L}$ of iodobenzene $(0.001 \mathrm{mmol}), 20 \mu \mathrm{L}$ of trifluoroacetic acid $(0.26$ $\mathrm{mmol}), 0.046 \mathrm{~g}$ of Selectfluor $(0.13 \mathrm{mmol})$ in $2 \mathrm{~mL}$ of 1,1,1,3,3,3-hexafluoropropan-2-ol and $0.2 \mathrm{~mL}$ of water $(0.05 \mathrm{M})$. Purification by MPLC (2:98 to 10:90 EtOAc:hexanes) afforded the product as a yellow solid (0.023 g, 92\%). The spectral data matched that reported by Driver and co-workers: ${ }^{18} \mathrm{mp}=58{ }^{\circ} \mathrm{C} ;{ }^{1} \mathrm{H} \mathrm{NMR}\left(500 \mathrm{MHz}, \mathrm{CDCl}_{3}\right) \delta 8.37(\mathrm{~d}, J=5.0 \mathrm{~Hz}, 1 \mathrm{H})$, $8.16-8.06(\mathrm{~m}, 2 \mathrm{H}), 7.88(\mathrm{~d}, J=7.7 \mathrm{~Hz}, 1 \mathrm{H}), 7.55-7.45(\mathrm{~m}, 3 \mathrm{H}), 7.23(\mathrm{dd}, J=7.8,5.0 \mathrm{~Hz}, 1 \mathrm{H}), 2.46-2.28(\mathrm{~m}, 4 \mathrm{H}), 2.23$ $-2.12(\mathrm{~m}, 2 \mathrm{H}), 2.05(\mathrm{dt}, J=12.0,5.7 \mathrm{~Hz}, 2 \mathrm{H}) ;{ }^{13} \mathrm{C}$ NMR $\left(125 \mathrm{MHz}, \mathrm{CDCl}_{3}\right) \delta 183.5(\mathrm{C}), 169.5(\mathrm{C}), 145.9(\mathrm{CH}), 145.7(\mathrm{C})$, $132.5(\mathrm{C}), 131.1(\mathrm{CH}), 128.7(\mathrm{CH}), 128.3(\mathrm{CH}), 126.9(\mathrm{CH}), 122.3(\mathrm{CH}), 62.0(\mathrm{C}), 35.8\left(\mathrm{CH}_{2}\right), 27.9\left(\mathrm{CH}_{2}\right)$. ATR-FTIR (thin film): 2954, 2870, 1509, 1442, 1409, 1256, 1182, 783, 771, $693 \mathrm{~cm}^{-1}$. HRMS (ESI) $\mathrm{m} / z$ calculated for $\mathrm{C}_{17} \mathrm{H}_{17} \mathrm{~N}_{2}(\mathrm{M}+\mathrm{H})^{+}$: 249.1392, found: 249.1387.

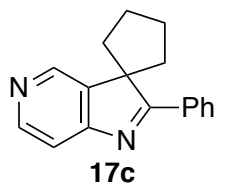

2'-Phenylspiro[cyclopentane-1,3'-pyrrolo[3,2-c]pyridine] 17c. ${ }^{18}$ The optimized procedure was followed using $0.025 \mathrm{~g}$ of 4-amino-3-vinylpyridine $16 \mathrm{c}(0.10 \mathrm{mmol}), 2.3 \mu \mathrm{L}$ of iodobenzene $(0.020 \mathrm{mmol}), 20 \mu \mathrm{L}$ of trifluoroacetic acid $(0.26 \mathrm{mmol})$, $0.092 \mathrm{~g}$ of Selectfluor $(0.26 \mathrm{mmol})$ in $2 \mathrm{~mL}$ of $1,1,1,3,3,3$-hexafluoropropan-2-ol and $0.2 \mathrm{~mL}$ of water $(0.05 \mathrm{M})$. This substrate required an increased reaction time of $16 \mathrm{~h}$. Purification by MPLC (2:98 EtOAc:hexanes to 10:90 MeOH:EtOAc) afforded the product as a colorless oil $(0.019 \mathrm{~g}, 76 \%)$. The spectral data matched that reported by Driver and co-workers: ${ }^{18}$ ${ }^{1} \mathrm{H}$ NMR $\left(500 \mathrm{MHz}, \mathrm{CDCl}_{3}\right) \delta 8.66(\mathrm{~s}, 1 \mathrm{H}), 8.60(\mathrm{~d}, J=5.0 \mathrm{~Hz}, 1 \mathrm{H}), 8.15-8.10(\mathrm{~m}, 2 \mathrm{H}), 7.59(\mathrm{~d}, J=5.1 \mathrm{~Hz}, 1 \mathrm{H}), 7.51$ $(\mathrm{qd}, J=8.7,7.7,3.6 \mathrm{~Hz}, 3 \mathrm{H}), 2.46(\mathrm{dt}, J=13.2,8.1 \mathrm{~Hz}, 2 \mathrm{H}), 2.31-2.21(\mathrm{~m}, 4 \mathrm{H}), 2.01-1.92(\mathrm{~m}, 2 \mathrm{H}) ;{ }^{13} \mathrm{C} \mathrm{NMR}(125 \mathrm{MHz}$, $\left.\mathrm{CDCl}_{3}\right) \delta 186.9(\mathrm{C}), 159.6(\mathrm{C}), 149.2(\mathrm{CH}), 145.2(\mathrm{C}), 142.3(\mathrm{CH}), 131.9(\mathrm{C}), 131.6(\mathrm{CH}), 128.9(\mathrm{CH}), 128.8(\mathrm{CH}), 115.8$ (CH), $63.0(\mathrm{C}), 36.8\left(\mathrm{CH}_{2}\right), 27.5\left(\mathrm{CH}_{2}\right)$. ATR-FTIR (thin film): 2958, 2874, 1599, 1515, 1460, 1261, 1019, 833, 774, 695 $\mathrm{cm}^{-1}$. HRMS (ESI) $\mathrm{m} / z$ calculated for $\mathrm{C}_{17} \mathrm{H}_{17} \mathrm{~N}_{2}(\mathrm{M}+\mathrm{H})^{+}: 249.1392$, found: 249.1385 .

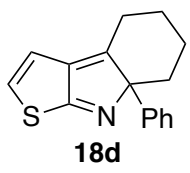

3b-Phenyl-3b,5,6,7-tetrahydro-4H-thieno[2,3-b]indole 18d. The optimized procedure was followed using $0.026 \mathrm{~g}$ of aniline $16 \mathrm{~d}(0.10 \mathrm{mmol}), 2.3 \mu \mathrm{L}$ of iodobenzene $(0.020 \mathrm{mmol}), 20 \mu \mathrm{L}$ of trifluoroacetic acid $(0.26 \mathrm{mmol}), 0.092 \mathrm{~g}$ of Selectfluor ( $0.26 \mathrm{mmol}$ ) in $2 \mathrm{~mL}$ of 1,1,1,3,3,3-hexafluoropropan-2-ol and $0.2 \mathrm{~mL}$ of water $(0.05 \mathrm{M})$. Purification by MPLC (2:98 to 10:90 EtOAc:hexanes) afforded the product as a green oil $(0.019 \mathrm{~g}, 74 \%):{ }^{1} \mathrm{H} \mathrm{NMR}\left(500 \mathrm{MHz}, \mathrm{CDCl}_{3}\right) \delta 7.33-$ $7.28(\mathrm{~m}, 2 \mathrm{H}), 7.27-7.22(\mathrm{~m}, 3 \mathrm{H}), 7.05(\mathrm{~d}, J=6.3 \mathrm{~Hz}, 1 \mathrm{H}), 6.56(\mathrm{~d}, J=6.3 \mathrm{~Hz}, 1 \mathrm{H}), 3.27(\mathrm{dq}, J=13.2,2.7 \mathrm{~Hz}, 1 \mathrm{H}), 3.15$ $-3.06(\mathrm{~m}, 1 \mathrm{H}), 2.40(\mathrm{td}, J=13.0,5.8 \mathrm{~Hz}, 1 \mathrm{H}), 2.08-1.96(\mathrm{~m}, 1 \mathrm{H}), 1.77-1.69(\mathrm{~m}, 1 \mathrm{H}), 1.36(\mathrm{tt}, J=13.5,3.2 \mathrm{~Hz}, 1 \mathrm{H})$, $1.25-1.20(\mathrm{~m}, 1 \mathrm{H}), 1.12(\mathrm{td}, J=13.5,3.4 \mathrm{~Hz}, 1 \mathrm{H}) ;{ }^{13} \mathrm{C} \mathrm{NMR}\left(125 \mathrm{MHz}, \mathrm{CDCl}_{3}\right) \delta 177.4(\mathrm{C}), 162.0(\mathrm{C}), 139.5(\mathrm{C}), 137.9$ $(\mathrm{C}), 135.6(\mathrm{CH}), 128.9(\mathrm{CH}), 127.6(\mathrm{CH}), 126.9(\mathrm{CH}), 114.8(\mathrm{CH}), 94.5(\mathrm{C}), 38.9\left(\mathrm{CH}_{2}\right), 28.5\left(\mathrm{CH}_{2}\right), 28.4\left(\mathrm{CH}_{2}\right), 22.2\left(\mathrm{CH}_{2}\right)$. ATR-FTIR (thin film): 2934, 2855, 1652, 1527, 1493, 1446, 763, 698, $668 \mathrm{~cm}^{-1}$. HRMS (ESI) $\mathrm{m} / z$ calculated for $\mathrm{C}_{16} \mathrm{H}_{16} \mathrm{NS}$ $(\mathrm{M}+\mathrm{H})^{+}: 254.1003$, found: 254.1003 . 


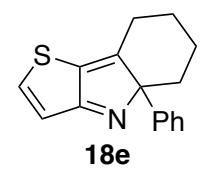

8a-Phenyl-6,7,8,8a-tetrahydro-5H-thieno[3,2-b]indole 18e. The optimized procedure was followed using $0.026 \mathrm{~g}$ of aniline $16 \mathrm{e}(0.10 \mathrm{mmol}), 2.3 \mu \mathrm{L}$ of iodobenzene $(0.020 \mathrm{mmol}), 20 \mu \mathrm{L}$ of trifluoroacetic acid $(0.26 \mathrm{mmol}), 0.092 \mathrm{~g}$ of Selectfluor $(0.26 \mathrm{mmol})$ in $2 \mathrm{~mL}$ of 1,1,1,3,3,3-hexafluoropropan-2-ol and $0.2 \mathrm{~mL}$ of water $(0.05 \mathrm{M})$. Purification by MPLC (2:98 to 30:70 EtOAc:hexanes) afforded the product as a yellow oil $(0.017 \mathrm{~g}, 66 \%):{ }^{1} \mathrm{H} \mathrm{NMR}\left(500 \mathrm{MHz}, \mathrm{CDCl}_{3}\right) \delta 7.65(\mathrm{~d}$, $J=5.7 \mathrm{~Hz}, 1 \mathrm{H}), 7.32-7.28(\mathrm{~m}, 2 \mathrm{H}), 7.27-7.22(\mathrm{~m}, 3 \mathrm{H}), 6.62(\mathrm{~d}, J=5.8 \mathrm{~Hz}, 1 \mathrm{H}), 3.27(\mathrm{dq}, J=13.5,2.6 \mathrm{~Hz}, 1 \mathrm{H}), 3.00$ (ddt, $J=13.5,4.2,1.9 \mathrm{~Hz}, 1 \mathrm{H}), 2.42(\mathrm{td}, J=13.1,5.8 \mathrm{~Hz}, 1 \mathrm{H}), 2.06-1.93(\mathrm{~m}, 1 \mathrm{H}), 1.78-1.68(\mathrm{~m}, 1 \mathrm{H}), 1.36(\mathrm{qt}, J=13.4$, $3.1 \mathrm{~Hz}, 1 \mathrm{H}), 1.25(\mathrm{qt}, J=13.0,4.2 \mathrm{~Hz}, 1 \mathrm{H}), 1.08(\mathrm{td}, J=13.4,3.4 \mathrm{~Hz}, 1 \mathrm{H}) ;{ }^{13} \mathrm{C} \mathrm{NMR}\left(125 \mathrm{MHz}, \mathrm{CDCl}_{3}\right) \delta 178.6(\mathrm{C}), 161.8$ $(\mathrm{C}), 147.5(\mathrm{CH}), 138.0(\mathrm{C}), 129.9(\mathrm{C}), 128.8(\mathrm{CH}), 127.6(\mathrm{CH}), 126.9(\mathrm{CH}), 118.1(\mathrm{CH}), 93.3(\mathrm{C}), 38.8\left(\mathrm{CH}_{2}\right), 28.2\left(\mathrm{CH}_{2}\right)$, $28.0\left(\mathrm{CH}_{2}\right), 22.2\left(\mathrm{CH}_{2}\right)$. ATR-FTIR (thin film): 2928, 2854, 1683, 1549, 1495, 1446, 764, 697, $677 \mathrm{~cm}^{-1}$. HRMS (ESI) $\mathrm{m} / z$ calculated for $\mathrm{C}_{16} \mathrm{H}_{16} \mathrm{NS}(\mathrm{M}+\mathrm{H})^{+}: 254.1003$, found: 254.1001 .

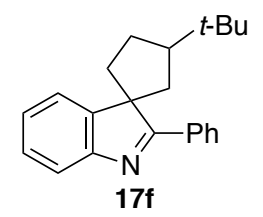

3-(tert-Butyl)-2'-phenylspiro[cyclopentane-1,3'-indole] 17f. ${ }^{18}$ The optimized procedure was followed using $0.030 \mathrm{~g}$ of aniline $16 \mathbf{f}(0.10 \mathrm{mmol}), 0.11 \mu \mathrm{L}$ of iodobenzene $(0.001 \mathrm{mmol}), 20 \mu \mathrm{L}$ of trifluoroacetic acid $(0.26 \mathrm{mmol}), 0.046 \mathrm{~g}$ of Selectfluor (0.13 mmol) in $2 \mathrm{~mL}$ of 1,1,1,3,3,3-hexafluoropropan-2-ol and $0.2 \mathrm{~mL}$ of water (0.05 M). Purification by MPLC (2:98 to 5:95 EtOAc:hexanes) afforded the product, a colorless oil, as a 1:1 ratio of diastereomers $(0.028 \mathrm{~g}, 93 \%)$. The spectral data matched that reported by Driver and co-workers. ${ }^{18}$ Diagnostic peaks for the one isomer: ${ }^{1} \mathrm{H} \mathrm{NMR}(500 \mathrm{MHz}$, $\left.\mathrm{CDCl}_{3}\right) \delta 8.20-8.14(\mathrm{~m}, 1 \mathrm{H}), 7.71(\mathrm{~d}, J=2.9 \mathrm{~Hz}, 0.5 \mathrm{H}), 7.56-7.40(\mathrm{~m}, 4 \mathrm{H}), 7.37(\mathrm{tdd}, J=7.6,4.2,1.2 \mathrm{~Hz}, 1 \mathrm{H}), 7.30-$ $7.22(\mathrm{~m}, 1 \mathrm{H}), 2.58-2.44(\mathrm{~m}, 2 \mathrm{H}), 2.44-1.94(\mathrm{~m}, 4 \mathrm{H}), 1.77(\mathrm{dd}, J=12.9,6.3 \mathrm{~Hz}, 0.5 \mathrm{H}), 1.06(\mathrm{~s}, 4.5 \mathrm{H}) ;{ }^{13} \mathrm{C} \mathrm{NMR}(125$ $\left.\mathrm{MHz}, \mathrm{CDCl}_{3}\right) \delta 182.6(\mathrm{C}), 153.1(\mathrm{C}), 150.9(\mathrm{C}), 132.8(\mathrm{C}), 130.5(\mathrm{CH}), 128.6(\mathrm{CH}), 128.39(\mathrm{CH}), 127.44(\mathrm{CH}), 126.0(\mathrm{CH})$, $121.2(\mathrm{CH}), 120.7(\mathrm{CH}), 63.1(\mathrm{C}), 53.2(\mathrm{CH}), 39.4\left(\mathrm{CH}_{2}\right), 36.4\left(\mathrm{CH}_{2}\right), 32.4(\mathrm{C}), 29.3\left(\mathrm{CH}_{2}\right), 28.1\left(\mathrm{CH}_{3}\right)$. Diagnostic peaks for the other isomer: ${ }^{1} \mathrm{H}$ NMR $\left(500 \mathrm{MHz}, \mathrm{CDCl}_{3}\right) \delta 8.16-8.10(\mathrm{~m}, 1 \mathrm{H}), 7.70(\mathrm{~d}, J=2.9 \mathrm{~Hz}, 0.5 \mathrm{H}), 1.66(\mathrm{dd}, J=12.9,6.5$ $\mathrm{Hz}, 0.5 \mathrm{H}), 0.99(\mathrm{~s}, 4.5 \mathrm{H}) ;{ }^{13} \mathrm{C} \mathrm{NMR}\left(125 \mathrm{MHz}, \mathrm{CDCl}_{3}\right) \delta 153.0(\mathrm{C}), 150.2(\mathrm{C}), 130.4(\mathrm{CH}), 128.6(\mathrm{CH}), 128.4(\mathrm{CH}), 127.4$ $(\mathrm{CH}), 125.9(\mathrm{CH}), 121.0(\mathrm{CH}), 62.5(\mathrm{C}), 52.6(\mathrm{CH}), 38.3\left(\mathrm{CH}_{2}\right), 34.4\left(\mathrm{CH}_{2}\right), 32.1(\mathrm{CH}), 28.1\left(\mathrm{CH}_{2}\right), 28.0\left(\mathrm{CH}_{3}\right)$. ATR-FTIR (thin film): 2958, 2869, 1521, 1465, 1456, 1442, 1365, 1265, 772, 750, $694 \mathrm{~cm}^{-1}$. HRMS (ESI) $\mathrm{m} / z$ calculated for $\mathrm{C}_{22} \mathrm{H}_{26} \mathrm{~N}$ $(\mathrm{M}+\mathrm{H})^{+}: 304.2065$, found: 304.2061 .

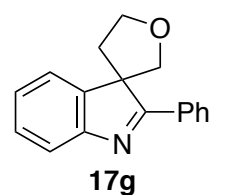

2'-Phenyl-4,5-dihydro-2H-spiro[furan-3,3'-indole] $\mathbf{1 7 g} .{ }^{18}$ The general procedure was followed using $0.025 \mathrm{~g}$ of aniline $16 \mathrm{~g}(0.10 \mathrm{mmol}), 2.3 \mu \mathrm{L}$ of iodobenzene $(0.020 \mathrm{mmol}), 20 \mu \mathrm{L}$ of trifluoroacetic acid $(0.26 \mathrm{mmol}), 0.046 \mathrm{~g}$ of Selectfluor $(0.13 \mathrm{mmol})$ in $2 \mathrm{~mL}$ of 1,1,1,3,3,3-hexafluoropropan-2-ol and $0.2 \mathrm{~mL}$ of water $(0.05 \mathrm{M})$. Purification by MPLC (2:98 to 5:95 EtOAc:hexanes) afforded the product as a white solid $(0.024 \mathrm{~g}, 96 \%)$. The spectral data matched that reported by Driver and co-workers: ${ }^{18} \mathrm{mp}=77{ }^{\circ} \mathrm{C} ;{ }^{1} \mathrm{H}$ NMR $\left(500 \mathrm{MHz}, \mathrm{CDCl}_{3}\right) \delta 8.26(\mathrm{dd}, J=6.7,3.1 \mathrm{~Hz}, 2 \mathrm{H}), 7.69(\mathrm{~d}, J=7.7 \mathrm{~Hz}$, $1 \mathrm{H}), 7.52-7.48(\mathrm{~m}, 3 \mathrm{H}), 7.46(\mathrm{~d}, J=7.3 \mathrm{~Hz}, 1 \mathrm{H}), 7.39(\mathrm{t}, J=7.6 \mathrm{~Hz}, 1 \mathrm{H}), 7.28(\mathrm{t}, J=7.5 \mathrm{~Hz}, 1 \mathrm{H}), 4.53(\mathrm{td}, J=8.6,2.5$ $\mathrm{Hz}, 1 \mathrm{H}), 4.47(\mathrm{~d}, J=9.5 \mathrm{~Hz}, 1 \mathrm{H}), 4.22(\mathrm{td}, J=9.5,6.6 \mathrm{~Hz}, 1 \mathrm{H}), 4.03(\mathrm{~d}, J=9.6 \mathrm{~Hz}, 1 \mathrm{H}), 2.68(\mathrm{ddd}, J=13.2,10.3,8.1 \mathrm{~Hz}$, 1H), 2.18 (ddd, $J=13.0,6.5,2.6 \mathrm{~Hz}, 1 \mathrm{H}) ;{ }^{13} \mathrm{C} \mathrm{NMR}\left(125 \mathrm{MHz}, \mathrm{CDCl}_{3}\right) \delta 180.7(\mathrm{C}), 153.6(\mathrm{C}), 145.7(\mathrm{C}), 131.7(\mathrm{C}), 131.0$ $(\mathrm{CH}), 128.8(\mathrm{CH}), 128.5(\mathrm{CH}), 128.2(\mathrm{CH}), 126.4(\mathrm{CH}), 121.1(\mathrm{CH}), 120.9(\mathrm{CH}), 75.9\left(\mathrm{CH}_{2}\right), 69.9\left(\mathrm{CH}_{2}\right), 62.8(\mathrm{C}), 38.2$ 
$\left(\mathrm{CH}_{2}\right)$. ATR-FTIR (thin film): 3061, 2976, 2929, 2865, 1523, 1465, 1213, 1075, 1030, 924, 771, 750, $697 \mathrm{~cm}^{-1}$. HRMS (ESI) $\mathrm{m} / z$ calculated for $\mathrm{C}_{17} \mathrm{H}_{16} \mathrm{NO}(\mathrm{M}+\mathrm{H})^{+}: 250.1232$, found: 250.1228 .

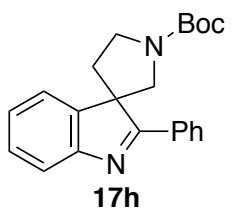

tert-Butyl 2-phenylspiro[indole-3,3'-pyrrolidine]-1'-carboxylate $17 \mathbf{h}^{18}{ }^{18}$ The optimized procedure was followed using $0.035 \mathrm{~g}$ of aniline $16 \mathrm{~h}(0.10 \mathrm{mmol}), 2.3 \mu \mathrm{L}$ of iodobenzene $(0.020 \mathrm{mmol}), 20 \mu \mathrm{L}$ of trifluoroacetic acid $(0.26 \mathrm{mmol}), 0.046$ $\mathrm{g}$ of Selectfluor $(0.13 \mathrm{mmol})$ in $2 \mathrm{~mL}$ of 1,1,1,3,3,3-hexafluoropropan-2-ol and $0.2 \mathrm{~mL}$ of water $(0.05 \mathrm{M})$. This substrate required an reduced reaction time of $1 \mathrm{~h}$. Purification by MPLC (2:98 to 10:90 EtOAc:hexanes) afforded the product, a colorless oil, as a 1.7:1 ratio of rotamers $(0.018 \mathrm{~g}, 53 \%)$. The spectral data matched that reported by Driver and co-workers. ${ }^{18}$ Diagnostic peaks for the major rotamer: ${ }^{1} \mathrm{H}$ NMR $\left(500 \mathrm{MHz}, \mathrm{CDCl}_{3}\right) \delta 8.04(\mathrm{~s}, 1.3 \mathrm{H}), 7.71(\mathrm{~d}, J=7.7 \mathrm{~Hz}, 1 \mathrm{H}), 7.50(\mathrm{~s}$, $3 \mathrm{H}), 7.41(\mathrm{t}, J=7.7 \mathrm{~Hz}, 2 \mathrm{H}), 7.31-7.24(\mathrm{~m}, 1 \mathrm{H}), 4.16-4.09(\mathrm{~m}, 0.73 \mathrm{H}), 4.00-3.74(\mathrm{~m}, 2 \mathrm{H}), 3.61(\mathrm{~d}, J=11.9 \mathrm{~Hz}, 0.68 \mathrm{H})$, $2.71(\mathrm{p}, J=10.9,10.2 \mathrm{~Hz}, 1 \mathrm{H}), 2.03(\mathrm{dt}, J=11.1,5.3 \mathrm{~Hz}, 1 \mathrm{H}), 1.47(\mathrm{~s}, 5.7 \mathrm{H}) ;{ }^{13} \mathrm{C} \mathrm{NMR}\left(126 \mathrm{MHz}, \mathrm{CDCl}_{3}\right) \delta 178.5(\mathrm{C})$, $154.4(\mathrm{C}), 153.1(\mathrm{C}), 145.7(\mathrm{C}), 132.4(\mathrm{C}), 130.9(\mathrm{CH}), 128.8(\mathrm{CH}), 128.4(\mathrm{CH}), 128.1(\mathrm{CH}), 126.5(\mathrm{CH}), 121.2(\mathrm{CH}), 120.9$ $(\mathrm{CH}), 80.2(\mathrm{C}), 62.2(\mathrm{C}), 53.1\left(\mathrm{CH}_{2}\right), 45.4\left(\mathrm{CH}_{2}\right), 34.4\left(\mathrm{CH}_{2}\right), 28.5\left(\mathrm{CH}_{3}\right)$. Diagnostic peaks for the minor rotamer: ${ }^{1} \mathrm{H} \mathrm{NMR}$ $\left(500 \mathrm{MHz}, \mathrm{CDCl}_{3}\right) \delta 8.01(\mathrm{~s}, 0.7 \mathrm{H}), 4.19(\mathrm{~d}, J=9.0 \mathrm{~Hz}, 0.40 \mathrm{H}), 3.68(\mathrm{~d}, J=10.8 \mathrm{~Hz}, 0.41 \mathrm{H}), 1.55(\mathrm{~s}, 3.6 \mathrm{H}) ;{ }^{13} \mathrm{C} \mathrm{NMR}(125$ $\left.\mathrm{MHz}, \mathrm{CDCl}_{3}\right) \delta 178.4(\mathrm{C}), 52.6\left(\mathrm{CH}_{2}\right), 45.5\left(\mathrm{CH}_{2}\right), 35.4\left(\mathrm{CH}_{2}\right), 29.7\left(\mathrm{CH}_{3}\right)$. ATR-FTIR (thin film): 2975, 2928, 1697, 1456, 1393, 1366, 1165, 1123, 756, $696 \mathrm{~cm}^{-1}$. HRMS (ESI) $\mathrm{m} / z$ calculated for $\mathrm{C}_{22} \mathrm{H}_{25} \mathrm{~N}_{2} \mathrm{O}_{2}(\mathrm{M}+\mathrm{H})^{+}: 349.1916$, found: 349.1911.

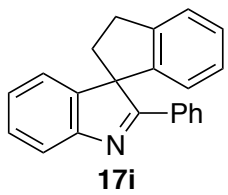

2'-Phenyl-2,3-dihydrospiro[indene-1,3'-indole] $17 \mathbf{i}^{18}{ }^{18}$ The optimized procedure was followed using $0.030 \mathrm{~g}$ of aniline $\mathbf{1 6 \mathbf { i }}$ $(0.10 \mathrm{mmol}), 0.11 \mu \mathrm{L}$ of iodobenzene $(0.001 \mathrm{mmol}), 20 \mu \mathrm{L}$ of trifluoroacetic acid $(0.26 \mathrm{mmol}), 0.046 \mathrm{~g}$ of Selectfluor $(0.13$ $\mathrm{mmol})$ in $2 \mathrm{~mL}$ of 1,1,1,3,3,3-hexafluoropropan-2-ol and $0.2 \mathrm{~mL}$ of water $(0.05 \mathrm{M})$. Purification by MPLC (2:98 to 5:95 EtOAc:hexanes) afforded the product as a colorless oil $(0.025 \mathrm{~g}, 85 \%)$. The spectral data matched that reported by Driver and co-workers: ${ }^{18}{ }^{1} \mathrm{H}$ NMR $\left(500 \mathrm{MHz}, \mathrm{CDCl}_{3}\right) \delta 7.75(\mathrm{~d}, J=7.7 \mathrm{~Hz}, 1 \mathrm{H}), 7.71(\mathrm{~d}, J=7.7 \mathrm{~Hz}, 2 \mathrm{H}), 7.45(\mathrm{~d}, J=7.6 \mathrm{~Hz}, 1 \mathrm{H})$, 7.37 (pentet, $J=7.4 \mathrm{~Hz}, 2 \mathrm{H}), 7.31(\mathrm{t}, J=7.8 \mathrm{~Hz}, 2 \mathrm{H}), 7.27(\mathrm{~d}, J=8.2 \mathrm{~Hz}, 1 \mathrm{H}), 7.14(\mathrm{t}, J=7.4 \mathrm{~Hz}, 1 \mathrm{H}), 7.10(\mathrm{~d}, J=7.4 \mathrm{~Hz}$, $1 \mathrm{H}), 7.06(\mathrm{t}, J=7.5 \mathrm{~Hz}, 1 \mathrm{H}), 6.63(\mathrm{~d}, J=7.7 \mathrm{~Hz}, 1 \mathrm{H}), 3.52(\mathrm{dt}, J=17.1,8.8 \mathrm{~Hz}, 1 \mathrm{H}), 3.33(\mathrm{ddd}, J=16.5,9.5,3.0 \mathrm{~Hz}, 1 \mathrm{H})$, $2.79(\mathrm{dt}, J=13.6,9.2 \mathrm{~Hz}, 1 \mathrm{H}), 2.37(\mathrm{ddd}, J=12.2,8.9,3.0 \mathrm{~Hz}, 1 \mathrm{H}) ;{ }^{13} \mathrm{C} \mathrm{NMR}\left(125 \mathrm{MHz}, \mathrm{CDCl}_{3}\right) \delta 181.6(\mathrm{C}), 153.2(\mathrm{C})$, $147.7(\mathrm{C}), 144.3(\mathrm{C}), 143.9(\mathrm{C}), 132.8(\mathrm{C}), 130.5(\mathrm{CH}), 128.6(\mathrm{CH}), 128.5(\mathrm{CH}), 128.0(\mathrm{CH}), 127.9(\mathrm{CH}), 127.5(\mathrm{CH}), 126.1$ $(\mathrm{CH}), 125.4(\mathrm{CH}), 123.5(\mathrm{CH}), 121.7(\mathrm{CH}), 120.9(\mathrm{CH}), 69.1(\mathrm{C}), 35.9\left(\mathrm{CH}_{2}\right), 31.9\left(\mathrm{CH}_{2}\right)$. ATR-FTIR (thin film): 3064, $2927,2853,1524,1455,1442,1342,1264,1214,1182,1156,1111,1012,745,693 \mathrm{~cm}^{-1}$. HRMS (ESI) $\mathrm{m} / z$ calculated for $\mathrm{C}_{22} \mathrm{H}_{18} \mathrm{~N}(\mathrm{M}+\mathrm{H})^{+}: 296.1439$, found: 296.1434 .

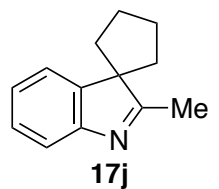

2'-Methylspiro[cyclopentane-1,3'-indole] $\mathbf{1 7} \mathbf{j}^{22}$ The optimized procedure was followed using $0.019 \mathrm{~g}$ of aniline $\mathbf{1 6 j}(0.10$ $\mathrm{mmol}), 2.3 \mu \mathrm{L}$ of iodobenzene $(0.020 \mathrm{mmol}), 20 \mu \mathrm{L}$ of trifluoroacetic acid $(0.26 \mathrm{mmol}), 0.046 \mathrm{~g}$ of Selectfluor $(0.13 \mathrm{mmol})$ in $2 \mathrm{~mL}$ of 1,1,1,3,3,3-hexafluoropropan-2-ol and $0.2 \mathrm{~mL}$ of water $(0.05 \mathrm{M})$. Purification by MPLC (2:98 to 5:95 EtOAc:hexanes) afforded the product as a yellow oil $(0.017 \mathrm{~g}, 94 \%)$. The spectral data matched that reported by Driver and co-workers: ${ }^{22}{ }^{1} \mathrm{H}$ NMR $\left(500 \mathrm{MHz}, \mathrm{CDCl}_{3}\right) \delta 7.50(\mathrm{~d}, J=7.6 \mathrm{~Hz}, 1 \mathrm{H}), 7.34-7.24(\mathrm{~m}, 2 \mathrm{H}), 7.16(\mathrm{t}, J=7.2 \mathrm{~Hz}, 1 \mathrm{H}), 2.29(\mathrm{~s}$, $3 \mathrm{H}), 2.15-1.94(\mathrm{~m}, 6 \mathrm{H}), 1.79(\mathrm{dd}, J=12.0,5.7 \mathrm{~Hz}, 2 \mathrm{H}) ;{ }^{13} \mathrm{C} \mathrm{NMR}\left(125 \mathrm{MHz}, \mathrm{CDCl}_{3}\right) \delta 187.4(\mathrm{C}), 153.6(\mathrm{C}), 147.2(\mathrm{C})$, 
$127.3(\mathrm{CH}), 125.2(\mathrm{CH}), 121.3(\mathrm{CH}), 119.5(\mathrm{CH}), 63.8(\mathrm{C}), 35.3\left(\mathrm{CH}_{2}\right), 26.9\left(\mathrm{CH}_{2}\right), 15.9\left(\mathrm{CH}_{3}\right)$. ATR-FTIR (thin film): 2954, 2867, 1716, 1573, 1465, 1455, 1377, 1260, 1201, 1016, 773, $749 \mathrm{~cm}^{-1}$. HRMS (ESI) $\mathrm{m} / z$ calculated for $\mathrm{C}_{13} \mathrm{H}_{16} \mathrm{~N}$ $(\mathrm{M}+\mathrm{H})^{+}: 186.1283$, found: 186.1283 .

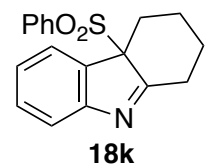

4a-(Phenylsulfonyl)-2,3,4,4a-tetrahydro-1 $\mathrm{H}$-carbazole $18 \mathrm{k} .{ }^{18}$ The optimized procedure was followed using $0.031 \mathrm{~g}$ of aniline $16 \mathrm{k}(0.10 \mathrm{mmol}), 2.3 \mu \mathrm{L}$ of iodobenzene $(0.020 \mathrm{mmol}), 20 \mu \mathrm{L}$ of trifluoroacetic acid $(0.26 \mathrm{mmol}), 0.046 \mathrm{~g}$ of Selectfluor $(0.13 \mathrm{mmol})$ in $2 \mathrm{~mL}$ of 1,1,1,3,3,3-hexafluoropropan-2-ol and $0.2 \mathrm{~mL}$ of water $(0.05 \mathrm{M})$. Purification by MPLC (2:98 to 5:95 EtOAc:hexanes) afforded the product as a yellow foam $(0.024 \mathrm{~g}, 76 \%)$. The spectral data matched that reported by Driver and co-workers: ${ }^{18}{ }^{1} \mathrm{H}$ NMR $\left(500 \mathrm{MHz}, \mathrm{CDCl}_{3}\right) \delta 7.71(\mathrm{~d}, J=7.0 \mathrm{~Hz}, 1 \mathrm{H}), 7.36(\mathrm{t}, J=7.4 \mathrm{~Hz}, 1 \mathrm{H}), 7.28-7.20$ $(\mathrm{m}, 4 \mathrm{H}), 7.14(\mathrm{q}, J=7.8,7.3 \mathrm{~Hz}, 3 \mathrm{H}), 3.54-3.46(\mathrm{~m}, 1 \mathrm{H}), 3.35(\mathrm{td}, J=13.2,5.4 \mathrm{~Hz}, 1 \mathrm{H}), 2.99(\mathrm{dd}, J=13.0,4.3 \mathrm{~Hz}, 1 \mathrm{H})$, $2.58(\mathrm{dtd}, J=18.0,13.8,4.1 \mathrm{~Hz}, 1 \mathrm{H}), 2.40-2.32(\mathrm{~m}, 1 \mathrm{H}), 1.77(\mathrm{~d}, J=13.5 \mathrm{~Hz}, 1 \mathrm{H}), 1.60(\mathrm{td}, J=14.4,4.7 \mathrm{~Hz}, 1 \mathrm{H}), 1.55$ $-1.44(\mathrm{~m}, 1 \mathrm{H}) ;{ }^{13} \mathrm{C}$ NMR $\left(125 \mathrm{MHz}, \mathrm{CDCl}_{3}\right) \delta 179.4(\mathrm{C}), 155.1(\mathrm{C}), 135.9(\mathrm{C}), 134.7(\mathrm{C}), 133.7(\mathrm{CH}), 129.9(\mathrm{CH}), 128.8$ $(\mathrm{CH}), 127.9(\mathrm{CH}), 125.8(\mathrm{CH}), 124.8(\mathrm{CH}), 120.3(\mathrm{CH}), 78.9(\mathrm{C}), 33.0\left(\mathrm{CH}_{2}\right), 31.1\left(\mathrm{CH}_{2}\right), 28.9\left(\mathrm{CH}_{2}\right), 21.8\left(\mathrm{CH}_{2}\right)$. ATRFTIR (thin film): 2931, 2856, 1578, 1446, 1305, 1288, 1158, 1141, 1088, 1075, 772, 755, 722, 686, $608 \mathrm{~cm}^{-1}$. HRMS (ESI) $m / z$ calculated for $\mathrm{C}_{18} \mathrm{H}_{18} \mathrm{NO}_{2} \mathrm{~S}(\mathrm{M}+\mathrm{H})^{+}: 312.1058$, found: 312.1050 .

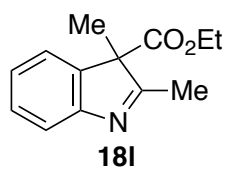

Ethyl 2,3-dimethyl-3H-indole-3-carboxylate 181. ${ }^{18}$ The optimized procedure was followed using $0.022 \mathrm{~g}$ of aniline $\mathbf{1 6 1}$ $(0.10 \mathrm{mmol}), 2.3 \mu \mathrm{L}$ of iodobenzene $(0.020 \mathrm{mmol}), 20 \mu \mathrm{L}$ of trifluoroacetic acid $(0.26 \mathrm{mmol}), 0.046 \mathrm{~g}$ of Selectfluor $(0.13$ $\mathrm{mmol})$ in $2 \mathrm{~mL}$ of 1,1,1,3,3,3-hexafluoropropan-2-ol and $0.2 \mathrm{~mL}$ of water $(0.05 \mathrm{M})$. Purification by MPLC (2:98 to 5:95 EtOAc:hexanes) afforded the product as a yellow oil $(0.013 \mathrm{~g}, 63 \%)$. The spectral data matched that reported by Driver and co-workers: ${ }^{18}{ }^{1} \mathrm{H}$ NMR $\left(500 \mathrm{MHz}, \mathrm{CDCl}_{3}\right) \delta 7.54(\mathrm{~d}, J=7.7 \mathrm{~Hz}, 1 \mathrm{H}), 7.40-7.32(\mathrm{~m}, 2 \mathrm{H}), 7.24-7.17(\mathrm{~m}, 1 \mathrm{H}), 4.16(\mathrm{dq}, J$ $=10.9,7.1 \mathrm{~Hz}, 1 \mathrm{H}), 4.03(\mathrm{dq}, J=10.9,7.1 \mathrm{~Hz}, 1 \mathrm{H}), 2.36(\mathrm{~s}, 3 \mathrm{H}), 1.58(\mathrm{~s}, 3 \mathrm{H}), 1.15(\mathrm{t}, J=7.1 \mathrm{~Hz}, 3 \mathrm{H}) ;{ }^{13} \mathrm{C} \mathrm{NMR}(125 \mathrm{MHz}$, $\left.\mathrm{CDCl}_{3}\right) \delta 181.4(\mathrm{C}), 170.5(\mathrm{C}), 155.0(\mathrm{C}), 140.4(\mathrm{C}), 128.8(\mathrm{CH}), 125.7(\mathrm{CH}), 122.2(\mathrm{CH}), 120.2(\mathrm{CH}), 63.8(\mathrm{C}), 61.7\left(\mathrm{CH}_{2}\right)$, $19.7\left(\mathrm{CH}_{3}\right), 16.8\left(\mathrm{CH}_{3}\right), 14.0\left(\mathrm{CH}_{3}\right)$. ATR-FTIR (thin film): 2980, 2930, 1732, 1582, 1456, 1376, 1256, 1217, 1107,1020 , $861,757,668 \mathrm{~cm}^{-1}$. HRMS (ESI) $\mathrm{m} / z$ calculated for $\mathrm{C}_{13} \mathrm{H}_{16} \mathrm{NO}_{2}(\mathrm{M}+\mathrm{H})^{+}: 218.1181$, found: 218.1177 .

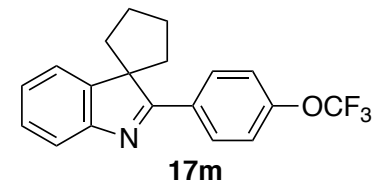

2'-(4-(trifluoromethoxy)phenyl)spiro[cyclopentane-1,3'-indole] $\mathbf{1 7} \mathbf{m}^{18}$ The optimized procedure was followed using $0.033 \mathrm{~g}$ of aniline $16 \mathrm{~m}(0.10 \mathrm{mmol}), 2.3 \mu \mathrm{L}$ of iodobenzene $(0.020 \mathrm{mmol}), 20 \mu \mathrm{L}$ of trifluoroacetic acid $(0.26 \mathrm{mmol}), 0.046$ $\mathrm{g}$ of Selectfluor $(0.13 \mathrm{mmol})$ in $2 \mathrm{~mL}$ of 1,1,1,3,3,3-hexafluoropropan-2-ol and $0.2 \mathrm{~mL}$ of water $(0.05 \mathrm{M})$. Purification by MPLC (2:98 to 5:95 EtOAc:hexanes) afforded the product as a yellow oil $(0.033 \mathrm{~g}, 98 \%)$. The spectral data matched that reported by Driver and co-workers: ${ }^{18}{ }^{1} \mathrm{H}$ NMR $\left(500 \mathrm{MHz}, \mathrm{CDCl}_{3}\right) \delta 8.14(\mathrm{~d}, J=8.5 \mathrm{~Hz}, 2 \mathrm{H}), 7.68(\mathrm{~d}, J=7.6 \mathrm{~Hz}, 1 \mathrm{H}), 7.40$ $(\mathrm{d}, J=7.4 \mathrm{~Hz}, 1 \mathrm{H}), 7.36(\mathrm{t}, J=7.7 \mathrm{~Hz}, 1 \mathrm{H}), 7.32(\mathrm{~d}, J=8.5 \mathrm{~Hz}, 2 \mathrm{H}), 7.25(\mathrm{t}, J=7.4 \mathrm{~Hz}, 1 \mathrm{H}), 2.38(\mathrm{dt}, J=13.7,7.0 \mathrm{~Hz}$, 2H), $2.21(\mathrm{q}, J=8.0 \mathrm{~Hz}, 4 \mathrm{H}), 1.94(\mathrm{dd}, J=12.8,6.5 \mathrm{~Hz}, 2 \mathrm{H}) ;{ }^{13} \mathrm{C} \mathrm{NMR}\left(125 \mathrm{MHz}, \mathrm{CDCl}_{3}\right) \delta 181.1(\mathrm{C}), 152.8(\mathrm{C}), 150.7$ $(\mathrm{C}), 150.1(\mathrm{C}), 131.3(\mathrm{C}), 129.9(\mathrm{CH}), 127.5(\mathrm{CH}), 126.2(\mathrm{CH}), 121.5\left(\mathrm{q}, J_{C F}=258.3 \mathrm{~Hz}, \mathrm{C}\right), 121.0(\mathrm{CH}), 120.8(\mathrm{CH}), 120.7$ $(\mathrm{CH}), 63.2(\mathrm{C}), 36.7\left(\mathrm{CH}_{2}\right), 27.5\left(\mathrm{CH}_{2}\right) ;{ }^{19} \mathrm{~F}$ NMR $\left(282 \mathrm{MHz}, \mathrm{CDCl}_{3}\right) \delta-58.1$. ATR-FTIR (thin film): 2957, 2875, 1506, $1256,1208,1165,750 \mathrm{~cm}^{-1}$. HRMS (ESI) $\mathrm{m} / z$ calculated for $\mathrm{C}_{19} \mathrm{H}_{17} \mathrm{~F}_{3} \mathrm{NO}(\mathrm{M}+\mathrm{H})^{+}: 332.1262$, found: 332.1262 . 


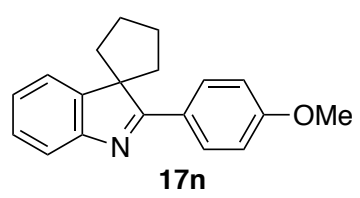

$\mathbf{2}^{\prime}$-(4-Methoxyphenyl)spiro[cyclopentane-1,3'-indole] $17 \mathbf{n} .^{18}$ The optimized procedure was followed using $0.028 \mathrm{~g}$ of aniline $16 \mathrm{n}(0.10 \mathrm{mmol}), 0.11 \mu \mathrm{L}$ of iodobenzene $(0.001 \mathrm{mmol}), 20 \mu \mathrm{L}$ of trifluoroacetic acid $(0.26 \mathrm{mmol}), 0.046 \mathrm{~g}$ of Selectfluor $(0.13 \mathrm{mmol})$ in $2 \mathrm{~mL}$ of 1,1,1,3,3,3-hexafluoropropan-2-ol and $0.2 \mathrm{~mL}$ of water $(0.05 \mathrm{M})$. Purification by MPLC (2:98 to 5:95 EtOAc:hexanes) afforded the product as a yellow oil $(0.027 \mathrm{~g}, 96 \%)$. The spectral data matched that reported by Driver and co-workers: ${ }^{18}{ }^{1} \mathrm{H}$ NMR $\left(500 \mathrm{MHz}, \mathrm{CDCl}_{3}\right) \delta 8.07(\mathrm{~d}, J=8.5 \mathrm{~Hz}, 2 \mathrm{H}), 7.65(\mathrm{~d}, J=7.7 \mathrm{~Hz}, 1 \mathrm{H}), 7.38(\mathrm{~d}, J=$ $7.4 \mathrm{~Hz}, 1 \mathrm{H}), 7.33(\mathrm{t}, J=7.6 \mathrm{~Hz}, 1 \mathrm{H}), 7.20(\mathrm{t}, J=7.4 \mathrm{~Hz}, 1 \mathrm{H}), 7.00(\mathrm{~d}, J=8.5 \mathrm{~Hz}, 2 \mathrm{H}), 3.88(\mathrm{~s}, 3 \mathrm{H}), 2.41(\mathrm{dt}, J=13.2,7.3$ $\mathrm{Hz}, 2 \mathrm{H}), 2.22(\mathrm{td}, J=8.9,8.0,4.3 \mathrm{~Hz}, 4 \mathrm{H}), 1.93(\mathrm{dt}, J=12.7,5.7 \mathrm{~Hz}, 2 \mathrm{H}) ;{ }^{13} \mathrm{C} \mathrm{NMR}\left(125 \mathrm{MHz}, \mathrm{CDCl}_{3}\right) \delta 182.3(\mathrm{C}), 161.5$ $(\mathrm{C}), 153.1(\mathrm{C}), 150.2(\mathrm{C}), 130.1(\mathrm{CH}), 127.4(\mathrm{CH}), 125.4(\mathrm{CH}), 125.3(\mathrm{C}), 120.9(\mathrm{CH}), 120.2(\mathrm{CH}), 114.0(\mathrm{CH}), 63.0(\mathrm{C})$, $55.4\left(\mathrm{CH}_{3}\right), 37.2\left(\mathrm{CH}_{2}\right), 27.6\left(\mathrm{CH}_{2}\right)$. ATR-FTIR (thin film): 2955, 1603, 1506, 1456, 1254, 1169, 837, $750 \mathrm{~cm}^{-1}$. HRMS (ESI) $m / z$ calculated for $\mathrm{C}_{19} \mathrm{H}_{20} \mathrm{NO}(\mathrm{M}+\mathrm{H})^{+}: 278.1545$, found: 278.1541.
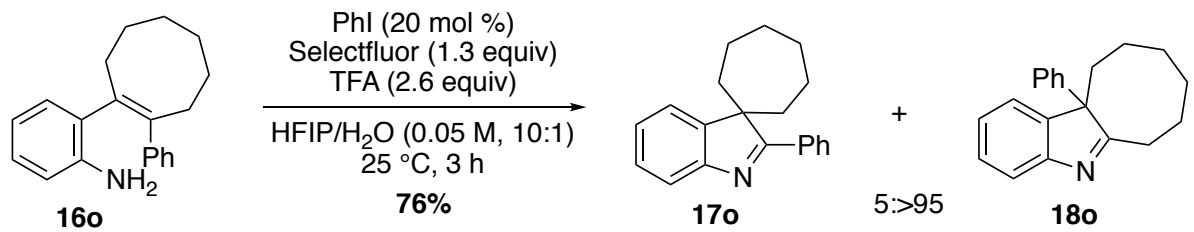

(s21)

11a-Phenyl-7,8,9,10,11,11a-hexahydro-6H-cycloocta[b]indole $180 .{ }^{18}$ The optimized procedure was followed using 0.028 $\mathrm{g}$ of aniline $160(0.10 \mathrm{mmol}), 2.3 \mu \mathrm{L}$ of iodobenzene $(0.020 \mathrm{mmol}), 20 \mu \mathrm{L}$ of trifluoroacetic acid $(0.26 \mathrm{mmol}), 0.046 \mathrm{~g}$ of Selectfluor $(0.13 \mathrm{mmol})$ in $2 \mathrm{~mL}$ of 1,1,1,3,3,3-hexafluoropropan-2-ol and $0.2 \mathrm{~mL}$ of water $(0.05 \mathrm{M})$. Analysis of the reaction mixture using ${ }^{1} \mathrm{H}$ NMR revealed only the formation of 180. Purification by MPLC (2:98 to 10:90 EtOAc:hexanes) afforded 180 as a yellow oil $(0.0021 \mathrm{~g}, 76 \%)$. The spectral data matched that reported by Driver and co-workers: ${ }^{18}{ }^{1} \mathrm{H}$ NMR $\left(500 \mathrm{MHz}, \mathrm{CDCl}_{3}\right) \delta 7.61(\mathrm{~d}, J=7.7 \mathrm{~Hz}, 1 \mathrm{H}), 7.32(\mathrm{t}, J=7.6 \mathrm{~Hz}, 1 \mathrm{H}), 7.28-7.17(\mathrm{~m}, 3 \mathrm{H}), 7.14(\mathrm{t}, J=7.4 \mathrm{~Hz}, 1 \mathrm{H}), 7.06$ $(\mathrm{dd}, J=7.8,3.7 \mathrm{~Hz}, 3 \mathrm{H}), 2.84-2.71(\mathrm{~m}, 2 \mathrm{H}), 2.48(\mathrm{tdt}, J=13.9,9.4,3.9 \mathrm{~Hz}, 2 \mathrm{H}), 1.95(\mathrm{ddp}, J=14.5,9.5,5.7,5.1 \mathrm{~Hz}$, 2H), 1.67 (dtd, $J=13.9,7.2,3.5 \mathrm{~Hz}, 1 \mathrm{H}), 1.61-1.39(\mathrm{~m}, 3 \mathrm{H}), 1.37-1.27(\mathrm{~m}, 1 \mathrm{H}), 0.97-0.88(\mathrm{~m}, 1 \mathrm{H}) ;{ }^{13} \mathrm{C} \mathrm{NMR}(125$ $\left.\mathrm{MHz}, \mathrm{CDCl}_{3}\right) \delta 192.0(\mathrm{C}), 155.2(\mathrm{C}), 145.3(\mathrm{C}), 139.7(\mathrm{C}), 128.8(\mathrm{CH}), 127.8(\mathrm{CH}), 127.1(\mathrm{CH}), 126.1(\mathrm{CH}), 125.6(\mathrm{CH})$, 122.4 (CH), $120.1(\mathrm{CH}), 65.8(\mathrm{C}), 30.9\left(\mathrm{CH}_{2}\right), 29.9\left(\mathrm{CH}_{2}\right), 28.9\left(\mathrm{CH}_{2}\right), 26.6\left(\mathrm{CH}_{2}\right), 25.2\left(\mathrm{CH}_{2}\right), 22.9\left(\mathrm{CH}_{2}\right)$. ATR-FTIR (thin film): 3057, 2925, 2854, 1699, 1567, 1464, 1455, 1445, 1252, 1034, 761, 743, $697 \mathrm{~cm}^{-1}$. HRMS (ESI) $\mathrm{m} / z$ calculated for $\mathrm{C}_{20} \mathrm{H}_{22} \mathrm{~N}(\mathrm{M}+\mathrm{H})^{+}: 276.1752$, found: 276.1744 .

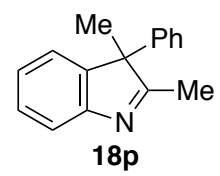

2,3-Dimethyl-3-phenyl-3H-indole 18p. ${ }^{22}$ The optimized procedure was followed using $0.022 \mathrm{~g}$ of aniline $16 \mathrm{p}(0.10 \mathrm{mmol})$, $2.3 \mu \mathrm{L}$ of iodobenzene $(0.020 \mathrm{mmol}), 20 \mu \mathrm{L}$ of trifluoroacetic acid $(0.26 \mathrm{mmol}), 0.046 \mathrm{~g}$ of Selectfluor $(0.13 \mathrm{mmol})$ in 2 $\mathrm{mL}$ of 1,1,1,3,3,3-hexafluoropropan-2-ol and $0.2 \mathrm{~mL}$ of water $(0.05 \mathrm{M})$. Purification by MPLC (2:98 to 5:95 EtOAc:hexanes) afforded the product as a yellow oil $(0.019 \mathrm{~g}, 86 \%)$. The spectral data matched that reported by Driver and co-workers: ${ }^{22}{ }^{1} \mathrm{H}$ $\operatorname{NMR}\left(500 \mathrm{MHz}, \mathrm{CDCl}_{3}\right) \delta 7.61(\mathrm{~d}, J=7.7 \mathrm{~Hz}, 1 \mathrm{H}), 7.36-7.29(\mathrm{~m}, 1 \mathrm{H}), 7.29-7.21(\mathrm{~m}, 3 \mathrm{H}), 7.15(\mathrm{t}, J=7.4 \mathrm{~Hz}, 1 \mathrm{H}), 7.09$ $(\mathrm{d}, J=7.3 \mathrm{~Hz}, 1 \mathrm{H}), 7.06-7.01(\mathrm{~m}, 2 \mathrm{H}), 2.13(\mathrm{~s}, 3 \mathrm{H}), 1.69(\mathrm{~s}, 3 \mathrm{H}) ;{ }^{13} \mathrm{C}$ NMR $\left(125 \mathrm{MHz}, \mathrm{CDCl}_{3}\right) \delta 187.2(\mathrm{C}), 154.5(\mathrm{C})$, $147.1(\mathrm{C}), 139.4(\mathrm{C}), 128.9(\mathrm{CH}), 127.9(\mathrm{CH}), 127.2(\mathrm{CH}), 126.1(\mathrm{CH}), 125.7(\mathrm{CH}), 122.5(\mathrm{CH}), 120.1(\mathrm{CH}), 61.8(\mathrm{C}), 20.3$ $\left(\mathrm{CH}_{3}\right), 15.9\left(\mathrm{CH}_{3}\right)$. ATR-FTIR (thin film): 2969, 2926, 1578, 1495, 1445, 1374, 1240, 1028, 1014, 772, $746,697 \mathrm{~cm}^{-1}$. HRMS (ESI) $m / z$ calculated for $\mathrm{C}_{16} \mathrm{H}_{16} \mathrm{~N}(\mathrm{M}+\mathrm{H})^{+}: 222.1283$, found: 222.1278 . 

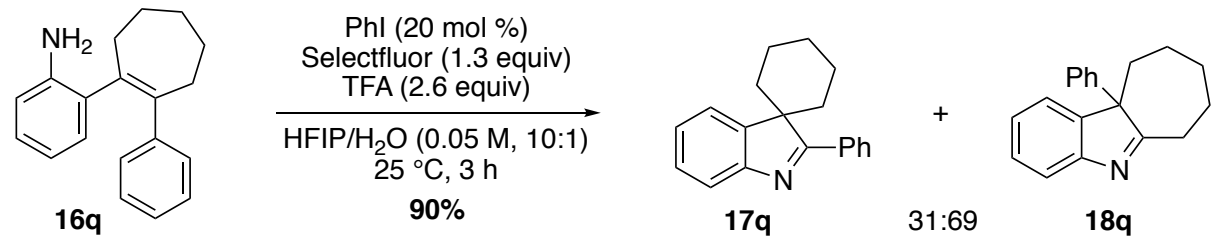

(s22)

2'-Phenylspiro[cyclohexane-1,3'-indole] $17 \mathbf{q} .{ }^{24}$ The optimized procedure was followed using $0.026 \mathrm{~g}$ of aniline $\mathbf{1 6 q}(0.10$ $\mathrm{mmol}), 2.3 \mu \mathrm{L}$ of iodobenzene $(0.020 \mathrm{mmol}), 20 \mu \mathrm{L}$ of trifluoroacetic acid $(0.26 \mathrm{mmol}), 0.046 \mathrm{~g}$ of Selectfluor $(0.13 \mathrm{mmol})$ in $2 \mathrm{~mL}$ of $1,1,1,3,3,3$-hexafluoropropan-2-ol and $0.2 \mathrm{~mL}$ of water $(0.05 \mathrm{M})$. Analysis of the reaction mixture using ${ }^{1} \mathrm{H} N M R$ revealed a 31:69 mixture of 17q and 18q. Purification by MPLC (2:98 to 5:95 EtOAc:hexanes) afforded the product as a colorless oil $(0.007 \mathrm{~g}, 28 \%)$. The spectral data matched that reported by Toste and co-workers: ${ }^{24}{ }^{1} \mathrm{H} \mathrm{NMR}\left(500 \mathrm{MHz}, \mathrm{CDCl}_{3}\right)$ $\delta 8.04(\mathrm{dd}, J=6.7,3.1 \mathrm{~Hz}, 2 \mathrm{H}), 7.83(\mathrm{~d}, J=7.5 \mathrm{~Hz}, 1 \mathrm{H}), 7.73(\mathrm{~d}, J=7.7 \mathrm{~Hz}, 1 \mathrm{H}), 7.52-7.45(\mathrm{~m}, 3 \mathrm{H}), 7.40(\mathrm{t}, J=7.6 \mathrm{~Hz}$, $1 \mathrm{H}), 7.24(\mathrm{t}, J=7.9 \mathrm{~Hz}, 1 \mathrm{H}), 2.29(\mathrm{td}, J=13.4,4.5 \mathrm{~Hz}, 2 \mathrm{H}), 2.09-1.96(\mathrm{~m}, 3 \mathrm{H}), 1.88-1.81(\mathrm{~m}, 2 \mathrm{H}), 1.59-1.48(\mathrm{~m}, 1 \mathrm{H})$, $1.44(\mathrm{~d}, J=13.5 \mathrm{~Hz}, 2 \mathrm{H}) ;{ }^{13} \mathrm{C} \mathrm{NMR}\left(125 \mathrm{MHz}, \mathrm{CDCl}_{3}\right) \delta 184.0(\mathrm{C}), 153.8(\mathrm{C}), 146.2(\mathrm{C}), 134.0(\mathrm{C}), 130.0(\mathrm{CH}), 128.6$ $(\mathrm{CH}), 128.4(\mathrm{CH}), 127.7(\mathrm{CH}), 124.9(\mathrm{CH}), 124.4(\mathrm{CH}), 121.3(\mathrm{CH}), 58.5(\mathrm{C}), 31.2\left(\mathrm{CH}_{2}\right), 25.2\left(\mathrm{CH}_{2}\right), 21.7\left(\mathrm{CH}_{2}\right)$; ATRFTIR (thin film): 2925, 2863, 1520, 1452, 1264, 1030, 773, 748, 693, $673 \mathrm{~cm}^{-1}$. HRMS (ESI) $\mathrm{m} / z$ calculated for $\mathrm{C}_{19} \mathrm{H}_{20} \mathrm{~N}$ $(\mathrm{M}+\mathrm{H})^{+}: 262.1596$, found: 262.1596 .

10a-Phenyl-6,7,8,9,10,10a-hexahydrocyclohepta[b]indole 17q. ${ }^{18}$ Purification by MPLC (2:98 to 10:90 EtOAc:hexanes) afforded $17 \mathbf{q}$ as a yellow solid $(0.016 \mathrm{~g}, 62 \%)$. The spectral data matched that reported by Driver and co-workers: $:^{18} \mathrm{mp}=$ $98^{\circ} \mathrm{C} ;{ }^{1} \mathrm{H}$ NMR $\left(500 \mathrm{MHz}, \mathrm{CDCl}_{3}\right) \delta 7.50(\mathrm{~d}, J=7.7 \mathrm{~Hz}, 1 \mathrm{H}), 7.28-7.15(\mathrm{~m}, 4 \mathrm{H}), 7.15-7.11(\mathrm{~m}, 2 \mathrm{H}), 7.06(\mathrm{~d}, J=4.3$ $\mathrm{Hz}, 2 \mathrm{H}), 2.99$ (ddd, $J=15.0,7.7,4.2 \mathrm{~Hz}, 1 \mathrm{H}), 2.70(\mathrm{ddd}, J=15.0,9.1,4.0 \mathrm{~Hz}, 1 \mathrm{H}), 2.50(\mathrm{ddd}, J=14.3,8.3,2.0 \mathrm{~Hz}, 1 \mathrm{H})$, 2.12 (ddd, $J=14.4,10.1,2.1 \mathrm{~Hz}, 1 \mathrm{H}), 1.79-1.69(\mathrm{~m}, 2 \mathrm{H}), 1.69-1.57(\mathrm{~m}, 2 \mathrm{H}), 1.57-1.47(\mathrm{~m}, 1 \mathrm{H}), 1.12$ (dddd, $J=14.8$, 11.1, 7.4, 4.4 Hz, 1H); ${ }^{13} \mathrm{C}$ NMR (125 MHz, $\left.\mathrm{CDCl}_{3}\right) \delta 190.4(\mathrm{C}), 153.9(\mathrm{C}), 146.4(\mathrm{C}), 140.1(\mathrm{C}), 129.1(\mathrm{CH}), 127.7(\mathrm{CH})$, 127.0 (CH), $125.8(\mathrm{CH}), 125.6(\mathrm{CH}), 122.0(\mathrm{CH}), 120.0(\mathrm{CH}), 66.5(\mathrm{C}), 34.4\left(\mathrm{CH}_{2}\right), 32.7\left(\mathrm{CH}_{2}\right), 29.9\left(\mathrm{CH}_{2}\right), 27.1\left(\mathrm{CH}_{2}\right)$, $25.1\left(\mathrm{CH}_{2}\right)$. ATR-FTIR (thin film): 2927, 2854, 1572, 1558, 1494, 1456, 1345, 765, 752, 743, $700 \mathrm{~cm}^{-1}$. HRMS (ESI) $\mathrm{m} / z$ calculated for $\mathrm{C}_{19} \mathrm{H}_{20} \mathrm{~N}(\mathrm{M}+\mathrm{H})^{+}: 262.1596$, found: 262.1595 .
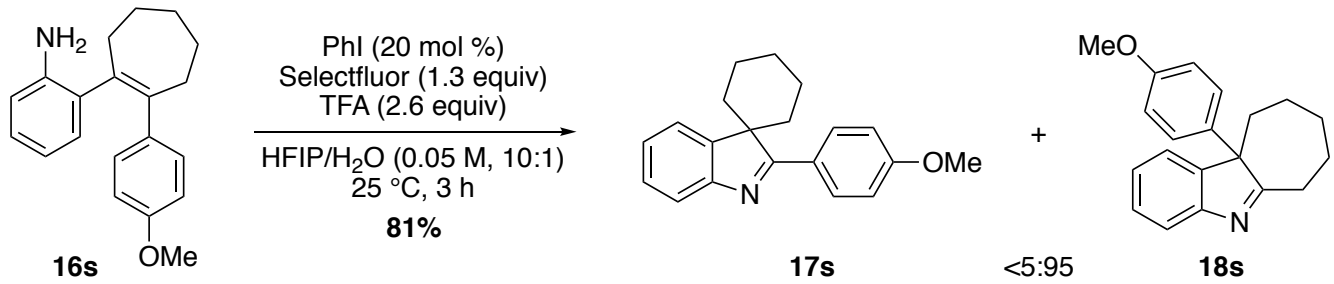

(s23)

10a-(4-Methoxyphenyl)-6,7,8,9,10,10a-hexahydrocyclohepta[b]indole $18 \mathrm{~s} .{ }^{18}$ The optimized procedure was followed using $0.029 \mathrm{~g}$ of aniline $16 \mathrm{~s}(0.10 \mathrm{mmol}), 2.3 \mu \mathrm{L}$ of iodobenzene $(0.020 \mathrm{mmol}), 20 \mu \mathrm{L}$ of trifluoroacetic acid $(0.26 \mathrm{mmol})$, $0.046 \mathrm{~g}$ of Selectfluor $(0.13 \mathrm{mmol})$ in $2 \mathrm{~mL}$ of 1,1,1,3,3,3-hexafluoropropan-2-ol and $0.2 \mathrm{~mL}$ of water $(0.05 \mathrm{M}) .{ }^{1} \mathrm{H} \mathrm{NMR}$ revealed only the formation of 18s. Purification by MPLC (2:98 to 10:90 EtOAc:hexanes) afforded 18s as a green oil (0.0024 g, 81\%). The spectral data matched that reported by Driver and co-workers: ${ }^{18}{ }^{1} \mathrm{H}$ NMR $\left(500 \mathrm{MHz}, \mathrm{CDCl}_{3}\right) \delta 7.52(\mathrm{~d}, J=$ $7.7 \mathrm{~Hz}, 1 \mathrm{H}), 7.24(\mathrm{td}, J=7.3,1.9 \mathrm{~Hz}, 1 \mathrm{H}), 7.10-7.04(\mathrm{~m}, 4 \mathrm{H}), 6.82(\mathrm{~d}, J=2.3 \mathrm{~Hz}, 1 \mathrm{H}), 6.81(\mathrm{~d}, J=2.0 \mathrm{~Hz}, 1 \mathrm{H}), 3.75(\mathrm{~s}$, $3 \mathrm{H}), 3.00(\mathrm{ddd}, J=14.8,7.7,4.1 \mathrm{~Hz}, 1 \mathrm{H}), 2.71(\mathrm{ddd}, J=14.8,9.2,4.0 \mathrm{~Hz}, 1 \mathrm{H}), 2.49$ (ddd, $J=14.3,8.3,2.0 \mathrm{~Hz}, 1 \mathrm{H}), 2.12$ (ddd, $J=14.4,10.1,2.1 \mathrm{~Hz}, 1 \mathrm{H}), 1.77(\mathrm{qt}, J=10.2,3.5 \mathrm{~Hz}, 2 \mathrm{H}), 1.72-1.60(\mathrm{~m}, 2 \mathrm{H}), 1.57(\mathrm{td}, J=11.1,9.3,3.3 \mathrm{~Hz}, 1 \mathrm{H})$, $1.13(\mathrm{dtd}, J=14.7,8.4,2.1 \mathrm{~Hz}, 1 \mathrm{H}) ;{ }^{13} \mathrm{C} \mathrm{NMR}\left(125 \mathrm{MHz}, \mathrm{CDCl}_{3}\right) \delta 190.6(\mathrm{C}), 158.5(\mathrm{C}), 153.8(\mathrm{C}), 146.8(\mathrm{C}), 131.9(\mathrm{C})$, 127.6 (CH), $126.8(\mathrm{CH}), 125.6(\mathrm{CH}), 121.9(\mathrm{CH}), 119.9(\mathrm{CH}), 114.4(\mathrm{CH}), 65.8(\mathrm{C}), 55.2\left(\mathrm{CH}_{3}\right), 34.5\left(\mathrm{CH}_{2}\right), 32.6\left(\mathrm{CH}_{2}\right)$, $29.9\left(\mathrm{CH}_{2}\right), 27.1\left(\mathrm{CH}_{2}\right), 25.1\left(\mathrm{CH}_{2}\right)$. ATR-FTIR (thin film): 2926, 2853, 1692, 1608, 1510, 1456, 1250, 1182, 1033, 795, $766,747 \mathrm{~cm}^{-1}$. HRMS (ESI) $\mathrm{m} / z$ calculated for $\mathrm{C}_{20} \mathrm{H}_{22} \mathrm{NO}(\mathrm{M}+\mathrm{H})^{+}: 292.1701$, found: 292.1698 .

24. Lackner, A. D.; Samant, A. V.; Toste, F. D. J. Am. Chem. Soc. 2013, 135, 14090. 

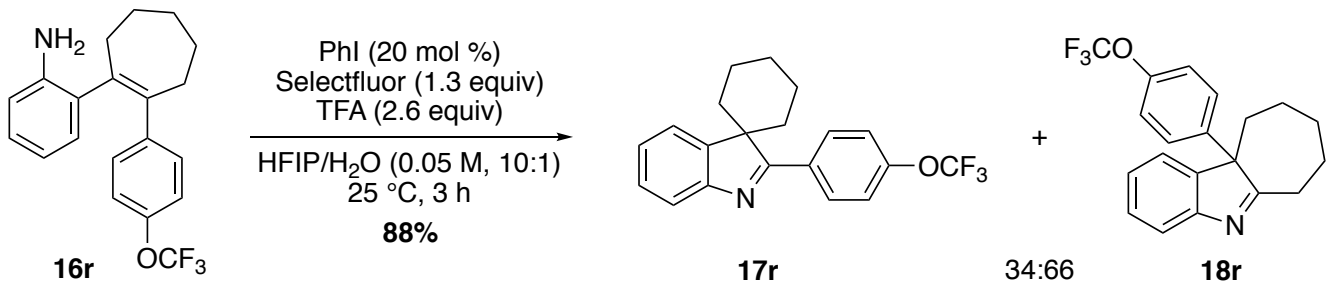

(s24)

2'-(4-(Trifluoromethoxy)phenyl)spiro[cyclohexane-1,3'-indole] $17 \mathbf{r}^{18}{ }^{18}$ The optimized procedure was followed using $0.035 \mathrm{~g}$ of aniline $16 \mathrm{r}(0.10 \mathrm{mmol}), 2.3 \mu \mathrm{L}$ of iodobenzene $(0.020 \mathrm{mmol}), 20 \mu \mathrm{L}$ of trifluoroacetic acid $(0.26 \mathrm{mmol}), 0.046$ $\mathrm{g}$ of Selectfluor $(0.13 \mathrm{mmol})$ in $2 \mathrm{~mL}$ of 1,1,1,3,3,3-hexafluoropropan-2-ol and $0.2 \mathrm{~mL}$ of water $(0.05 \mathrm{M})$. Analysis of the reaction mixture using ${ }^{1} \mathrm{H}$ NMR revealed a $66: 34$ mixture of 18r and 17r. Purification by MPLC (2:98 to 5:95 EtOAc:hexanes) afforded 17r as a yellow oil $(0.010 \mathrm{~g}, 30 \%)$. The spectral data matched that reported by Driver and coworkers: ${ }^{18}{ }^{1} \mathrm{H}$ NMR $\left(500 \mathrm{MHz}, \mathrm{CDCl}_{3}\right) \delta 8.14(\mathrm{~d}, J=8.5 \mathrm{~Hz}, 2 \mathrm{H}), 7.84(\mathrm{~d}, J=7.5 \mathrm{~Hz}, 1 \mathrm{H}), 7.74(\mathrm{~d}, J=7.7 \mathrm{~Hz}, 1 \mathrm{H}), 7.42$ $(\mathrm{t}, J=7.6 \mathrm{~Hz}, 1 \mathrm{H}), 7.33(\mathrm{~d}, J=8.3 \mathrm{~Hz}, 2 \mathrm{H}), 7.25(\mathrm{t}, J=7.5 \mathrm{~Hz}, 1 \mathrm{H}), 2.25(\mathrm{td}, J=13.3,4.4 \mathrm{~Hz}, 2 \mathrm{H}), 2.03(\mathrm{dtt}, J=13.5,9.9$, $3.5 \mathrm{~Hz}, 3 \mathrm{H}), 1.85(\mathrm{dt}, J=14.2,3.9 \mathrm{~Hz}, 2 \mathrm{H}), 1.53(\mathrm{dtd}, J=18.0,13.4,11.8,6.6 \mathrm{~Hz}, 1 \mathrm{H}), 1.44(\mathrm{~d}, J=13.3 \mathrm{~Hz}, 2 \mathrm{H}) ;{ }^{13} \mathrm{C} \mathrm{NMR}$ $\left(125 \mathrm{MHz}, \mathrm{CDCl}_{3}\right) \delta 182.2(\mathrm{C}), 153.6(\mathrm{C}), 150.4(\mathrm{C}), 146.1(\mathrm{C}), 132.5(\mathrm{C}), 130.3(\mathrm{CH}), 127.8(\mathrm{CH}), 125.2(\mathrm{CH}), 124.4$ $(\mathrm{CH}), 121.5(\mathrm{CH}), 120.5\left(\mathrm{q}, J_{C F}=256 \mathrm{~Hz}, \mathrm{C}\right), 120.6(\mathrm{CH}), 58.4(\mathrm{C}), 31.2\left(\mathrm{CH}_{2}\right), 25.2\left(\mathrm{CH}_{2}\right), 21.7\left(\mathrm{CH}_{2}\right)$. ATR-FTIR $($ thin film): 2935, 2866, 1506, 1467, 1255, 1211, 1166, 1018, 851, 774, $751 \mathrm{~cm}^{-1}$. HRMS (ESI) $\mathrm{m} / z$ calculated for $\mathrm{C}_{20} \mathrm{H}_{19} \mathrm{NOF}_{3}$ $(\mathrm{M}+\mathrm{H})^{+}: 346.1419$, found: 346.1416 .

10a-(4-(Trifluoromethoxy)phenyl)-6,7,8,9,10,10a-hexahydrocyclohepta[b]indole $18{ }^{18}{ }^{18}$ Purification by MPLC (2:98 to 5:95 EtOAc:hexanes) afforded $18 \mathrm{r}$ as a green oil $(0.020 \mathrm{~g}, 58 \%)$. The spectral data matched that reported by Driver and coworkers: ${ }^{18}{ }^{1} \mathrm{H}$ NMR $\left(500 \mathrm{MHz}, \mathrm{CDCl}_{3}\right) \delta 7.54(\mathrm{~d}, J=7.7 \mathrm{~Hz}, 1 \mathrm{H}), 7.27(\mathrm{td}, J=7.5,1.4 \mathrm{~Hz}, 1 \mathrm{H}), 7.20-7.15(\mathrm{~m}, 2 \mathrm{H}), 7.14$ $-7.08(\mathrm{~m}, 3 \mathrm{H}), 7.06(\mathrm{dd}, J=7.5,1.3 \mathrm{~Hz}, 1 \mathrm{H}), 3.02(\mathrm{ddd}, J=14.7,7.5,4.1 \mathrm{~Hz}, 1 \mathrm{H}), 2.68(\mathrm{ddd}, J=14.8,9.2,3.9 \mathrm{~Hz}, 1 \mathrm{H})$, $2.48($ ddd, $J=14.3,8.5,2.0 \mathrm{~Hz}, 1 \mathrm{H}), 2.19$ (ddd, $J=14.3,9.9,2.1 \mathrm{~Hz}, 1 \mathrm{H}), 1.76$ (tdd, $J=9.7,7.4,4.4 \mathrm{~Hz}, 2 \mathrm{H}), 1.68$ (dddd, $J=19.4,8.5,6.7,2.8 \mathrm{~Hz}, 2 \mathrm{H}), 1.60-1.50(\mathrm{~m}, 1 \mathrm{H}), 1.12(\mathrm{dtd}, J=15.0,8.7,2.1 \mathrm{~Hz}, 1 \mathrm{H}) ;{ }^{13} \mathrm{C} \mathrm{NMR}\left(125 \mathrm{MHz}, \mathrm{CDCl}_{3}\right) \delta$ $189.8(\mathrm{C}), 153.9(\mathrm{C}), 148.2(\mathrm{C}), 145.9(\mathrm{C}), 138.8(\mathrm{C}), 128.0(\mathrm{CH}), 127.2(\mathrm{CH}), 125.8(\mathrm{CH}), 122.0(\mathrm{CH}), 121.5(\mathrm{CH}), 120.4$ $\left(\mathrm{q}, J_{C F}=255 \mathrm{~Hz}, \mathrm{C}\right), 120.2(\mathrm{CH}), 65.9(\mathrm{C}), 34.4\left(\mathrm{CH}_{2}\right), 32.5\left(\mathrm{CH}_{2}\right), 29.9\left(\mathrm{CH}_{2}\right), 27.3\left(\mathrm{CH}_{2}\right), 24.9\left(\mathrm{CH}_{2}\right)$. ATR-FTIR (thin film): 2930, 2856, 1696, 1507, 1456, 1258, 1211, 1166, 1018, 767, 748, $668 \mathrm{~cm}^{-1}$. HRMS (ESI) $\mathrm{m} / z$ calculated for $\mathrm{C}_{20} \mathrm{H}_{19} \mathrm{NOF}_{3}(\mathrm{M}+\mathrm{H})^{+}: 346.1419$, found: 346.1415 .

\section{Diastereoselective experiments.}

\section{A. Screening of reaction conditions}

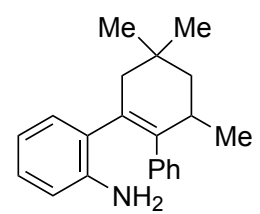

$16 t$

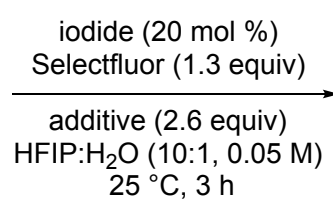

$25{ }^{\circ} \mathrm{C}, 3 \mathrm{~h}$

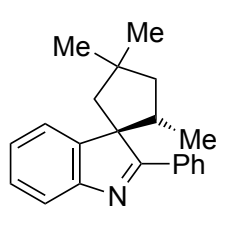

$17 \mathrm{t}$ (s25)

To a solution of aniline $\mathbf{1 6 t}$ (1.0 equiv), RI (20 mol \%), and an additive in $2 \mathrm{~mL}$ of solvent, was added the oxidant as a single portion. After $3 \mathrm{~h}$, the reactives were quenched by the additional of $3 \mathrm{~mL}$ of a saturated aq soln of $\mathrm{Na}_{2} \mathrm{~S}_{2} \mathrm{O}_{3}$ and $3 \mathrm{~mL}$ of saturated aq soln of $\mathrm{NaHCO}_{3}$. The resulting mixture was extracted with $3 \times 5 \mathrm{~mL}$ of EtOAc. The combined organic phases were washed with $10 \mathrm{~mL}$ of brine. The resulting organic phase was dried over $\mathrm{Na}_{2} \mathrm{SO}_{4}$ and filtered through Celite. The filtrate was concentrated under reduced pressure, and the residue was analyzed using ${ }^{1} \mathrm{H}$ NMR spectroscopy $\operatorname{using} \mathrm{CH}_{2} \mathrm{Br}_{2}$ as the internal standard. 
Table s2. Correlation of diastereoselectivity with catalyst structure.

\begin{tabular}{|c|c|c|c|c|}
\hline entry & $\mathrm{RI}$ & additive & yield, $\%{ }^{a}$ & d.r. ${ }^{a}$ \\
\hline 1 & $\mathrm{R}=\mathrm{H}$ & TFA & 62 & $79: 21$ \\
\hline 2 & $\mathrm{R}=\mathrm{Me}$ & TFA & 64 & $72: 28$ \\
\hline 3 & $\mathrm{R}=\mathrm{iPr}$ & TFA & 65 & $67: 33$ \\
\hline 4 & & TFA & 68 & $48: 52$ \\
\hline 5 & $\mathrm{R}=\mathrm{OMe}$ & & 61 & $81: 19$ \\
\hline 6 & $\mathrm{R}=\mathrm{CO}_{2} \mathrm{Me}$ & TFA & 39 & $76: 24$ \\
\hline 7 & & TFA & 20 & $76: 24$ \\
\hline 8 & $n$-BuI & TFA & 40 & $80: 20$ \\
\hline 9 & 2-iodopropane & TFA & 32 & $81: 19$ \\
\hline 10 & $\mathrm{MeI}$ & TFA & 20 & $82: 18$ \\
\hline 11 & $\mathrm{PhI}$ & $p$-TsOH & 31 & $73: 27$ \\
\hline 12 & $\mathrm{PhI}$ & TMSTFA & 71 & $81: 19$ \\
\hline 13 & $n$-BuI & TMSTFA & 57 & $81: 19$ \\
\hline 14 & MeI & TMSTFA & 61 & $84: 16$ \\
\hline $15^{\mathrm{b}}$ & & TMSTFA & 49 & $84: 16$ \\
\hline $16^{\mathrm{b}}$ & MeI & TMSTFA & 51 & $88: 12$ \\
\hline $17^{\mathrm{b}, \mathrm{c}}$ & MeI & TMSTFA & 33 & $64: 36$ \\
\hline 18 & none & none & 20 & $64: 36$ \\
\hline
\end{tabular}

\section{B. Characterization data for $3 H$-indole $17 t$.}

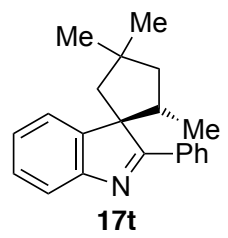

2,4,4-Trimethyl-2'-phenylspiro[cyclopentane-1,3'-indole] 17t. The original optimized procedure was followed using $0.029 \mathrm{~g}$ of aniline $16 \mathrm{t}(0.10 \mathrm{mmol}), 2.3 \mu \mathrm{L}$ of iodobenzene $(0.020 \mathrm{mmol}), 20 \mu \mathrm{L}$ of trifluoroacetic acid $(0.26 \mathrm{mmol}), 0.046$ $\mathrm{g}$ of Selectfluor $(0.13 \mathrm{mmol})$ in $2 \mathrm{~mL}$ of 1,1,1,3,3,3-hexafluoropropan-2-ol and $0.2 \mathrm{~mL}$ of water $(0.05 \mathrm{M})$. Purification by MPLC (2:98 to 5:95 EtOAc:hexanes) afforded the product, a yellow oil, as a 3.7:1 diastereomers (0.018 g, 62\%): Diagnostic peaks for the major diastereomer: ${ }^{1} \mathrm{H} \mathrm{NMR}\left(500 \mathrm{MHz}, \mathrm{CDCl}_{3}\right) \delta 8.02-7.98(\mathrm{~m}, 1.50 \mathrm{H}), 7.63(\mathrm{~d}, J=7.5 \mathrm{~Hz}, 0.76 \mathrm{H}), 7.51$ $-7.40(\mathrm{~m}, 3.96 \mathrm{H}), 7.37-7.30(\mathrm{~m}, 1.01 \mathrm{H}), 7.30-7.23(\mathrm{~m}, 1.16 \mathrm{H}), 2.91(\mathrm{dt}, J=13.5,6.8 \mathrm{~Hz}, 0.75 \mathrm{H}), 2.71(\mathrm{~d}, J=15.0 \mathrm{~Hz}$, $0.83 \mathrm{H}), 2.04-1.86(\mathrm{~m}, 2.08 \mathrm{H}), 1.72(\mathrm{dd}, J=12.9,6.3 \mathrm{~Hz}, 0.91 \mathrm{H}), 1.40(\mathrm{~s}, 2.35 \mathrm{H}), 1.28(\mathrm{~s}, 2.40 \mathrm{H}), 0.60(\mathrm{~d}, J=7.0 \mathrm{~Hz}$, 2.36H); ${ }^{13} \mathrm{C}$ NMR $\left(125 \mathrm{MHz}, \mathrm{CDCl}_{3}\right) \delta 183.5(\mathrm{C}), 153.5(\mathrm{C}), 147.8(\mathrm{C}), 136.9(\mathrm{C}), 129.9(\mathrm{CH}), 128.8(\mathrm{CH}), 128.3(\mathrm{CH})$, $127.4(\mathrm{CH}), 126.2(\mathrm{CH}), 121.2(\mathrm{CH}), 120.4(\mathrm{CH}) 69.7(\mathrm{C}), 49.5\left(\mathrm{CH}_{2}\right), 48.5\left(\mathrm{CH}_{2}\right), 45.9(\mathrm{CH}), 39.3(\mathrm{C}), 31.4\left(\mathrm{CH}_{3}\right), 29.5$ $\left(\mathrm{CH}_{3}\right), 13.8\left(\mathrm{CH}_{3}\right)$. Diagnostic peaks for the minor diastereomer: ${ }^{1} \mathrm{H}$ NMR $\left(500 \mathrm{MHz}, \mathrm{CDCl}_{3}\right) \delta 8.05-8.02(\mathrm{~m}, 0.44 \mathrm{H})$, 
$7.67(\mathrm{~d}, J=7.6 \mathrm{~Hz}, 0.24 \mathrm{H}), 2.98(\mathrm{dt}, J=13.3,6.6 \mathrm{~Hz}, 0.21 \mathrm{H}), 2.63(\mathrm{~d}, J=14.7 \mathrm{~Hz}, 0.23 \mathrm{H}), 1.82(\mathrm{dd}, J=12.8,6.1 \mathrm{~Hz}$, $0.25 \mathrm{H}), 1.35(\mathrm{~s}, 0.66 \mathrm{H}), 1.31(\mathrm{~s}, 0.70 \mathrm{H}), 0.36(\mathrm{~d}, J=6.6 \mathrm{~Hz}, 0.63 \mathrm{H}) ;{ }^{13} \mathrm{C} \mathrm{NMR}\left(125 \mathrm{MHz}, \mathrm{CDCl}_{3}\right) \delta 1130.1(\mathrm{CH}), 128.4$ $(\mathrm{CH}), 128.2(\mathrm{CH}), 127.45(\mathrm{CH}), 125.4(\mathrm{CH}), 123.0(\mathrm{CH}), 120.9(\mathrm{CH}), 70.3(\mathrm{C}), 49.4\left(\mathrm{CH}_{2}\right), 48.1\left(\mathrm{CH}_{2}\right), 42.6(\mathrm{CH}), 38.6$ (C), $32.1\left(\mathrm{CH}_{3}\right), 31.1\left(\mathrm{CH}_{3}\right), 13.2\left(\mathrm{CH}_{3}\right)$. ATR-FTIR (thin film): 2955, 2931, 2888, 1464, 1456, 770, 750, $694 \mathrm{~cm}^{-1}$. HRMS (ESI) $m / z$ calculated for $\mathrm{C}_{21} \mathrm{H}_{24} \mathrm{~N}(\mathrm{M}+\mathrm{H})^{+}: 290.1909$, found: 290.1909 .

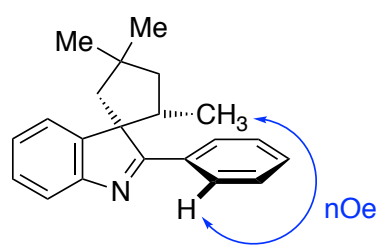

The identity of the major diastereomer and connectivity was determined using nOe.

\section{Spectral Data}

A. Spectral data for boronic acid pinacol ester s1p $s-45$

B. Spectral data for ortho-alkenyl substituted anilines 11 and 16.....................................46

C. Spectral data for $3 H$-indoles. $s-114$ 
$500 \mathrm{MHz}, \mathrm{CDCl}_{3}$

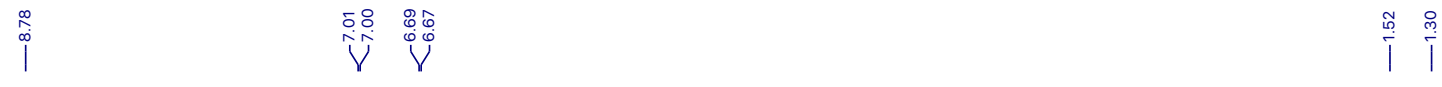

$$
{ }_{\mathrm{SHBoc}}^{\mathrm{Bpin}}
$$

s1p

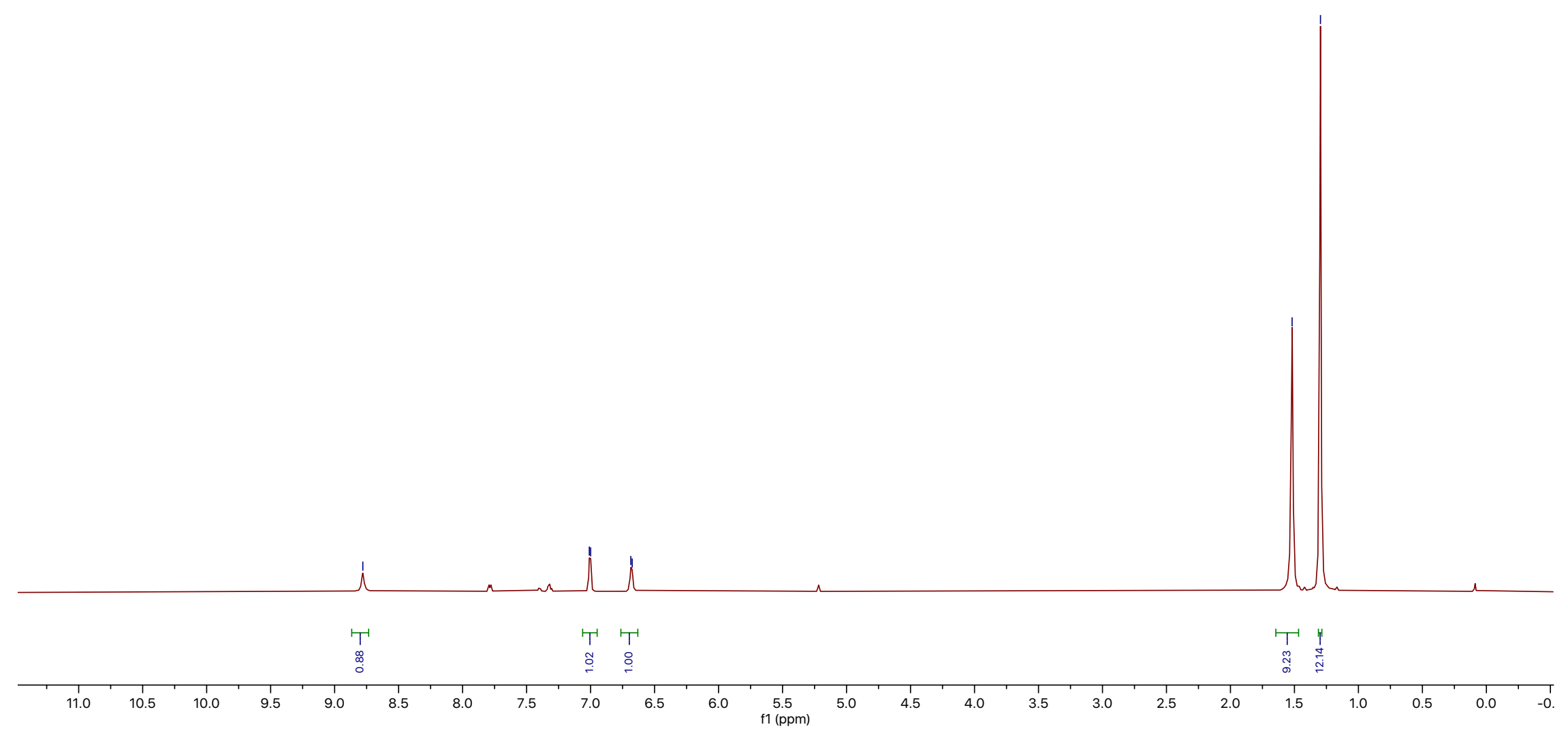


$500 \mathrm{MHz}, \mathrm{CDCl}_{3}$
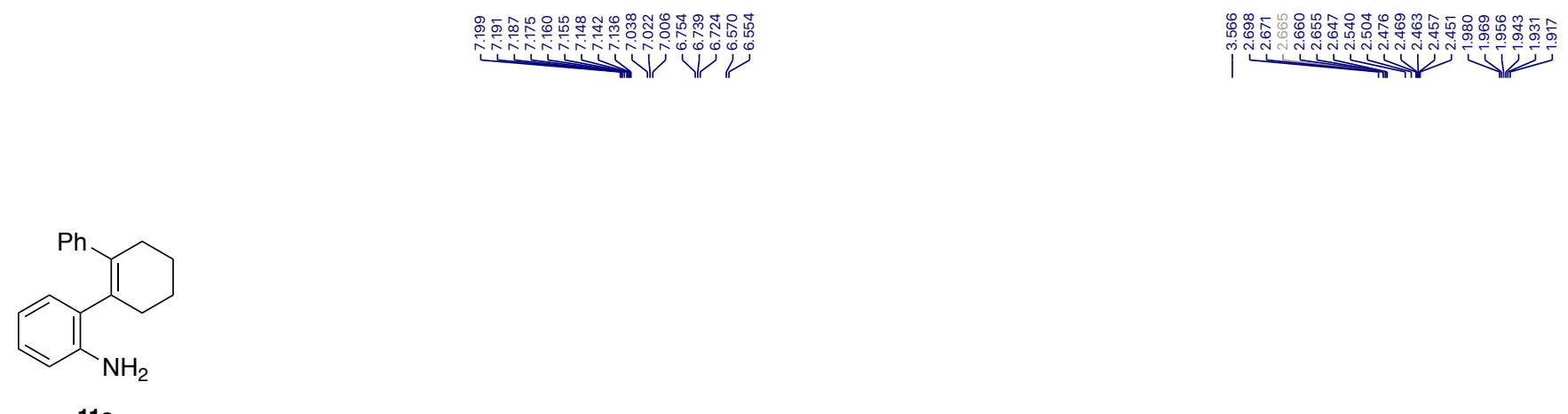

11a

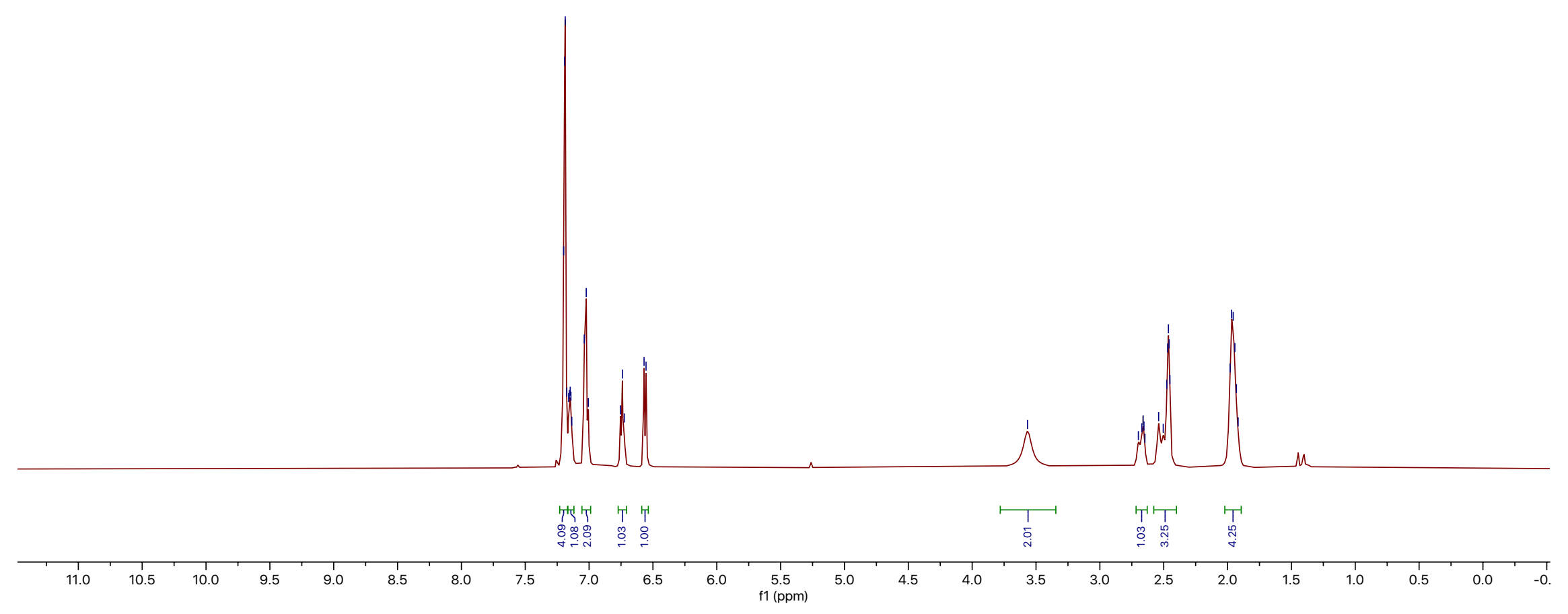


$125 \mathrm{MHz}, \mathrm{CDCl}_{3}$

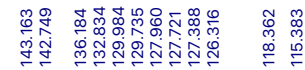

$\underbrace{2}$

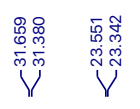

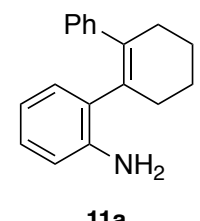

$11 a$

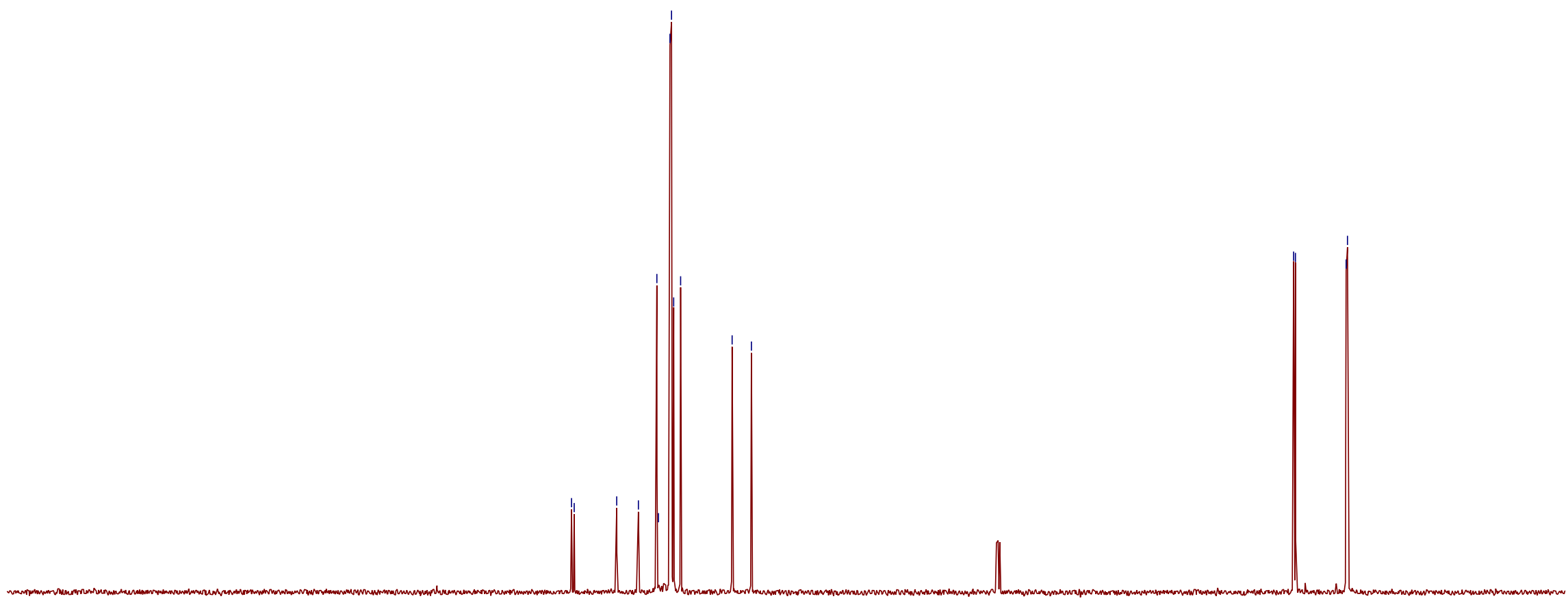

30

220

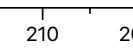

$190 \quad 180$
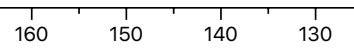

$120 \quad 110$
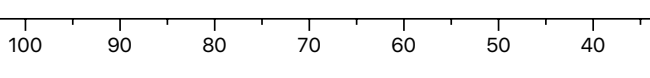
$500 \mathrm{MHz}, \mathrm{CDCl}_{3}$

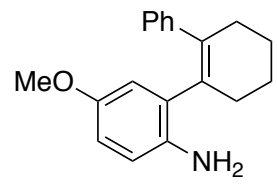

11b

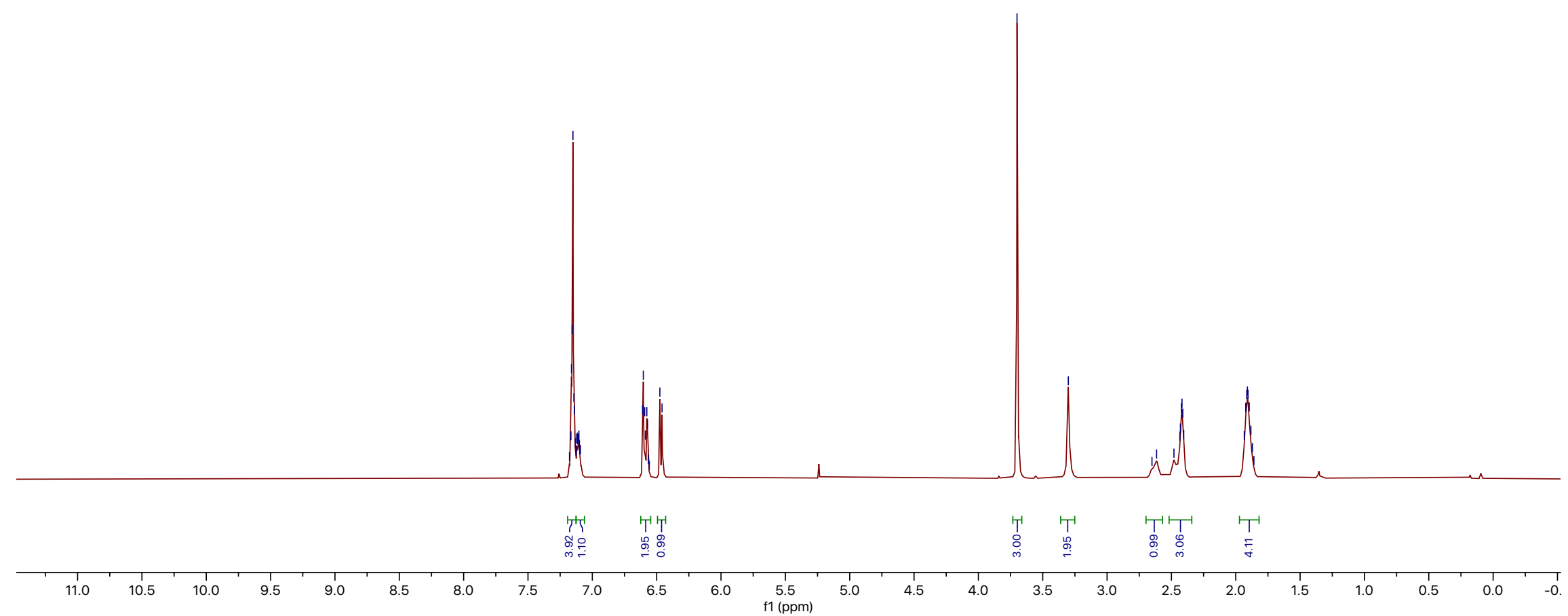


$125 \mathrm{MHz}, \mathrm{CDCl}_{3}$

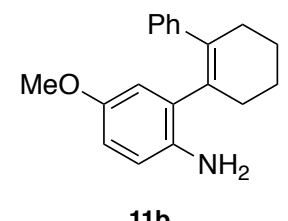

11b

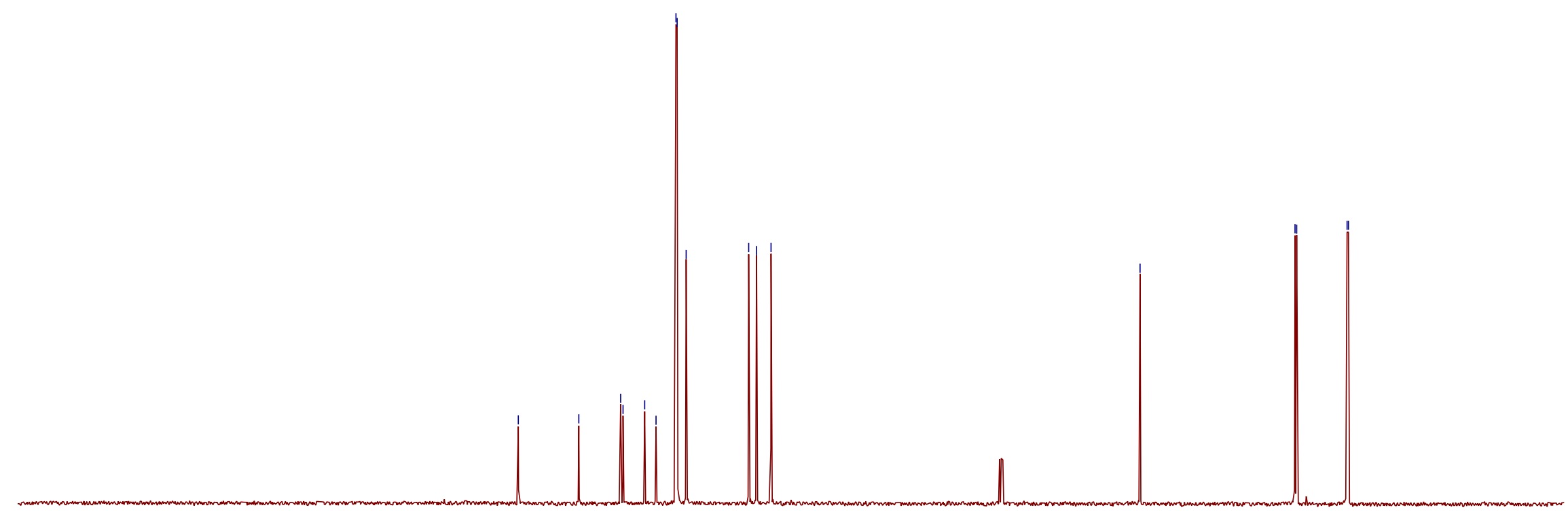

30

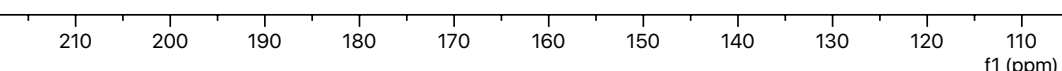
f1 (ppm)
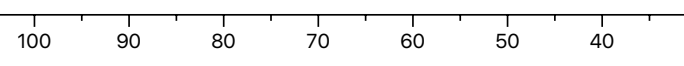
$500 \mathrm{MHz}, \mathrm{CDCl}_{3}$
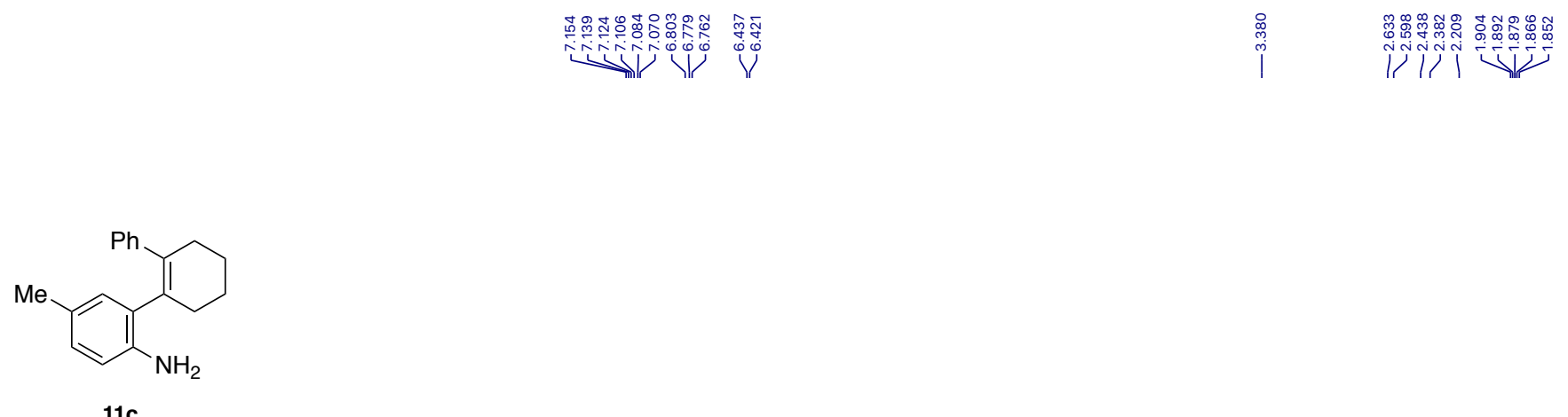

$1 \mathrm{c}$

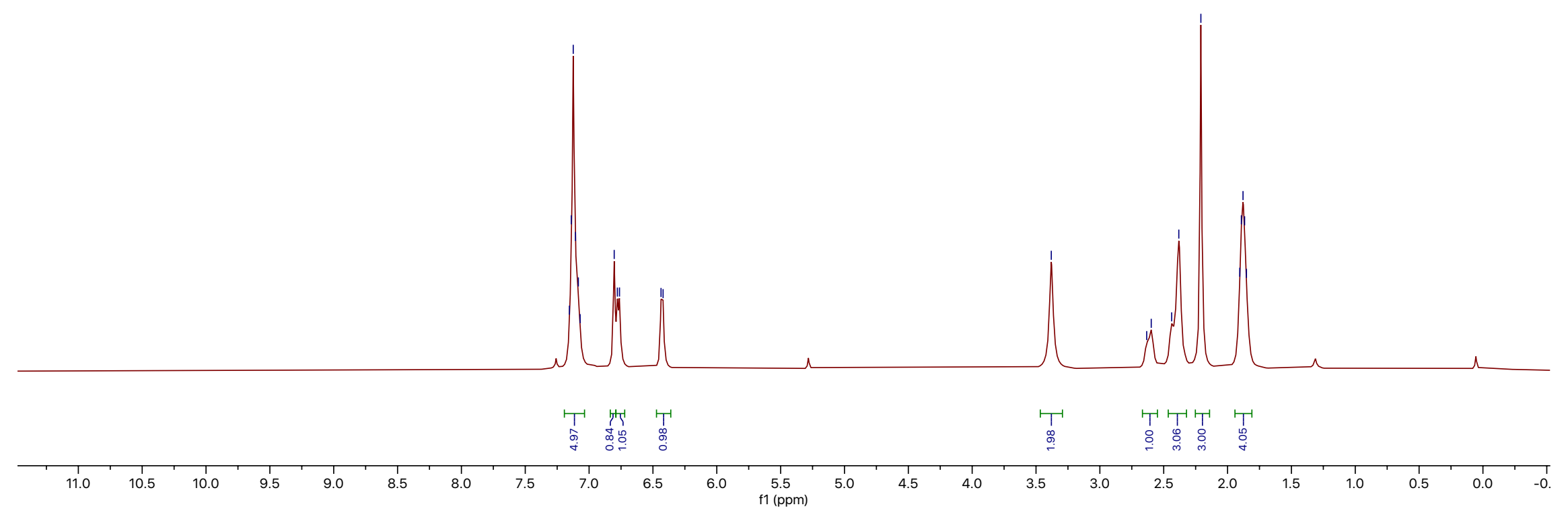


$125 \mathrm{MHz}, \mathrm{CDCl}_{3}$
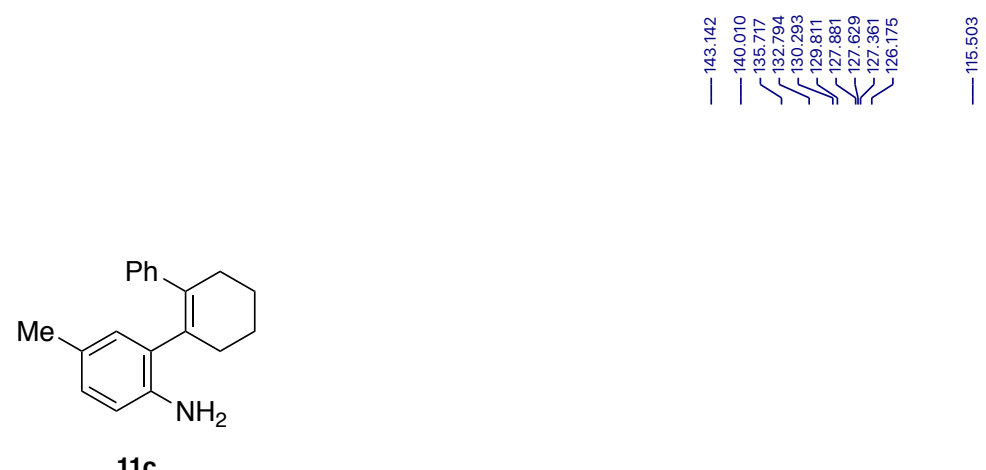

$11 \mathrm{c}$
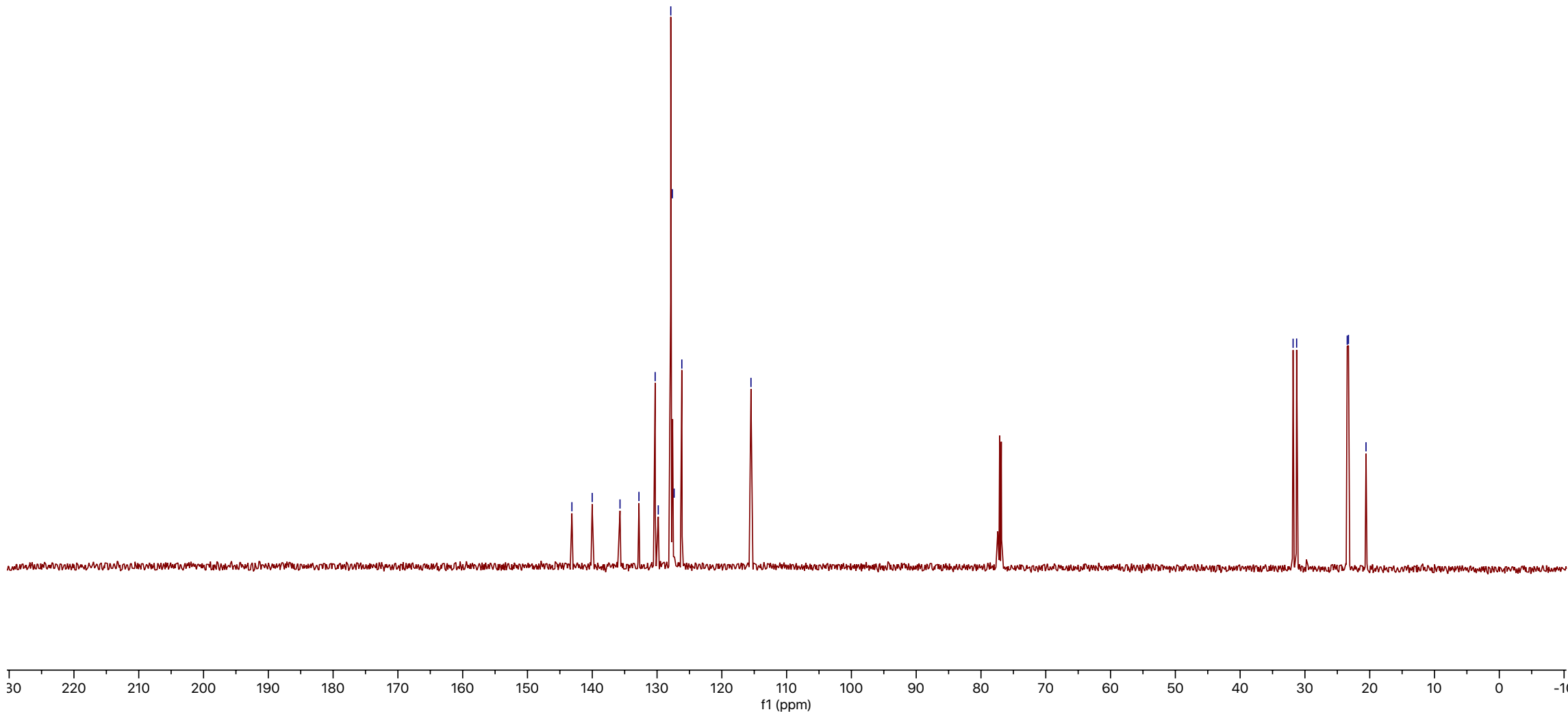
$500 \mathrm{MHz}, \mathrm{CDCl}_{3}$
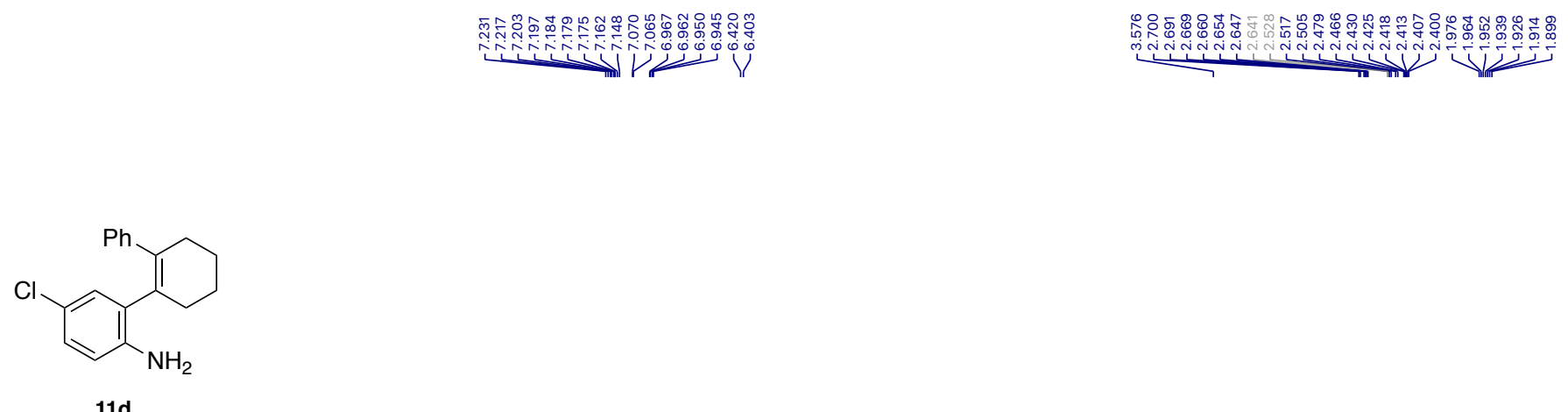

11d

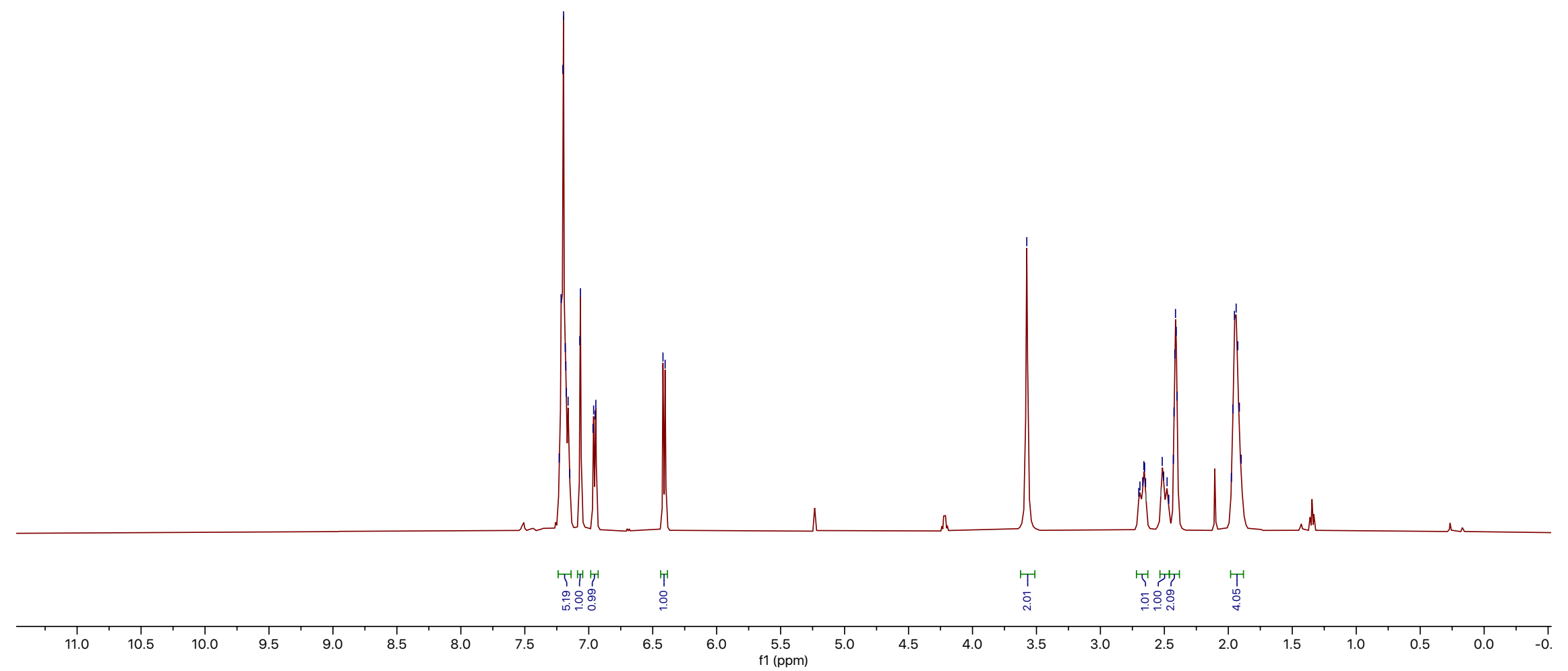


$125 \mathrm{MHz}, \mathrm{CDCl}_{3}$

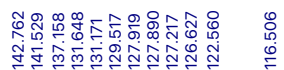

11

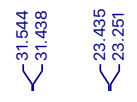

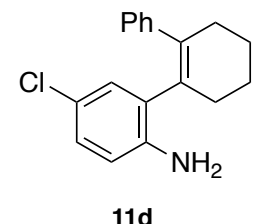

11d
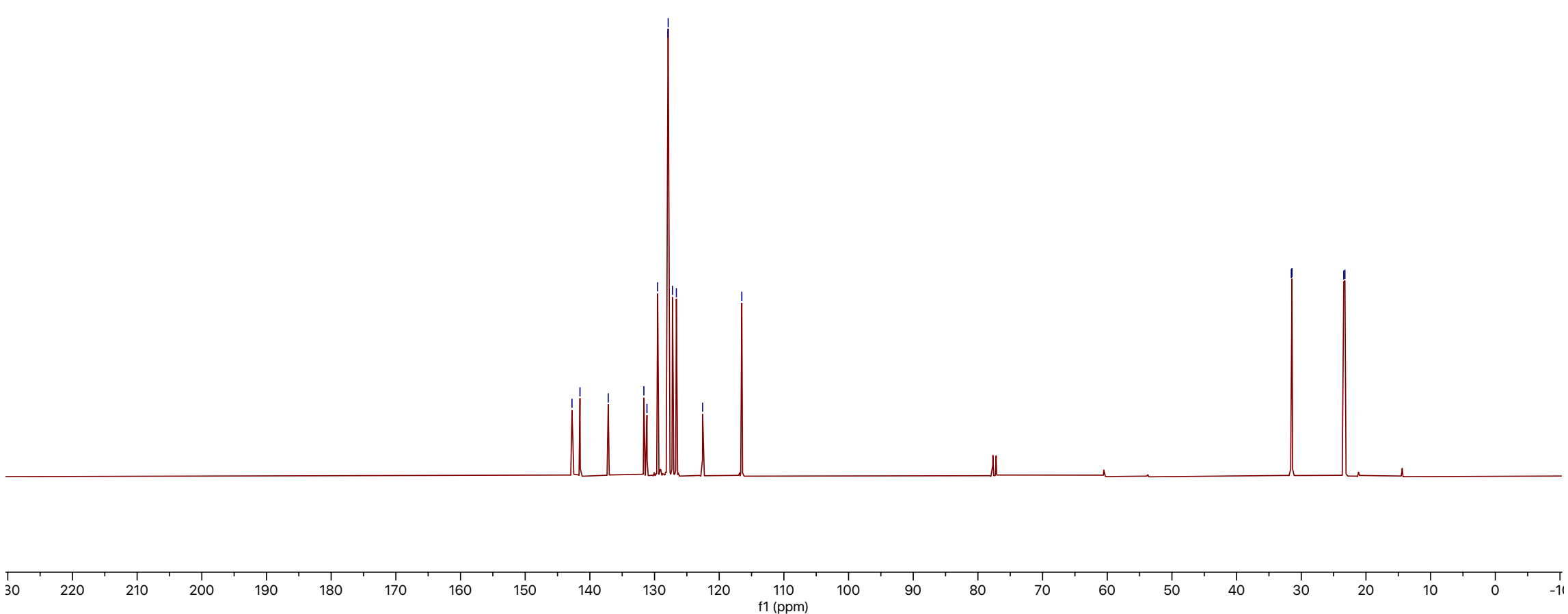
$500 \mathrm{MHz}, \mathrm{CDCl}_{3}$

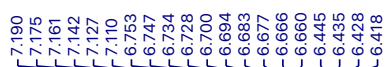

䒹

年

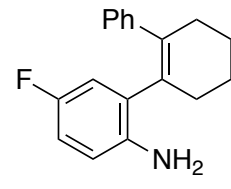

$11 \mathrm{e}$

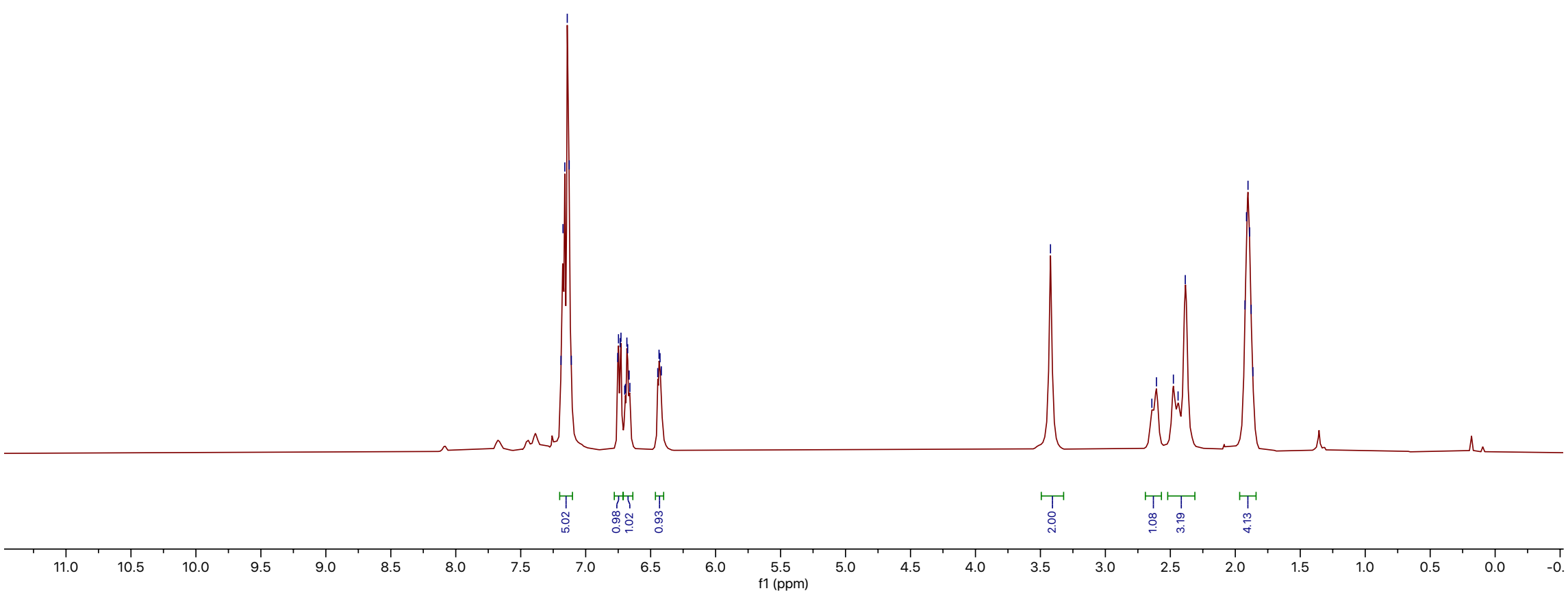


$125 \mathrm{MHz}, \mathrm{CDCl}_{3}$

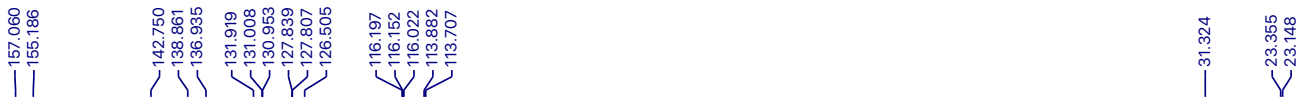

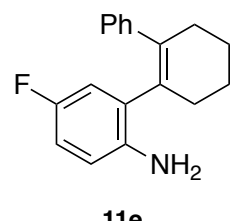

11e

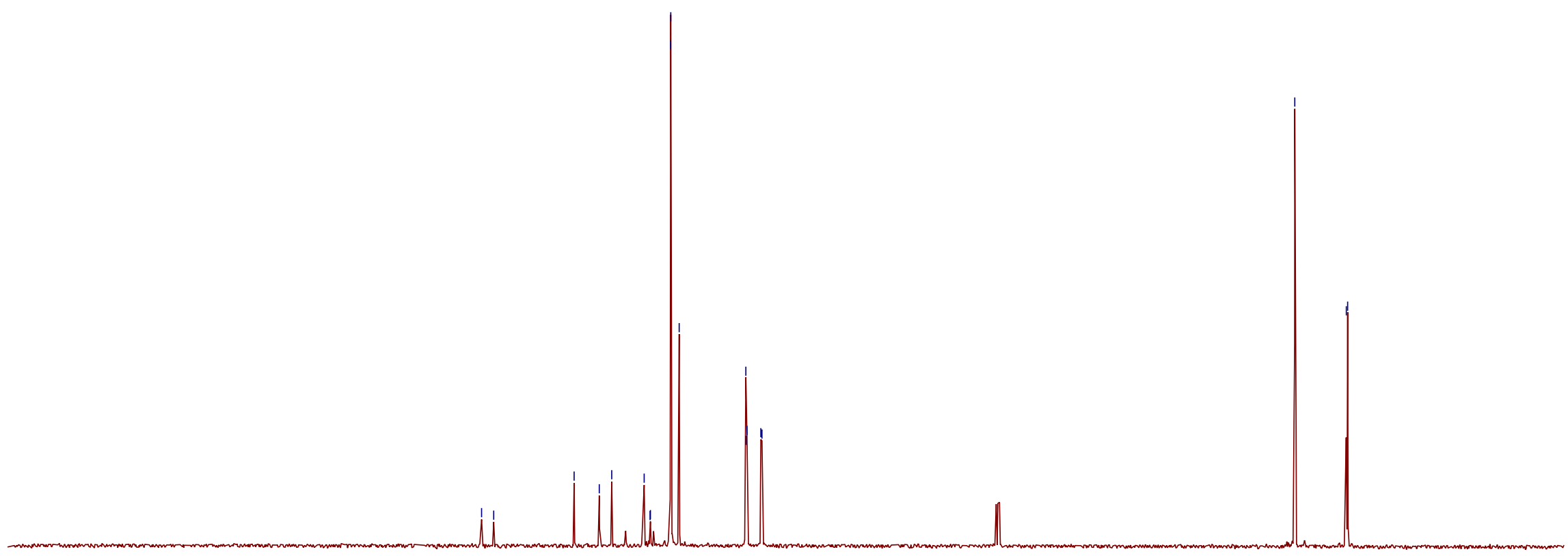

30

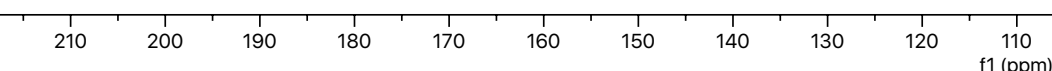
110
$\mathrm{f} 1(\mathrm{ppm})$
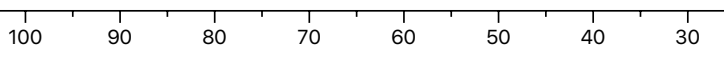
$500 \mathrm{MHz}, \mathrm{CDCl}_{3}$

|

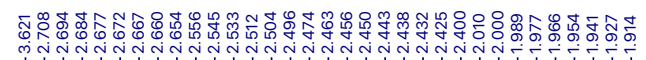

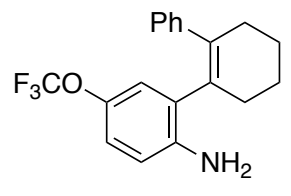

$11 f$

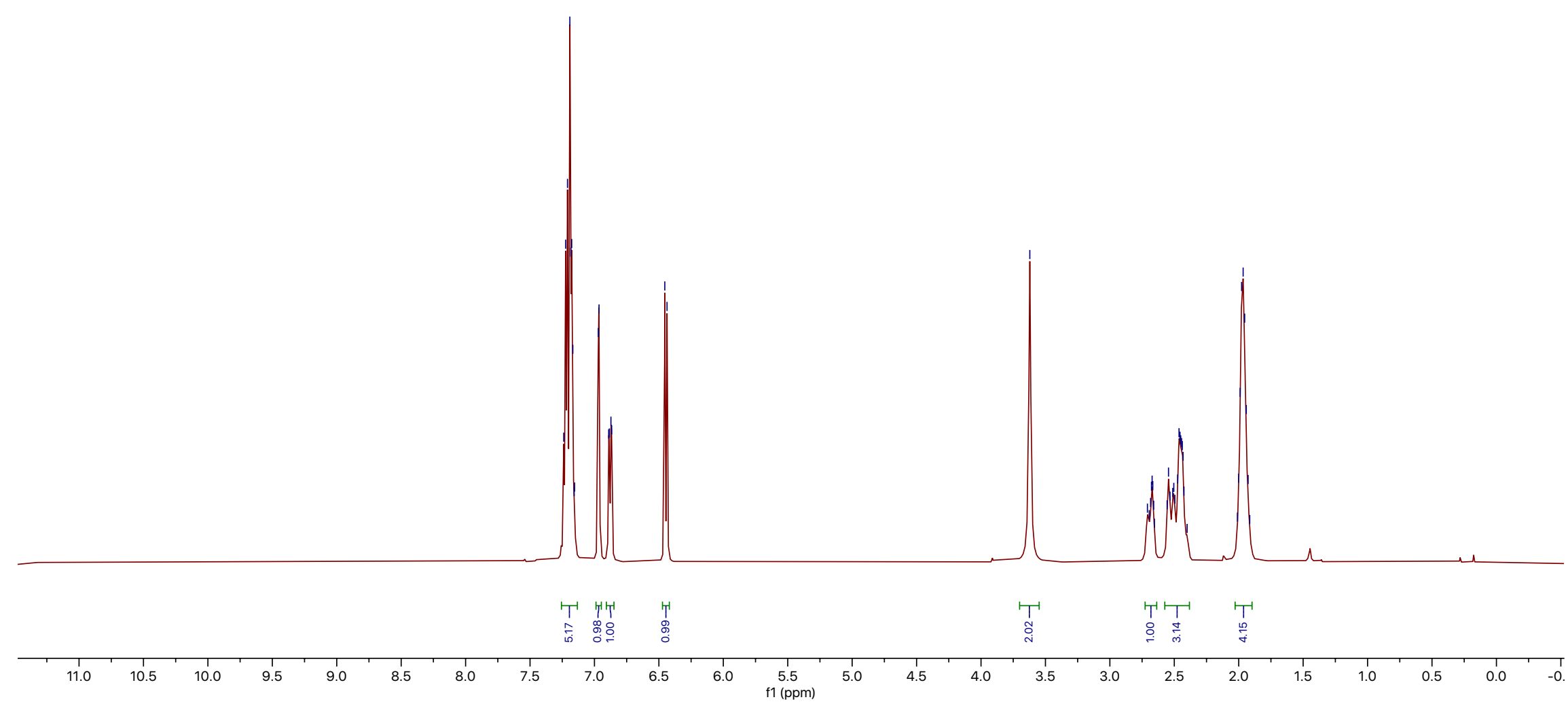


$125 \mathrm{MHz}, \mathrm{CDCl}_{3}$

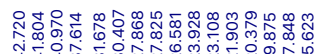 \\ 王手商}

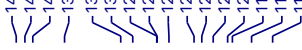

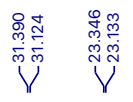

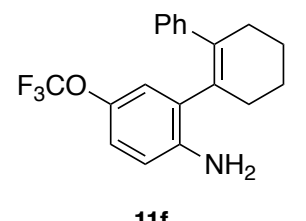

$11 f$
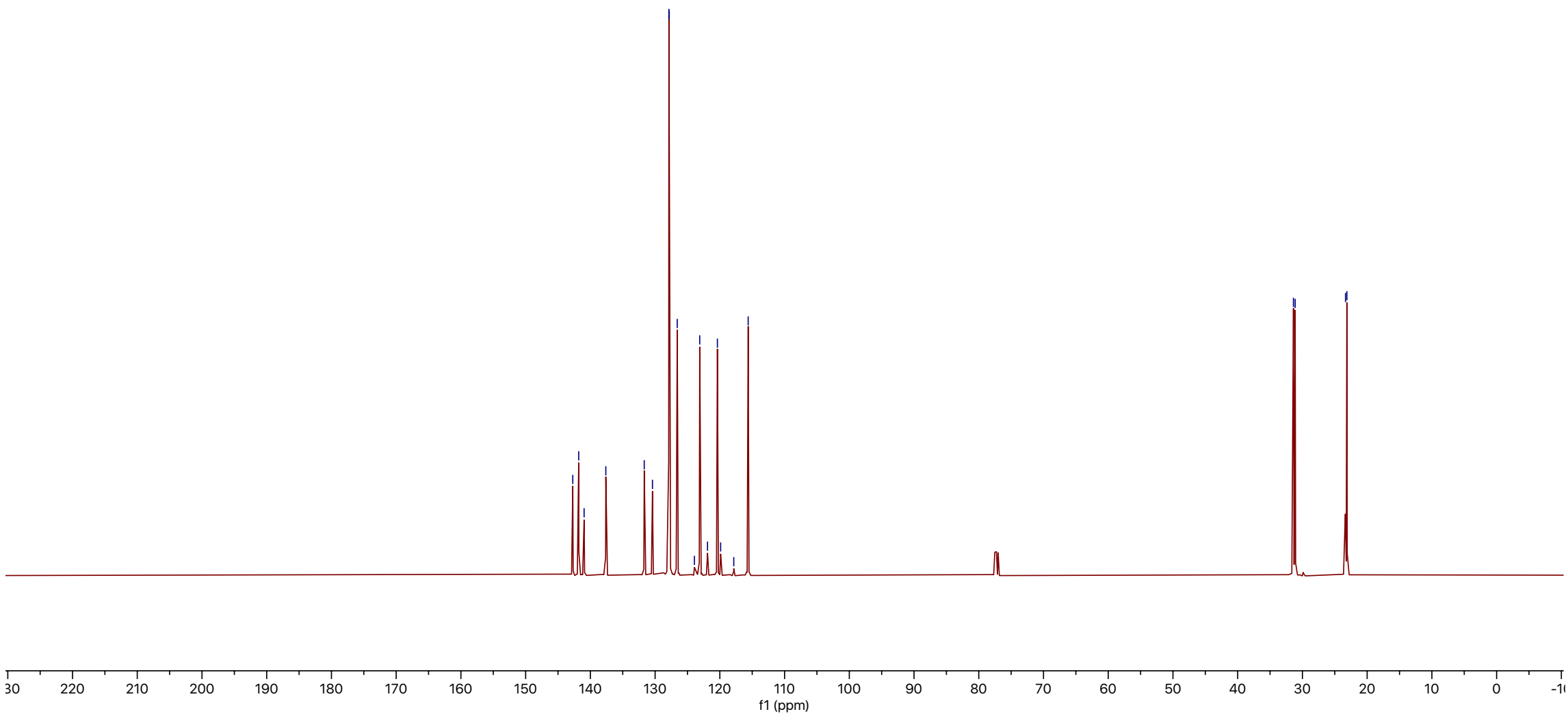
$500 \mathrm{MHz}, \mathrm{CDCl}_{3}$
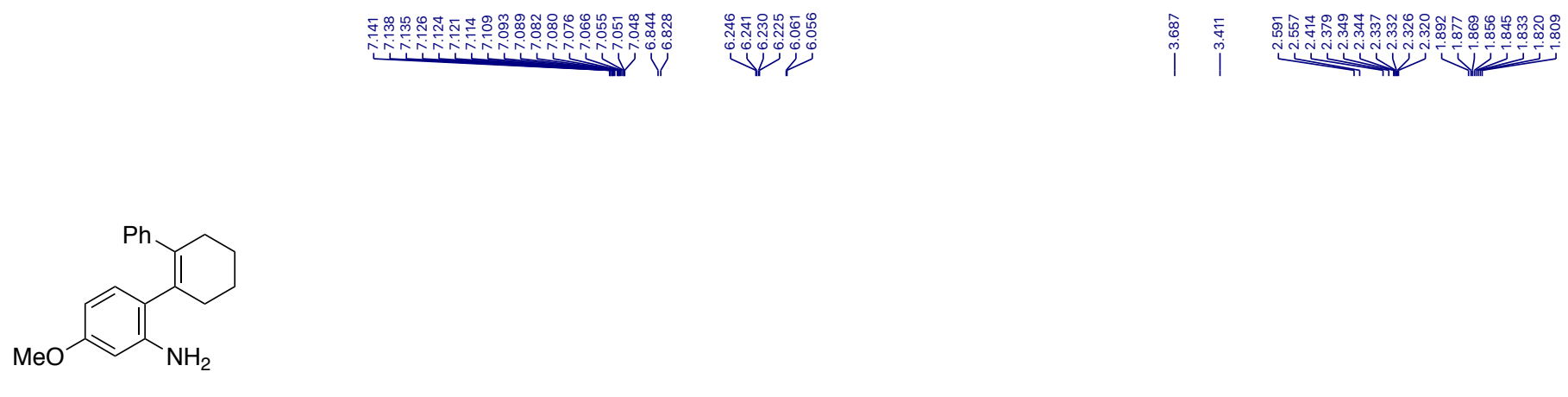

$11 \mathrm{~g}$

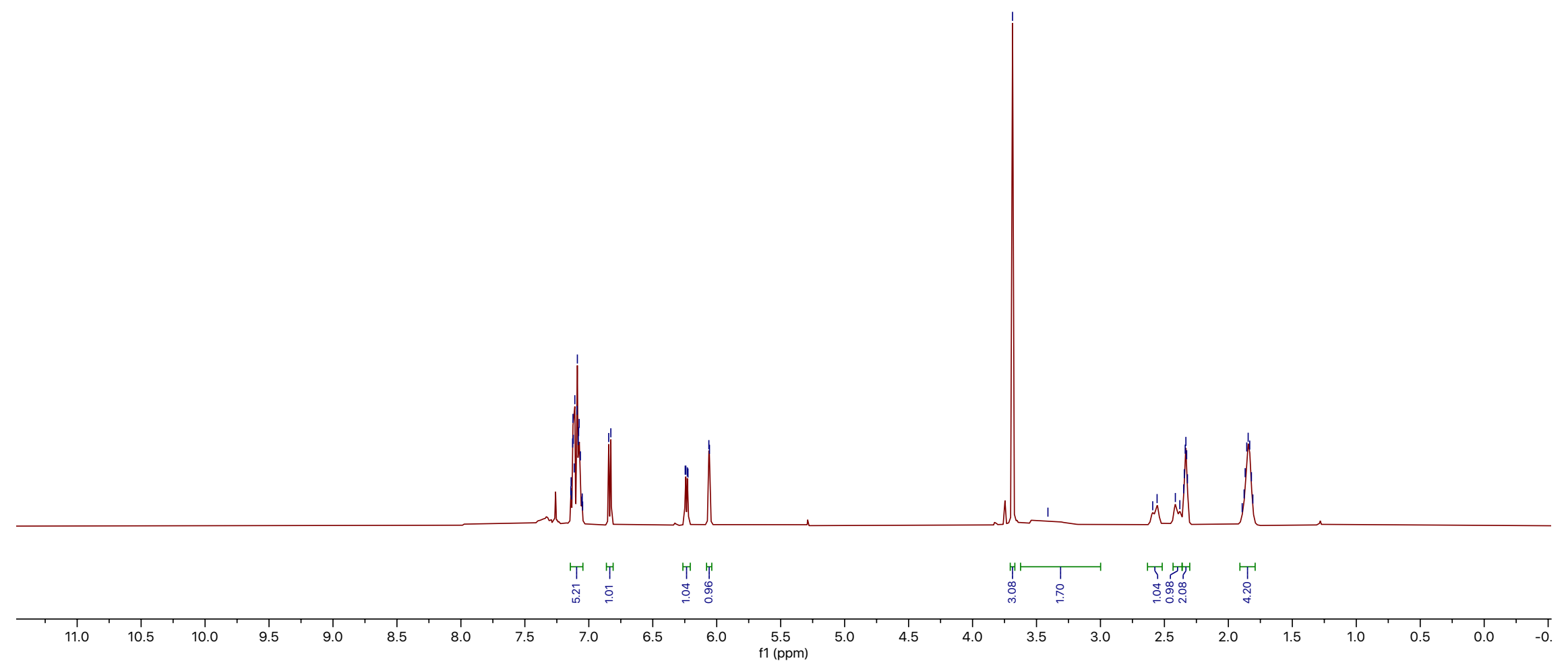


$125 \mathrm{MHz}, \mathrm{CDCl}_{3}$

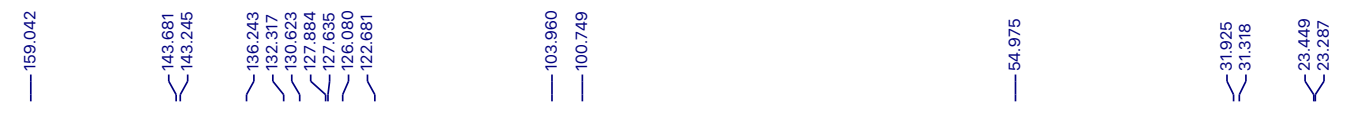

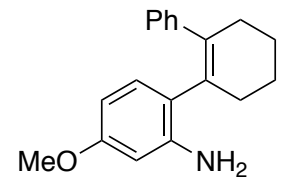

$11 \mathrm{~g}$
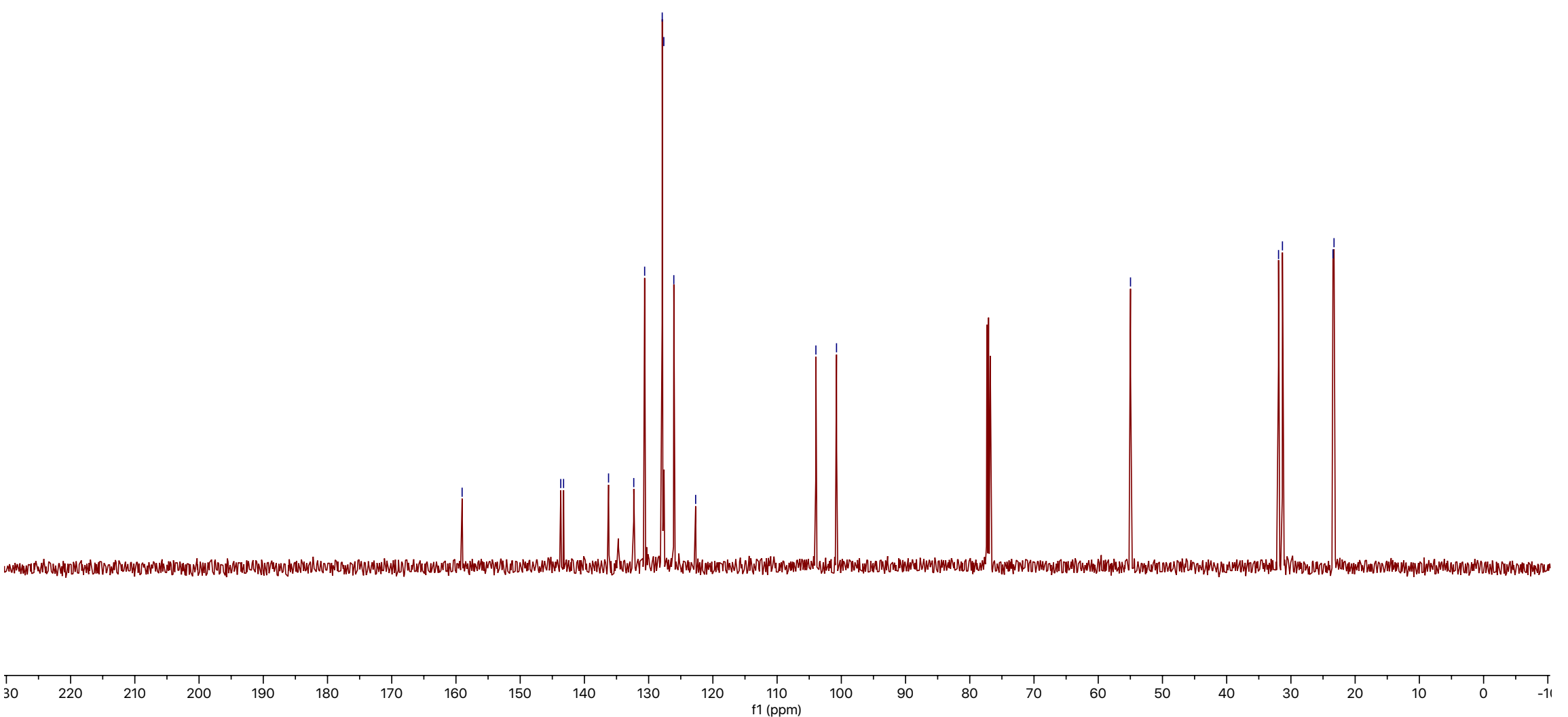
$500 \mathrm{MHz}, \mathrm{CDCl}_{3}$
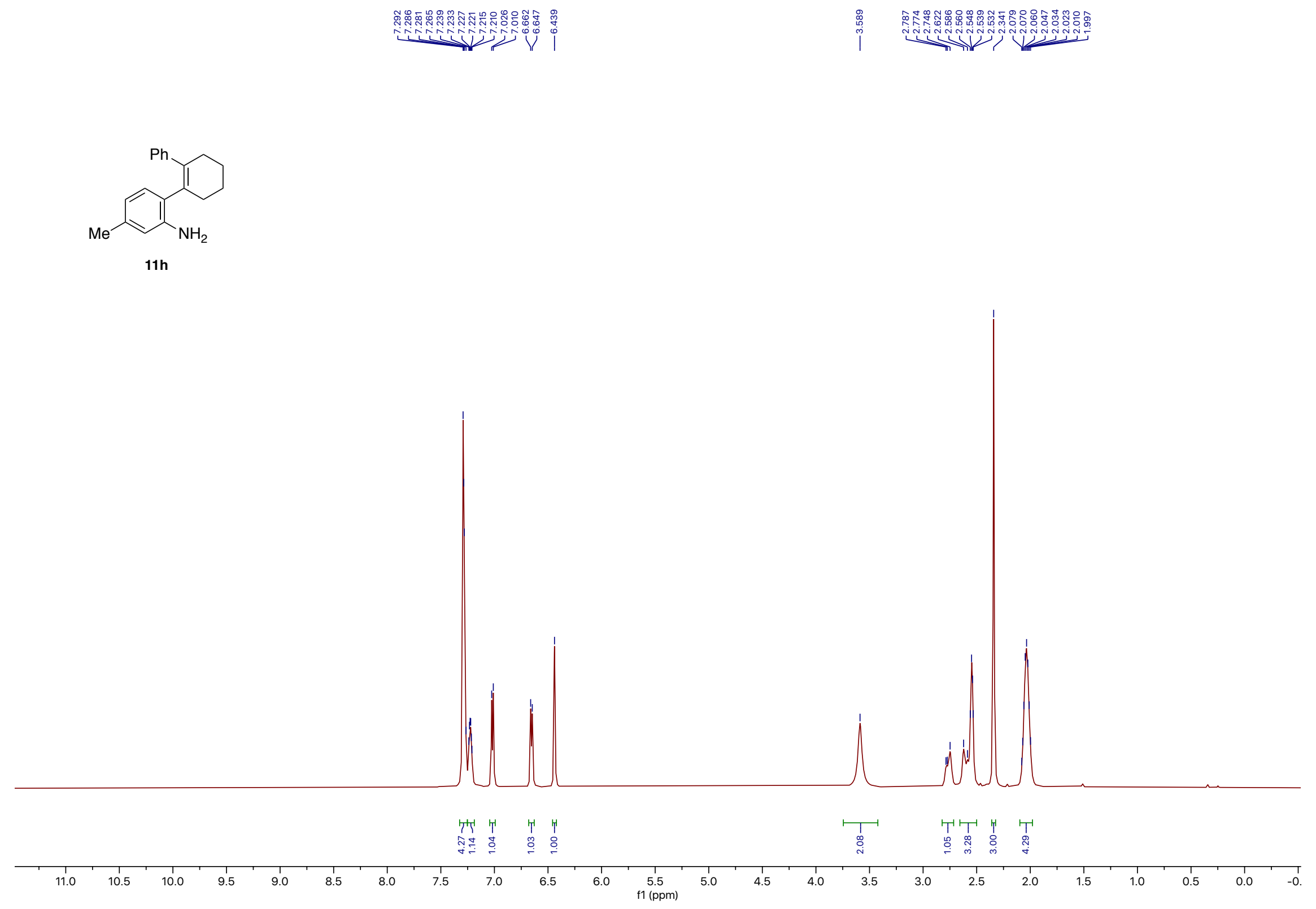
$125 \mathrm{MHz}, \mathrm{CDCl}_{3}$
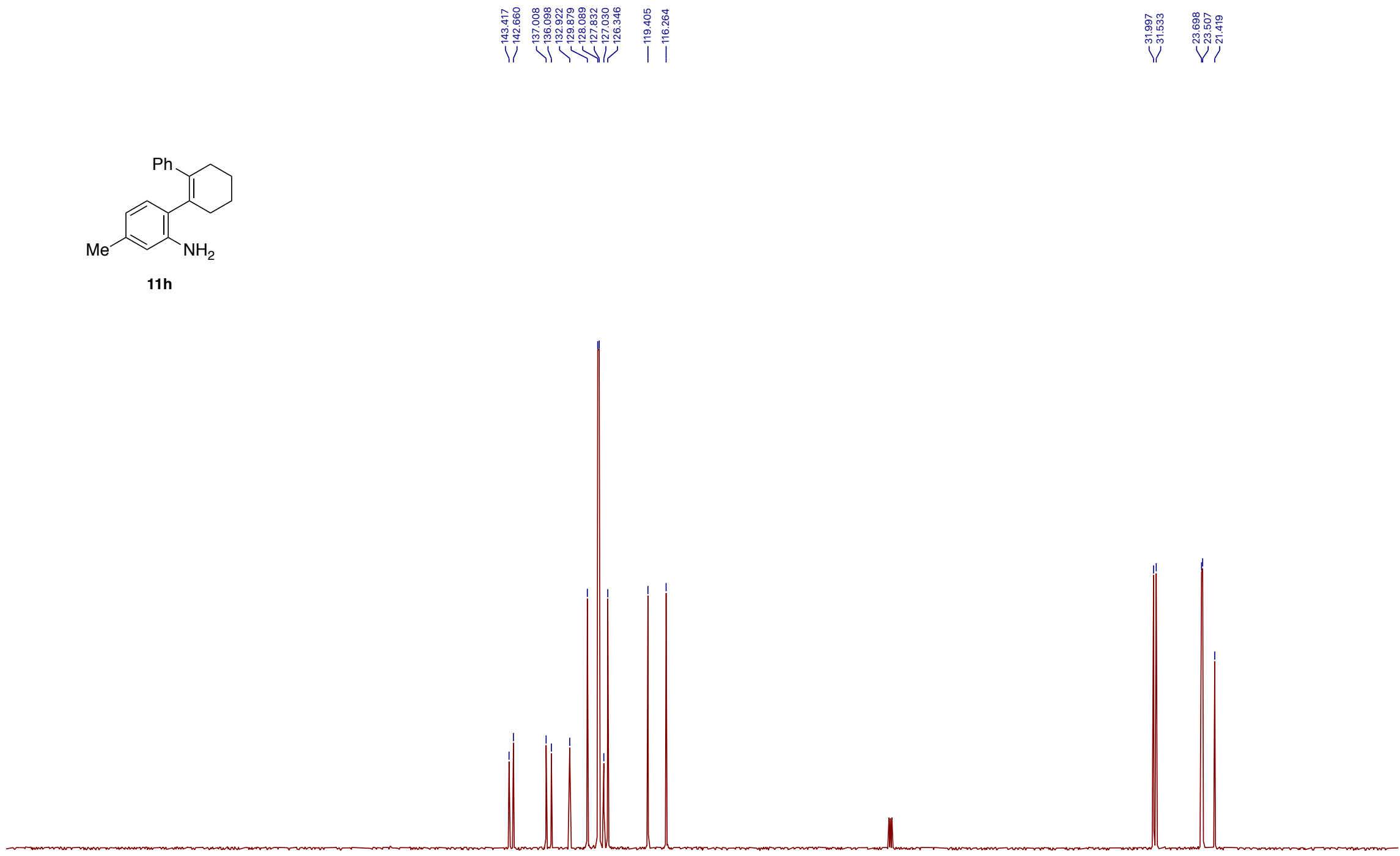

30
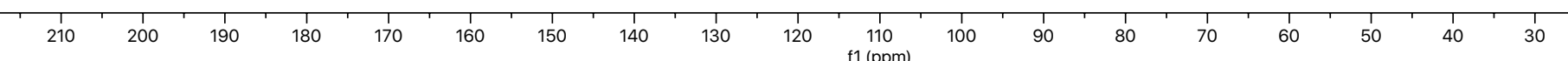
$500 \mathrm{MHz}, \mathrm{CDCl}_{3}$
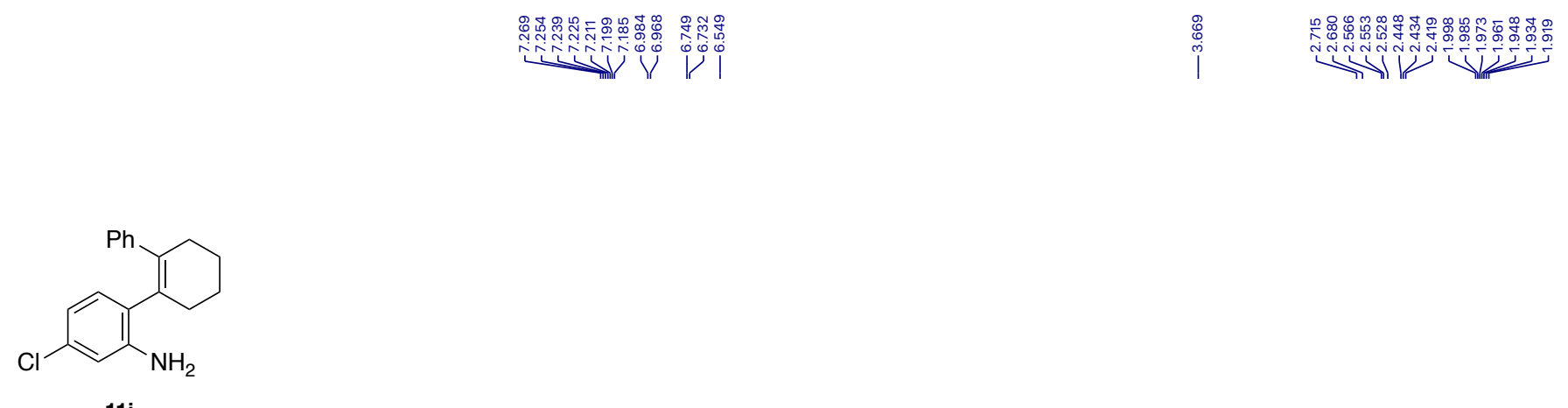

$11 i$

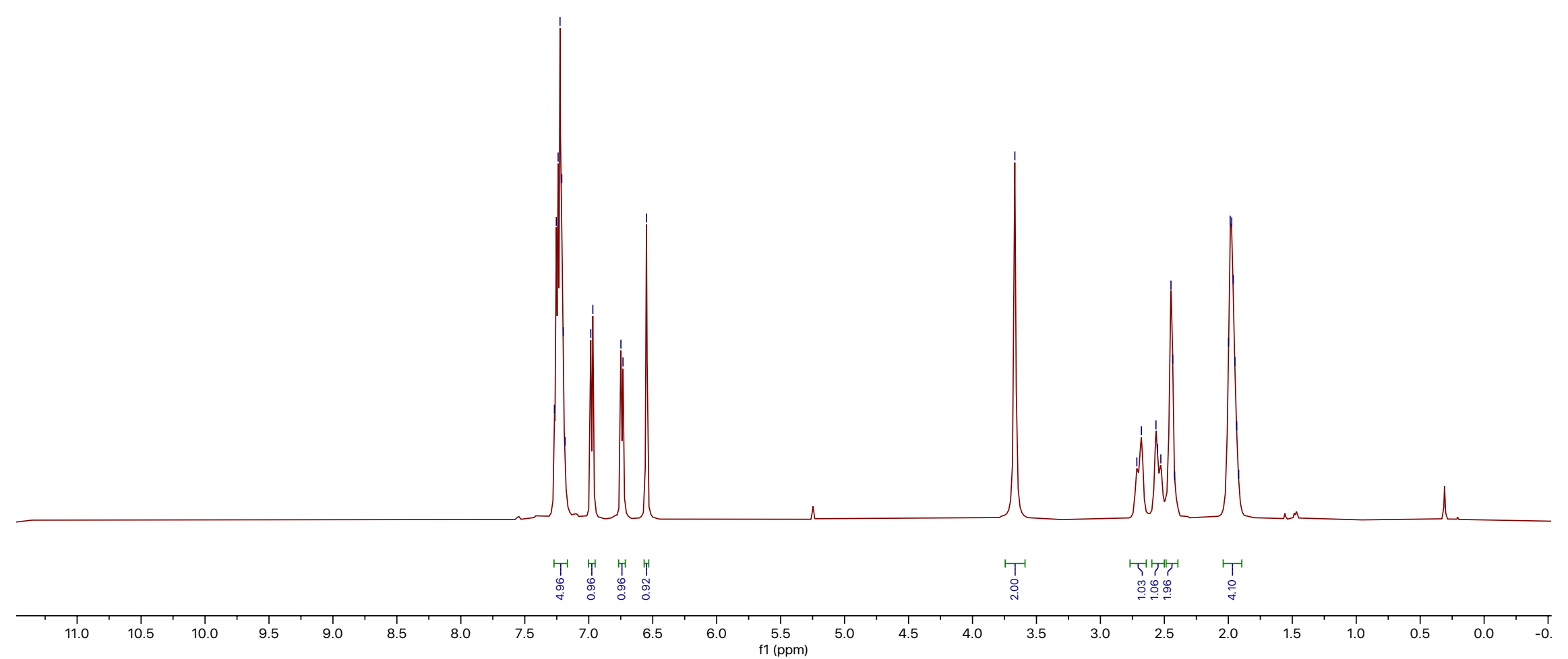


$125 \mathrm{MHz}, \mathrm{CDCl}_{3}$

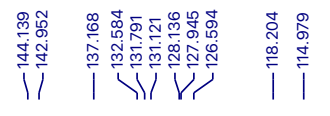

V

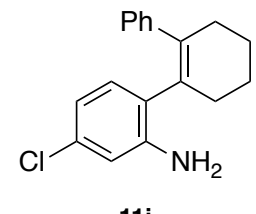

$11 i$

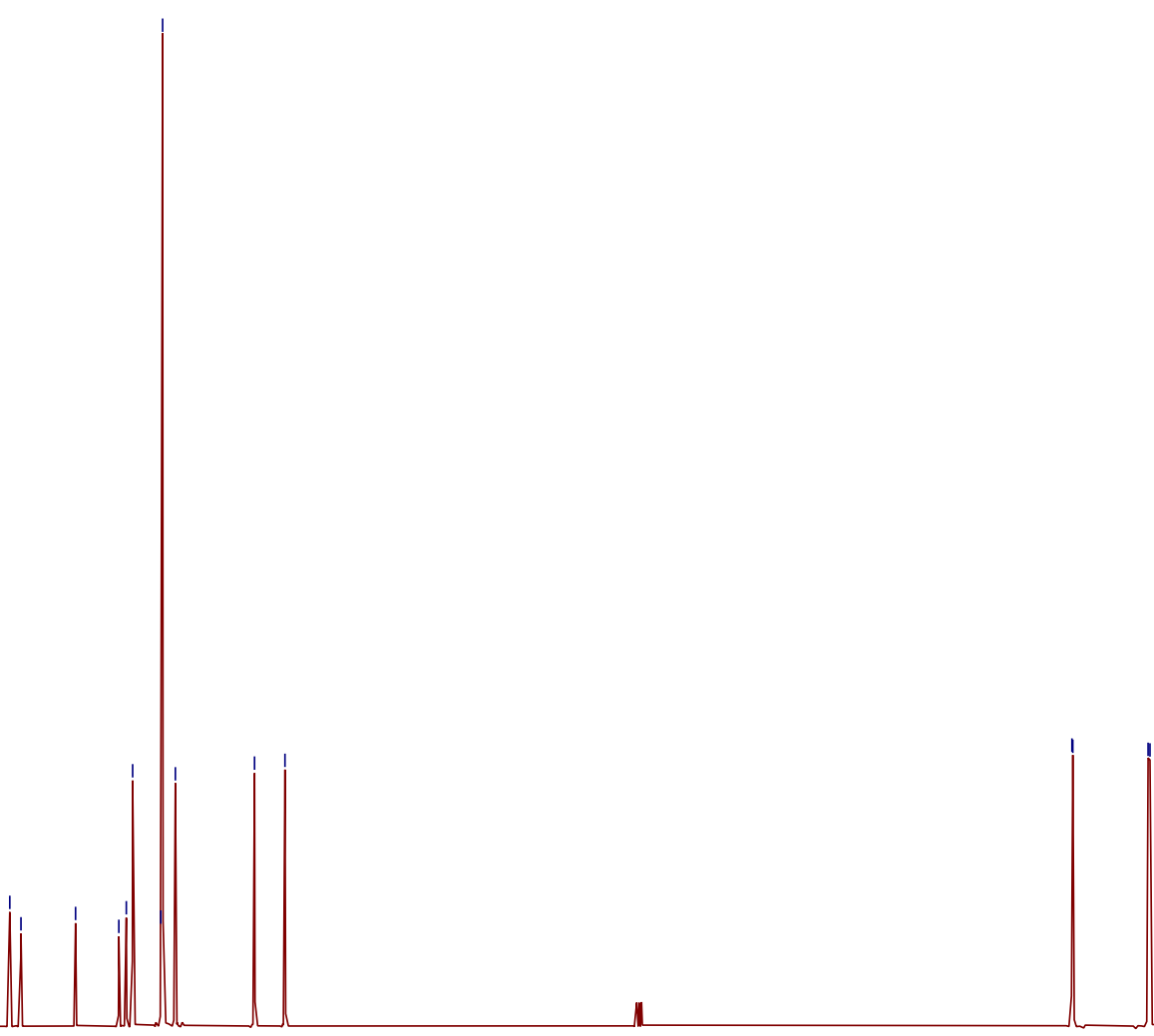

30

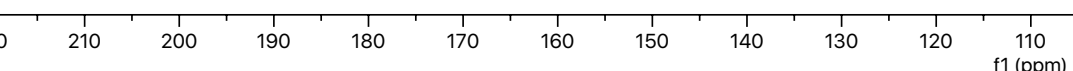
110
$\mathrm{f} 1(\mathrm{ppm})$
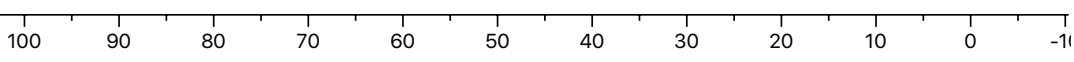
$500 \mathrm{MHz}, \mathrm{CDCl}_{3}$
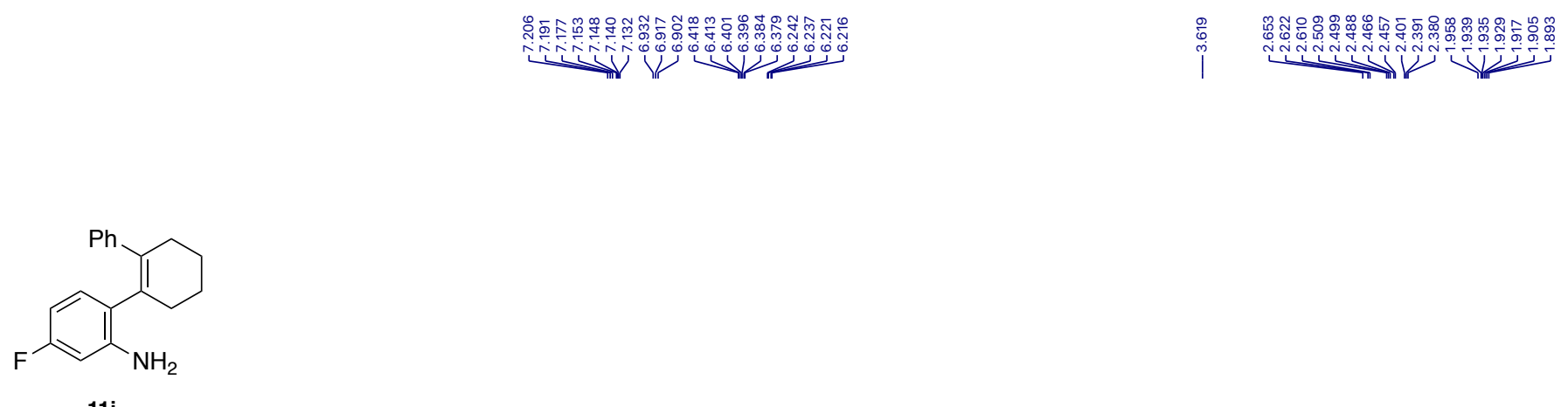

11j

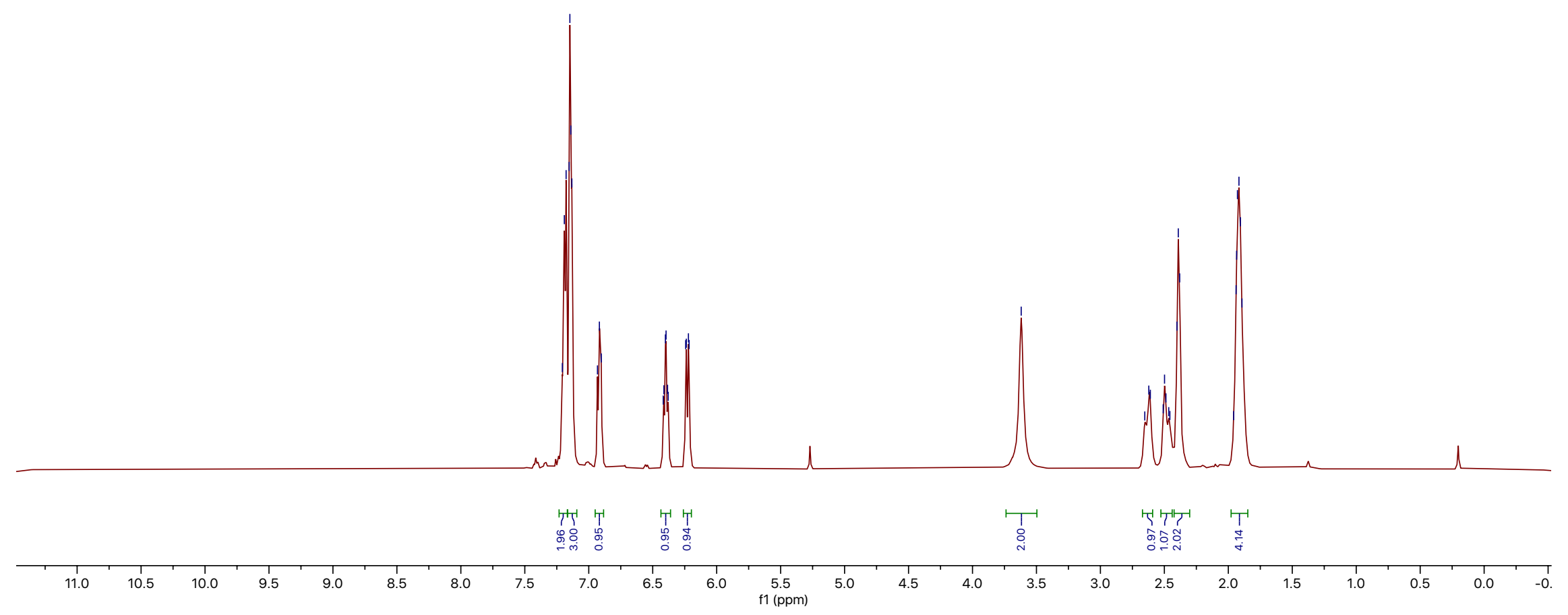


$125 \mathrm{MHz}, \mathrm{CDCl}_{3}$
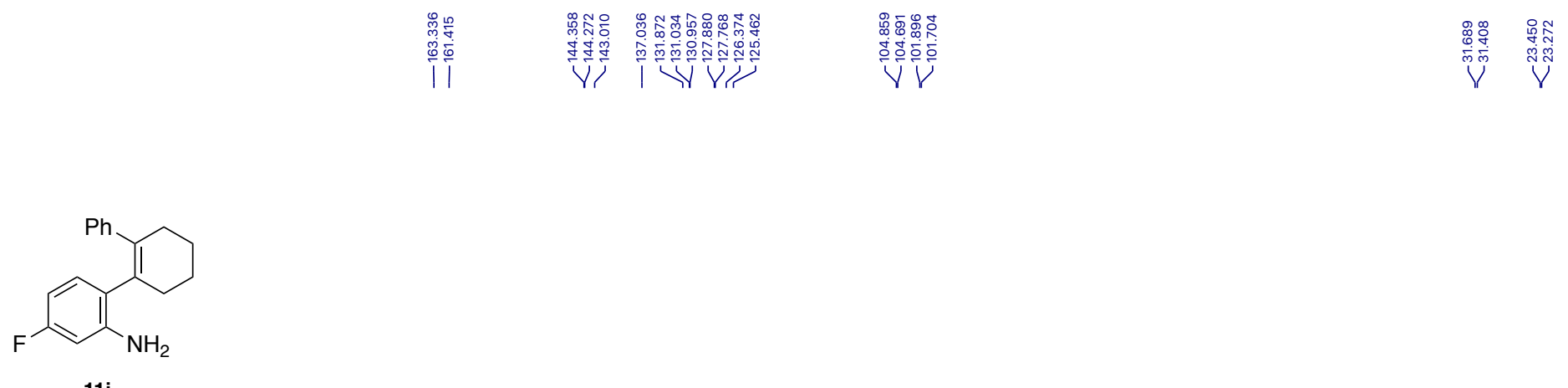

11j

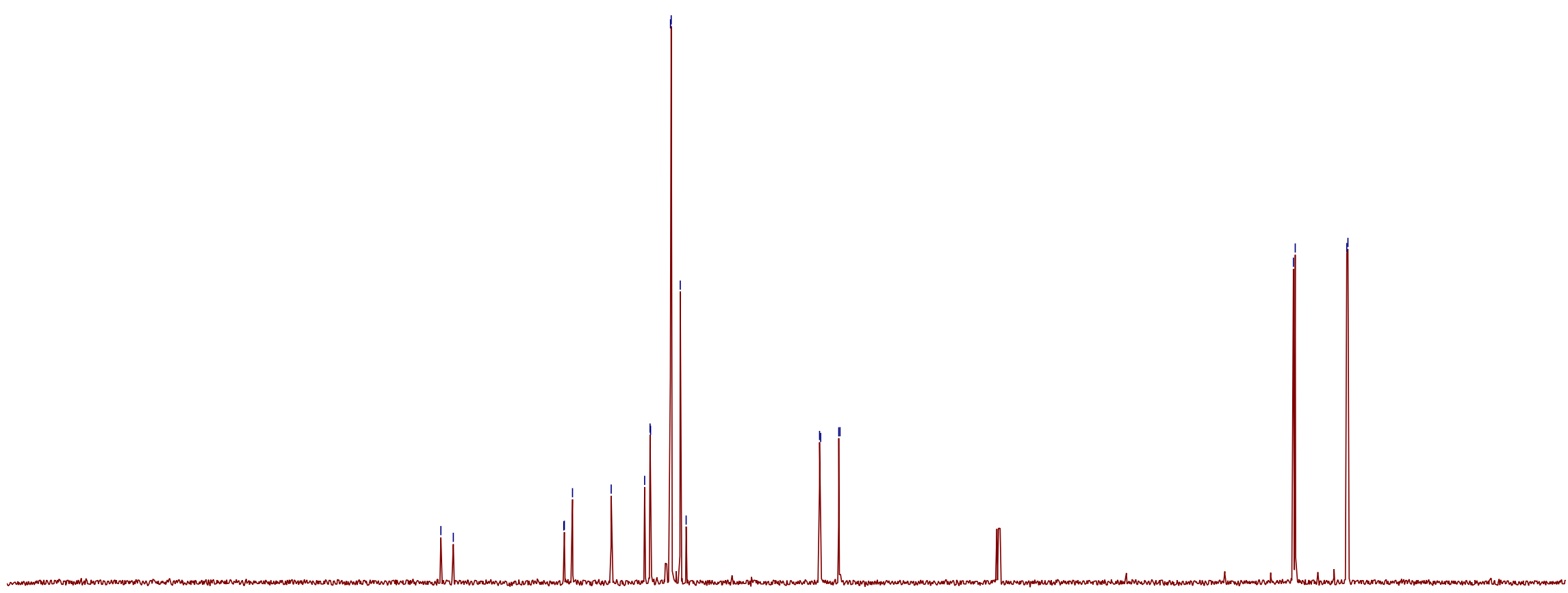

30
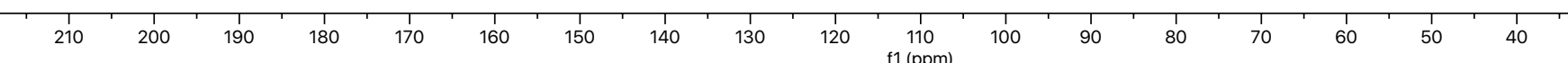
$500 \mathrm{MHz}, \mathrm{CDCl}_{3}$

年

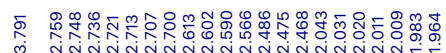

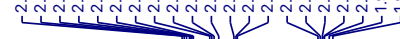

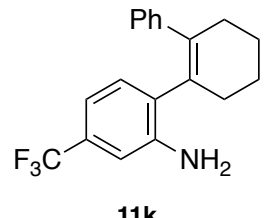

$11 k$

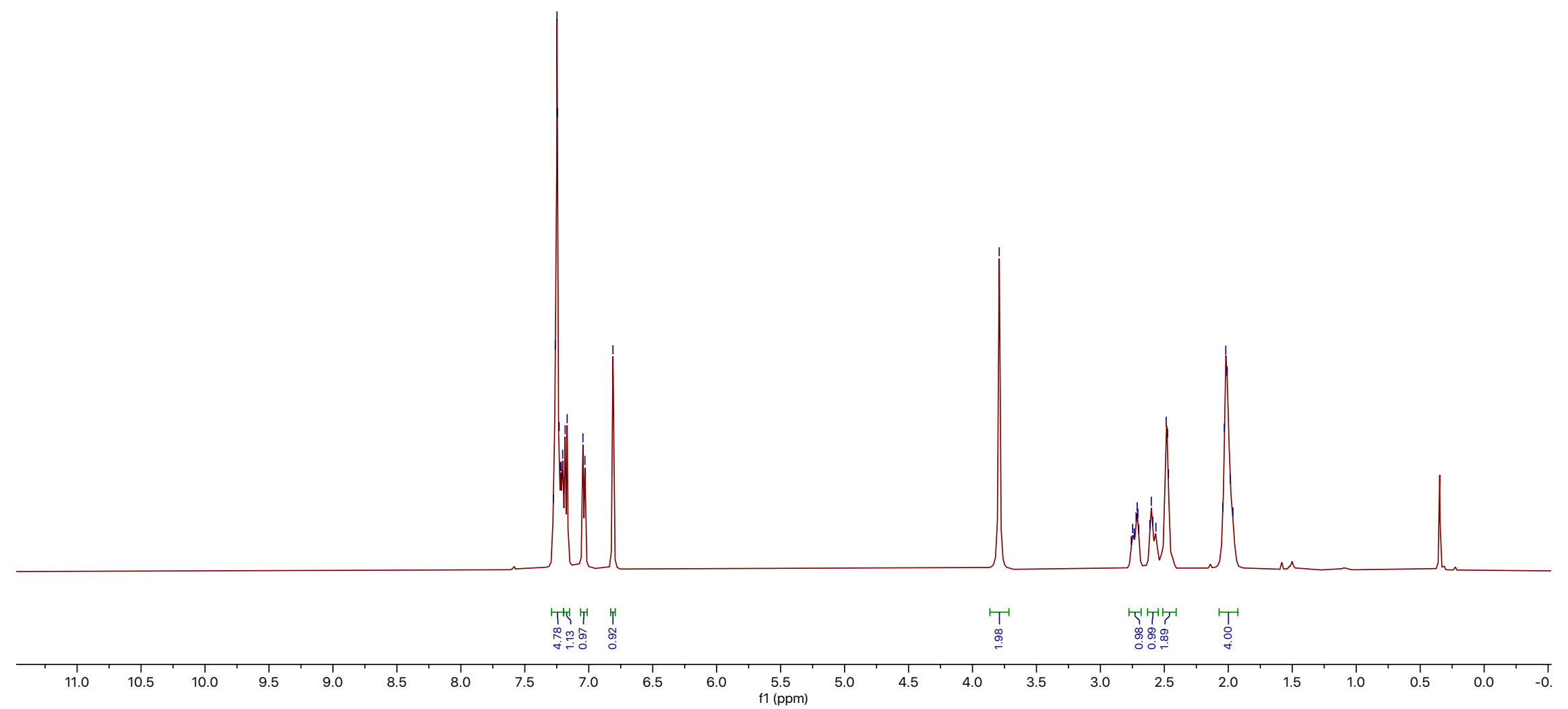


$125 \mathrm{MHz}, \mathrm{CDCl}_{3}$

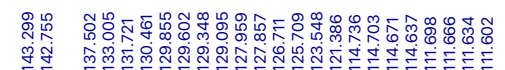

$V^{2}$
V

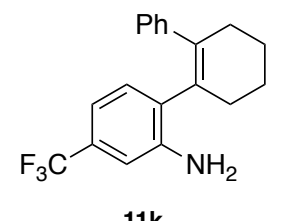

11k

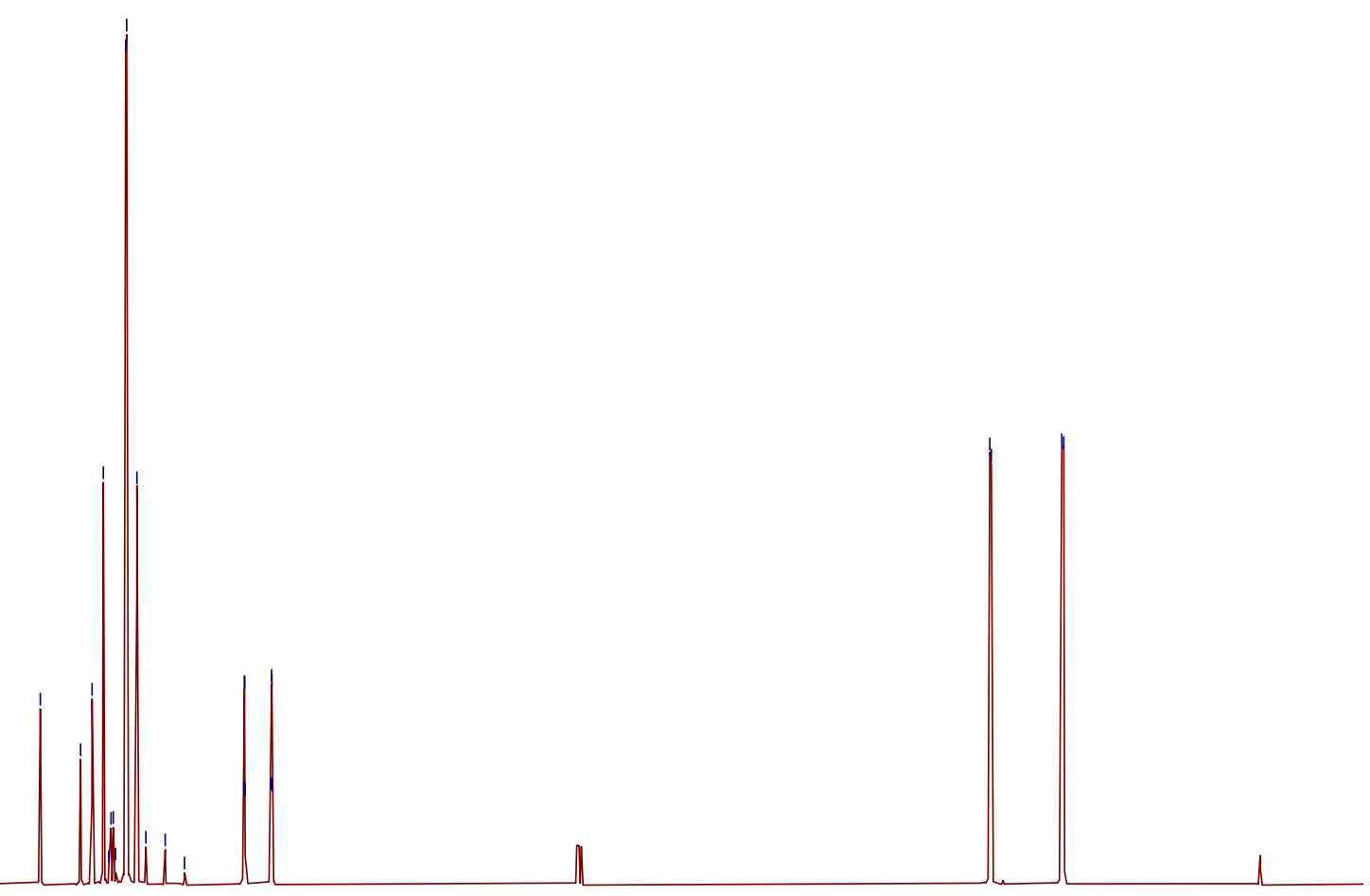

30

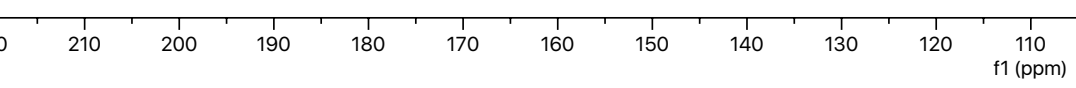
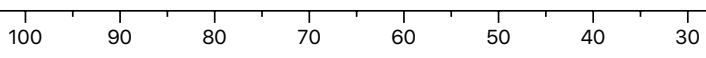
$500 \mathrm{MHz}, \mathrm{CDCl}_{3}$
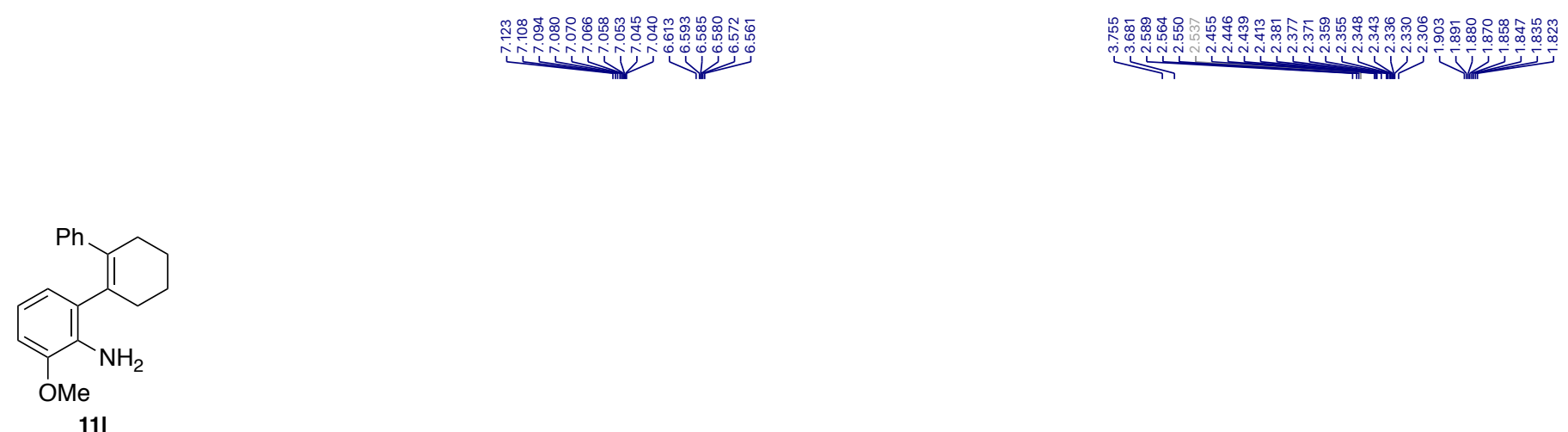

11

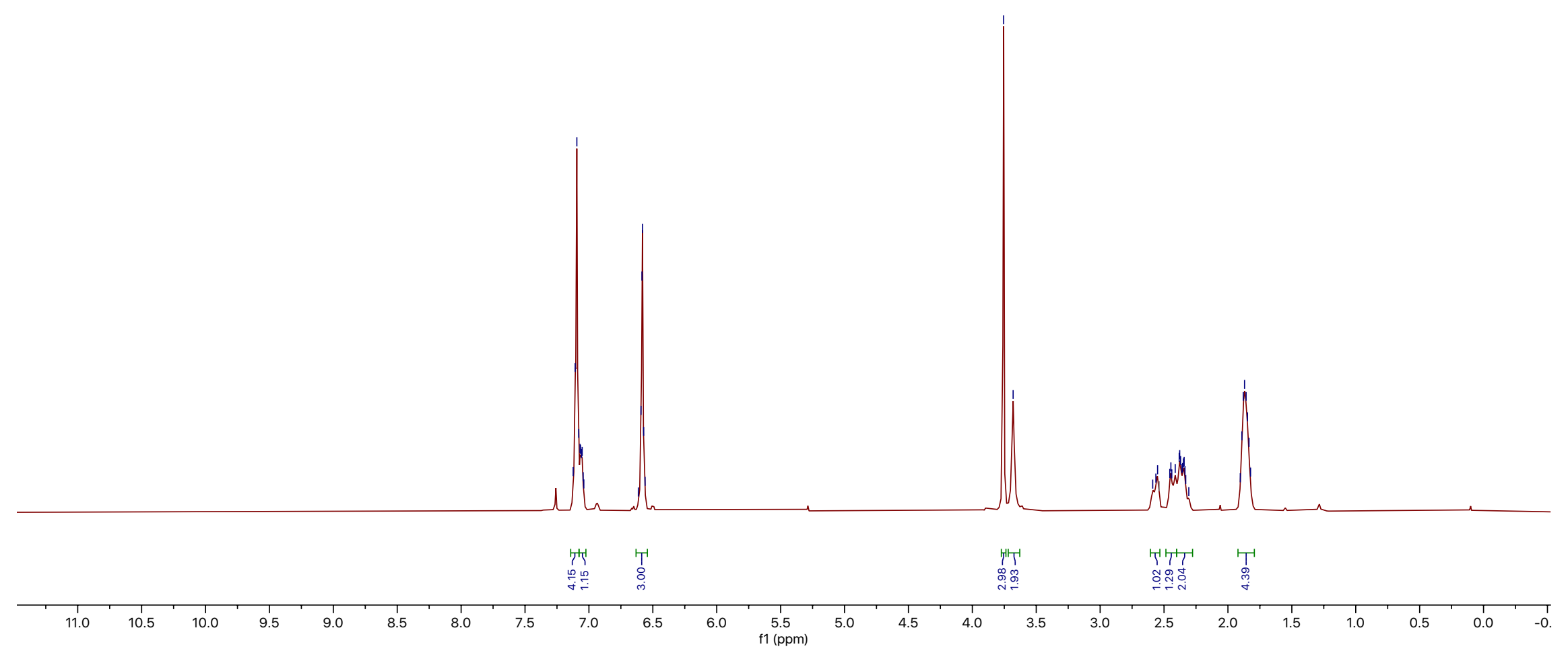


$125 \mathrm{MHz}, \mathrm{CDCl}_{3}$
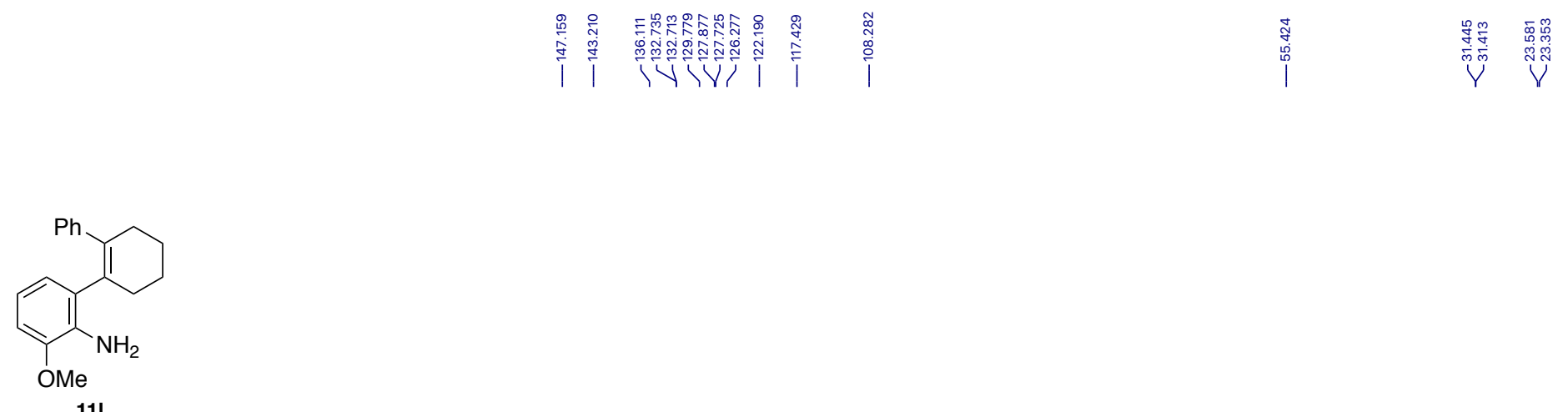

11
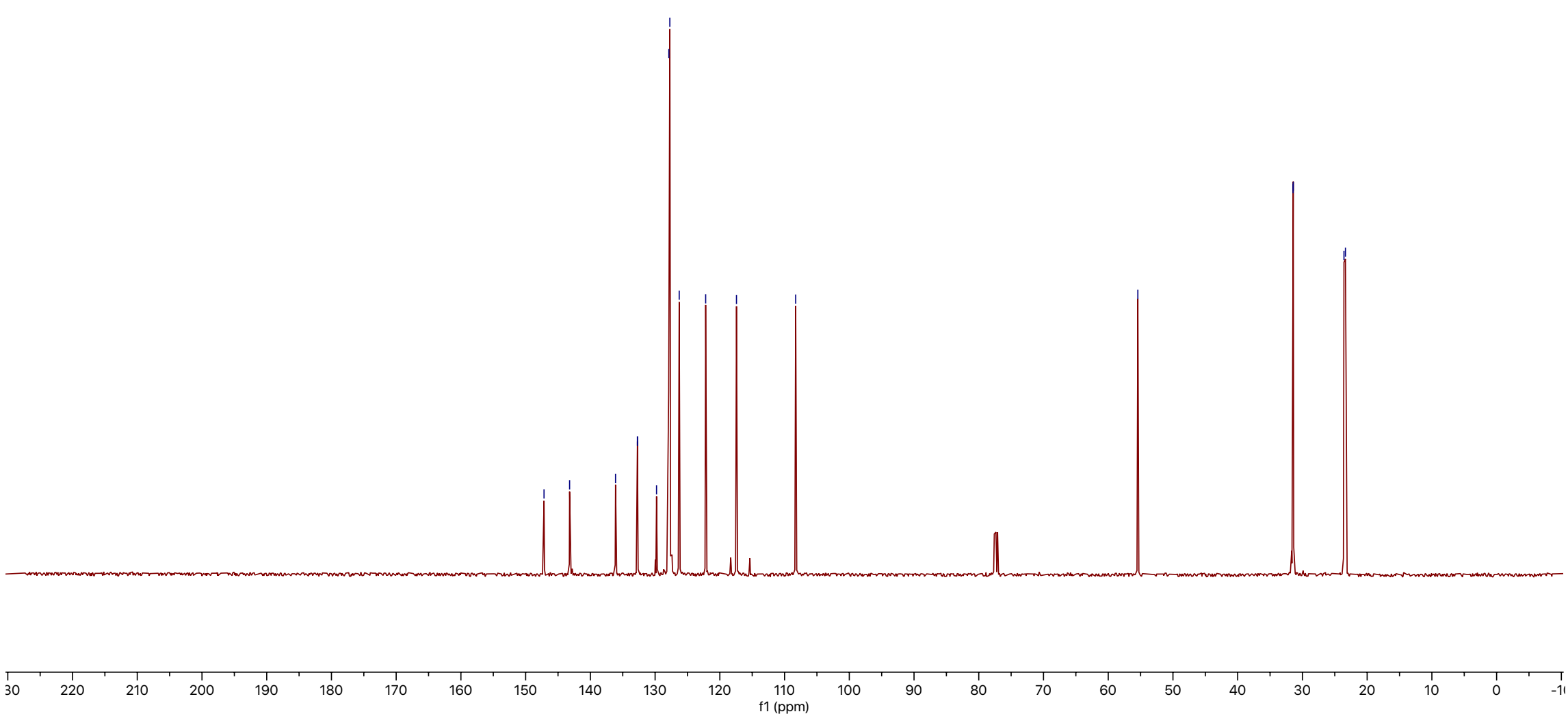
$500 \mathrm{MHz}, \mathrm{CDCl}_{3}$

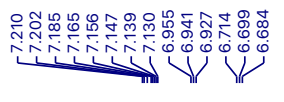

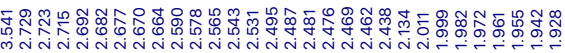
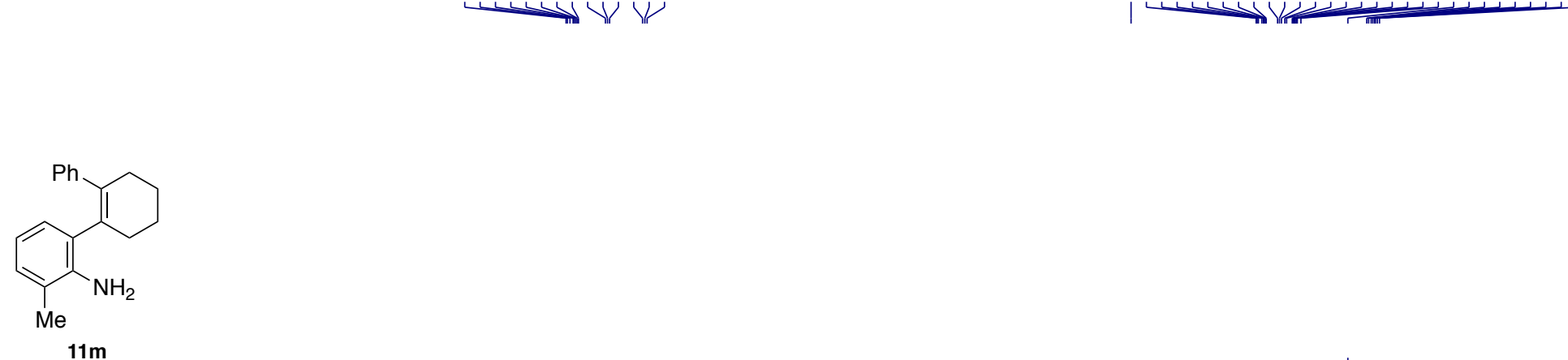

$11 \mathrm{~m}$

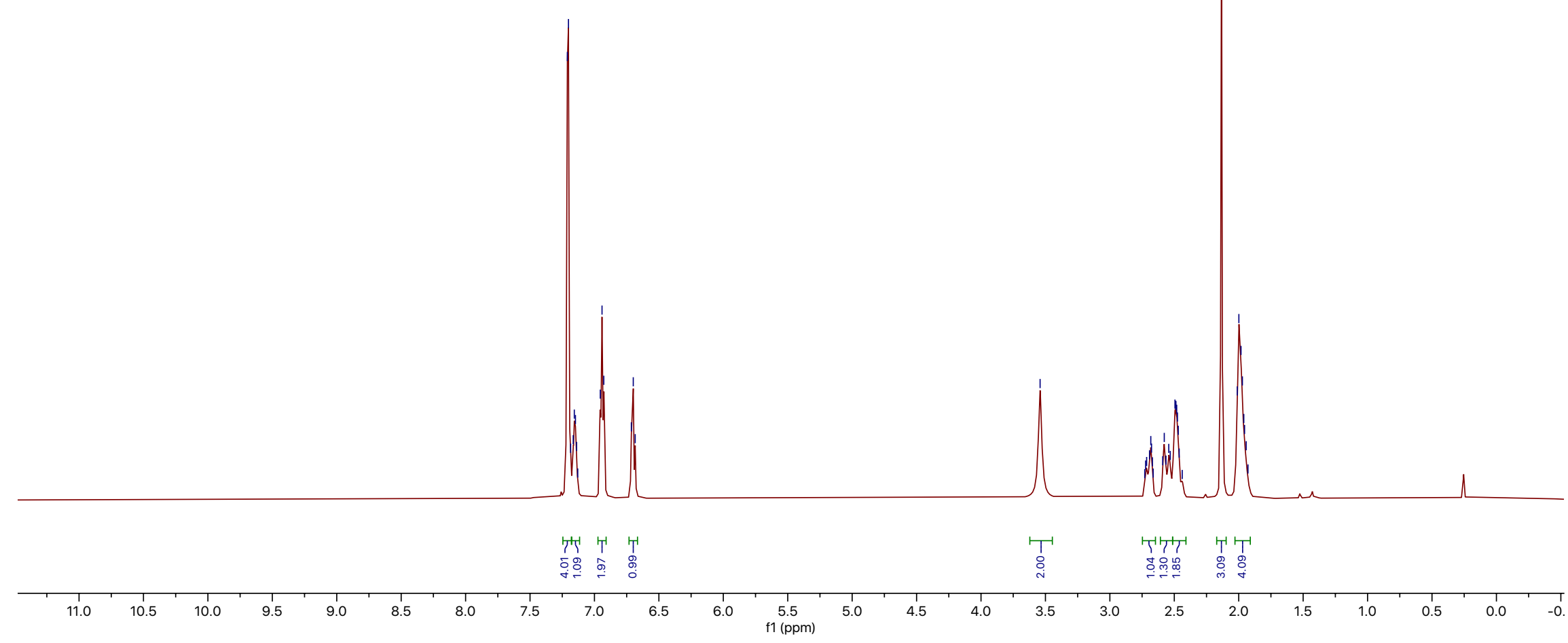


$125 \mathrm{MHz}, \mathrm{CDCl}_{3}$

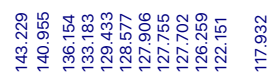

|

ํํำ

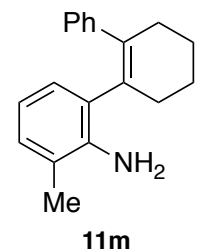

$11 \mathrm{~m}$
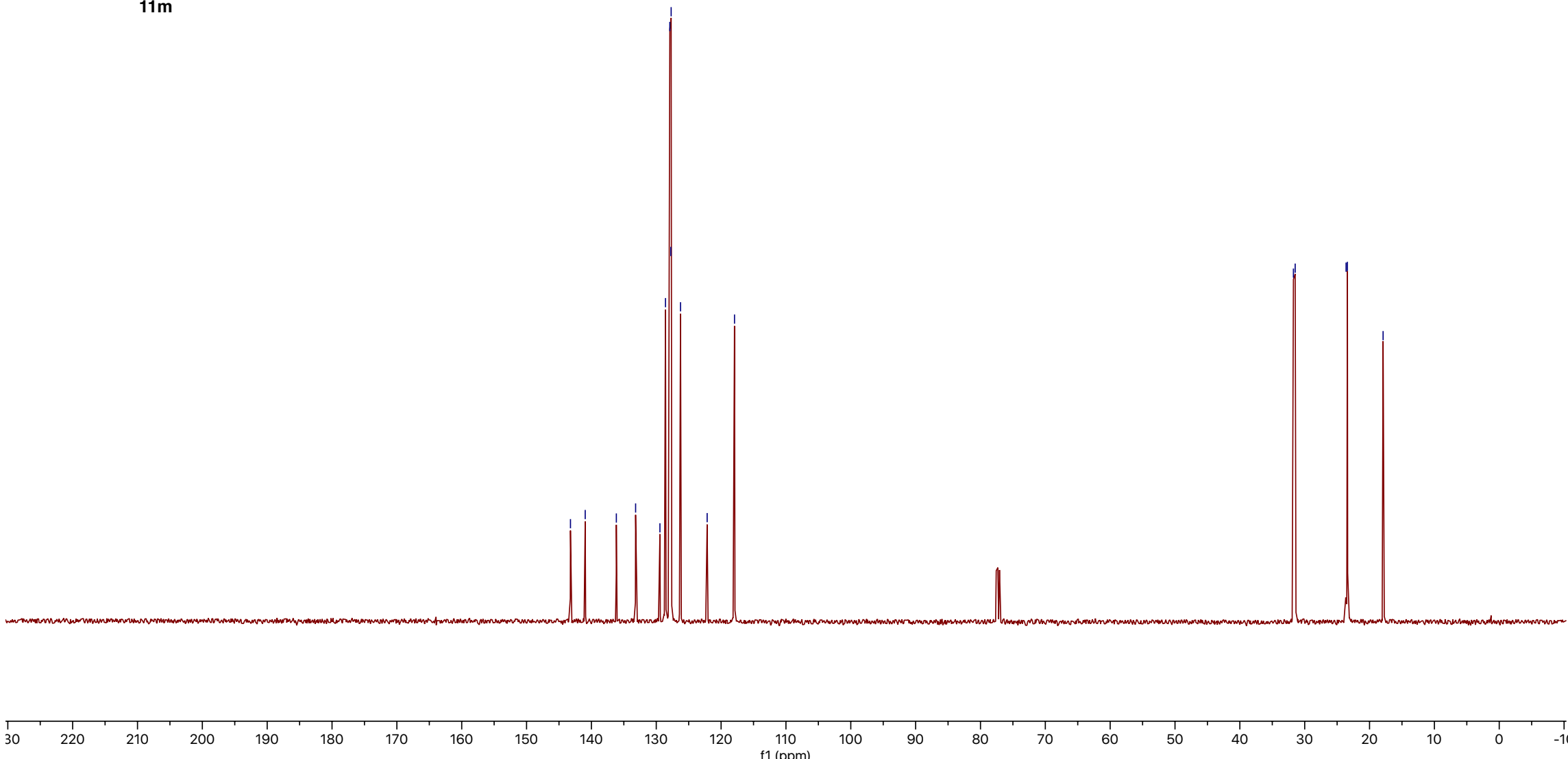
$500 \mathrm{MHz}, \mathrm{CDCl}_{3}$
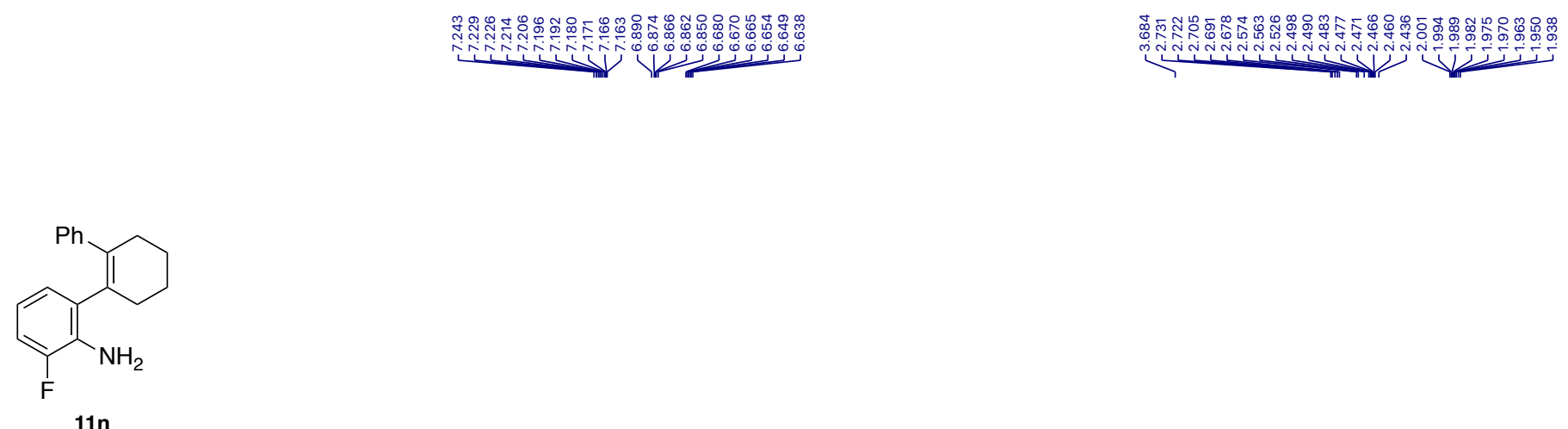

$11 n$

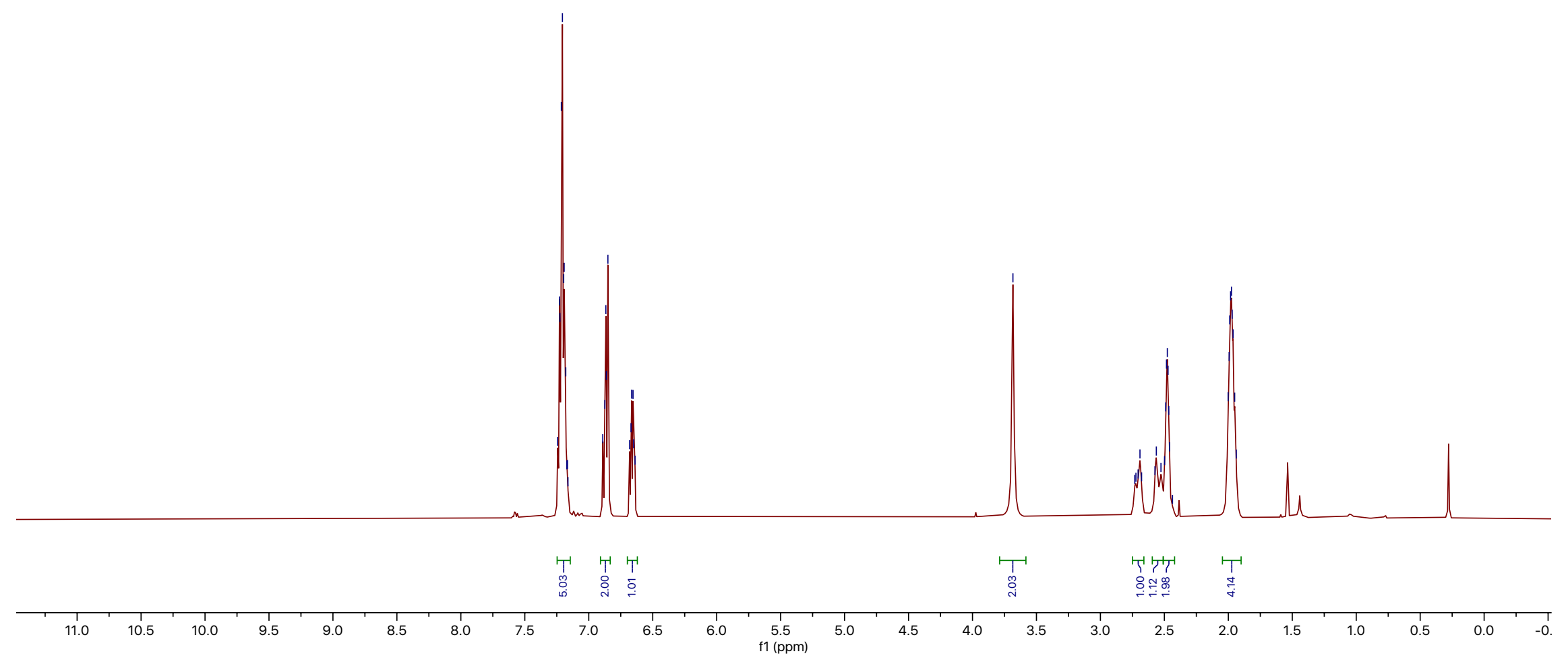


$125 \mathrm{MHz}, \mathrm{CDCl}_{3}$

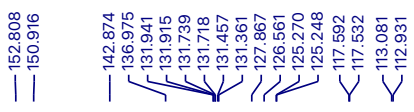

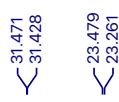

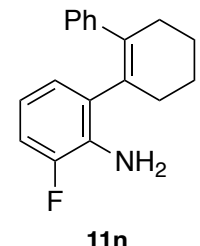

$11 n$

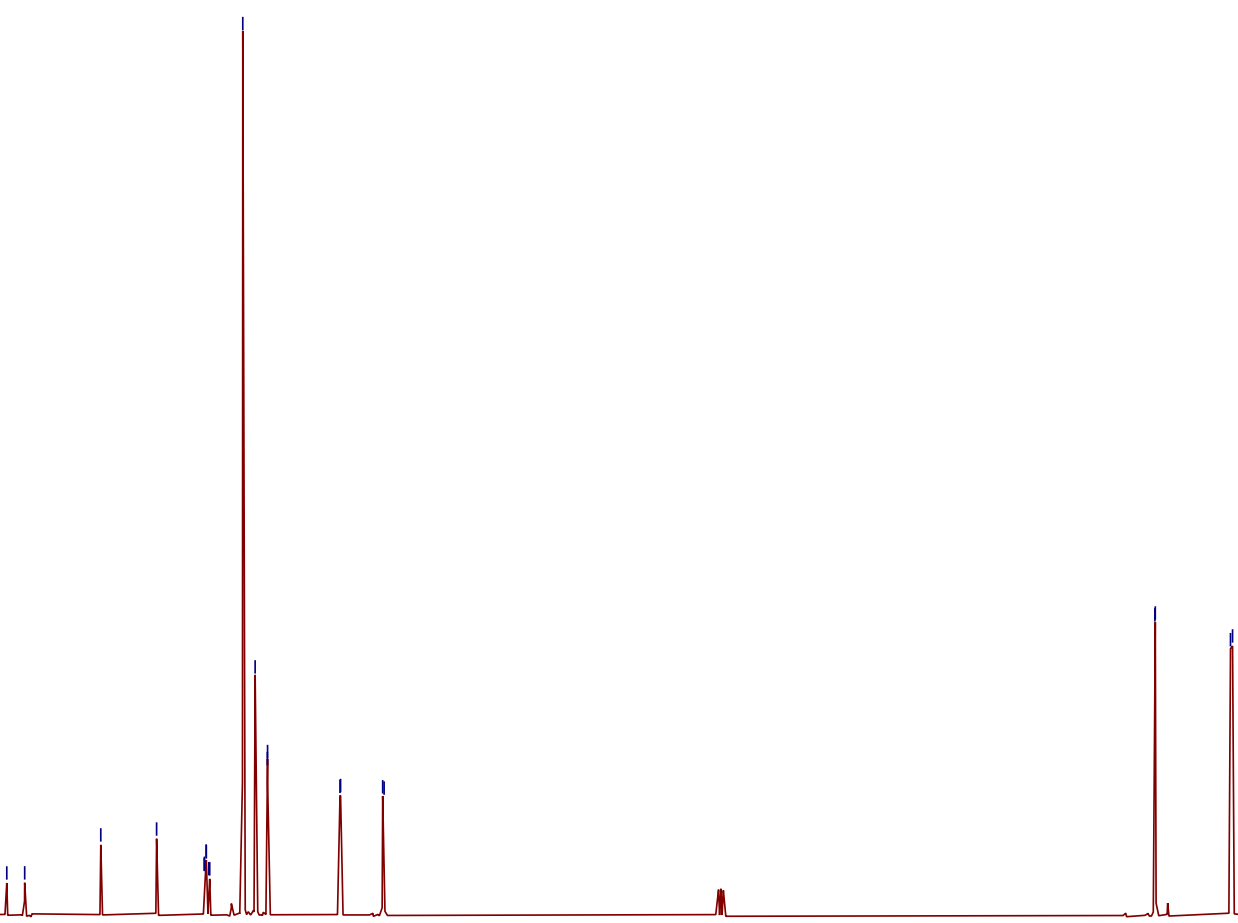

30

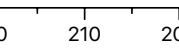

$200 \quad 190$

180

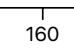

150

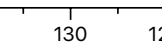

$20 \quad 110$
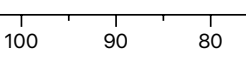

1
$70 \quad 60$
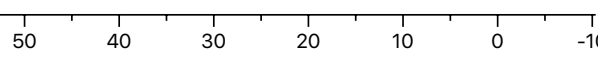
$500 \mathrm{MHz}, \mathrm{CDCl}_{3}$

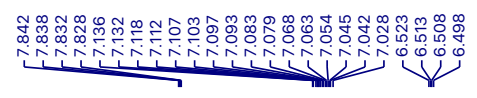

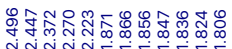

11114

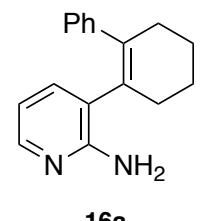

$16 a$

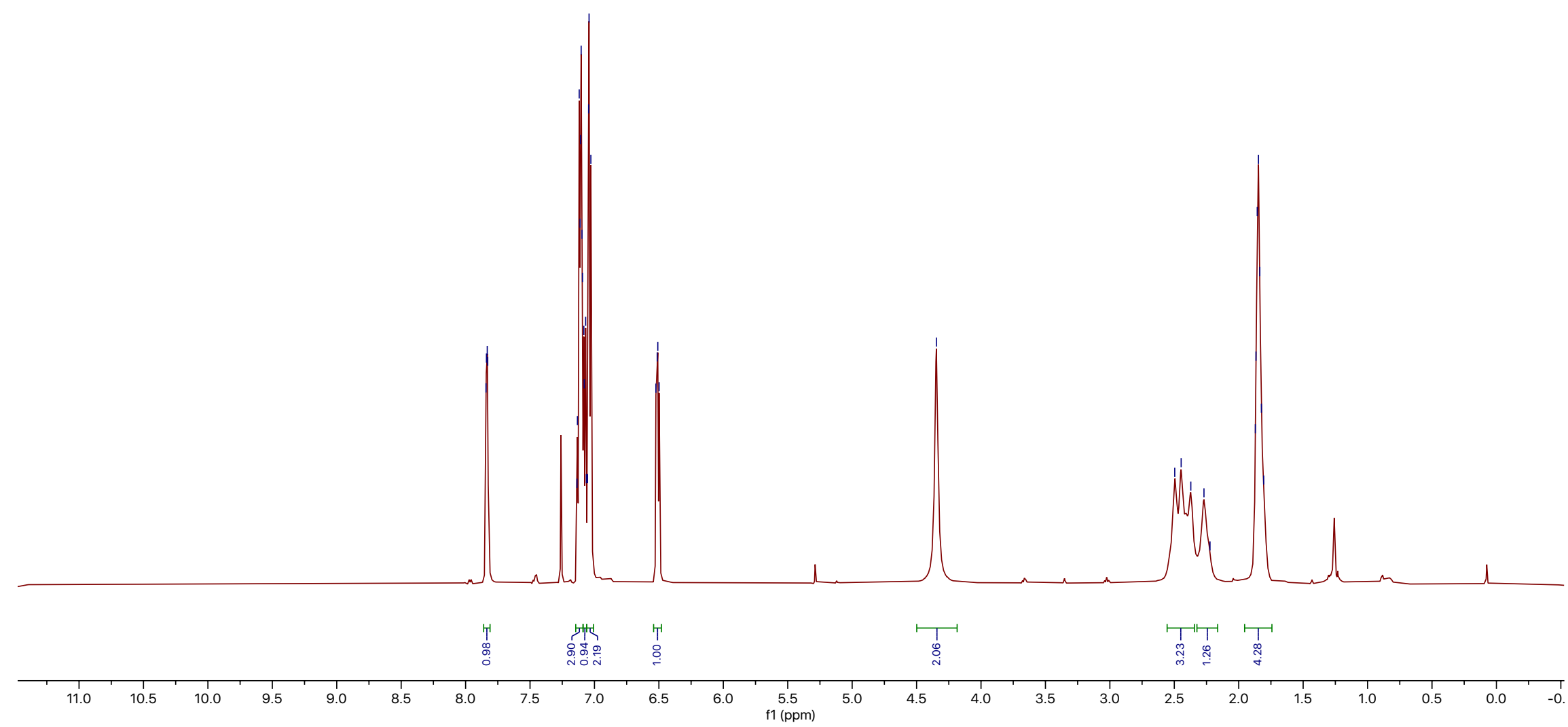


$125 \mathrm{MHz}, \mathrm{CDCl}_{3}$

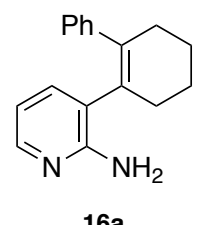

$16 a$

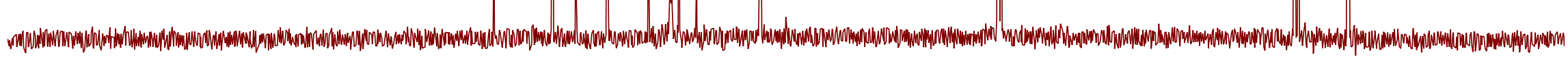

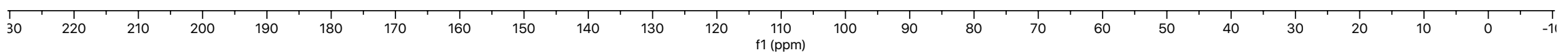


$500 \mathrm{MHz}, \mathrm{CDCl}_{3}$
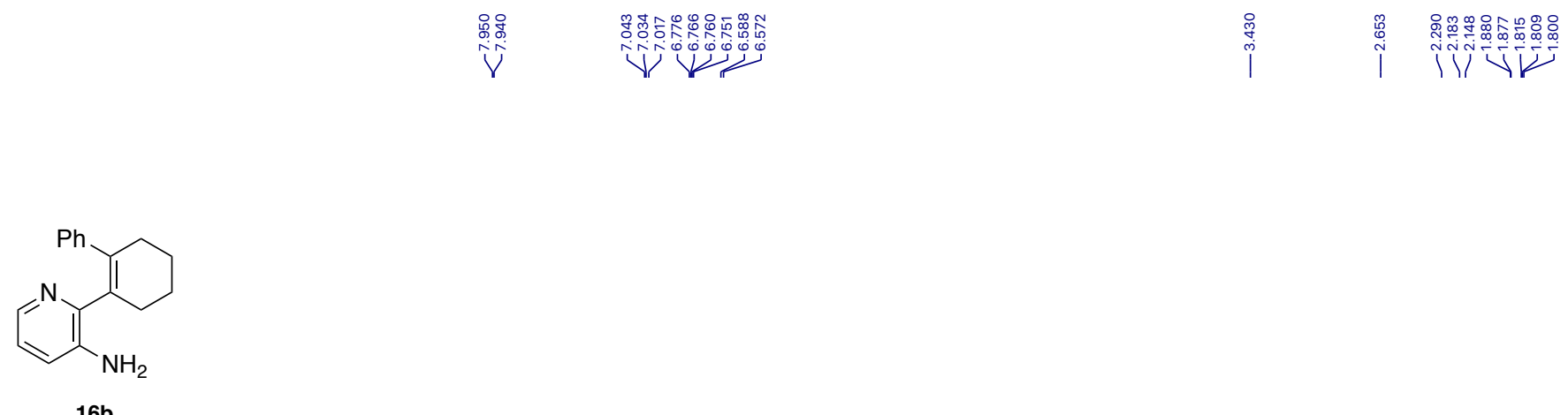

$16 b$

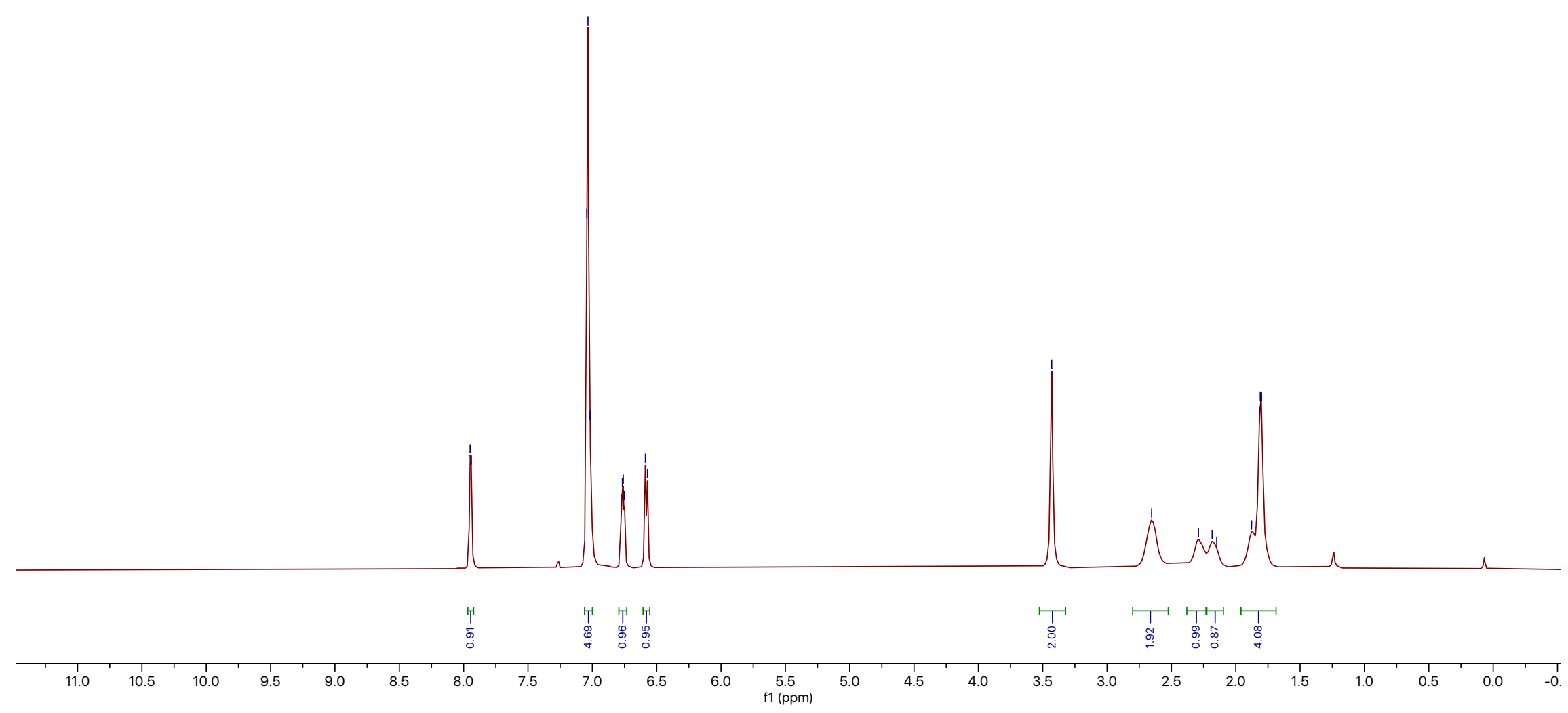


$125 \mathrm{MHz}, \mathrm{CDCl}_{3}$

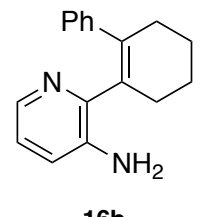

$16 b$
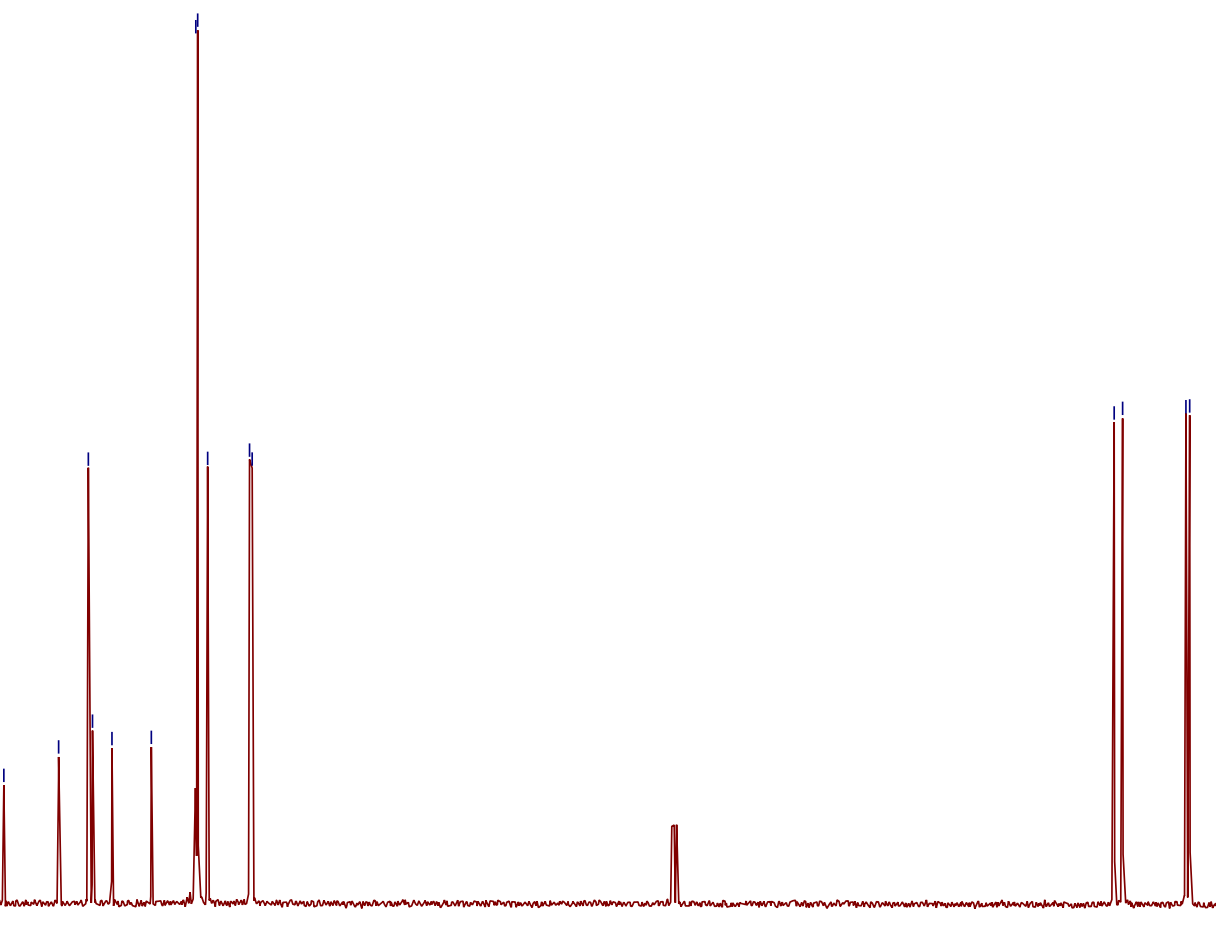

30
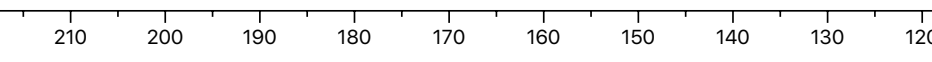

110
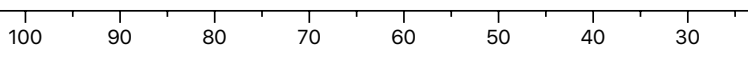
$500 \mathrm{MHz}, \mathrm{CDCl}_{3}$
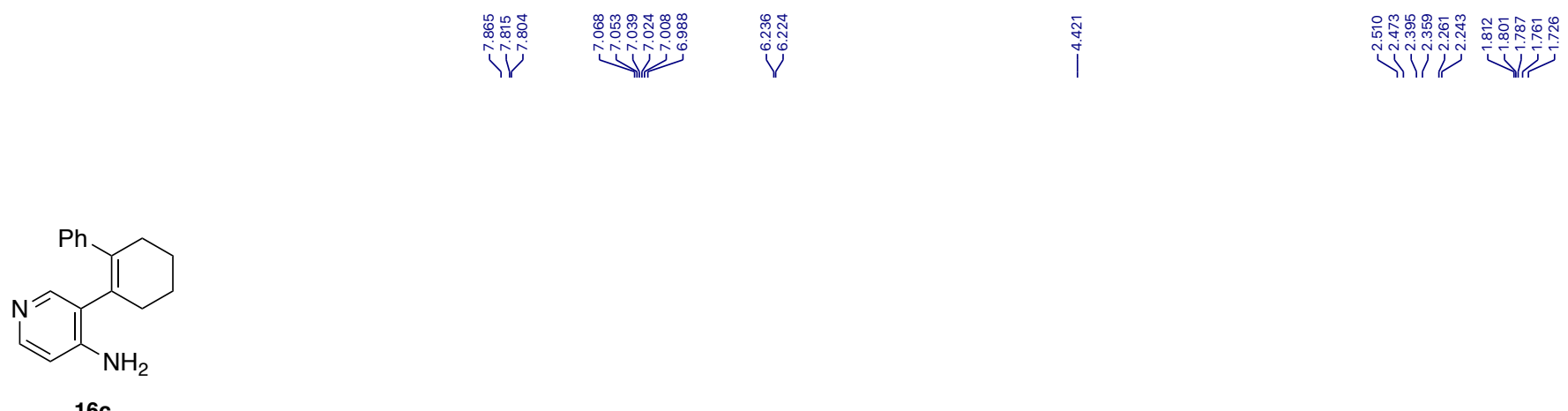

$16 c$

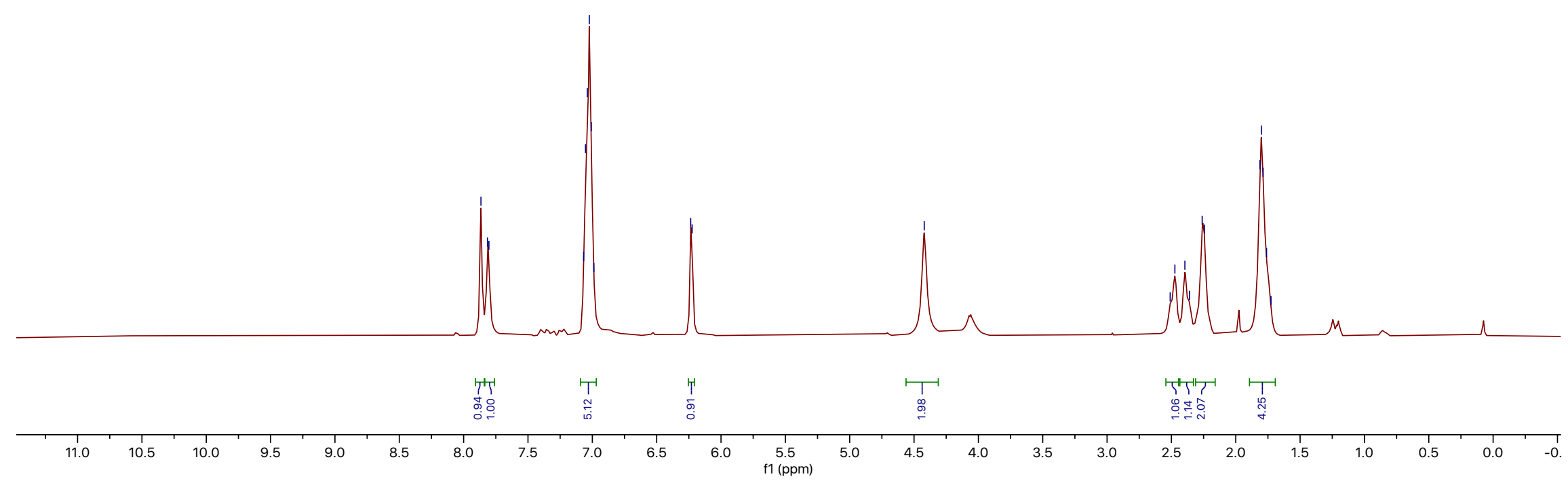


$125 \mathrm{MHz}, \mathrm{CDCl}_{3}$

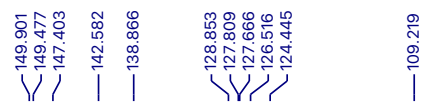
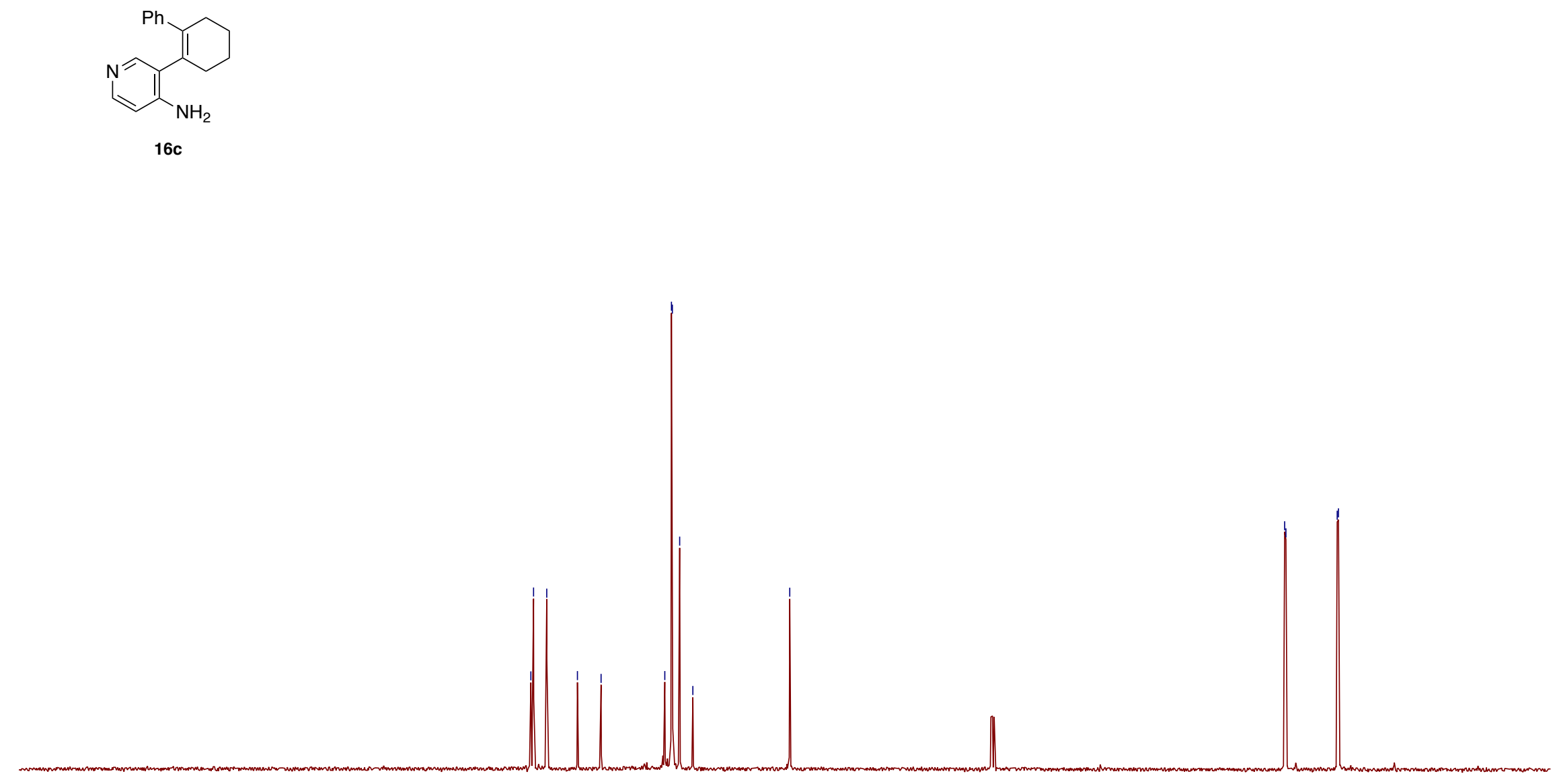

30
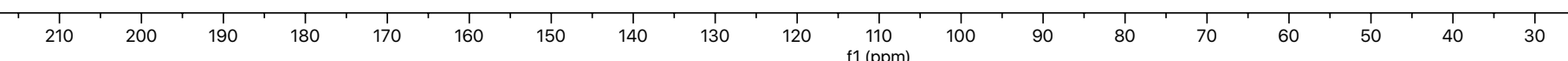
$500 \mathrm{MHz}, \mathrm{CDCl}_{3}$
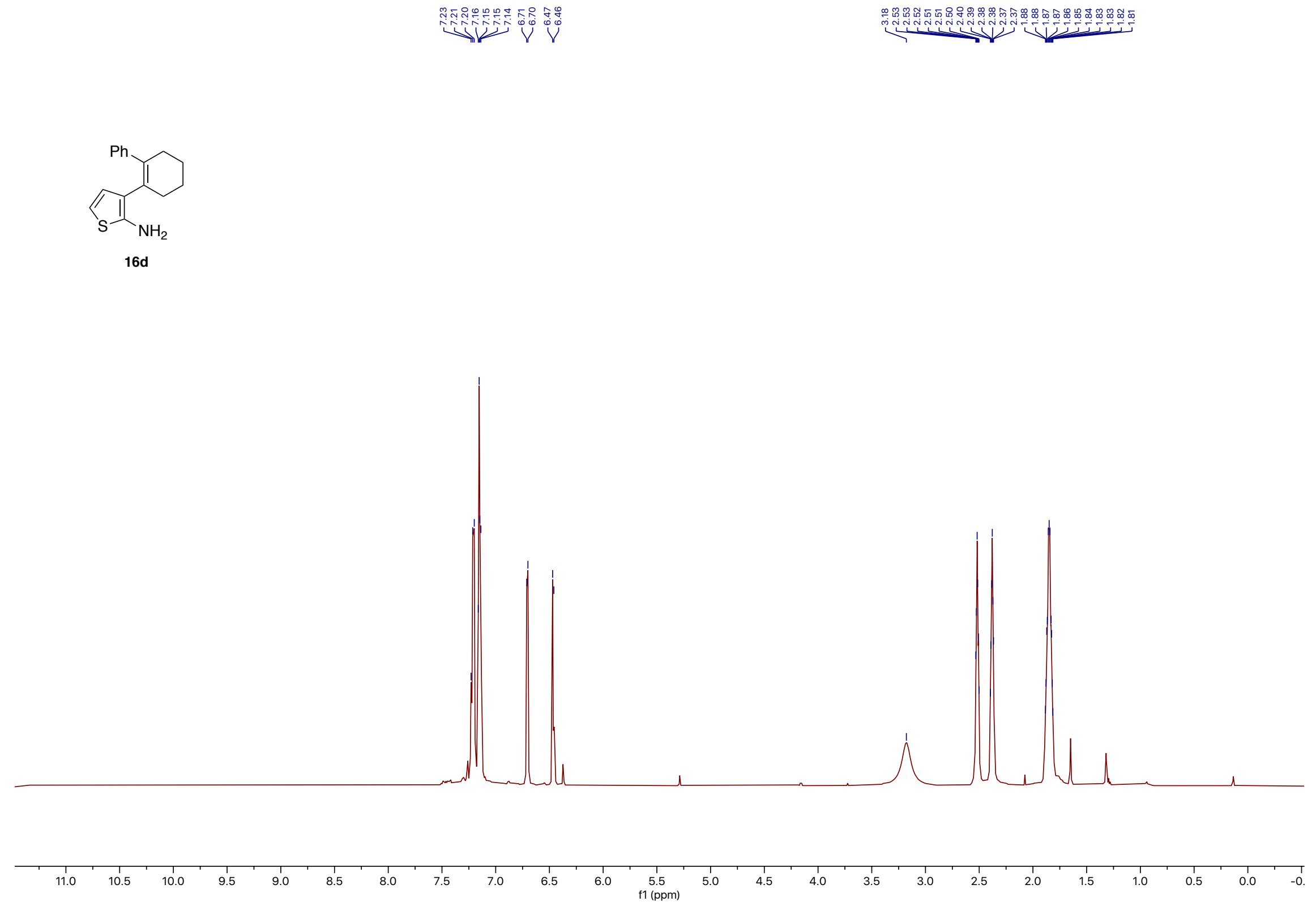
$125 \mathrm{MHz}, \mathrm{CDCl}_{3}$
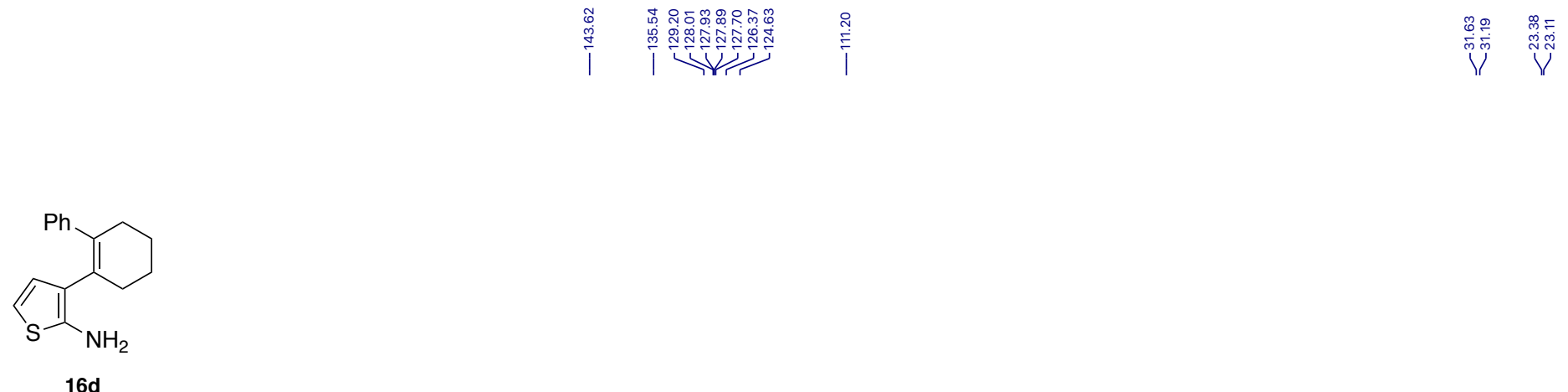

$16 d$
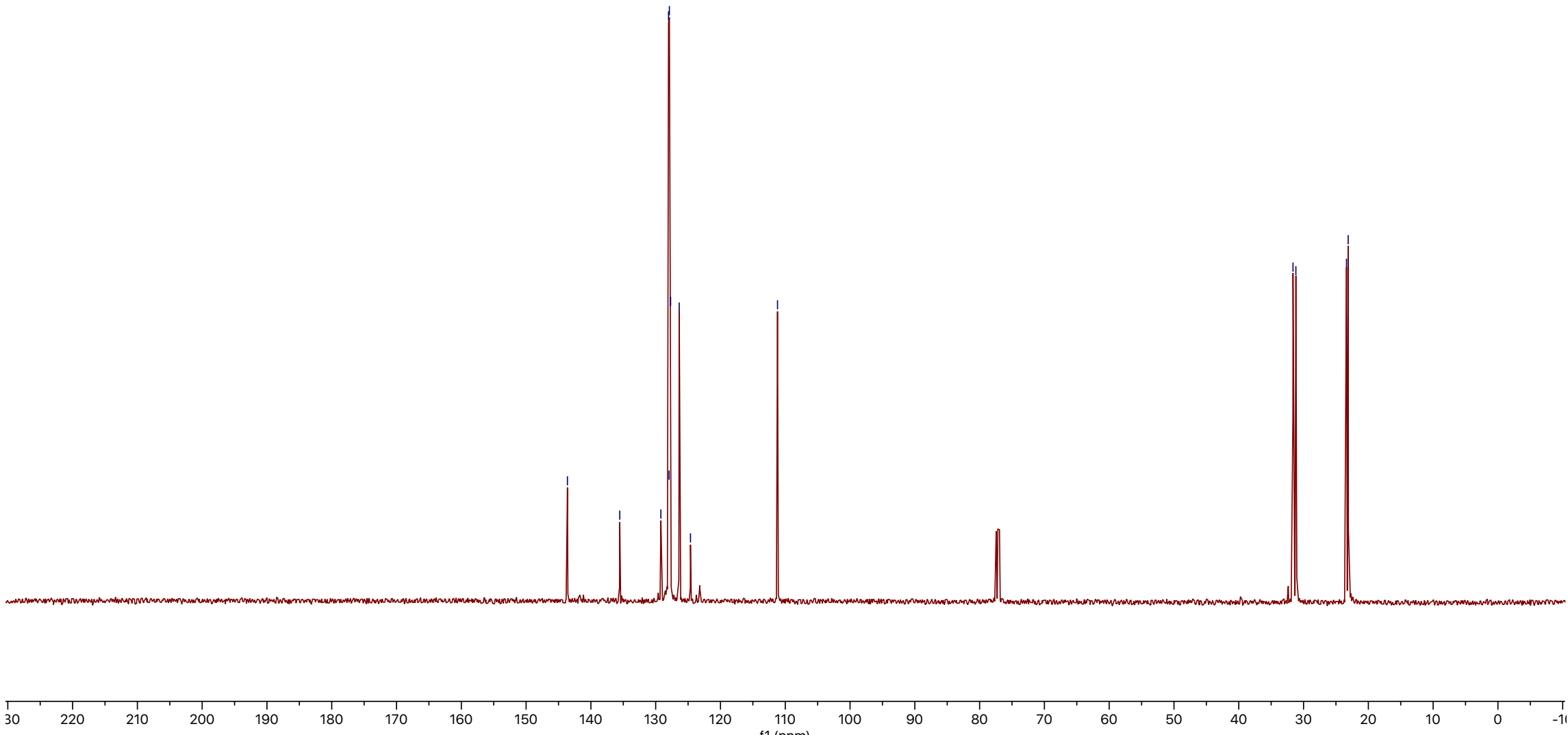
$500 \mathrm{MHz}, \mathrm{CDCl}_{3}$
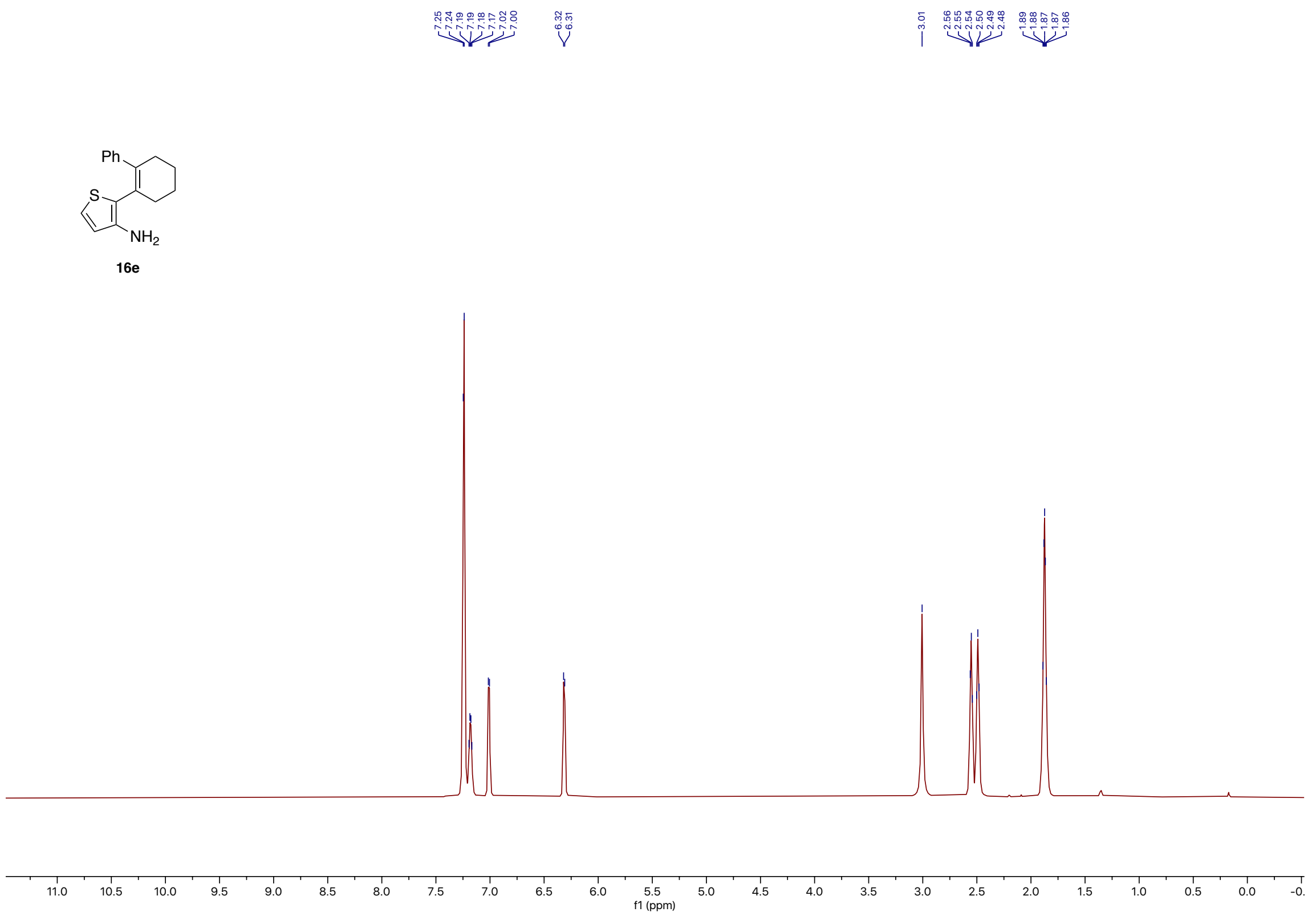
$125 \mathrm{MHz}, \mathrm{CDCl}_{3}$
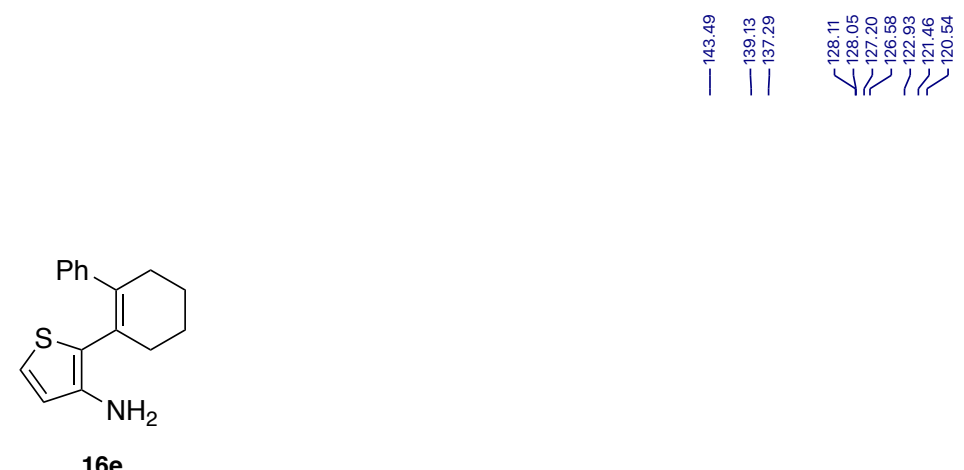

$16 e$

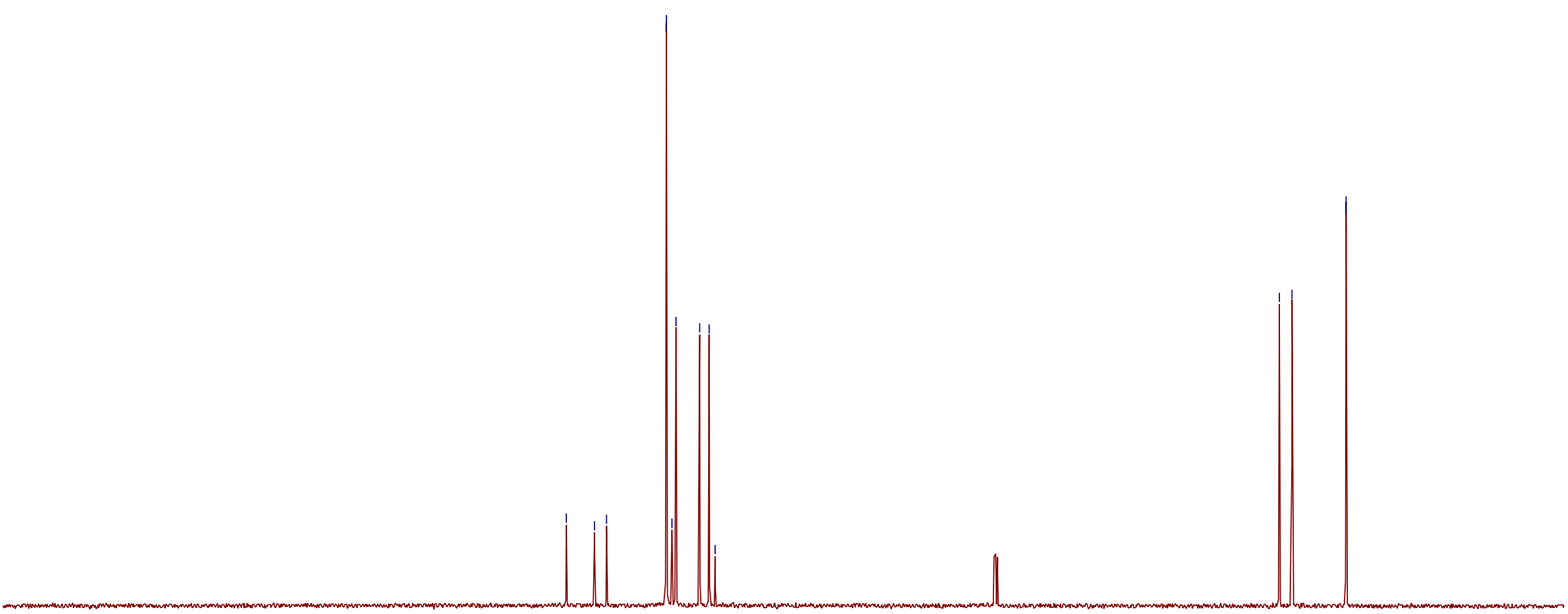

30

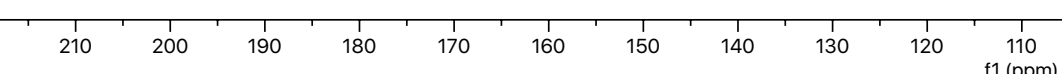
110
f1 (ppm)
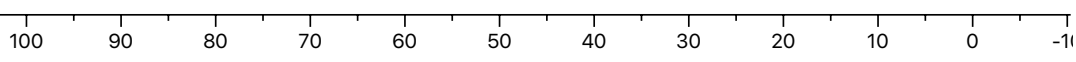
$500 \mathrm{MHz}, \mathrm{CDCl}_{3}$

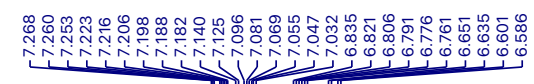

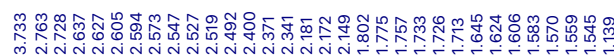

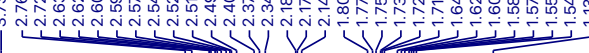
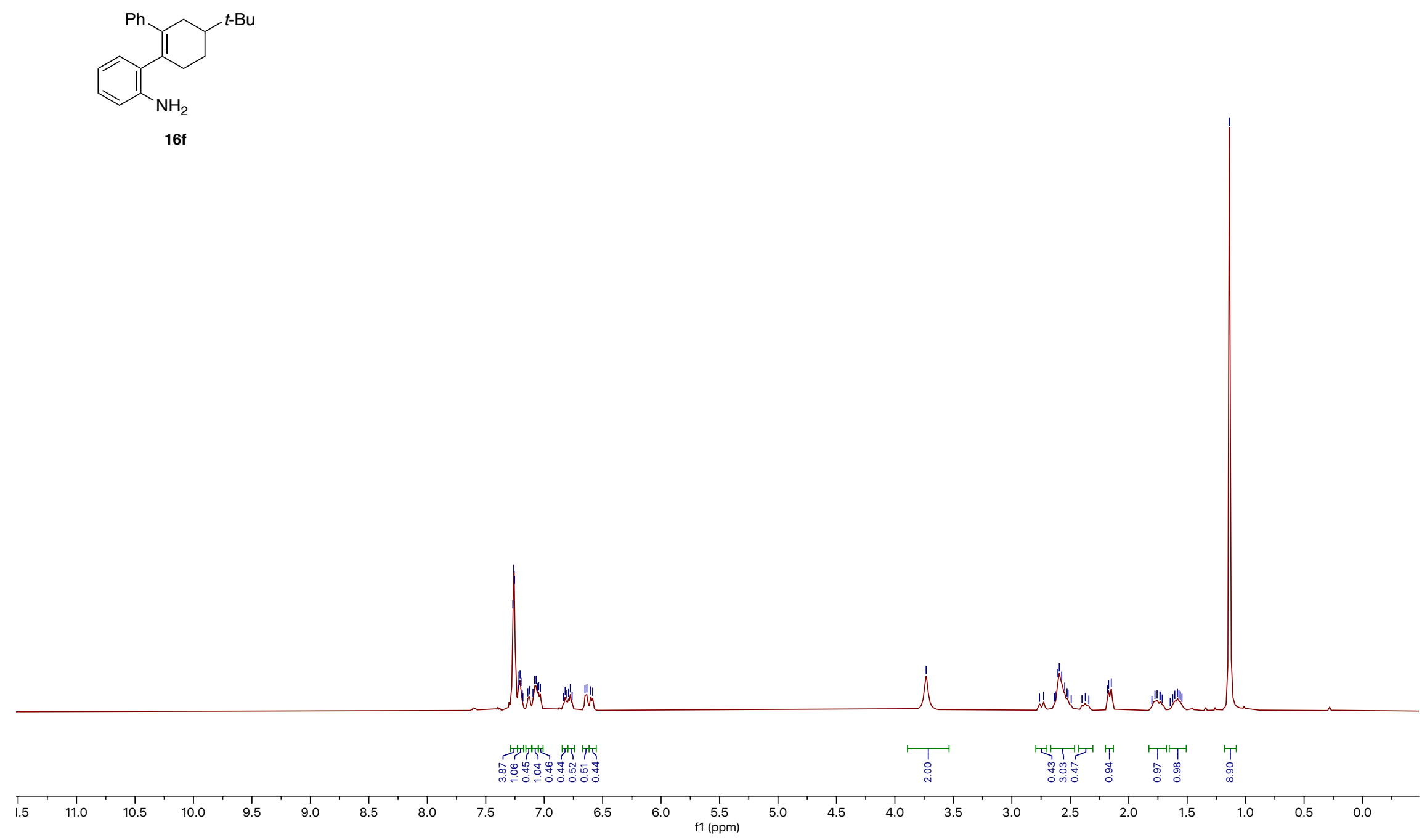
$125 \mathrm{MHz}, \mathrm{CDCl}_{3}$

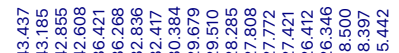

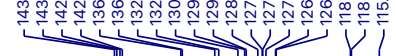

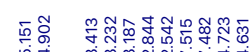

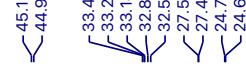

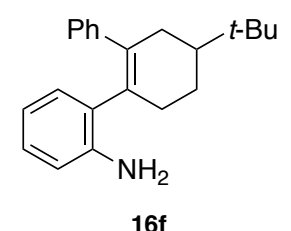

$16 f$

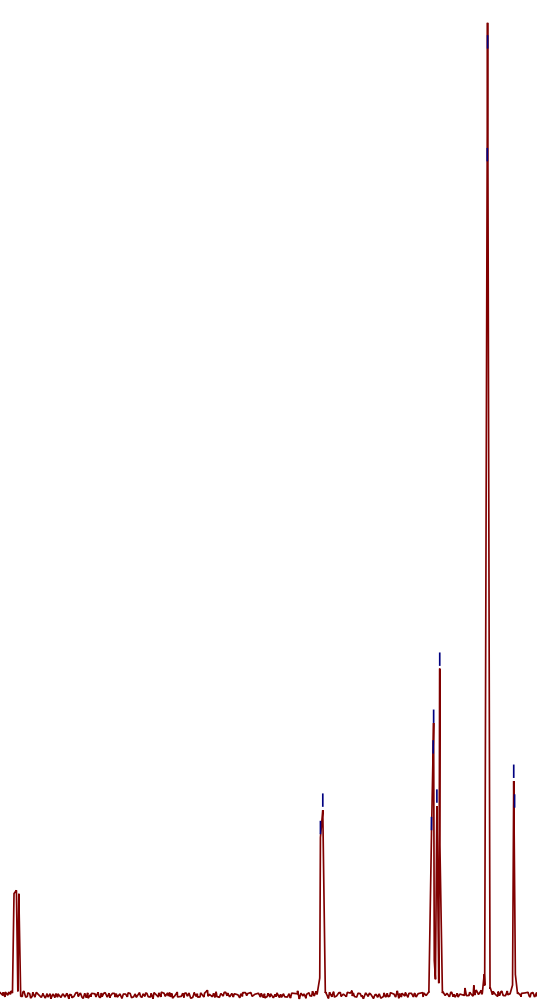

30
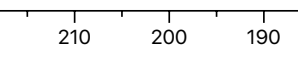

180

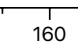

150
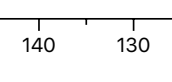

120

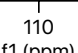

$100 \quad 90$
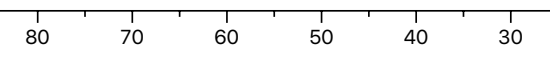
$500 \mathrm{MHz}, \mathrm{CDCl}_{3}$

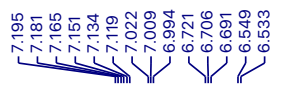

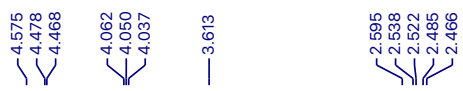

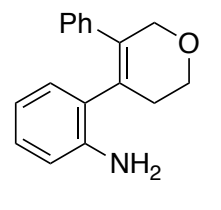

$16 \mathrm{~g}$

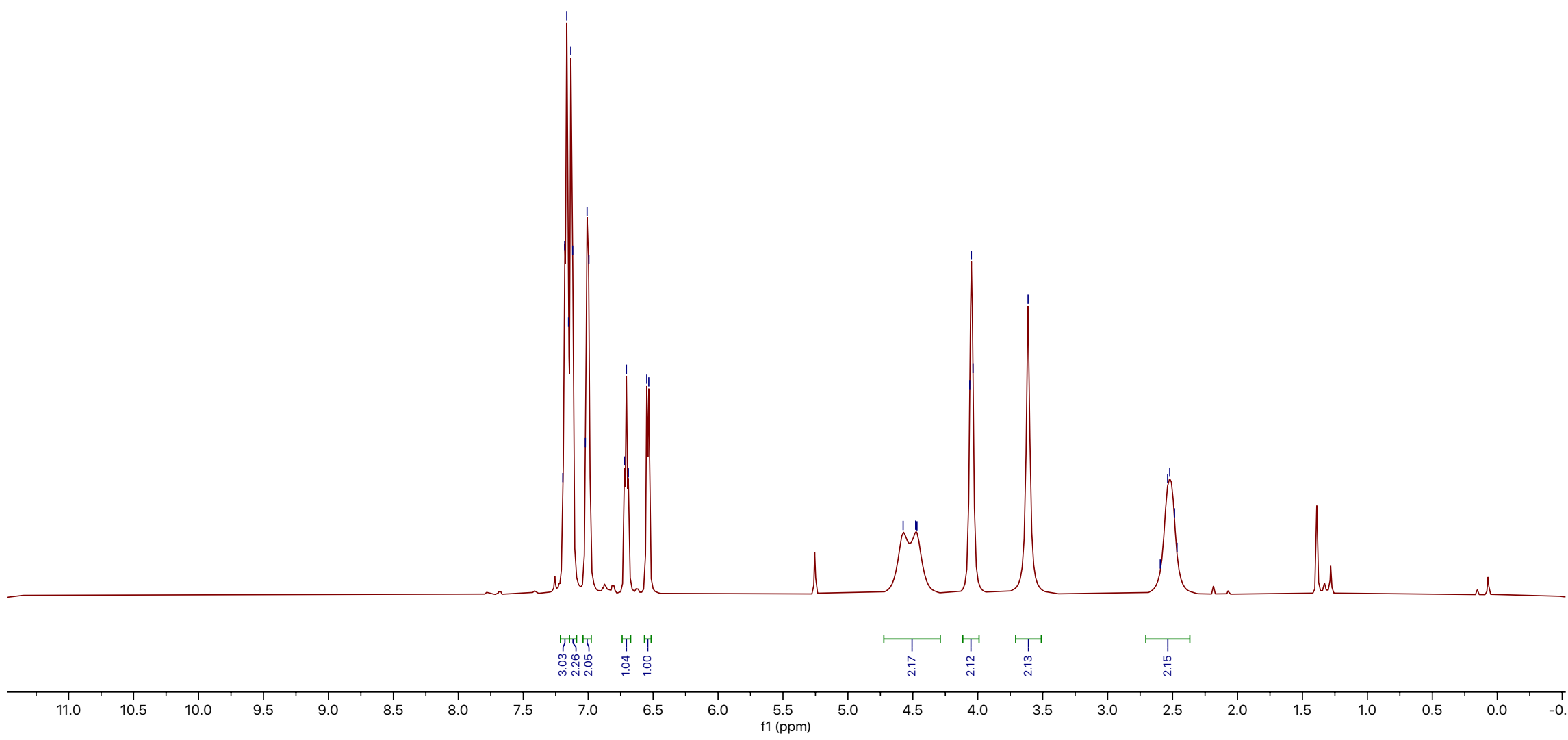


$125 \mathrm{MHz}, \mathrm{CDCl}_{3}$

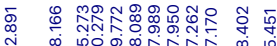
ป
| | | |

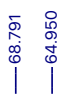
$\left.\right|_{\substack{\infty \\ 0 \\ 0}} ^{\substack{0 \\ 0}}$

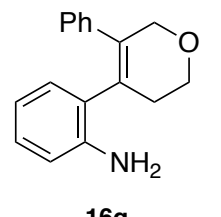

$16 \mathrm{~g}$
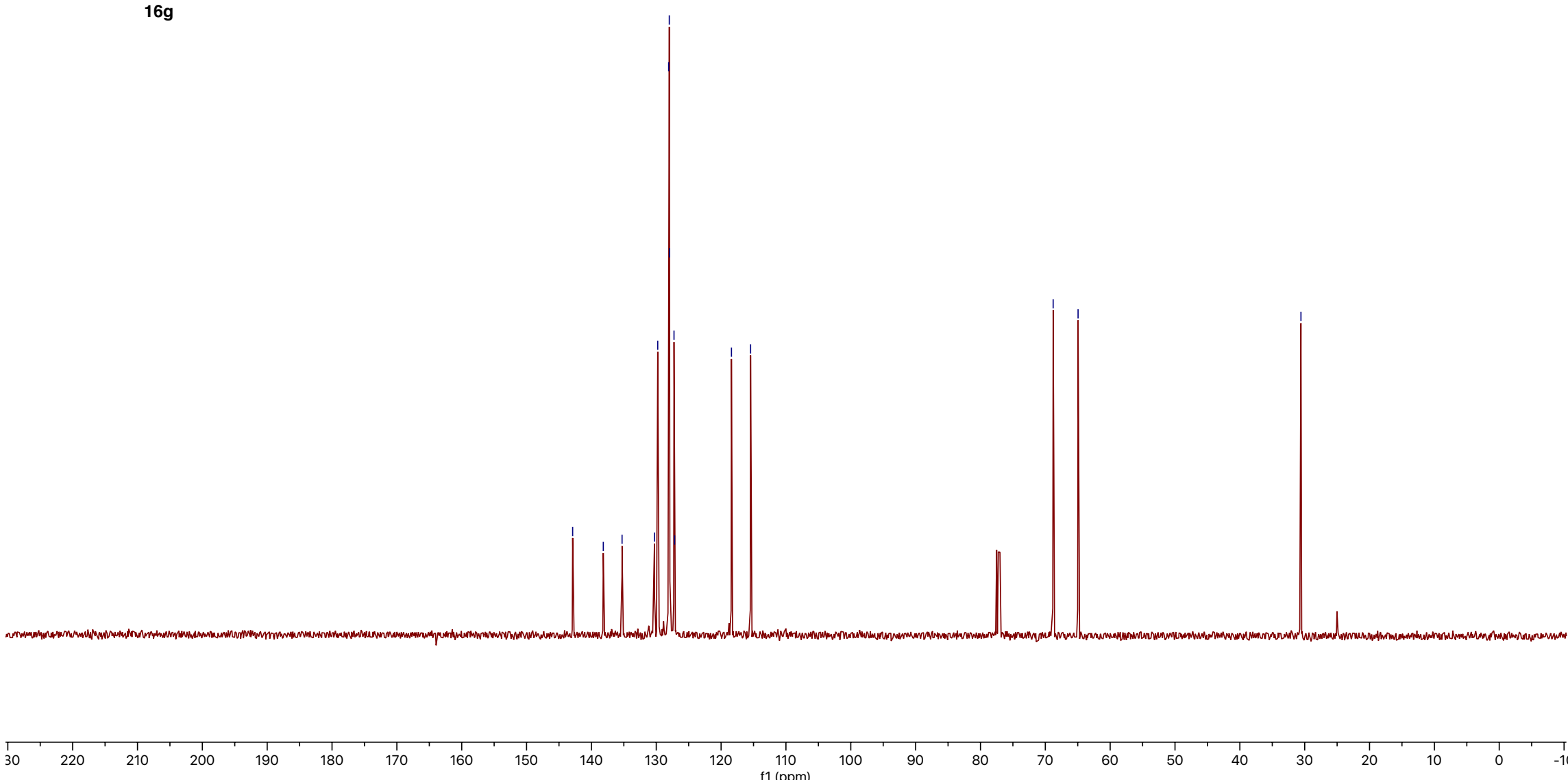
$500 \mathrm{MHz}, \mathrm{CDCl}_{3}$

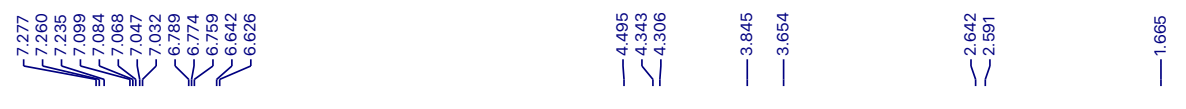

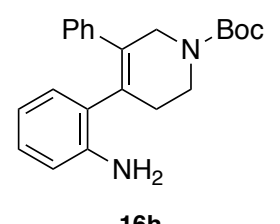

$16 \mathrm{~h}$

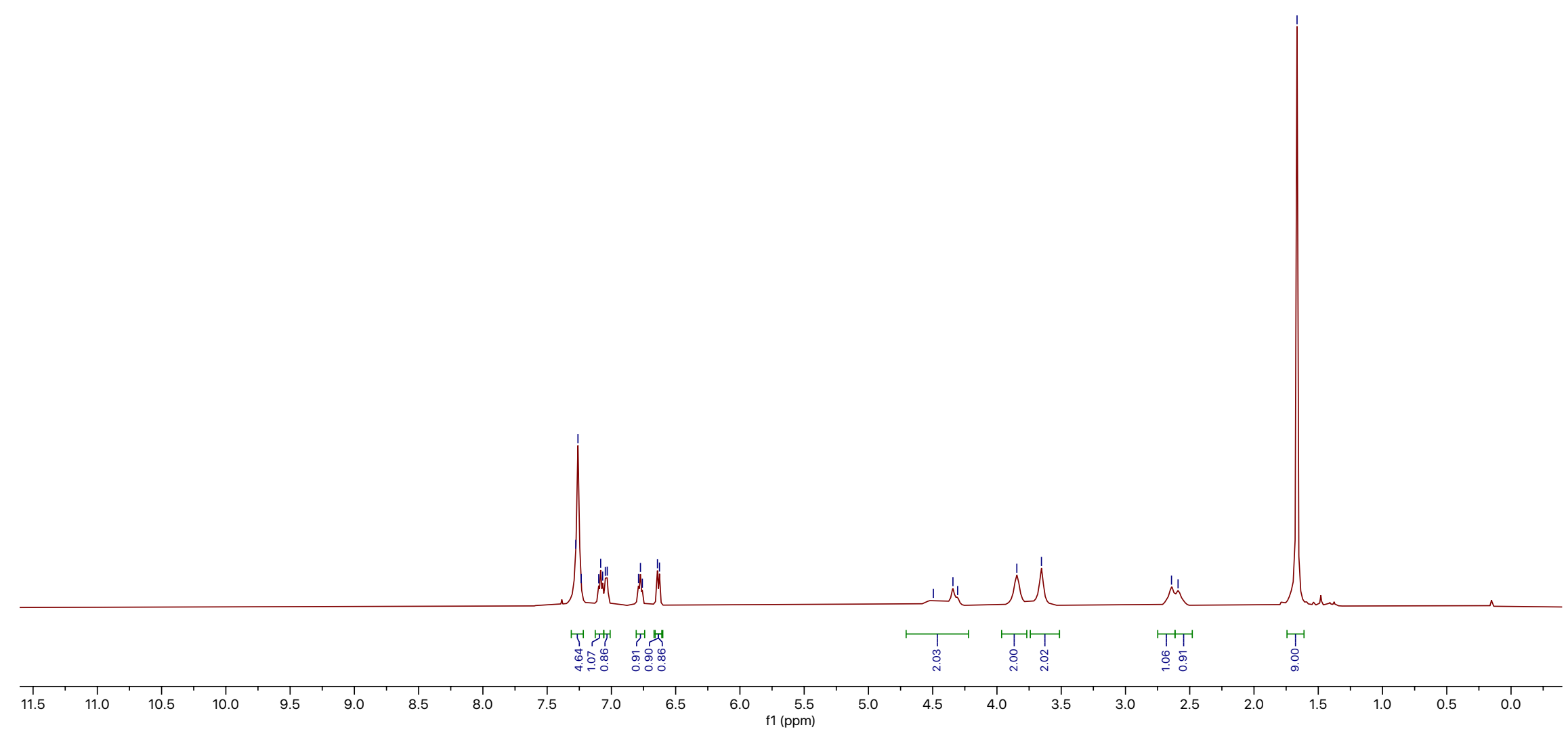


$125 \mathrm{MHz}, \mathrm{CDCl}_{3}$
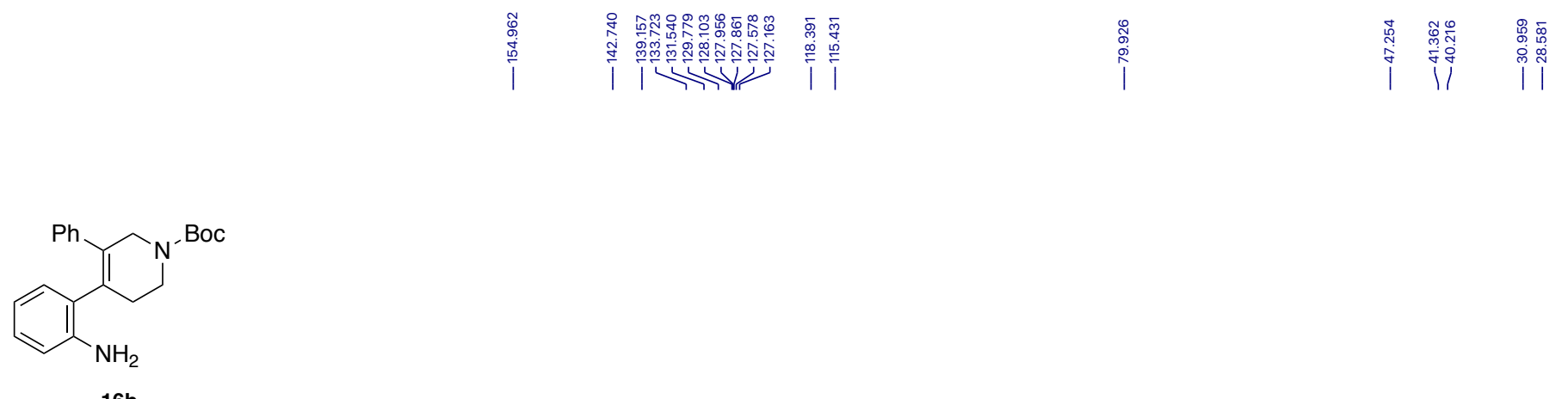

$16 \mathrm{~h}$
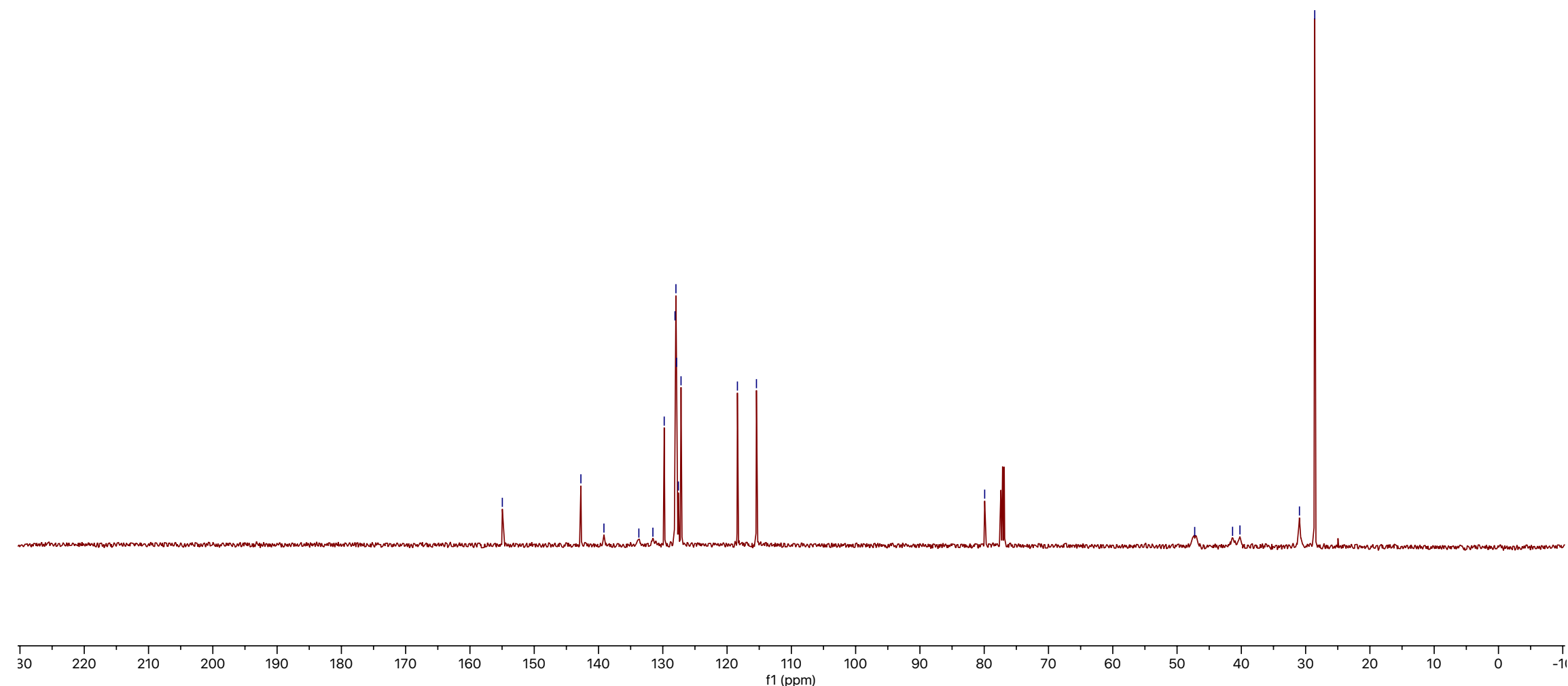
$500 \mathrm{MHz}, \mathrm{CDCl}_{3}$

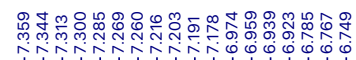
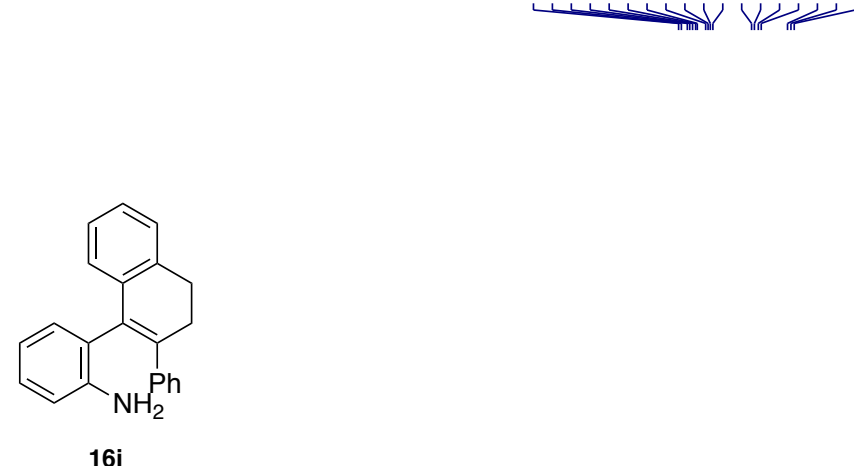

$16 i$

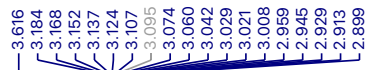

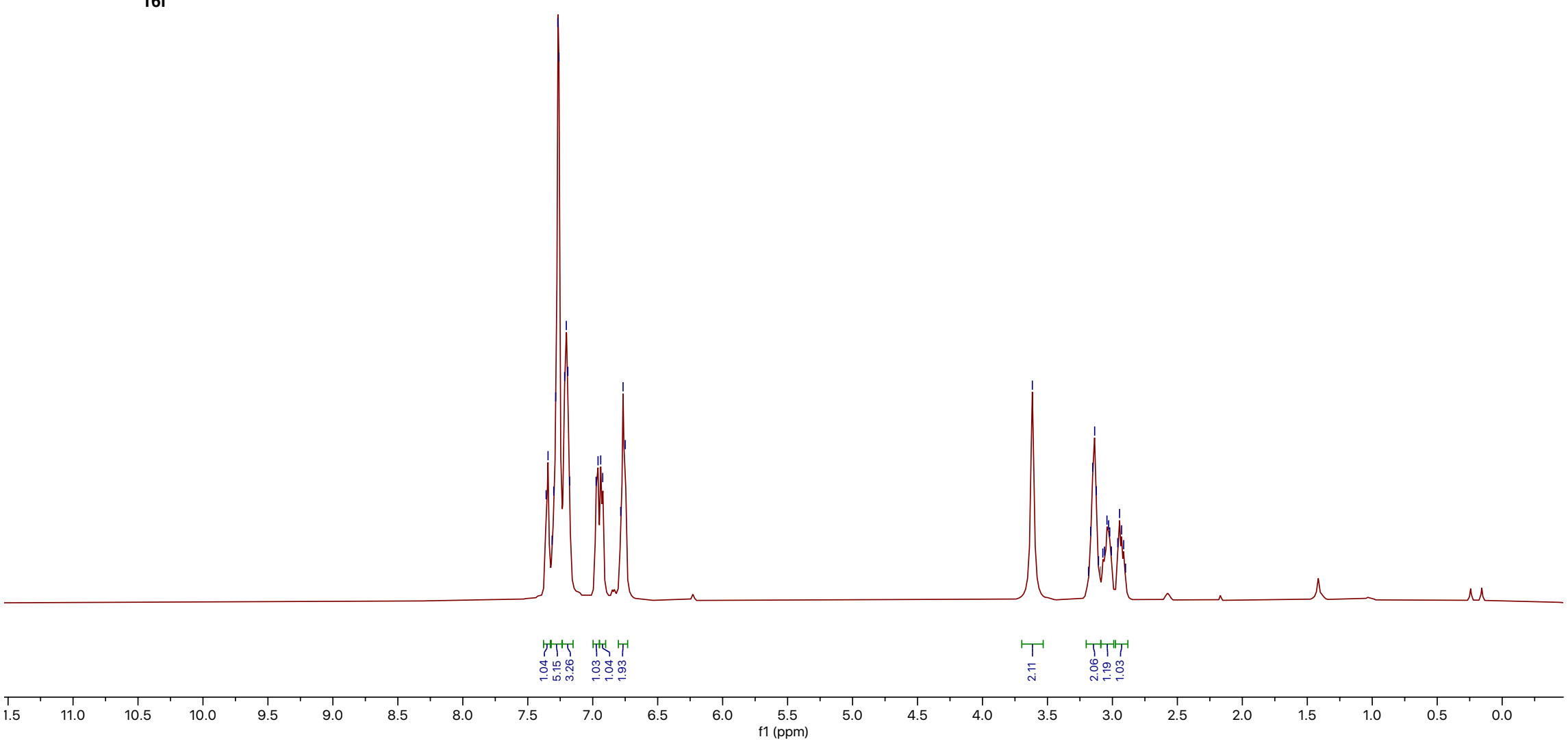


$125 \mathrm{MHz}, \mathrm{CDCl}_{3}$

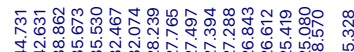

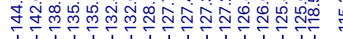

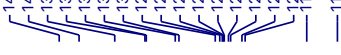

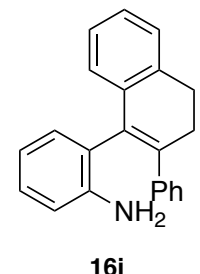

$16 i$

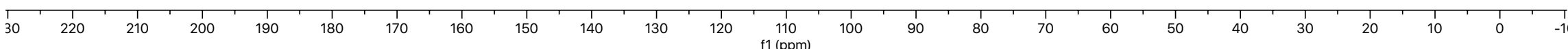


$500 \mathrm{MHz}, \mathrm{CDCl}_{3}$
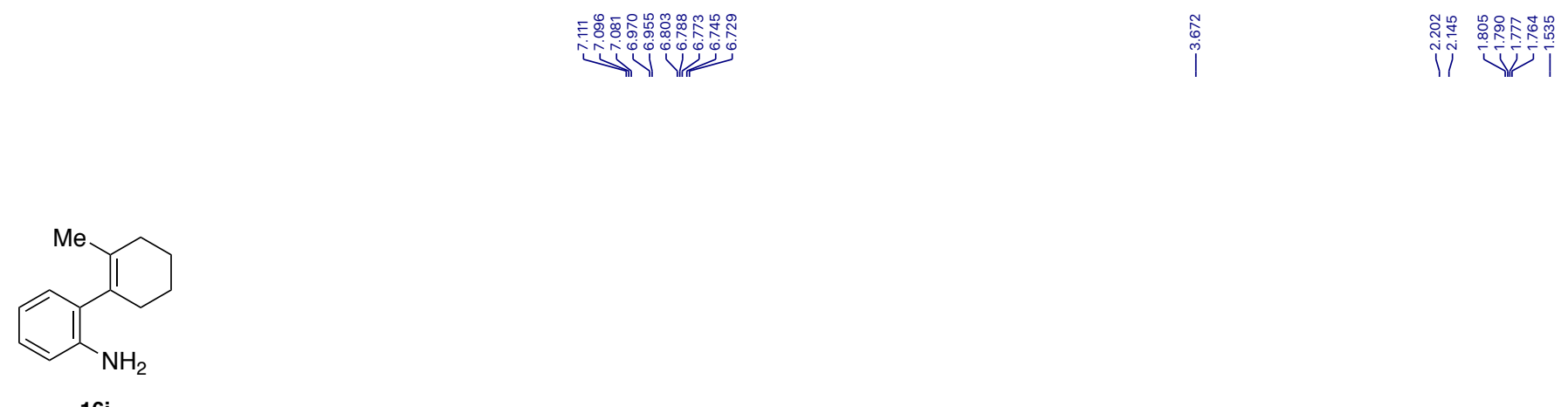

16j

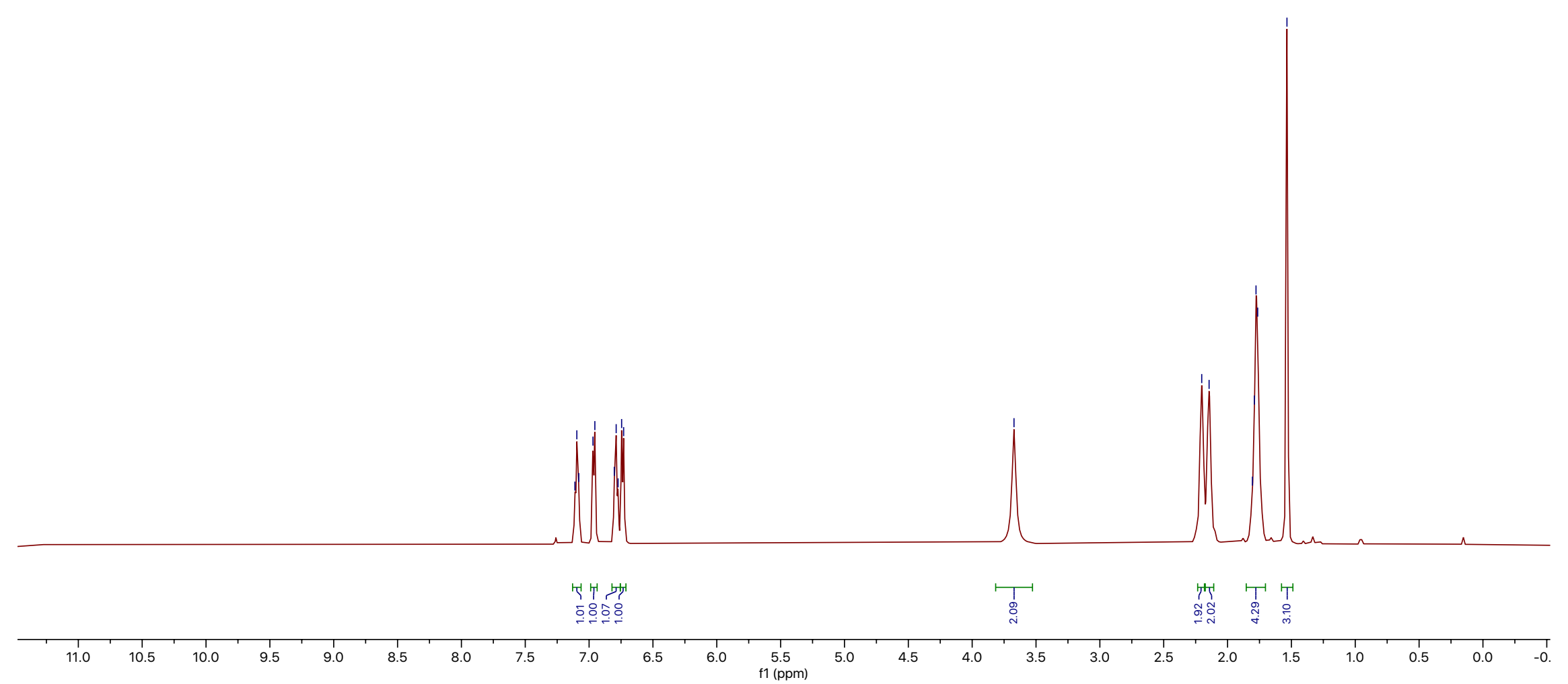


$125 \mathrm{MHz}, \mathrm{CDCl}_{3}$

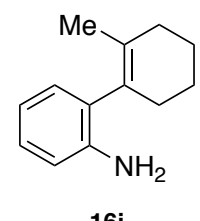

16j
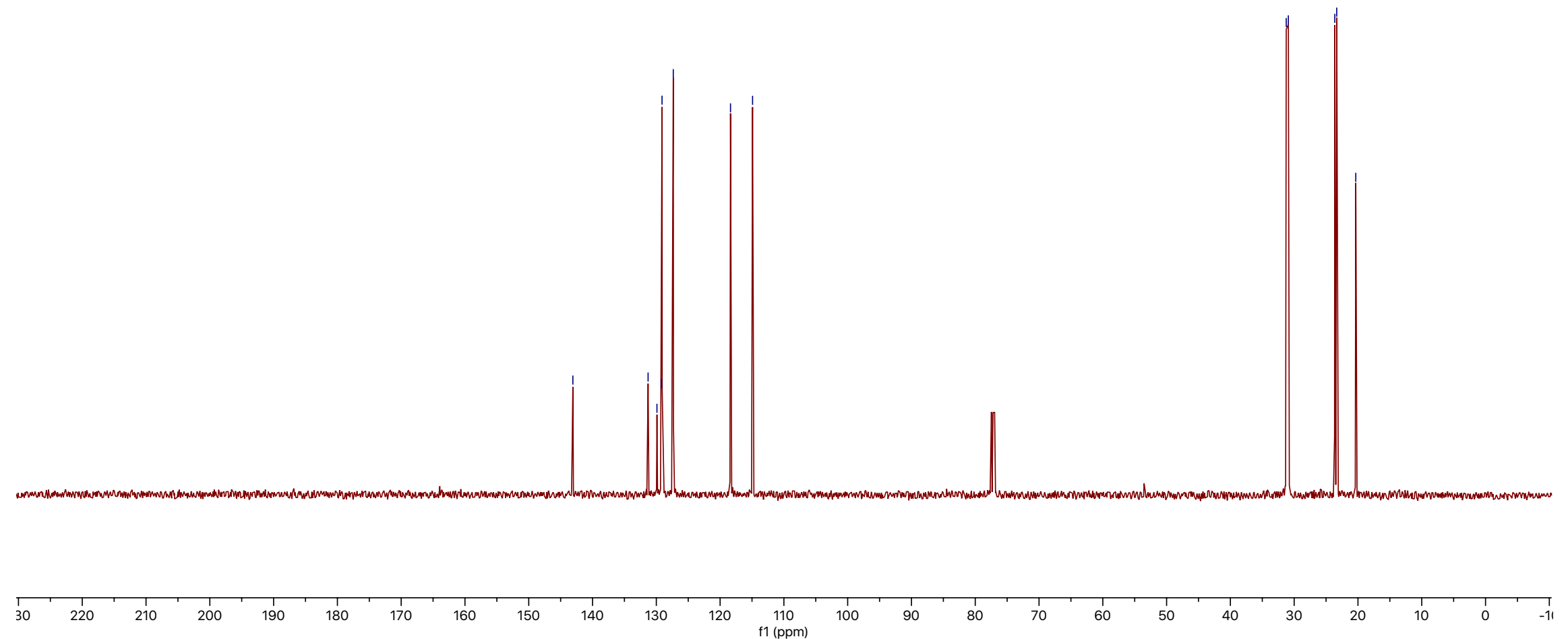
$500 \mathrm{MHz}, \mathrm{CDCl}_{3}$
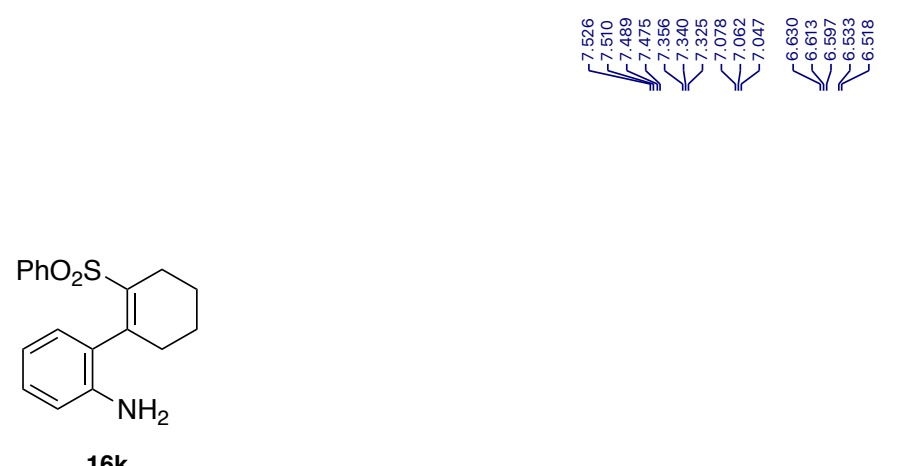

$16 k$
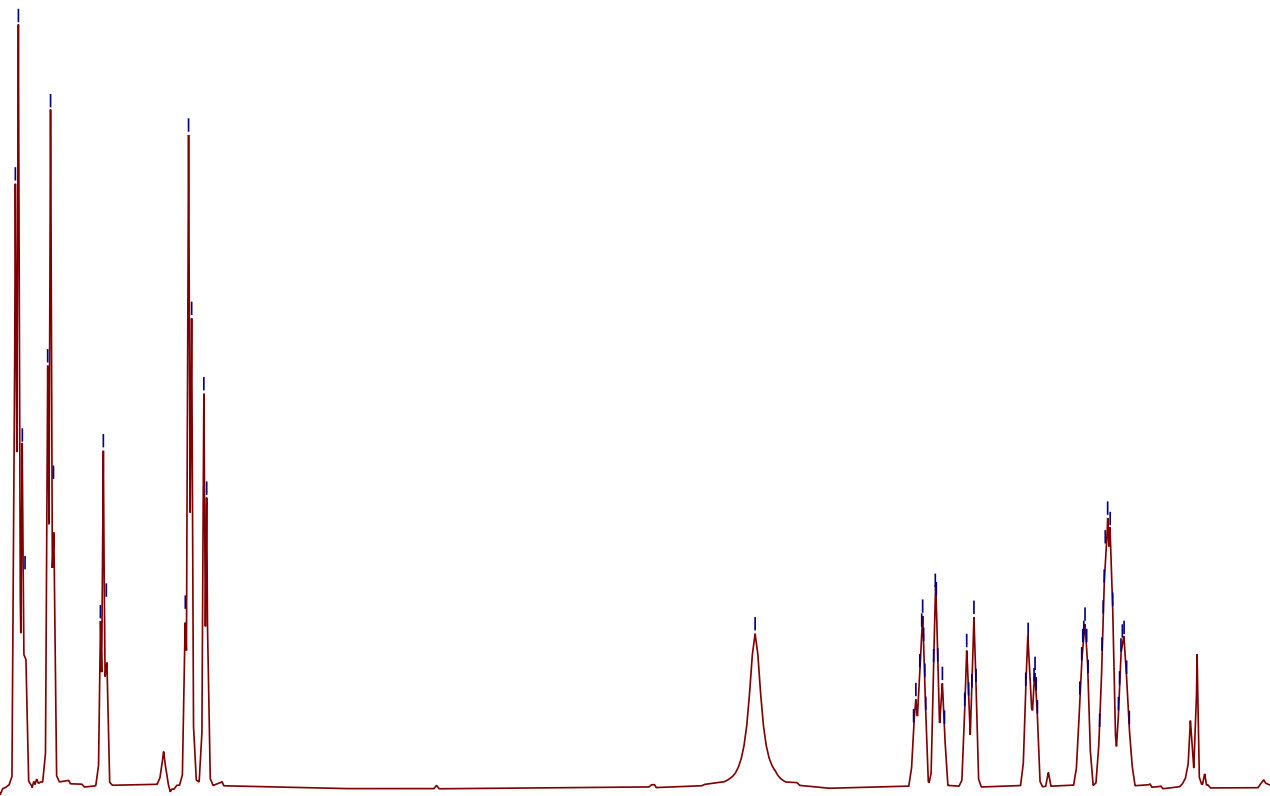

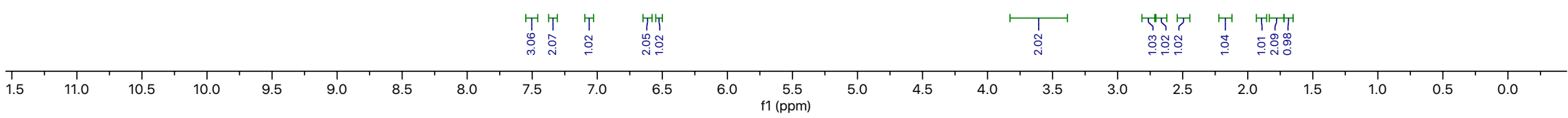


$125 \mathrm{MHz}, \mathrm{CDCl}_{3}$
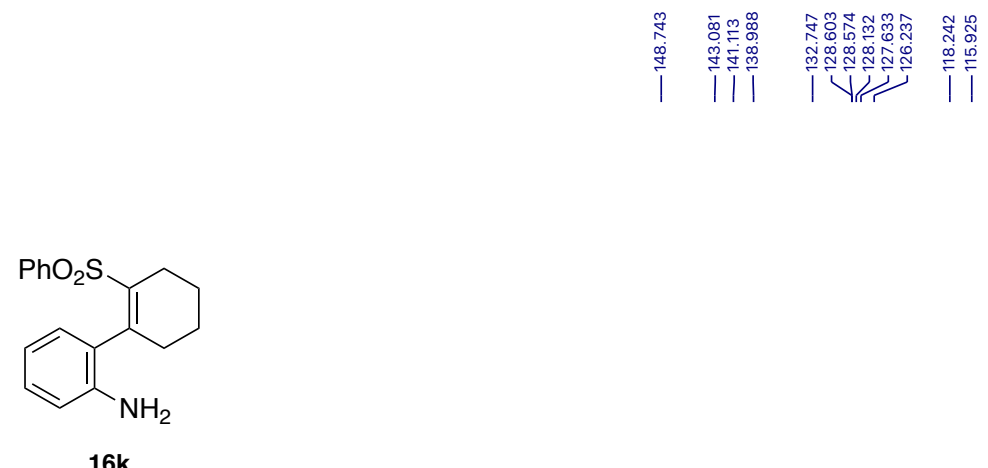

$16 k$

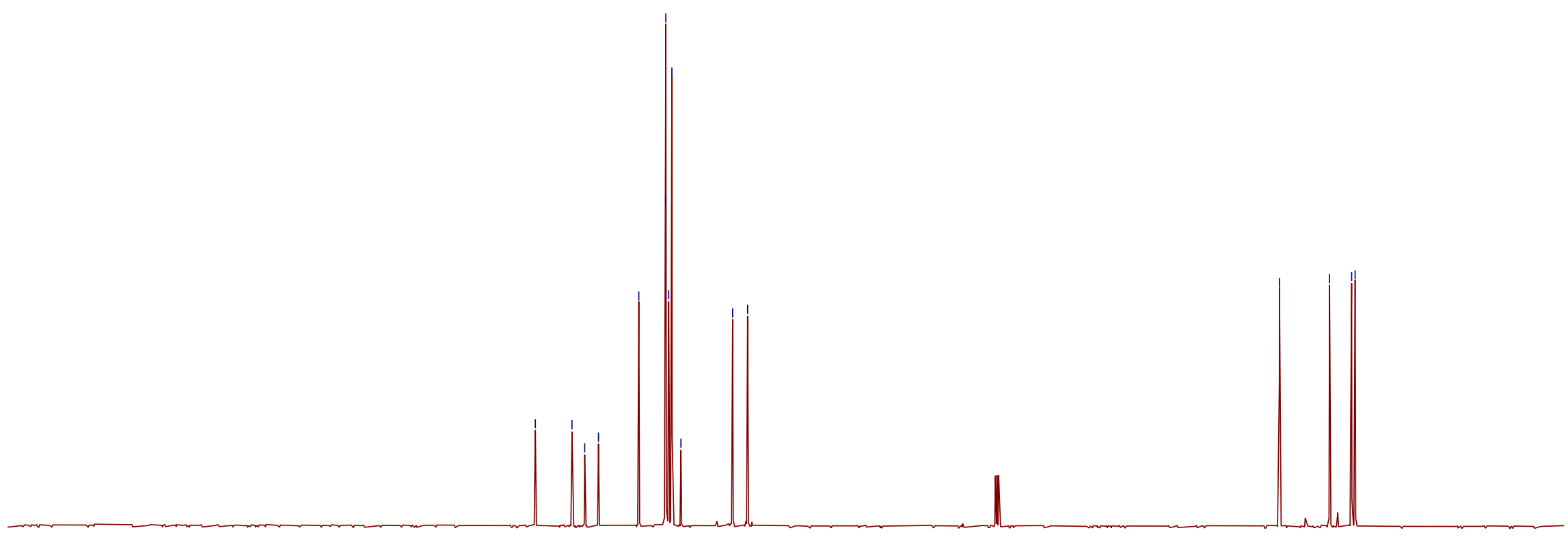

30

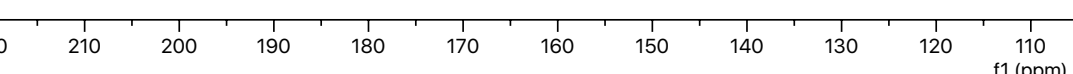
110
$\mathrm{f} 1(\mathrm{ppm})$
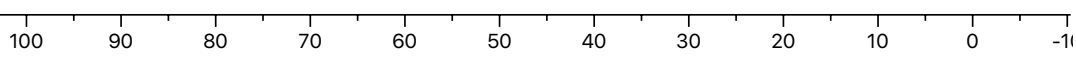
$500 \mathrm{MHz}, \mathrm{CDCl}_{3}$

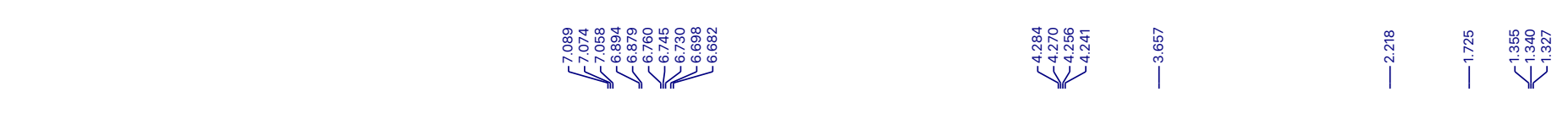<smiles>CCOC(=O)/C(C)=C(\C)c1ccccc1N</smiles>

$16 \mathrm{I}$

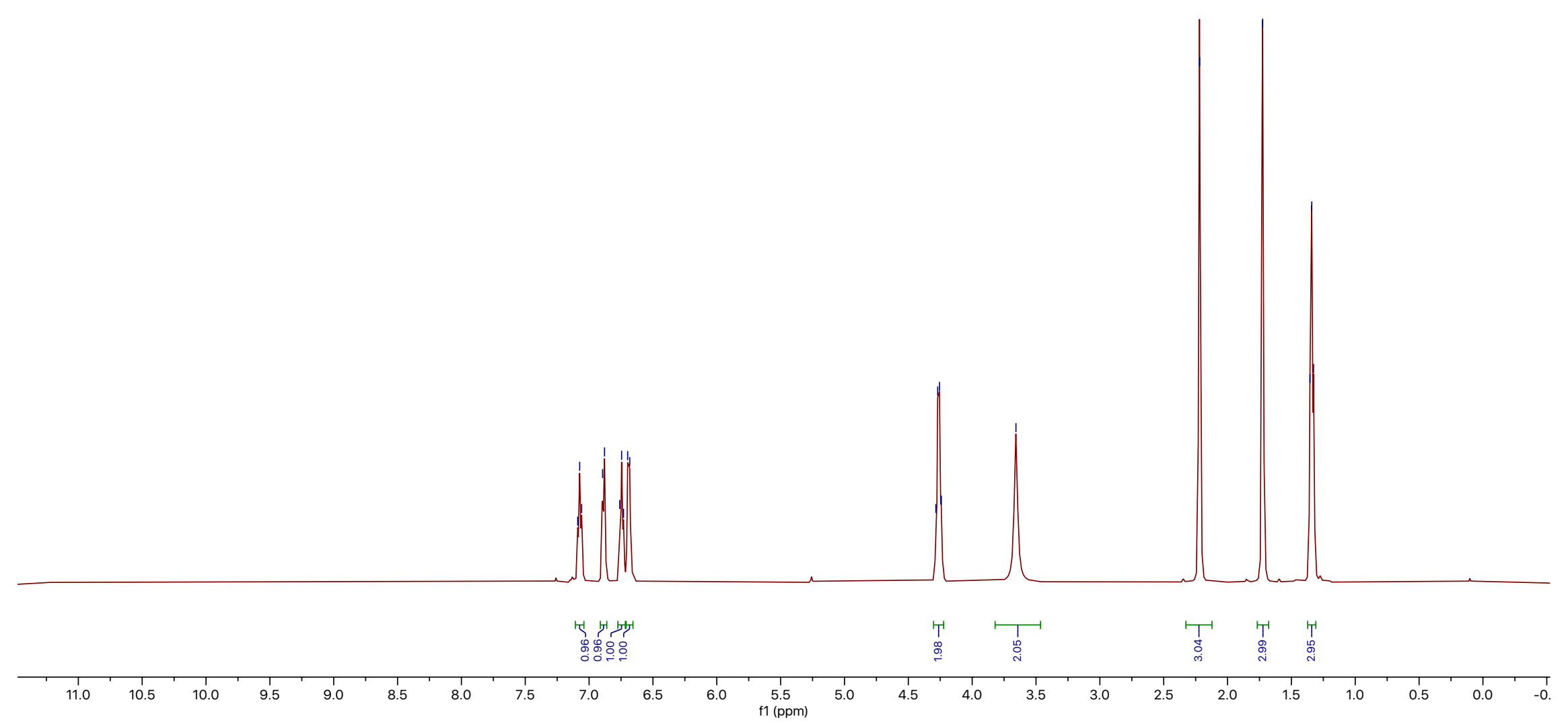


$125 \mathrm{MHz}, \mathrm{CDCl}_{3}$
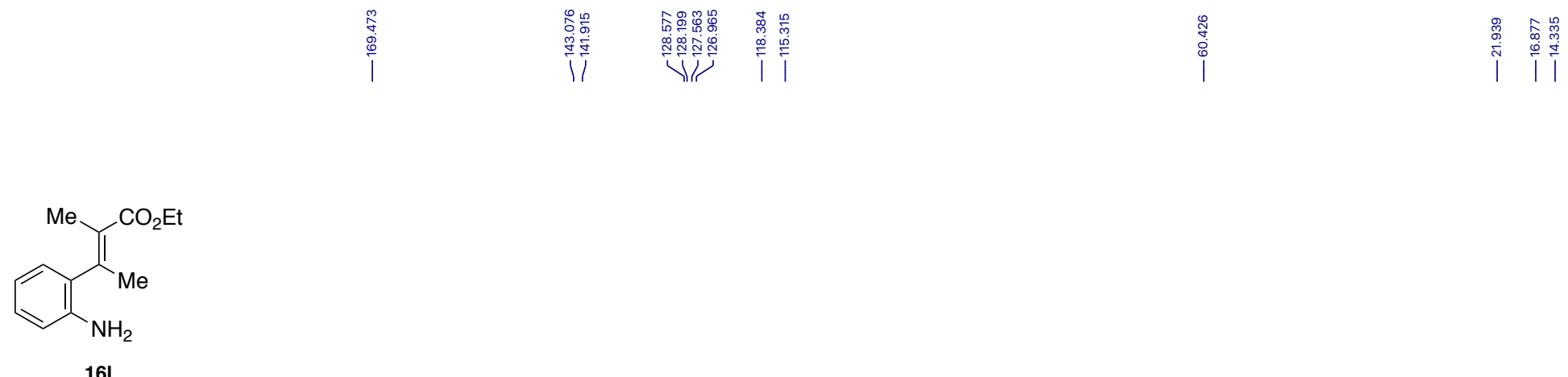

$16 I$
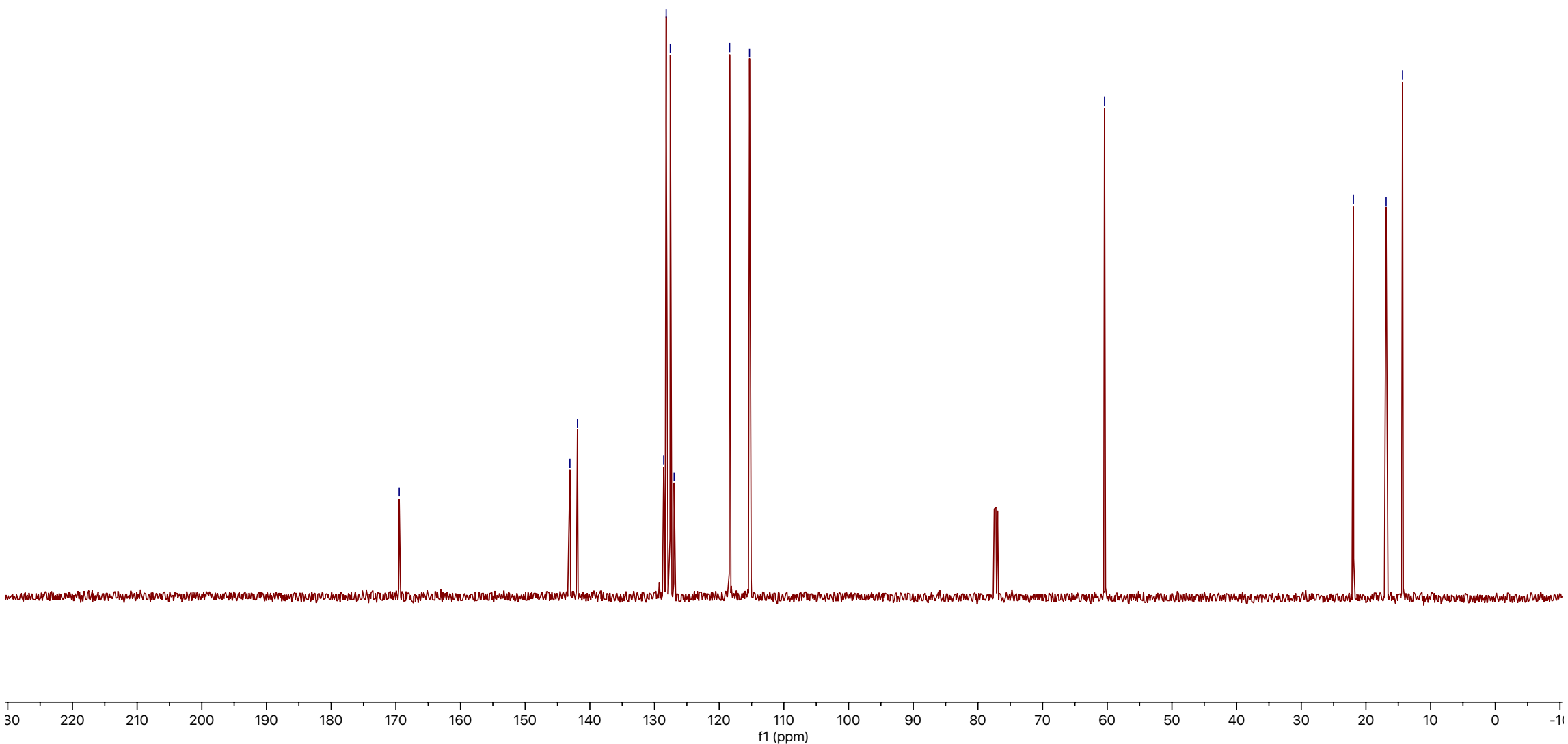
$500 \mathrm{MHz}, \mathrm{CDCl}_{3}$
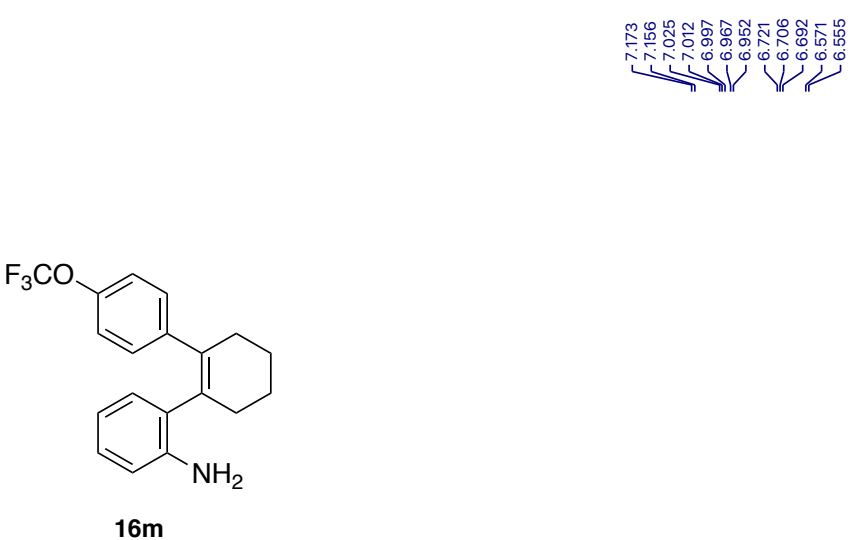

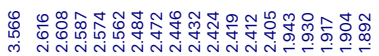

1

$16 \mathrm{~m}$

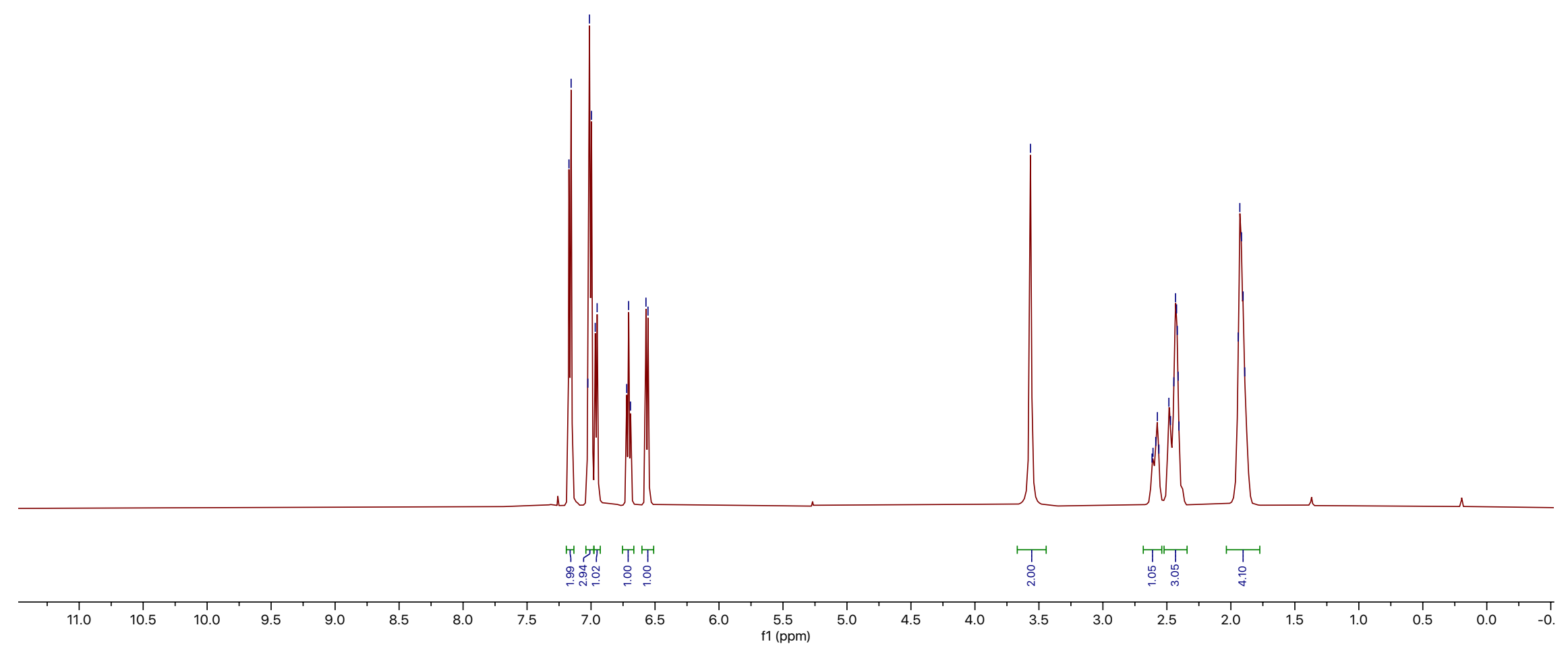


$125 \mathrm{MHz}, \mathrm{CDCl}_{3}$

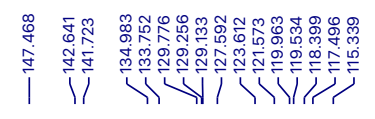

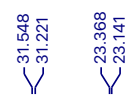

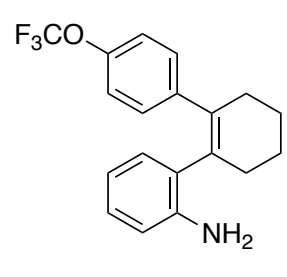

$16 m$

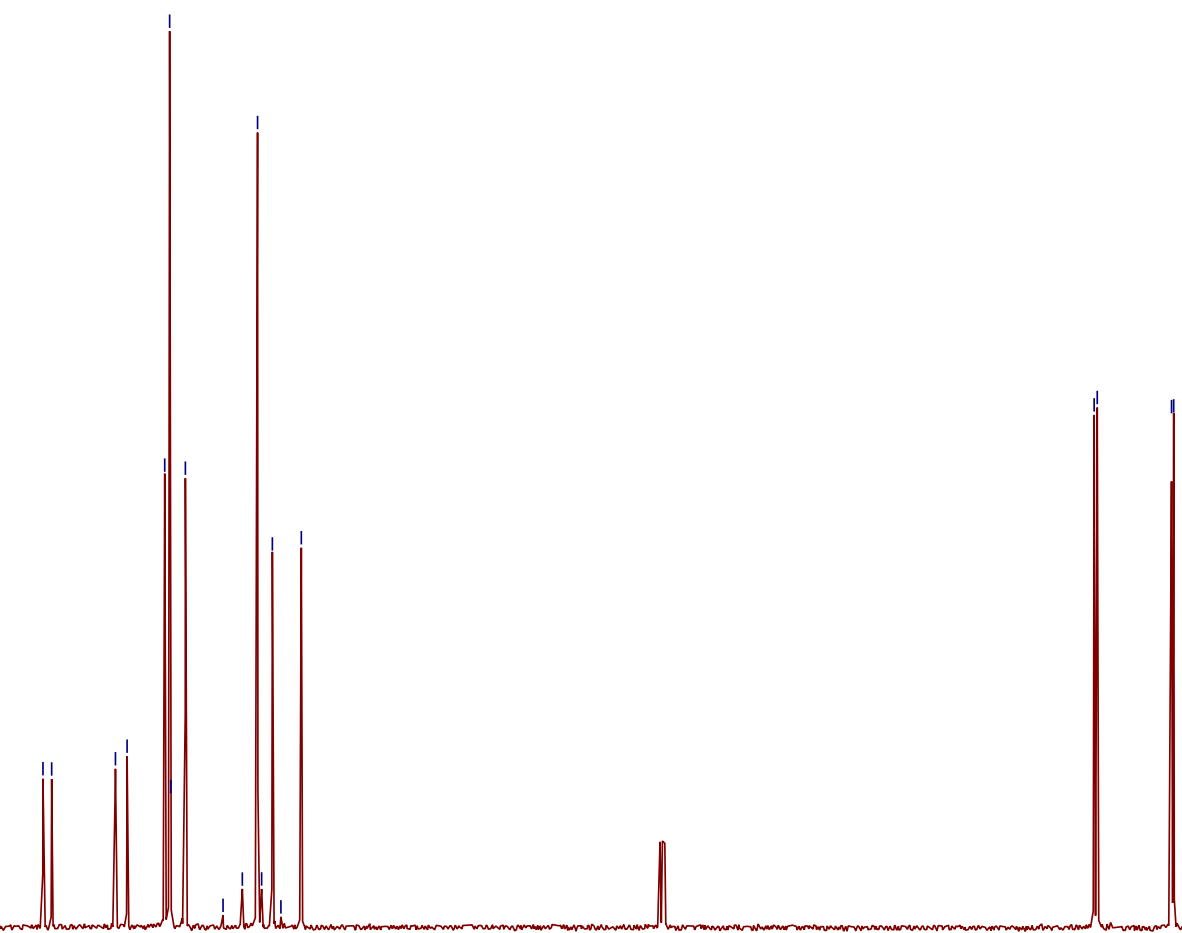

30
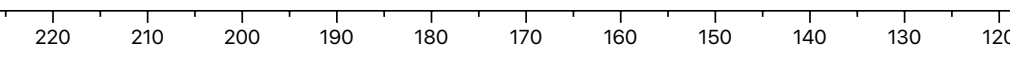

110
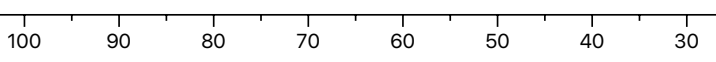
$500 \mathrm{MHz}, \mathrm{CDCl}_{3}$
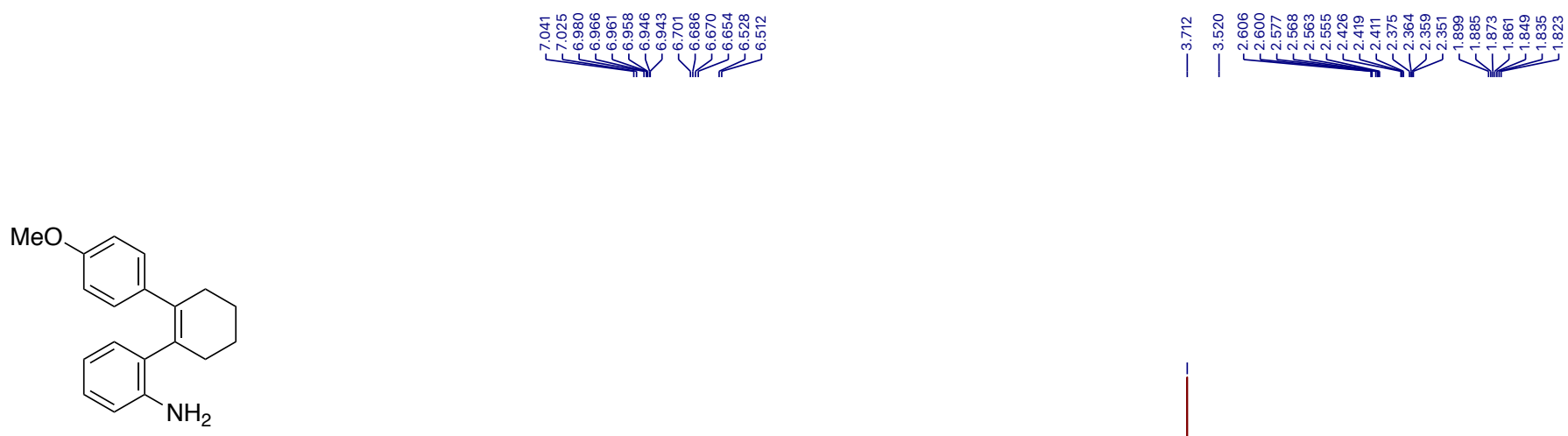

16n

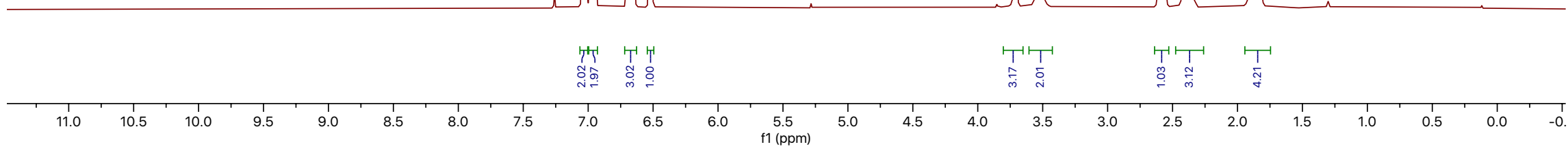


$125 \mathrm{MHz}, \mathrm{CDCl}_{3}$
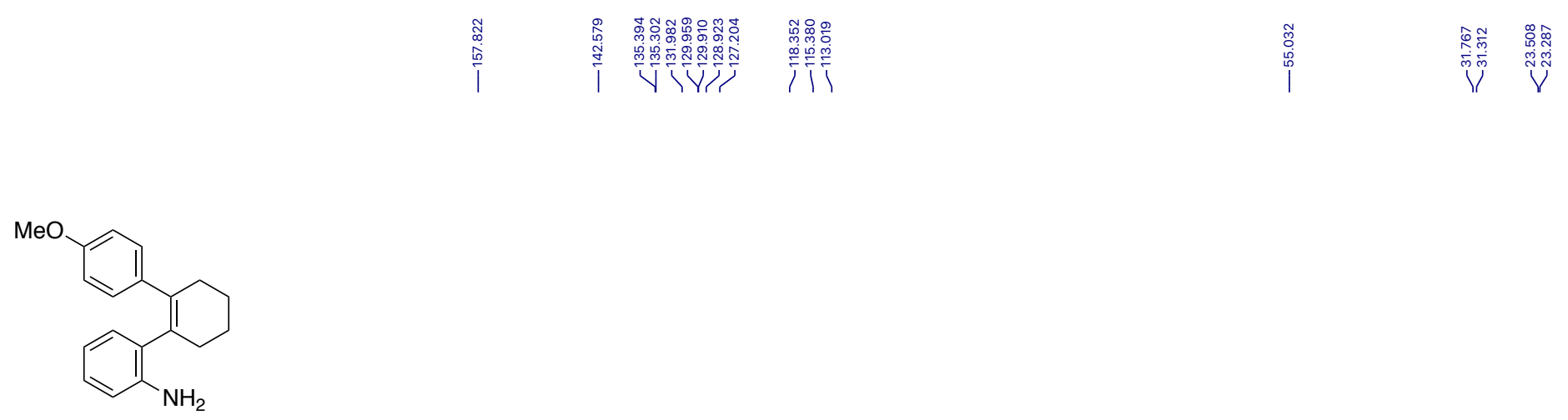

16n
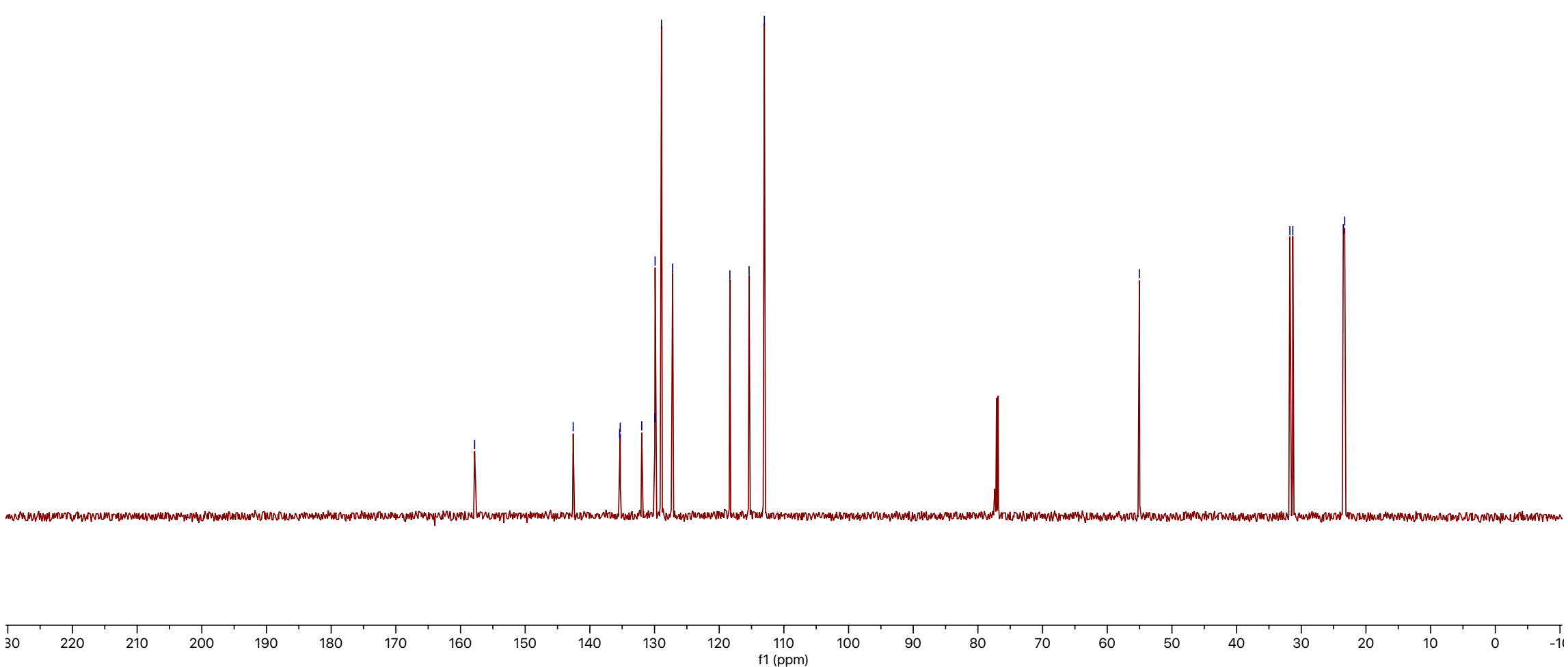
$500 \mathrm{MHz}, \mathrm{CDCl}_{3}$

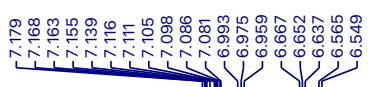

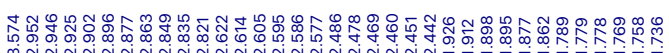
inininivivi
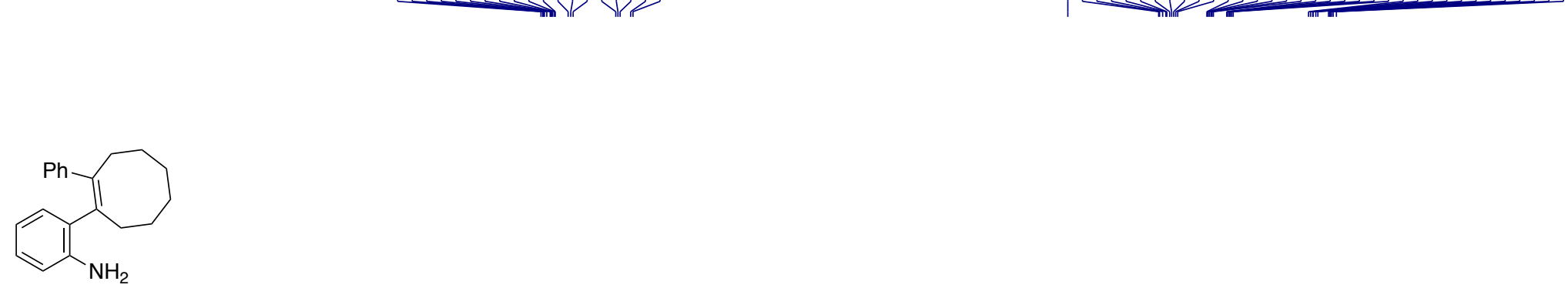

160

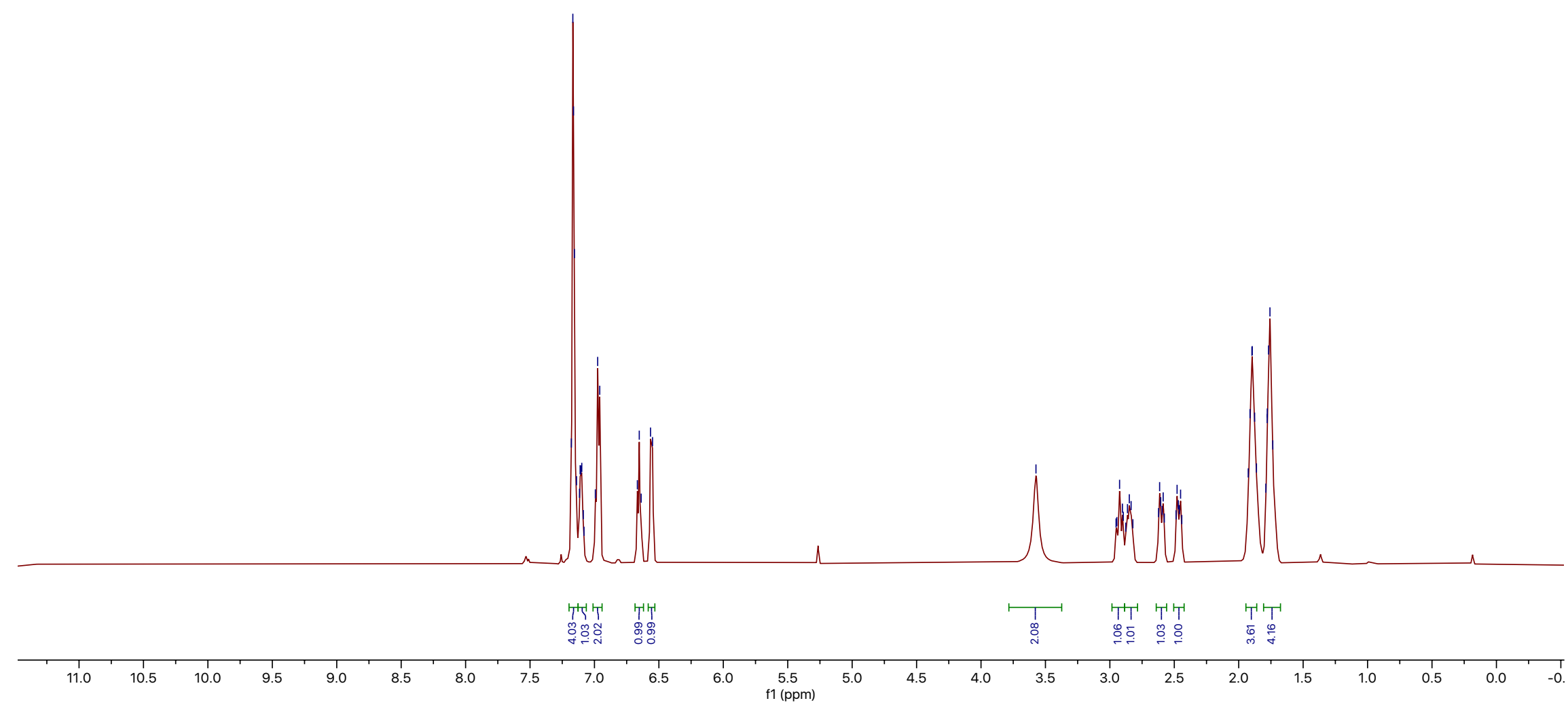


$125 \mathrm{MHz}, \mathrm{CDCl}_{3}$

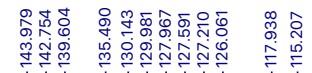

门া

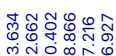

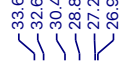

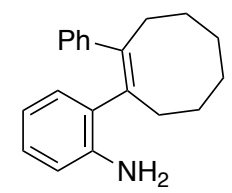

160

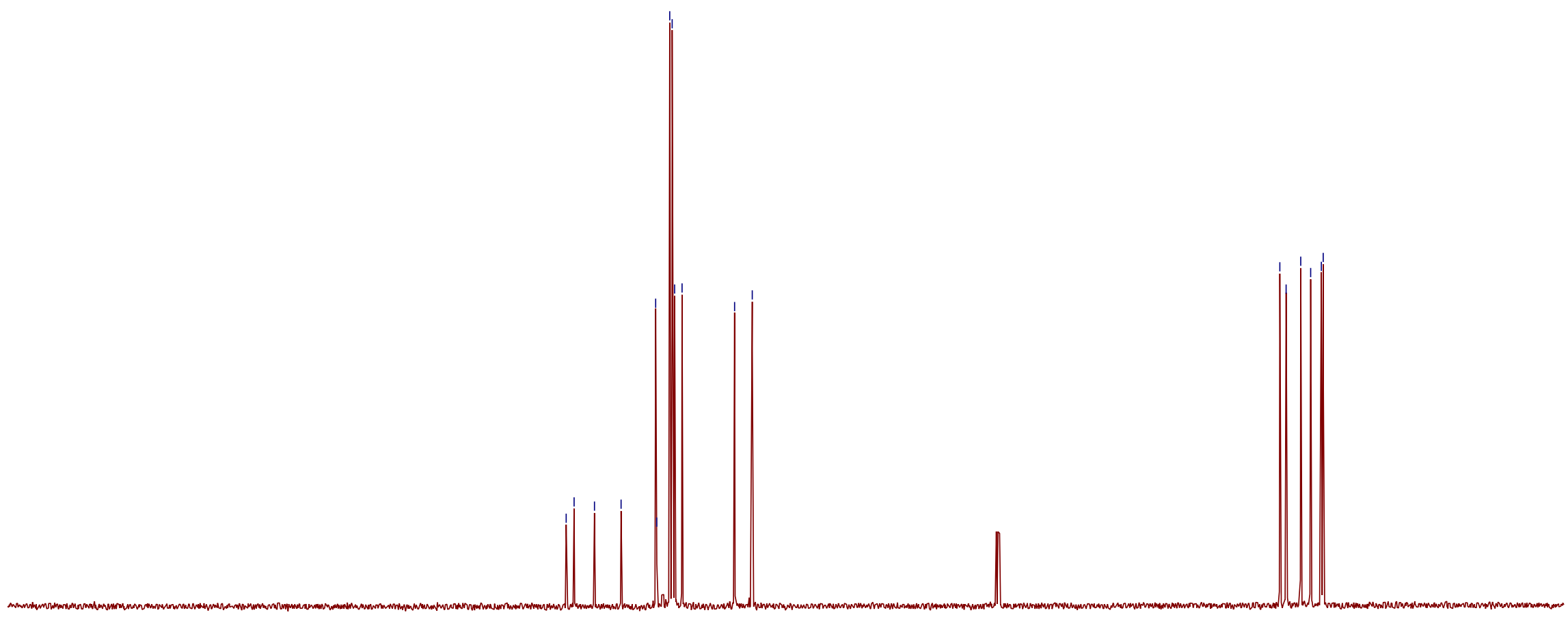

30
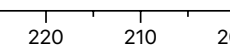

$190 \quad 180$
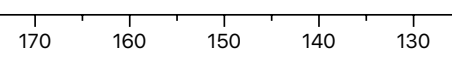

$120 \quad 110$
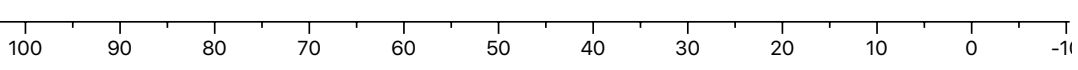
$500 \mathrm{MHz}, \mathrm{CDCl}_{3}$

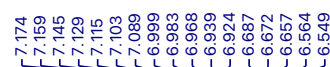

8
0

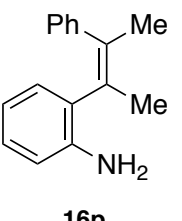

$16 p$

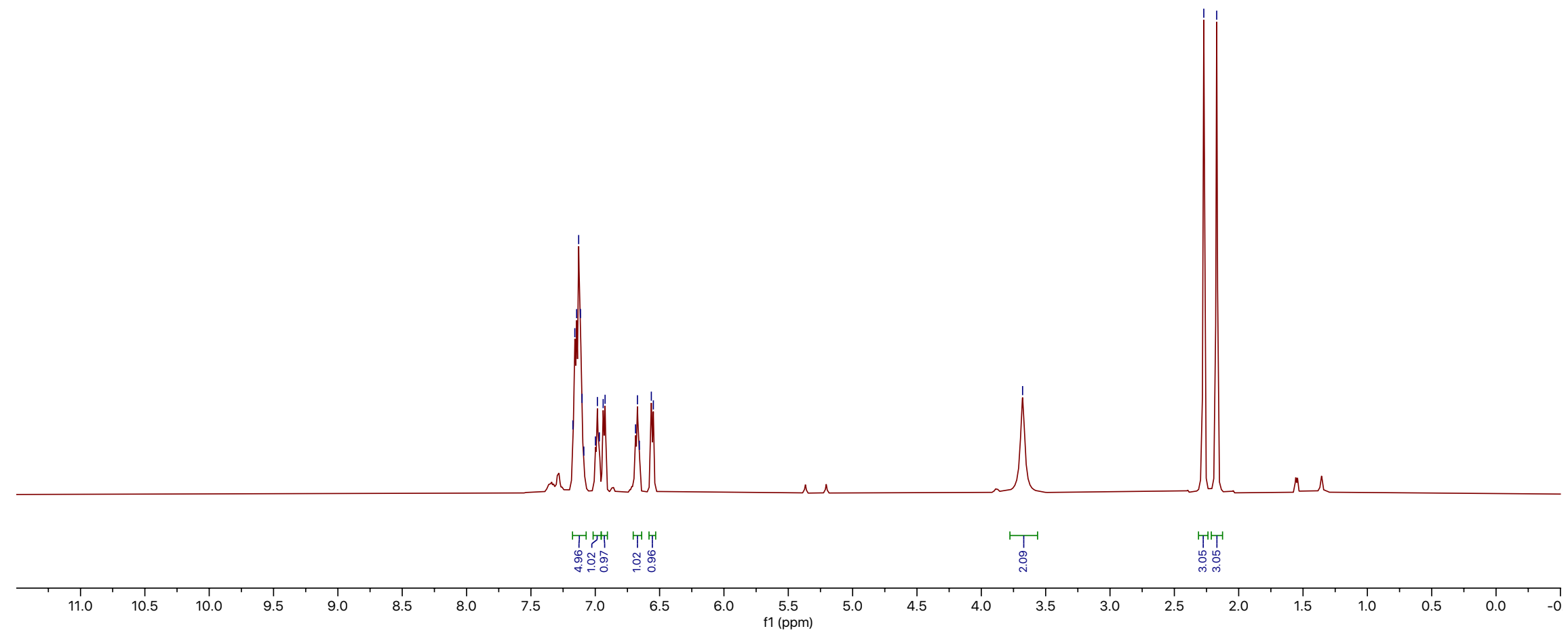


$125 \mathrm{MHz}, \mathrm{CDCl}_{3}$

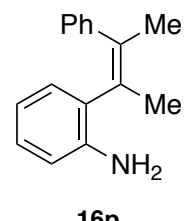

$16 p$
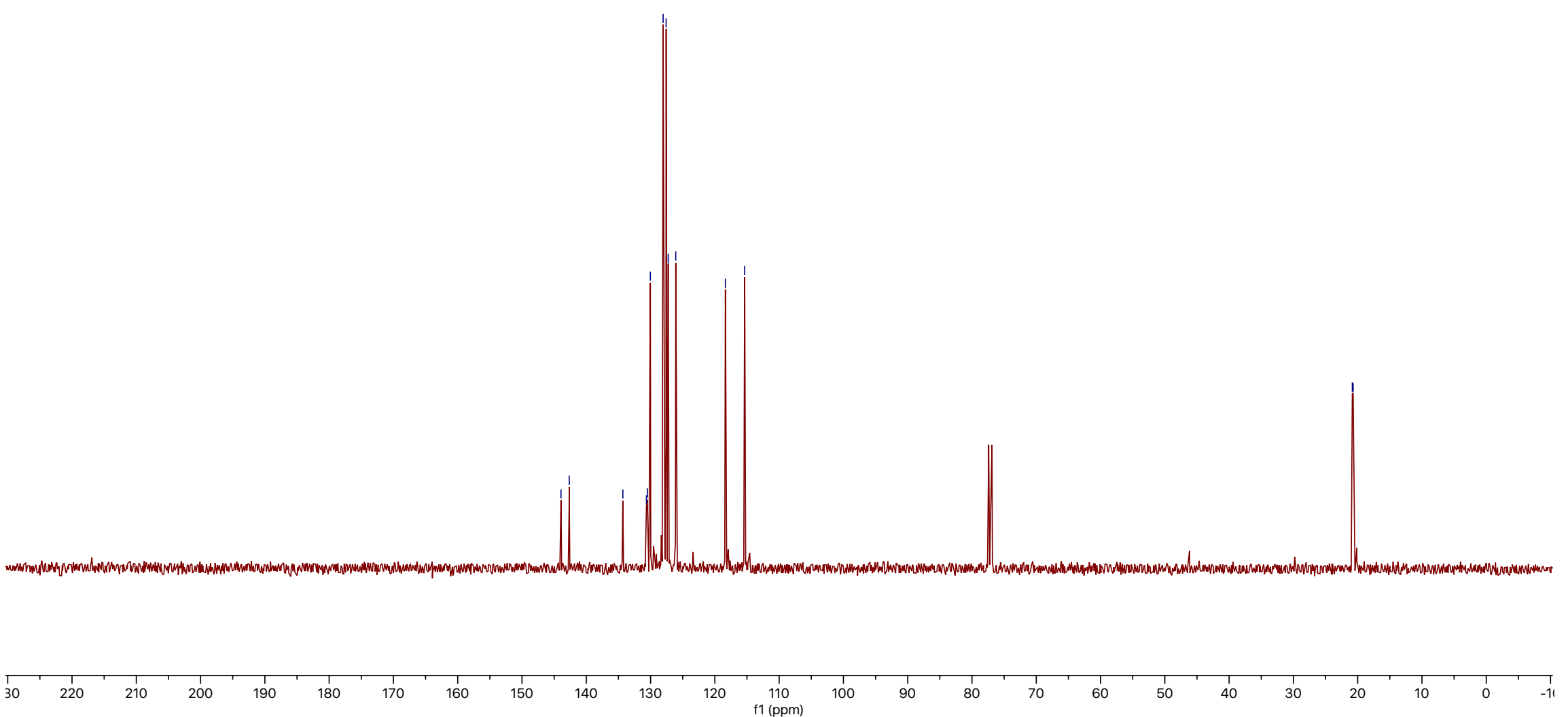
$500 \mathrm{MHz}, \mathrm{CDCl}_{3}$

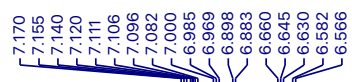

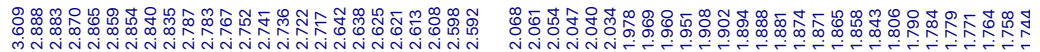

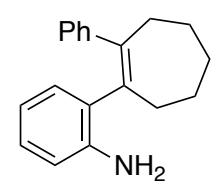

$16 q$

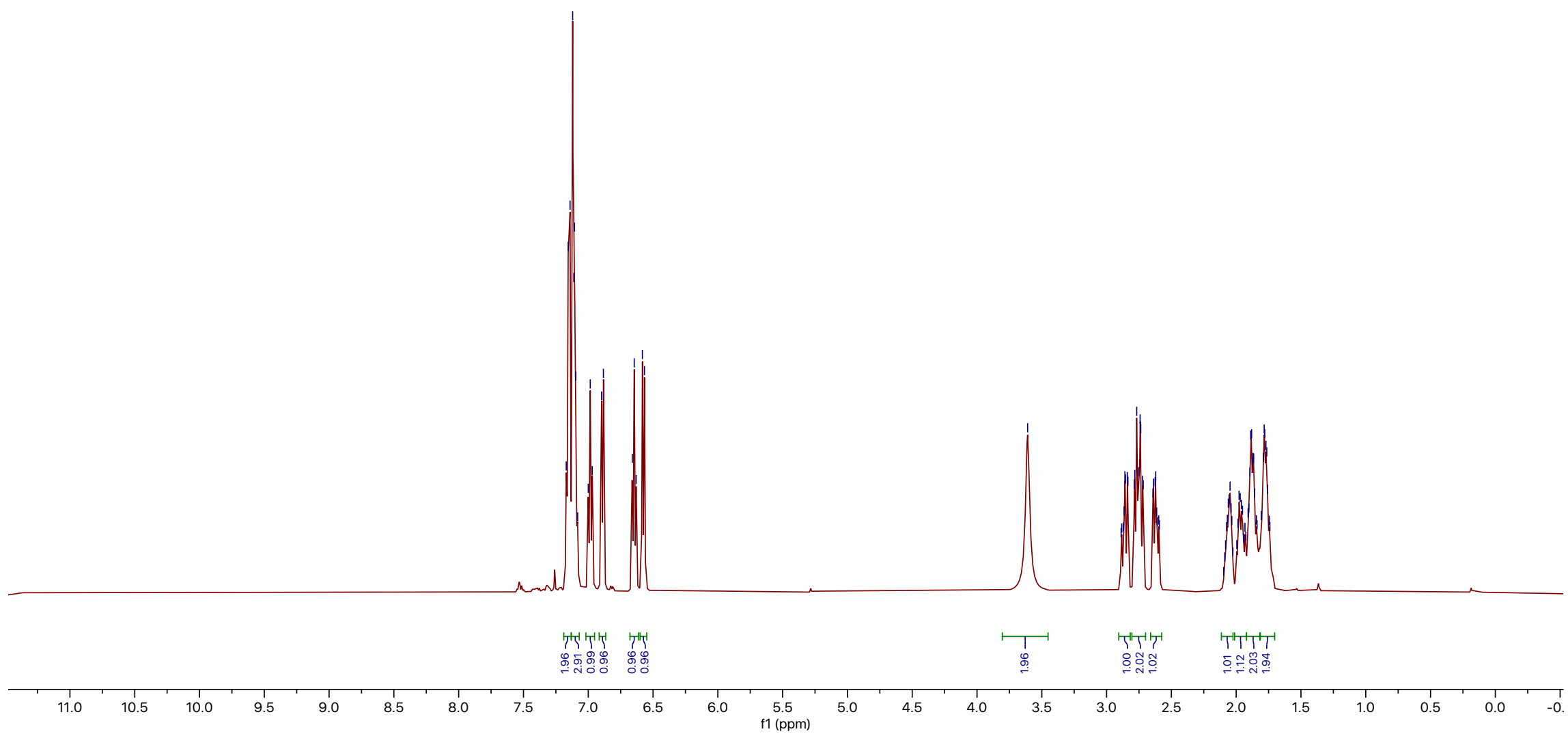


$125 \mathrm{MHz}, \mathrm{CDCl}_{3}$

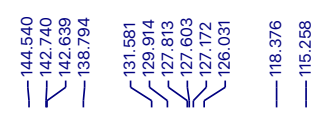

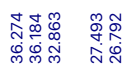

Vi

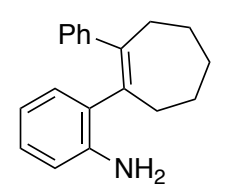

$16 q$
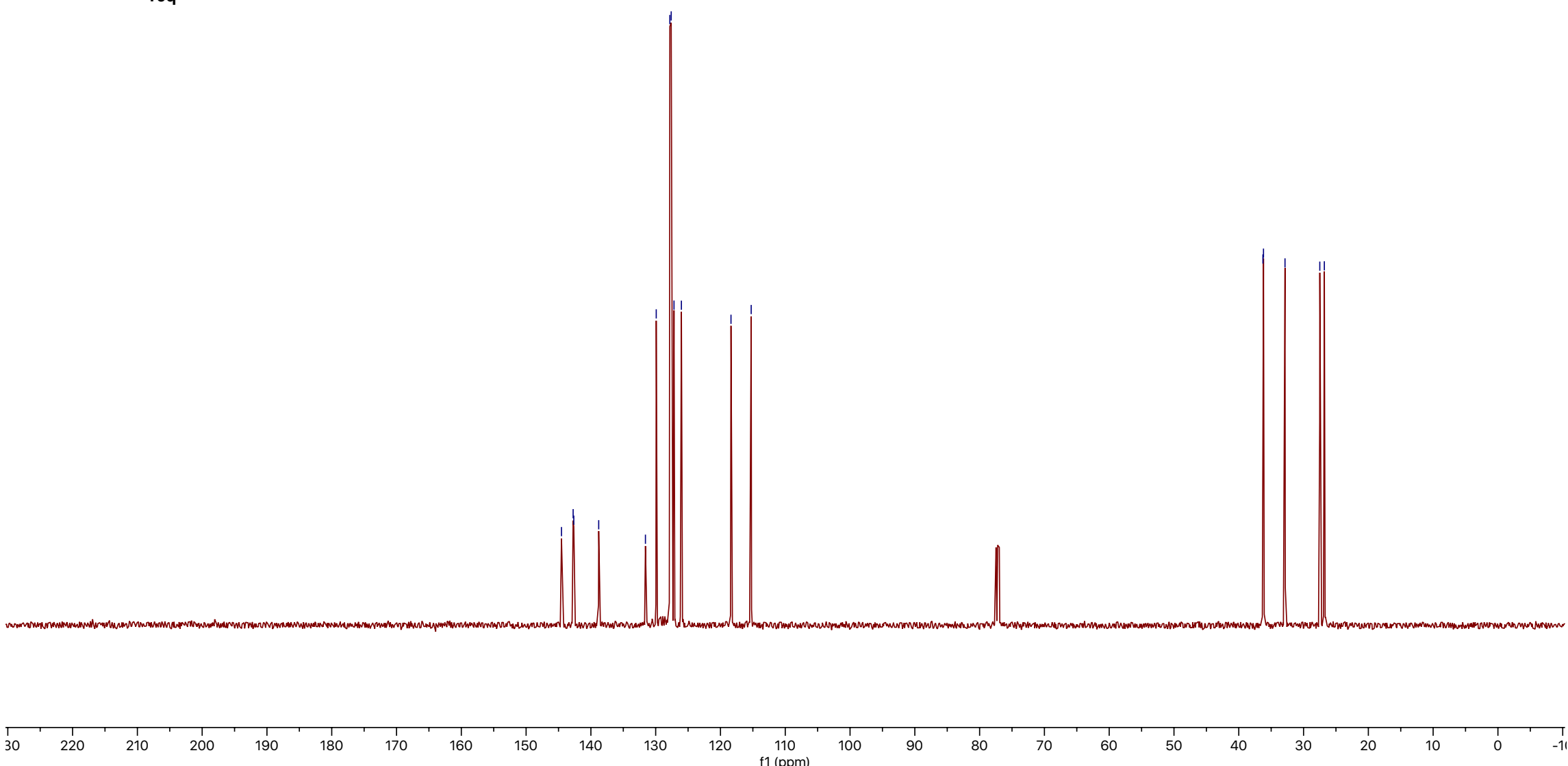
$500 \mathrm{MHz}, \mathrm{CDCl}_{3}$

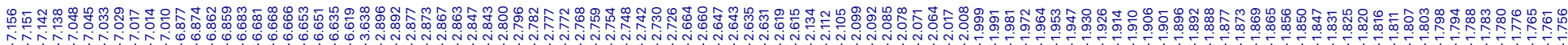
TIIIm

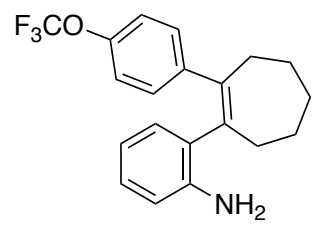

$16 r$

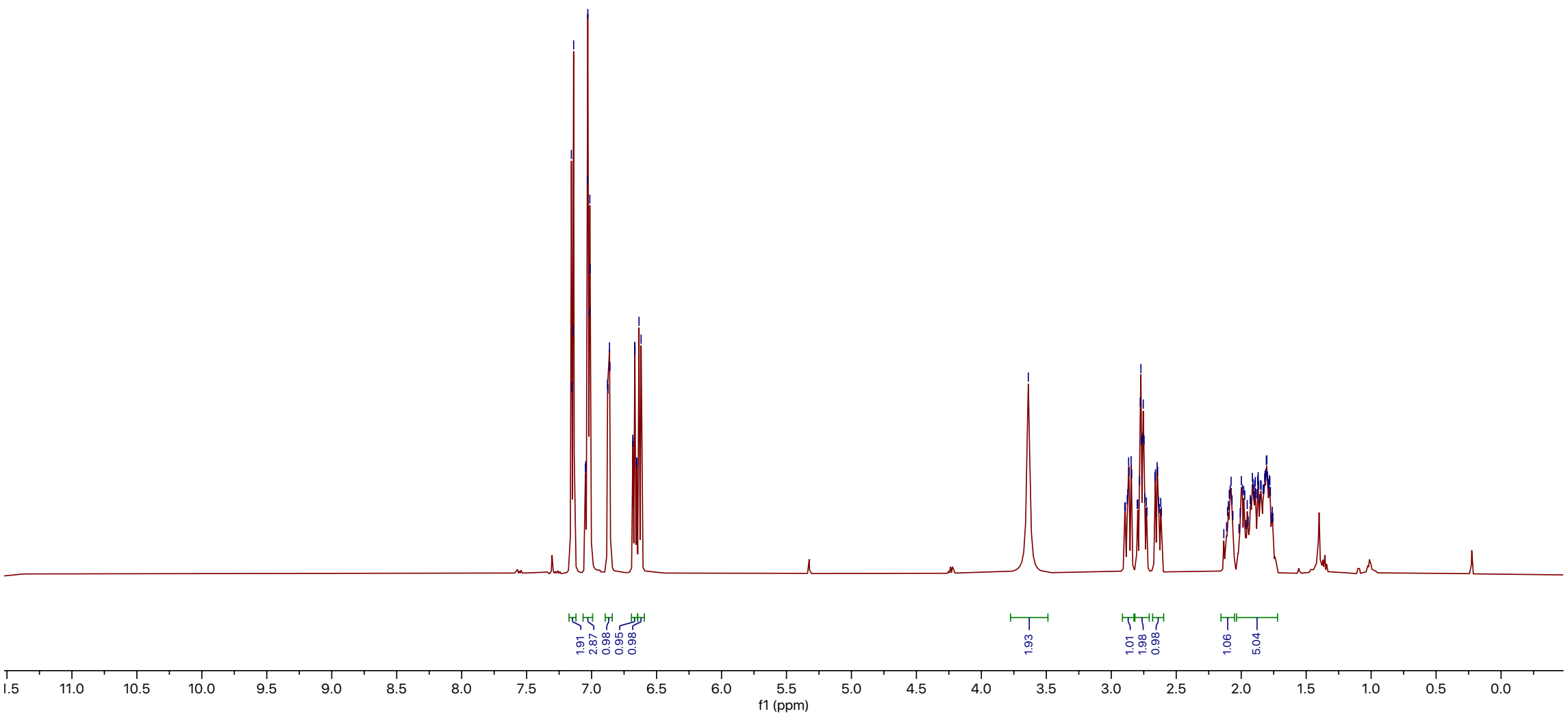


$125 \mathrm{MHz}, \mathrm{CDCl}_{3}$

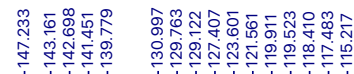

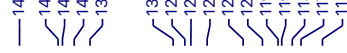

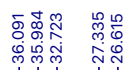

Иं।

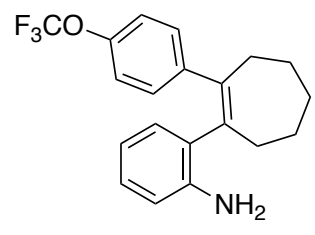

$16 r$

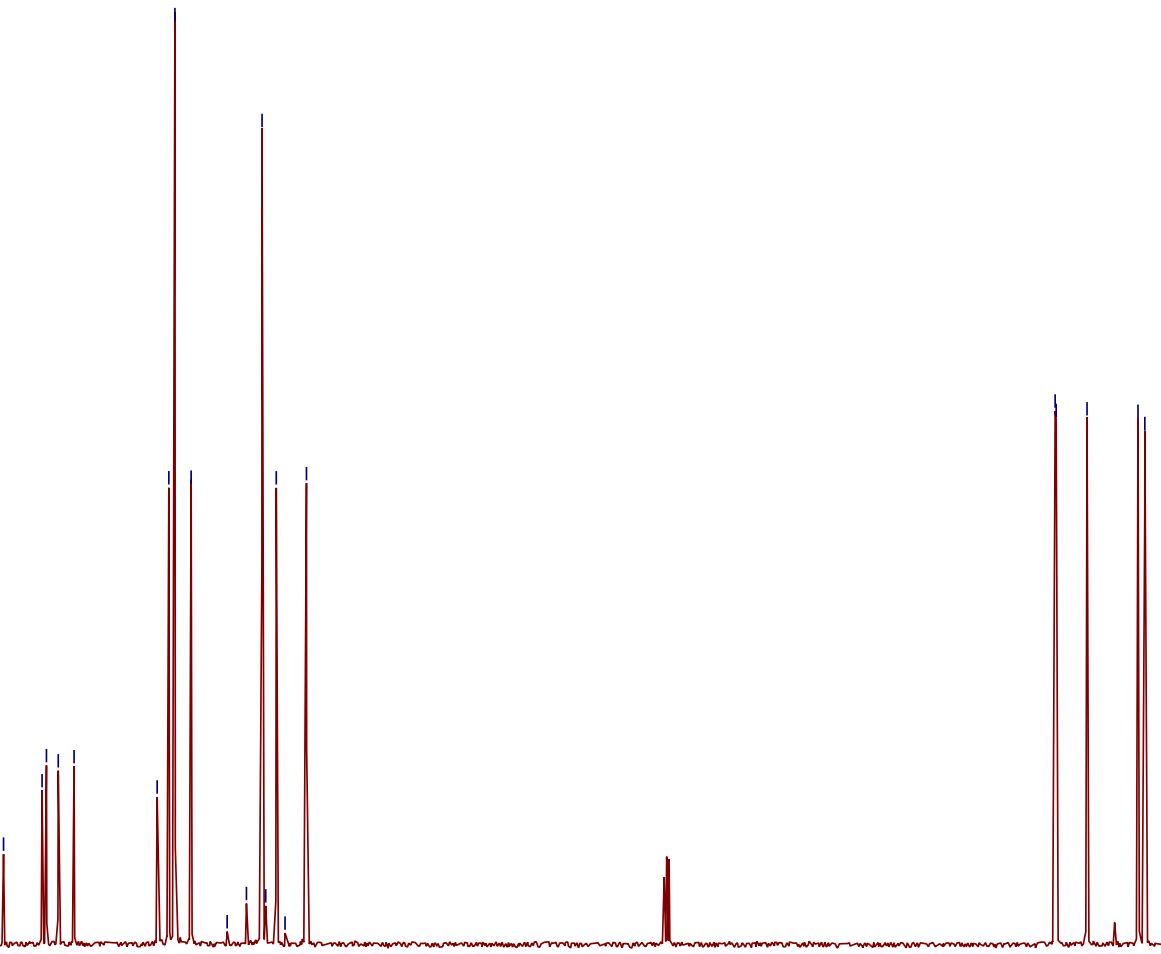

30

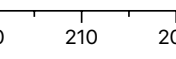

200
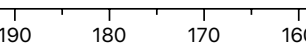

150
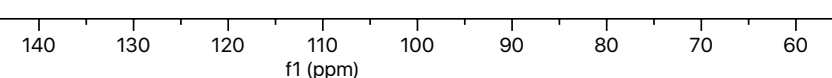
$500 \mathrm{MHz}, \mathrm{CDCl}_{3}$
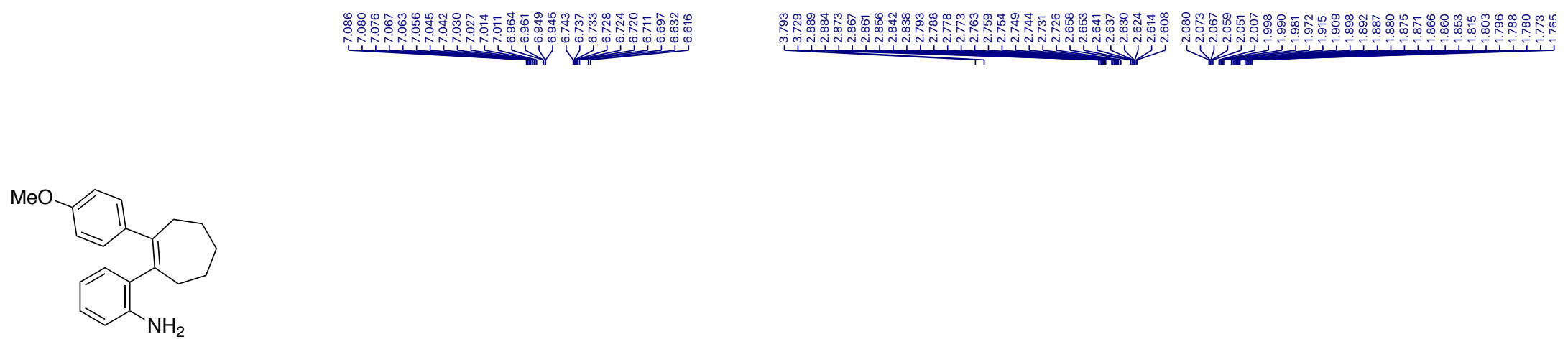

$16 s$

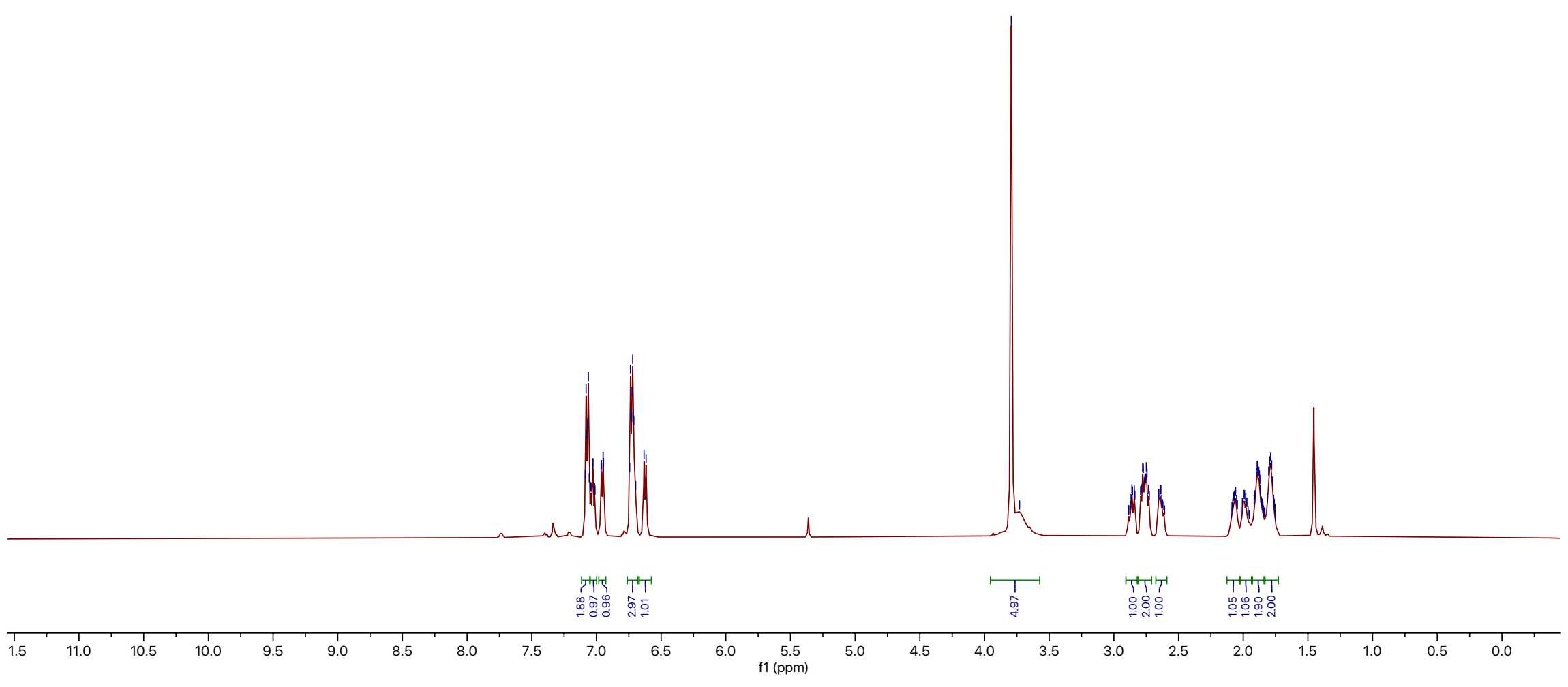


$125 \mathrm{MHz}, \mathrm{CDCl}_{3}$

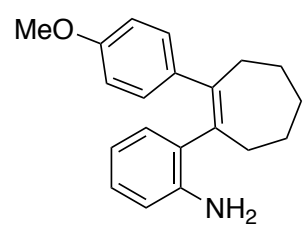

$16 s$
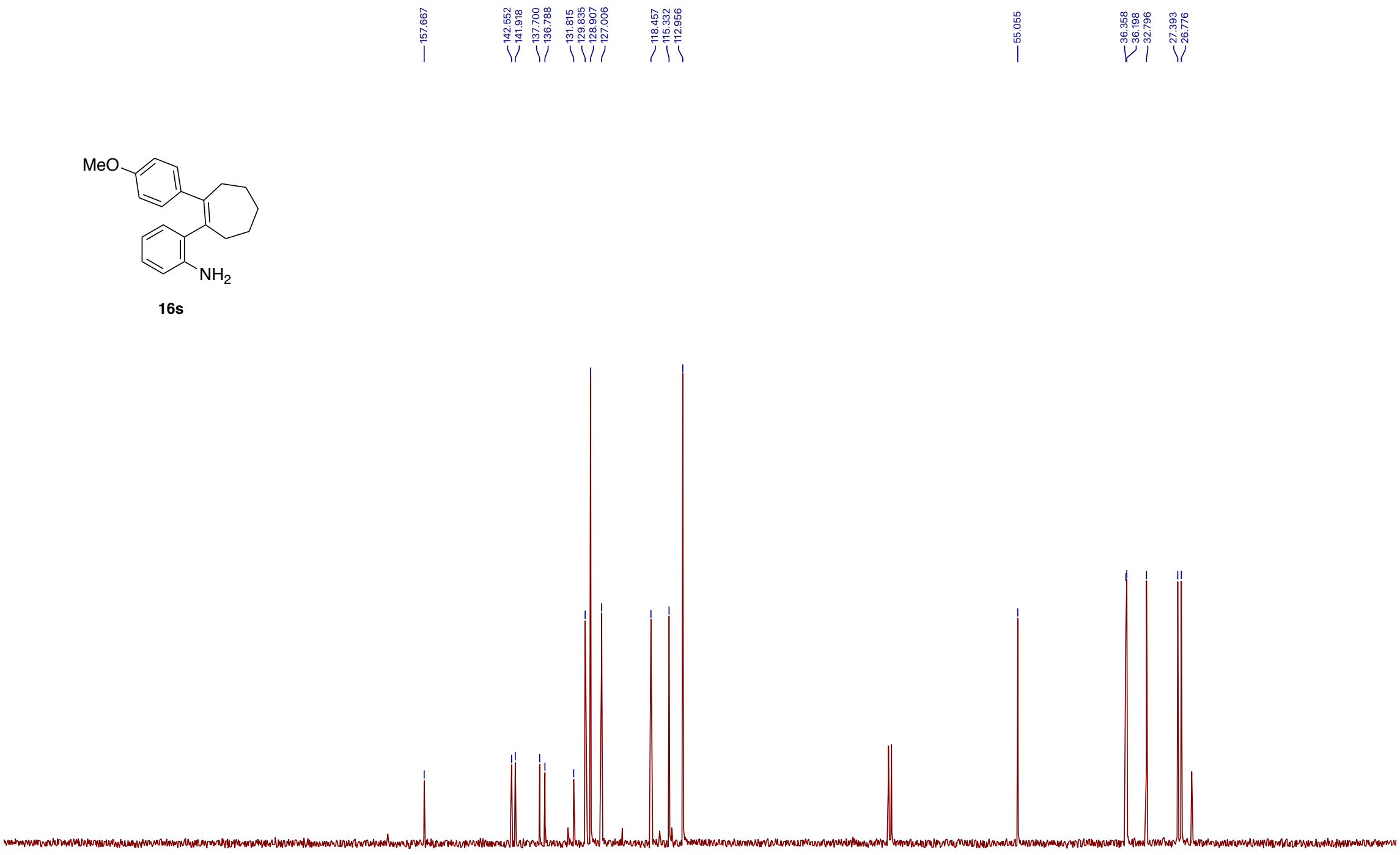

30

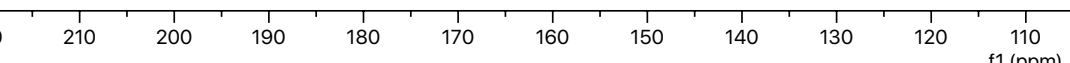

110
f1 (ppm)
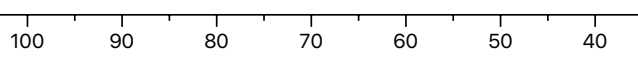
$500 \mathrm{MHz}, \mathrm{CDCl}_{3}$

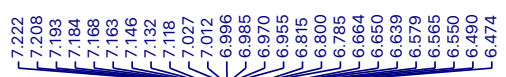

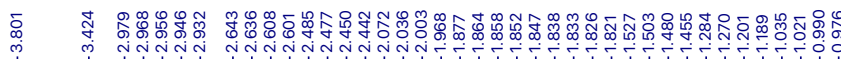

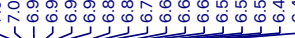

$1 \underbrace{N}$

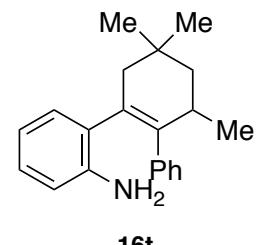

$16 t$

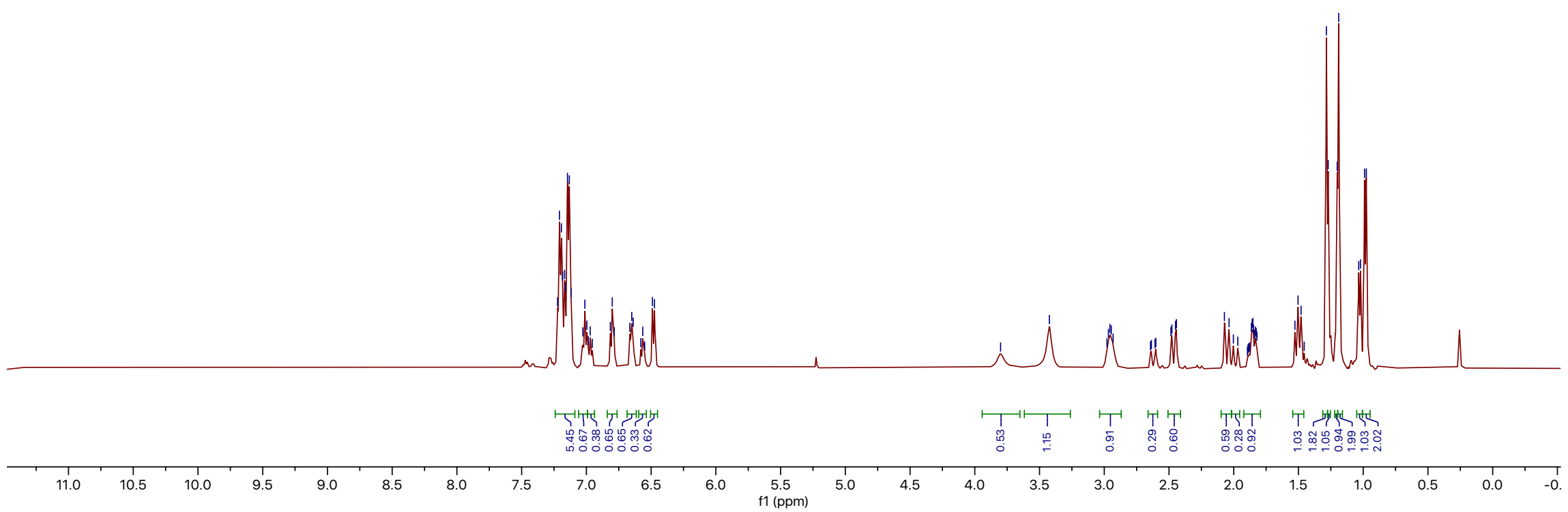


$125 \mathrm{MHz}, \mathrm{CDCl}_{3}$
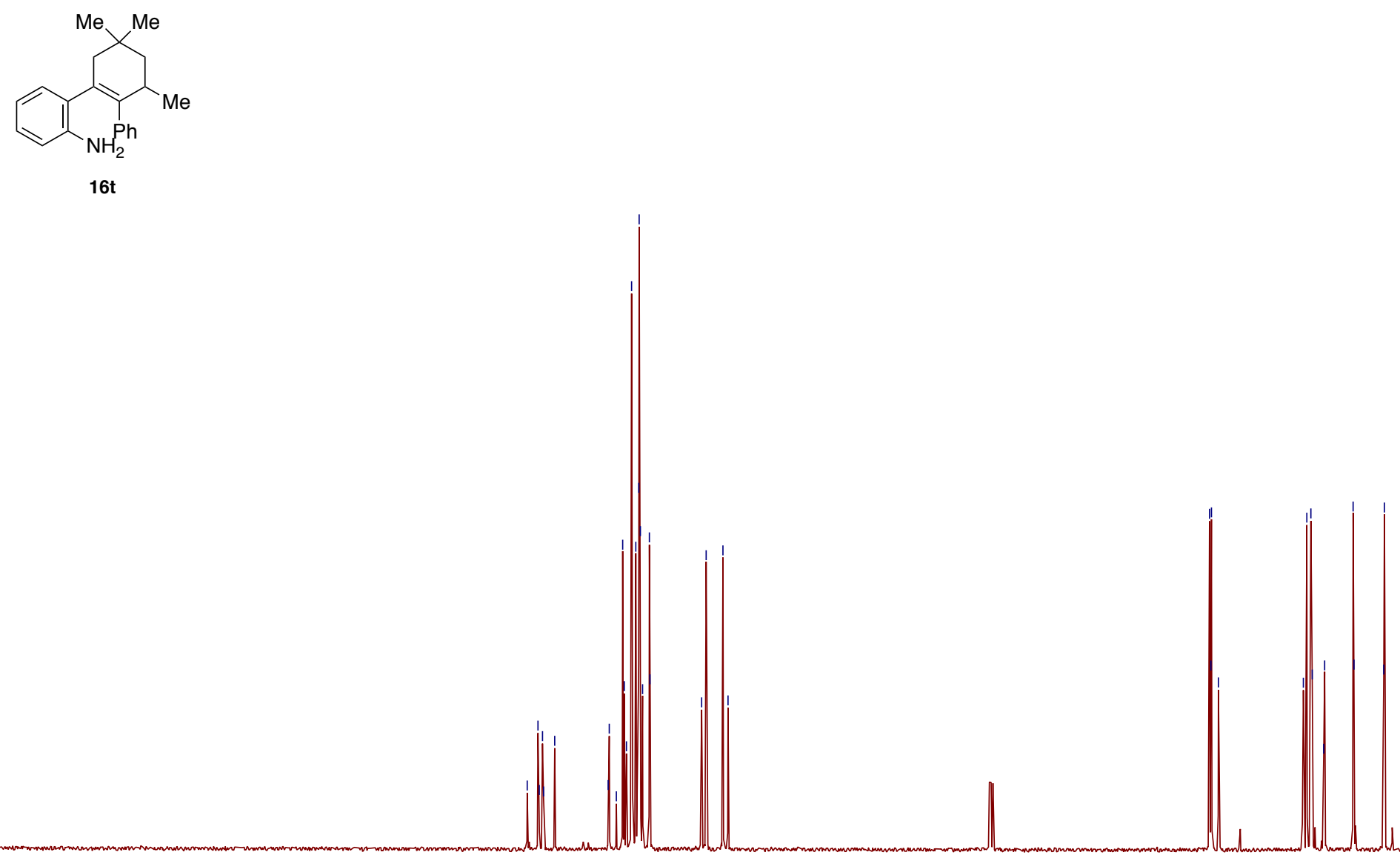

30

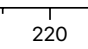

$210 \quad 200$
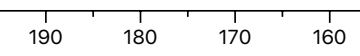

150
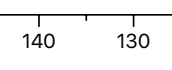

120

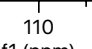

$100 \quad 90$

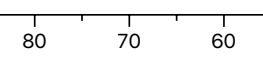

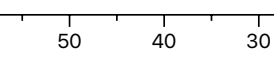

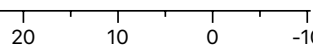


$500 \mathrm{MHz}, \mathrm{CDCl}_{3}$

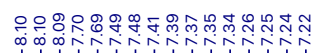

$V^{\infty}$

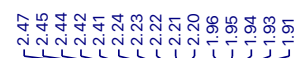

皮
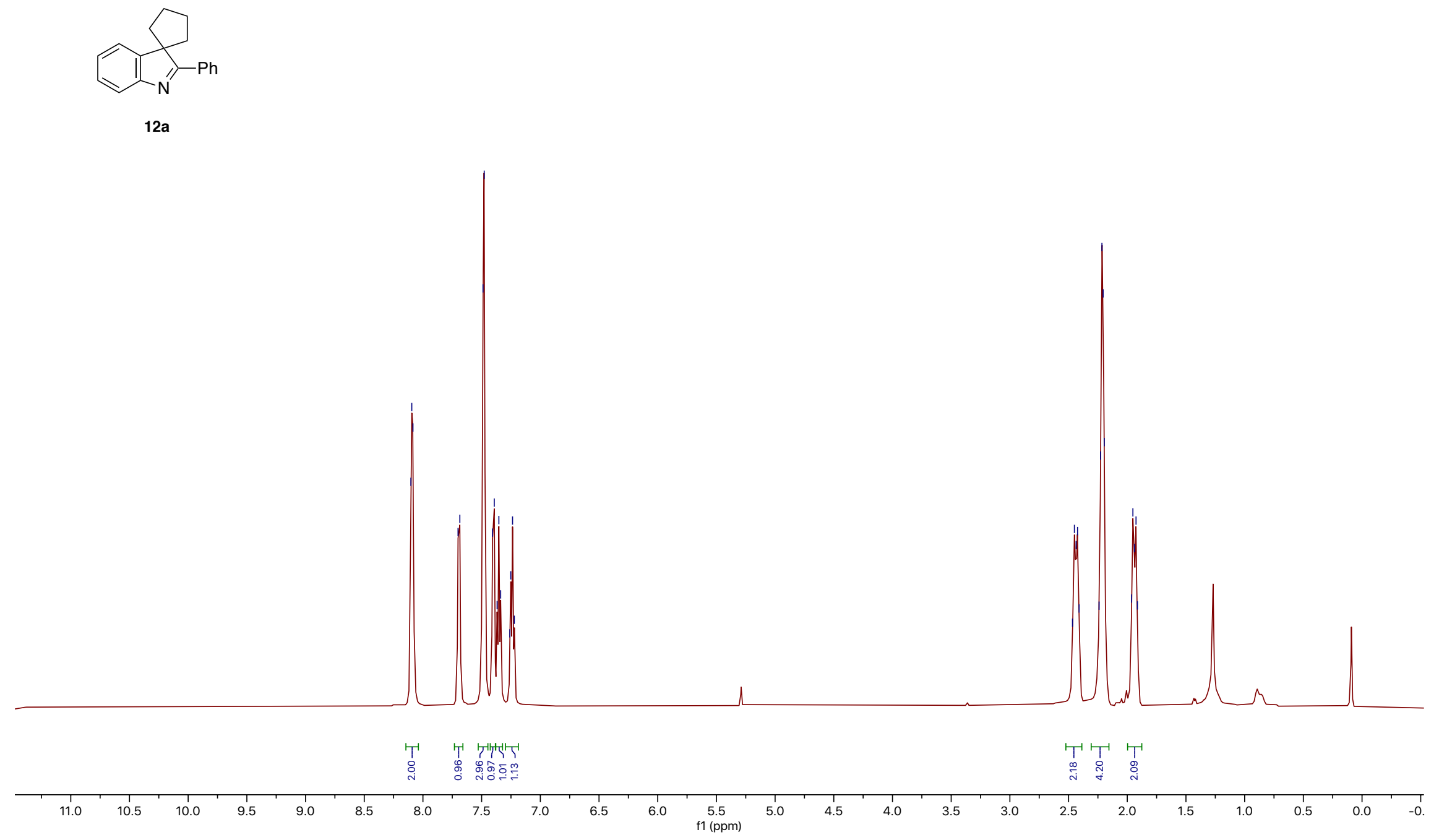
$125 \mathrm{MHz}, \mathrm{CDCl}_{3}$

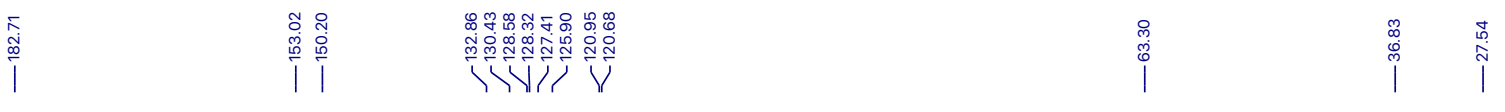

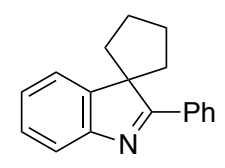

$12 a$
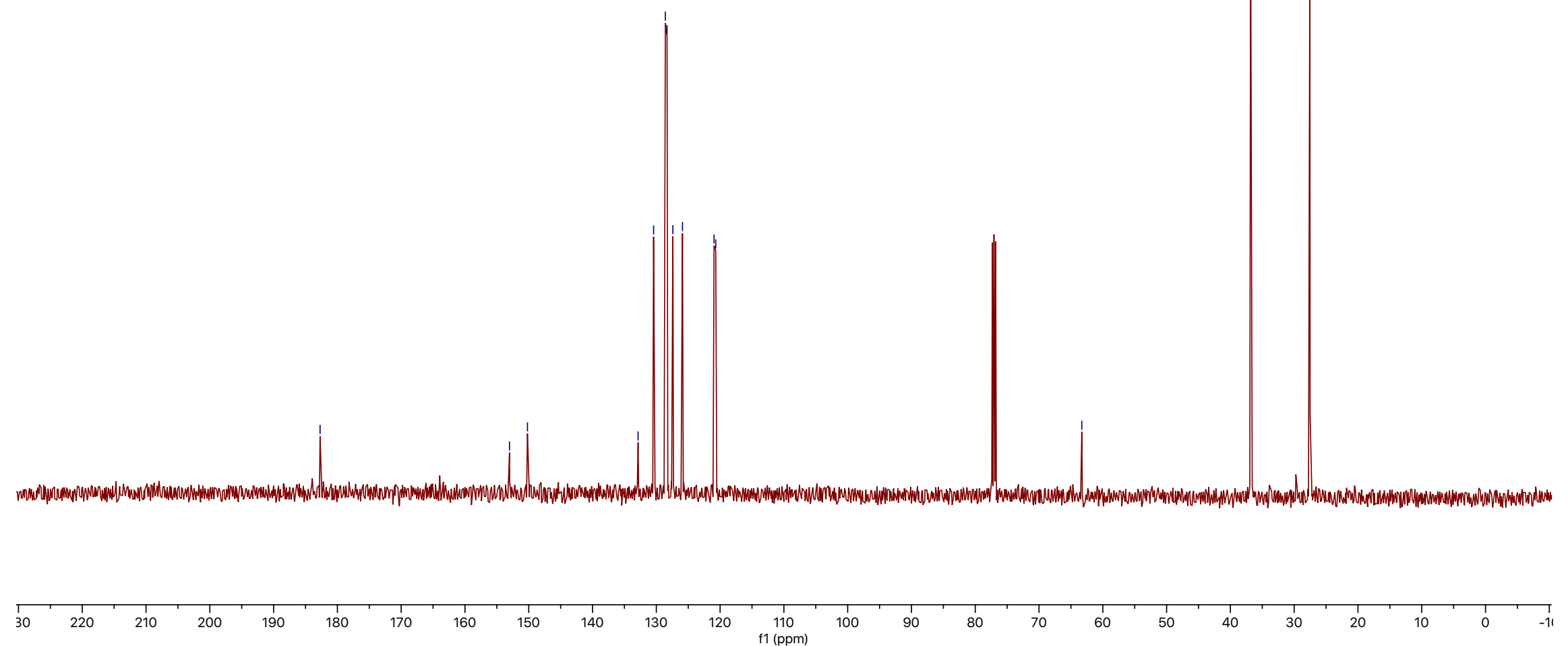
$500 \mathrm{MHz}, \mathrm{CDCl}_{3}$
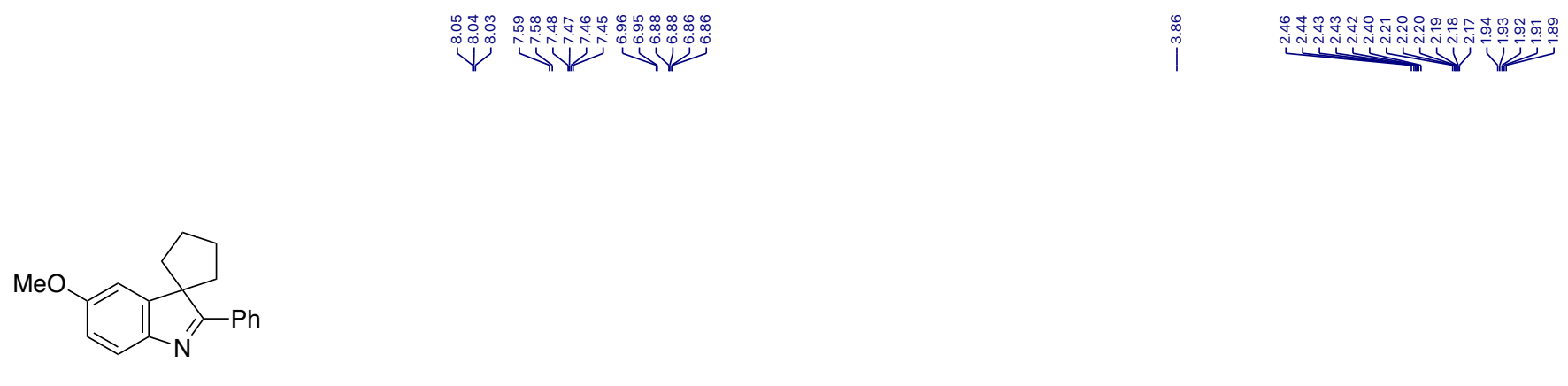

$12 b$

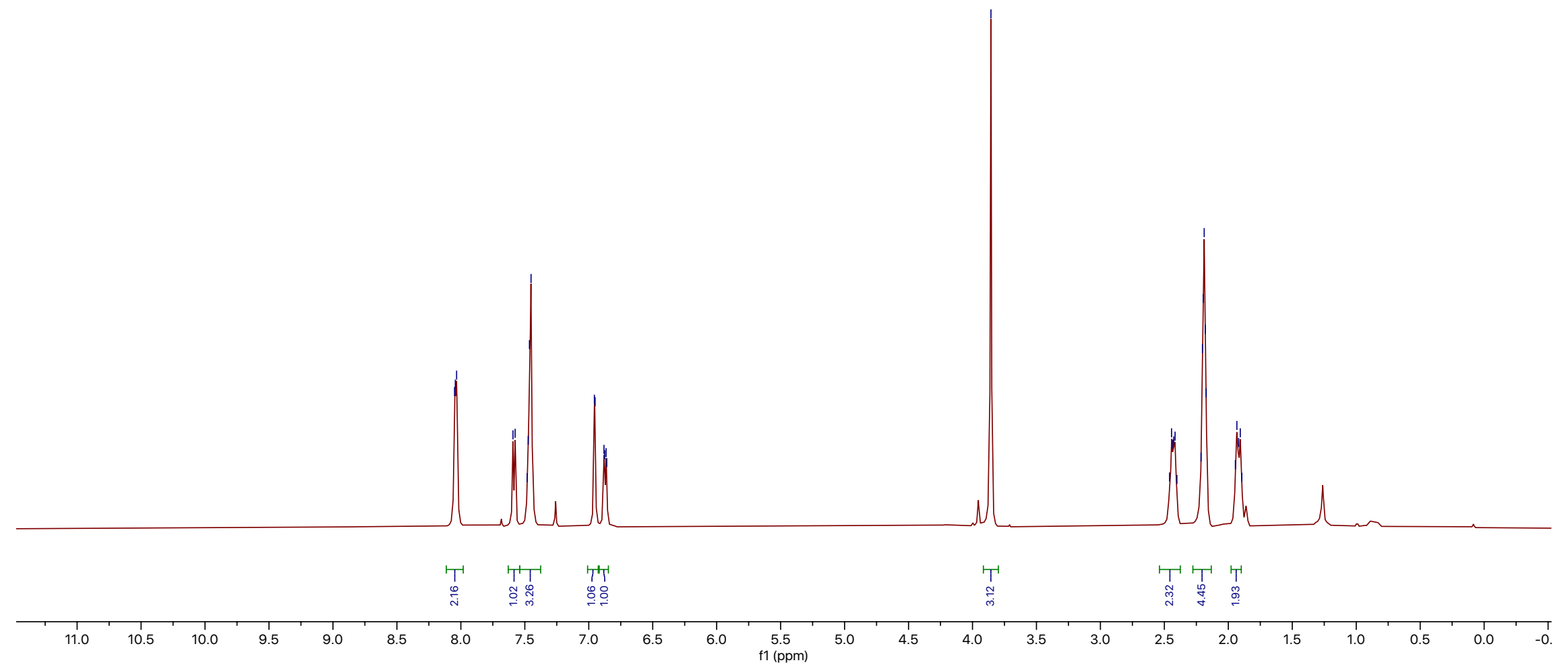


$125 \mathrm{MHz}, \mathrm{CDCl}_{3}$

|

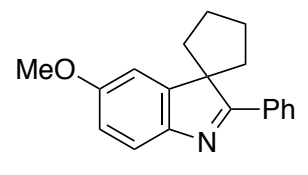

$12 b$
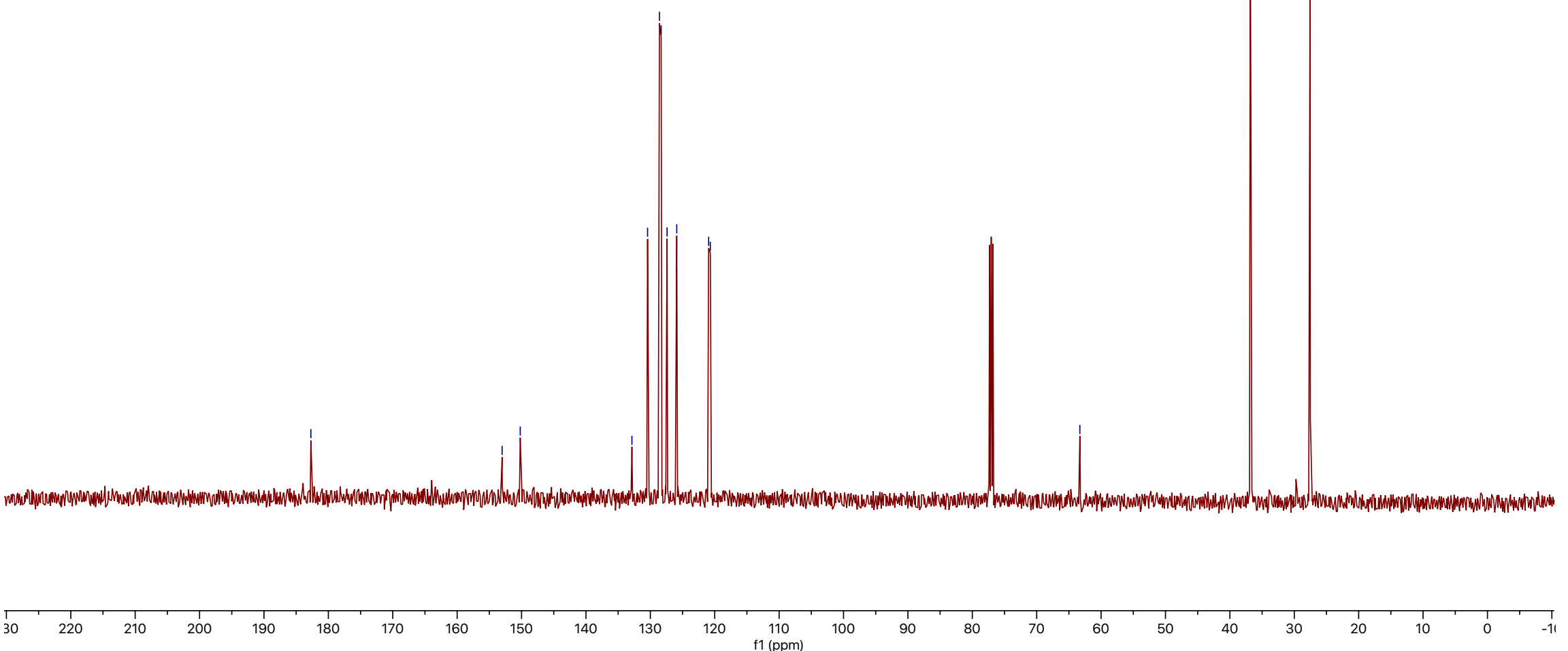
$500 \mathrm{MHz}, \mathrm{CDCl}_{3}$

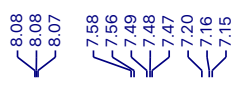

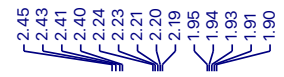

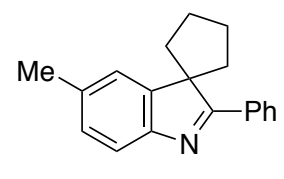

12c

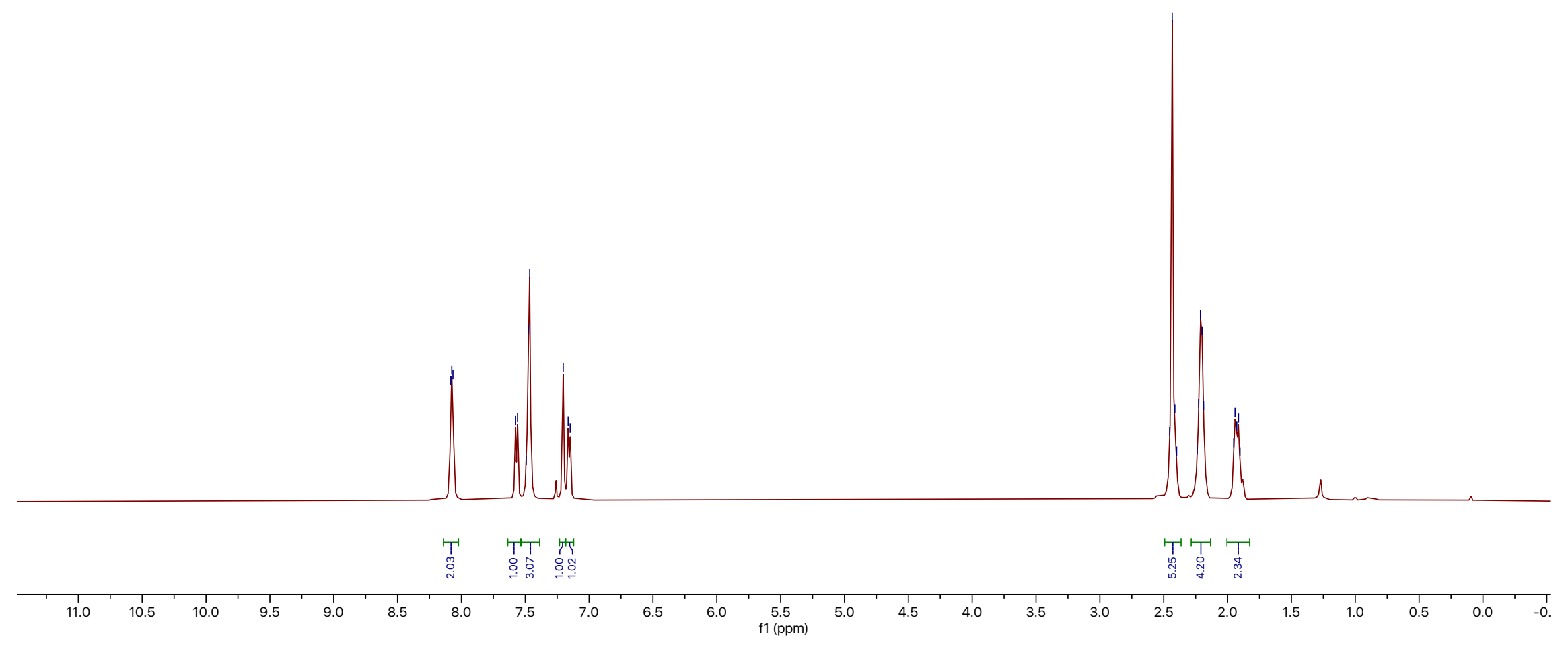


$125 \mathrm{MHz}, \mathrm{CDCl}_{3}$

|

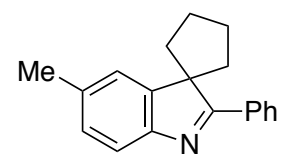

12c
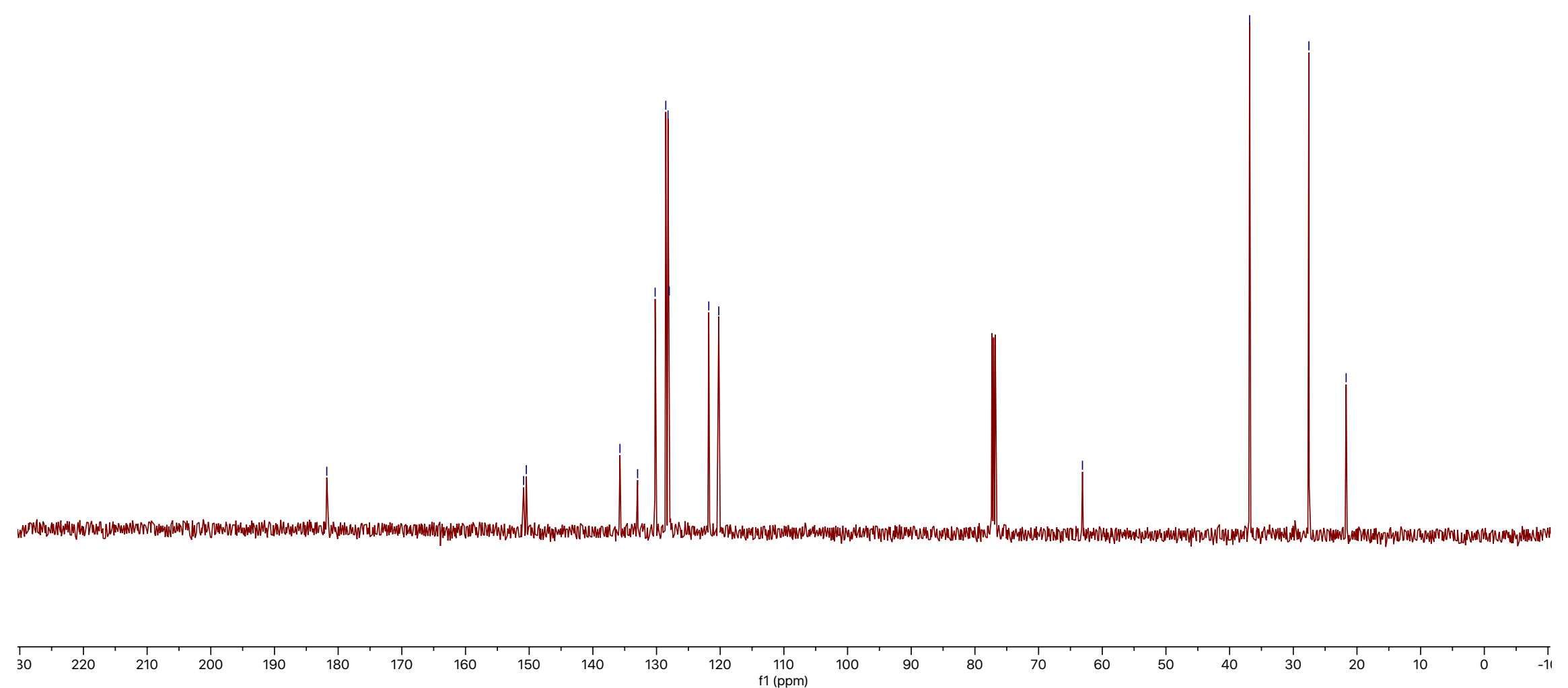
$500 \mathrm{MHz}, \mathrm{CDCl}_{3}$
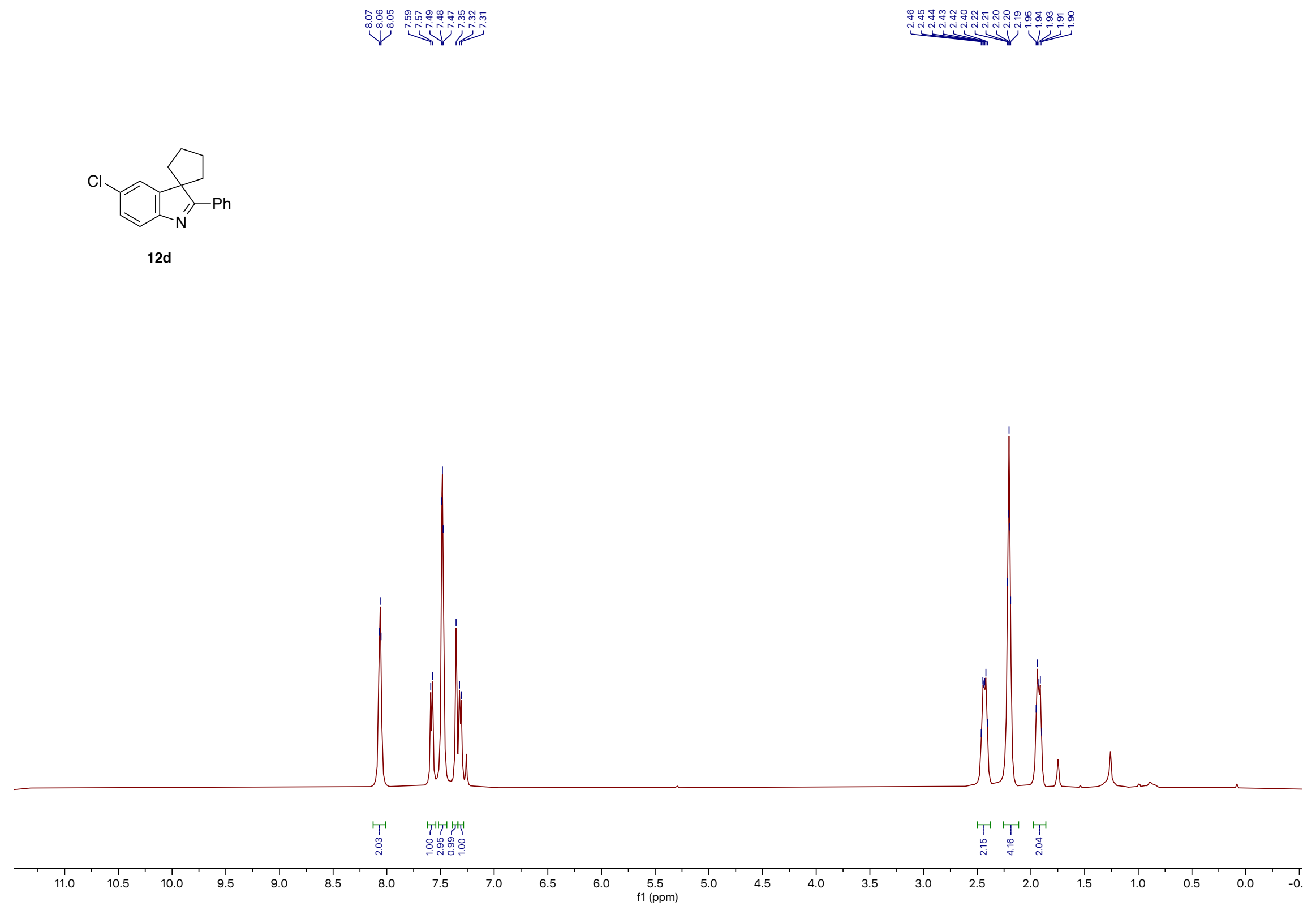
$125 \mathrm{MHz}, \mathrm{CDCl}_{3}$

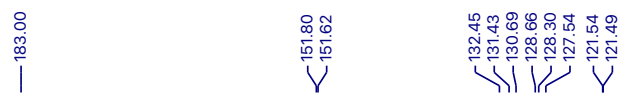

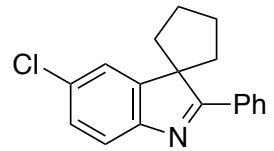

$12 d$

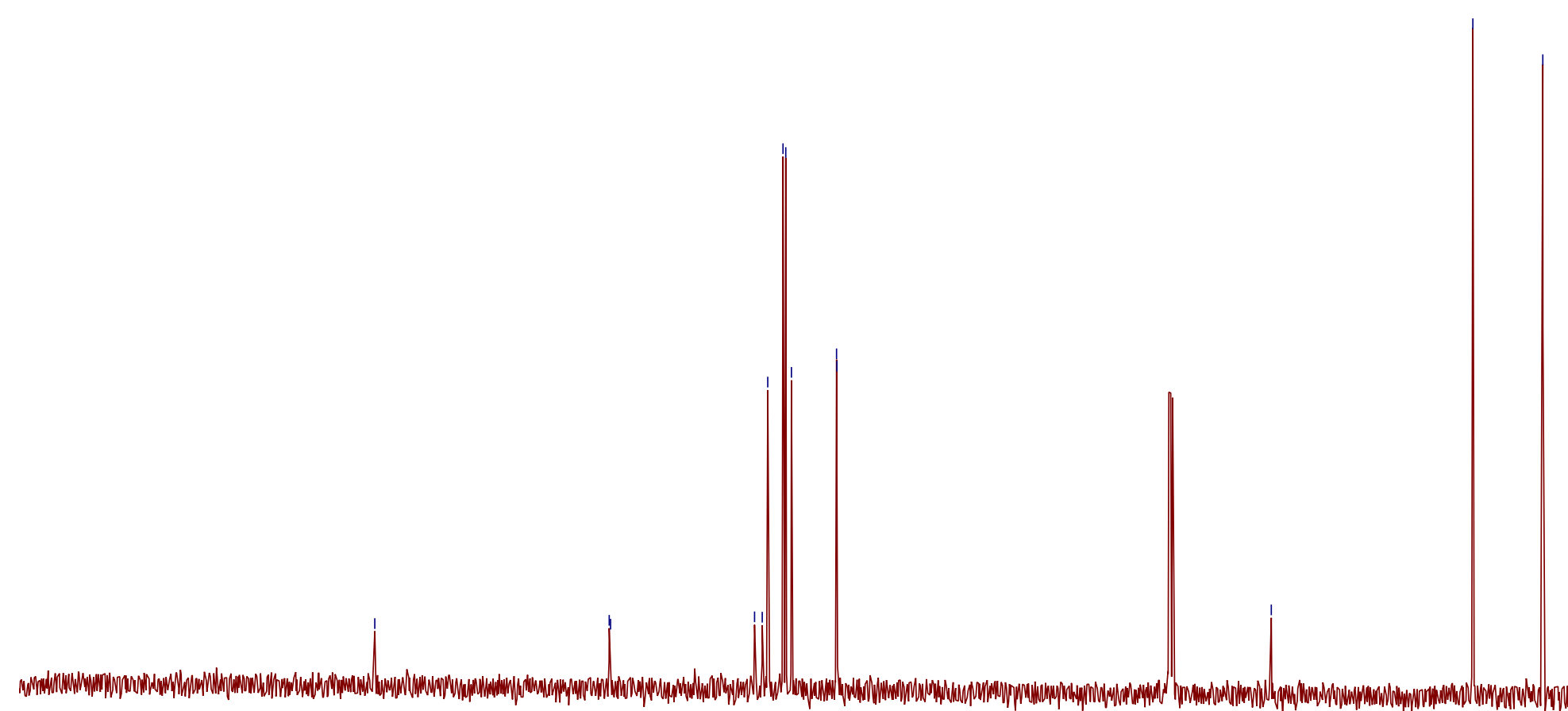

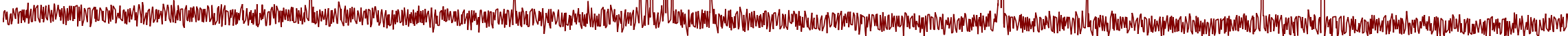

30

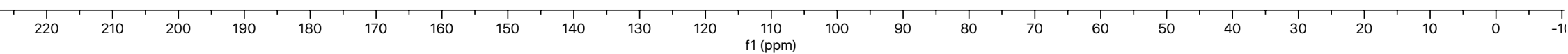


$500 \mathrm{MHz}, \mathrm{CDCl}_{3}$

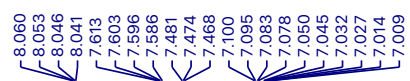

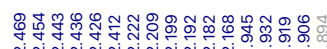

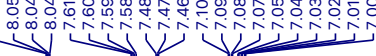

VIVI
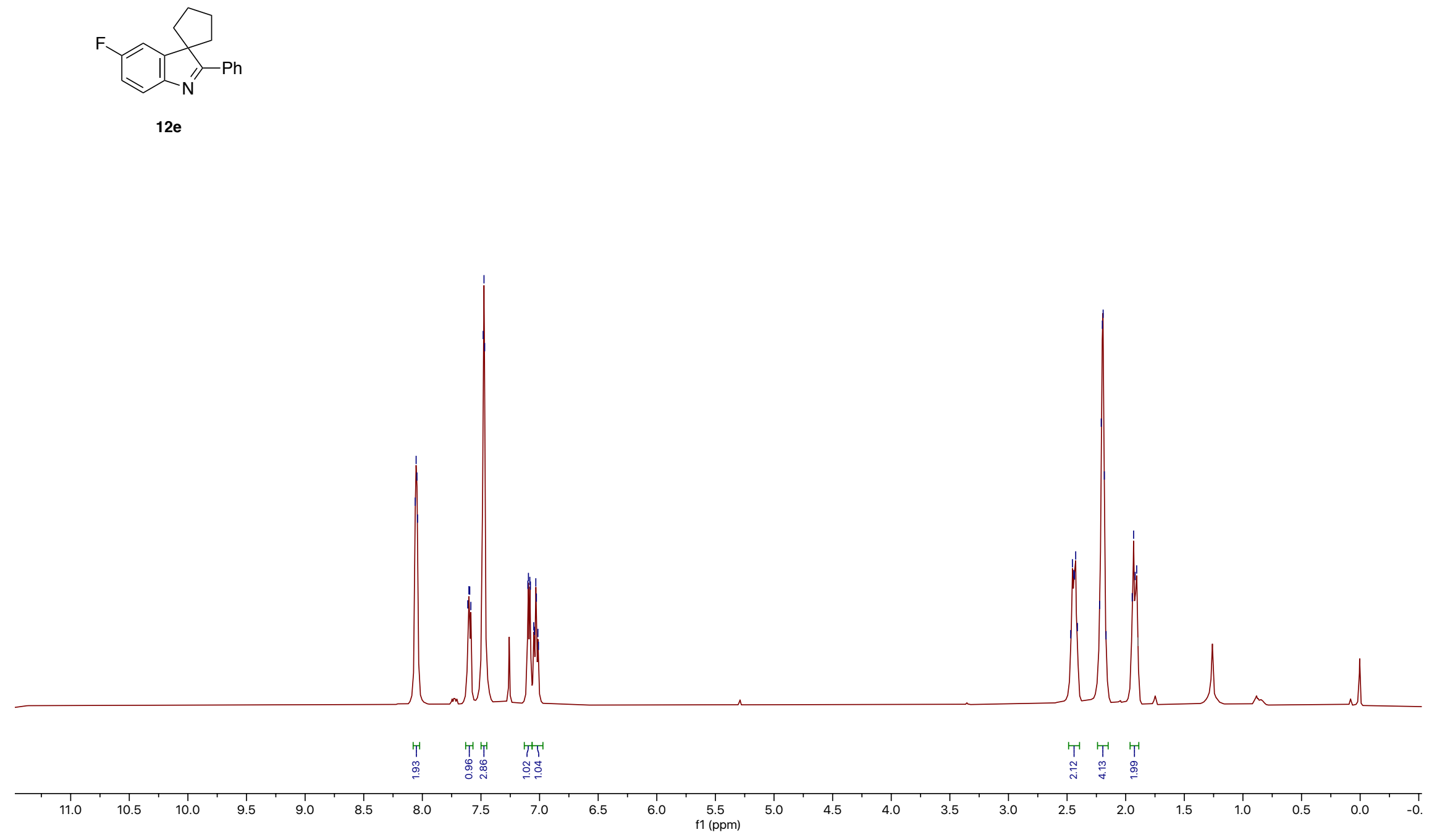
$125 \mathrm{MHz}, \mathrm{CDCl}_{3}$

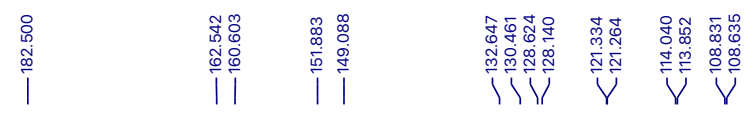

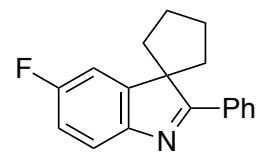

$12 \mathrm{e}$
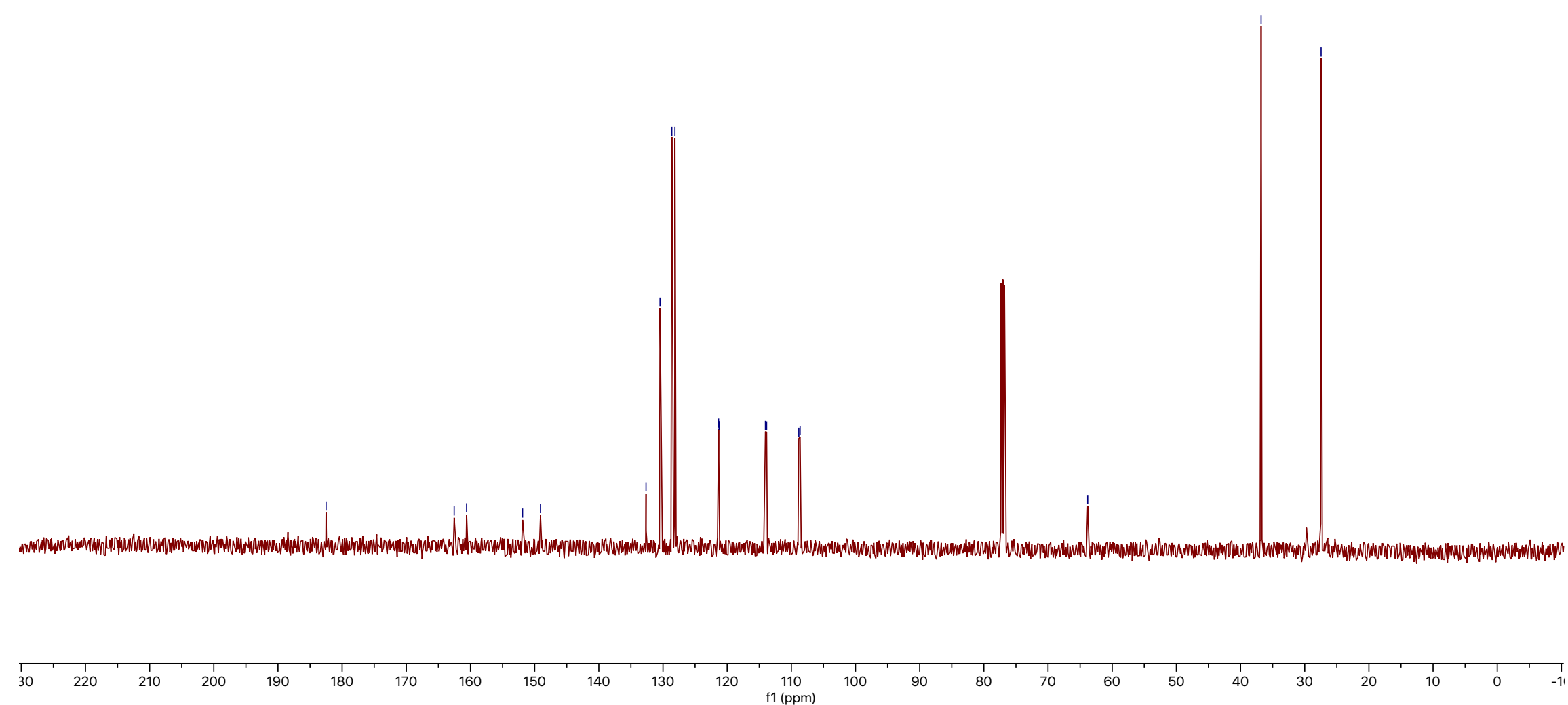
$500 \mathrm{MHz}, \mathrm{CDCl}_{3}$
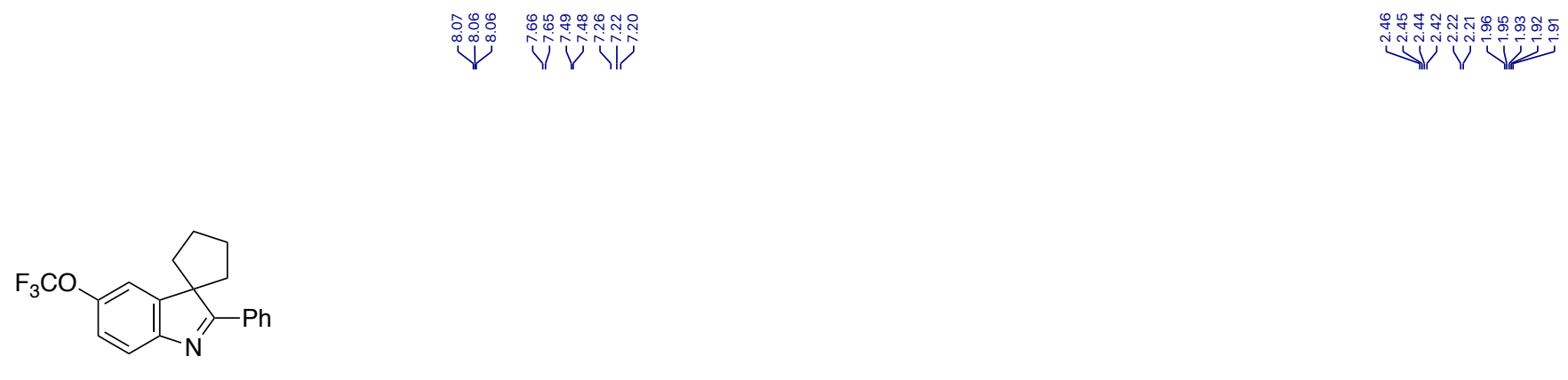

$12 f$

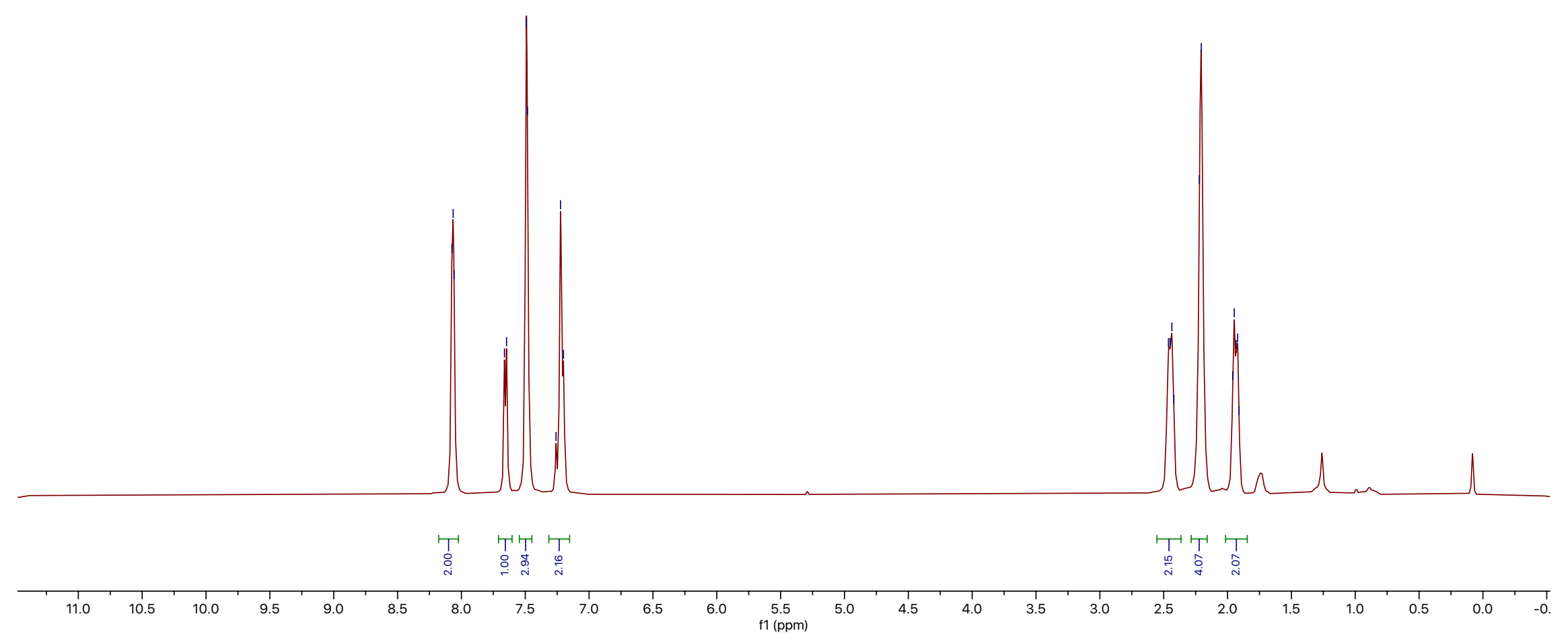


$125 \mathrm{MHz}, \mathrm{CDCl}_{3}$
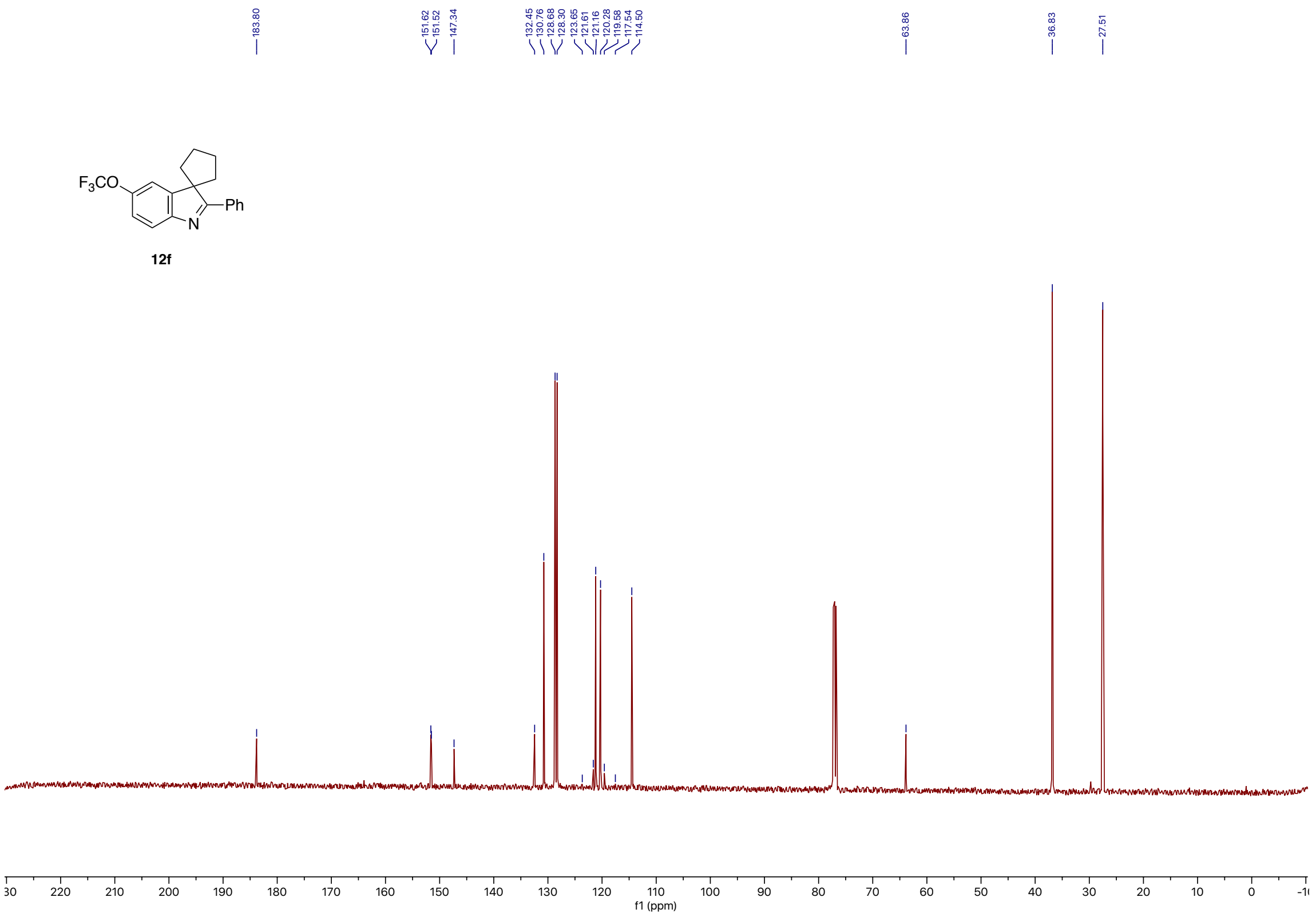
$500 \mathrm{MHz}, \mathrm{CDCl}_{3}$
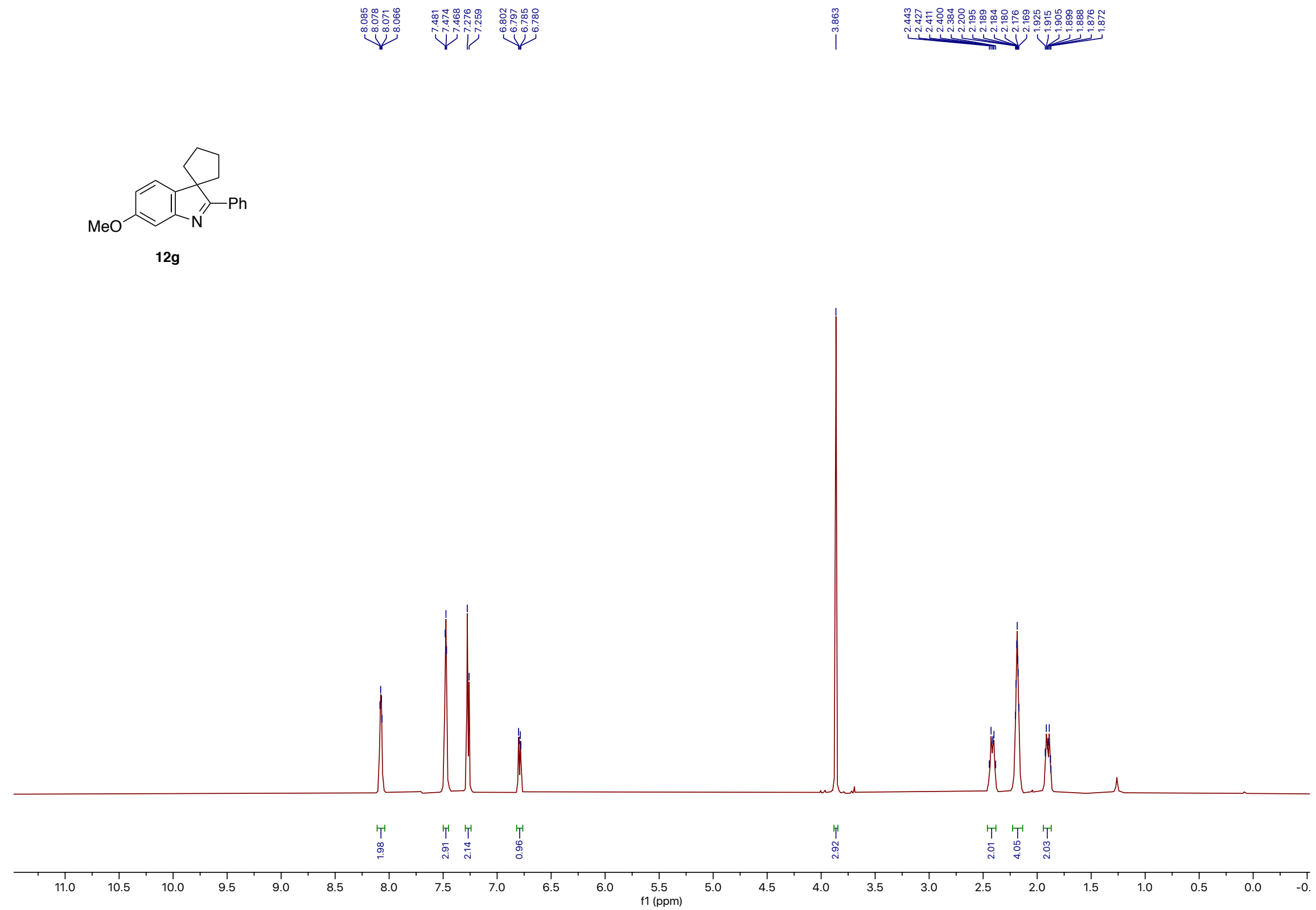
$125 \mathrm{MHz}, \mathrm{CDCl}_{3}$

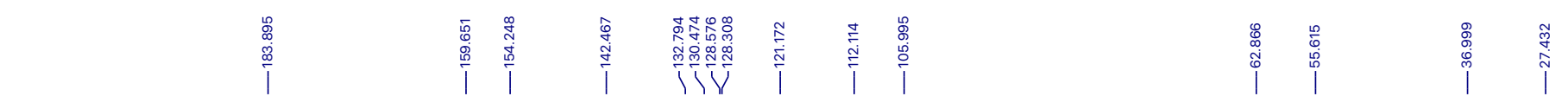

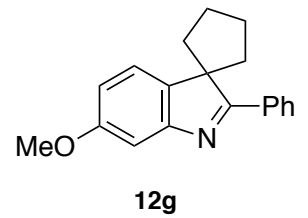

(1)

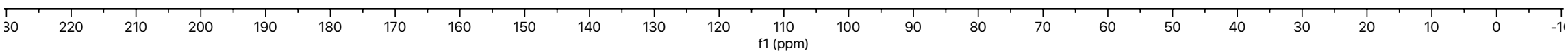


$500 \mathrm{MHz}, \mathrm{CDCl}_{3}$
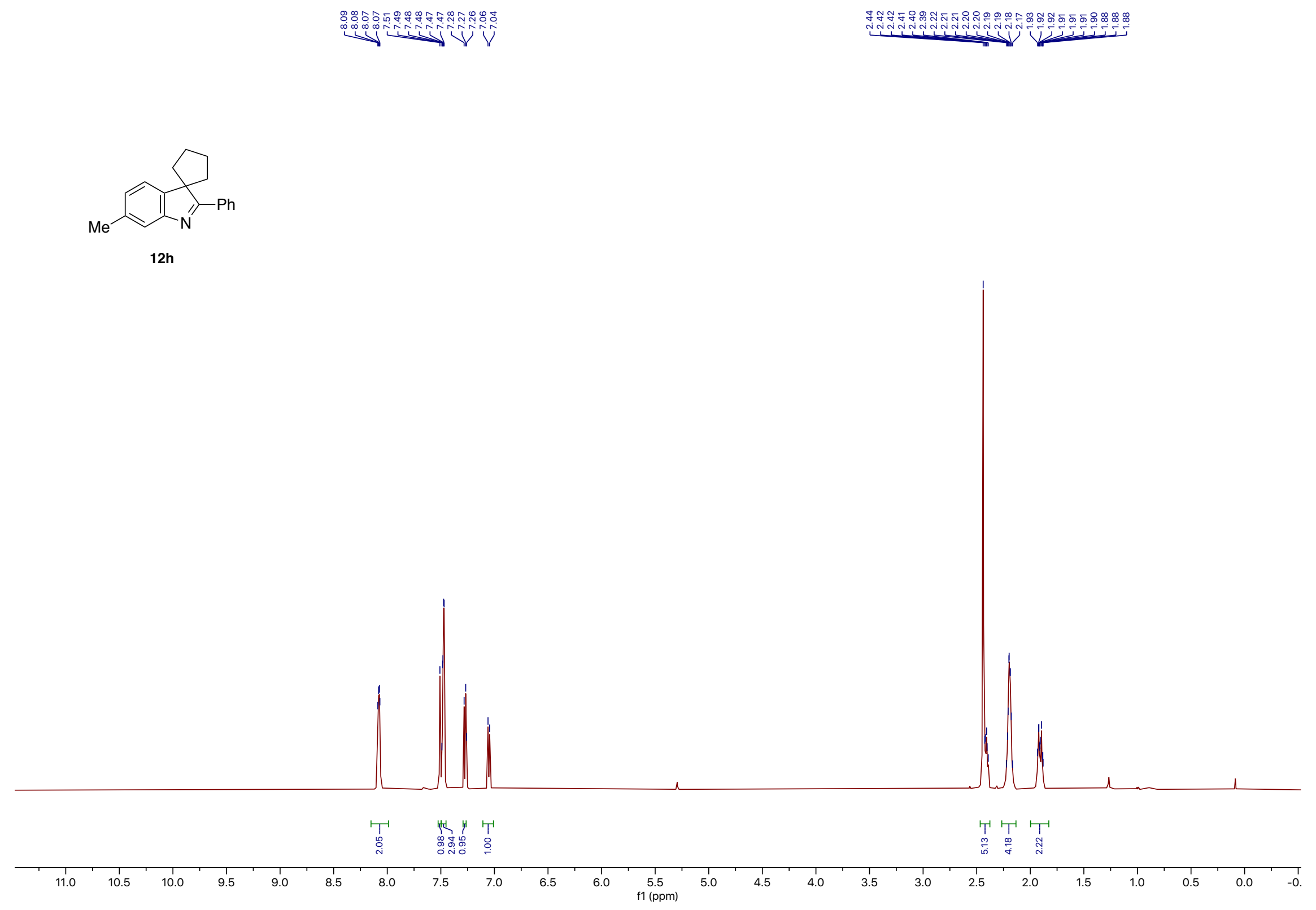
$125 \mathrm{MHz}, \mathrm{CDCl}_{3}$

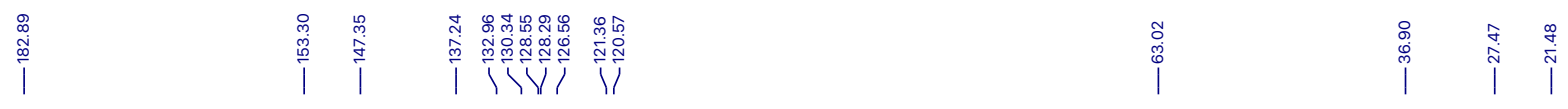

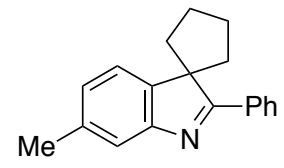

$12 \mathrm{~h}$
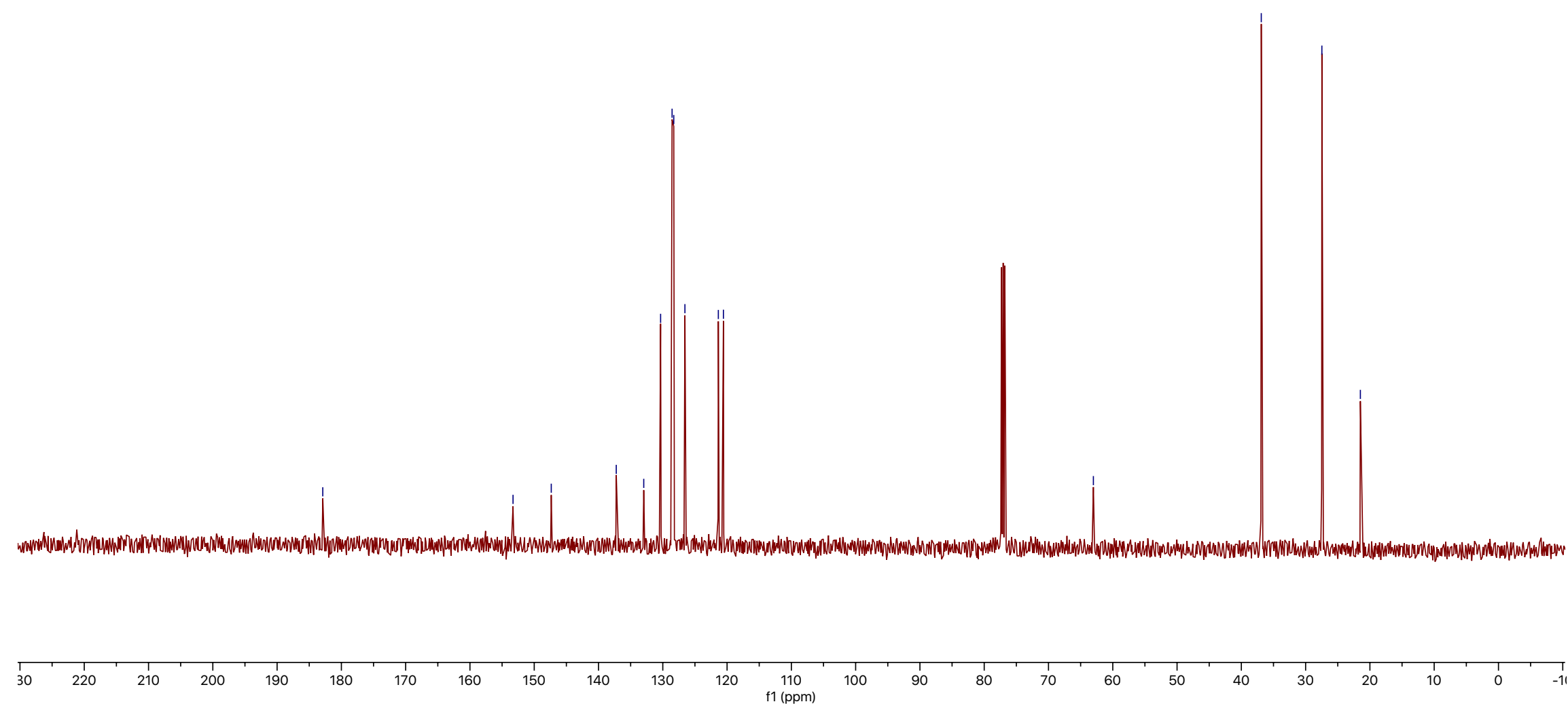
$500 \mathrm{MHz}, \mathrm{CDCl}_{3}$

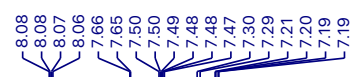

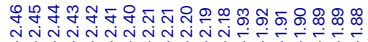

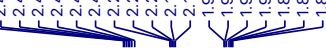

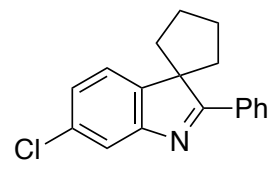

12i

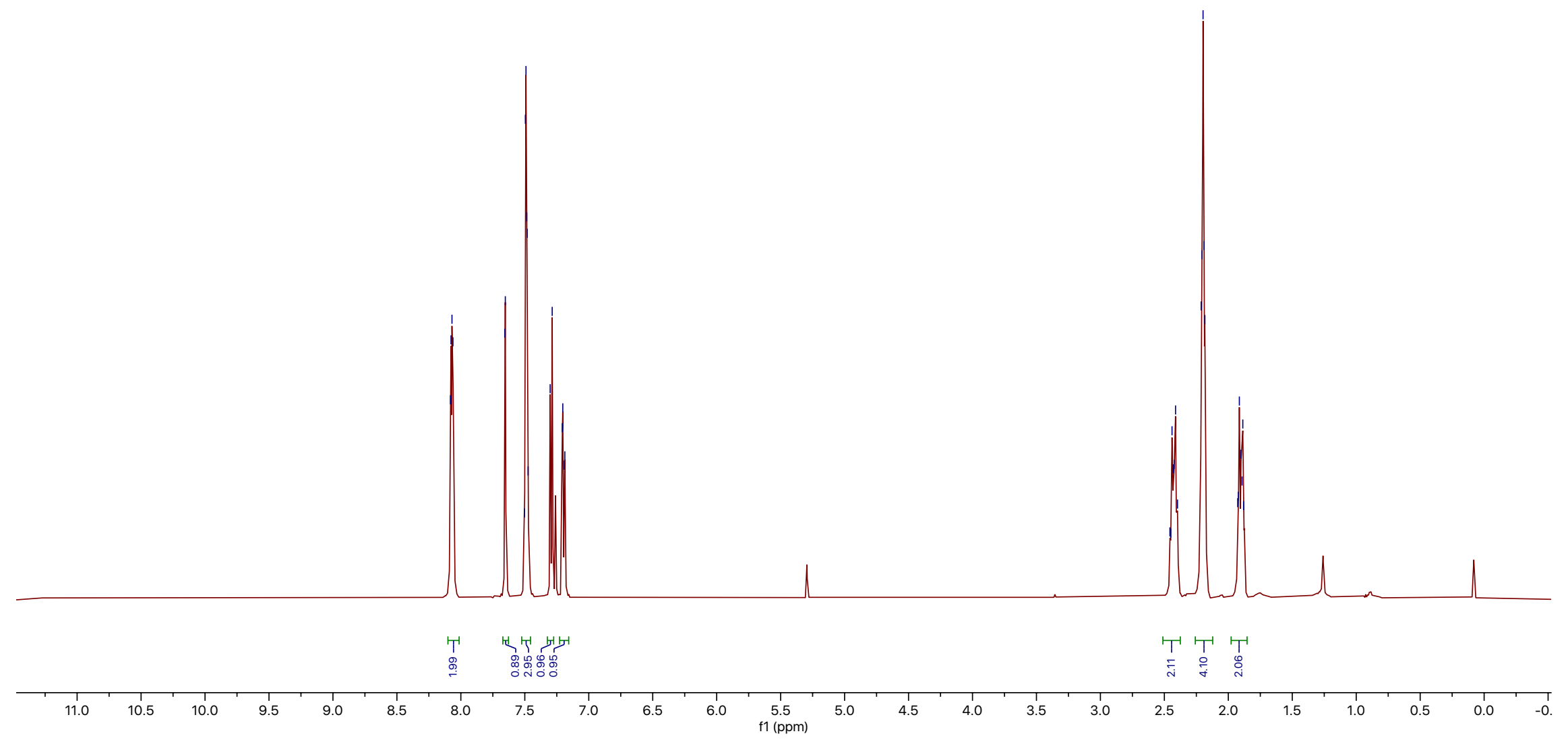


$125 \mathrm{MHz}, \mathrm{CDCl}_{3}$

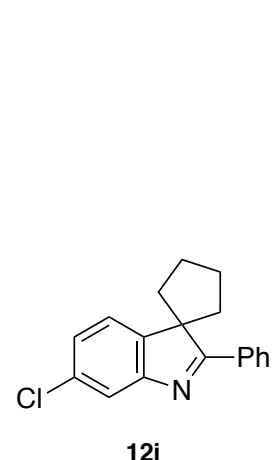

l|l l
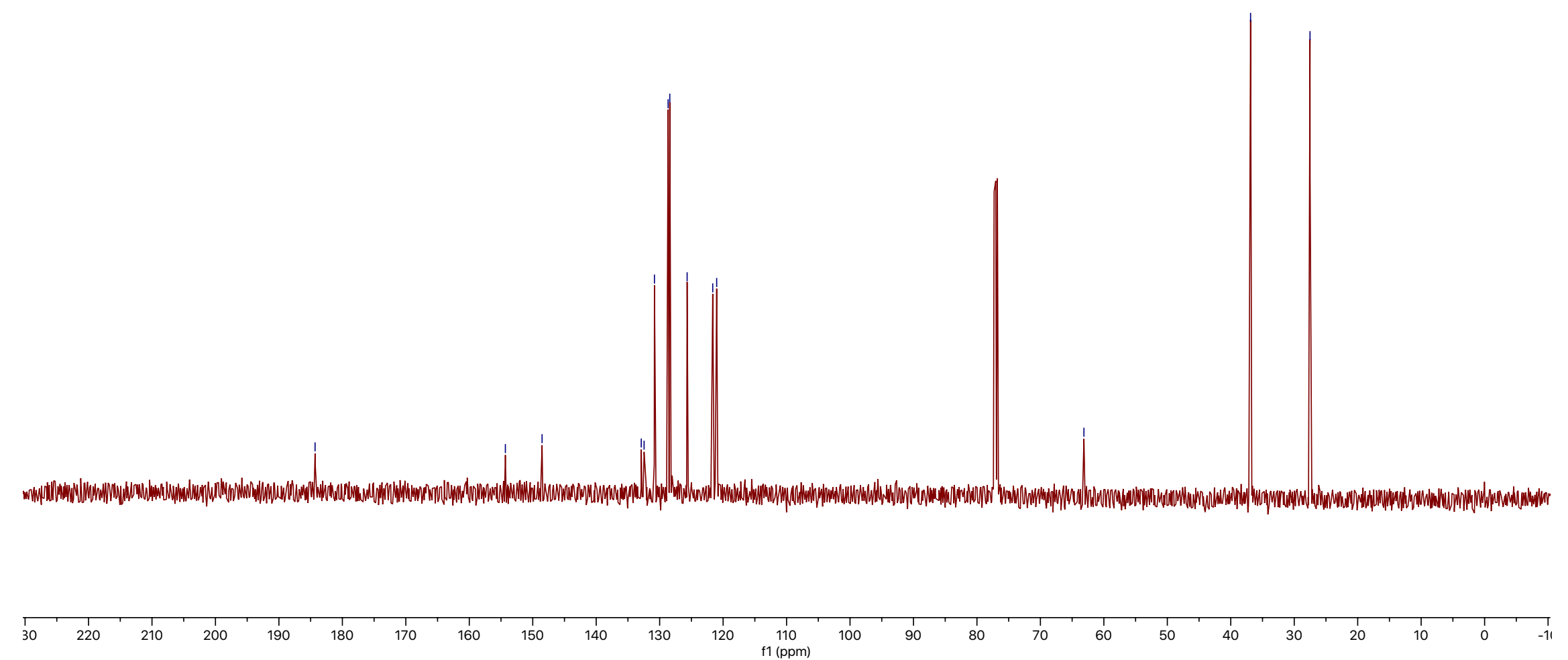
$500 \mathrm{MHz}, \mathrm{CDCl}_{3}$

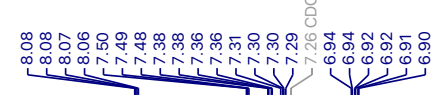

$\longrightarrow$

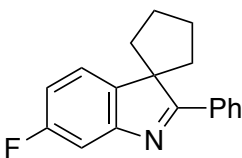

12j

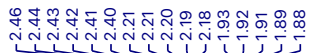

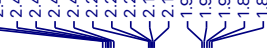

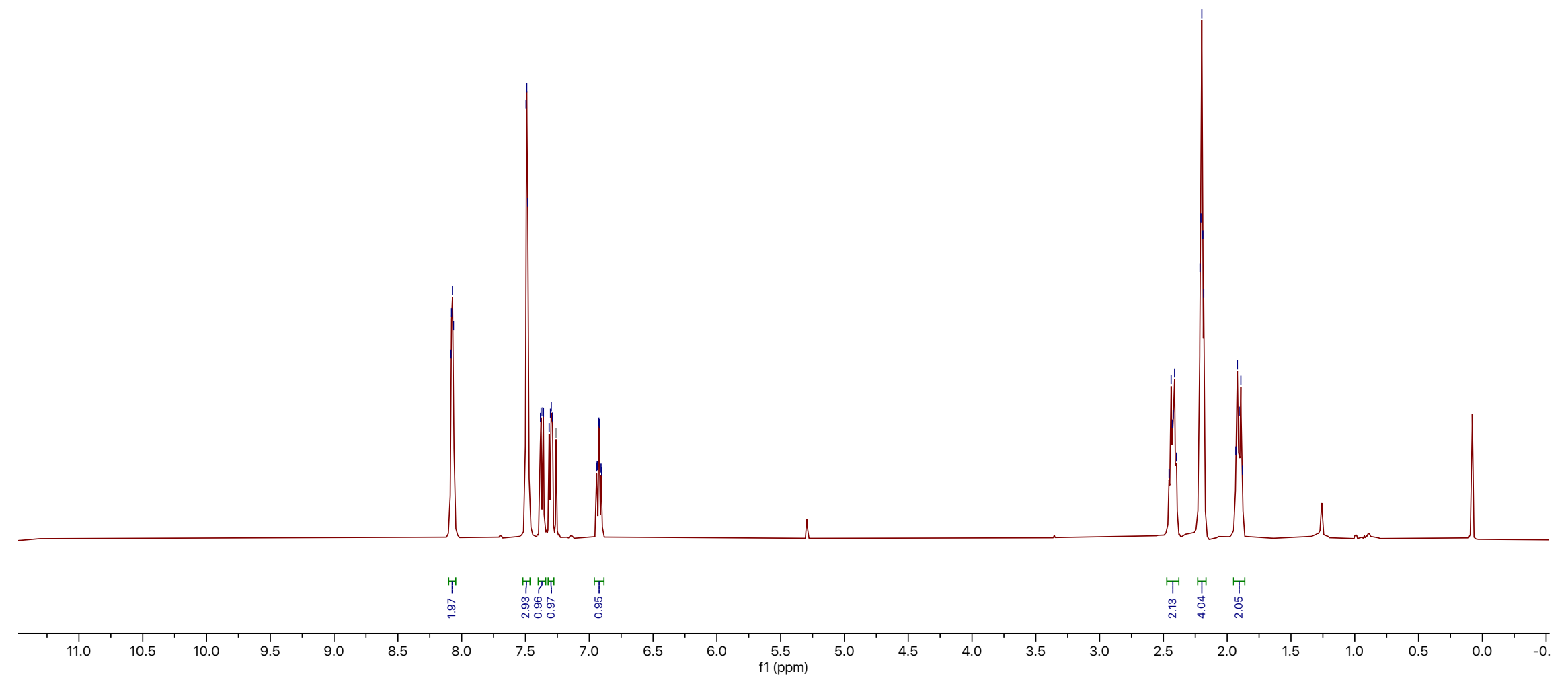


$125 \mathrm{MHz}, \mathrm{CDCl}_{3}$

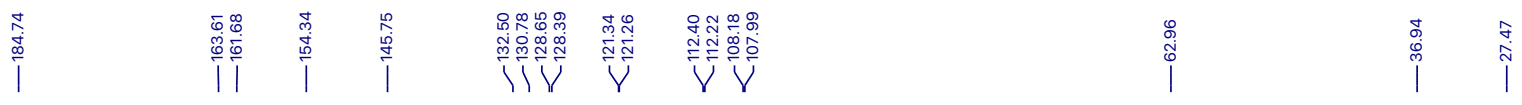

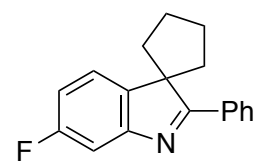

12j
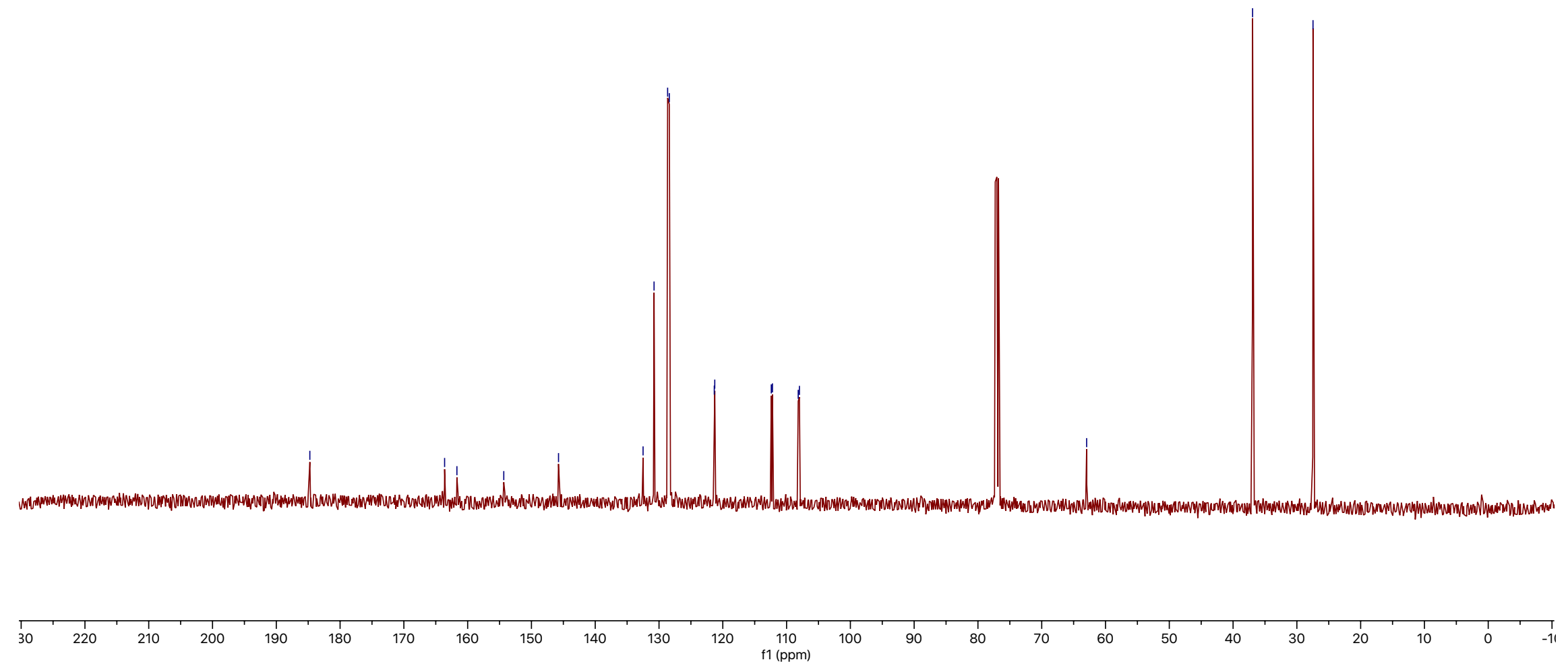
$500 \mathrm{MHz}, \mathrm{CDCl}_{3}$
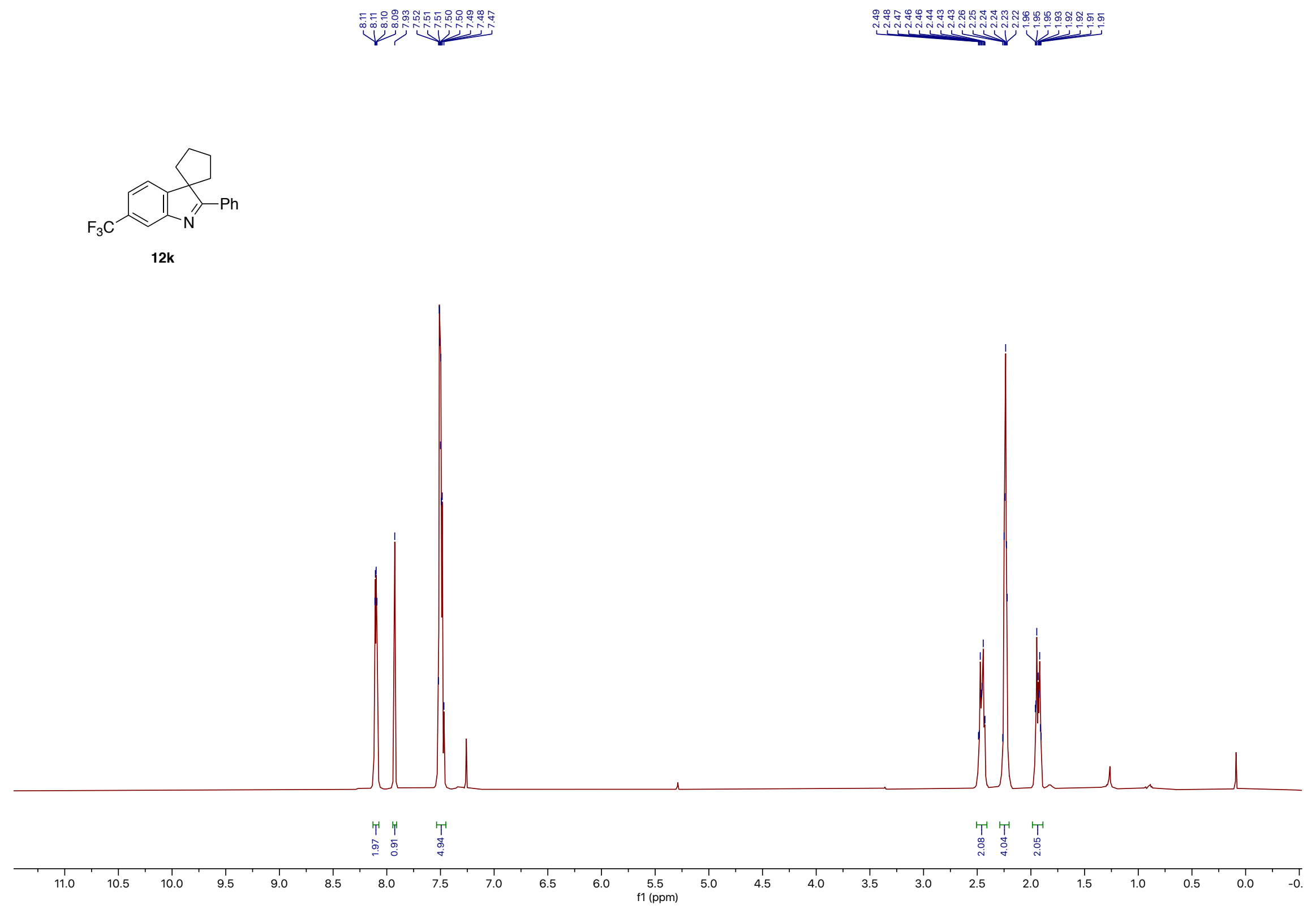
$125 \mathrm{MHz}, \mathrm{CDCl}_{3}$
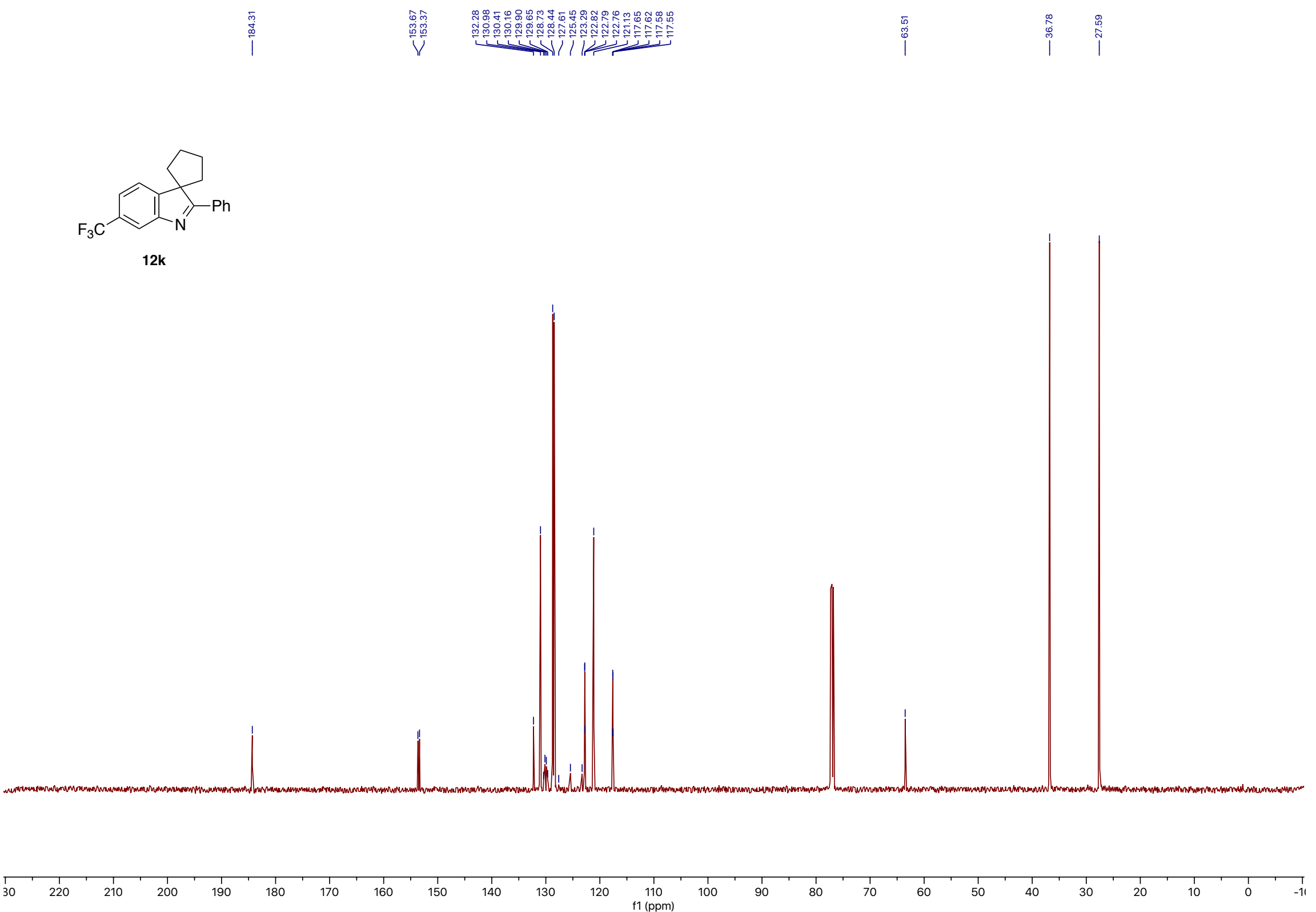
$500 \mathrm{MHz}, \mathrm{CDCl}_{3}$
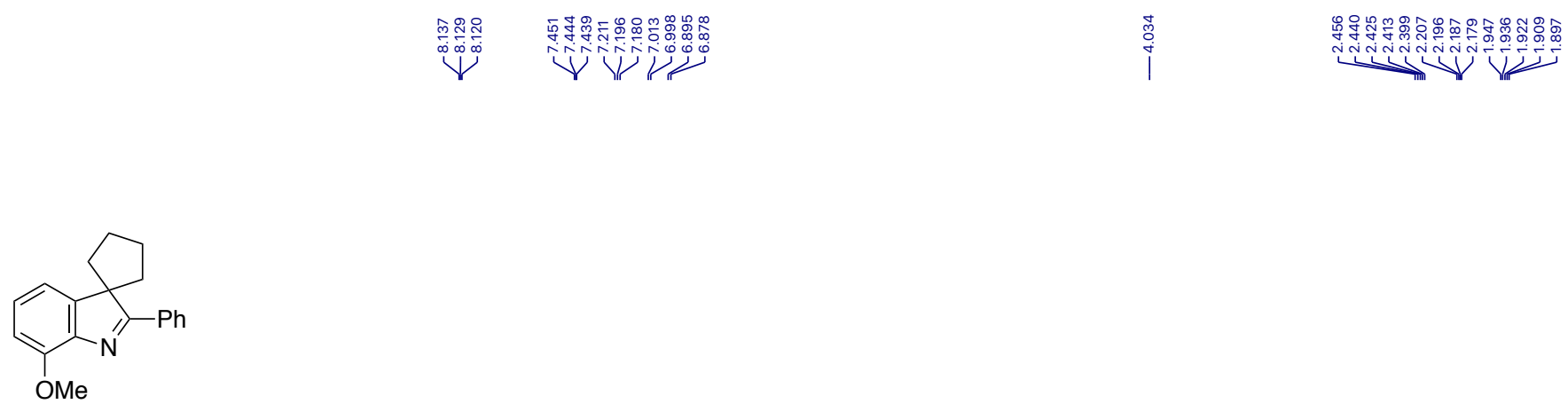

121

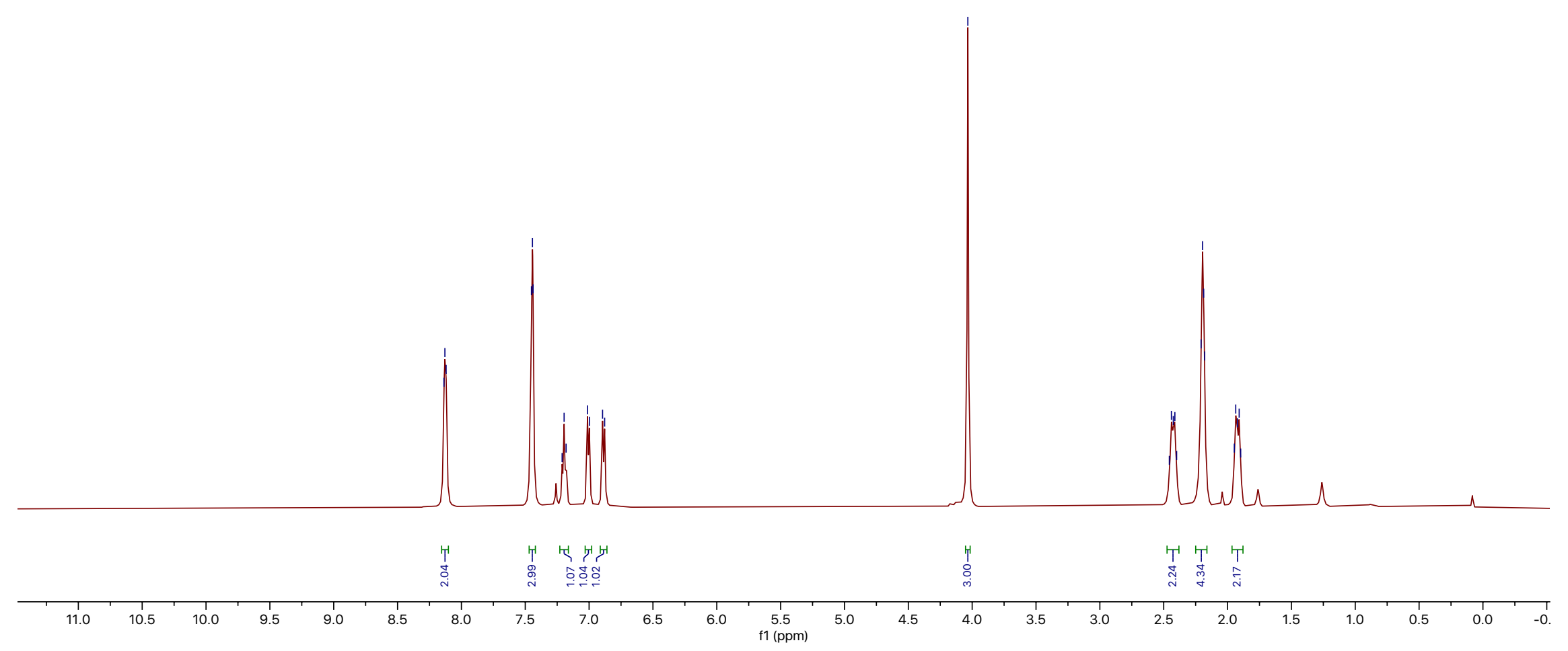


$125 \mathrm{MHz}, \mathrm{CDCl}_{3}$

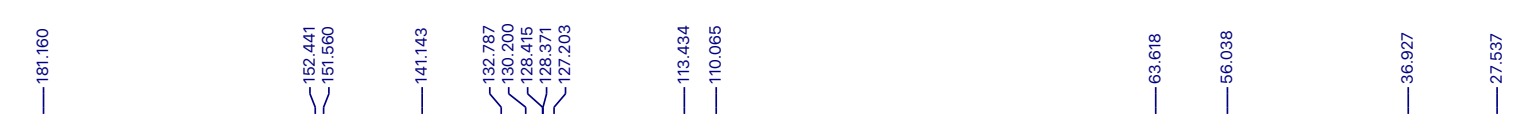

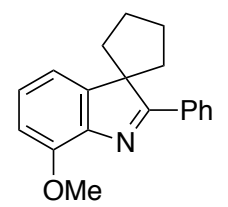

12
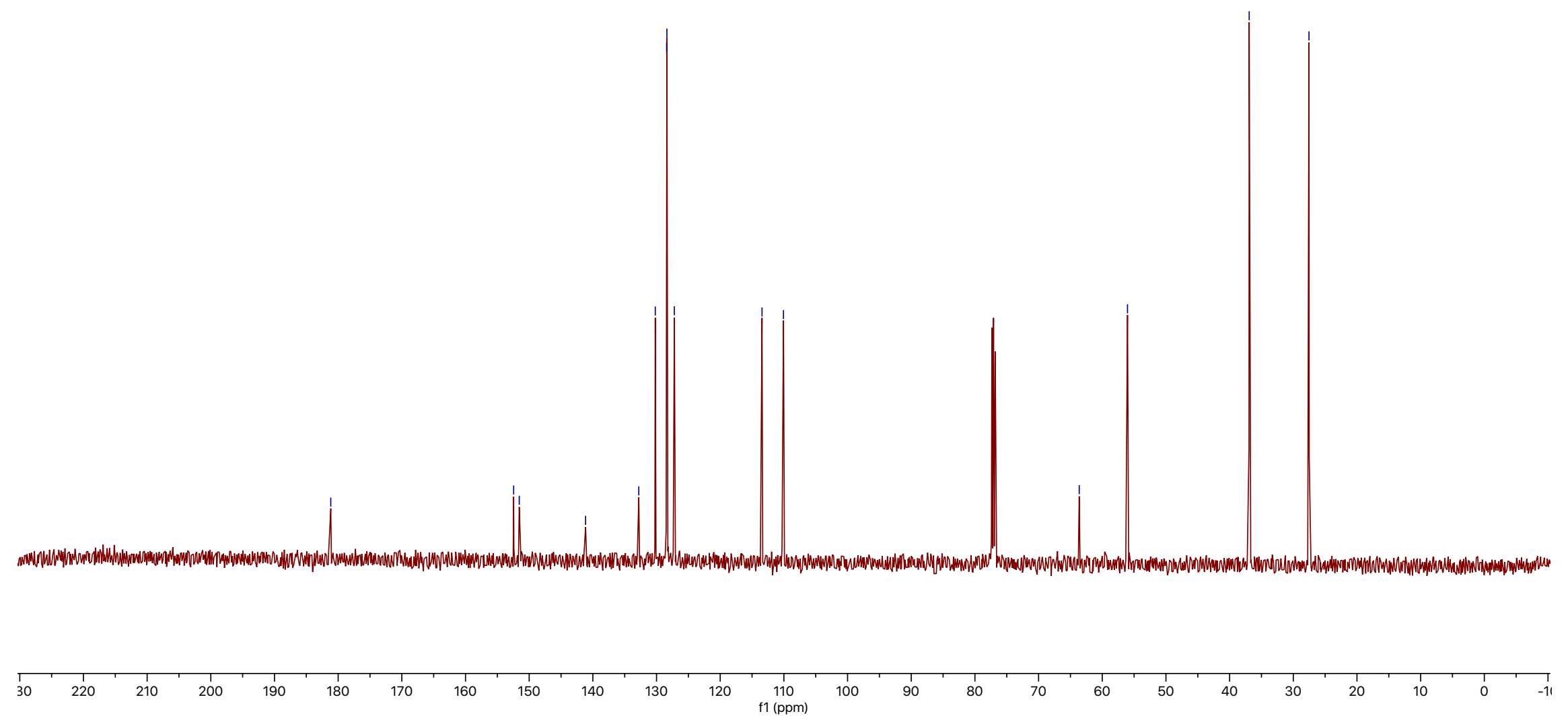
$500 \mathrm{MHz}, \mathrm{CDCl}_{3}$
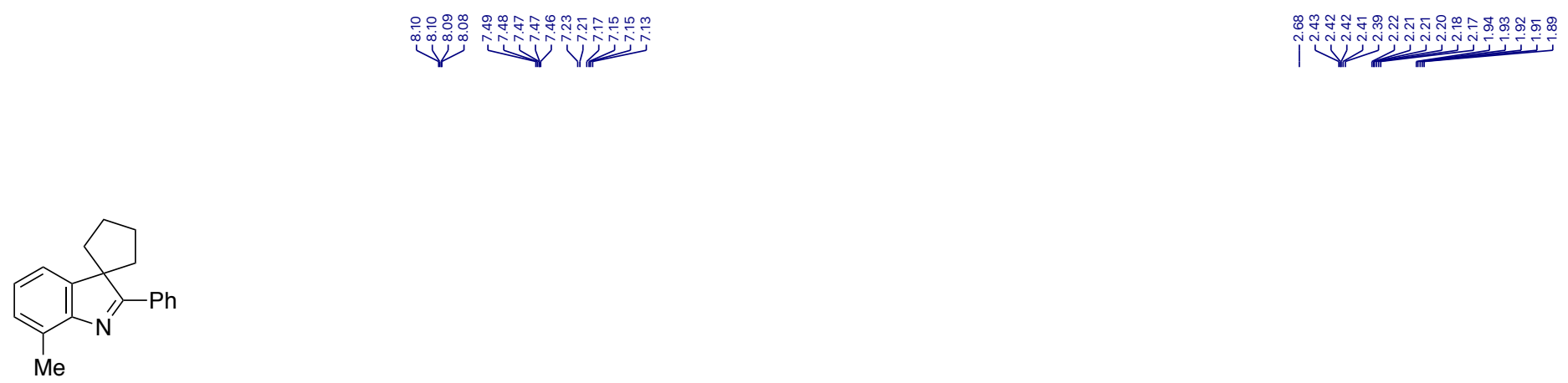

$12 m$

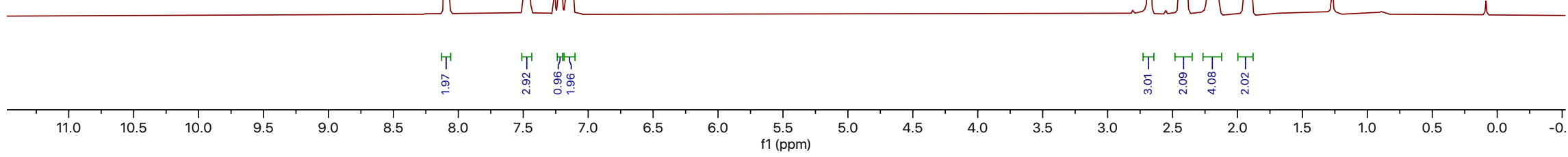


$125 \mathrm{MHz}, \mathrm{CDCl}_{3}$

I

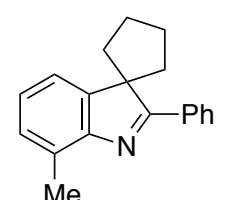

$12 m$
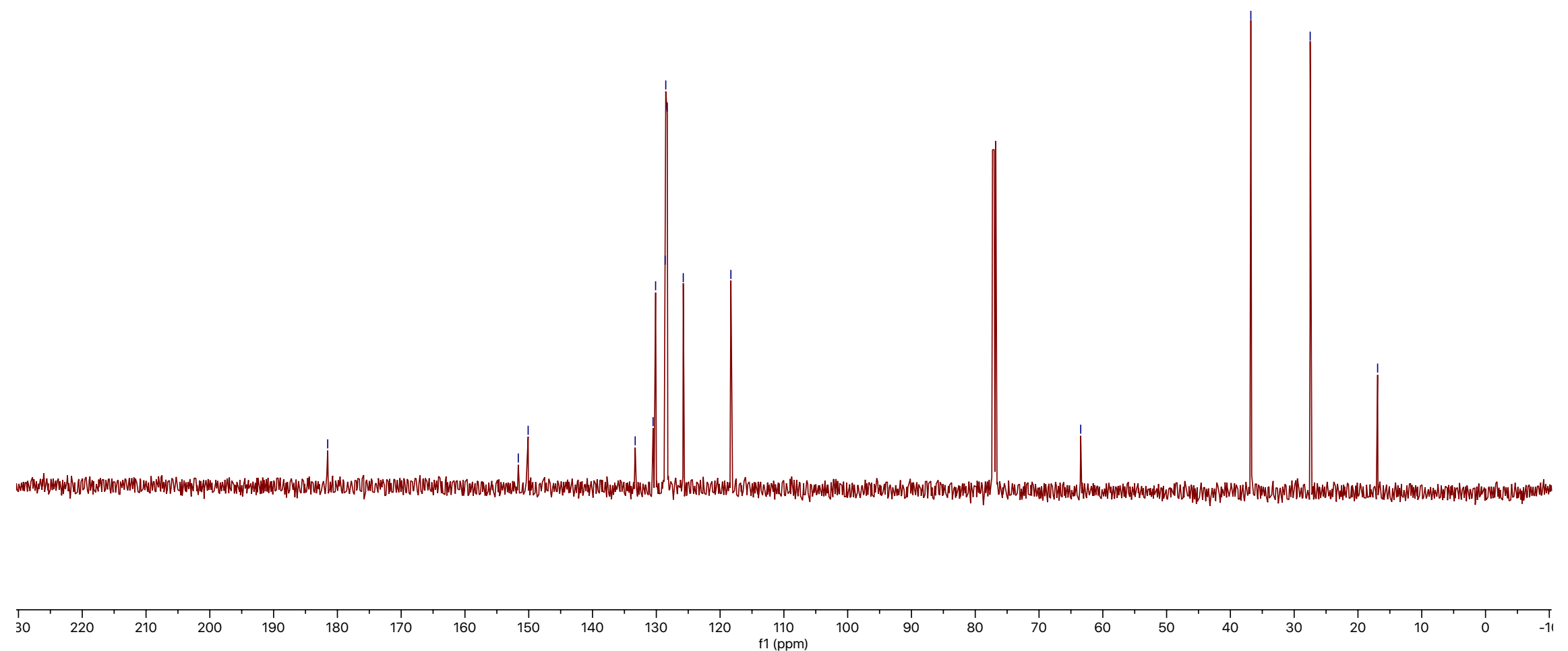
$500 \mathrm{MHz}, \mathrm{CDCl}_{3}$
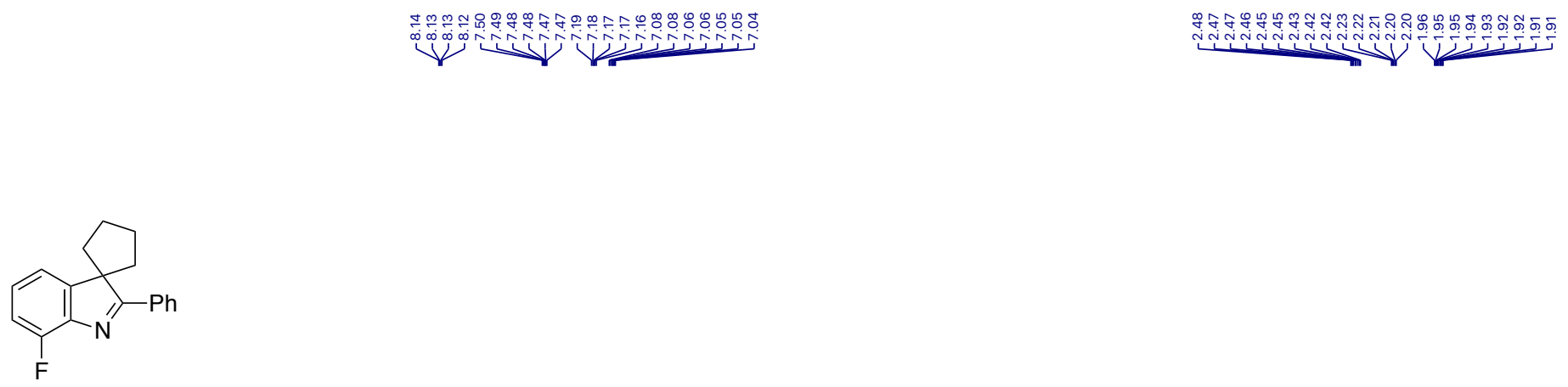

$12 n$

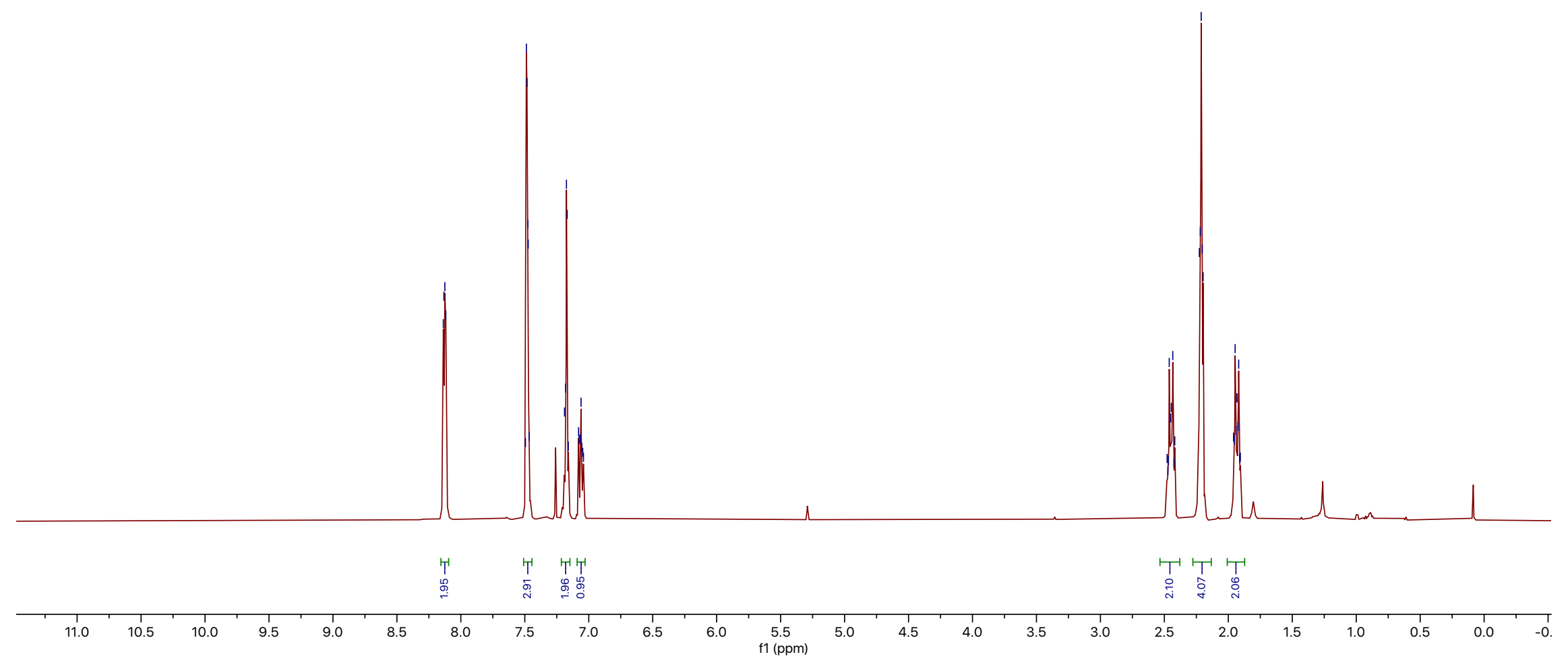


$125 \mathrm{MHz}, \mathrm{CDCl}_{3}$

I

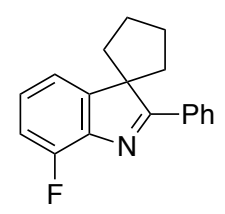

12n
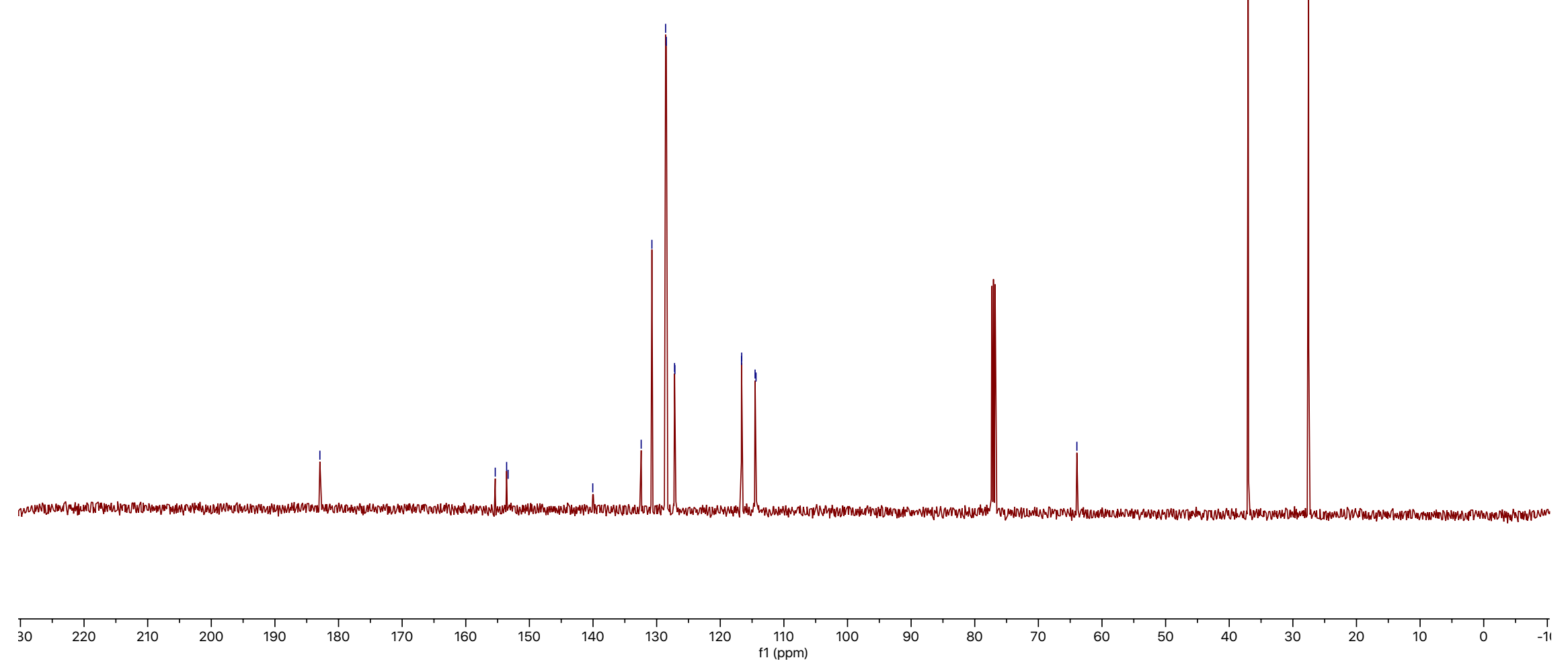
$500 \mathrm{MHz}, \mathrm{CDCl}_{3}$

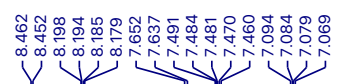

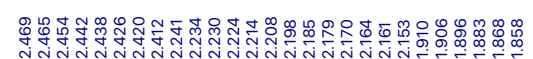
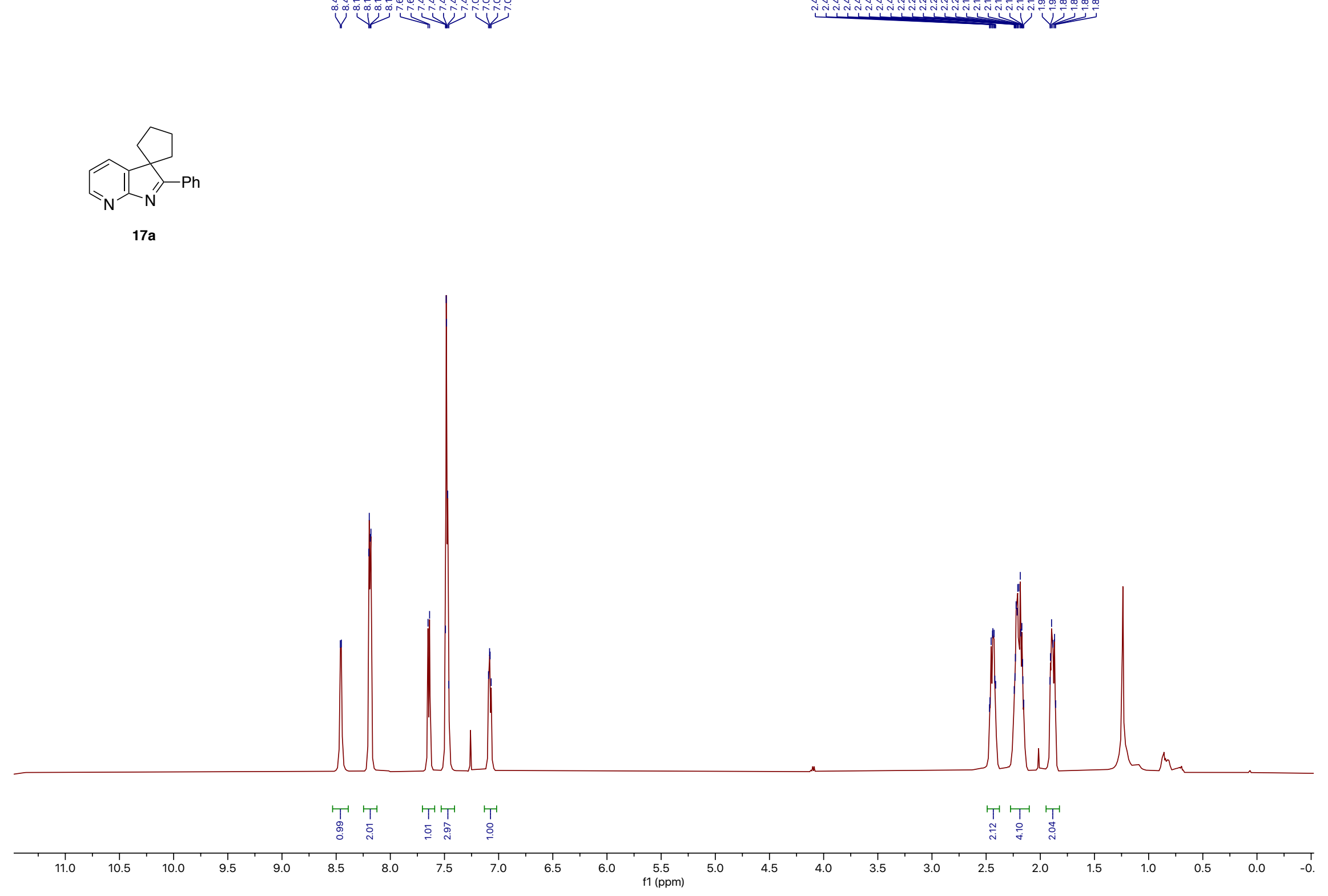
$125 \mathrm{MHz}, \mathrm{CDCl}_{3}$

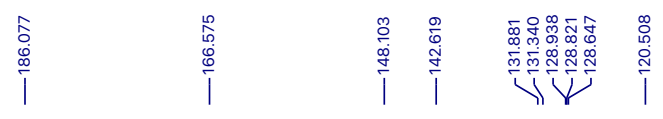

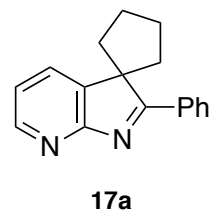

$17 a$
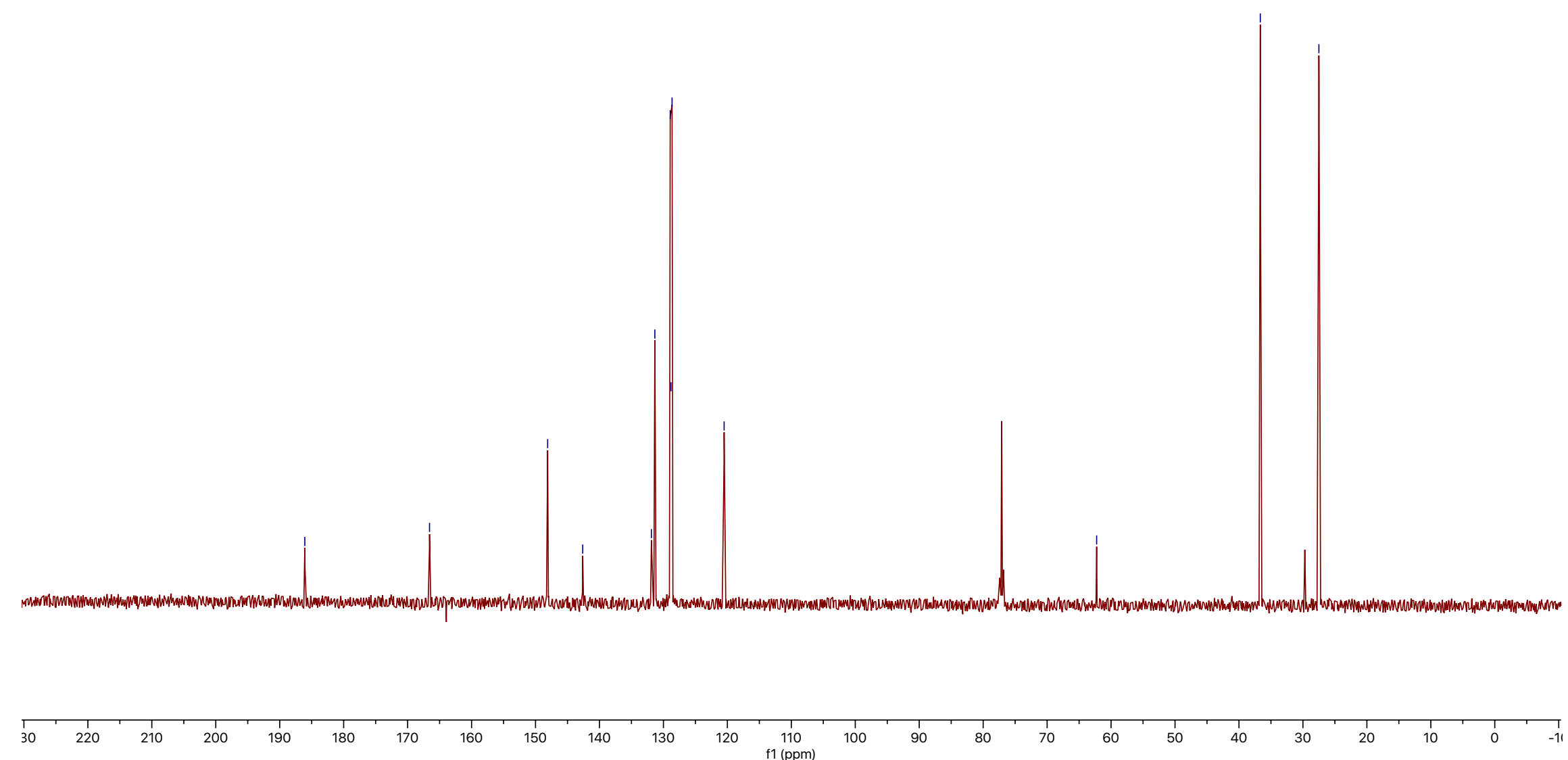
$500 \mathrm{MHz}, \mathrm{CDCl}_{3}$
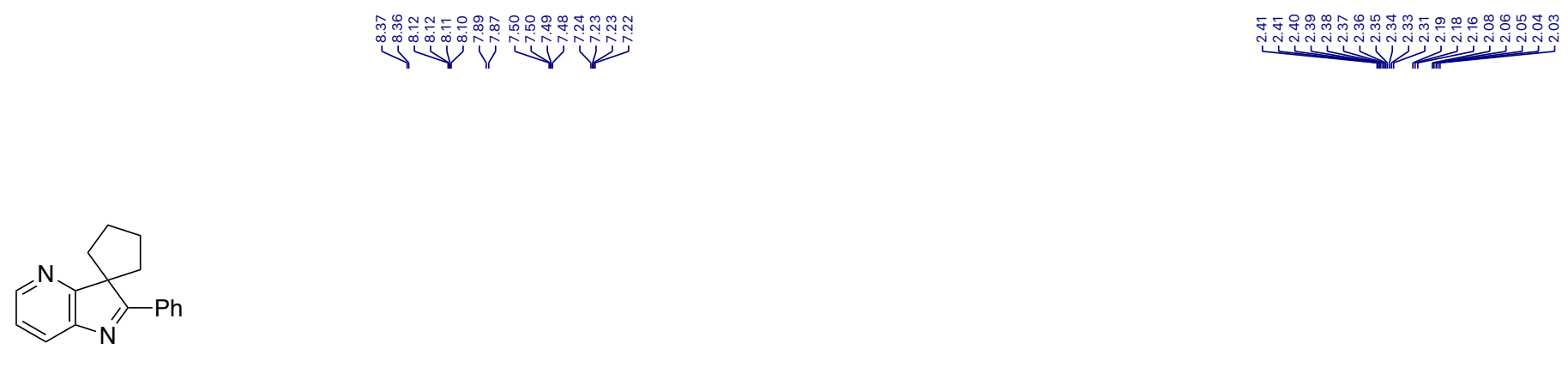

$17 b$

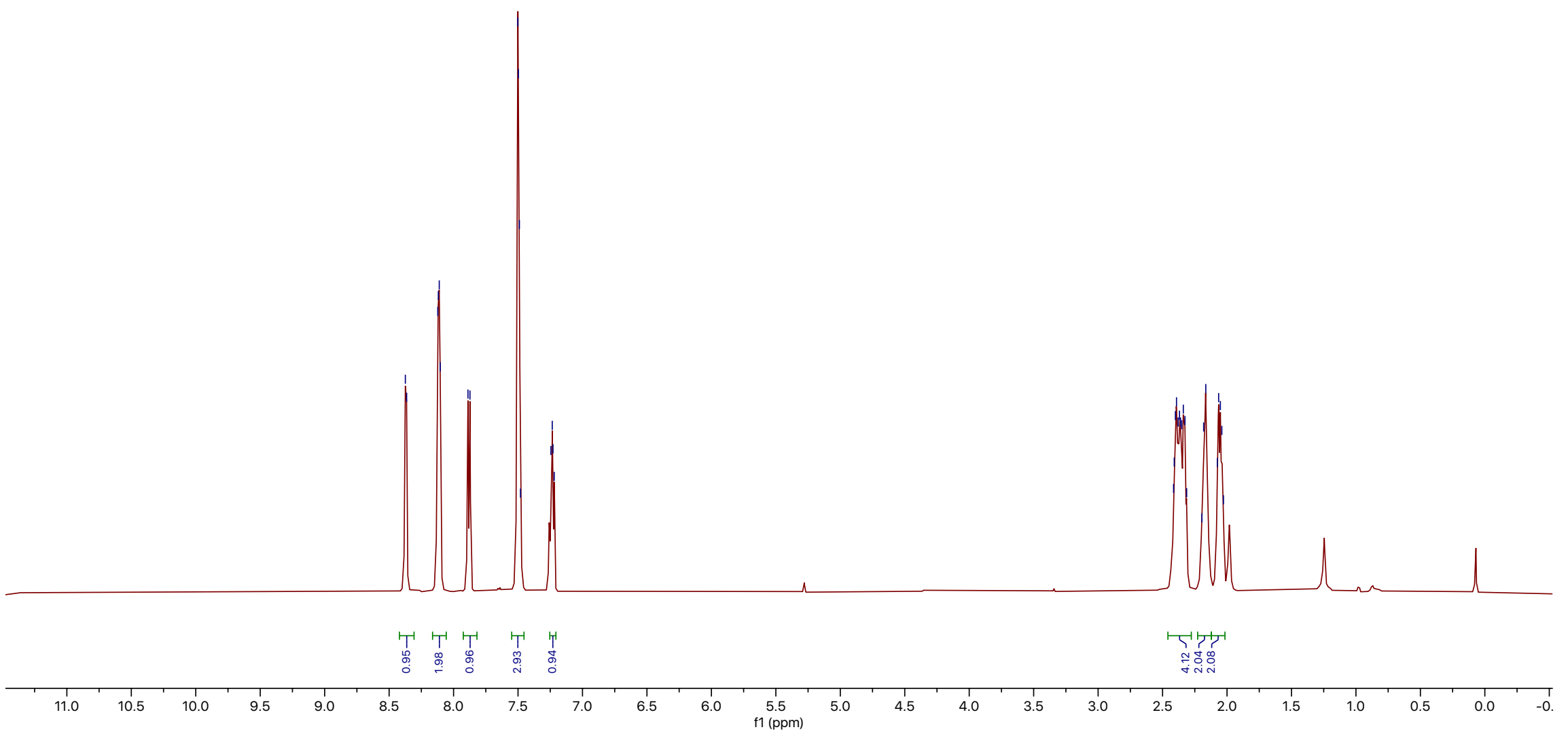


$125 \mathrm{MHz}, \mathrm{CDCl}_{3}$

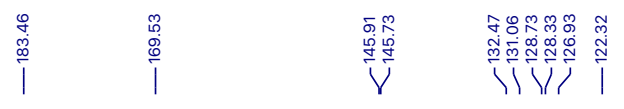
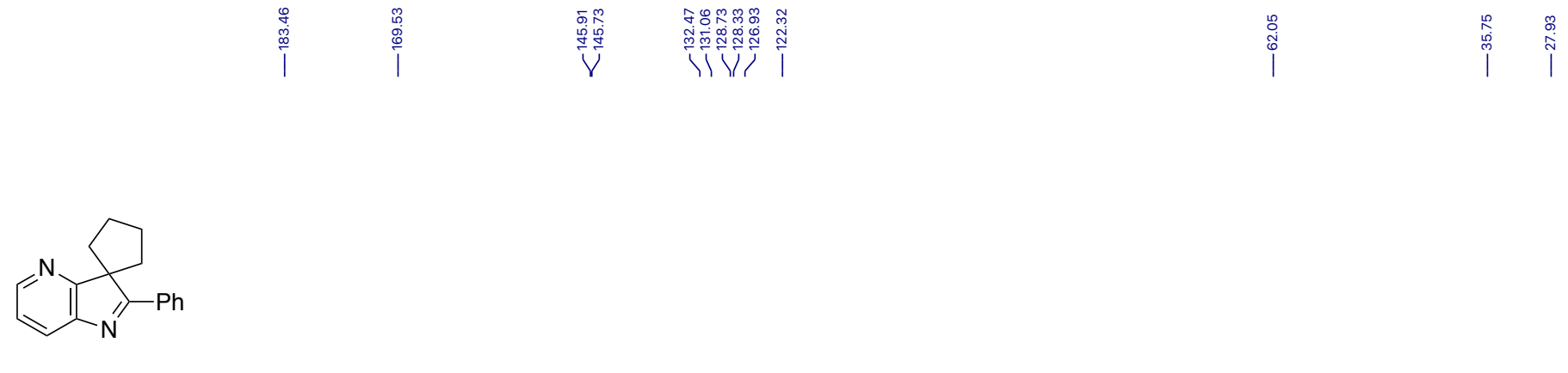

17b
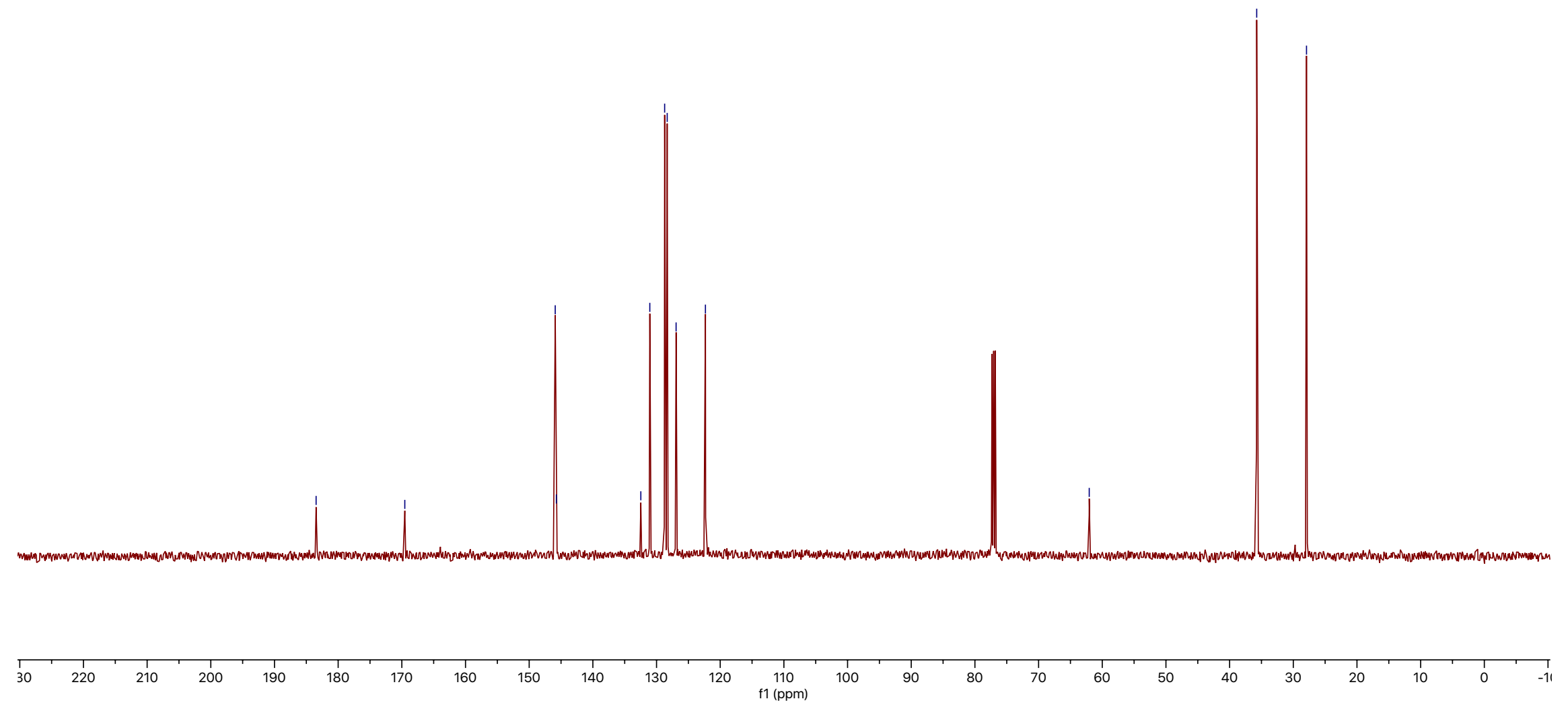
$500 \mathrm{MHz}, \mathrm{CDCl}_{3}$

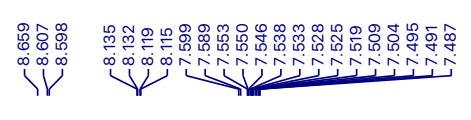

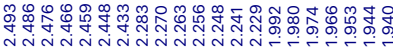

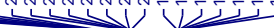

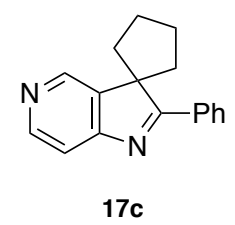

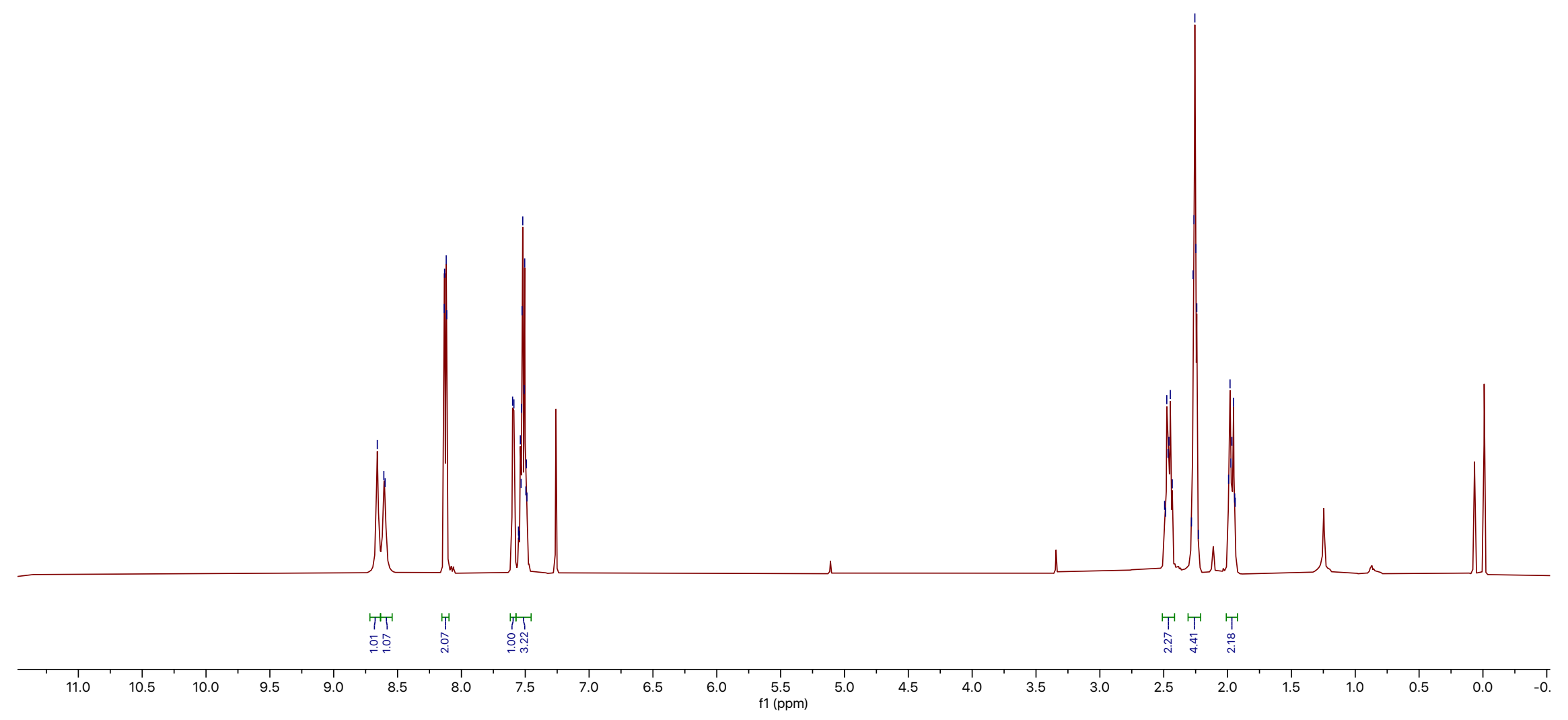


$125 \mathrm{MHz}, \mathrm{CDCl}_{3}$

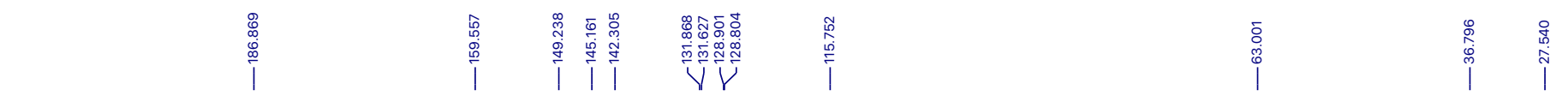
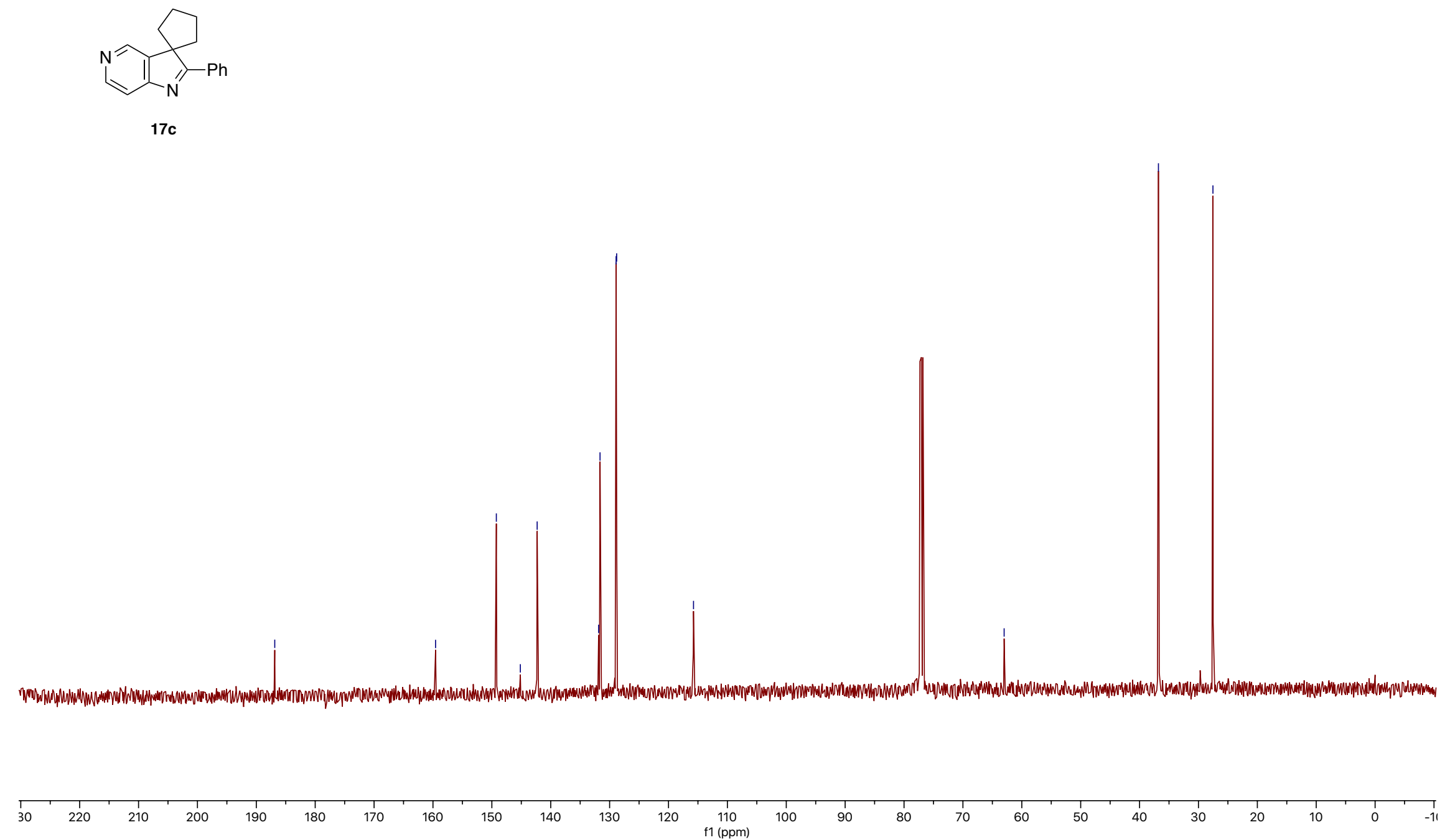
$500 \mathrm{MHz}, \mathrm{CDCl}_{3}$
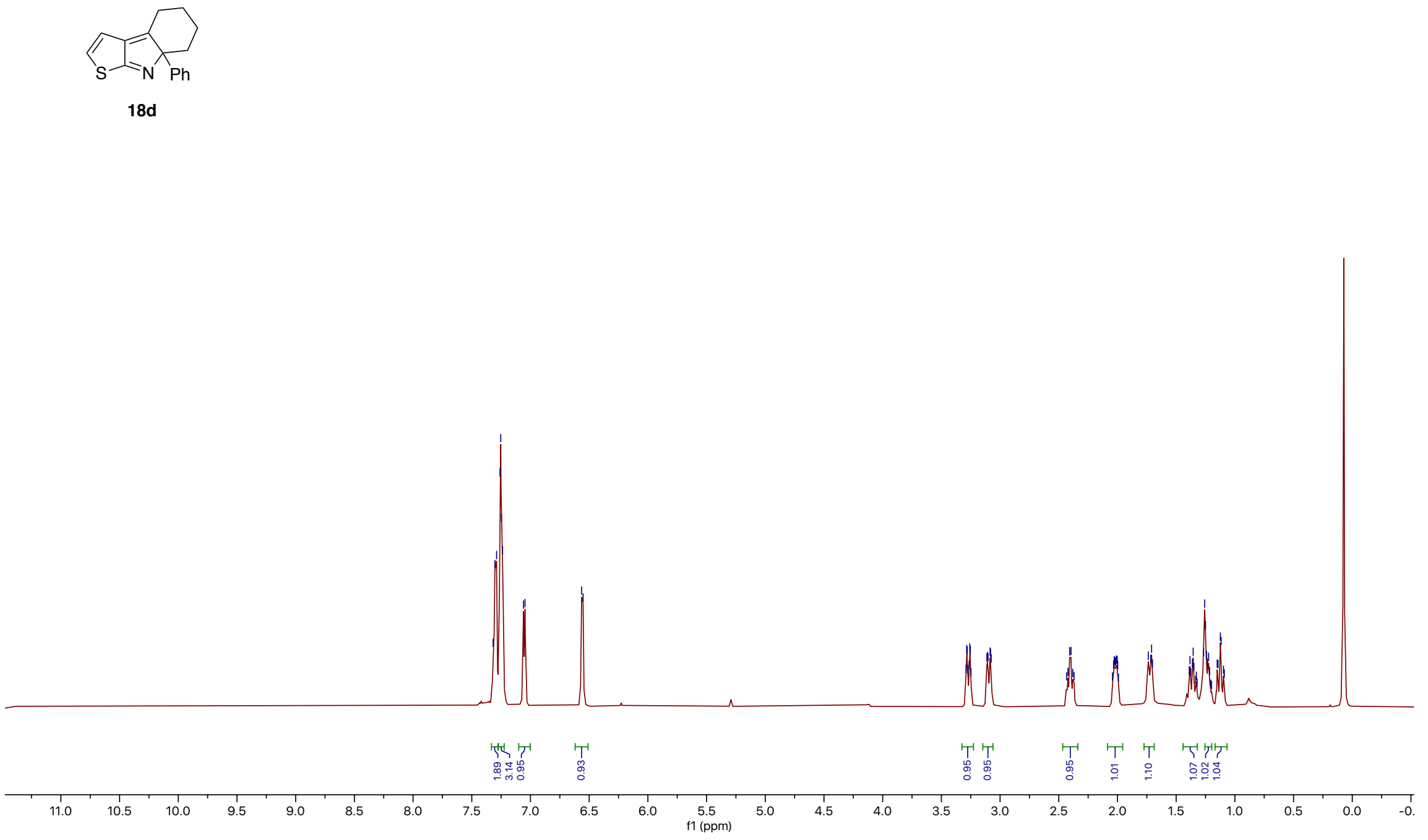
$125 \mathrm{MHz}, \mathrm{CDCl}_{3}$

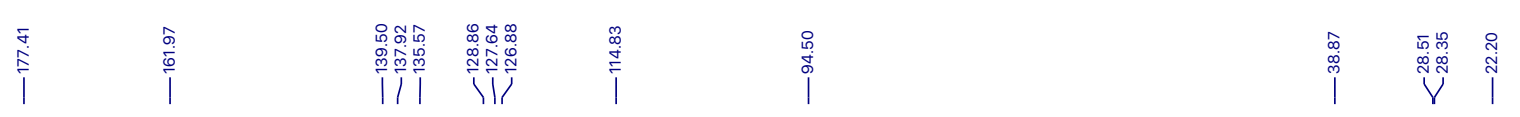

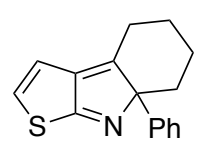

18d
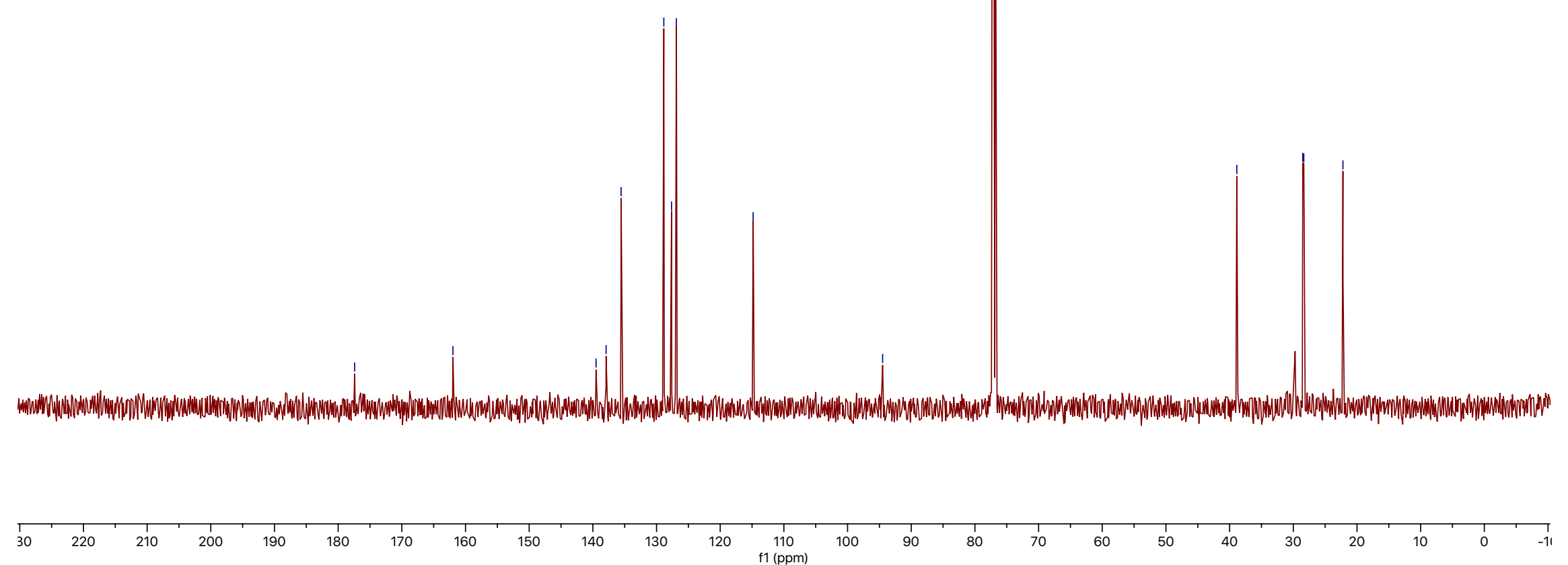
$500 \mathrm{MHz}, \mathrm{CDCl}_{3}$

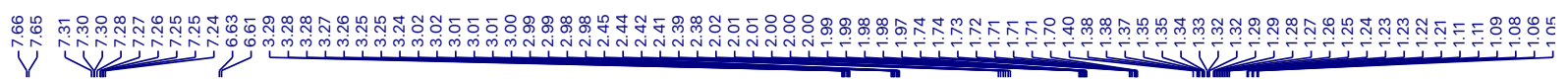

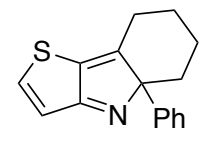

$18 \mathrm{e}$

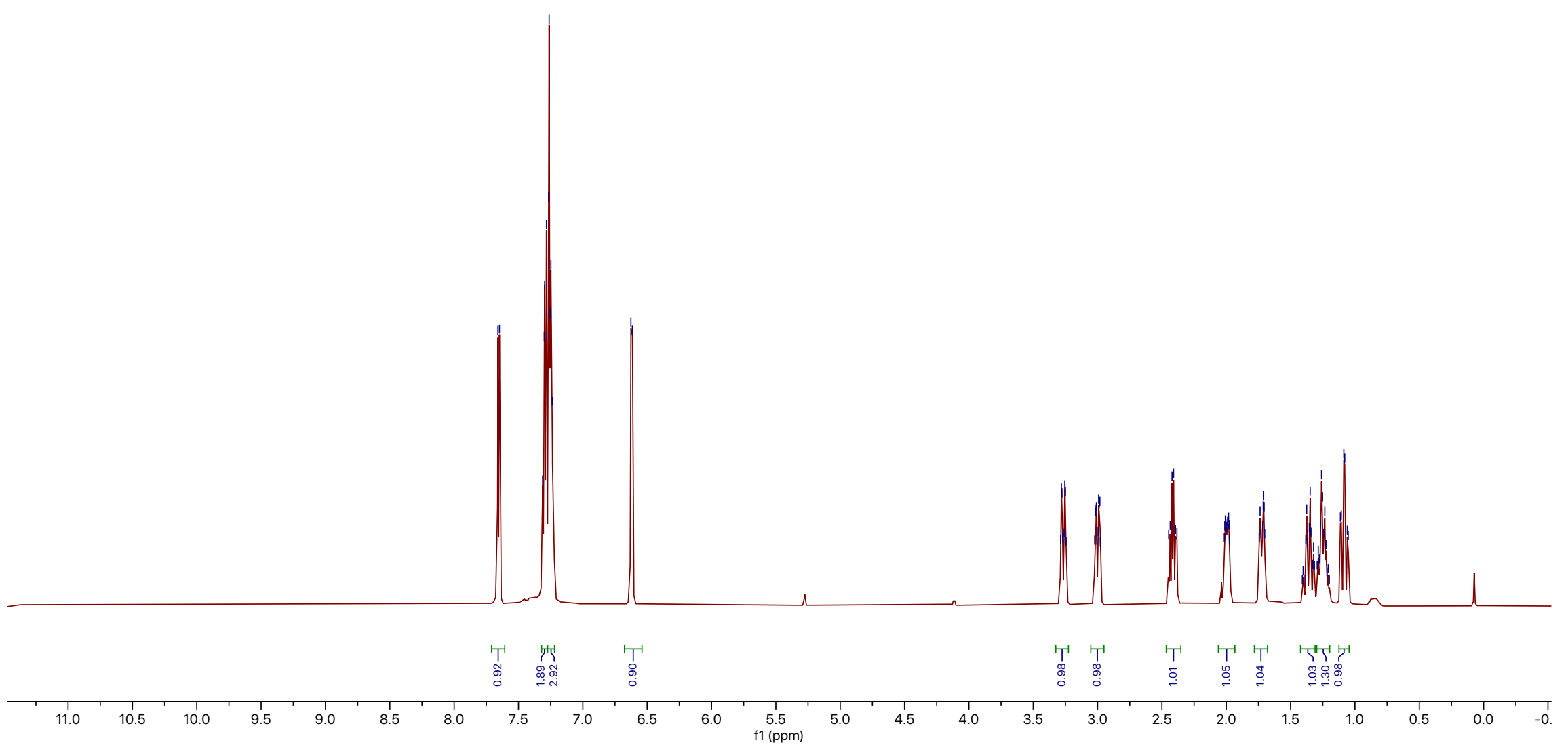


$125 \mathrm{MHz}, \mathrm{CDCl}_{3}$

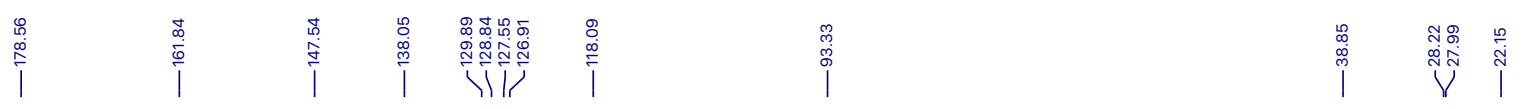

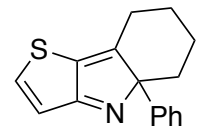

$18 \mathrm{e}$
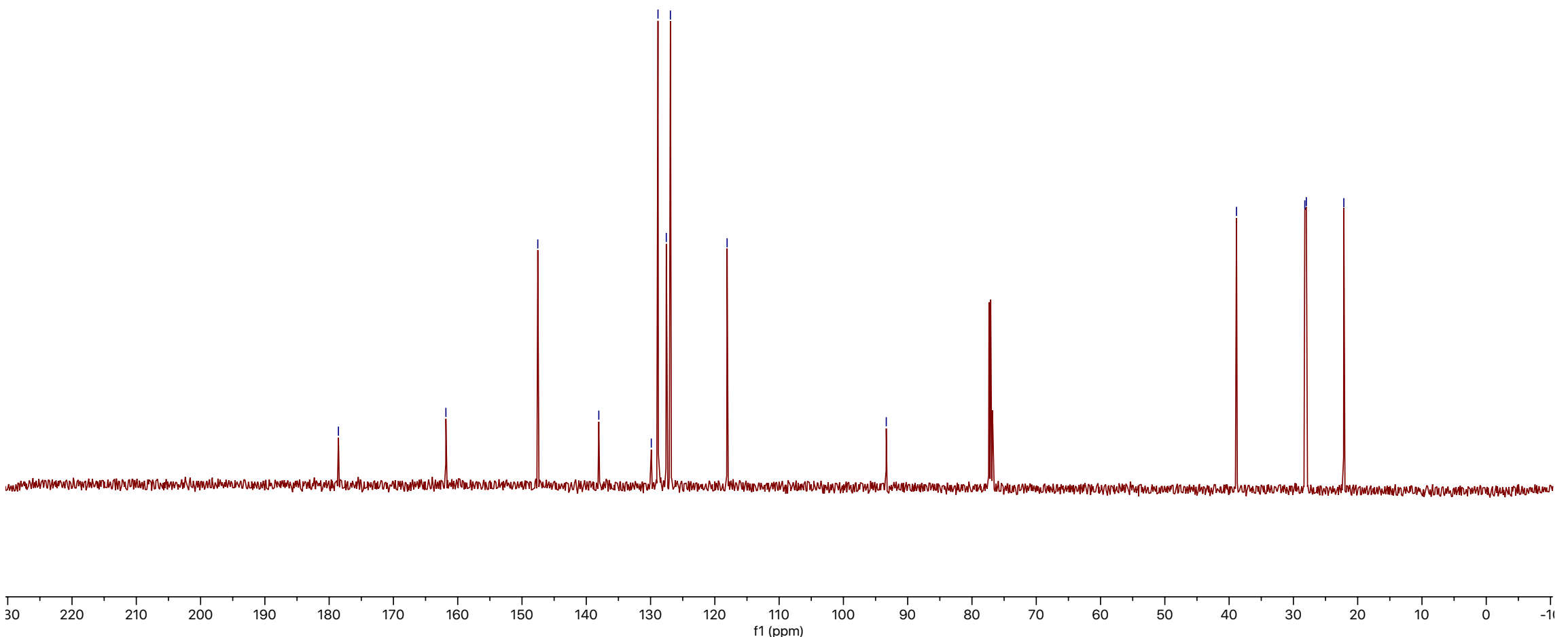
$500 \mathrm{MHz}, \mathrm{CDCl}_{3}$
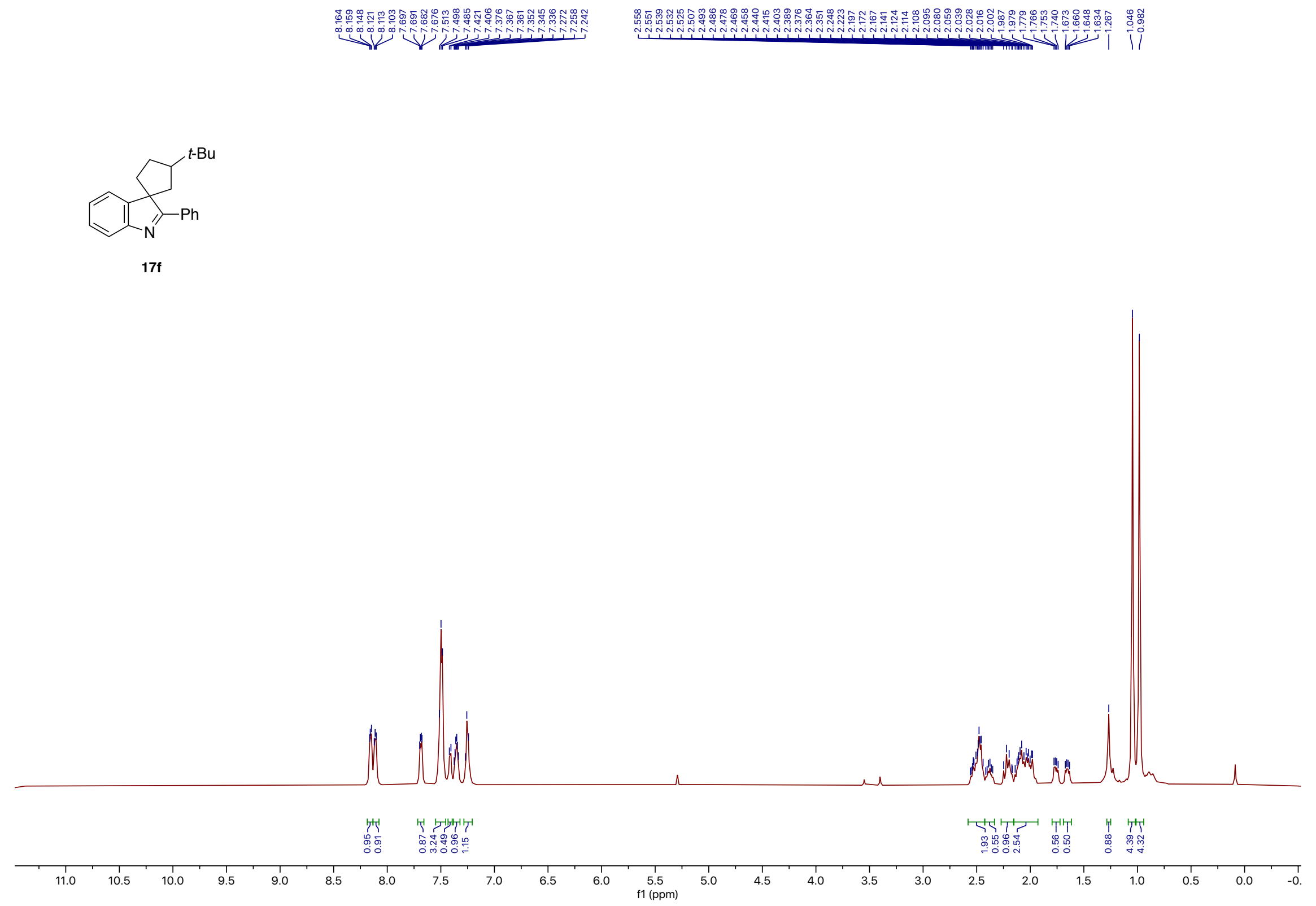
$125 \mathrm{MHz}, \mathrm{CDCl}_{3}$
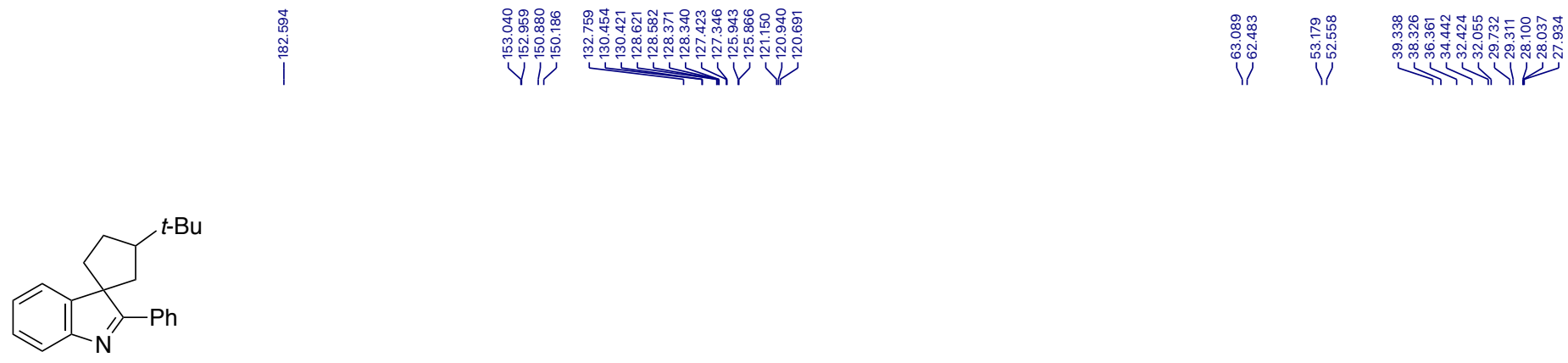

$17 f$
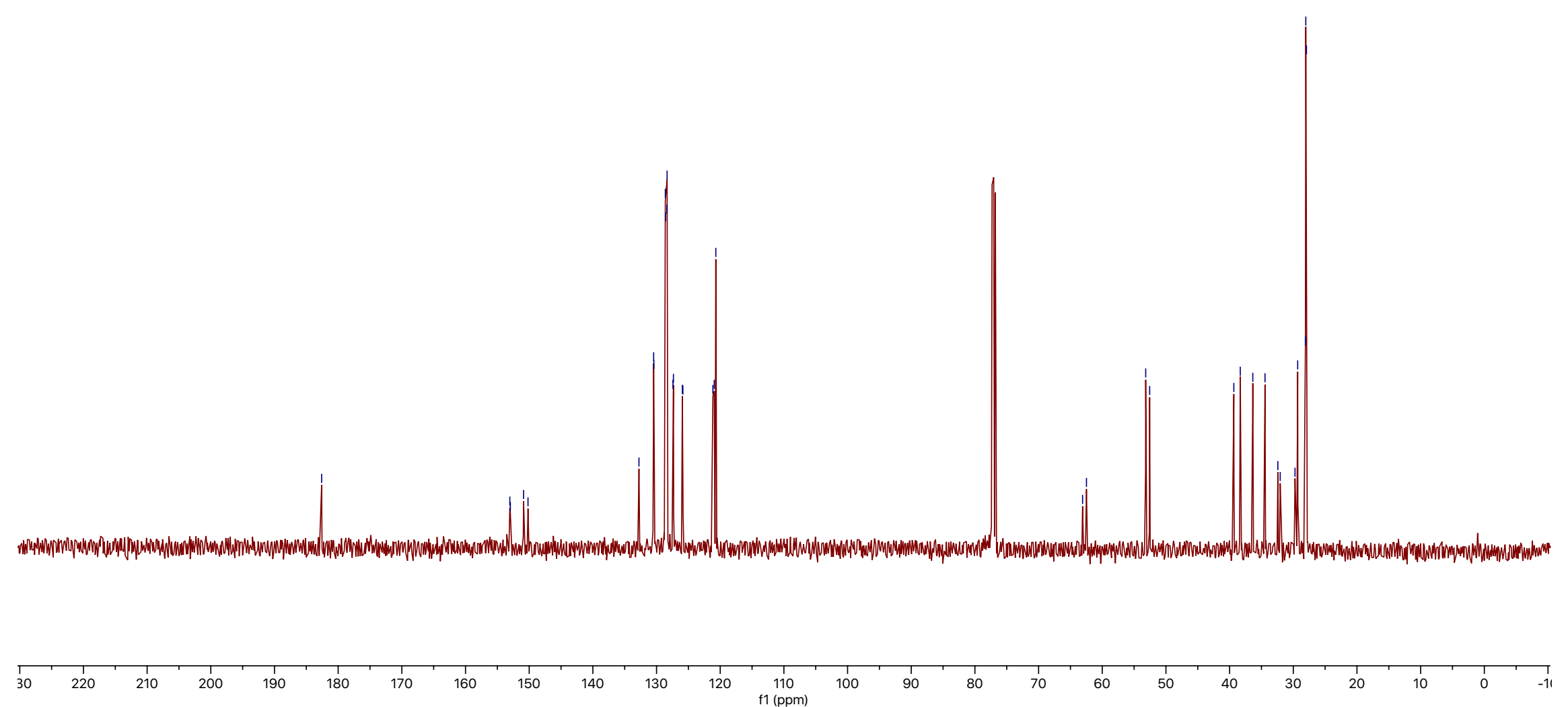
$500 \mathrm{MHz}, \mathrm{CDCl}_{3}$

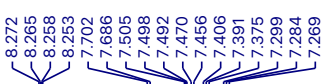

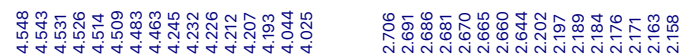
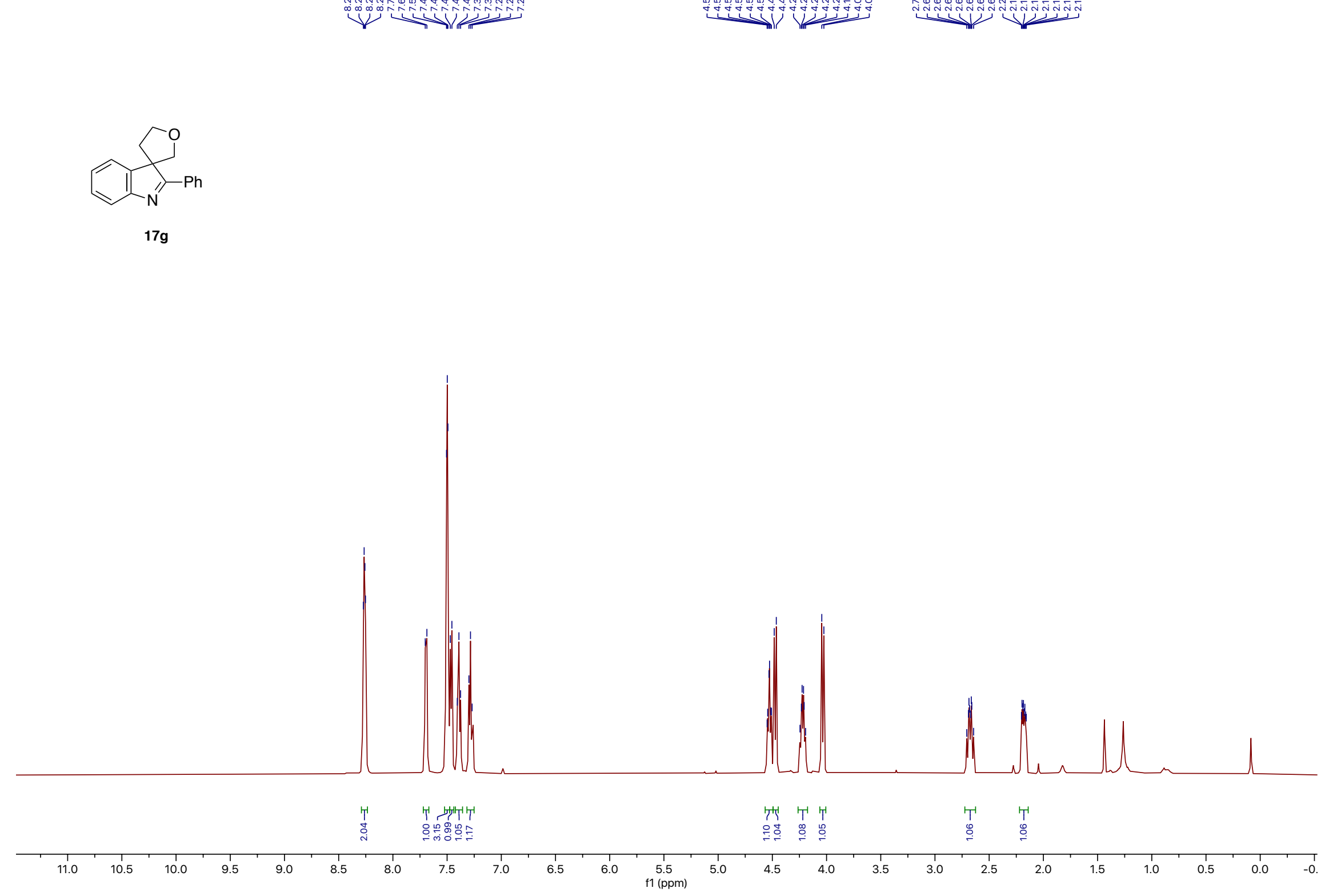
$125 \mathrm{MHz}, \mathrm{CDCl}_{3}$

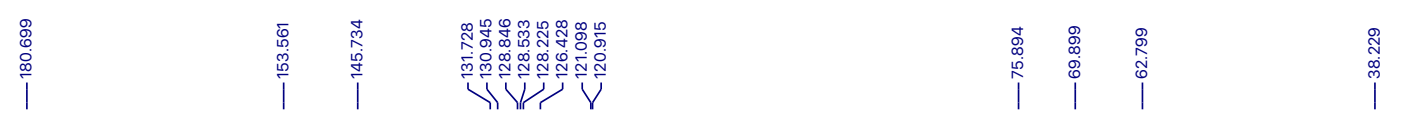

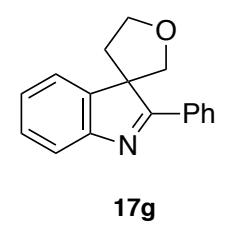

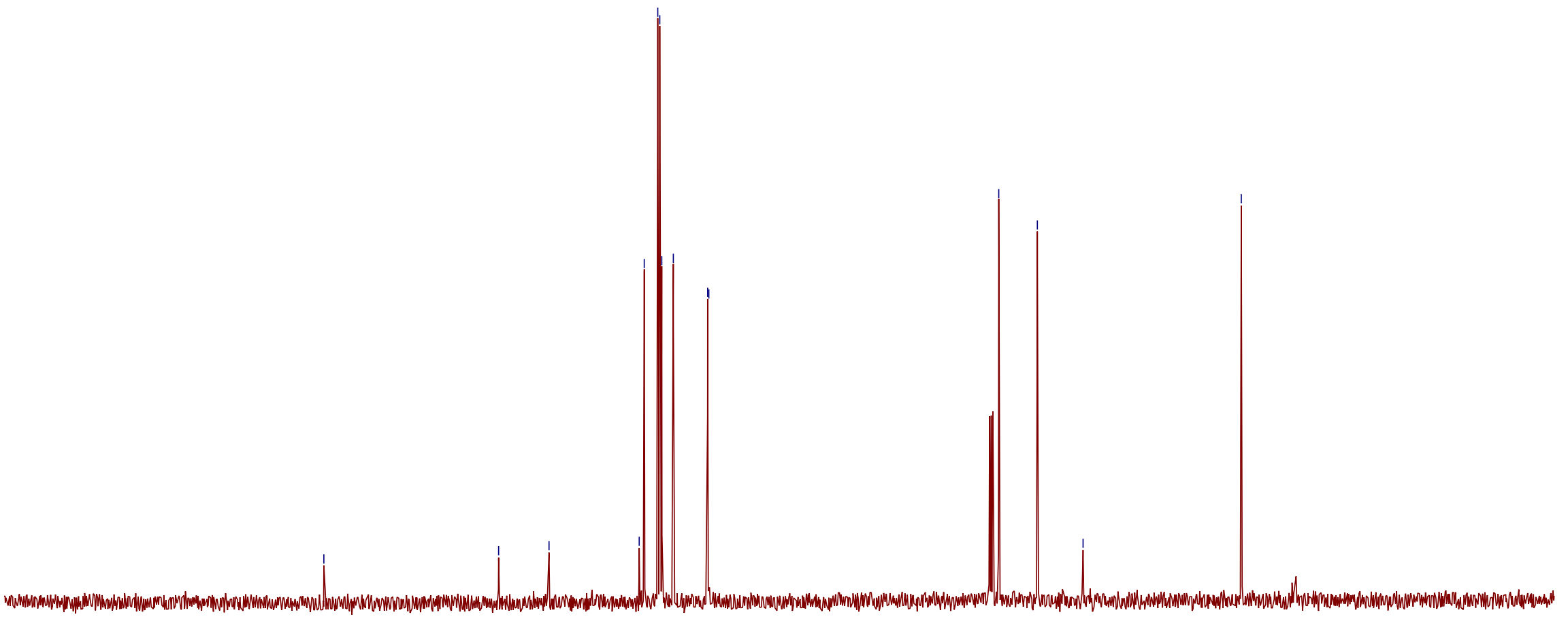

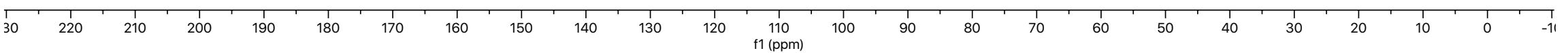


$500 \mathrm{MHz}, \mathrm{CDCl}_{3}$

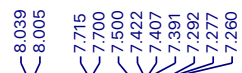

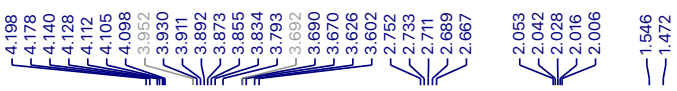

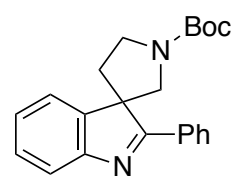

17h
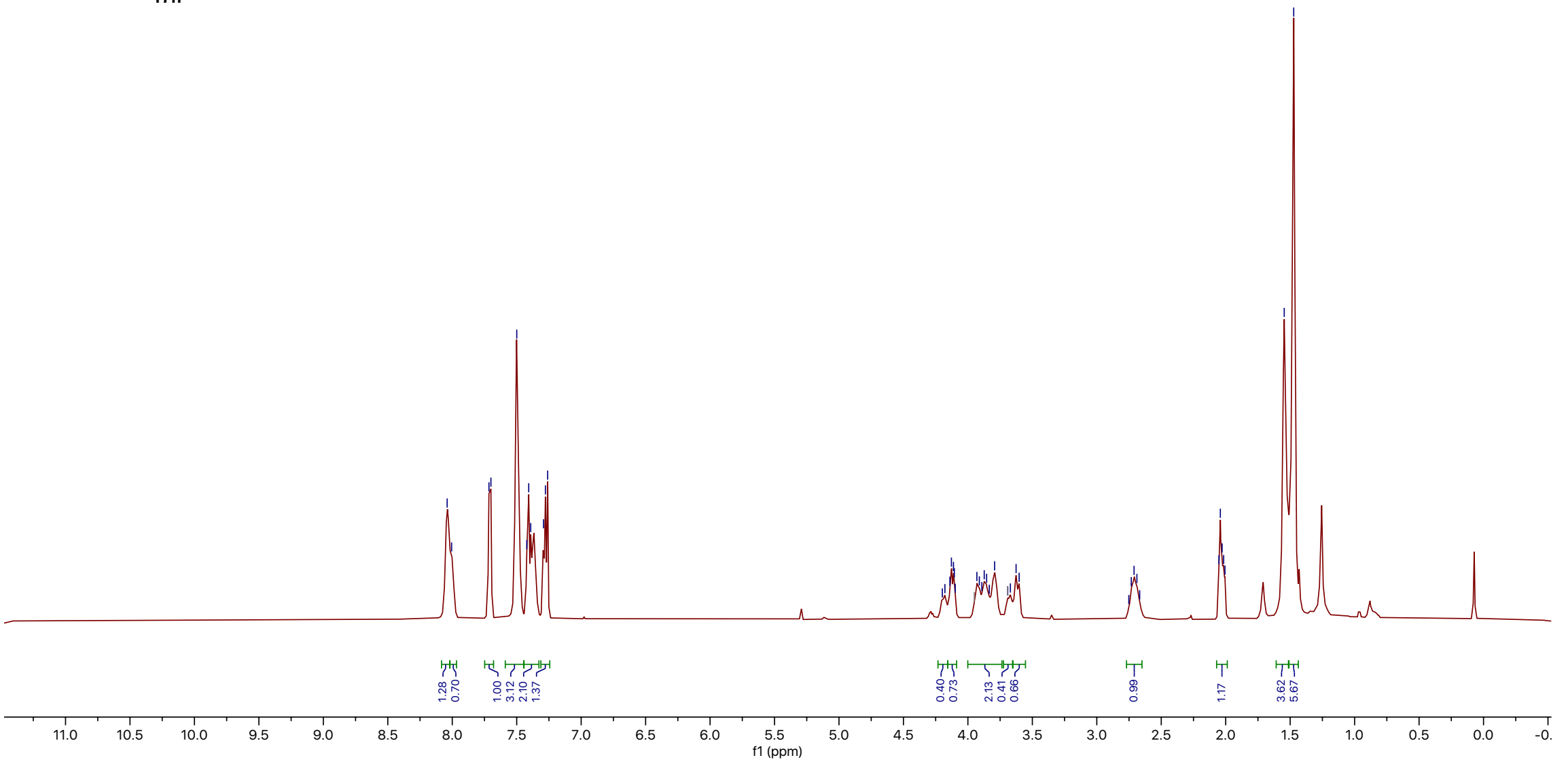
$125 \mathrm{MHz}, \mathrm{CDCl}_{3}$

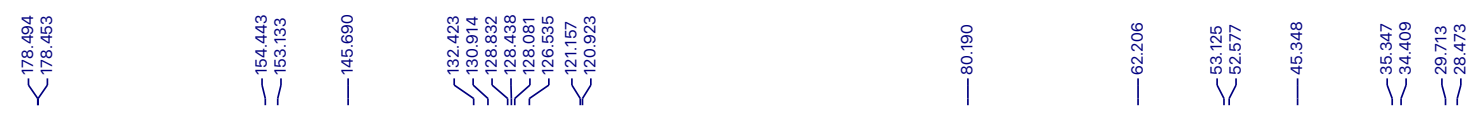

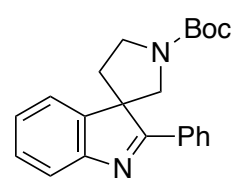

17h
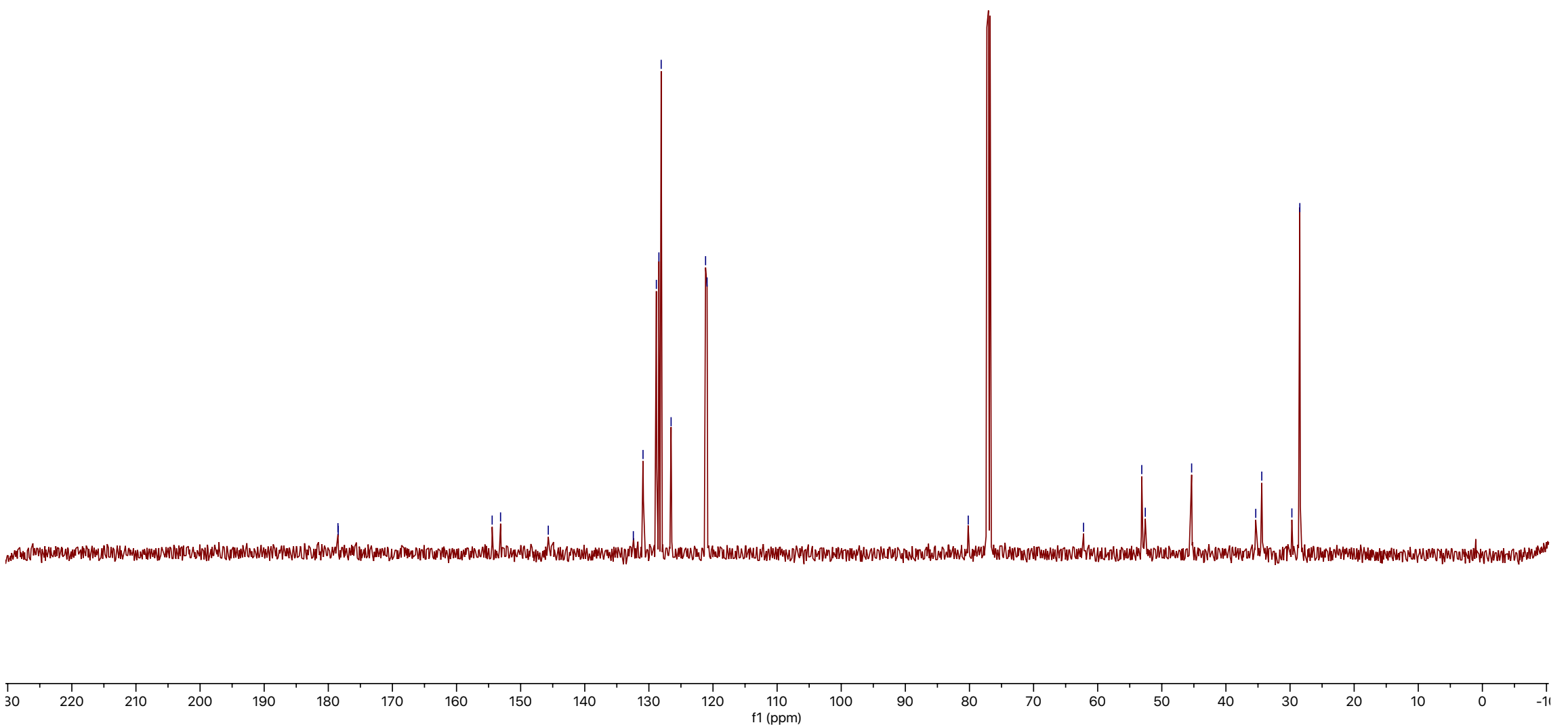
$500 \mathrm{MHz}, \mathrm{CDCl}_{3}$
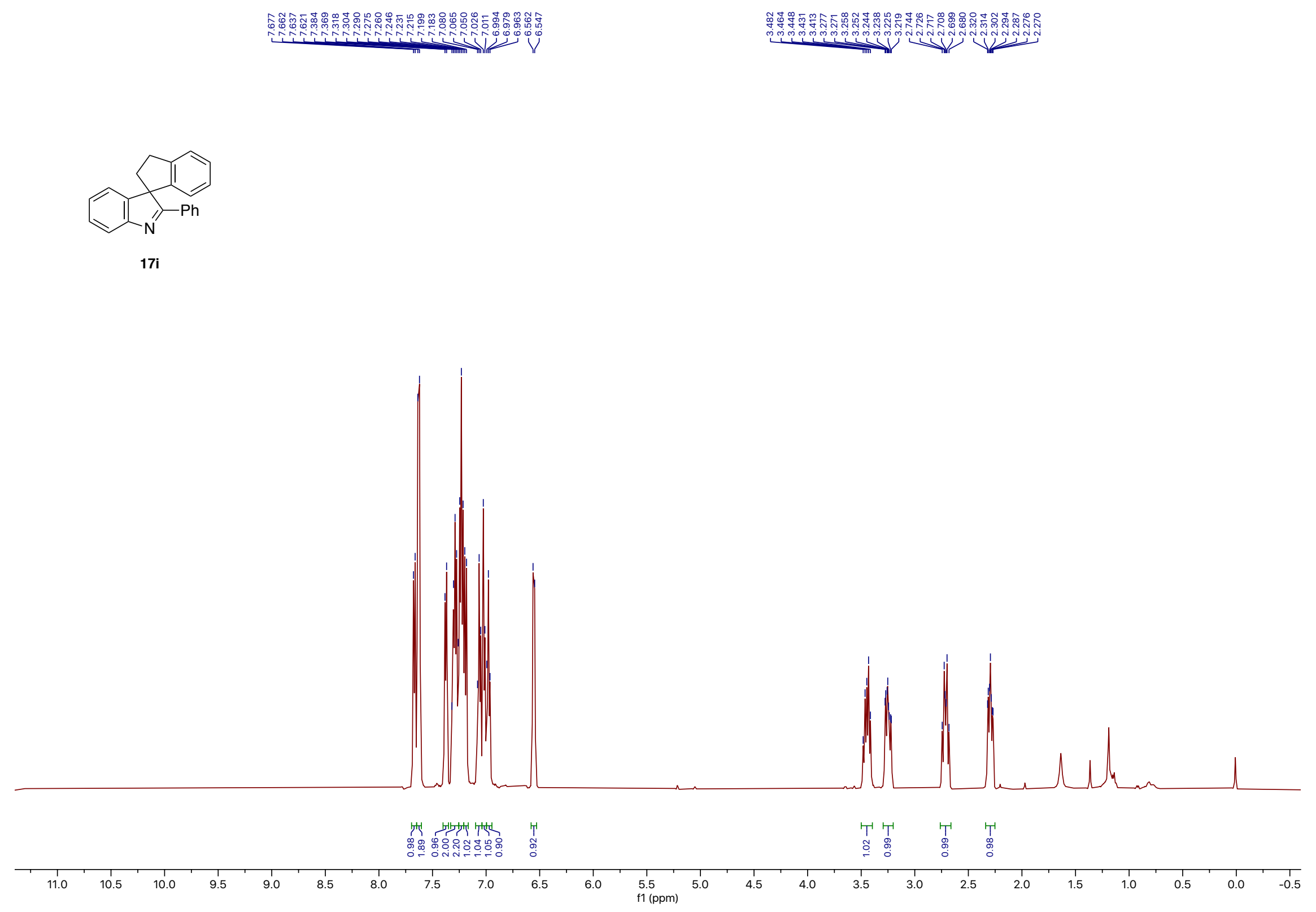
$125 \mathrm{MHz}, \mathrm{CDCl}_{3}$

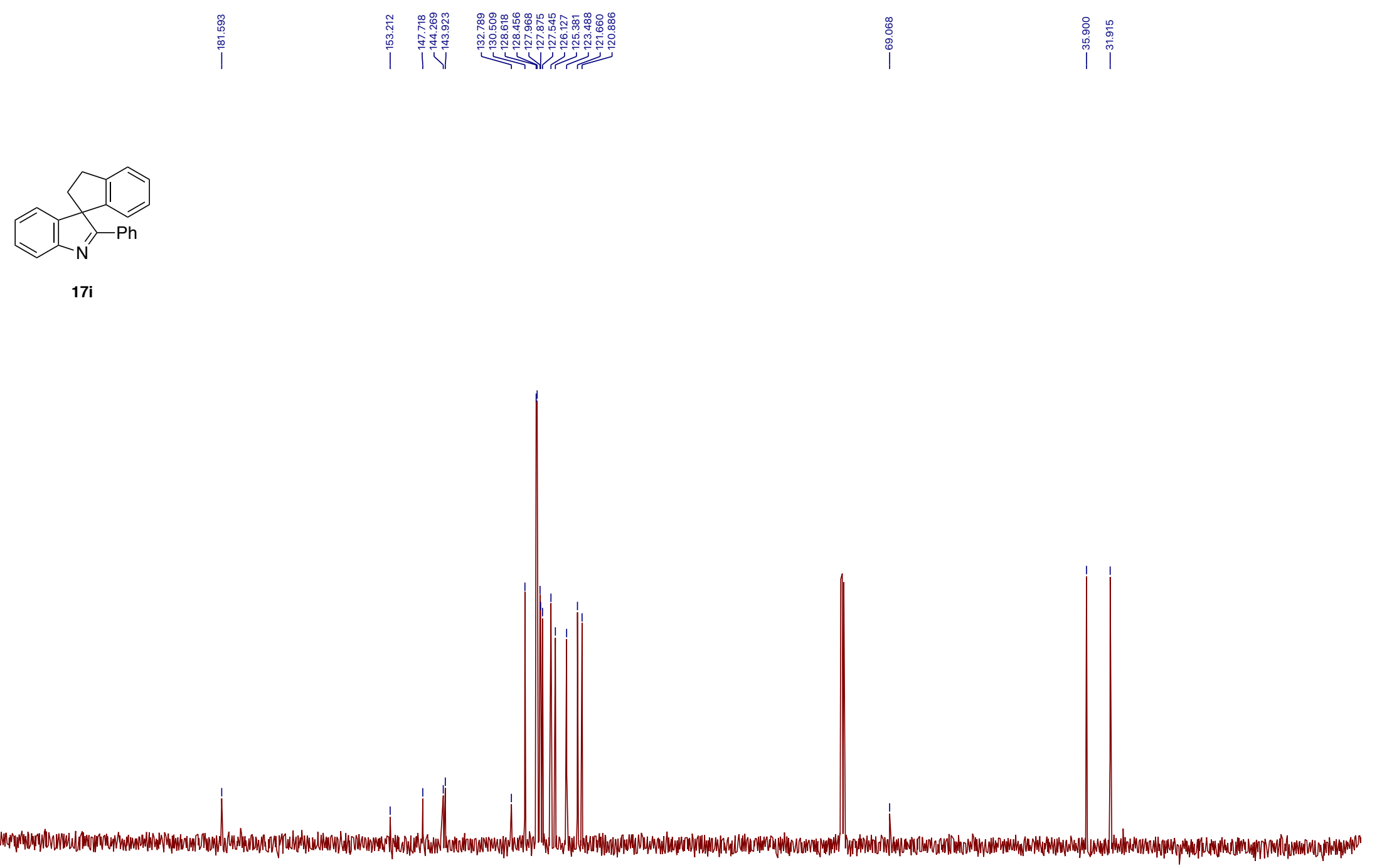

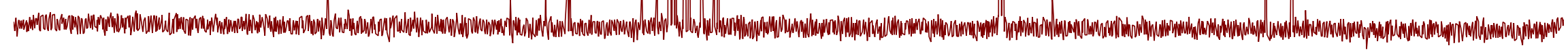

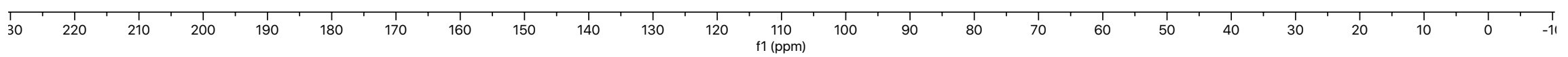


$500 \mathrm{MHz}, \mathrm{CDCl}_{3}$

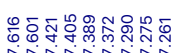

迕许

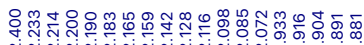

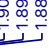

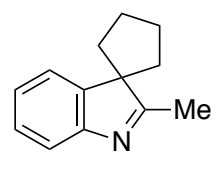

17j

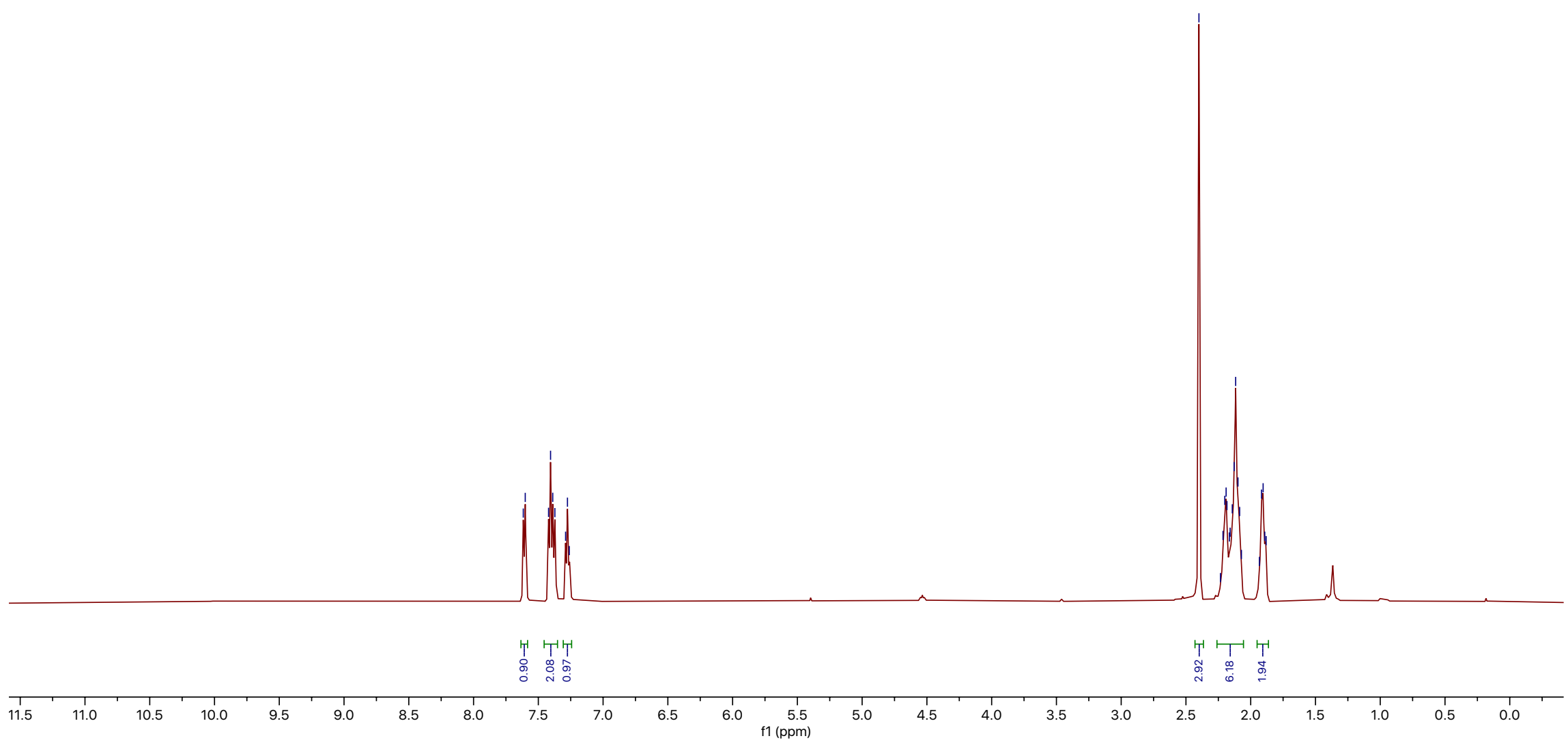


$125 \mathrm{MHz}, \mathrm{CDCl}_{3}$

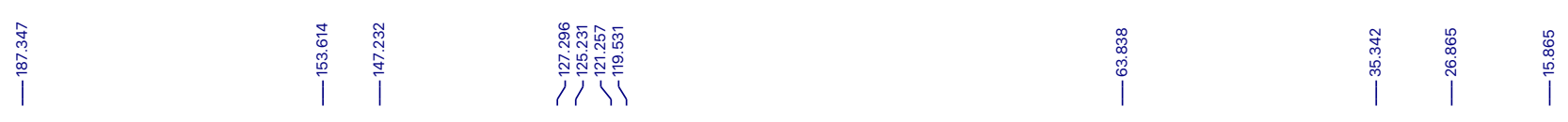

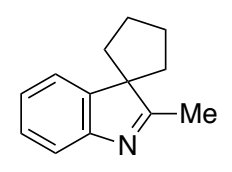

17j

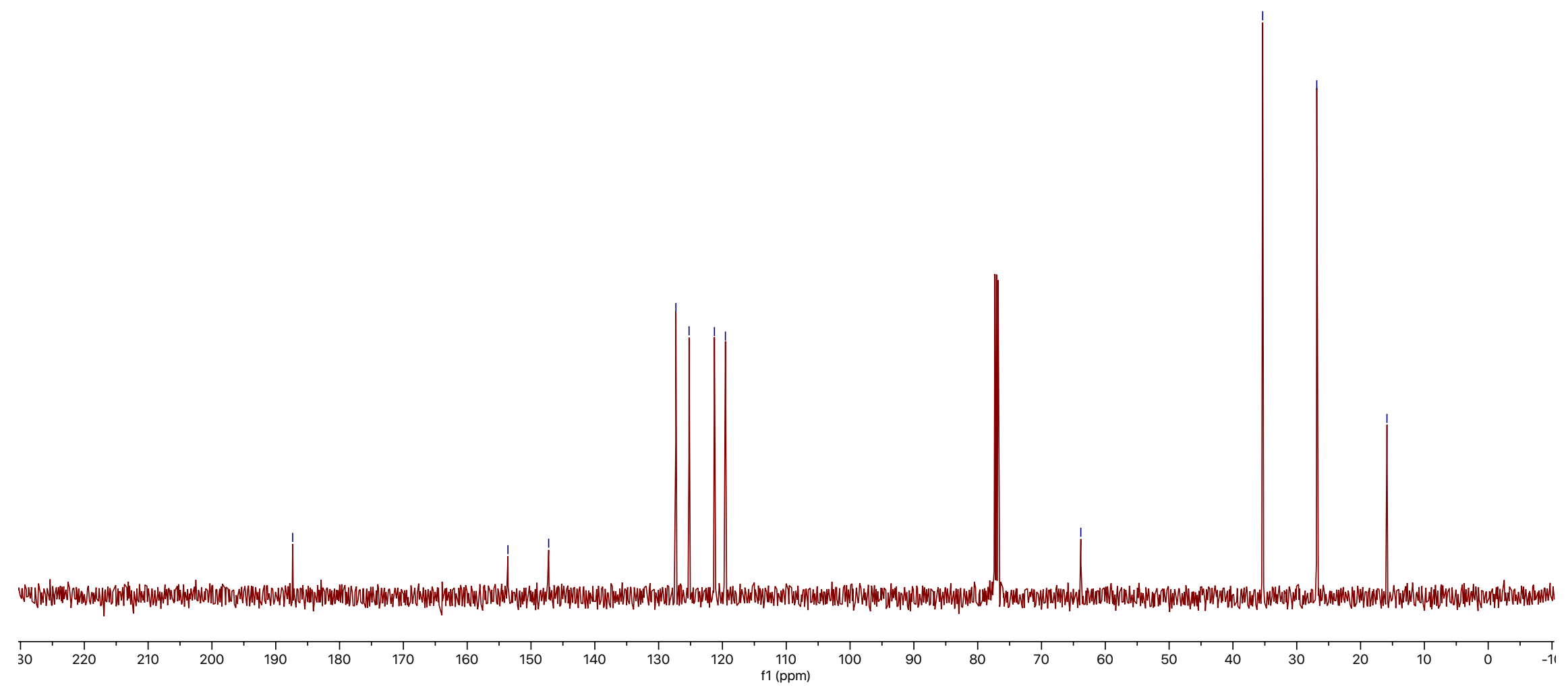


$500 \mathrm{MHz}, \mathrm{CDCl}_{3}$

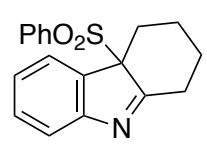

$18 \mathrm{k}$

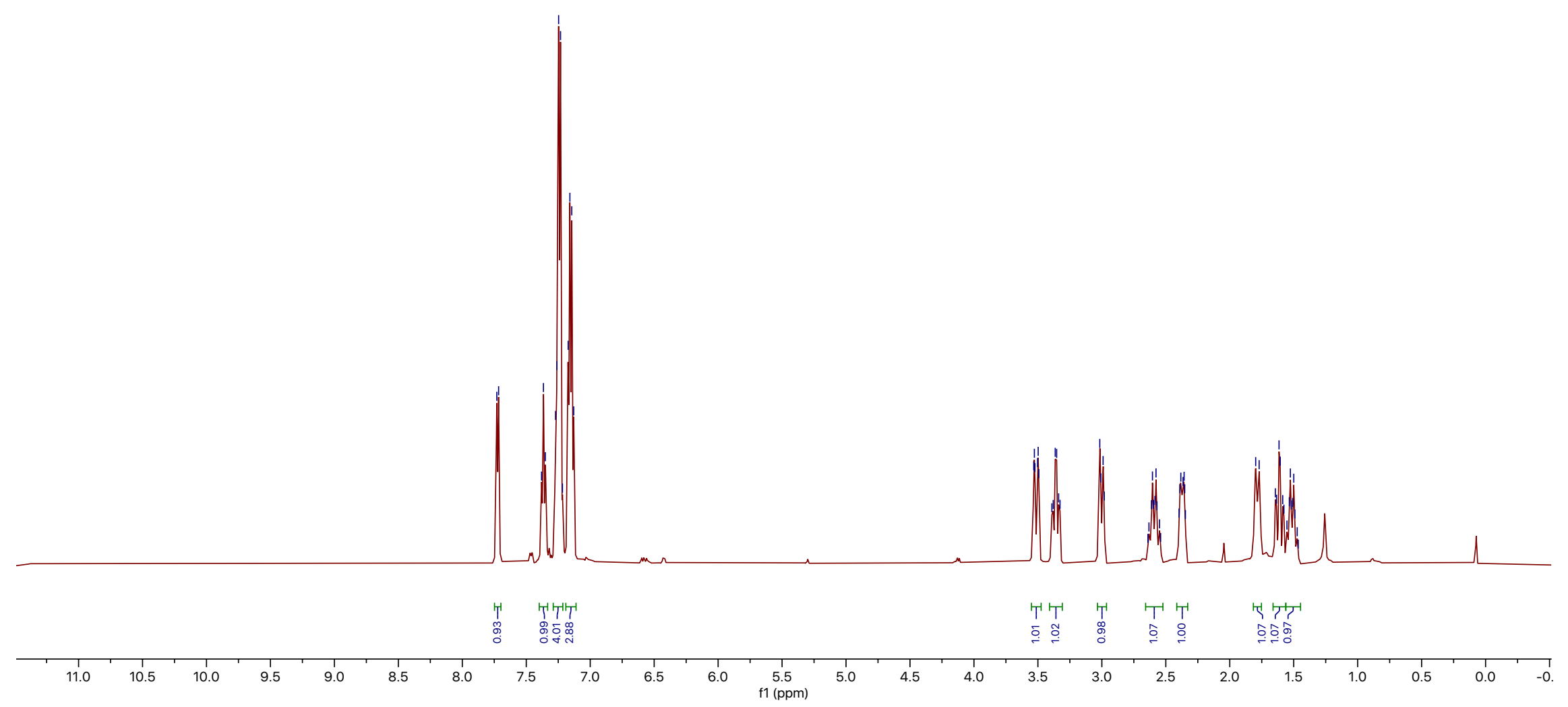


$125 \mathrm{MHz}, \mathrm{CDCl}_{3}$

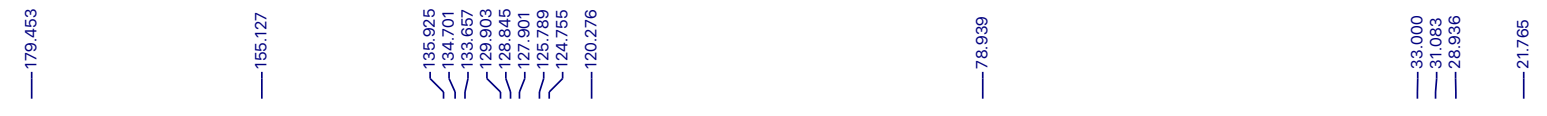

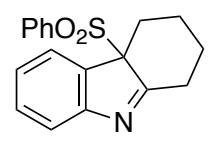

$18 k$
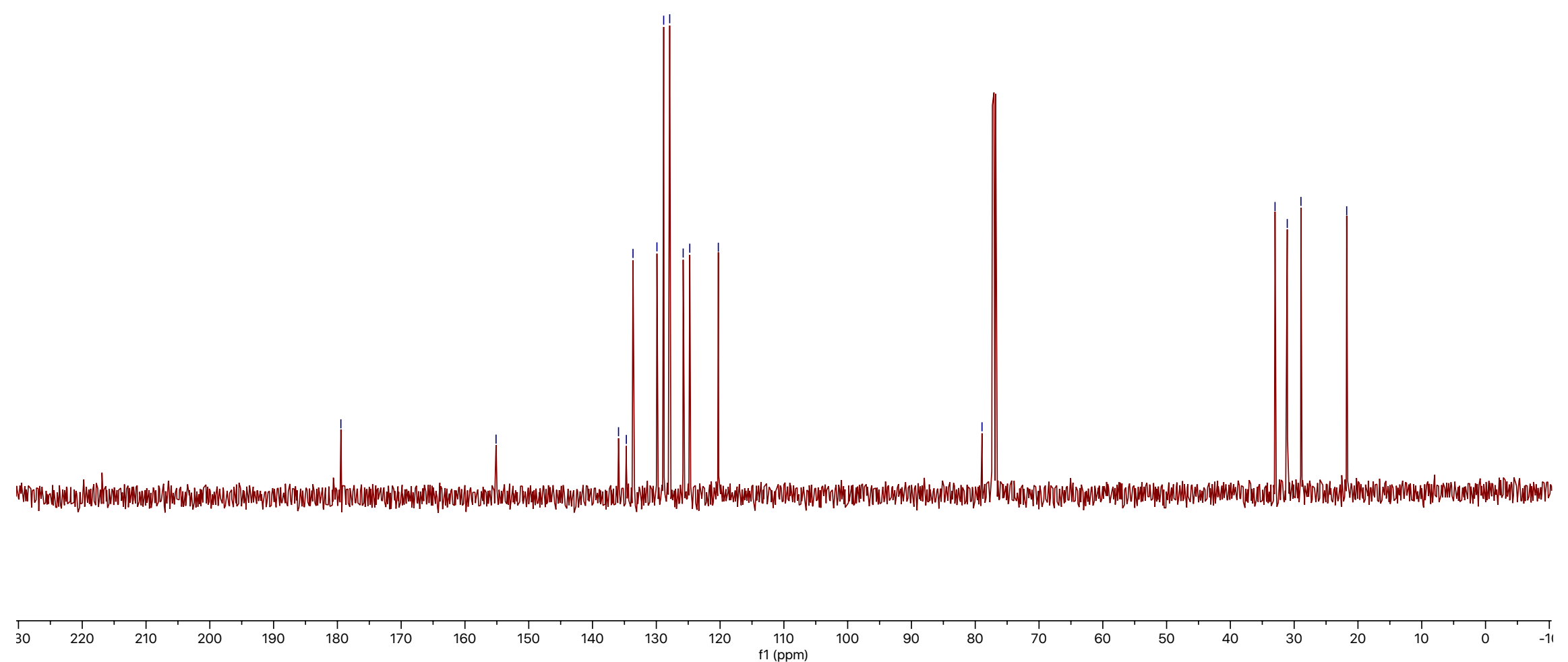
$500 \mathrm{MHz}, \mathrm{CDCl}_{3}$

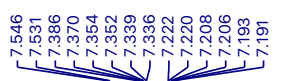

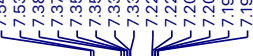

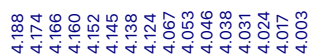

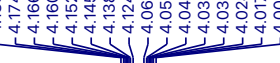

\section{|}<smiles>CCOC(=O)C1(C)C(C)=Nc2ccccc21</smiles>

181

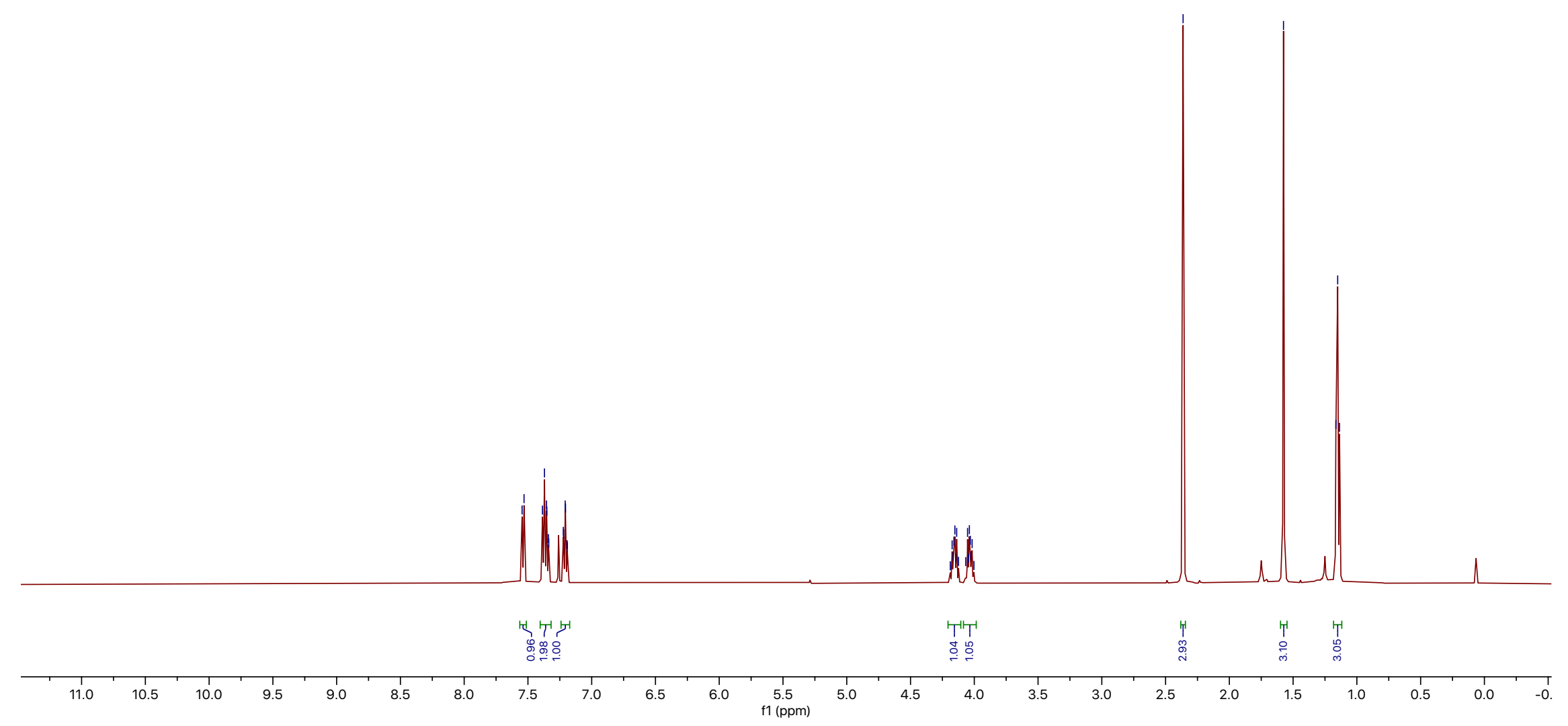


$125 \mathrm{MHz}, \mathrm{CDCl}_{3}$

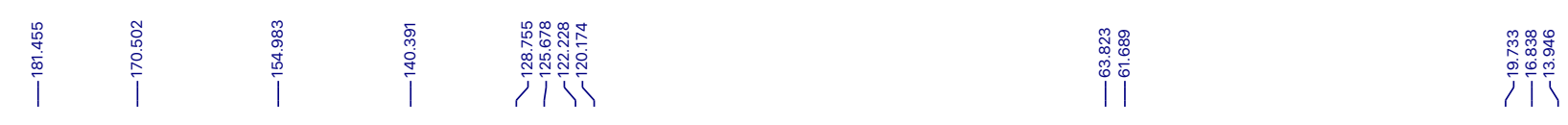

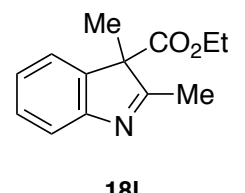

181
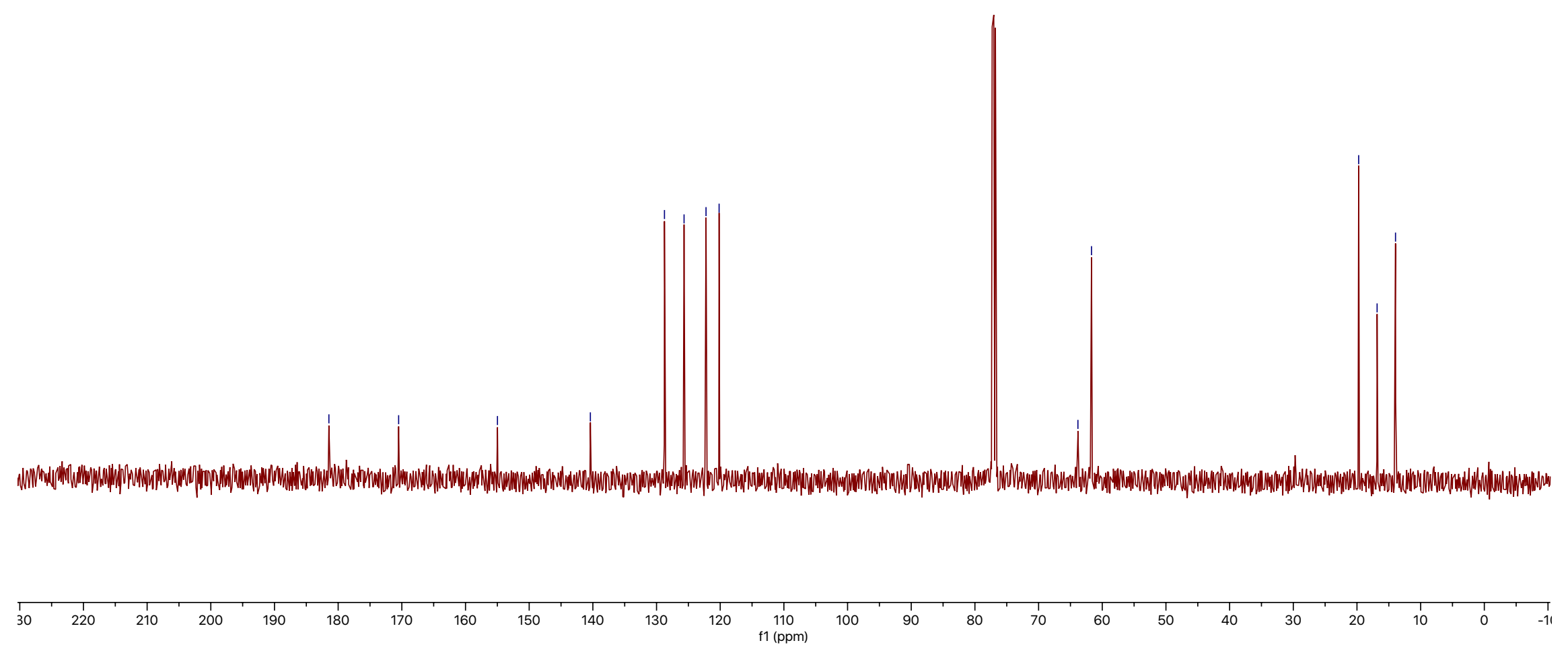
$500 \mathrm{MHz}, \mathrm{CDCl}_{3}$

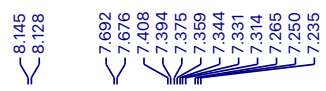

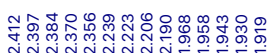
ำ
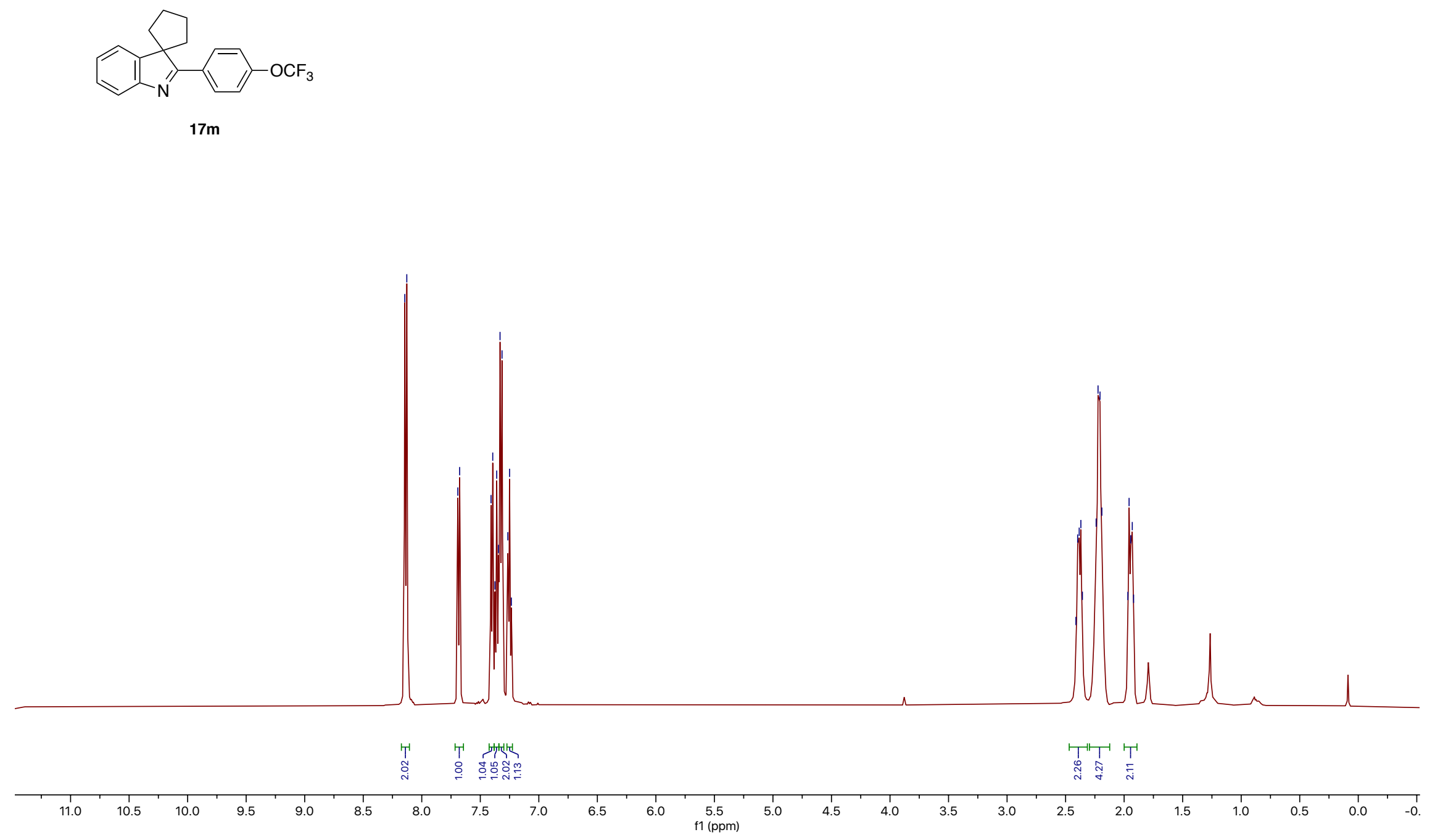
$125 \mathrm{MHz}, \mathrm{CDCl}_{3}$
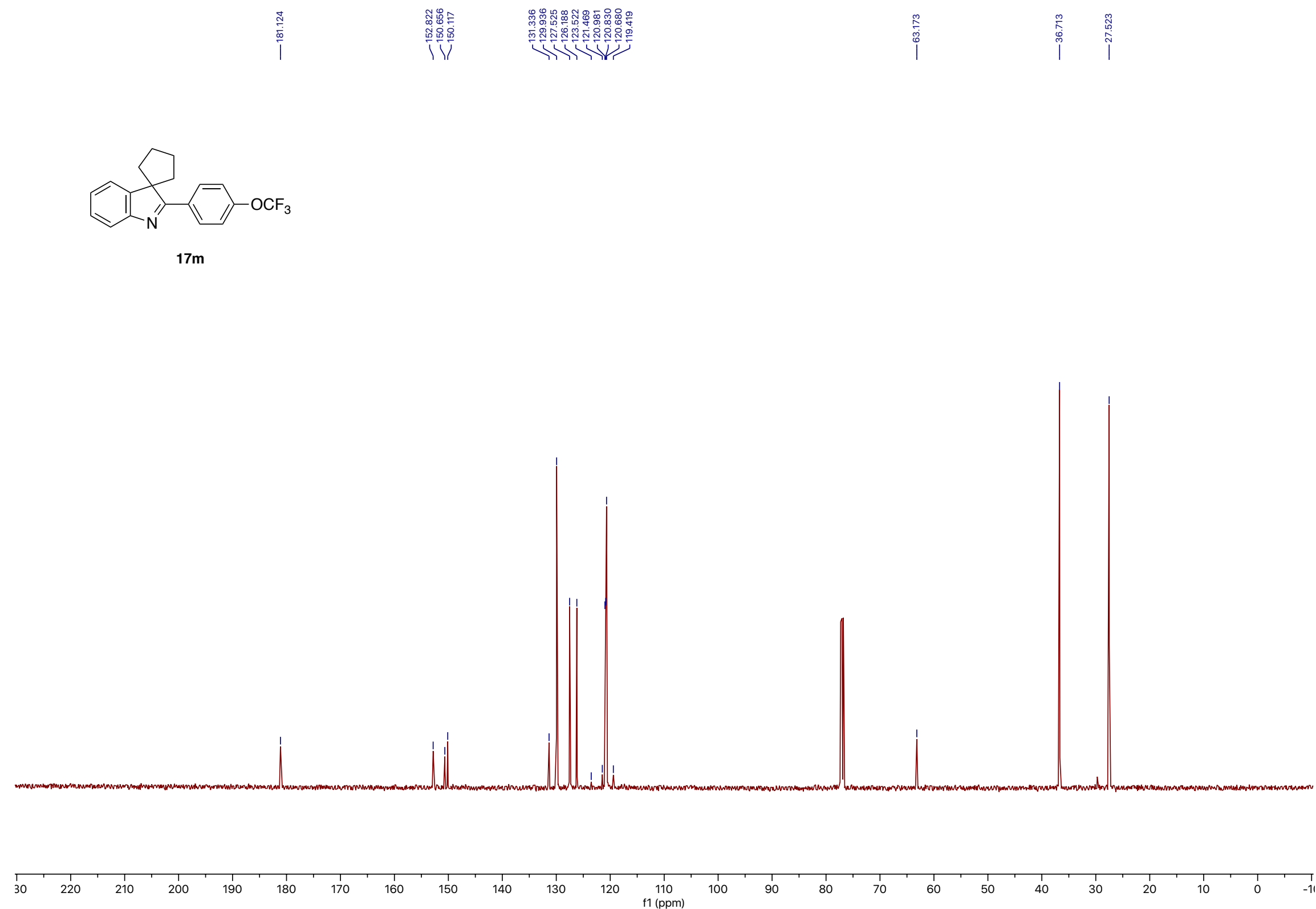
$500 \mathrm{MHz}, \mathrm{CDCl}_{3}$
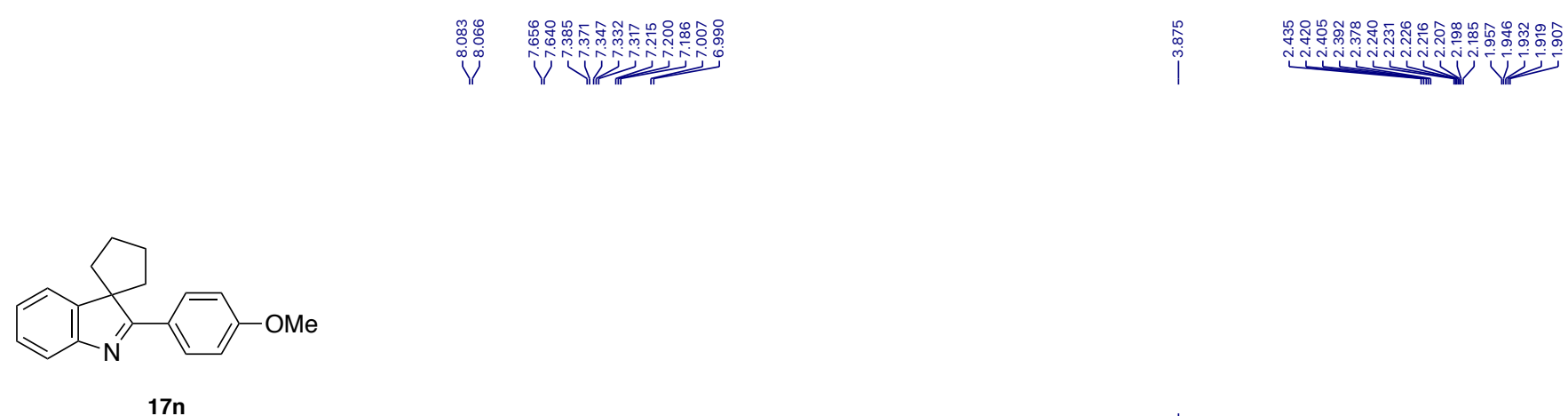

$17 n$

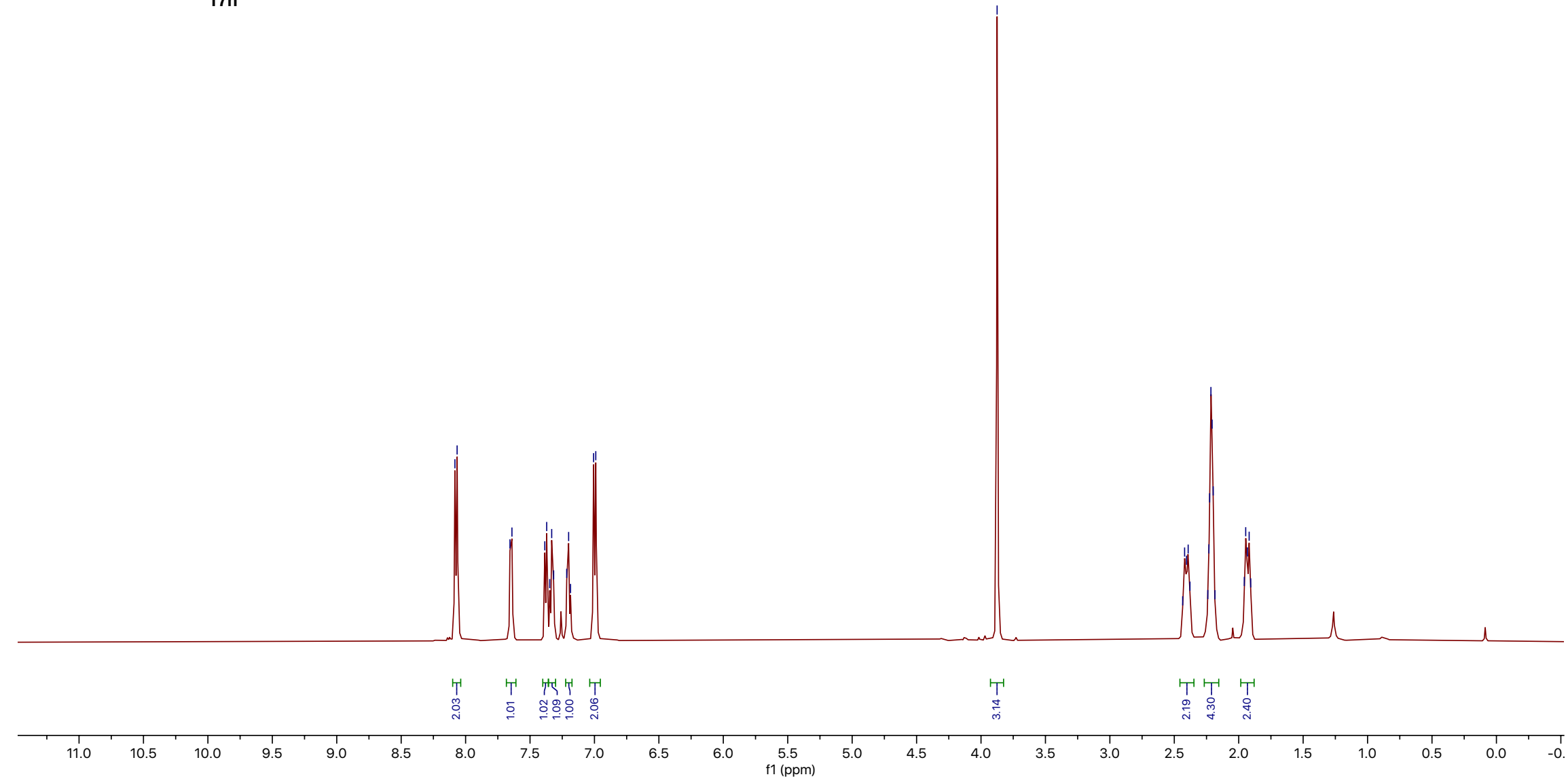


$125 \mathrm{MHz}, \mathrm{CDCl}_{3}$

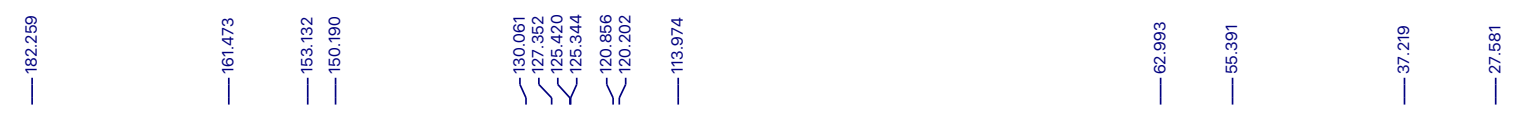

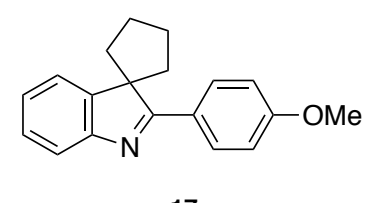

$17 n$
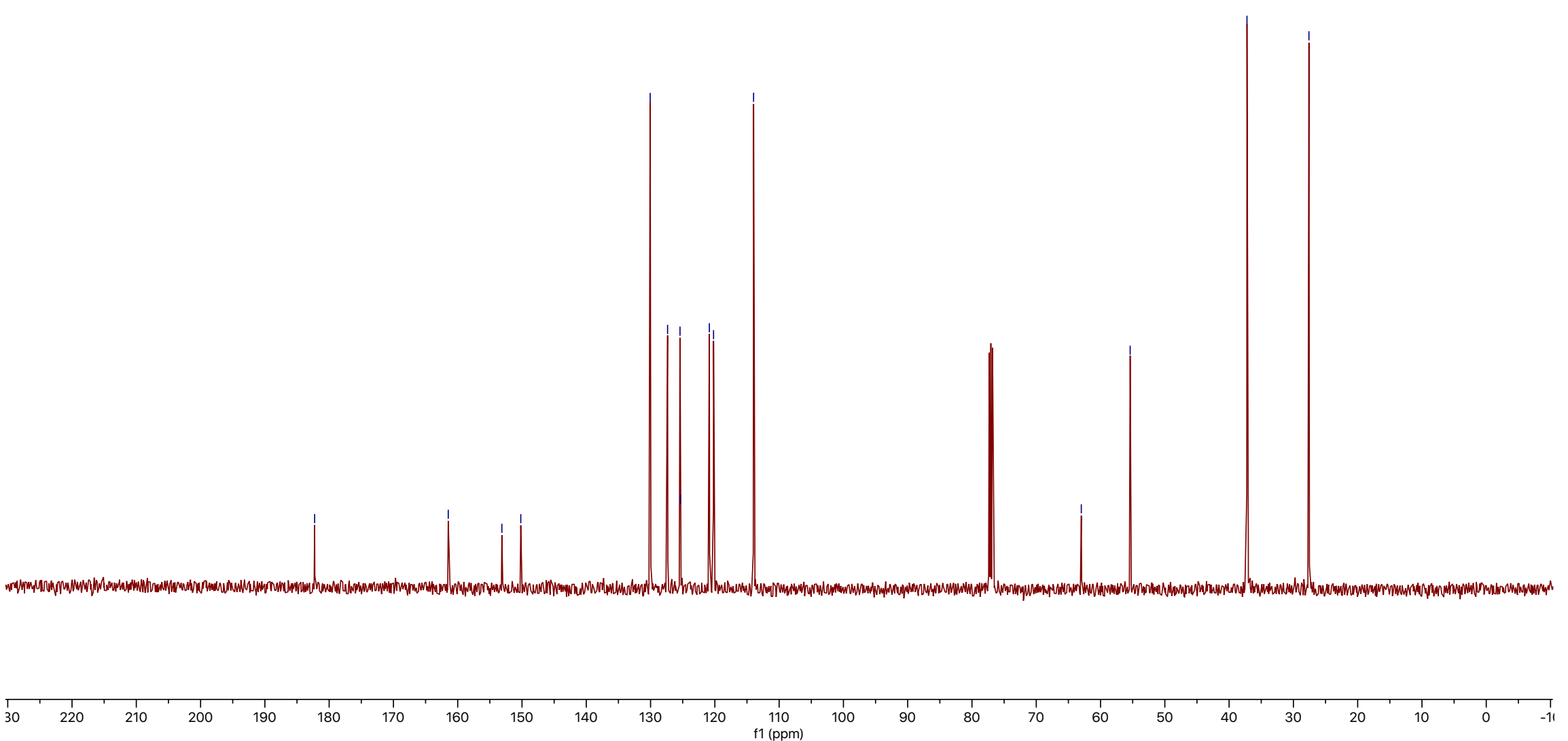
$500 \mathrm{MHz}, \mathrm{CDCl}_{3}$

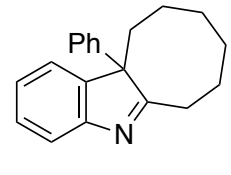

180

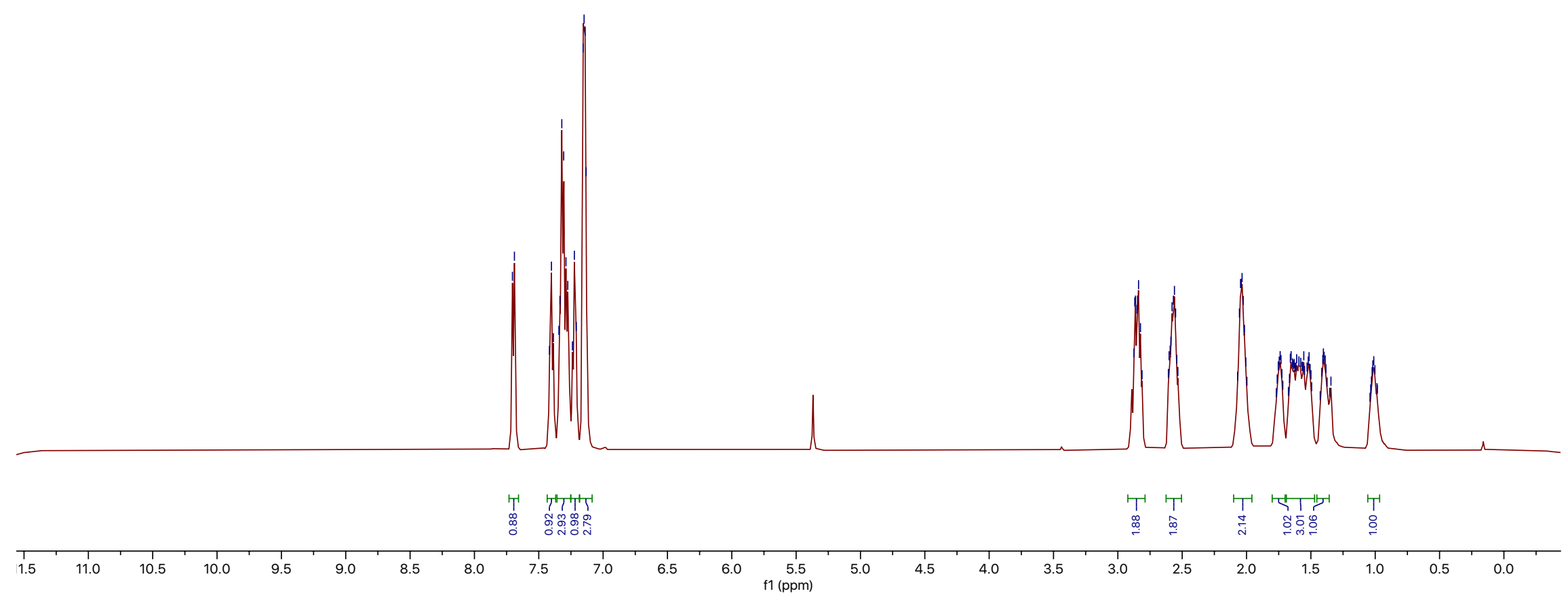


$125 \mathrm{MHz}, \mathrm{CDCl}_{3}$

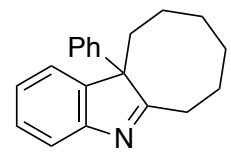

180
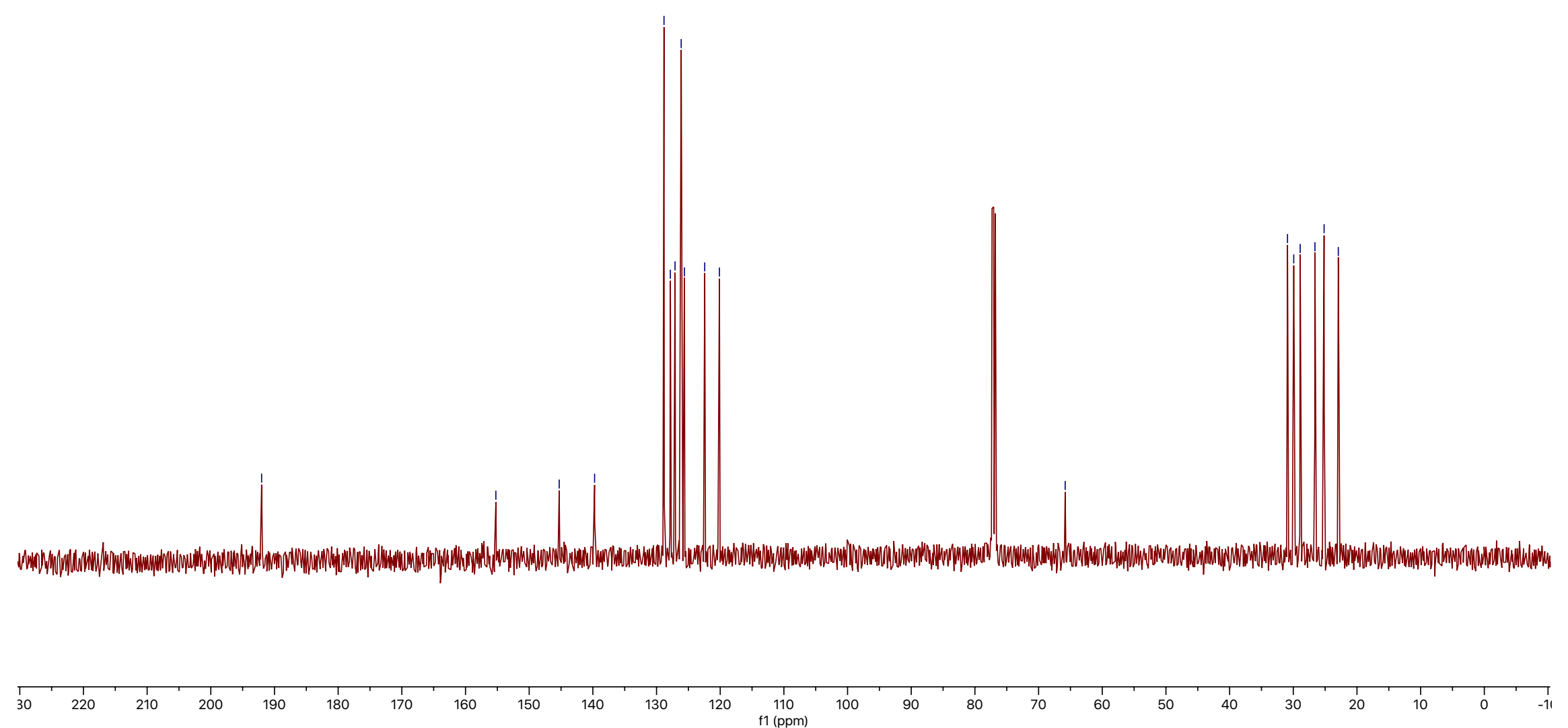
$500 \mathrm{MHz}, \mathrm{CDCl}_{3}$

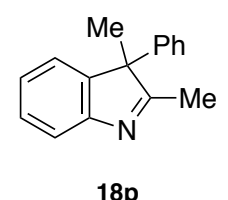

$18 p$

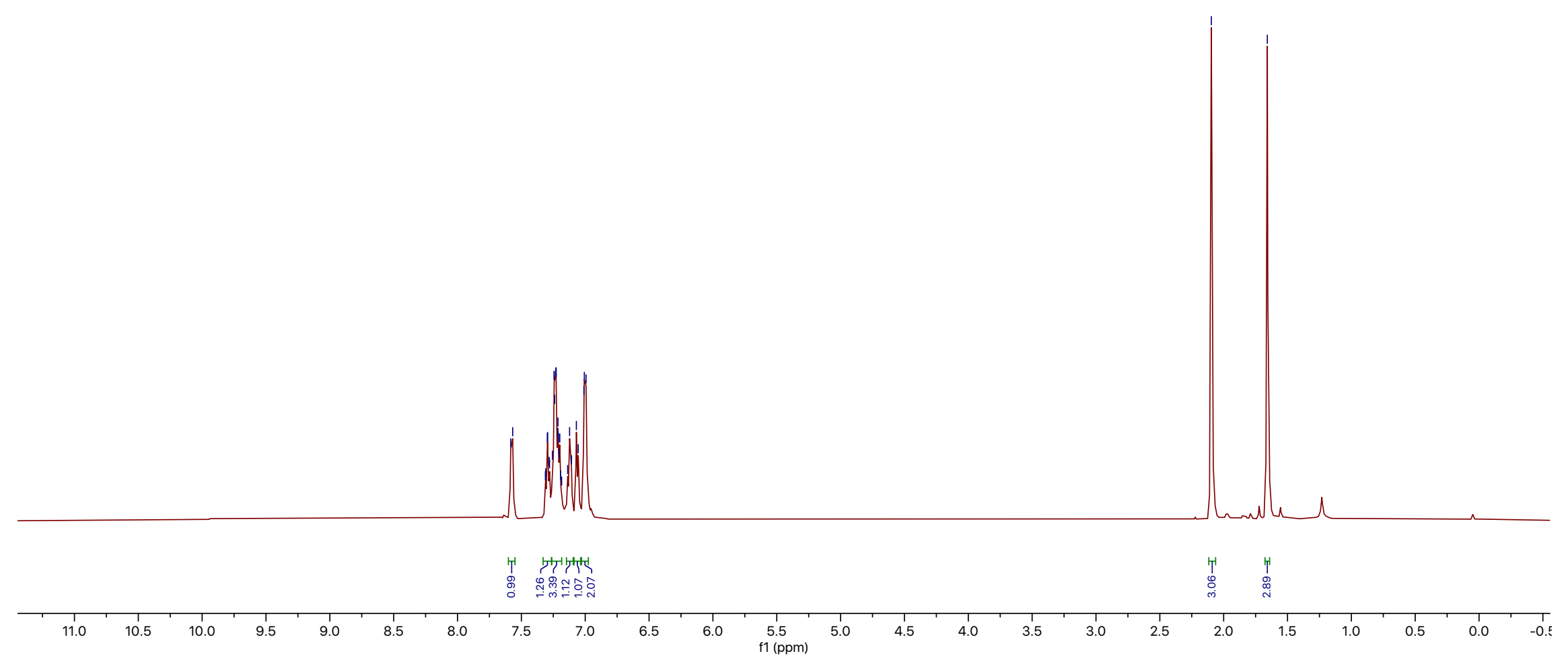


$125 \mathrm{MHz}, \mathrm{CDCl}_{3}$

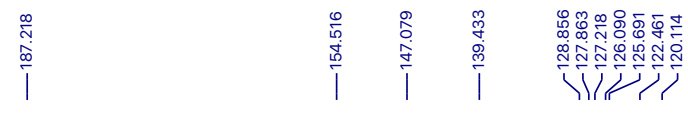

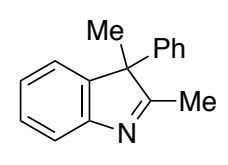

$18 p$
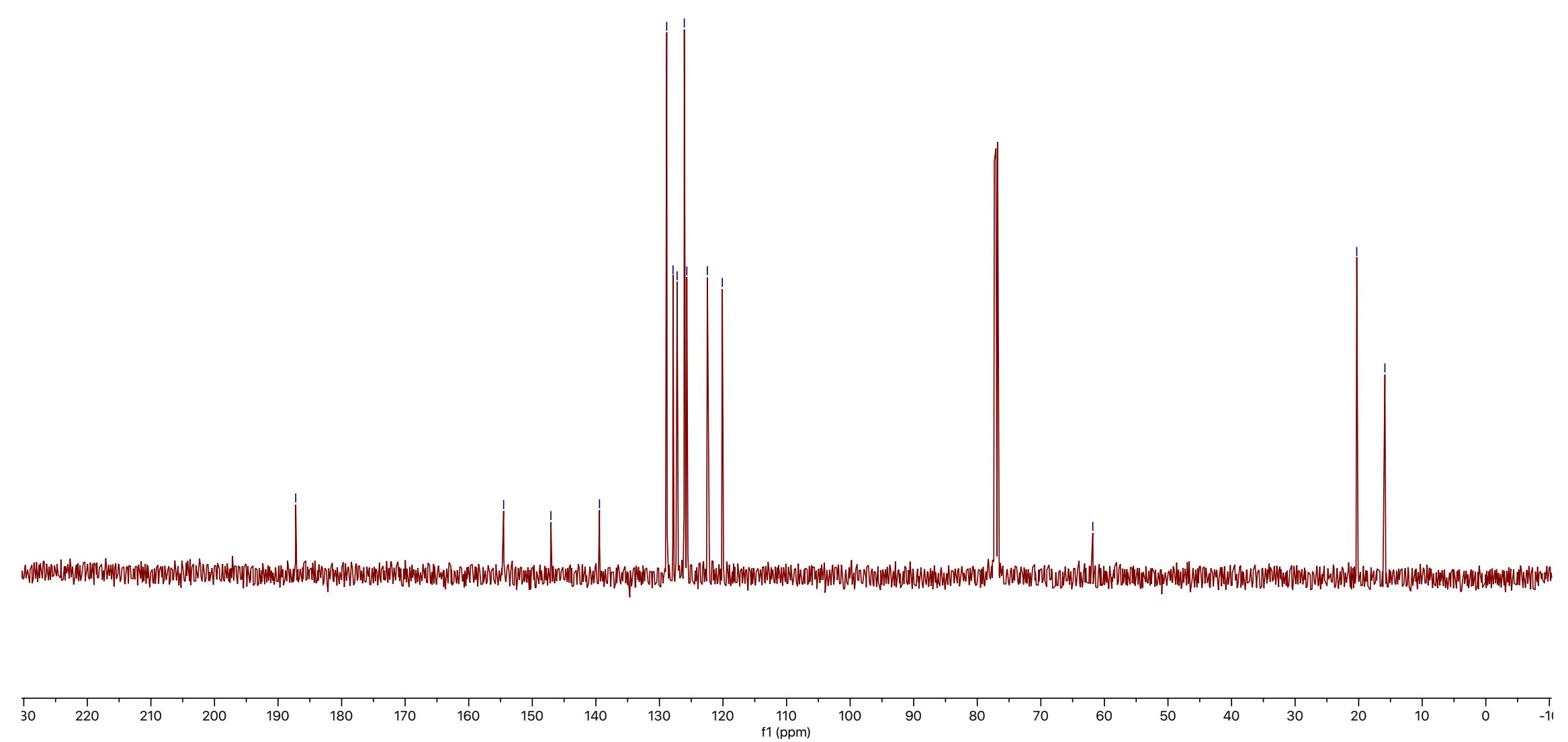
$500 \mathrm{MHz}, \mathrm{CDCl}_{3}$

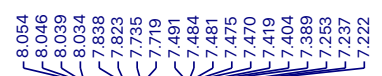

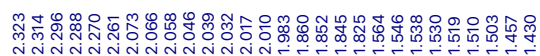
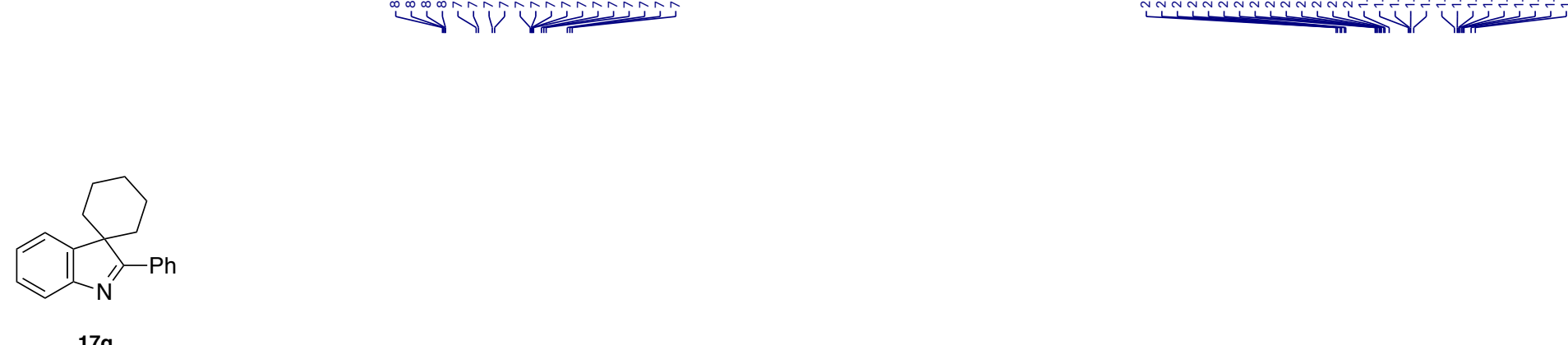

$17 q$

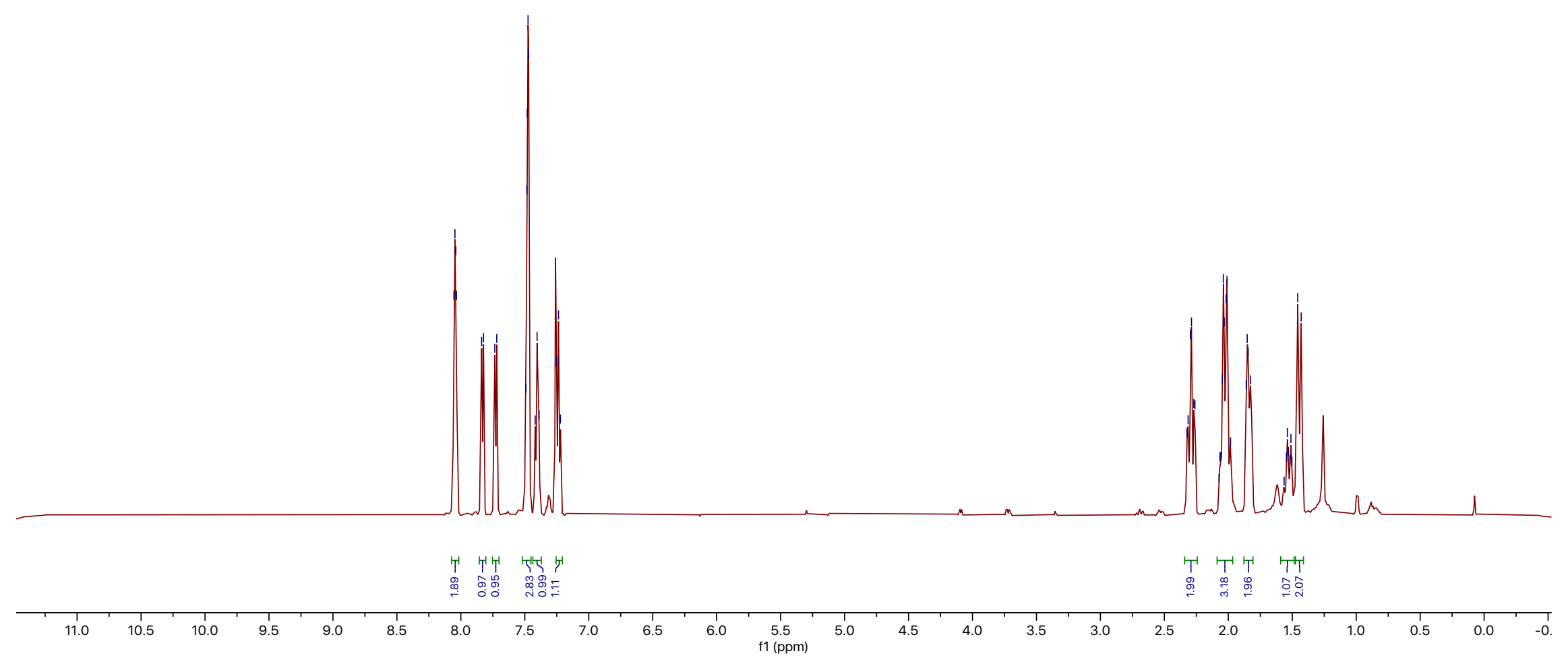


$125 \mathrm{MHz}, \mathrm{CDCl}_{3}$
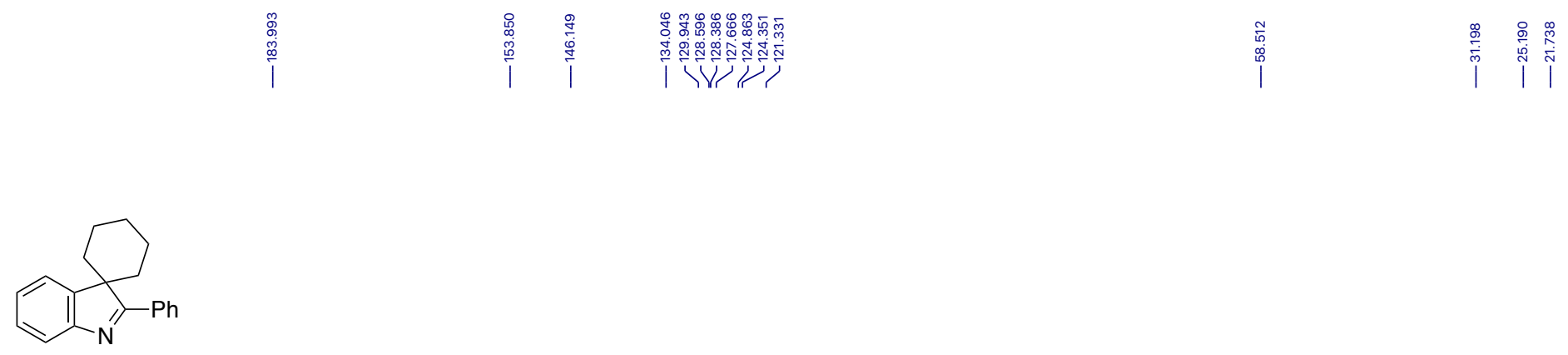

$17 q$
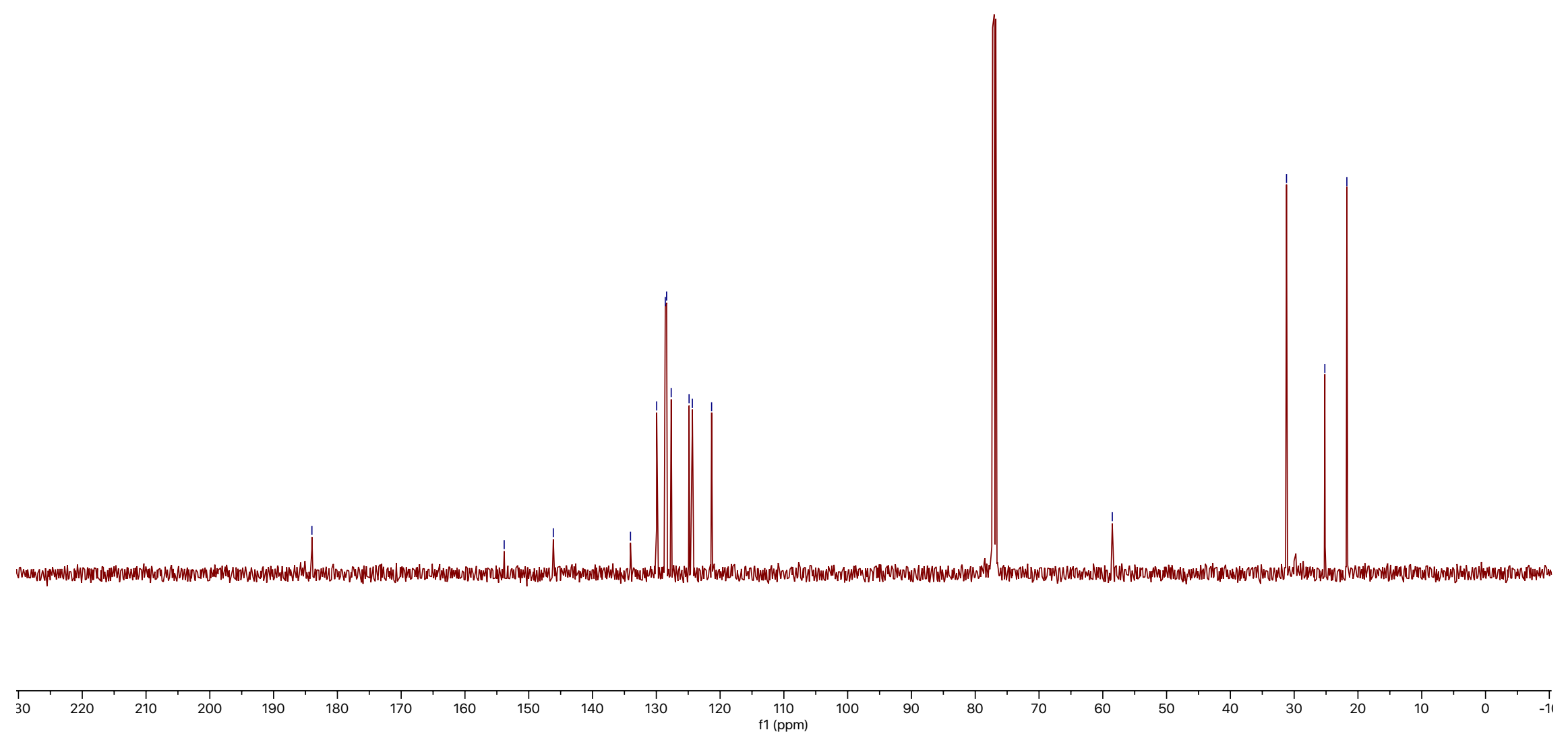
$500 \mathrm{MHz}, \mathrm{CDCl}_{3}$

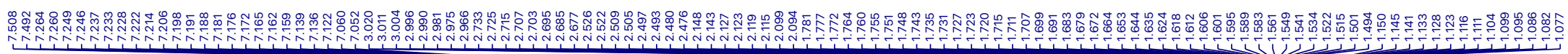<smiles>c1ccc(C23CCCCCC2=Nc2ccccc23)cc1</smiles>

$18 q$

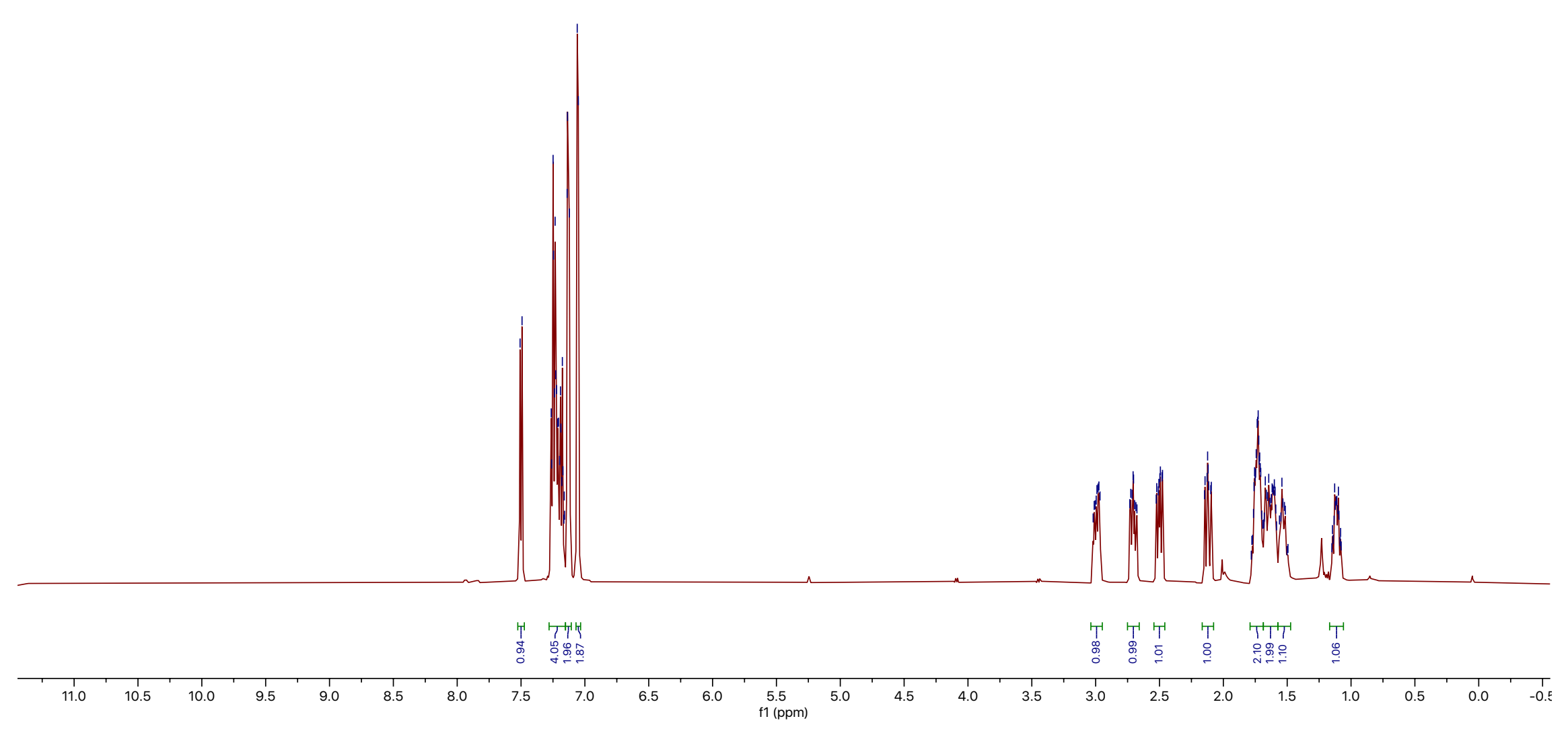


$125 \mathrm{MHz}, \mathrm{CDCl}_{3}$

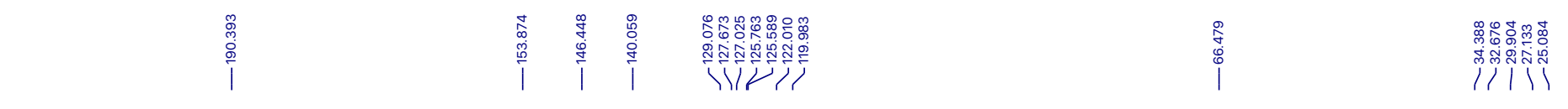

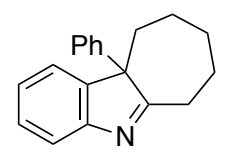

$18 q$
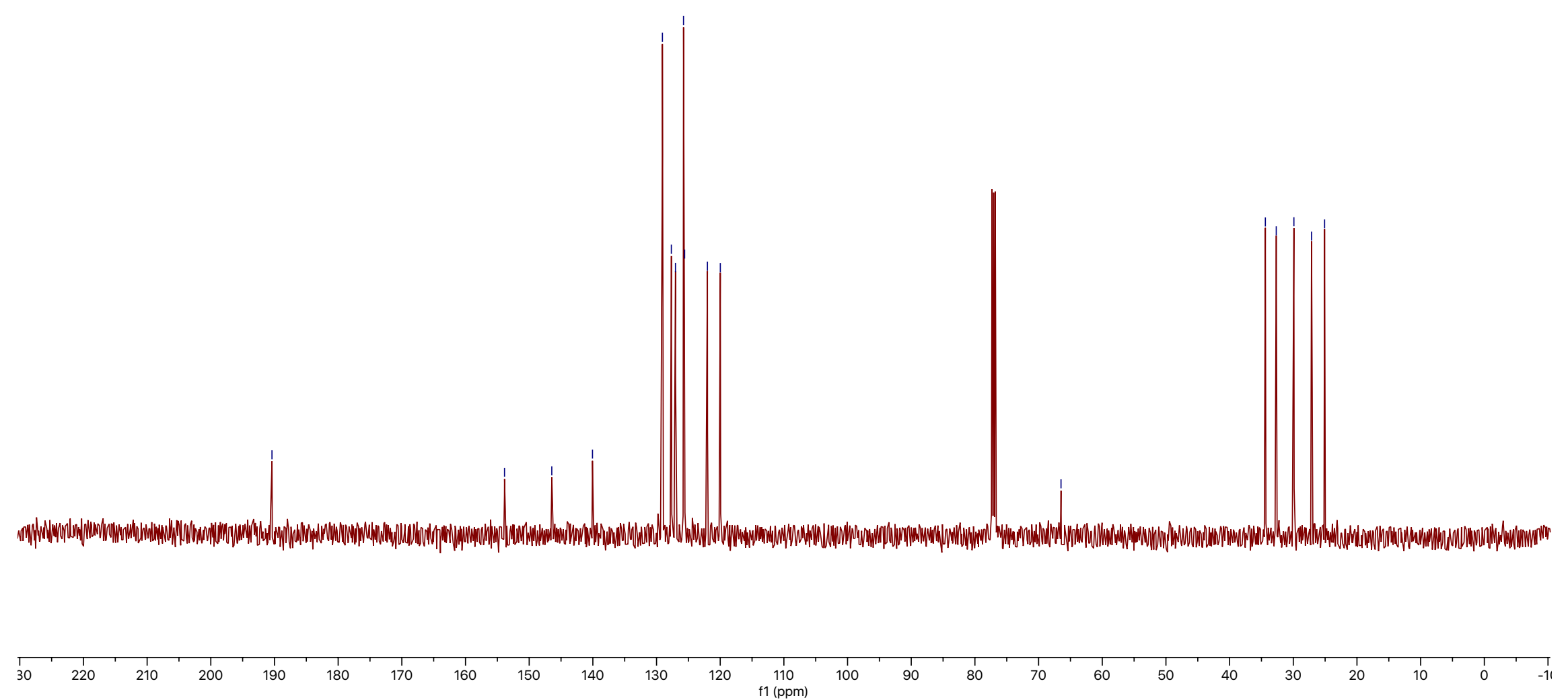
$500 \mathrm{MHz}, \mathrm{CDCl}_{3}$

$\mathrm{MeO}$

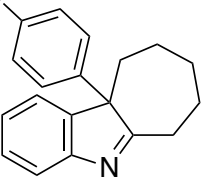

$18 \mathrm{~s}$

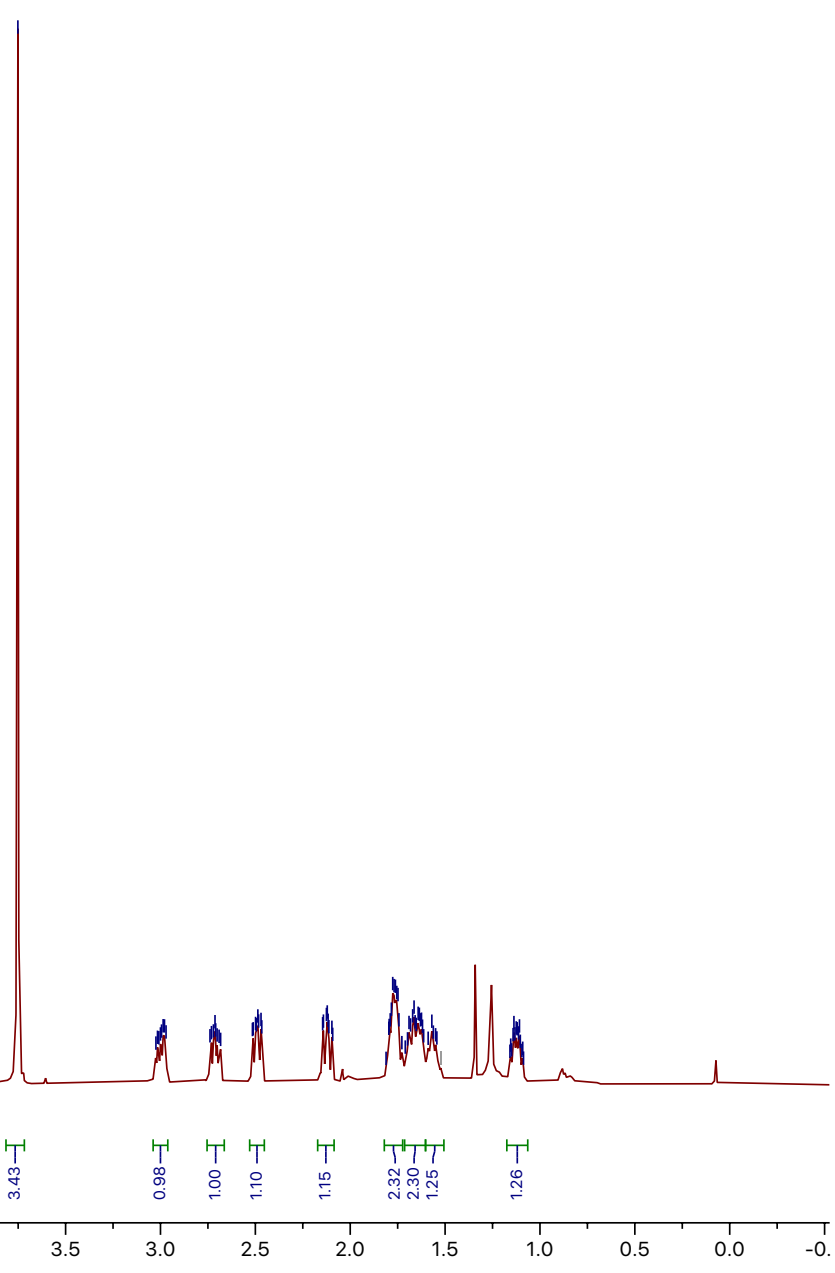


$125 \mathrm{MHz}, \mathrm{CDCl}_{3}$

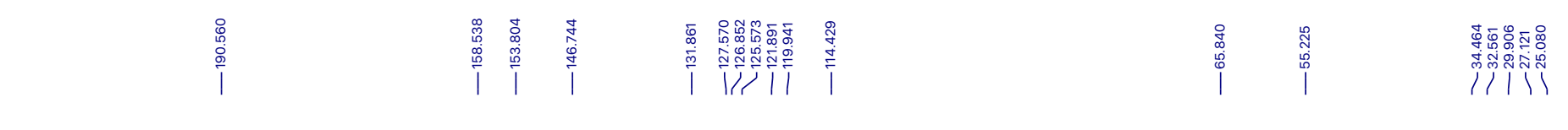

$\mathrm{MeO}$

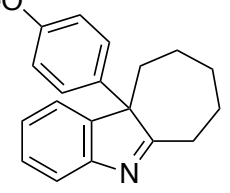

$18 \mathrm{~s}$

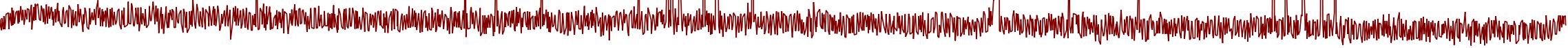

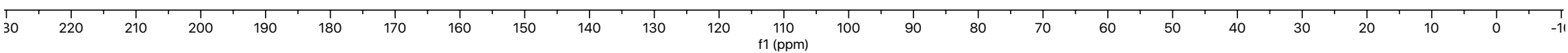


$500 \mathrm{MHz}, \mathrm{CDCl}_{3}$

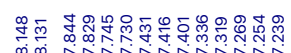

V Viv

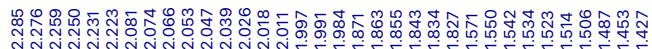

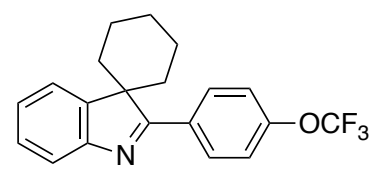

$17 r$

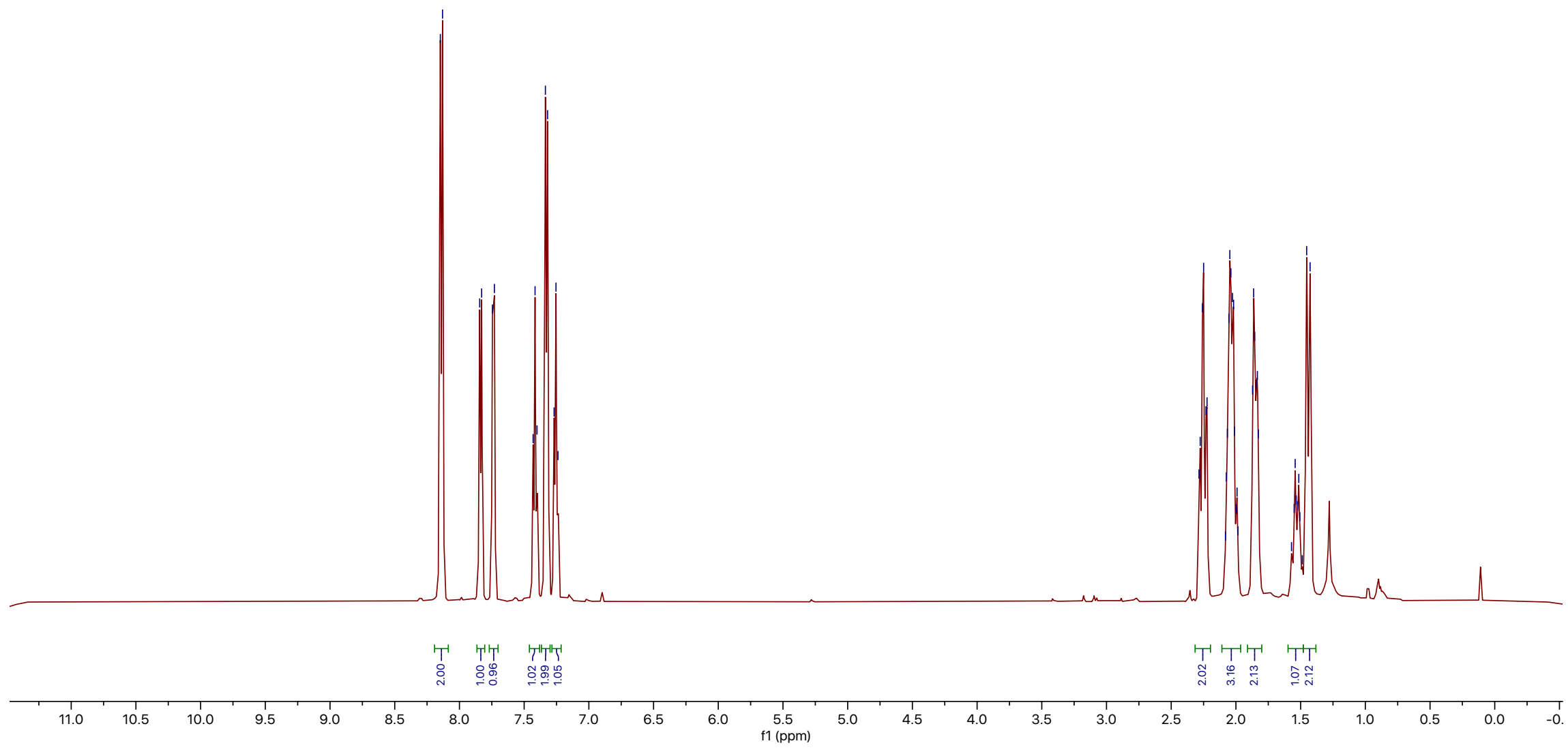


$125 \mathrm{MHz}, \mathrm{CDCl}_{3}$

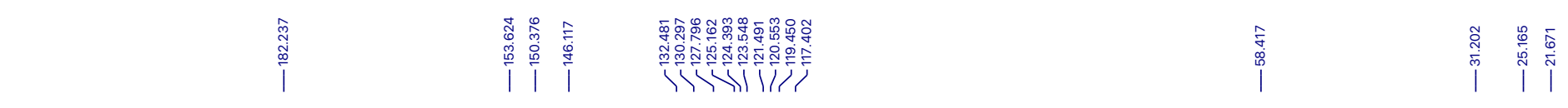

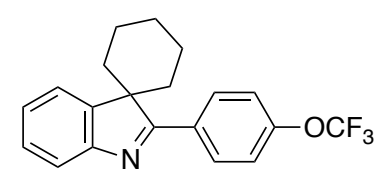

$17 \mathrm{r}$

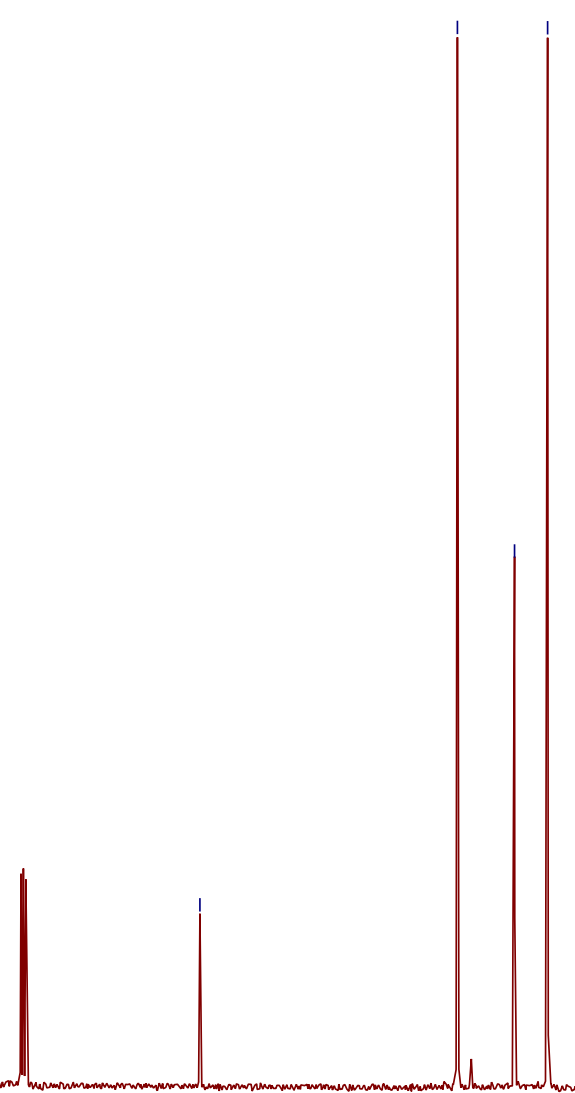

30

220

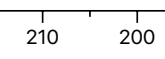

190

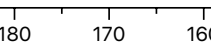

$160 \quad 150$
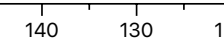

$120 \quad 110$
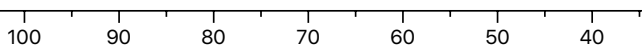
$500 \mathrm{MHz}, \mathrm{CDCl}_{3}$

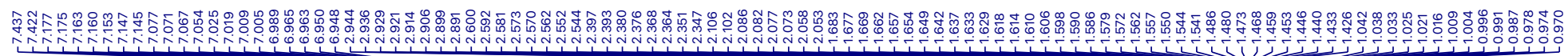

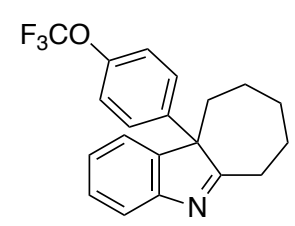

$18 \mathrm{r}$

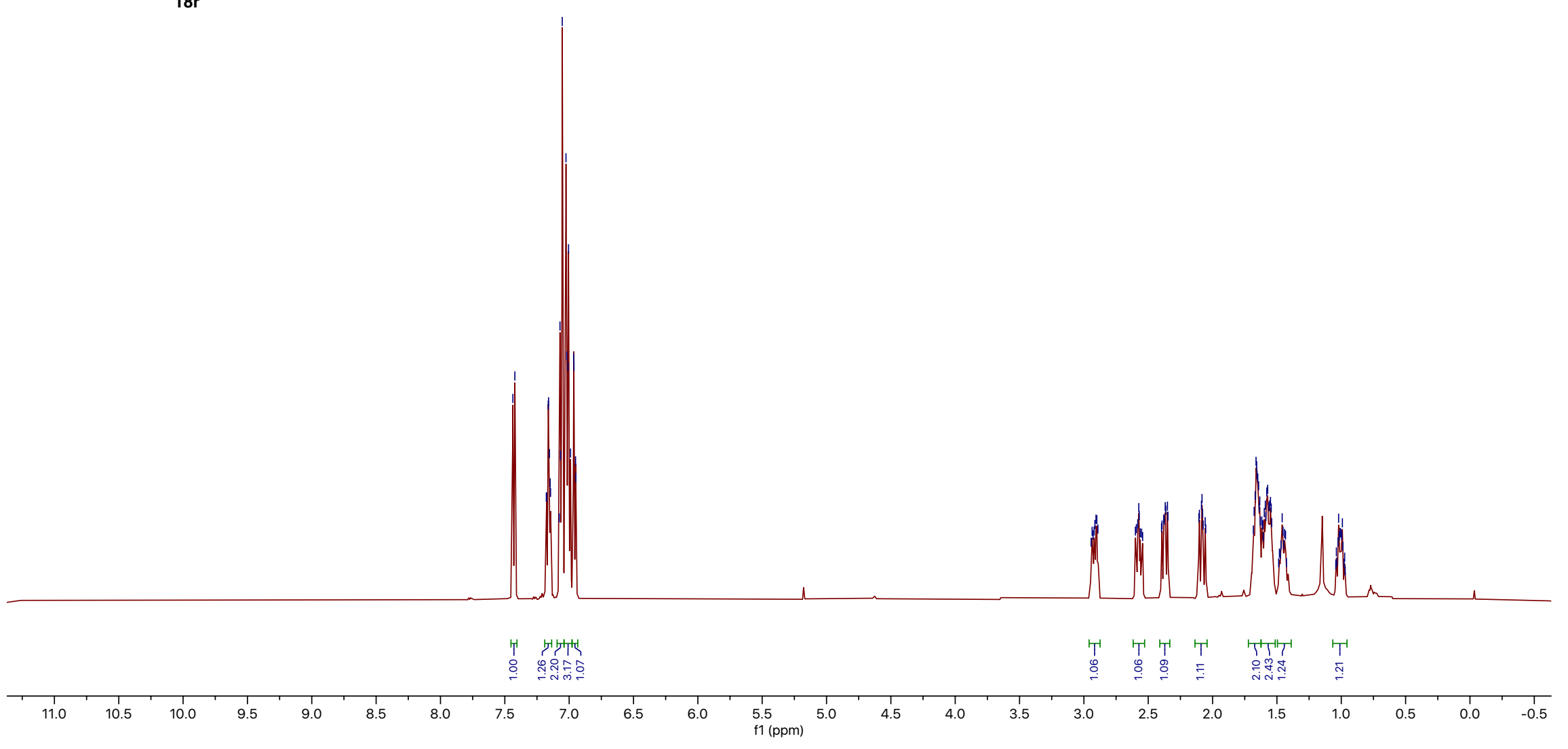


$125 \mathrm{MHz}, \mathrm{CDCl}_{3}$

(1)

$\overbrace{\substack{\infty \\ 0}}^{\substack{\infty \\ 0}}$

$\mathrm{F}_{3} \mathrm{CO}$

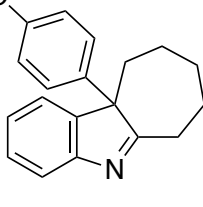

$18 r$

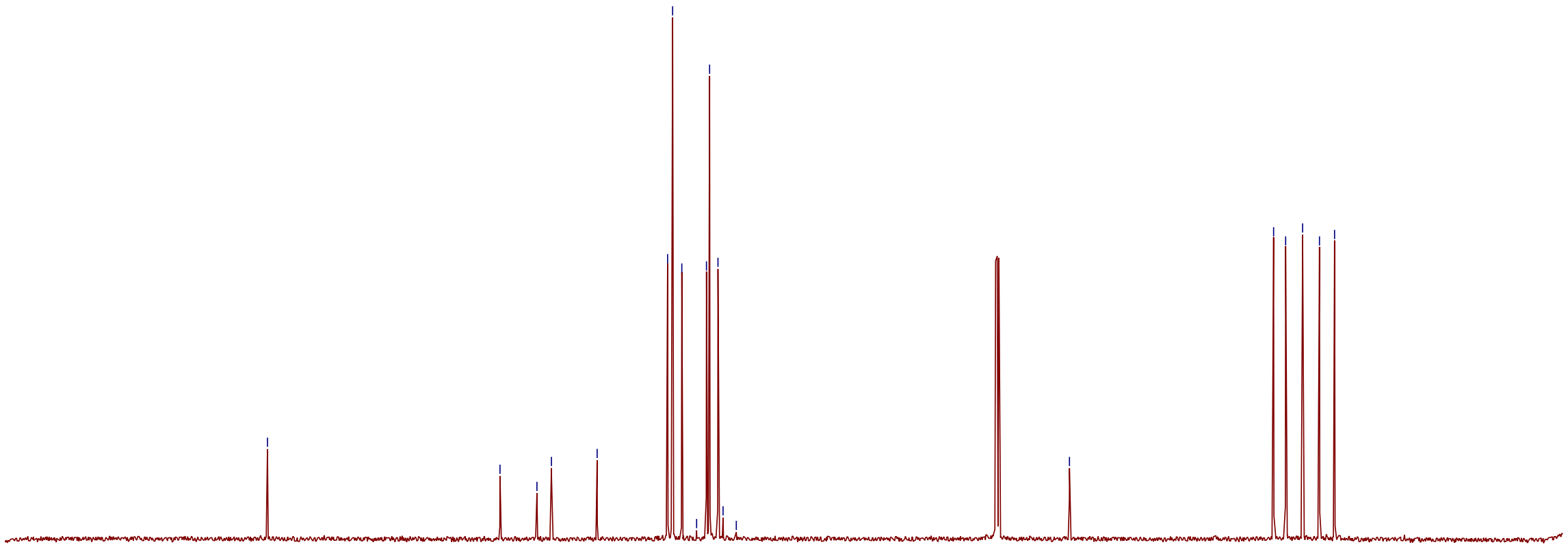

30
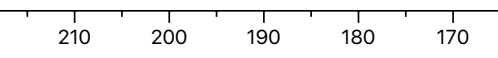

160
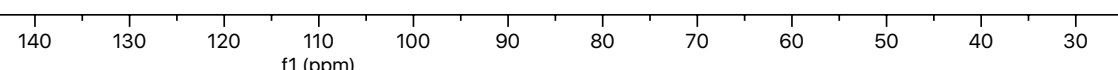
$500 \mathrm{MHz}, \mathrm{CDCl}_{3}$
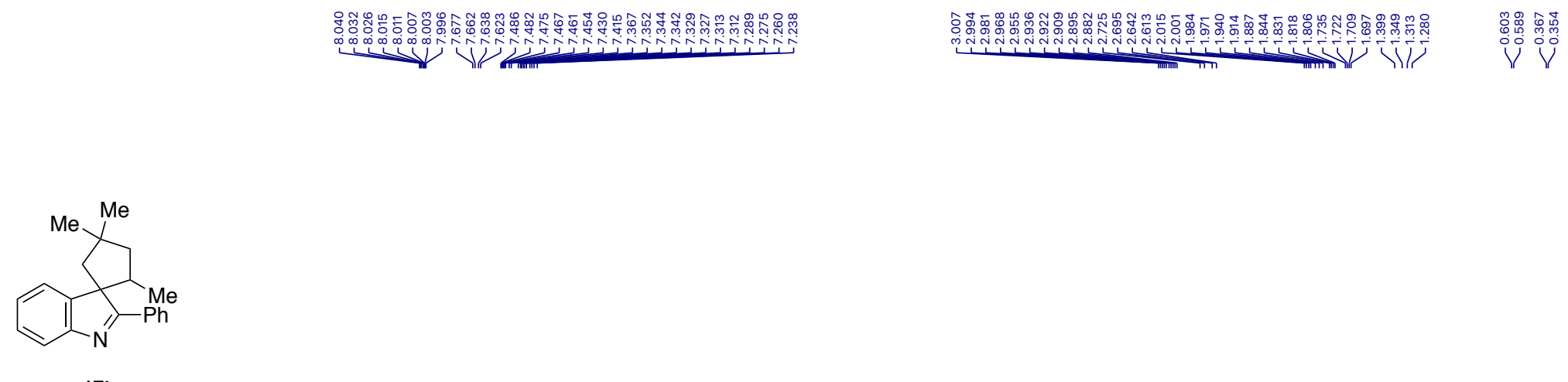

$17 \mathrm{t}$

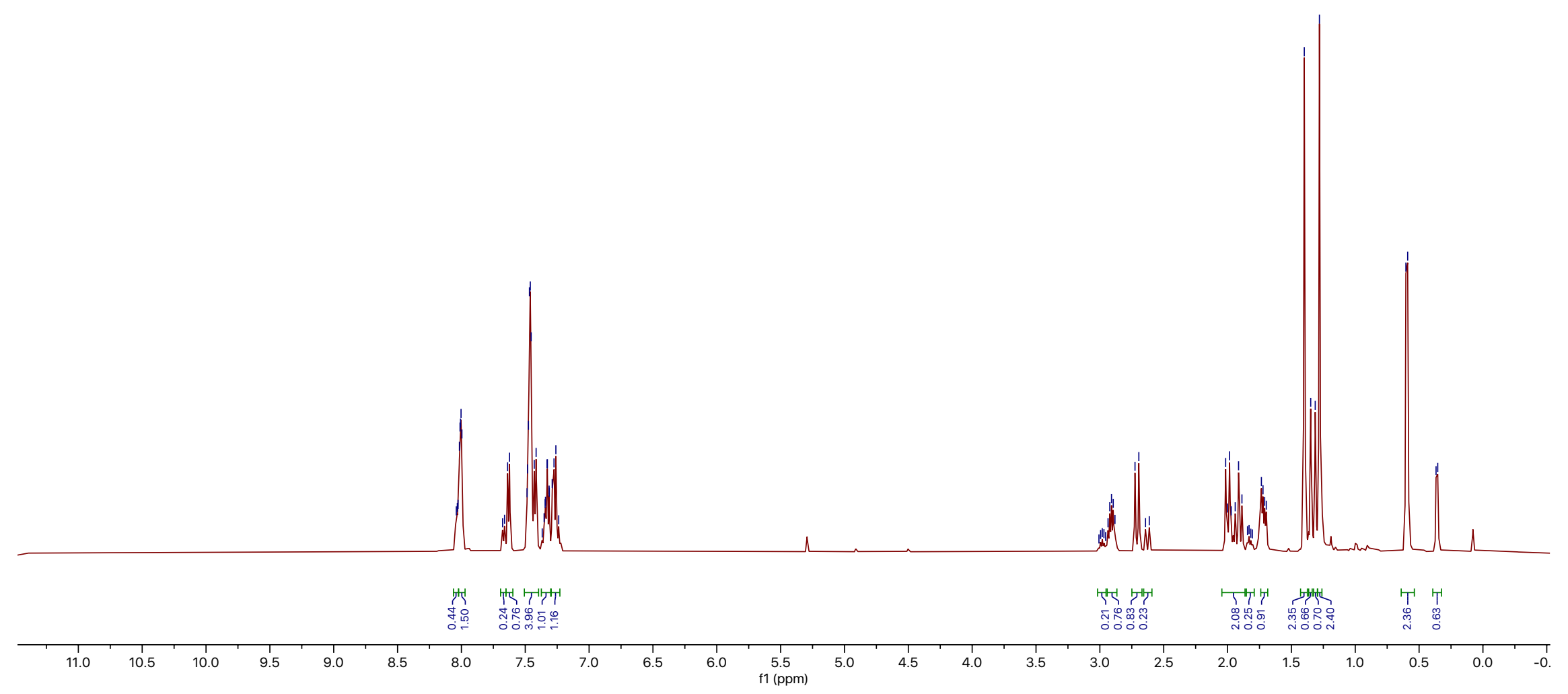


$125 \mathrm{MHz}, \mathrm{CDCl}_{3}$

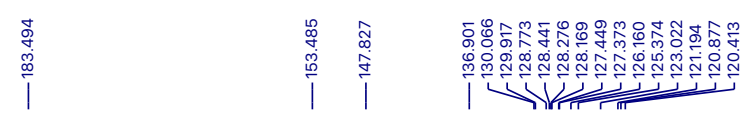

约

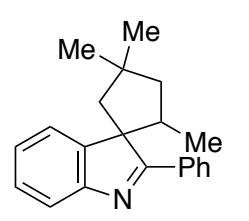

$17 t$

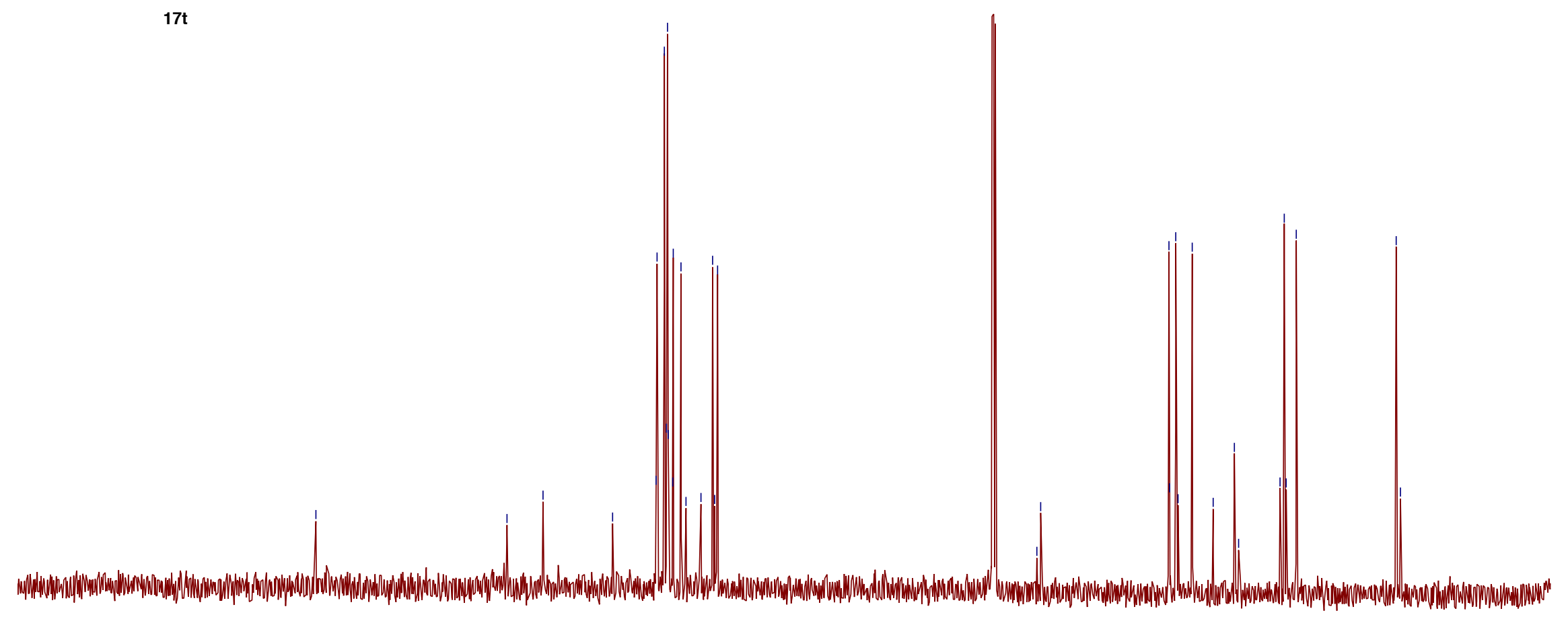

30

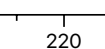

$210 \quad 200$

$\begin{array}{ll}190 & 180\end{array}$

$170 \quad 160$

150

$140 \quad 130$

120

$100 \quad 90$

$80+70 \quad 60$

$50+40$

30 
$500 \mathrm{MHz}, \mathrm{CDCl}_{3}$

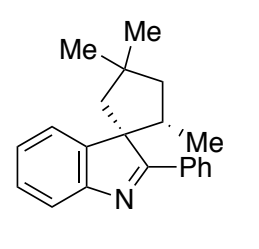

17t $d r=88: 12$

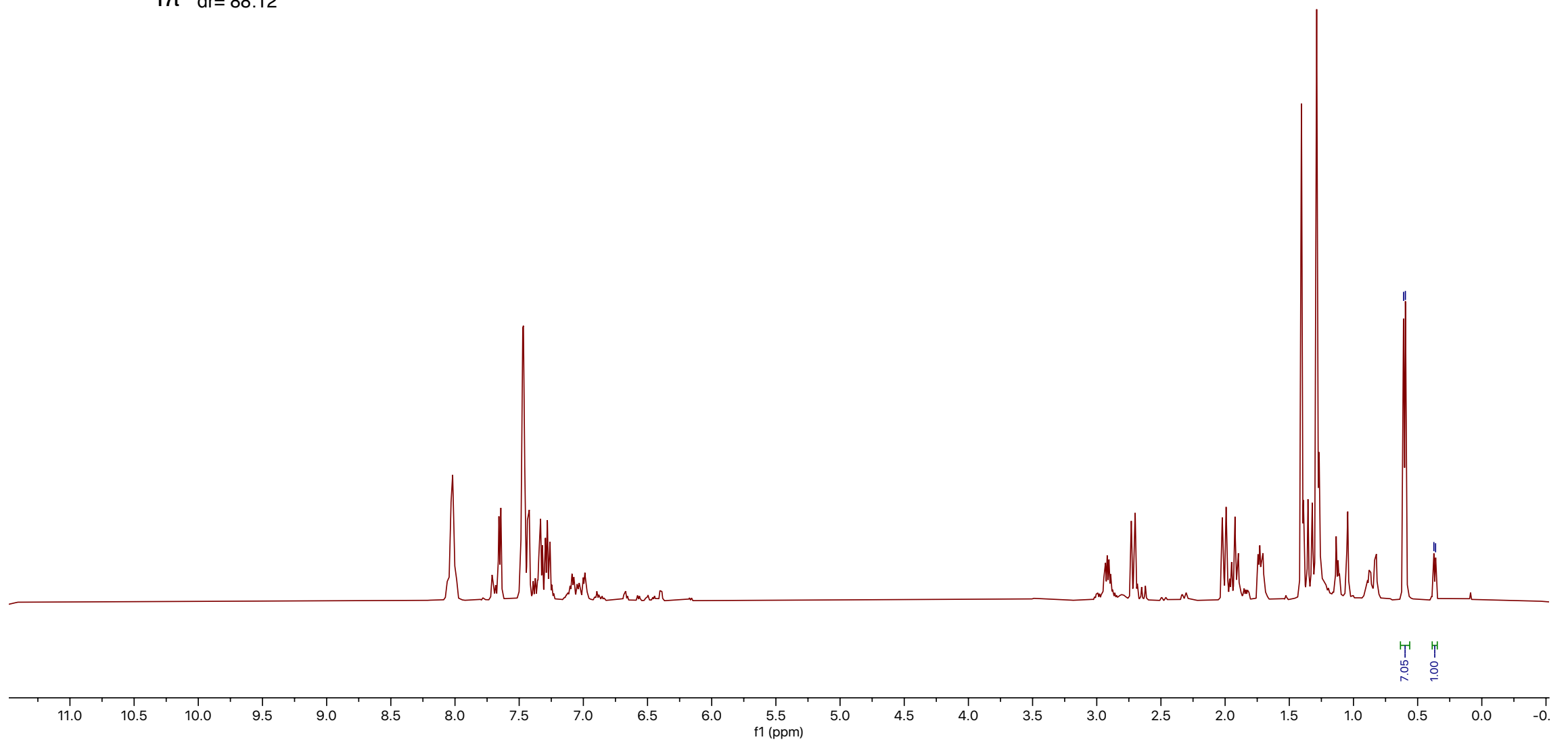

